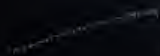



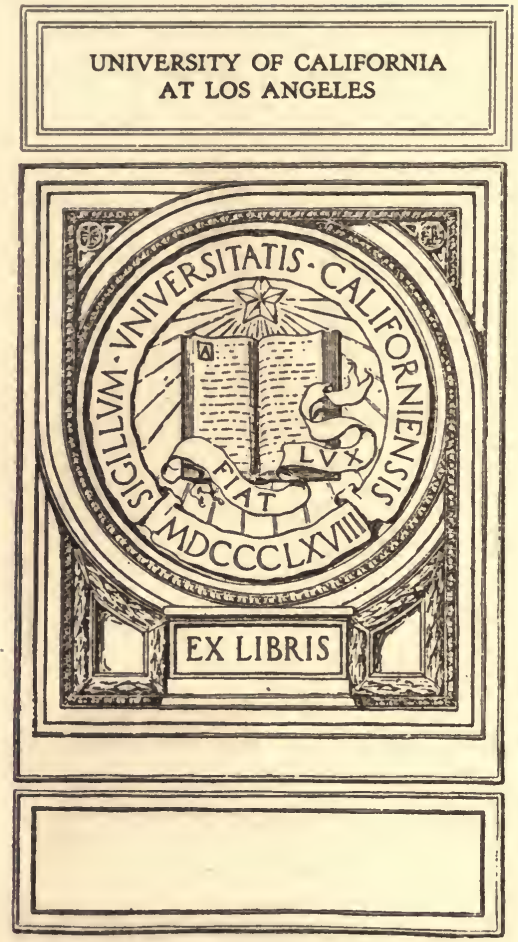


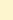


THE ANATOMY OF WOODY PLANTS 


\section{THE UNIVERSITY OF CHICAGO PRESS CHICAGO, ILLINOIS}

THE BAKER \& TAYLOR COMPANY NEW YORK

THE CAMBRIDGE UNIVERSITY PRESS LONDON

THE MARUZEN-KABUSHIKI-KAISHA TOKYO, OSAKA, KYOTO, FUKUOKA, SENDAI THE COMMERCIAL PRESS, LIMITED SHANGHAI 


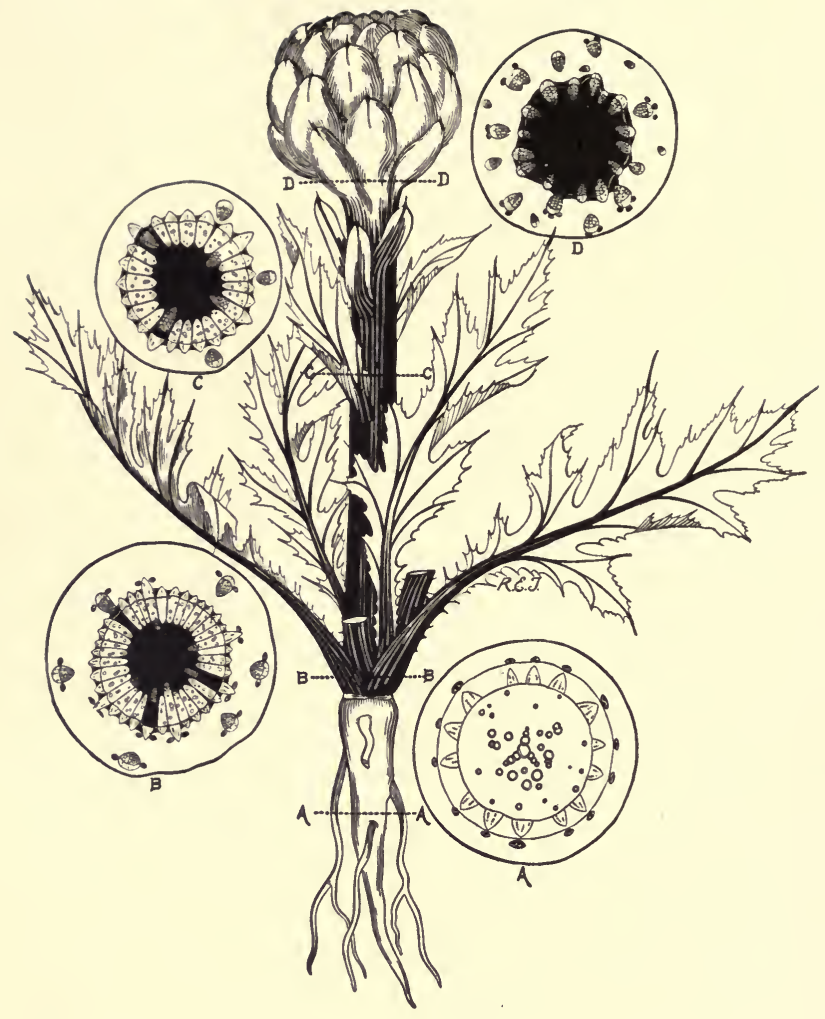

Diagrammatic figure of the French artichoke, Cynara Scolymus, showing distribution of oil canals in the various organs and regions. For explanation see chapter xxxi. 


\title{
THE ANATOMY OF
} WOODY PLANTS

\author{
By \\ EDWARD CHARLES JEFFREY
}

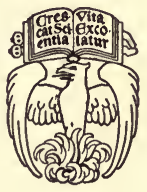

THE UNIVERSITY OF CHICAGO PRESS CHICAGO, ILLINOIS 
COPYRIGHT I 9 I 7 BY EDWARD CHARLES JEFFREY ALL RIGHTS RESERVED. PUBLISHED OCTOBER 1917

Fourth Impression December 1930

COMPOSED AND PRINTED BY THE UNIVERSITY OF CHICAGO PRESS CHICAGO, ILLINOIS, U.S.A.

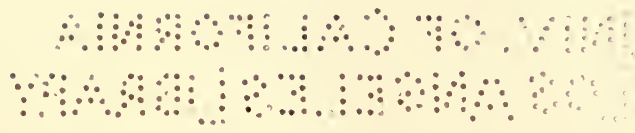




\section{5372}

\section{PREFACE}

It is now forty years since De Bary's classic Comparative Anatomy of the Vegetative Organs of the Phanerogams and Ferns made its appearance. In the interval much has been added to our knowledge, particularly in the paleobotanical and experimental fields. The doctrine of descent, too, has now reached a degree of prominence and importance which it did not possess in De Bary's time. As a consequence, it is desirable that the general subject of the anatomy of the woody or so-called vascular plants should be reviewed, with special reference to its historical and experimental aspects. This is perhaps all the more desirable as an effective counterpoise to the extreme mechanistic tendencies of the time. It will accordingly serve a useful purpose to indicate how large a part of the organization of existing plants is an inheritance from their ancestors of earlier geological times.

In De Bary's textbook both paleobotany and development are deliberately eschewed. The first of these is now essential for any adequate comprehension of comparative anatomy in its all-important evolutionary aspects. It is abundantly clear that the most fruitful results from the standpoint of the doctrine of descent are to be derived from the comparative study of extinct and existing plants belonging to the same orders, families, or genera. It is, moreover, obvious that the living forms cannot be interpreted without a knowledge of their past, and that to an even greater degree the organization of fossil plants is a closed book to those who are unfamiliar with the anatomy of allied and still living types. The wide range of facts which must of necessity be covered calls for a somewhat brief and even elementary treatment. Fortunately, since De Bary's time, it has become more and more evident that the study of the development of organs and tissues throws little trustworthy light on the processes of evolution, and consequently that aspect of our subject need receive no more attention than was vouchsafed to it by the great German anatomist nearly half a century ago. 
In the seventeenth chapter are summarized the important general principles derived from the investigation of related living and extinct organisms. The beginning of the studies leading to the formulation of these anatomical canons stands largely to the credit of French and English paleobotanists. Since they have worked mainly with Paleozoic types, their activities have been preponderantly in the direction of comparisons between the organization of the earlier cryptogams and gymnosperms and their still living survivors. It has been in some measure the good fortune of American anatomists to continue the lines of investigation thus begun and to extend them to the study of Mesozoic and still living gymnosperms. The extremely harmonious conclusions resulting from the anatomical comparison of both Paleozoic and Mesozoic forms with their surviving descendants have justified the extension of the same principles to the evolutionary investigation of other woody plants (particularly to the angiosperms), concerning the geological past of which we are still ignorant. The canons formulated in chapter xvii have as a consequence been regarded of such importance that any conclusions not in harmony with them have ordinarily not been considered in the following pages unless they have held the ground for many years or are at the present time advocated by anatomists of great eminence: On account of the large field covered in the present necessarily elementary work, this procedure has been regarded as essential, and it is hoped that, with the explanation offered, it will not appear to the reader too dogmatic.

The author has been fortunate in utilizing the services of his students in the preparation and illustration of this volume. $\mathrm{He}$ is particularly indebted to Mr. R. E. Torrey for his skilful and artistic execution of a large number of the figures. Help in this respect has also been supplied by Mr. R. C. Staebner and Mr. Charles Drechsler. Miss Ruth Cole has rendered invaluable aid in the onerous task of preparing the text and reading the proof. Miss Edith S. Whitaker has also assisted in the preparation of the index.

To Professor M. A. Chrysler, of the University of Maine, the author owes illustrations of secondary growth in monocotyledons, 
and to Miss Eloise Gerry, of the United States Forest Service, an admirable photograph elucidating the "bars of Sanio" in coniferous wood. Last but not least, the author records the valuable services of Mr. James Austin, assistant in the laboratories, in connection with the preparation of the numerous photographic illustrations. Dr. D. H. Scott and his publishers, Messrs. A. and C. Black, have very kindly permitted the reproduction of a number of figures from the admirable Studies in Fossil Botany, which are among the comparatively few illustrations in the present work which are not original. To Dr. R. T. Jackson the author is indebted for the opportunity of photographing for illustration a number of the sections of carboniferous plants under his care in the Botanical Museum.

Professor John M. Coulter has been good enough to read the proofs as the present volume passed through the press. To my friend Mr. R. W. Sayles, Director of the Geological Museum, I am indebted for valuable criticisms in the field of paleoclimatology and also for other essential aid. The author is alone responsible for the views expressed and for any errors.

Botanical Laboratories, Harvard University

June, I9I 7 



\section{CONTENTS}

CHAPTER

PAGE

I. The Cell . . . . . . . . . . . . . . I

II. The Tissue Systems . . . . . . . . . . . . 8

III. The Fibrovascular Tissues: Wood-General . . 14

IV. The Fibrovascular Tissues: Secondary Wood-TraCHEIDS AND FIBERS . . . . . . . . . . . . 24

V. The Fibrovascular Tissues: Secondary Wood-ParenCHYMA . . . . . . . . . . . 37

VI. The Fibrovascular Tissues: Secondary Wood-Rays . 6i

VII. The Fibrovascular Tissues: Secondary Wood-Vessels 92

ViII. The Fibrovascular Tissues: Phloem . . . . . . 107

IX. The EpIDERMIS * . . . . . . . . . . . . I26

X. The Fundamental Tissues . . . . . . . . . . 132

XI. Definttions of the Organs . . . . . . . . . 136

XII. THE ROOT . . . . . . . . . . . . . . . 143

XIII. The Stem . . . . . . . . . . . . . . . $\mathrm{I}_{2}$

XIV. The Leaf . . . . . . . . . . . . . . 199

XV. The Microsporangium . . . . . . . . . . . 214

XVI. The Megasporangium and Seed . . . . . . . 223

XVII. The Canons of Comparative Anatomy . . . . . . 234

XVIII. The Lycopsida And Pteropsida * . . . . . . . 244

XIX. The LycopodialfS . . . . . . . . . . 252

XX. The Equisetales (Including Sphenophyllales) . . . 264

XXI. The Filicales . . . . . . . . . . . . . . 277

XXII. The Archigymospermae: Cycadofilicales and CycaDALES . . . . . . . . . . . . . . 292

XXIII. The Archigymospermae: Cordaitales and Ginkgoales 305

XXIV. The Metagymnospermae: Coniferales . . . . 317

XXV. The Metagymospermae: Gnetales . . . . . . . 357

XXVI. The Angrosperms . . . . . . . . . . . . 373 
CHAPTER

XXVII. The Woody Dicotyledons . • . . . . . . . 379

XXVIII. The Herbaceous Dicotyledons . . . . . . . . 387

XXIX. The Monocotyledons . . . . . . . . . . . 409

XXX. Anatomcal Structure and Climatic Evolution . . . 4I 7

XXXI. Evolutionary Principles Exhibited by the Compositae 433

XXXII. Anatomcal Technique . . . . . . . . . . . 444

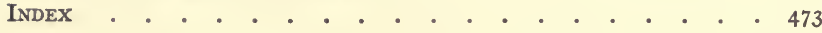




\section{CHAPTER I}

\section{THE CELL}

It has been recognized since the seventeenth century that living beings, particularly plants, are composed of cells. The English investigators Hooke and Grew in the latter half of that century noted the fact that the body of vegetable organisms was often constituted of minute chambers, and to these Hooke first gave the name of cells. Grew was apparently the originator of the term "tissue," and he compared the organization of plants with the woven texture of lace. A clear conception of the cellular structure of living beings was not, however, reached until nearly two centuries later. Plants differ from animals in the fact that their gross internal organization is of relatively slight scientific importance compared with the more obvious bony and muscular structures of the animal body. The anatomy of plants is thus essentially a matter for microscopic investigation. Some knowledge of the general features of the structure of the cell in plants is accordingly necessary as a preliminary to the more detailed pursuit of anatomy.

The cell in vascular plants has certain features which bring it into sharp contrast with the corresponding unit of structure in the higher animals. The essential substance of all living cells is protoplasm, simply distinguished from inanimate matter by the possession of a capacity for change and reproduction, which does not characterize matter devoid of life. In the higher plants the protoplasm does not show the large degree of solidity which is a feature of the animal cell, but is ordinarily reduced to a thin vesicle surrounding a larger or smaller central cavity known as the vacuole. The bladder-like protoplasmic vesicle is rendered possible in plants by a containing, supporting, and likewise more resistant envelope called the cell wall. This wall is not nitrogenous in its chemical composition, as is true of the living protoplasmic body, but is, primitively at least, a ternary compound, containing carbon, oxygen, and hydrogen, the first-named element being the most abundant. 
Fig. I will make clear the organization of cells in an immature seed pod or pericarp of an iris. Each element obviously consists of a large central cavity as noted above, variously known as hydroplastid, hydroleucite, tonoplast, and vacuole. The last designation seems best for descriptive purposes. Surrounding the median space, which in life contains water with various substances in

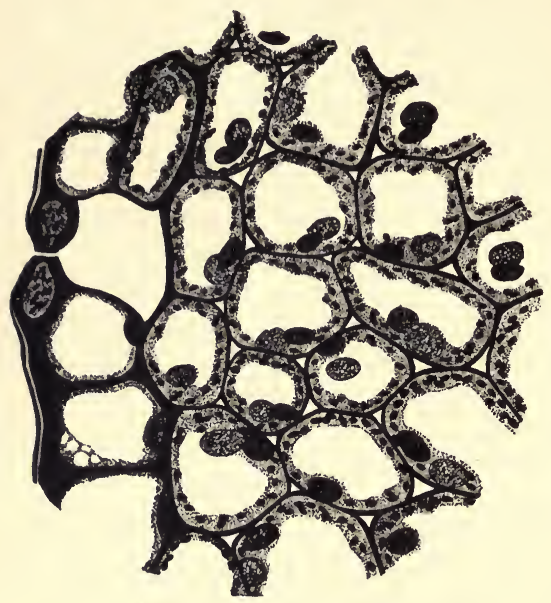

Fic. 1.-Transverse section of young pericarp of Iris species, showing organization of cells of epidermal and fundamental tissues. solution, is the protoplasmic utricle. The nature of the latter varies in different cells. In the elements to the left side of the illustration the protoplasm appears as a somewhat minutely granular substance, while in the cells to the right the structural organization of the protoplasm is marked by the presence of oval bodies, the chloroplastids, which in life color are green. A dark body of larger size is to be seen somewhere against the cell wall and imbedded in the protoplasm. This is the nucleus, one of the most important organs of the cell, which appears to preside over all its changes and activities. Another body, generally larger and lighter than the nucleus, is frequently present in the cells under discussion-the oilplastid or eleoplast. This is conspicuously spongy in its structure. We may now turn with advantage to the organization of the cell wall. On the left side of the illustration the common outer wall is limited by a distinct membrane, the cuticle, which is chemically different from the rest of the wall substance and is viable to 
gases but not to fluids. Below the cuticle lies the cell wall proper, in this case composed of cellulose. The cuticle and its underlying wall are continuous except in the region of certain apertures, the stomata, one of which is represented in the figure. From the stoma there passes inward an air space, which quickly divides into fine canals lying in the angles between the internal cells. These are intercellular spaces and are a practically unfailing accompaniment of the living elements in vascular plants.

The next illustration (Fig. 2) visualizes the conditions in a harder tissue, namely, one of the wood rays of a dicotyledon. Here the mass of cells present individually somewhat rounded contours, and in the resulting angular interstices appear the intercellular spaces. The protoplasm in the cells under consideration is much denser than in the elements of the wall of the ovary figured above, and accordingly the nucleus lies near the geometrical center of the cell, as is commonly the case in highly protoplasmic elements, in contrast to its

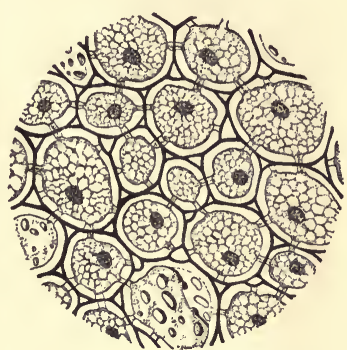

FIG. 2.-Tangential section of cells of a ray in a dicotyledon (Drimys species), showing pitting in relation to cells and intercellular spaces.

peripheral position in those in which the protoplasm is only a bounding utricle. The cell wall in the present instance is, relatively to the size of the cells, much thicker than in the first figure. As a consequence of this increased thickness of the wall, special devices are necessary for the purpose of permitting interchanges between the cellular elements and their environment. The wall is thin in certain definite regions for the attainment of this end, and these locally thin spots are known as pits. It is clear that the pit of one cell always coincides with a corresponding pit in a neighboring element. Further, it is obvious that, although the cells are in close relation to one another by means of their pits, these are prevented from becoming actual holes by the persistence of the middle lamella or cement substance as the membrane of the pits. Not all the pits, however, meet a corresponding depression. It 
will be observed that there are well-developed pits toward the intercellular aërating spaces. These are known as air pits and have no counterpart on the side of the air space. They are commonly found wherever the thickening of the cell wall makes difficult the interchanges between the living protoplasm and the outside air. In the figure under consideration the cells are united by the cement substance of the middle lamella, which appears dark and contains

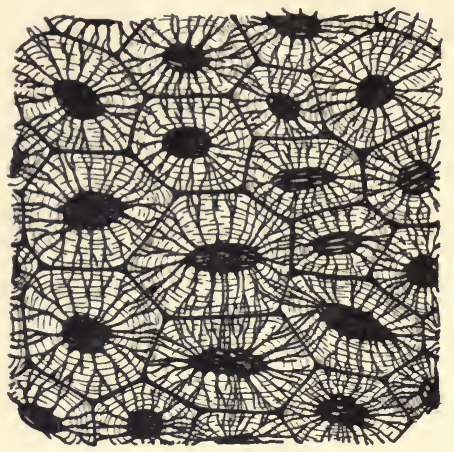

Fig. 3.- Section of inner pericarp ("stone") of a peach, showing cells with extremely thick and layered walls as well as numerous elongated pits. the air spaces within its substance. Most of the cells have had both the top and the bottom walls removed by the plane of section, but in one or two the cell wall meets the eye. Here the pits are seen in face view, and it becomes clear that they vary somewhat in size and are characteristically outlined by a single contour. On account of this condition, which results from the fact that the bounding walls do not overhang, pits of this type are called simple pits. Communicating pores of this kind are found in cells which in their functional condition contain living protoplasm.

Let us now turn to the examination of cells in which the wall is so greatly thickened that the space containing the protoplasmic body becomes much reduced in size. Fig. 3 reproduces some of the elements of a peach stone which has been softened by a prolonged sojourn in hydrofluoric acid. The protoplasmic structures have been largely consumed in the thickening of the cell wall. The latter shows well-marked indications of layering, a feature often present when much thickening has taken place. The layering doubtless has some numerical relation to the age of the wall in days. The pits are of special interest in this instance. Their correspondence in adjacent cells is as marked as in the former 
illustration. In many cases two or more near-lying pits of the same cellular element become confluent as they pass inward, and open into the cavity of the cell by a common aperture. The cement

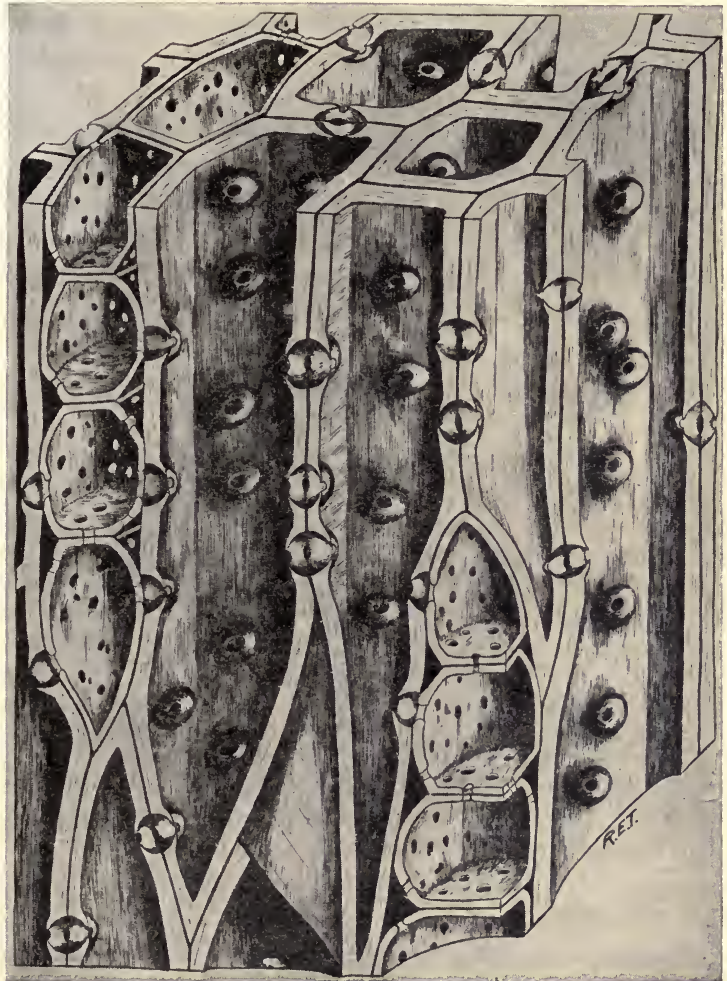

Fig. 4.-Wood of the spruce (Picea canadensis), showing ray cells with simple pits and longitudinal water-conducting elements with bordered pits.

substance is strongly developed here and the thickened walls of the cells are lignified. The pits, in spite of the complications noted above, are to be regarded as simple pits. 
The examples of cells hitherto considered have been those which in their functional condition contain living protoplasm. It is now necessary to refer to those very important histological elements of vascular plants, which in the mature condition normally contain no protoplasm and serve to conduct water. Elements of this nature are typically much elongated and are provided with a different sort of intercommunicating pitting than that found in the case of those which in the active state shelter a protoplasmic body. In the accompanying figure (Fig. 4) are seen a number of cells of this sort cemented together by the middle lamella (repre-

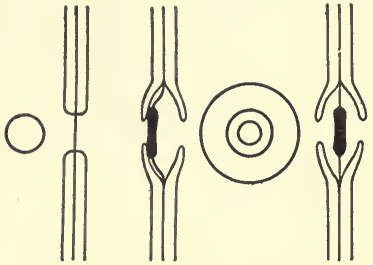

FIG. 5.-Diagram of simple and bordered pits in face and profile views. In the center a bordered pit from heartwood is shown.

sented as a black framework around the cell walls). The pits which bring about intercommunication are figured as occurring on two of the four nearly parallel sides of the tracheids or water-conducting cells. Their openings have overhanging margins, and the membrane, the presence of which precludes the possibility of actual openings, is much thickened in the middle to constitute the so-called torus. The presence of overhanging margins clearly distinguishes this type of pore from that found in the walls of living cells. The cement substance uniting the cells with one another is in general lignified (that is, has undergone that somewhat complex and obscure modification chemically known as lignification) like the cell walls which it holds together, but is likewise partially in the pectic or mucilaginous condition, a state which causes it to absorb hematoxylin strongly. In the region of the pit membranes the middle lamella becomes pectic cellulose, and here water passes through the walls much more readily than it does elsewhere. On either side of the figure is seen a ray of the wood, the cells of which are in relation with the tracheids by means of pits. It is clear that the pits which bring about intercommunication are bordered on the side of the elongated element of the wood (the tracheid) and are 
simple on the side of the medullary-ray cells. Pits of this nature are called half-bordered.

In Fig. 5 simple and bordered pits are represented diagrammatically side by side and from both profile and face view. Obviously the simple pits in face view are single in contour, while those which are bordered have a triple concentric outline, the outermost circle corresponding to the boundary of the broad membrane of the pit, the innermost to the narrow mouth, and the intermediate representing the outline of the torus. The distinction between simple and bordered pits is an extremely important one, particularly in the lower groups of vascular plants. In higher forms the distinction is of less value, but the presence of bordered pores in vascular elements in general still indicates that an important function of the element so provided is the transport of water. It should be further noted that elements with bordered pits are usually without intercellular spaces, while those in which the pits are of the simple type normally possess such aërating cavities. Exceptions to this statement are found ordinarily only in plants which have become highly specialized in connection with resistance to drought. 


\section{CHAPTER II}

\section{THE TISSUE SYSTEMS}

The cells in higher plants are generally grouped into well-marked systems of elements which are known as the tissues. Tissues are sometimes defined as aggregations of cells performing a similar function. This definition is, however, open to some objections, as is also that which describes a tissue as a mass of cells of similar origin. Characterization of tissues, either by their functions or by their mode of origin, seems less desirable than a definition which makes clear that the aggregations of cells have a common organization. From the standpoint of evolution it is the structural features of the tissues which are of the greatest significance. In works dealing with so-called physiological plant anatomy the functions of the tissues rather than their peculiarities of structure are naturally most emphasized. From the point of view of the doctrine of descent functional features are of less significance, since it is precisely these which are the most readily modified and as a consequence furnish the least valuable indications of the course of evolutionary development in any given large group. Because the present work deals with anatomy from the outlook of evolution, structural organization in the case of the tissues stands in the foreground, although, of course, the question of the functioning of the various tissue systems cannot be left out of view.

It is an interesting general fact that the boundaries between the tissue systems are much more marked in the plants which are geologically older and lower in the scale of evolution than they are in the higher seed plants, the conifers, and the angiosperms. Further, the more conservative organs of the higher forms-namely, the root and leaf-exemplify a sharper delimitation of the tissue systems than does that most progressive of all plant parts, the stem.

For the purpose of the present work the tissues of plants may be divided into three distinct systems, which can be most easily identified by reference to the accompanying figure (Fig. 6) of a 
transverse section of the creeping stem of Pteris aquilina. Throughout the area of the figure are scattered oval masses, the fibrovascular bundles. These are very sharply marked off from the rest of the tissues by a boundary appearing as a dark circumscribing line. On the outside of the stem is the integumentary system, or

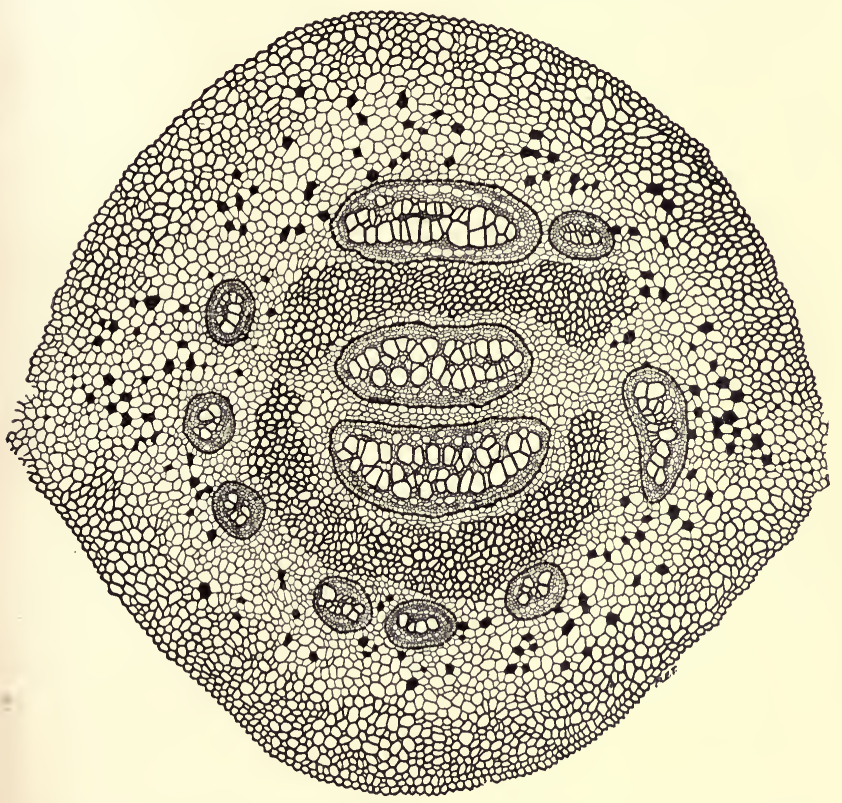

Fig. 6.-Transverse section of the rootstock of Pteris aquilina, showing three categories of tissue - namely, epidermal (external), vascular, and fundamental.

epidermal tissue, consisting of a single layer of cells. The epidermis is characteristically uniseriate in the lower forms, and only in some of the higher vascular plants does it become a multiple layer. The remaining structures of the stem of the bracken fern belong to the fundamental system. The next illustration (Fig. 7) shows a part of the stem of Pteris more highly magnified, so that the details of 
structure may be more easily discerned. External is the single layer of the epidermis, made up of cells with thick, heavily pitted walls. With the greater magnification employed, the cellular organization becomes very obvious. Underneath the epidermal layer is situated the fundamental system, in turn clearly outlined against the fibrovascular tissues by a striking boundary composed of a single series of cells with dark tanniniferous contents. This is the most internal layer of the fundamental system and is known either as the endodermis or as the phloeoterma (the latter term in its etymology indicating the inner layer of the cortex or fundamental tissue). The fundamental tissues are characterized locally by

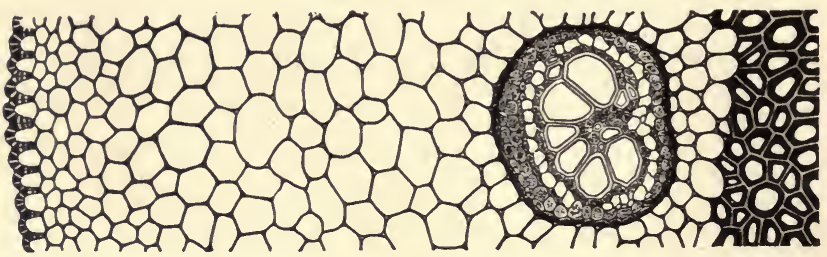

FIG. 7.-A portion of a transverse section of the rootstock of Pteris aquilina more highly magnified, showing the three tissue systems.

certain bands of dark-brown skeletal tissue, which are very characteristic of the lower vascular plants and subserve to a large extent the mechanical function which in the higher plants is attended to by the fibrovascular system. Those regions of the fundamental system which are not mechanical in their nature take over the function of storage and are crowded with granules of starch forming a cordon around the periphery of the cells. Centrally the elements of storage show the presence of a dark-brown substance in the region of the vacuole. This is tannin-like in its nature and is commonly present in the fundamental system of ferns. The fibrovascular structures stand out sharply from the rest of the tissues and are obviously much more complicated in their organization than are those previously considered. The fibrovascular aggregations of cells are of the greatest anatomical importance, both because their very complexity of structure supplies many valuable 
features for comparison which may be utilized in the study of evolution, and because their conservatism makes them on the whole the least variable of the elements entering into the composition of the higher plants. Likewise, by reason of their resistance to decay, they are more likely to be preserved as fossils. Beyond emphasizing the complexity of the fibrovascular system, it need not be further considered at the present time.

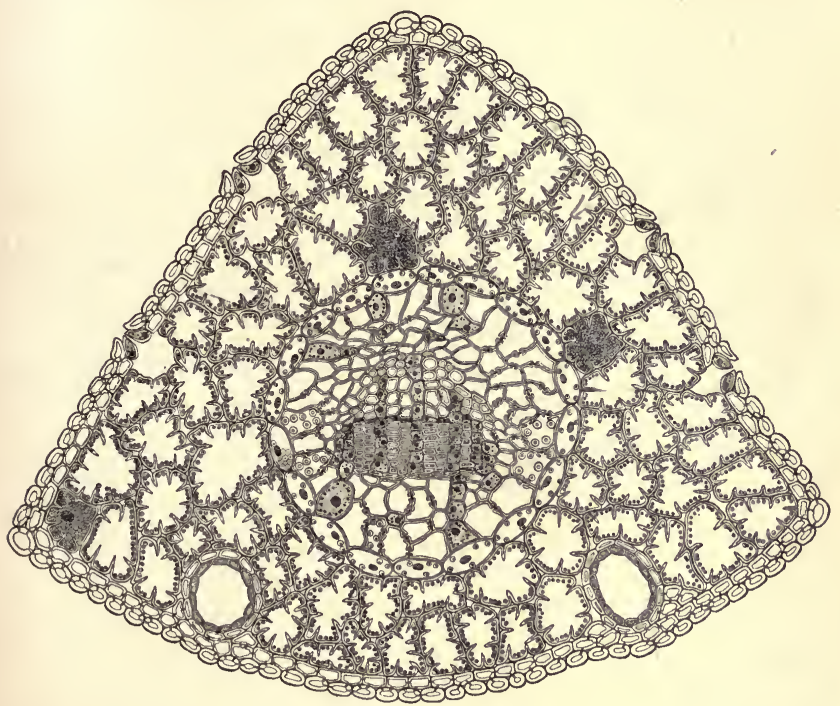

FIG. 8.-Transverse section of the leaf of the white pine (Pinus Strobus), showing the three tissue systems in a leaf.

We may next consider the tissue arrangements in another of the plant organs, namely, the leaf. In the figure (Fig. 8) is represented the transverse section of the needle of Pinus strobus. Externally the epidermis forms a boundary of a single row of cells, continuous except where interrupted by the occurrence of stomatic openings. Beneath the epidermis lies a layer which is ordinarily known as the hypoderma and is more strongly developed in fossil 
than in living pines. The fibrovascular strand is sharply bounded in the median region by the endodermis, a layer circular in configuration and well developed in Pinus, though often absent in the higher conifers. The organization of the fibrovascular strand need not particularly occupy our attention at this stage, as it will be considered in detail more appropriately in the sequel. It is enough to note that its upper or woody part is composed of empty cells often showing bordered pits-in other words, of tracheids. These

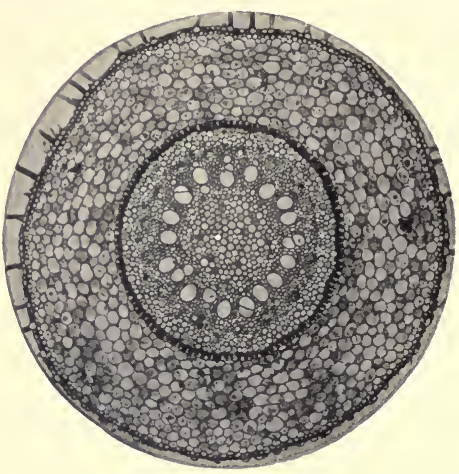

Fig. 9.-Transverse section of the root of the sarsaparilla (Smilax), showing the three systems of tissues in root organs.

side of the leaf, conspicuous secretory spaces, the resin canals. In the root of the pine, as in the leaf, the same sharp distinction between the fibrovascular structures and the fundamental tissues is present. In the stem of the conifers generally, however, the limit between the tissues belonging to the central conducting cylinder (the fibrovascular system) has become obsolete and can be judged to have been formerly present only on theoretical grounds.

In the case of the root of vascular plants in general, from the lowest to the highest, the limit between conducting or fibrovascular tissues and the fundamental system is usually very distinct and is one of the features which so clearly and universally mark the root as the most conservative of the organs of plants. Fig. 9 illustrates 
the situation in this respect for the monocotyledons, which may on strong grounds be considered as the highest of the seed plants. Externally is the piliferous layer, from which the root hairs are derived, and which may be considered in a general way as the equivalent of the epidermis of the stem and the leaf. The central region is occupied by the fibrovascular system, sharply limited by the endodermis, composed of cells ordinarily thick-walled. Between the endodermal limiting membrane and the piliferous layer lies the cortex of the root, and this corresponds to the fundamental category of tissues in the case of the stem and leaf.

It will be clear from the account given above that there are three tissue systems in plants which are very. distinct in lower forms and in the less changeable and more conservative parts. Of these the epidermal tissues are always clearly limited both toward the outside and also in relation to the tissue system which lies inside. The boundaries dividing the fibrovascular from the fundamental tissues are often less plainly indicated, and in the case of the higher groups of plants, particularly those in which the secondary growth is strongly developed, may disappear altogether; in such cases the limits of the tissues can only be inferred from comparative and developmental anatomy. 


\section{CHAPTER III}

\section{THE FIBROVASCULAR TISSUES: WOOD-GENERAL}

Since the fibrovascular tissues are on the whole the most important in the organization of the higher plants both from the evolutionary and from the physiological standpoint, it will be well to begin with their anatomy. The most conspicuous and bestdeveloped portion of the fibrovascular system in land plants is the wood. The aggregation of elements assembled under this heading affords also the best exemplification of the process of evolution in the higher plants as the result of the progressive development of the principle of division of labor and the gradual adaptations of plants to the more complicated conditions of life obtaining in later geological times. The resistance of the wood to the organisms of decay is greater than that of any other common plant tissue except those possessing cutinized or suberized cell walls. We have, consequently, in the woody structures past and present an almost perfect biological document, carrying back the history of plants in relation to their changing conditions of environment into remote epochs of our earth's history.

In beginning the discussion of the organization of wood it will be well to direct our attention in the first instance to the contrasts in structure presented by woods of ancient and modern types. The first illustration (Fig. Io) shows us the situation in the oak. The wood is conspicuously marked into areas by boundaries running at right angles. Crossing the figure from top to bottom are rows of large openings which represent the vessels or water-conducting tubes of our modern forest trees. It is clear that these are large only along the lines which mark the beginning of each year's growth. Farther out the vessels become suddenly of much smaller caliber. Not only is the ligneous structure of the oak transversely banded by reason of the strikingly larger size of the vessels which signalize the spring development, but also by an equally significant change in the diameter and the distribution of other more or less highly 
differentiated elements, to be described in a later chapter. At right angles to the annual zones of growth and crossing these are the large wood rays. These are extremely conspicuous structures, and as a result of a variation in the rate of growth due to their presence they bring about very evident depressions on the faces of the annual rings. The intervals between the large wood rays are occupied by more numerous linear storage bands, which are but a single row of cells in thickness and are known as uniseriate rays.

A marked contrast to the wood of the oak is presented. by the ligneous organization of the Paleozoic gymnosperm Cordaites, illustrated in Fig. II. Here the annual rings, so clearly present in the oak as a result of a differentiation in the size and

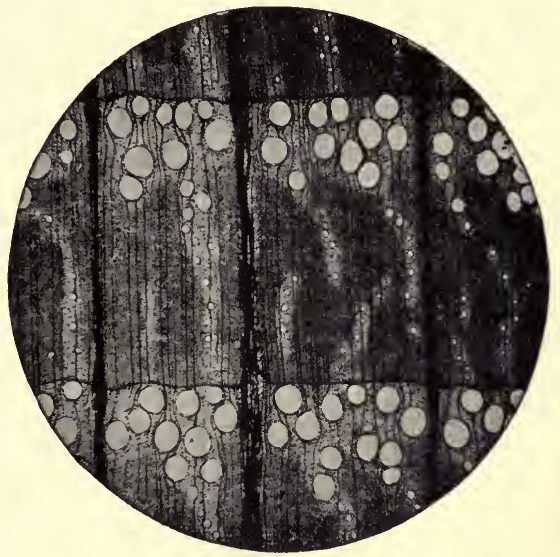

Fig. I0.-Transverse section of the wood of the red oak (Quercus rubra), showing annual rings and highly differentiated structure which characterizes the organization of the woody cylinder in modern trees.

character of the elements corresponding to regularly recurring annual changes, are conspicuous by their absence. This situation is directly correlated with the more equable annual cycle of remote geological times. We find illustrated in the case of Cordaites absolutely no indication of seasonal changes in temperature or variations in other important conditions of a periodic or seasonal nature. Not only is the wood monotonously the same as one passes from the inner regions to the exterior layers, but it likewise shows slight differentiation in the direction from left to right. Large rays of the oak type are quite absent, and the radial storage strands are entirely linear in their nature. 
Having noted the varieties involved in wood structure in correlation to the more variable conditions of environment present in modern times, we may now with advantage direct our attention to other features of organization which characterize the evolution of the woody cylinder in the higher plants. Fig. I 2 illustrates the transverse section of the wood of a lepidodendrid, an ancient

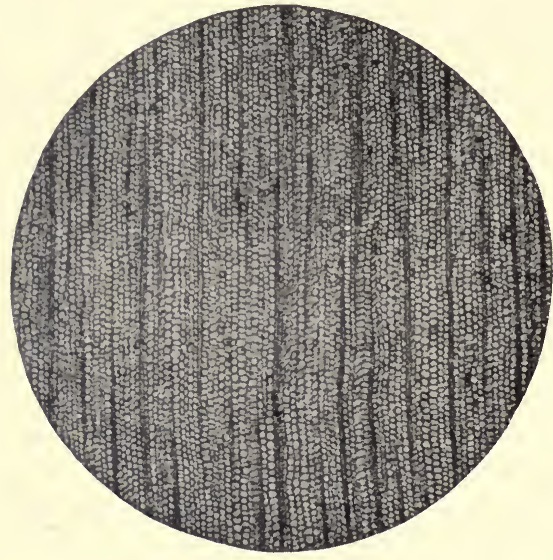

Fig. Ir.-Wood of a Paleozoic gymnosperm from Prince Edward Island, Canada, showing absence of annual rings and extremely simple organization. tree of the Paleozoic age. It is clear in this case that the wood has a circular outline corresponding to that of the stem as a whole. Although there is no indication of the existence of annual increments of growth, the wood is obviously divided into a central mass, in which the cells are irregularly disposed, surrounded by a zone regularly seriate and marked by the presence of wood rays. The portion of the wood which shows no linear disposition of its elements is known as the primary wood. This region of the wood is sometimes designated the "cryptogamic" or "old" wood, because it is particularly characteristic of the organization of vascular cryptogams and of the older groups of plants generally. As will be made clear later, the structure and mode of development of wood of this category is of considerable importance from the evolutionary point of view. The zone of secondary wood, outside the primary or cryptogamic ligneous core, is conspicuous by reason of its regular radial seriation and the presence of storage bands 
called wood rays. The secondary wood need not further occupy us in the present chapter.

Turning our attention now to the longitudinal organization of the primary wood, we find it characterized by the presence of certain elements appearing in a somewhat regular sequence. The general situation is represented in Fig. I3. On the left of the diagrammatic illustration lies an elongated thin-walled cell marked by the presence of spiral strengthening bands. In its present state this element is devoid of protoplasmic substance, but in an earlier phase, as indicated in the next figure (Fig. I4), living matter was present and specially aggregated in the regions of the thickened spiral bands. To the right lies a second element in which the strengthening horizontal ridges, which reinforce from the inside the generally thin walls of the water-conducting cell, are nearer to one another and

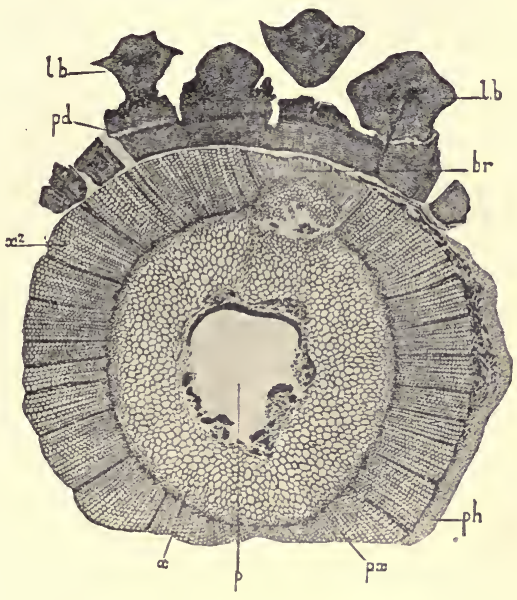

FIG. I 2.-Transverse section of a stem of a lepidodendrid trunk from the Paleozoic (after Scott), showing the strong distinction between secondary (radially seriate) and primary (unseriate) xylem characteristic of ancient forms.

in some instances are more or less united. By accentuation of the condition of approximation, fusion between the bands results and we have as a consequence the presence of the scalariform or reticulate tracheid. In general, among the Pteridophyta this is the extreme stage of evolution of the tracheary cells of the primary wood, but occasionally among the more complicated and extinct vascular cryptogams, and characteristically in all seed plants, the final state of the primary wood is characterized by the presence of 
the pitted element, marking a distinct advance on the scalariform and reticulate tracheids of the primary ligneous organization of the ferns and their allies. In the case of the primary wood those elements which have in their walls thickenings of the nature of rings and spirals are ordinarily designated the protoxylem. This

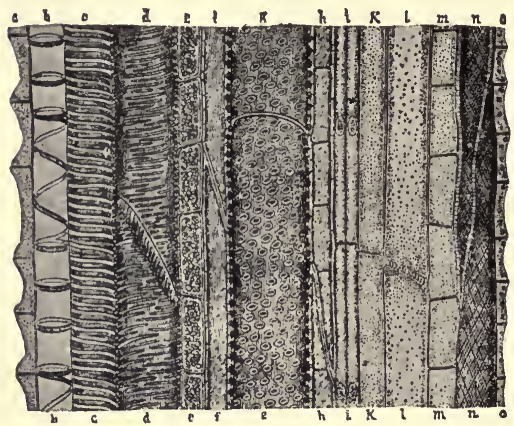

Fig. 13.-Diagrammatic longitudinal section of the fibrovascular tissues of a dicotyledon (after Sachs), showing organization of primary wood.

characterization of the first-formed portion of the primary wood is not always justified by the structures present, because in slowly growing organs and in subterranean parts even of rapid development typical ringed and spiral elements may be nearly or quite absent. Usually, however, the protoxylem as defined is the ligneous structure present when the organ is undergoing rapid elongation and by its constitution permits of accommodation by stretching to correspond with the increase in length. Its elements as a consequence are frequently drawn out and almost obliterated. The cells of the primary wood which are thickened in the reticulate, scalariform, or pitted manner are formed after elongation has ceased, since by their organization they are incapable of increasing their
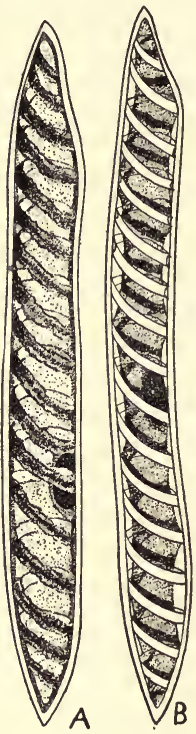

FIG. I4.-Diagrammatic views of a young element of the primary wood. In $A$ the normal condition is shown, while in $B$ the protoplasm has been caused to contract by means of plasmolysis. 
length. Elements in the aggregation belonging to this category are known as the metaxylem or, more specifically, as the primary metaxylem.

In the preceding paragraph, for the sake of convenience it has been assumed that the order of development of the primary wood is always in the same direction. As a matter of fact the time and the order of appearance of the elements in this, from the evolutionary standpoint, highly significant tissue vary within certain important limits. We may first consider the most ancient order of seriation of the constituents of the primary woodthat found in the stems of the most antique plants and in the roots of all vascular organisms from the lowest to the highest. Fig. I5

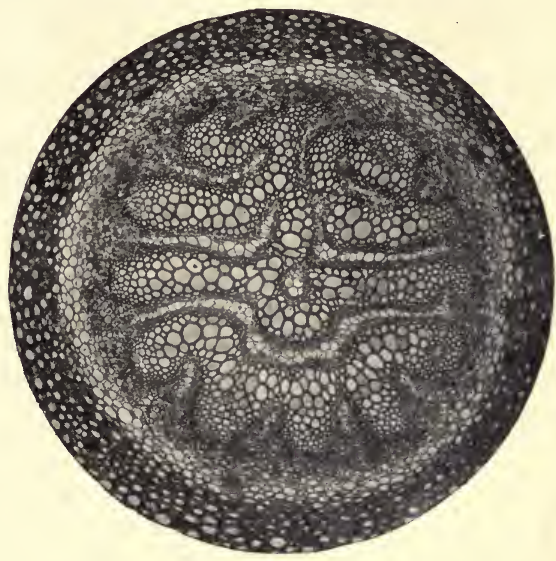

FIG. I5.-Transverse section of the upright stem of Lycopodium clavatum, showing centripetal or centrad development of the primary wood; the smaller elements represent the protoxylem. illustrates the organization of the wood in a stem of the common club moss, Lycopodium. The tissues of the xylem constitute a sort of star, the points of which are occupied by the small-sized elements of the protoxylem. As the rays of the star broaden inwardly, there is a transition from protoxylem to metaxylem. The situation becomes more clear by reference to transverse sections of the root in a fern shown in Fig. r6a. Spiral sculpture marks the small elements on the outside, while toward the center of the organ the typical sculpture of the metaxylem becomes more and more conspicuous. In the club mosses and their allies, as well as in all roots, the seriation in the development of the elements of the primary wood is very 
generally from the exterior toward the center, and the metaxylem, as a consequence, is more axial or central in position than the protoxylem. This mode of development of the primary wood is characteristic of the most ancient plants, the lycopods and their allies, and is likewise universally present in the most conservative organ of all plants, the root. When the primary woody tissues develop from the outer region inward, as indicated above, they are said to be exarch. The situation just described will become more apparent by reference to Fig. $16 b$, which shows $16 a$ in an immature

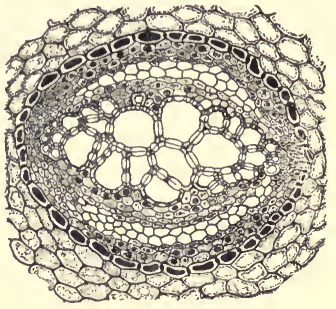

$a$

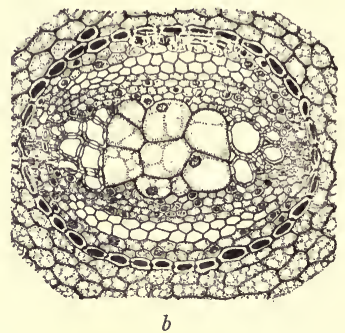

$b$

FIg. 16.- $-a$, transverse section of the bundle of an old root of Osmunda cinnamomea, showing primary wood complete; $b$, younger condition of the same with central region (metaxylem) of bundle still immature and containing protoplasm.

condition. The outer regions of the oval mass of xylem are alone developed, the center being still occupied with thin cells filled with protoplasm.

In the stem organs of the ferns and lower gymnosperms a somewhat different mode of development of the primary wood is characteristically present. This may be illustrated by reference to one of the fibrovascular strands of the bracken fern, Pteris aquilina. The smallest elements of the wood are situated in the woody tissue constituting the center of the bundle. As in the case of Lycopodium and its allies, the smaller first-formed cells belong to the protoxylem. The situation which presents itself in the later development of the woody strand of ferns, however, is usually quite different from that found in the lycopod series. In the ferns the tissues of the primary metaxylem, instead of lying entirely toward the center of the organ 
in the exarch condition featured in the lowest vascular plants, characteristically surround the first-formed ringed and spiral elements (the protoxylem). This situation so frequently presented by the ferns and lower gymnosperms is designated as mesarch. The longitudinal topography of the bundle in this type is shown in Fig. I7.

Fig. I3, described at the outset, pictures the relative position of the constituents of the primary wood in the stem of the higher seed plants, the Gnetales, the Coniferales, and the angiosperms. In this case, since the seriation of the successive elements is always outward from an internal starting-point, the primary wood is known as endarch. This condition is the typical one for all the higher plants, and no form characterized by it can, without the clearest evidence, be

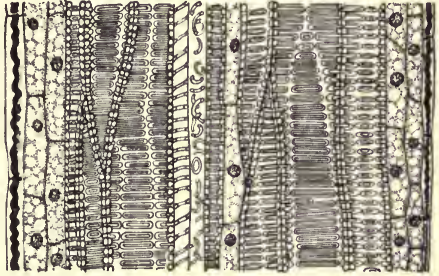

Fig. 17.-Longitudinal section of a bundle from the rootstock of Pteris aquilina, showing the elements of the protoxylem in the center of the wood. regarded as low in the scale of plants. Although the endarch condition is a feature of the development of the stem and generally of the leaf in the highest plants, it is important to emphasize at this stage that the root, even in the most advanced organisms, betrays its extreme conservatism by adhering in its primary structures to the mode of development and seriation of the elements characteristic of the most ancient known plants, the lycopods and their allies. Fig. I8 reveals the organization of a young root of the American larch. We may disregard in the present connection all but the central tissues of the root. Right and left can be distinguished two spaces, the resin canals. Inside of each of these two secretory cavities lies a cluster of protoxylem, distinguishable by the small size of its elements. Toward the center from each aggregation of protoxylem extends the metaxylem, which in the young condition represented in the figure has not yet become joined in the center. 
It may be summarily stated in regard to the present chapter that wood in the older types is much simpler in structure and less differentiated than in modern forms. Further, in most cases it is necessary to distinguish between primary and secondary wood.

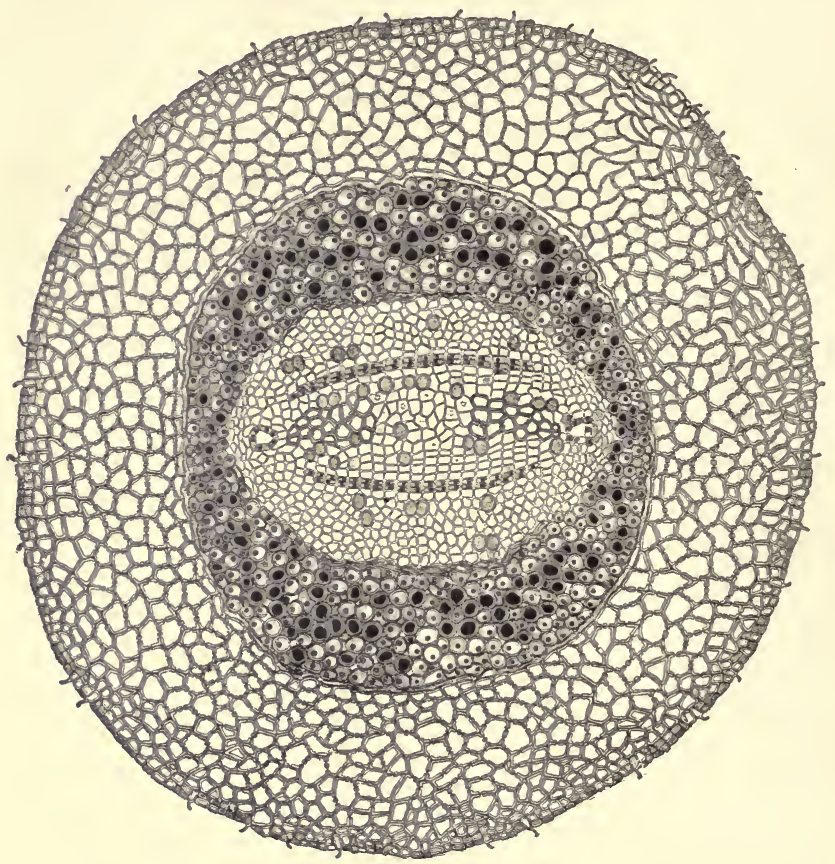

FIg. 18.-Transverse section of the young root of the American larch. The primary wood has not yet been developed in the central region of the root. The endodermis separates the central or fibrovascular region of the root from the external fundamental tissue or cortex.

The former is first to appear and is characterized by the lack of distinct seriation in its elements. The secondary wood, by contrast, is formed later and the cells which constitute its structure are arranged in rows corresponding to radii; files of storage cells, 
known as wood rays, are a marked feature of its organization. The primary wood consists typically of two regions: one composed of elements with ringed and spiral thickenings and capable of elongation in accordance with the growth of the organs; the other constituted of tracheary cells thickened in a reticulate, scalariform, or pitted fashion and, as a consequence, incapable of extension to meet the needs of lengthening parts. The former region of the primary wood is designated the protoxylem and the latter the. metaxylem. The order of development of the protoxylem and metaxylem differs significantly in different groups of plants. In the very oldest forms the progress of differentiation is entirely toward the center (lycopods and their allies). In the ferns and the lower gymnosperms the sequence is first central or centripetal and later peripheral or centrifugal, with the result that the protoxylem occupies a median position. In the higher gymnosperms and in the angiosperms the inward order of development has nearly or quite disappeared, and, as a consequence, the protoxylem lies on the inside of the metaxylem, which is formed characteristically outward or centrifugally. Technically these three types of organization of the primary wood are designated exarch, mesarch, and endarch. Finally, it may be recalled that the primary wood of the root of all vascular plants has the exarch organization of the older types of stems and exemplifies the fact that the root in this respect as in so many others (to be shown in the sequel) is the most conservative of plant organs. 


\section{CHAPTER IV}

THE FIBROVASCULAR TISSUES: SECONDARY WOODTRACHEIDS AND FIBERS

Very important constituents of all woody organizations are the tracheids and fibers. These present a wide range of structure from the lower forms to the higher and illustrate some interesting general evolutionary principles. Fig. I9 is a highly magnified transverse view of the wood of the white pine (Pinus strobus) in the region of transition from one annual ring to the next. Woods of

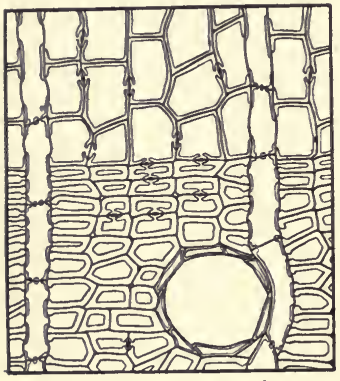

FIG. 19.-Part of a transverse section of the wood of the white pine (Pinus Strobus), showing radial and tangential pitting of the tracheids.

this type are very simple and consist mostly of elongated tapering elements with bordered pits in their walls and known as tracheids. The only features of organization not tracheary in their nature are the rays and the resin cavity. The tracheids are distinctly of two kinds. Some are large and thinner-walled and begin the annual ring as the so-called spring elements. Others, thicker as to their walls and with a smaller lumen or central cavity, constitute the summer tracheids. The two kinds of tracheids are in further contrast with one another because of the position of the bordered pits on their walls. In the spring elements the pits are confined to the radial walls-that is, those sides of the fibers which are either in actual contact with, or are parallel to, rays. In the case of the summer tracheary cells pits are predominant on the tangential walls which are at right angles to the rays. In addition to being in communication with one another by means of bordered pits, the tracheids both of the spring and of the summer wood are likewise related to the rays by 
pits which are bordered on the side of the tracheids and are simple on the side of the elements of the rays.

In order to form an adequate conception of the nature of the fibrous or tracheary elements of coniferous woods it is necessary to view them in isolation from the tissues of which they form so essential a part. It will be advantageous to consider a simpler condition first and then to proceed to the more complex situation presented by the elongated elements of the wood of the pine. In Fig. 20 are shown tracheids of the Big Tree (Sequoia gigantea) belonging to the spring and summer growth respectively. On the left, one of the spring tracheids is seen from its radial face. The cell is obviously bluntly tapering at the ends and has a length many times that of its diameter. The pits which ornament the radial aspect are of two sizes. The larger bordered pits are those which connect tracheid with tracheid. The smaller pores bring about relations between the rays and the tracheids. The latter are bordered only on the tracheary side, although this feature is naturally not obvious in the illustration. The tracheid is represented as still surrounded by its cement substance, which is indicated by a heavier line. On the
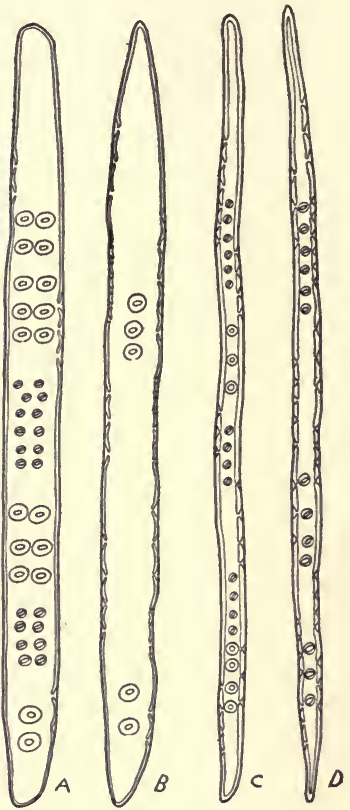

FIG. 20.-Tracheids of the Big Tree (Sequoia gigantea). Explanation in the text. lateral or tangential walls of the cell may be seen the profile aspect of other bordered pits. These are tangential pits and are very rarely present in the spring wood of conifers. Fig. $20 b$ reproduces the appearance of the same tracheid from the tangential side. The general configuration of the fibrous element is now much more pointed and only a few pits can be seen in face. On the side 
walls in this position numerous pores may be distinguished, in profile, forming the principal means of communication between tracheid and tracheid and the sole one between tracheid and ray. To the right of the figure are to be seen the radial $c$ and tangential $d$ views of a summer tracheid. The narrower lumen and thicker

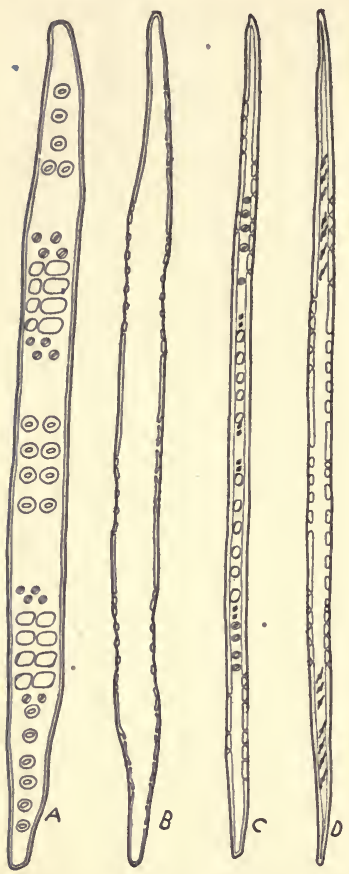

Fig. 21.-Tracheids of the pine. Explanation in the text.

walls are conspicuous. In the case of the pitting the most marked fea- ture of contrast with the spring fibers is the greater number of bordered pits occurring on the tangential walls.

The situation in Pinus may now be advantageously considered. Figs. $2 \mathrm{I} a$ and $b$ reproduce the spring tracheary elements of this genus from the same aspects as represented in the case of Sequoia. Beginning with the spring element on the left, it is clear that the pits in relation to the rays show a considerable degree of differentiation, since they consist of two categories-namely, small, distinctly bordered pores which form the intermediary between marginal ray cells and tracheids, and large, angular, scarcely bordered apertures uniting the central ray cells with the tracheids. The more pointed tangential contour of the tracheid in $b$ surrounds an area entirely free from pits, a situation nearly universal for the spring elements of coniferous woods. In the lateral walls of $b$ numerous radial pits are seen in profile. On the right of the figure are shown the corresponding views of the summer tracheids in Pinus. The narrower diameter and thicker walls, as well as the numerous tangential pores, clearly differentiate elements terminating the annual growth from those formed at the beginning of the year. 
Tangential pits are so obviously and constantly a criterion of structure at the end of the annual rings in conifers that they may be used for the purpose of distinguishing the yearly increments in tropical species, in which, by reason of the slightly marked seasonal conditions, the zonal woody bands are indistinctly indicated or even apparently absent. This is notably the case, for example, in the tropical or subtropical species of the genus Araucaria.

The phenomenon of annual rings is very closely correlated with the appearance of tangential pits, and the general phenomena in this respect are worthy of much greater attention than has hitherto been devoted to them. The incipient yearly zones in trunks of trees of earlier ages are the clearest indication of the progressive climatic refrigeration of the earth, and these become ever more marked in later geological times. Secondly, it will be made clear in the sequel that the modification of the annual ring in response to inclement seasonal conditions has been on the whole the most important factor in the evolutionary development of plants from the earlier epochs to the present. A consideration of the organization of the wood in a gymnosperm without annual rings-that is, a seed plant of Paleozoic time-is of extreme interest in the present connection. The tracheids of the secondary wood of the gymnosperms of this age, without exception so far as is at present known, had the pitting confined to the radial walls; as a consequence water could move, easily at any rate, only in a spiral and tangentially through the tracheary elements. The truth of this situation may readily be grasped by reference to Fig. II, in chapter iii, representing wood of the Paleozoic gymnosperm Cordaites. Details of the structure of the wood in this genus can be gathered from the inspection of Fig. 22 in the present chapter. It is clear that the tracheids communicate with one another and with the cells of the uniseriate rays by radial pits. Pits are conspicuously absent on the tangential walls of all the fibrous elements of the wood. It will be obvious from the facts put forward in this paragraph that the distinction between spring and summer tracheids did not exist in the case of Paleozoic woods; in other words, the modifications in structure and pitting which have become a fixed feature of the organization of the summer tracheids of trees of the Mesozoic 
and Tertiary had not yet made their appearance in the Paleozoic age. It has been suggested that the function of the tangential pits in the summer wood is the rapid supply of water to the

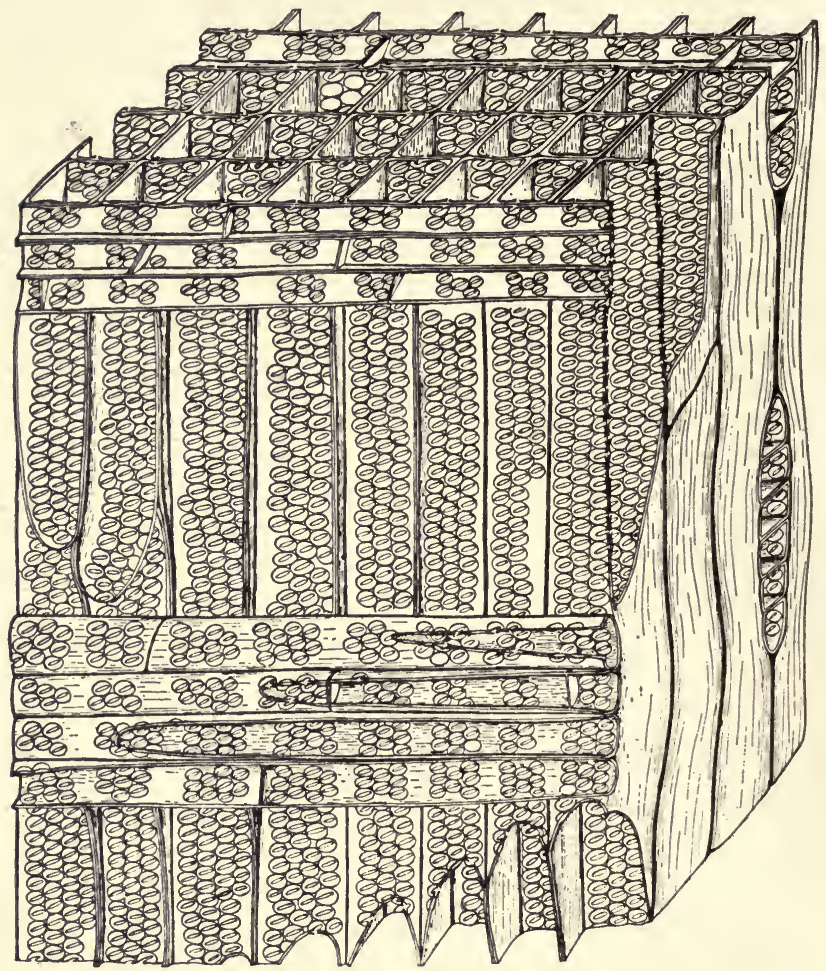

FIG. 22.-Wood of Paleozoic gymnosperm Cordaites. Explanation in the text

cambium, or zone of growth, in the reawakening of life in the trunk after the winter period of rest. It is admissible also to interpret the greater mechanical efficiency which marks the summer wood of trees possessing annual rings as of advantage in resisting the 
winter storms of later geologic times. It is unlikely that the trees of the Paleozoic age were exposed to the often furious air currents which characterize the present age of extreme physiographic and climatic differentiation of the surface of our earth.

It is convenient at this point to introduce a general statement in regard to the organization of the wood of Paleozoic and Mesozoic forms so far as the tracheary elements of the secondary wood are concerned. In the case of the giant club mosses or lepidodendrids of the earlier forests, the secondary growth consisted entirely of tracheids with scalariform markings similar to those characteristic of the primary wood of many forms. These elements were provided with pits on both their radial and their tangential walls, in this respect offering a distinct contrast, as likewise in their sculpture, to the gymnosperms of the same period, which, as has been indicated above, possessed only pitted tracheids in their secondary growth and had the pitting strictly confined to the radial surfaces of the elements. In the arboreal forms included under the general heading of sigillarians the scalariform sculpture often gave place to the pitted condition, but the pores, as in the lepidodendrids, were both radial and tangential in distribution. In the sphenophyllums and their allies, the calamites, the sculpture was transitional from scalariform to pitted and was confined to the radial aspect of the tracheids. In the true gymnosperms of earlier ages, including the Cycadofilicales (Pteridospermae) and their allies, as well as that group of forms included under the convenient cognomen of Cordaitales, the sculpture of the secondary elements of the wood was pitted (not scalariform) and was confined to the radial aspects of the cells. It is thus clear that in the great Paleozoic age the water-conducting elements of the secondary woody growth, so generally prominent in both vascular cryptogams and gymnosperms, were much more uniformly organized than is the case with plants of later geologic times.

The further course of evolution and differentiation in tracheary or fibrous elements of the secondary wood can be studied to greatest advantage in the angiosperms, which, although they first appeared in the later Mesozoic, did not become the predominant feature of the flora until Tertiary times. We may conveniently begin with 
the examination of the fibrous structures of the wood of the oak. Fig. 23 gives a general view of the organization of the wood in a red oak (Quercus rubra). Clearly it shows a much higher degree of complication than is found in the ligneous tissues of a conifer or

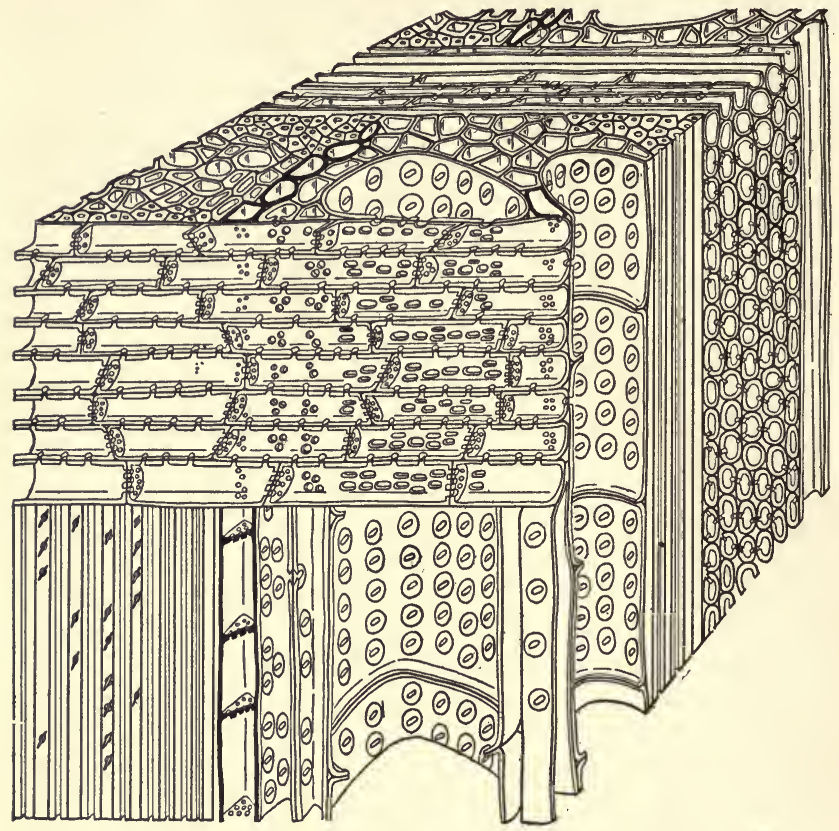

Fig. 23.-Wood of the red oak. Explanation in the text

forms still lower in the scale. The wood is crossed by conspicuous bands of storage tissue, the large rays so characteristic of the organization of oak wood. In the squares bounded by the annual rings and conspicuous rays lie the remaining structures. Of these the vessels are most outstanding and are very large at the beginning of the annual zones, becoming much smaller in caliber in the later-formed wood. The vessels are imbedded in radially directed 
bands of tracheids in intimate relation to the vascular elements which they surround. Laterally to the stripes of tracheids in a general radial direction lie other denser areas made up of mechanical elements known as fiber-tracheids. There is scarcely any marked boundary between the fibertracheid and the tracheid proper, although the extreme conditions of both are distinct. Fig. 24 represents these two kinds of elements as seen in longitudinal aspect in a maceration of the wood. The tracheid is much shorter and broader, has thinner walls, and is abundantly pitted, both radially and tangentially, with pores provided with oblique mouths. In the case of the fiber-tracheids we have to do with elements of considerable length and narrow lumen, inclosed by rather thick walls. The pits are much more scanty, but are equally distributed, as in the case of tracheids proper, on both radial and tangential walls. A marked feature of the pits in these elements is the extreme length of their oblique mouths, which much exceeds the diameter of the pit membrane. This situation becomes the rule in tracheary elements devoted more to the mechanical than to the water-conducting function.

In the mass of woods of the dicotyledons the occurrence of tracheids proper and extremely differentiated mechanical elements side by side with gradual transitions is not seen. Usually the elongated elements of the wood other than vessels (to be discussed in a later chapter) are of a more or less definite mechanical nature and have, as a conse-

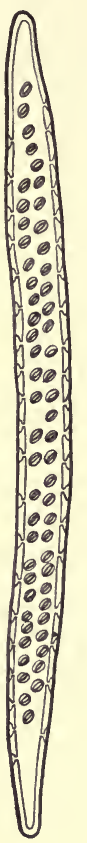

FIG. 24.-Tracheids and fibertracheids of the oak. quence, lost more or less completely the characteristics of tracheids. Fig. 25 illustrates several types of fibertracheids and similar structures. In $a$ is seen a tracheid 


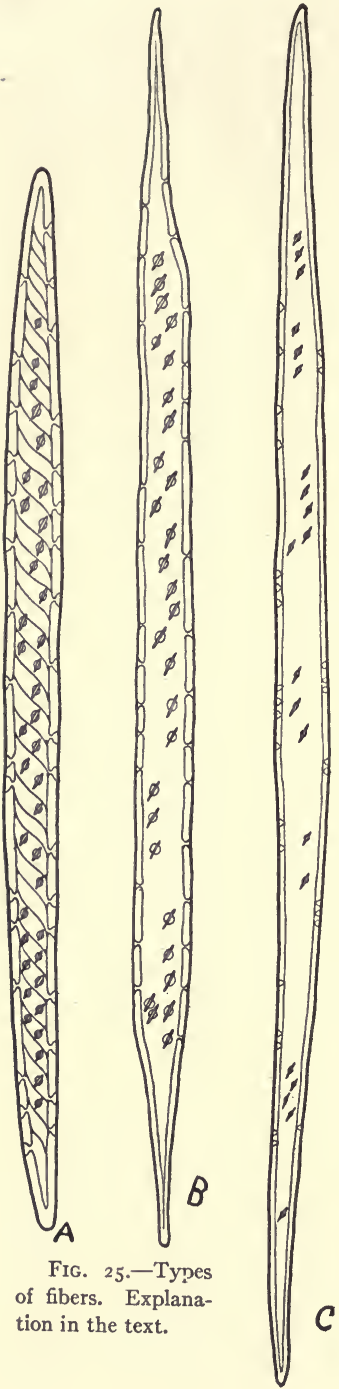

of Ephedra, a low representative of the Gnetales. The pitting is on all walls, and spiral bands are present on the inner surface. In $b$ is shown a fiber-tracheid from a species of Magnolia. Here again the pitting is not confined to the radial aspects of the element, but is also tangential. The apertures of the pits are much elongated and extend beyond the nearly circular outline of the pit membrane. In $c$ appears a type of mechanical element characteristic of the higher dicotyledons. In this form of fiber the pit membrane is exceedingly narrow and the mouth extremely elongated. As a result of this situation the pores of the fiber appear to be practically without a border. Mechanical elements of this type are usually known as libriform fibers. It has been considered by Strasburger and others that the libriform mechanical element is of a different morphological nature from the fiber-tracheid and the tracheid. The distinguished German morphologist was of the opinion that elements of this type were derived from the fusion of storage parenchyma cells. This view, however, as will be pointed out more appropriately later, does not harmonize with the general evolutionary sequence in the development of structures in the wood and, moreover, meets with serious difficulties $C$ even from the comparative stand- 
point. For example, in the northern species which are placed under the genus Fagus the mechanical elements are of the nature of fiber-tracheids and possess clearly bordered pits. In the antarctic species, on the other hand, the mechanical structures of the wood are libriform fibers. We have thus the remarkable situation that the fibrous elements in certain species of the same genus are derived from tracheids, while in others they are the result of the fusion and modification of storage parenchyma elements in the wood. The situation has only to be stated to make the absurdity of the interpretation obvious. Moreover, while in the woods of the Leguminosae the mechanical elements are, for the most part, of the nature of libriform
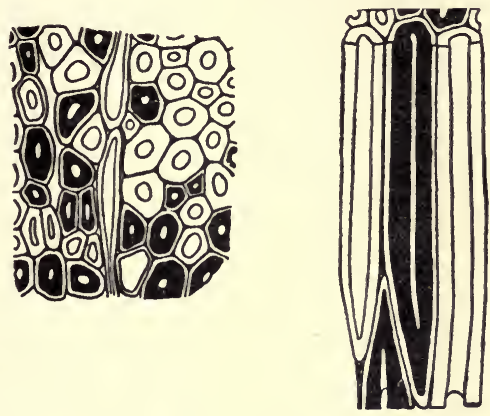

Fig. 26.-Mucilaginous fibers of the black fibers, in Bocoa provacensis

the mechanical elements are represented exceptionally by fibertracheids with clearly bordered pits. In certain Rosaceae the transition from fiber-tracheid to libriform fiber may take place within the limits of species (Rhodotypus kerrioides). The interpretation of the libriform fiber most in accord at once with the facts of comparative anatomy and with the general trend of evolution, as inferred from the comparison of existing with extinct types, is that it is the product of the extreme modification of the fiber-tracheid, just as the latter in turn takes its origin from the less specialized tracheid.

An interesting category of mechanical fibrous elements is supplied by the mucilaginous fibers found in many woods of widely separated systematic affinities. In these elements the inner portion of the wall has become more or less completely modified into a mucilaginous state which causes it to stain strongly with hematoxylin. Fibers of this type are found commonly in certain oaks 


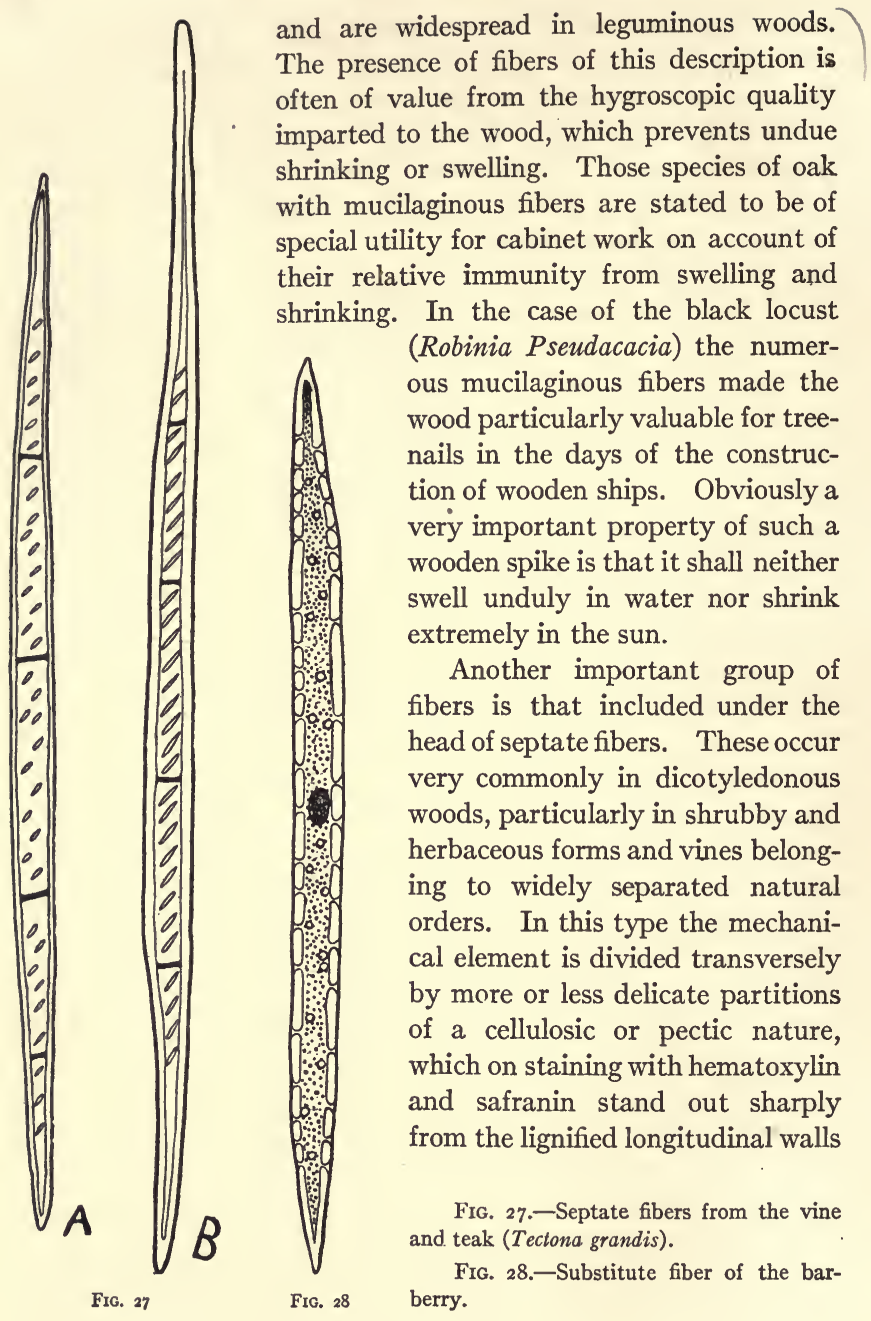


of the elements in question. The condition here present is represented in Fig. 27. To the left $(a)$ is shown a septate fiber from the grapevine, while on the right appears a similar element from teak (Tectona grandis). Elements of this nature are the probable basis of Strasburger's apparently badly founded view that parenchyma cells by their fusion have given rise to libriform fibers. The pits in septate fibers are usually much larger than in the libriform elements and are conspicuously simple.

A last interesting category of fibrous elements, also characteristic of extreme types, such as herbs, shrubs, and vines, is the so-called substitute fiber (Ersatzfaser of German authors). In elements of this nature septation is absent, but protoplasmic contents are present in the cell when it is fully developed and in a functional condition. Fig. 28 shows such an element from the wood of the barberry. The pits are both radial and tangential, although the former are obscured in illustration by the presence of protoplasm. The mechanical elements of nearly all dicotyledonous herbs belong in this category. The substitute fiber represents a convenient union of the functions of strength and storage of food in the same element, a condition particularly advantageous in the slender stems of vines and herbs.

The final paragraph of this chapter may appropriately be devoted to the subject of the distribution of the pitting in the fibers of the dicotyledons. It has been noted that in the conifers in general the pitting of the tracheary or fibrous elements is typically confined to the radial walls, except in the case of the cells terminating the year's growth (summer tracheids). The mechanical elements of the end of the annual ring have tangential as well as radial pits. In the case of the Paleozoic gymnosperms, since there are (with certain unimportant exceptions) no annual rings marking seasonal diversity, tangential pitting is entirely absent, and the pores of the tracheids are, as a consequence, exclusively radial. The dicotyledons and the Gnetales present a very different situation from that found in the seed plants characteristic of Mesozoic and Paleozoic times; for in the former the mechanical elements, whether of the nature of tracheids, fiber-tracheids, libriform fibers, 
substitute fibers, or septate fibers, are particularized by the fact that the pits or pores are not of exclusively radial distribution in any part of the wood, but likewise occur in numbers on the tangential aspects of the elements. This situation in the woods of later times is of special significance when considered in connection with other features of structure to be noted in a later chapter. 


\section{CHAPTER V}

\section{THE FIBROVASCULAR TISSUES: SECONDARY WOOD- PARENCHYMA}

Although the present chapter is to be devoted to the presence of parenchyma in the secondary wood, it will be advantageous to consider first the presence of cells belonging to this category in the primary xylem. Parenchymatous elements, as has been made clear in an earlier chapter, are those which are characterized in their functional condition by the presence of protoplasm, by simple pitting of their walls, and by their generally more or less isodiametric dimensions. Cells of this nature form a well-marked feature of primary wood= that is, ligneous tissues which are without rays and without regular disposition of their elements. The parenchyma of the ancient arboreal club

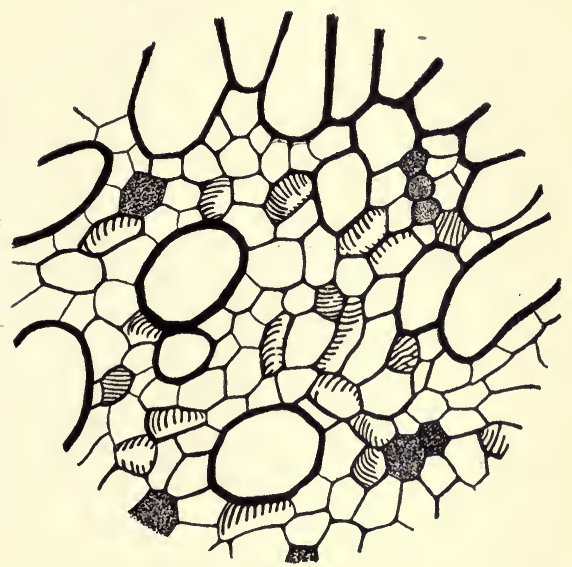

FIG. 29.-Origin of wood parenchyma in the primary wood of a lepidodendrid. mosses of the Paleozoic, the lepidodendrids, is of special interest from the standpoint of the doctrine of descent, for here the mode of origin of the parenchymatous elements of the primary wood is clearly indicated. Fig. 29 shows a transverse view of the primary woody tissues of a species of Lepidodendron from a "coal-ball" of the Carboniferous of Lancashire in England. A number of elements of large size and thick walls can be distinguished. These are the tracheids. In 
addition there can be made out other small cells which are not so markedly thickened. Finally, the tissues include cells with walls which are distinctly thin-the wood parenchyma. In the case of the cells with moderately thickened walls, where the bottom (or top) is included in the plane of section, scalariform thickenings can readily be distinguished. The thin-walled elements, when the lower

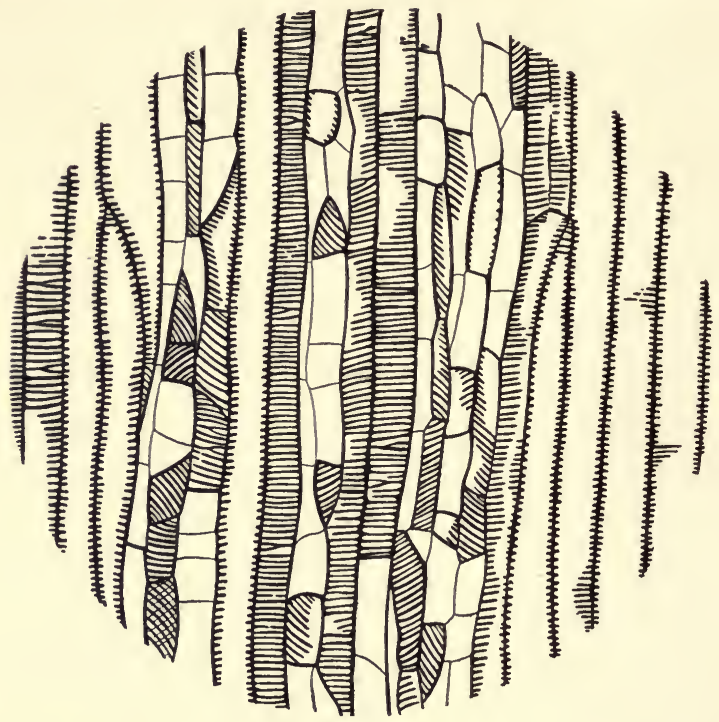

FIG. 30.-Longitudinal section of the primary wood of a lepidodendrid. Explanation in the text.

or upper wall is exposed in the preparation, show it to be without scalariform sculpture.

A highly important light is thrown on the facts recorded at the end of the preceding paragraph by the longitudinal section of the same primary wood exhibited in Fig. 30. Here may be seen a number of long structures with thick walls and scalariform sculpture. These are tracheids of the primary wood. Here and there may be distinguished other structures of similar length, 
which instead of being simple are septate. In some instances the short segments of the longer cells are obviously tracheary, since they have scalariform sculpture on their lateral and terminal walls. In other instances the septations of the long elements are quite thin-walled and without any scalariform or reticulate thickenings. Cells of the latter type are parenchymatous, and the origin of such elements in the case of the primary wood of Lepidodendron is clearly indicated. It is obvious that, as a consequence of the subdivision of originally long cells, tracheary in their character, two sorts of products result-namely, short thin-walled cells (the wood parenchyma), and equally abbreviated elements, sisters to these, which constitute merely segments of the subdivided tracheids. It thus becomes clear that the parenchymatous elements of the primary wood in the very ancient genus Lepidodendron are derived from the subdivision of elements which were primitively tracheids. It will be shown in subsequent paragraphs that the origin of wood parenchyma in the secondary xylem is likewise due to the septation of elements originally of the nature of tracheids. In the present connection the question naturally arises as to the derivation in turn of the tracheids. The following up of this problem would lead us too far into the field of purely speculative evolution. This much, however, may be stated, that, accepting the thallose liverworts as the probable starting-point of the evolution of vascular forms, it becomes clear that the mass of elaters (with spirally thickened walls) which constitute the columella in Pellia, Aneura, and some species of Anthoceros, etc., obviously constitute the prototype of the fibrovascular bundle of the ferns and higher forms. If this hypothesis is to be admitted, and it is the one assumed by the greater number of those who have worked on the somewhat meager facts which throw light on the probable origin of the Pteridophyta, it appears clear that in the spirally thickened elaters we have the originals of the spiral elements of the protoxylem. It follows that elaters are the forerunners of the tracheids, which in turn, by subdivision, as evidenced by the lepidodendrids, have given rise to the parenchymatous elements of the primary wood. There does not seem, however, to be any clear indication in the case of other and existing lycopodineous forms 
which throws light on this important subject. In the stem of the remaining vascular plants the problem has likewise become obscured by lapse of time and the blotting out of the original and primitive conditions. There seems to be no evidence in regard to the origin of parenchymatous elements in primary wood other than that in the stem of the lepidodendrids, described above. The modern descendants of the group do not furnish elucidation of the origin of parenchymatous elements, and the same statement appears to hold in the case of other living and extinct representatives of the Pteridophyta.

The secondary wood of the older and Paleozoic groups of plants was, so far as our present knowledge goes, entirely without parenchymatous or storage elements (with the exception of the so-called medullary rays to be dealt with in the next chapter). This is true not only of the arboreal cryptogams of the ancient period, the lepidodendrids, the sigillarias, the sphenophyllums, and calamites, but also of those lower and more primitive gymnosperms included under the general headings of Cycadofilicales (Pteridospermae) and Cordaitales. Not only are the Paleozoic vascular plants not known to manifest the presence of true xyliary parenchyma, but the same statement appears to hold in regard to the vascular plants of the early part of the Mesozoic - that is, the Trias. Parenchymatous elements have not yet been seen in any American wood of the earlier Mesozoic. It is only in the Jurassic period that parenchymatous cells clearly manifest themselves as a feature of the organization of the secondary wood, and at this time also the annual ring as a feature of organization of the woody cylinder first becomes well marked. There is, in fact, a distinct correlation between the appearance of true parenchymatous storage elements and the phenomenon of annual rings.

Before referring to true parenchymatous structures in secondary wood it will be well to make mention of structures which may readily be mistaken for these. In certain plants even of the Paleozoic age the rays have attached to their margins more or less elongated cells which are often of such length that they form longitudinal commissures between adjacent rays. A situation of this kind is found, for example, in the calamites and sphenophyllums 
and is quite commonly present in the genus Ginkgo, a living survivor of a group anciently numerous in genera and species. A parallel state appears in Pinus, a living genus which is represented by nearly a hundred species in the Northern Hemisphere, but which had probably three or four times as many species in the later Mesozoic. These vertical commissures between the rays must be considered in the same category with rays and do not represent veritable wood parenchyma.

Having made it clear that all parenchymatous elements in the wood cannot be regarded as true xylem parenchyma, we find it possible to discuss to greater advantage structures properly included under this heading. The first clear evidence in regard to in Picea.

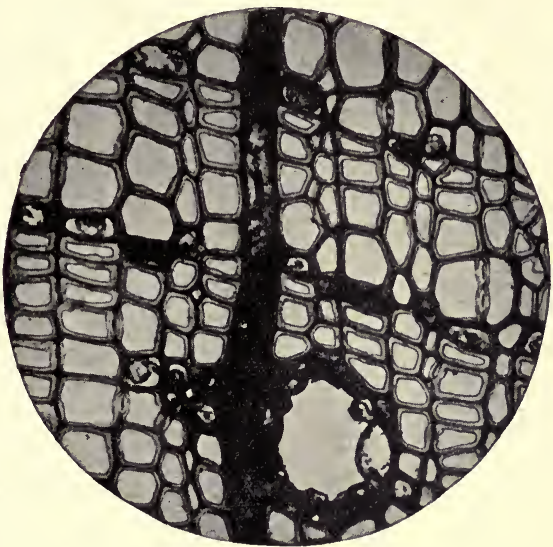
Fig. 3r.-Transverse section of the wood of the root the presence of cells belonging to this category dates from the Jurassic of Northern Europe as observed by Gothan and Holden. There is good reason to believe that the original region of appearance of the storage elements of the secondary wood is at the end of the annual rings. There is, in fact, no good evidence that true parenchymatous elements of the wood occur anywhere in forms not characterized by the presence of annual rings. The conditions accompanying the evolution of the storage elements of the xylem can best be studied in the lower representatives of the pine family or Abietineae. The genus Pinus itself, now known to be extremely ancient in its occurrence, does not exhibit the elements under consideration in a typical form. It is in the case of Picea, Larix, and Pseudotsuga 
that they manifest themselves in the most significant manner. The genus Picea will perhaps serve best to illustrate the conditions involved in the appearance of parenchymatous elements in the secondary wood. Fig. 32 illustrates the structure of a transverse view of the wood of the root of Picea canadensis. Parts of two annual rings are represented. The summer wood is easily recog-

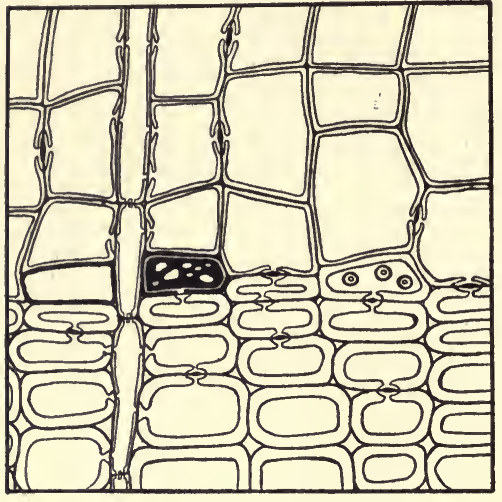

FIG. 32.-Detail of transverse section of the wood of the root in Picea. nized by the thicker walls of the tracheids and the tangential pits. It passes abruptly into the spring wood of the next annual increment, characterized by largesized thin-walled elements with entirely radial pitting. The crucial feature of the figure is presented by certain cells portrayed in black at the end of the annual ring, or, as it is commonly phrased, on the face of thesummerwood. These elements are parenchymatous and constitute the only representatives of this category of storage tissue in the wood of Picea. Fig. 33 presents a longitudinal aspect of the face of the summer wood in the same species. In order that a considerable length may be shown, three successive segments of the plane of section are depicted. In the median segment a row of short cells is seen. These have obviously simple pits in their walls in relation both to one another and to adjacent elements in the wood belonging to a different category. In other words, the cells under consideration are typical parenchymatous elements in longitudinal view. To the left lies another and lower segment. At the top of this is the continuation of one of the parenchymatous elements of the first-described segment. As we follow the series of short cells downward in the segment to the left the character of the elements 
changes. They now show bordered pitting instead of pores of the simple type. The right-hand longitudinal segment in the figure shows the region above that represented in the central segment. Here the continuation of the parenchymatous elements of the median segment is below, and this type of cell is again merged into a series of short tracheids derived from the segmentation of one of the normal longitudinal elements of the wood.

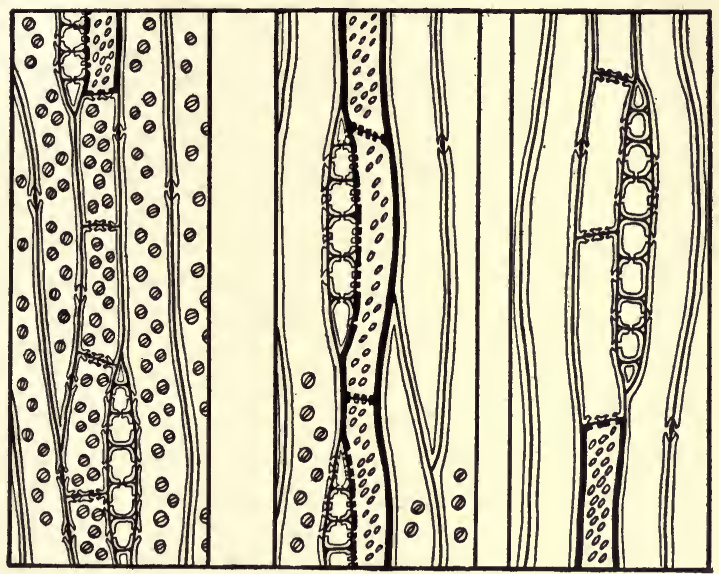

FIg. 33.-Longitudinal section of the wood of the root in Picea canadensis. Explanation in the text.

The general situation in regard to the mode of origin of longitudinal storage elements or wood parenchyma in the gymnosperms can perhaps be more readily illustrated by means of a diagram. Fig. 34 represents a ray, ordinary tracheids, and a septate or divided tracheid in relation to one another in the longitudinal aspect. The ray need not be specially considered, as the subject of the origin and organization of rays is to be discussed in the following chapter. Turning our attention to the normal tracheids represented in the diagram, we find it clear that these are characterized by the possession of pits both on the radial and on the 
tangential walls, a feature commonly found in the case of fibrous elements in the terminal region of the annual ring in the conifers and

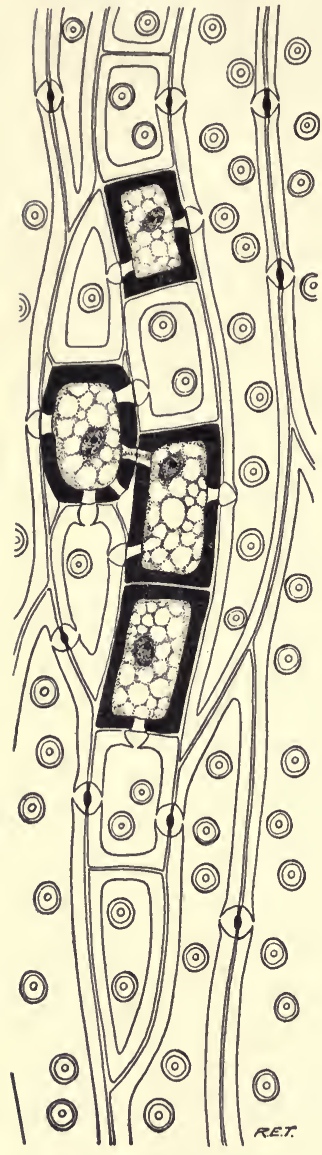

FIG. 34.-Diagrammatic representation of the origin of wood parenchyma in the Picea.

allied groups. The septate tracheid is the most striking feature of the diagram. In this element numerous transverse walls interrupt the continuity of the central lumen. Certain of these transverse partitions are characterized by the presence of bordered pits, while others offer to the eye pits of the simple type. In a third condition bordered and simple pits confront one another in the same wall. Those cells which communicate with the surrounding elements by means of simple pits are typically occupied by protoplasmic contents, while the elements possessing pores of the bordered category are invariably without living substance. It will be clear to the reader that, if the diagrammatic representation presented in Fig. 34 is correct, the parenchymatous elements of the wood come from the subdivision of the primordial elements which would in other cases and under different circumstances give rise to ordinary tracheids. At an early stage these elements became transversely septate, and in the segments so set off the protoplasm sometimes persists (when a typical parenchymatous element of the wood is the result); at other times it disappears with the complete differentiation of the walls surrounding it (in the case of so-called short tracheids). An interesting fact in this connection is the occurrence of parenchymatous storage elements in the same region of the wood where the tangential pits take their origin. It has been made 
clear in a former chapter that in the case of the Paleozoic gymnosperms there are at the same time no annual rings (typically at least) and the pitting of the tracheary elements of the wood is entirely radial in its disposition. In the gymnosperms of the Mesozoic age both annual rings and tracheids tangentially pitted in the terminal region of the yearly zones of growth become the rule. It is highly significant in this connection that the first appearance of true longitudinal storage parenchyma is from the Jurassic (Middle Mesozoic) onward, and that the storage cells take their origin in the terminal region of the annual rings and clearly show from their mode of development that they are the derivatives of tracheary elements, since all stages of transition between septate tracheids proper and files of parenchyma are found. It has been suggested by Strasburger that the tangential pitting of the summer tracheids is for the purpose of easily and rapidly supplying water to the cambium in the next opening period of growth. Such a hypothesis would accord well with the very definite correlation between tracheids of this type and the phenomenon of annual rings. If the condition of annual growth be accepted as the probable elucidation of the tangential pitting of tracheids in more modern gymnosperms, it is not difficult to put forward a similar claim for the terminal and tangential parenchyma which probably represents the primitive disposition of parenchymatous elements in gymnospermous woods. If the tangential pitting is for the purpose of supplying the awakening cambium with water, there can be little doubt that the later-appearing and correlated feature of terminal longitudinal storage cells is significant in connection with the need of the reviving initial cells of the zone of growth for readily available food.

In Pinus, as indicated above, there is no true wood parenchyma. In the allied genera, Picea, Pseudotsuga, and Larix, it has become clearly and unmistakably established and occurs everywhere in the wood of the root, even when it is absent or degenerate in the stem. As will be shown later, the root is the most conservative organ of plants. In the case of higher members of the pine family (Abietineae), Cedrus, Pseudolarix, Abies, and Tsuga, the parenchymatous elements are still found typically at the end of the annual ring, but they no longer normally show evidence of derivation from tracheids, 
other than in the fact that they are found in spindle-shaped or fusiform groups resembling in contour tracheary elements. Injured specimens of the woods of the four genera under discussion show (Fig. 35), however, the transition from short tracheids to true parenchyma cells as the result of the septation of elements laid down by the cambium as tracheids. This is an example of the

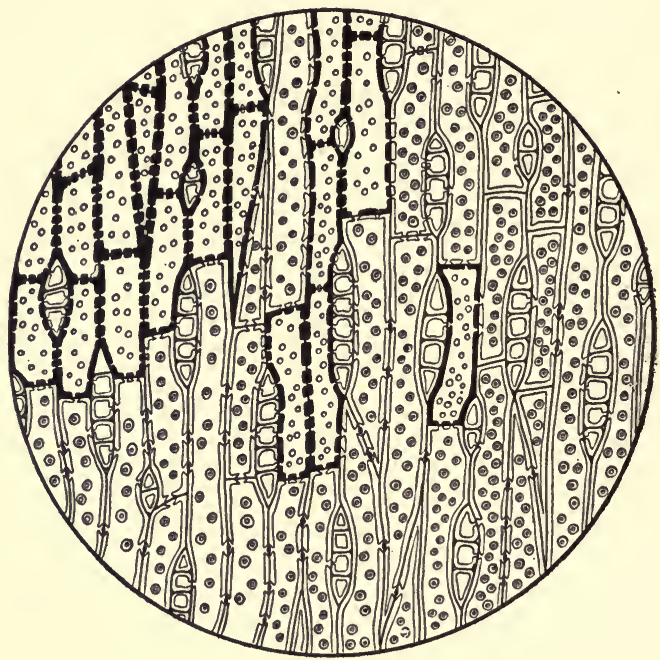

FIG. 35.-Injured wood of Tsuga canadensis, showing transition of tracheids to parenchyma.

recall of ancestral conditions as a corisequence of injury, a phenomenon extremely common in the case of plants with secondary growth. This situation is classified, as will be shown later, under the caption of the evolutionary doctrine of reversion.

Already in the higher representatives of the pinelike conifersnamely, Abies and Tsuga-the parenchymatous elements have not only lost all normal indications of derivation from tracheary elements at the end of the zone of annual growth, but have even begun to abandon the strictly terminal position in the yearly rings 
of wood. In certain species of Abies and Tsuga the location of the parenchyma is on the face of the summer wood only, while in others it is no longer strictly confined to this position. In the genus Abies, A. webbiana and A. cephalonica are characterized by longitudinal storage cells no longer wholly terminal in position but scattered throughout the annual growth. The same condition is present in Tsuga mertensiana and in smaller branches of $T$. canadensis. This type of disposition of parenchyma may conveniently be designated diffuse to distinguish it from the terminal condition of storage elements in their first appearance among the conifers. It is of interest to note that, so far as the matter has been investigated, both Abies and Tsuga in the root have terminal parenchyma exclusively, no matter what the situation may be in the case of the stem. The diffusion of parenchymatous cells throughout the annual ring in higher forms of seed plants has its significant analogy in the spreading of tangential pitting, at first confined to the later-formed tracheids of the summer wood, to the elements of the rest of the annual ring in higher types. Diffuse wood parenchyma never betrays its origin by normal transitions to septate tracheids. It is only in the case of wounding that its tracheary origin can be distinctly observed. The longitudinal storage cells, to which the name of wood parenchyma is given, always reveal their derivation from the fibrous elements, however, by the fact that they are grouped in series corresponding in length and outline to the pointed tracheids constituting primitively the sole longitudinal elements of the wood.

In the case of the abietineous genera Abies and Tsuga, cited above, it is quite clear that the storage elements of the woods have . in some instances departed from the exclusively terminal position and have become distributed throughout the annual ring. In those conifers belonging to the subtribes known as Taxodineae, Cupressineae, and Podocarpineae the parenchyma of the wood is characteristically diffuse and no longer, except by the grouping and contour of its elements, gives evidence of tracheary origin. Injuries in most cases reveal conditions of transition from septate tracheids to pointed rows of parenchymatous elements. In the subtribes Araucariineae and Taxineae parenchyma has disappeared from the 
normal structure of the wood in the vegetative stem, but is ordinarily clearly and sometimes even abundantly present in the diffuse condition in regions which are conservative of ancestral characteristics, such as the root, cone axis, etc. It may, moreover, be easily recalled as a consequence of experimental procedure. It is accordingly clear that, from the evolutionary standpoint at any rate, considerable care is necessary in deciding as to the primitive presence or absence of parenchymatous elements in the woody tissues of the conifers.

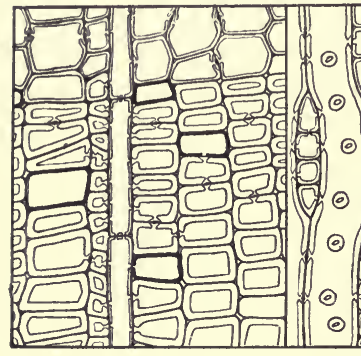

A

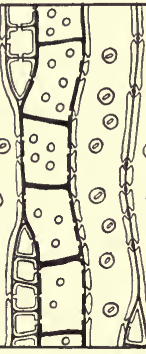

$B$

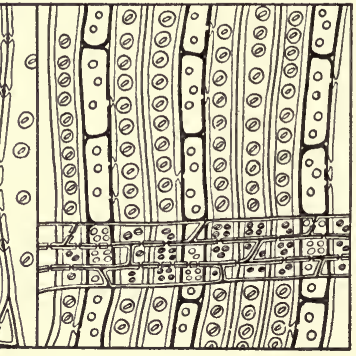

C

Fig. 36.-Diffuse parenchyma in a coniferous wood. Explanation in the text

Fig. 36 shows the topography of the diffuse condition of parenchyma in coniferous woods in three dimensions. In $a$ is seen the transverse view of a wood of this type. The longitudinal storage elements, or wood parenchyma, are represented with heavy black walls to bring out by contrast their distribution in the general structure and at the same time the frequent chemical difference of their walls from the tracheary tissues proper. In $b$ appears the tangential aspect of the storage elements, in which they present themselves in the greatest breadth and on the whole show most clearly by pits their relation to other elements of the wood. In the case of $c$ these important elements of wood structure are revealed in the radial aspect, and their diffuse disposition is as apparent as in the transverse plane of section. 
It will be clear from the account of the appearance of parenchymatous cells given in the preceding paragraphs that their origin in the primary tissues of the wood is revealed only in the case of that very ancient group of cryptogamous trees, the lepidodendrids of the Paleozoic age, where they are very clearly derived from the septation of tracheids. Further, in the case of the secondary wood, parenchymatous elements properly so called did not make their appearance before the Mesozoic period and are as distinctly correlated in their origin with the phenomenon of annual rings presented by plants of the Mesozoic and later geologic time as are tangential pits in the tracheids. As has been made clear in an earlier chapter, the secondary wood of Paleozoic gymnosperms was characterized, not only by the absence of annual rings, but equally clearly by the default of pits on the tangential walls of the tracheids and the entire absence of parenchymatous elements in the wood. The arrival of the phenomenon of annual rings can be most satisfactorily explained in the case of the known facts by the hypothesis of the gradual refrigeration of the surface of our earth, with the consequent appearance of a winter period of rest in vegetative activity. The phenomenon of annual growth soon brought into prominence the two striking features of terminal tangential pitting and terminal storage parenchyma. Both features of organization were later extended with greater or less completeness to the whole of the annual ring. It is further quite clear that parenchymatous storage elements came into being in the secondary wood by the septation of tracheids, precisely as has been shown to be the case in the similar elements of the primary wood (apparently only clearly indicated in the case of the lepidodendroid cryptogams of the Paleozoic age).

In the higher gymnosperms, including the greater number of conifers as well as the Gnetales, the distribution of the longitudinal storage elements of the secondary wood is typically and primitively diffuse; that is, such cells are not confined to the end of the recurring zones of growth, but are scattered throughout the annual ring. It is apparently not necessary to figure the situation for the Gnetales, since it corresponds so closely with that found in 
the higher Coniferales. Fig. 37 illustrates the distribution of the wood parenchyma in those dicotyledons in which the condition is diffuse. In $a$ is shown a transverse view of the secondary wood in the black walnut (Juglans nigra). The distribution of the storage cells is made clear by the outlining of their walls in solid black. In $b$ the same wood is depicted in longitudinal radial aspect, and the nature and distribution of the parenchymatous elements become doubly clear. A fact not without interest from the evolutionary

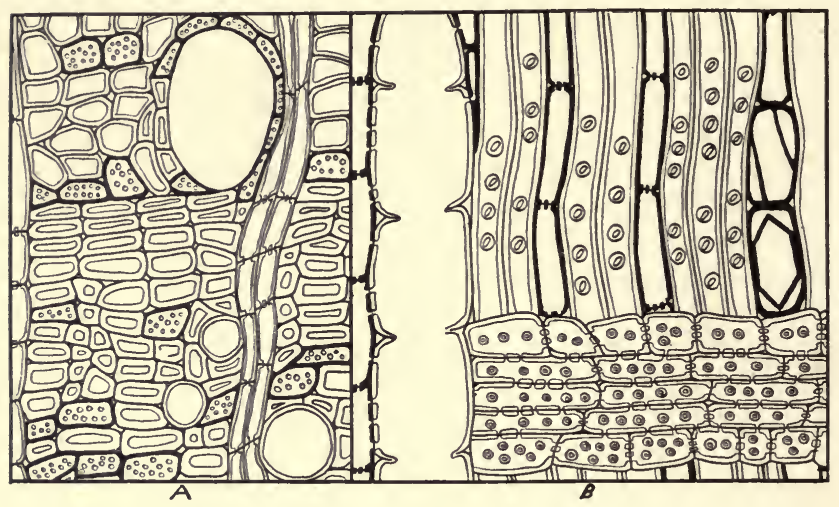

Fig. 37.-Diffuse parenchyma in Juglans. Explanation in the text

standpoint is the uniform distribution of parenchyma after this fashion in whole natural orders of dicotyledons. For example, in the Juglandaceae, Betulaceae, Fagaceae, Ebenaceae, Ericaceae, etc., the disposition of the longitudinal food-storing cells of the wood is entirely of the manner designated above as diffuse.

In other orders of dicotyledons, particularly those which are with some degree of unanimity assigned by systematists to a high position, a strikingly different mode of parenchymatous arrangement is found. This is notably the case in the Compositae, Verbenaceae, Oleaceae, etc. The situation in this type of distribution of parenchyma can be clearly indicated by reference to the ash. Fig. $38 a$ shows the grouping of the parenchyma at the end 
of the annual ring and around the vessels in this genus. These are the only situations in which parenchymatous cells are normally present in orders characterized by what may be conveniently termed vasicentric parenchyma. In $b$ the longitudinal view of a vessel with its parenchymatous jacket of vasicentric parenchyma (that is, parenchyma confined to the vicinity of the vessels) is indicated. The vasicentric distribution of parenchyma is, other things being equal, an indication of an advanced systematic position among the dicotyledons and is, as will be shown in the sequel, accompanied

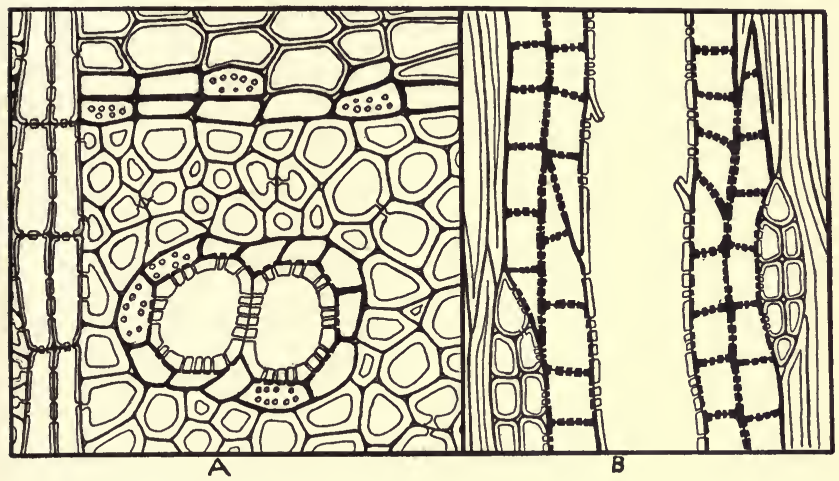

Frg. 38.-Vasicentric parenchyma in Fraxinus. Explanation in the text

by other features of organization in the tissues of the secondary wood which are somewhat generally accepted as indicating a high degree of specialization.

In dicotyledonous woods, with either of the two characteristic modes of distribution of the parenchymatous elements indicated in the preceding paragraphs, degeneracy may occur. As a consequence the longitudinal elements may somewhat rarely be absent altogether or may be confined to the end of the annual ring, the latter situation being much more commonly found. Fig. 39 illustrates the organization of the wood in Salix or Populus (or equally well that of Liriodendron or Magnolia) as regards the 
distribution of parenchymatous elements. In $a$ is shown the transverse view, and it is here clear that the cells in question, accentuated by the representation of their walls in black, are confined to a position at the end of the annual ring. Clearly, the vessels appearing in the figure are unaccompanied by any parenchymatous elements. In $b$ is represented the longitudinal radial aspects of the same wood, and it becomes clear that in this plane, too, the storage cells are confined to the terminal region of the annual ring, for none can be seen in relation to the vessels. The situation here indicated is not
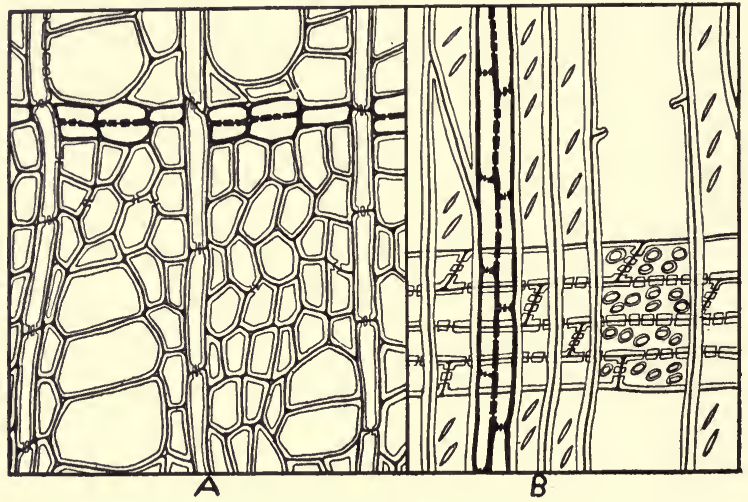

Fig. 39.-Terminal parenchyma in Populus. Explanation in the text

uncommon in the case of woods with reduced vasicentric parenchyma. As in so many other instances, the situation is made clear by reference to more conservative parts, such as the root and the first annual ring of the stem, or to injured material showing a reversion to the primitive condition. By such control of evidence it becomes clear that in the Salicaceae, as well as in Magnolia and Liriodendron among the Magnoliaceae, the characteristic mode of distribution of the storage cells is vasicentric, for they occur in this manner both in conservative organs and parts and likewise as the result of experimental injury. It cannot, of course, be too strongly emphasized in the case of comparative anatomical investigations that a wide view of any particular situation is essential to an ade- 
quate comprehension of a given problem. The truth of this statement will become more and more obvious as a result of repeated illustrations in the sequel. Much more rarely does the diffuse condition of parenchymatous disposition in the dicotyledons give rise by reduction to a state in which storage elements are to be found only at the end of the annual zones of growth. This situation is exemplified by certain species of the antarctic beech (Nothofagus), in which, in contrast to all the boreal species of the Fagaceae, the parenchyma is confined to the face of the summer wood and is not distributed throughout the annual ring, as is the rule for the family as a whole. Similar reasoning to that employed in the case of the Salicaceae and certain Magnoliaceae results in the correct conclusion as to the typical and primitive mode of occurrence of parenchymatous elements.

It is necessary to emphasize the different interpretations of terminal parenchyma which must be adopted in the case of the conifers and the dicotyledons. In the coniferous series the presence of wood parenchyma on the face of the summer wood is clearly a primitive phenomenon, both because of the comparative and historical data and because of the equally cogent evidence derived from the consideration of the origin of parenchyma cells in this position in the conifers. Clearly, terminal parenchyma in the gymnospermous series is in the act of origination in view of its almost imperceptible transition to septate tracheids. In the dicotyledons, on the other hand, comparative and experimental data alone, in the absence at the present time of any adequate information in regard to the historical evolution of woods of this type, lead to the conclusion that the occurrence of storage elements in the terminal region of the annual rings is rather the result of reduction from a more elaborate and advanced condition (diffuse or vasicentric) than one of primitive simplicity.

It is obvious that in general among the dicotyledons we need not expect to have as clear evidence in regard to the problem of the derivation of the longitudinal storage elements of the wood as in the case of the gymnosperms, and in particular the conifers, which present themselves to our gaze in so long and continuous a series in geologic time. Evidence for the origin of parenchymatous 
elements from the tracheary and other fibrous constituents of the secondary wood in the dicotyledonous angiosperms is sufficiently

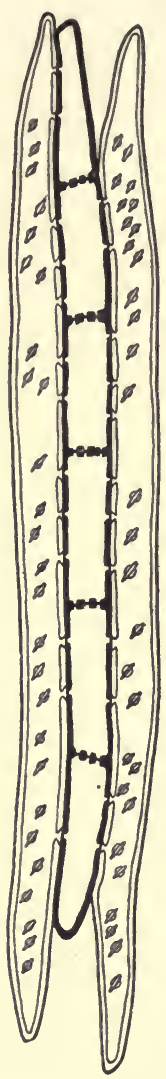

Frg. 40.-Longitudinal view of tracheids and parenchyma from the root of the alder. authentically supplied by the grouping of such elements in longitudinal terminally pointed groups possessing the exact configuration of tracheids or fibers. Fig. 40 illustrates this situation for the wood of the alder. To the right and left are seen fiber-tracheids which constitute the mechanical elements of the wood in this genus. Between these two cells lies a file of parenchymatous elements of the wood. It is clear that the latter, represented with heavy black boundaries, form a series which both in length and in contour corresponds with the adjacent fibrous elements. Normally there are no transitions from tracheids to parenchymatous cells in the case of the dicotyledons, and even experimental means do not in general suffice to bring about the clear production of parenchyma as the result of the progressive septation of the original tracheary elements of the wood. Some information on this subject, however, is furnished by the phenomena of injury in Liquidambar and Prumus. Here, as a response to experimental or accidental injuries, tangential rows of gum or mucilage canals are formed in the wood. About these canals may sometimes be detected short elements which grade from abbreviated tracheids, resulting from the septation of tracheids of normal length, to typical parenchymatous elements, characterized by persistent protoplasmic contents and simple pits in their walls.

Certain general characteristics of wood which depend as much as anything on the condition of the wood parenchyma may be conveniently introduced here. Fig. 4I is a photograph of the 
polished end of the trunk of an oak. The wood is clearly separated into two regions, a darker central and a pale peripheral. The deeply colored central region of the trunk constitutes the heartwood or duramen. The uncolored zone which surrounds this is the sapwood or alburnum. The dark-hued heartwood is extremely resistant to decay and constitutes the only material properly utilizable for exposed structures. In the case of dicotyledonous trees in general, one frequently notes even with the naked eye a difference in color between the heartwood and the sapwood. Sometimes this important distinction is not revealed to the eye, but becomes obvious only under microscopic investigation. Frequently the sap indi-

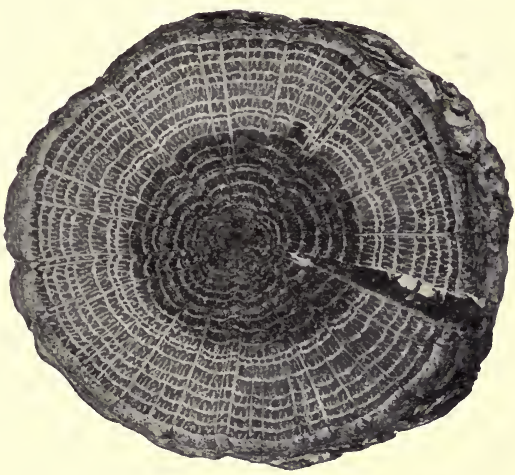

FIG. 4r.-Transverse view of an oak log showing heart- and sapwood.

cates its boundaries in the felled trunk by the discoloration brought about in its tissues either by oxidases or by fungi, or by both agencies united. In conifers, likewise, a distinction between a darker central heartwood and a surrounding pale-hued sapwood can be often recognized by the naked eye. The redwood or red cedar presents the contrast in color in a very marked manner. The larch and the spruce, which in the microscopic organization of their woods are practically identical, can be readily distinguished from one another by the gross aspect of their trunks. The larch has a dark-brown heartwood, while in the species of spruce the central region of the woody cylinder is in no way contrasted in color with the peripheral sapwood.

It will be convenient in connection with the discussion of the parenchymatous or storage elements of the wood to elucidate certain features of the microscopic organization of the heartwood 
as contrasted with the sapwood. In this connection it will be well to begin with a conifer. Fig. 42 represents side by side the microscopic aspects of heart and sap tissues in the case of the white pine (Pinus strobus). Beginning with the sapwood, which appears on the right of the illustration, it is clear that both its rays and the cells surrounding a resin space or canal are possessed of nuclei and likewise contain a somewhat granular living substance or protoplasm. Imbedded in the protoplasm are usually found oval bodies,

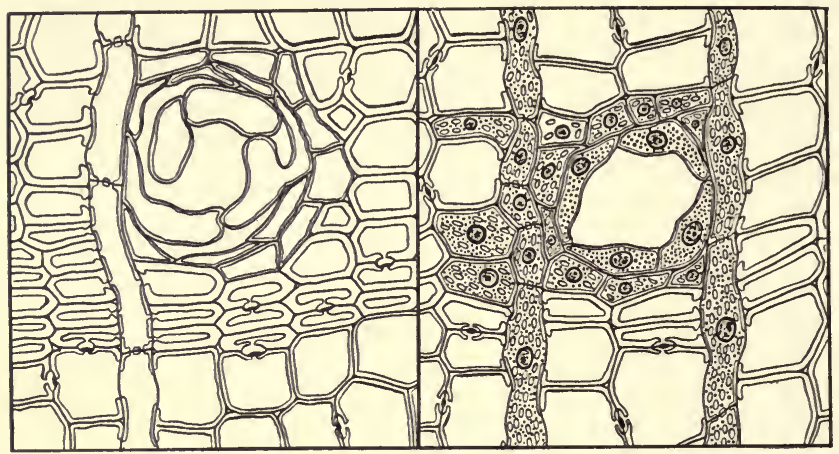

FIG. 42.-Sap- and heartwood of the pine. Explanation in the text

the grains of starch, which are lacking, indeed, only in the cells immediately surrounding the resin canal or space. By examination of the bordered pits of the tracheids it becomes evident that in the wood of the sap the membranes of the pits are central in position. Turning to the left side of the figure, we find represented the corresponding organization of the wood in the case of the heart. The elements distinguished by simple pits in the delineation on the right-in other words, the living cells-here have lost their living contents and are quite empty. Moreover, in the water-conducting cells, or tracheids, distinguished by the presence of bordered pits, we discover that the membranes of the pores with their thickened central region, or torus, are no longer median in position, but in general have become adherent to one side or the other. Further, the resin space has been stopped by an ingrowth known as tylosis. 
In other coniferous woods, particularly those higher in the scale than Pinus, the parenchymatous elements, whether radial or longitudinal in position, secrete antiseptic substances such as essential oils, tannin, etc., which preserve the heart structures from the decay resulting from the attacks of wood-destroying fungi. Fig. 43 throws light on the similar conditions as regards the organization of heart and sap in the oak, as an example of the dicotyledons. To the right, radial and longitudinal parenchymatous

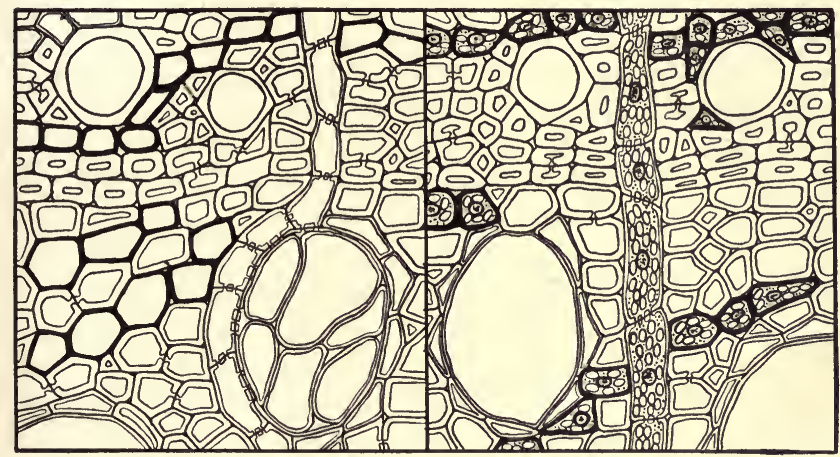

Fig. 43.-Heart- and sapwood in the oak. Explanation in the text

elements appear loaded with starch and provided with protoplasm and nucleus. The radial storage cells are represented with light walls, while the true wood parenchyma is delineated with thick, black, bounding membranes. Larger and smaller vessels are to be seen corresponding to the spring and summer region of the wood. The rest of the area is occupied by tracheids (larger and thinner-walled) and fiber-tracheids (narrower and with thicker walls). Turning now to the left, where the organization of the heartwood is indicated, we discover the same absence of contents in the radially and longitudinally directed elements with simple pits (in other words, the parenchymatous elements) as in the case of the similar structures of the pine. In this instance the larger vessel presents a certain analogy to the resin canal of the conifer by reason of the fact that it is occluded by an ingrowth in the form of 
a tylosis. An adhesion of the pit membranes to one overhanging margin or the other of the pit cannot be made out. Occasionally a torus is present in the relatively narrow membranes of the small bordered pits of the water-conducting elements of the dicotyledons, but it does not present the phenomenon of fusion with the margins of the pit characteristic of heartwood in conifers. The parenchymatous constituents of dicotyledonous woods in many cases give rise to highly efficient antiseptics in the case of the heartwood. In many instances, such as the oak, the blue gum, the quebracho, etc., large amounts of tannin are formed which serve as an effectual preservative. In other cases ulmic and even humic acids make their appearance and exercise greater or less inhibitive action on the organisms which ordinarily bring about the decay of woody structures. In teak we have the rare example of a structurally valuable dicotyledonous wood which in the transformation from heartwood to sapwood elaborates, not acid substances which exercise a corrosive action on metals, particularly on iron and steel, but an essential oil. The enduring heartwood of the teak (Tectona grandis) is consequently valuable above all others for naval construction by reason of its compatibility with iron and steel, since it neither corrodes this fundamental structural material of present naval architecture nor, in turn, is rotted by iron rust.

It will be apparent from the foregoing paragraphs that those longitudinal elements which subserve the function of storage in the woods of Mesozoic, Tertiary, and actual plants are of great evolutionary significance. Their importance in this respect can be gauged only after the rays or radial storage devices have been considered in the next chapter, and they will receive their final and fullest appreciation in connection with the highest groups of plants. It is obvious that the incentive to the development of longitudinal parenchymatous elements was the appearance of an annual winter period of rest which in later geological times, beginning with the earlier Mesozoic, with progressively greater emphasis marked the originally unvarying cycle of the year. The first parenchymatous elements came into being, so far as our knowledge at present goes, in the earlier Jurassic. Their primitive occurrence was at the end of the annual ring. In the conifers in this position 
they often clearly reveal their derivation from tracheids by almost imperceptible transitions into elements belonging to this category. The terminal situation of the primitive parenchymatous cells is probably of advantage to the cambium awakening from its winter sleep and standing much in need of instantly available food. In general, the tangential terminal parenchyma of the more primitive conifers has the same nutritive relation to the cambial elements as the similarly located tangential pitting of the tracheids has to the water supply of the cambial zone.

Later the longitudinal elements devoted to the function of storage, like the tangential pitting of the tracheids, became distributed throughout the annual ring. So long as there was no further differentiation of the elongated elements of the wood there was no incentive to further evolution on the part of the elements of the wood parenchyma. With the appearance of the vessel as the final expression of efficiency in the transport of water on the part of the wood and the correlated gradual loss of the aquiferous function on the part of the tracheids (which progressively gave rise to fiber-tracheids and libriform fibers, respectively more and more specialized in the mechanical direction), a new tendency found expression in the organization of the longitudinally oriented storage devices of the wood. In the case of the higher gymnosperms (Gnetales) and lower dicotyledons the fibrous elements of the wood are still largely capable, by reason of the presence of numerous bordered pits in their walls, of the transport of water. The higher dicotyledons, however, are in general characterized by the strict allocation of the function of movement of water to the vessels, and the tracheary structures of lower types become transformed into purely mechanical or partially mechanical and partially food-storing elements designated progressively as fiber-tracheids, libriform fibers, septate fibers, and substitute fibers. With the appearance of this situation the parenchymatous elements of the wood become more and more relegated to the vicinity of the vessels for their necessary supplies of all-important water. This situation receives its final morphological and evolutionary expression in the appearance of strictly localized vasicentric parenchyma in the case of high dicotyledonous groups, such as the Compositae, Sapindales, 
Verbenaceae, Oleaceae, etc. It must not be assumed, however, that the localization of the parenchyma about the vessels is strictly referable to the transformation of the tracheids into purely mechanical elements: It has been shown by Miss Holden in her interesting investigations on the Sapindales that it makes no difference whether the fibrous elements of the wood here are of the nature of tracheids (and hence are capable of conducting water) or are libriform and mechanical as regards the distribution of the elements of the wood parenchyma which in the group throughout are vasicentric. It is thus clear that the distribution of the parenchymatous cells in dicotyledonous woods is of morphological importance and is not physiologically conditioned by the nature of the fibrous portions of the wood, whether tracheary and with bordered pits or libriform and thus definitely relegated to purely mechanical functions.

It is finally important to note that not even the obvious facts of distribution can without proper discrimination be subjected to evolutionary inference. For example, in many dicotyledonous woods terminal parenchyma occurs which might be regarded as prima facie evidence of a primitive systematic position in view of the situation presented by the conifers, exhibiting parenchyma on the face of the summer wood. A comparative and experimental investigation of this situation makes it clear in the case of the dicotyledons that the terminal position of parenchyma is the result of degeneracy either from the vasicentric or from diffuse modes of parenchymatous distribution. 


\section{CHAPTER VI}

\section{THE FIBROVASCULAR TISSUES: SECONDARY WOOD-RAYS}

In the preceding chapter the subject of the origin of longitudinal storage cells has been discussed and to elements of this type the general appellation of wood parenchyma has been given. In the case of the ligneous tissues of vascular plants a much older type of storage device exists in the form of radially directed bands of masses of cells which often, with a high degree of impropriety, are designated medullary rays. This denomination is erroneous from the historical and evolutionary standpoint, since it is clear that in the first instance and in the earlier and primitive forms rays had no relation whatever to the pith or medulla and consequently cannot with any degree of propriety be designated

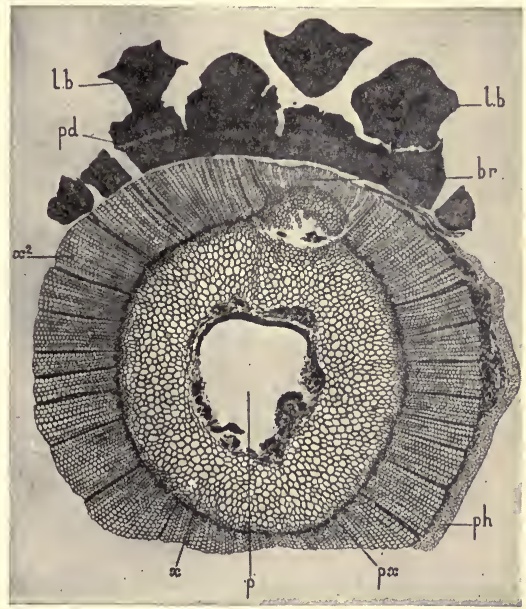

FIG. 44--Transverse section of the stem of a lepidodendrid, showing well-developed primary and secondary wood, the latter being radially seriate and provided with storage rays (after Scott).

as medullary. Fig. 44 illustrates the conditions obtaining in the case of the so-called medullary rays of the ancient genus Lepidodendron. The region of the pith $(p)$ is represented largely by an empty space, which is in turn surrounded by the primary wood. This is distinguished by the non-seriate and irregular arrangement 
of its cells in contrast to the regularly and radially disposed structures of the secondary xylem which lies just outside the primary region. It is clear from the figure here introduced that the so-called medullary rays which extend from the primary wood outward cannot be properly so designated, since they never come in contact with the pith. The same situation occurs in the stem

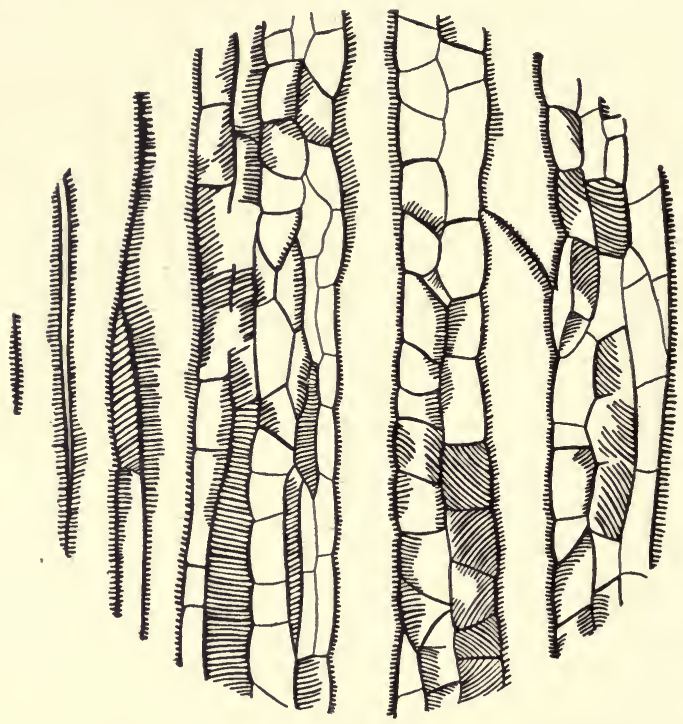

FIG. 45.-Longitudinal view of the primary wood of a lepidodendrid. Explanation in the text.

of many other extinct fernlike or gymnospermous plants with secondary growth. It is worth while to note, too, in this connection, that in the root of living plants in which the wood undergoes secondary increase its radially disposed bands of storage cells have nothing to do with a pith or medulla. It is accordingly evident from an examination of older and consequently more primitive forms, and likewise from the study of the organization of the conservative root structure of living groups, that the term medullary 
ray is a misnomer. The most appropriate name for the radial stripes of storage elements in the secondary wood is the merely descriptive one of wood ray.

The status of the ray having been preliminarily defined from the evolutionary standpoint there now remains the question of the origin of the horizontally directed bands of storage tissue which it is customary to consider under this head. It has been made clear that the lepidodendrids are the only plants which supply decisive evidencè as to the origin of the parenchymatous elements found universally in the primary wood of vascular plants. It will be well to recall

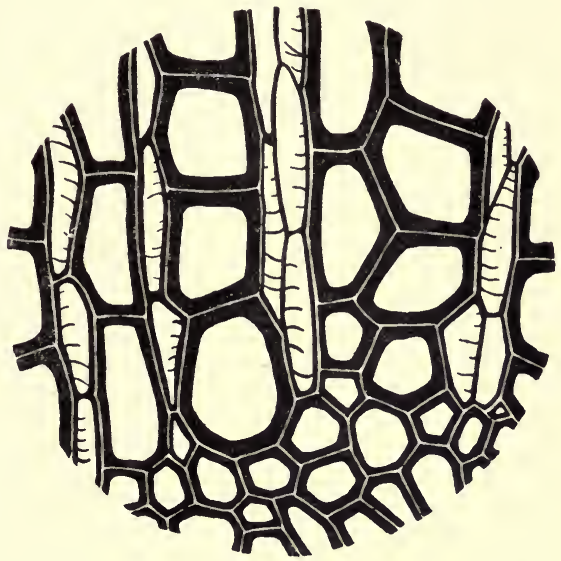

FIG. 46.-Transition from primary to secondary wood in a lepidodendrid. Description in the text. the situation here by

reference to Fig. 45, which shows a longitudinal view of the tracheary and allied structures of the first-formed wood of a specimen of the genus Lepidodendron from the Carboniferous of Lancashire, England. It is obvious that, in addition to the longer cells with reticulately thickened walls-the tracheids proper-there are numerous short elements with a similar kind of sculpture. In series with these are other cells again which are quite without the usual tracheary thickenings and which belong, in fact, not to the water-conducting system, but to the storage category. These are wood parenchyma. It is evident in the present instance that the storage cells of the primary wood have been derived from what were originally tracheids by septation or division and subsequent differentiation. It has been demonstrated in the previous chapter 
that the parenchymatous structures of the secondary wood are in the first place derived from modified tracheids.

In Fig. 46 is shown the region of transition from primary to secondary wood in a lepidodendrid, somewhat highly magnified. The secondary tissue is most characteristically distinguished by

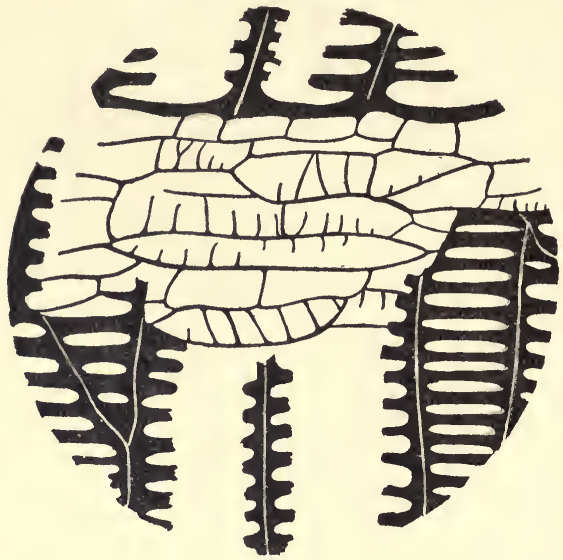

FIg. 47.-Radial view of the secondary wood of a lepidodendrid, showing tracheary origin of the rays. its rays running in alternation with bands of large tracheids. These radially directed stripes of storage elements at once attract attention by reason of the unusual organization of their component cells. The constituent units of the rays in this instance are reticulately thickened after the manner of tracheids and, in fact, differ from these only by their abbreviated length and the somewhat more delicate nature of their sculpture. There is, indeed, not the slightest doubt that in the case of the lepidodendrids the rays are largely, and in some instances wholly, composed of cells belonging to the category of tracheids. This situation is clear in radial sections taken lengthwise through the wood. Fig. 47 represents such a section from the root of the lepidodendroid type know as stigmaria. The heavy sculpture of the tracheids composing the mass of the wood can be easily made out. Running at right angles to the direction of the tracheary elements of the wood are the cells of a ray. These again show a considerable degree of scalariform thickening, only a few being completely devoid of this form of sculpture. It is obvious from the conditions described in the case of the lepidodendrids, a group of arboreal club mosses flourishing 
in the Paleozoic, that rays in this type were more or less largely composed of tracheids. It seems clear for this reason that the radial bands of storage parenchyma which constitute the rays in secondary wood, like the longitudinal parenchyma included under the caption of wood parenchyma, are derived from the modification of tracheary tissue. It thus becomes apparent that originally all the parenchymatous constituents of wood, whether primary or secondary or radial or longitudinal, in the first instance made their appearance by the modification of tracheary tissues. Tracheids, in fact, constitute the sole original feature of organization in woods, and the course of evolution expressing itself in continued differentiation has led to the derivation of all the other features of ligneous structure from this primary constituent. In other words, wood primitively was a purely water-conducting tissue, and the superadded mechanical and storage functions subserved by its organization in later geologic times resulted in appropriate modifications of the original tracheary elements. The derivation of the rays from tracheary tissues can be distinguished clearly only in the lepidodendrids and their allies. In others of the arboreal cryptogams which were so characteristic of the forests of the Paleozoic age no evidence of the origin of ray cells from tracheids has been observed. The same statement holds for the lower and ancient gymnosperms, the Cycadofilicales (Pteridospermae of Oliver and Scott) and the Cordaitales and their allies. These antique gymnospermous groups, as well as the arboreal cryptogams signalized above, had no storage tissues in their wood other than radial parenchyma; for the longitudinal parenchymatous elements known as wood parenchyma proper made their appearance only in connection with the seasonal refrigeration which became ever more pronounced during Mesozoic and later geologic time. The rays of the older plants with secondary growth were of two main types. In some instances (e.g., Cycadofilicales) they were composed of bands of cells several elements in width and greatly varying in height. In the Cordaitales and allied forms the rays were ordinarily uniseriate - that is, a single cell in width, in contrast to their often multiseriate and considerable height. In a general way it seems clear that woods of the first type are perpetuated in the still living, although much reduced, Cycadales, while the Cordaitales, 
according to common consent, find their successors in the conifers of the present age.

Since it is highly probable that the Cordaitales gave origin to the Coniferales, and since, as a consequence, they present us with

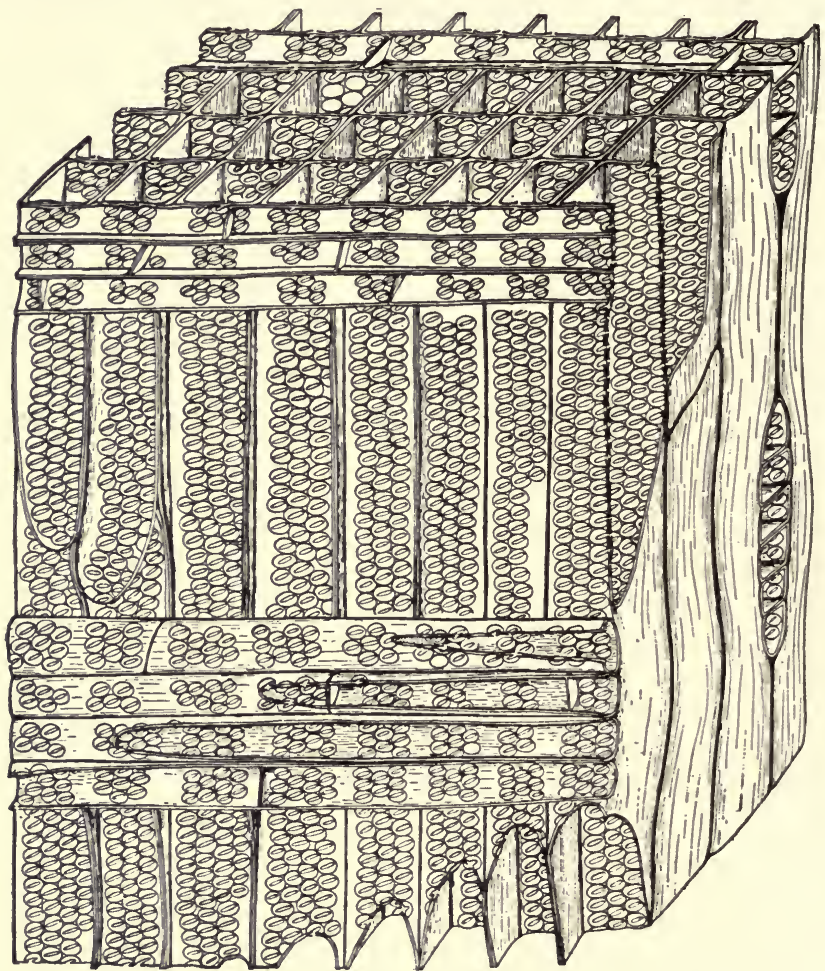

FIg. 48.-View of cordaitean wood in three dimensions. Explanation in the text.

the starting-point of the radial parenchyma of the latter, it will be well to consider the organization of this group in respect to the structure of the rays. Fig. 48 shows the structure of a cordaitean wood. The tracheids in this genus are usually extremely long; 
consequently only a portion of their length is represented in the figure. The pitting is somewhat characteristic and is ordinarily marked by the large number of pores and their consequent crowded and alternating arrangement. The rays cross the direction of the longitudinal elements and are characterized by their thin walls, which are, however, clearly pitted laterally in relation to the radial walls of the tracheids. It is to be noted in passing that there are no tangential pits on the walls of the longitudinal elements of the wood-a feature, as has been indicated in a previous chapter, very generally characteristic of the woods of the gymnosperms of the Paleozoic regardless of their affinities. In the transverse section of the wood the rays stand out distinctly as uniseriate files of cells, having their axes radially elongated. The rays are in lateral communication with the tracheids by half-bordered pits. Otherwise their thin walls are not characterized by the presence of pores. The tracheary elements of the wood are tangentially in communication by numerous radial pits, but their tangential walls are quite free from pores, so that in these ancient gymnosperms water could make progress in the trunk only in a tangential direction. The tangential view of the wood shows the radial pits of the tracheids as well as the lateral ones of the cells of the medullary rays in profile view.

After the discussion of the organization of rays in the Cordaitales we are in a favorable position to understand the condition in the conifers. In this connection it will be well to start with the most complicated condition in living representatives of the group, since the coniferous gymnosperms, as will be shown clearly in a subsequent chapter, constitute a reduction series with the more complex forms at the bottom and those with simpler organization at the top. Fig. 49 represents radial and tangential views of the wood of the white pine (Pinus strobus). Taking first the radial view shown in $a$, a number of important contrasts in organization to cordaitean woods are to be seen. First of all as regards the tracheids, or rather such part of them as is included in the field of view, it is clear that they are distinguished from similar structures in the Cordaitales by the smaller number of pits and the considerably larger size of these. Further, the pits in face view, instead of presenting the 
merely double contour of the more ancient group, are marked by triple concentric outlines. The outer circle corresponds to the boundary of the pit membrane, while the inner one outlines the mouth or aperture of the pit. The intermediate circular outline delimits the thickened central region of the torus, a structure, which so far as is known, was not present in Paleozoic gymnosperms. Its presence in the conifers is in all probability correlated in some

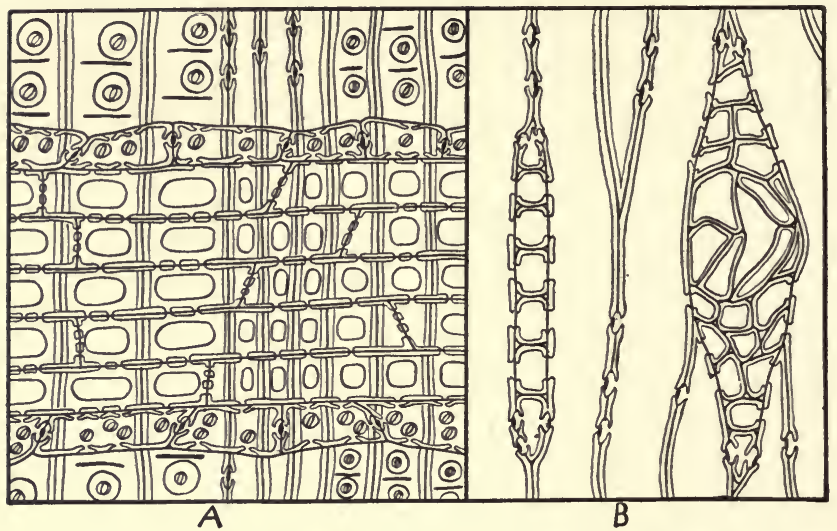

Fig. 49.-Radial and tangential sections of the wood of the white pine (Pinus Strobus). Explanation in the text.

way with the large size of the bordered pits; it has, in fact, as pointed out in an earlier chapter, been interpreted as a safety device useful in preventing the rupture of the broad pit membranes under extreme pressure. Not only are the pits of large size and more complex organization in the pine than in cordaitean forms, but the walls of the tracheary elements in their vicinity, particularly toward the ends of the tracheids, are distinguished by transverse bands of cellulosic or pectocellulosic material, and these are conveniently designated "bars of Sanio." They should not be confused with the trabeculae, radially directed lignified bars running transversely through the lumina of the cells of many gymnosperms living and extinct. (They are even found in some cases among the 
angiosperms.) There is good reason to believe that the "bars of Sanio" are an original and characteristic feature of the organization of the wood of the Coniferales and allied groups. The tracheids shown in the figure differ from those of the cordaitean forms by their periodic variation in size, those laid down in the beginning of the annual increment being of larger caliber than those coming into existence toward its close. The late tracheids are distinguished, as indicated in earlier pages, by their tangential pits seen in profile in the radial section. Turning our attention now to the ray itself, we see at once from the figure that a considerably greater degree of complication is present than that exemplified by the similar structure in the case of the Cordaitales. Manifestly the radial elements are of two kinds. First there are the cells which constitute the central region of the ray and which in life are characterized by protoplasmic and other contents. This situation is indicated by the copious simple pits which ornament the vertical and horizontal walls of the cells. The strong pitting is also a clear feature of difference from the Cordaitales where the walls of the ray cells are in general thin and unpitted. Laterally the central elements of the rays are related to the tracheids by means of very large, somewhat angular pits. The second type of element characteristic of the ray in Pinus is likewise distinguished by the nature of its pitting. All the pits seen on the walls, whether horizontal, vertical, or lateral (related to the tracheids), belong to the bordered type. The cells of the ray possessing this peculiar organization are typically marginal in position and, naturally, are quite without protoplasmic contents, except in the early stages of development. Such tracheary elements in coniferous rays are commonly designated marginal tracheids or simply marginal cells. They obviously permit the easy movement of water in the radial direction in those woods characterized by their presence.

In the tangential view presented in $b$, Fig. 49 , it is clear that there are two types of rays-namely, narrow uniseriate ones and broader ones tapering at either end to the uniseriate condition. The former are known as linear rays and the latter as fusiform rays. It is evident from the figure that the ray of greater width is characterized by the presence of a partly occluded cavity, a resin canal. 
The stopping up of the resin canal is explained by the fact that the section is taken from the heartwood, which, as has been pointed

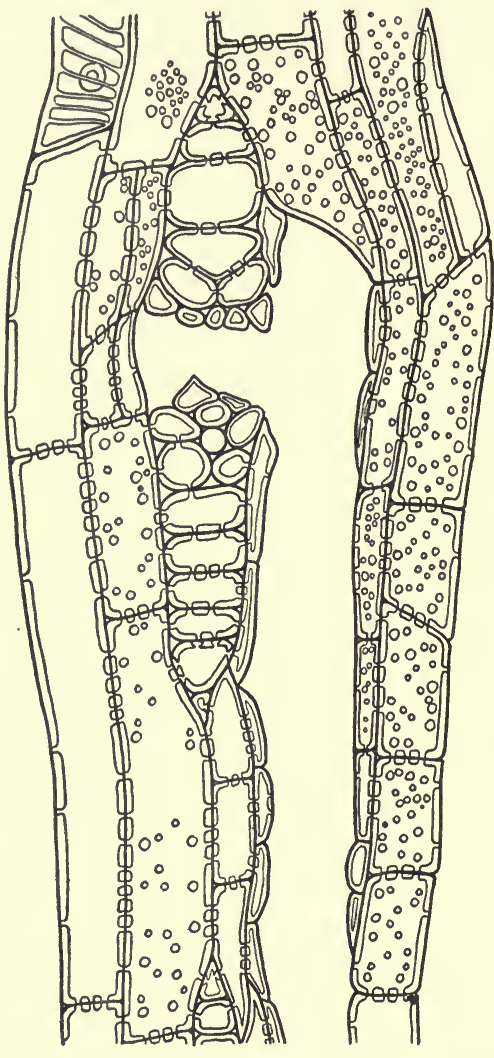

Fig. 50.-Tangential view of the wood of the Pseudotsuga. Explanation in the text. out in a former chapter, is distinguished by the phenomenon of tylosis or occlusion of the secretory spaces by means of ingrowths of the resiniferous parenchyma. In the case of the narrow or linear ray it is possible to distinguish in the tangential view, by means of their characteristic pitting, both the parenchymatous central cells and the tracheary marginal elements. The central elements with their simple pits are in relation to air spaces in the angles. The presence of both linear and fusiform rays is a constant feature of organization in the pine and its nearer allies. The linear rays are doubtless an older feature than the larger ones which contain the horizontal resin canals, since they resemble most nearly the radial parenchymatous structures of the Cordaitales. The fusiform rays serve to bring about a connection between the vertical resin canals in different annual rings, not 
only with one another, but often with the similar but larger secretory cavities which are present in the bark.

Fig. 50 illustrates the relation between the vertical and horizontal resin canals as seen in a vertical section of the wood of the Douglas fir (Pseudotsuga). To the left of the center lies a fusiform ray with its included resin space, which opens broadly on the right into a vertical secretory canal. The plane of section happens to lie near the end of the annual ring so that the cellular structures lying in view are almost entirely parenchymatous. The lining of the resiniferous spaces is evidently largely composed of elements with thick walls and bordered pits, a condition very commonly present in the representatives of the Pineae other than Pinus itself.

After the discussion of

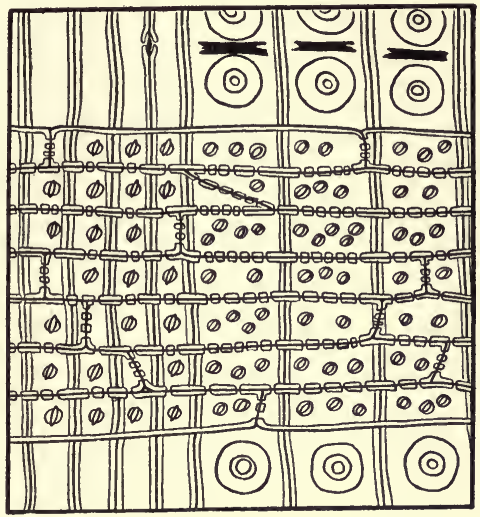

Fig. 51.-Ray of the balsam fir. Explanation in the text.

the pine and its nearer allies we may proceed with advantage to the description of the ray structures in coniferous woods of simpler organization. Fig. 5I illustrates the structure of the ray in the wood of the balsam fir (Abies balsamea). It is clear that in this case the cells of the rays are all of one kind and that the marginal tracheids are conspicuous by their absence. The parenchymatous elements which compose the rays in the genus under consideration are in relation to one another on both vertical and horizontal walls by numerous simple pits. The lateral walls show bordered pores such as ordinarily, except in certain species of Pinus, characterize the relation between rays and tracheids. The organization of the linear rays in conifers other than the Abietineae is in general of a simple nature; and in the higher 
subtribes, such as the Cupressineae, Taxodineae, and Taxineae, even the intercommunicating simple pits of the horizontal and vertical walls of the ray cells are clearly and often conspicuously absent. In such cases, as is to be expected, the wall of the ray elements is in general thinner and often curved.

An interesting condition of organization of the radial paren-

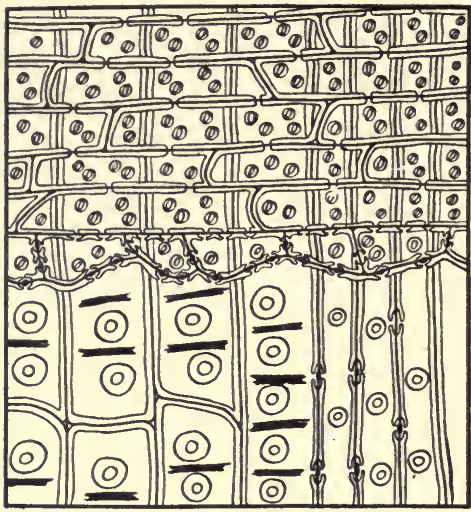

FIG. 52.-Ray of the Nootka cypress. Description in the text. chyma is presented by Chamaecyparis nootkatensis. Here, as is shown in Fig. $5^{2}$, the rays are frequently marked by the presence of tracheary elements on one or both margins. The illustration represents the radial aspect of the transition from summer to spring wood, and the features of structure are in general such as one would expect to find under the circumstances, except as regards ray organization. The normal presence of marginal tracheids in the species figured gains a special evolutionary significance from the fact that similar conditions are found in other representatives of the Taxodineae, Cupressineae, and the genus Abies among the Abietineae as a consequence of injury. For reasons which will be fully discussed in a subsequent chapter it seems quite clear that the structures which are found to appear as a result of injury in vascular plants with secondary growth are often of the nature of reversions to an ancestral condition. Fig. 53 illustrates in radial view the wood formed after injury in a root of the Big Tree (Sequoia gigantea). Parts of two annual rings are included, and the ray clearly shows features of structure which are abnormal for the Taxodineae. To the left below and in the spring wood can be seen several cells included in the substance of the ray which are 
clearly tracheary in their character, since they contrast with the adjoining elements both in the absence of protoplasmic contents and in the occurrence of bordered pits in all their walls. It is evident that in the case under discussion short tracheids may make their appearance among the elements of the radial parenchyma as a sequel to injury. The interesting investigations of Miss Holden

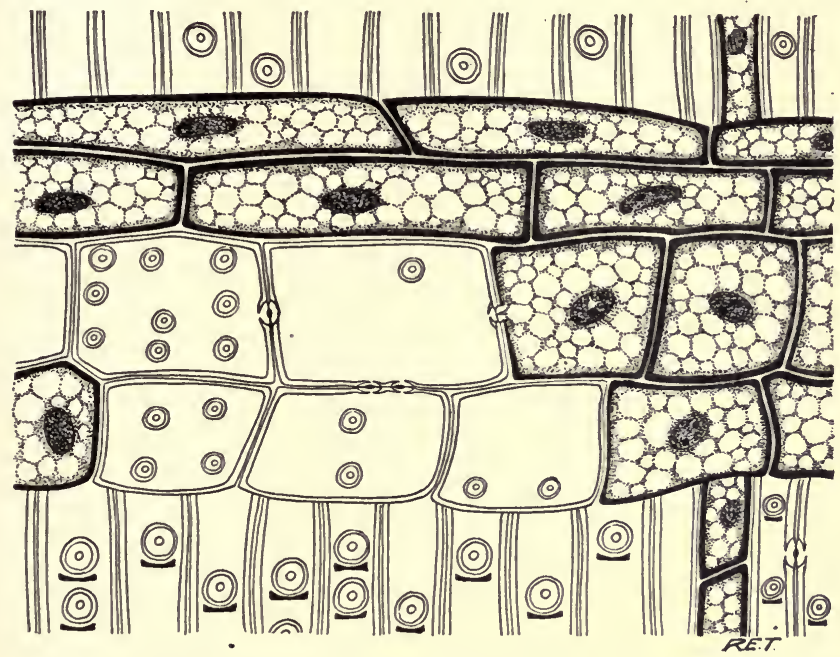

Frg. 53.-Ray from the injured root of Sequoia gigantea. Explanation in the text (after Holden).

on the Cupressineae and Taxodineae as a whole make it clear that abnormalities of this kind in these subtribes of coniferous gymnosperms are a common feature in.the wood formed after injury.

It follows from the statements and illustrations in connection with the last paragraph that normally in Chamaecyparis nootkatensis and traumatically in practically all representatives of the Cupressineae and Taxodineae, ray-tracheids are found such as are a feature of the normal structure of the wood in the lower members of the Abietineae. The most natural interpretation of this phenomenon is in connection with the biological doctrine of 
reversion. This general doctrine will receive particular consideration at a later stage and consequently need not be elucidated here. If the radial tracheids occurring under the conditions described in the foregoing paragraph are interpreted as a reversion to an ancestral condition, it follows that the simple type of ray found in Abies and in the Cupressineae or Taxodineae is by no means primitive,

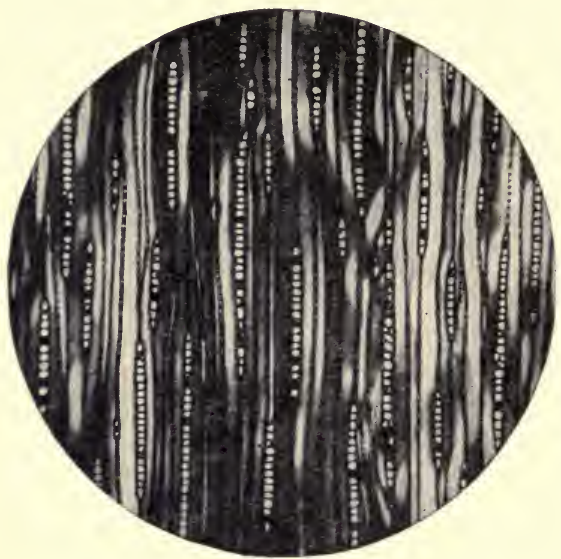

FIG. 54.-Tangential section of the normal wood of Cedrus Libani.

but is the result of simplification from the more complex state of organization of the ray, characteristic of the lower living Abietineae. Obviously an experimental as well as a purely anatomical investigation of ray structures is necessary for their complete morphological and evolutionary understanding.

Not only does one find in the case of certain coniferous woods of simpler organization evidence of derivation from ancestral types presenting the complication of marginal ray-tracheids, but likewise in the genus Cedrus among the Abieteae, which has normally only linear or uniseriate rays, fusiform radial structures containing horizontal resin canals are found. This situation is made clear by Figs. 54 and 55, which present tangential views of the normal and injured wood of Cedrus Libani (the cedar of Lebanon). In the traumatic or injured wood of cedar, which, so far as the geological record supplies us with definite evidence, is the oldest representative of the Abieteae or firlike conifers, we have clearly indicated a condition ensuing from injury which definitely unites the cedar 
with the genus Pinus, much older geologically and more complicated in the normal organization of the wood. It will be clear from the statement in this connection that fusiform rays have also an importance from the experimental standpoint, quite comparable with marginal tracheids, but less copiously expressed in traumatic phenomena.

The rays of the Cycadales and their allies present no features of special evolutionary interest, so far at any rate as is known at the present time; they may therefore be dismissed with the simple statement that they are typically multiseriate bands and not the linear structures constituting the primitive condition

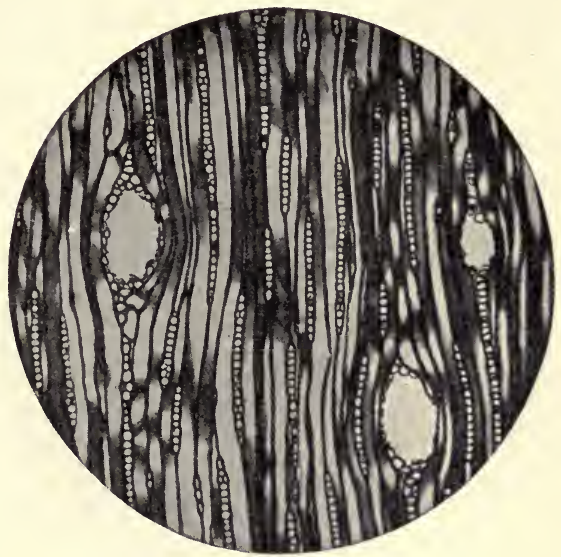

FIG. 55.-Tangential section of the wood of Cedrus Libani formed after injury.

of organization of the radial parenchyma for the Cordaitales, Coniferales, and Ginkgoales. The ray structures in the highest gymnosperms, the Gnetales, are best discussed in connection with the similar features of dicotyledonous woods, which they resemble in so many respects. This procedure is the more desirable because the living Gnetales are represented by a very small number of genera of widely separated geographical ranges.

At this point the dicotyledonous angiosperms may appropriately be considered in regard to the organization of their rays. Fig. $5^{6}$ reproduces a transverse section of the wood of the oak. The structure in this case is highly complicated and corresponds to a marked development of the principle of division of labor. The movement of water, the functions of strength and of storage, are all distinctly 
allocated to particular and highly differentiated categories of cells. In the present connection we are concerned only with the structures included under the heading of radial parenchyma. Clearly the rays in the oak are of two types, even as seen in transverse section. A small number are very broad and constitute a large bulk of storage tissue. In contrast to these in respect both to size and to number are linear rays, abundantly present in the figure.

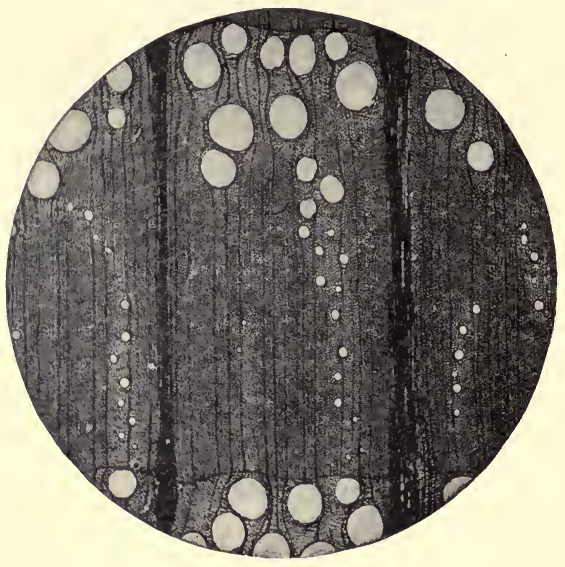

Fig. 56.-Transverse section of the wood of the red oak.

It is best in this instance to focus our attention on the composition of the uniseriate or linear rays and their relation to the various elements represented in the complex organization of the wood in the same genus, Quercus.

Fig. 57 represents the uniseriate rays in radial and tangential aspect. In $a$ is seen the tangential view.

The ray is obviously composed of cells which are all alike and related to one another and to the air spaces by simple pits. Halfbordered pits connect ray cells with tracheids. In $b$ and $c$ are shown radial aspects of the ray in relation to the various structural elements of the wood. On the left and right in $b$ files of vertical parenchyma cross its course and are related to the radial elements by groups of clustered pits. To the left of the middle of $b$ is shown a vessel (this type of element will be considered in the following chapter) which communicates with the radial storage elements by large, generally oval, pits. The rest of the width of $b$ is occupied by tracheids, which in turn communicate with the cells of the 
rays by small bordered pits. In $c$ is seen a region where the linear ray passes through a purely fibrous portion of the wood; and here there are no pits at all, since the mechanical elements which, as has been indicated in a former chapter, have been differentiated from the tracheids no longer supply water to the other structures of the wood. It will be clear from the foregoing account that the uniseriate or linear rays of the oak are of uniform and simple organization as regards their constituent elements, but that these are characterized by a variety of pitting corresponding to the high degree of differentiation of the wood
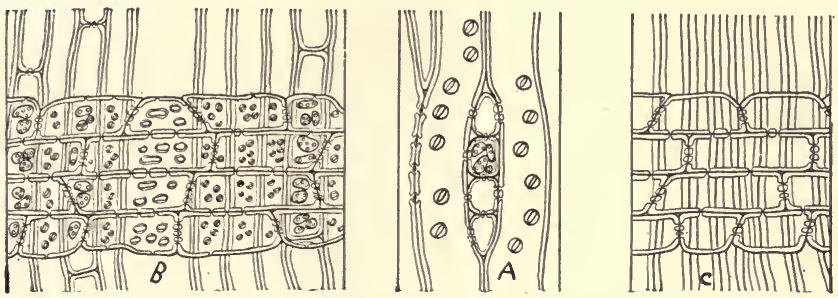

Fig. 57.-Longitudinal and transverse views of linear rays in the oak. Explanation in the text.

through which they pass. The large rays of the oak can better be considered at a later stage after a type showing a more generalized condition of radial organization has been examined.

It will be convenient and profitable to consider in the present connection the genus Casuarina, which occurs in the East Indian and Australasian regions, since here we find in various species all the main types of organization of the wood rays exemplified by the dicotyledons. First is presented the tangential, longitudinal view of the wood in Casuarina Fraseri. Here the structural conditions are manifestly very similar to those found in the oaks of northern latitudes, for there are two distinctly contrasted categories of raysnamely, the numerous linear or uniseriate and the sparse broad rays. In comparison with the wood of $C$. Fraseri, presenting a strong resemblance to that of a white or black oak (Fig. 58), is that of C. torulosa, shown in Fig. 59. Here the linear rays are as 
in C. Fraseri, but the large band of radial parenchyma is obviously not homogeneously parenchymatous, but is separated into pointed groups by the presence of interspersed fibers. In other words, instead of a continuous mass of storage tissue there is present an aggregation of rays of a certain size, separated from one another by strands of fibers. This condition of the large ray may be conveniently designated as aggregate. In Fig. 60 is represented

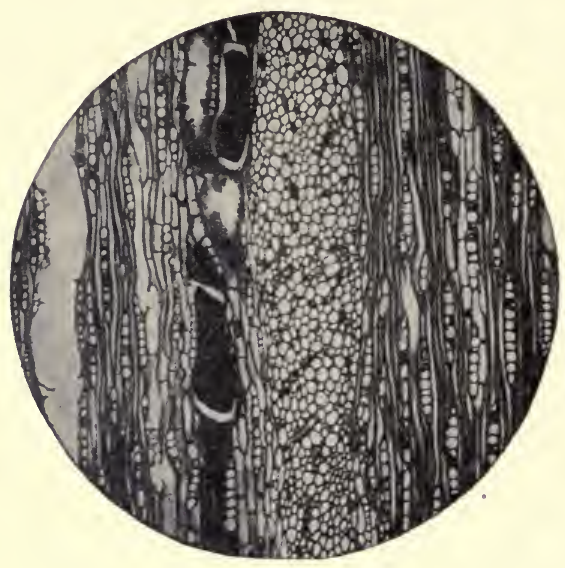

Fig. 58.-Tangential section of the wood of Casuarina Fraseri. Explanation in the text.

the tangential aspect of the wood in C. equisetifolia. In this type we no longer see a sharp contrast between large multiseriate or aggregate rays and small entirely uniseriate ones, but, as it were, a more democratic organization of the radial parenchyma, in which no extremely large radial parenchymatous masses. are found, as all grade almost imperceptibly into one another in size. The last-described condition of the rays, for reasons to be indicated later, is here designated as diffuse. We have thus in the single genus Casuarina three distinct types of radial parenchyma: first, the northern oak type in which huge rays stand in the sharpest contrast to narrow uniseriate bands; secondly, a condition in which the contrast still obtains as regards the dimensions of the rays, with the distinction that the large masses are not homogeneous but penetrated by bands of fibers; and, finally, there is to be noted a state or organization in which all rays are of moderate width and are scattered somewhat evenly throughout the tangential or transverse 
section of the wood. In the first condition the large rays are known as compound; in the second they are designated as aggregate, and in the third state, where there is generally no marked superiority in size and the rays intergrade almost imperceptibly, they are known as diffuse.

Of the three conditions of organization of radial parenchyma in the dicotyledons described above, the compound is extremely rare in trees but is commonly found in climbing and herbaceous types. The diffuse condition of the radial parenchyma is very common in forest trees, but is much less characteristic of plants of herbaceous texture. The genus Casuarina has purposely been chosen for the exemplification of the problems connected with the

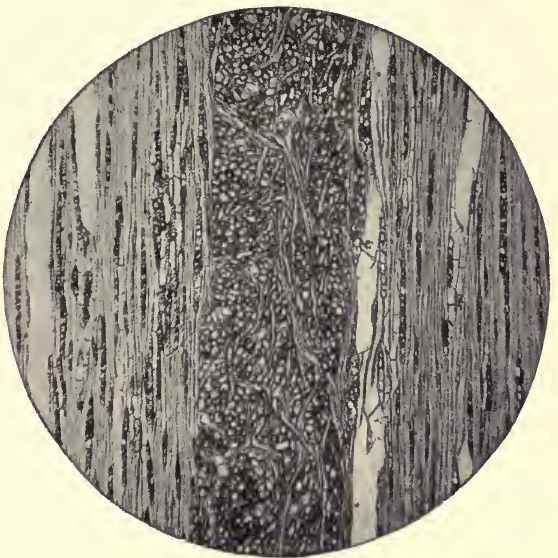

FIG. 59.- Tangential section of the wood of Casuarina torulosa. Explanationin the text. evolution of radial parenchyma in the dicotyledons, not because it is necessarily a primitive form, but because it shows the situation synoptically and, moreover, furnishes very clear evidence as to the relation of the various types to one another from the evolutionary standpoint.

An exposition of the interesting and important situation of the ray structures in the dicotyledons can best be approached by a diagrammatic comparison with the conditions presented by the conifers. Fig. 6r reproduces the essential features of organization of a coniferous stem with whorled leaves-for example, a Juniperus or a Cupressus. The leaves, three in number, are indicated in 
black on the periphery of the stem. Centrally placed is the pith, surrounded by the woody cylinder, which in turn is circled by the phloem and the cortex. The woody tissues are encroached

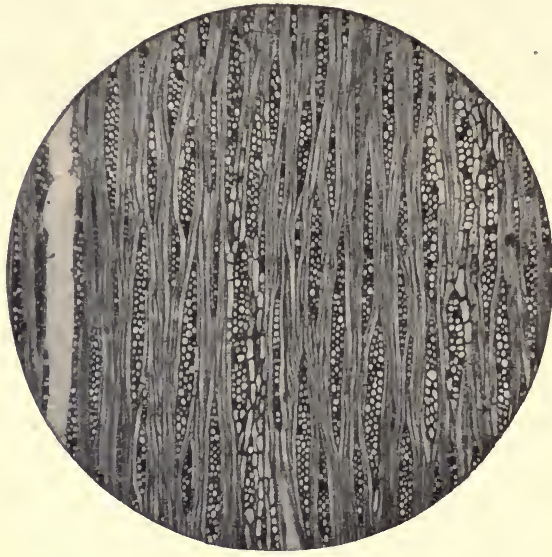

Fig. 60.-Tangential section of the wood of Casuarina equisetifolia. Explanation in the text.

upon by three deep bays extending from the medulla and subtended by the three leaves. These extensions from the pith mark the presence of the leaf gaps, interruptions in the continuity of the woody cylinder related to the passing out of the foliar traces. Later the intervals in the wood are covered by the activity of the cambium, so

that the cylinder becomes continuous in the second or third year of growth. Clearly there is no structural feature of importance in the woody cylinder related to the leaf trace other than the foliar gap. This is the general situation in the case of coniferous stems as well as in that of their Paleozoic ancestors, the Cordaitales.

Having diagrammatically compassed the organization of the stem in the conifers, we are in a position to consider the case of such a dicotyledon as Casuarina.

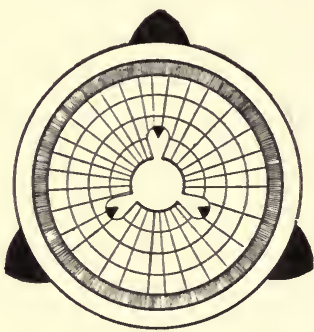

FIG. 6r.-Diagrammatic transverse section of a coniferous twig. Explanation in the text. In Fig. 62 arre reproduced the essential features of topography of a small branch in this genus. Leaves, as in the case of the conifer- 
ous diagram given previously, are represented in black on the periphery of the stem. The central pith is likewise encircled in turn by wood, phloem, and cortex. Here, too, there are excursions of the pith at six points, extending into the second annual increment of the wood; these subtend radially six corresponding leaves. In the diagram under discussion there are, however, two marked features of contrast to those presented in the foregoing scheme of a coniferous axis. First of all, the wood is characterized by the presence of vessels, and secondly by a broad radial stripe which extends from each leaf gap outward. This radial stripe contrasts with the rest of the woody cylinder by the absence of vessels and the clustering of rays. The radial parenchymatous stripes which lie in the region of the six radial bands corresponding to six leaves are not only more prominent than the linear and somewhat sparse rays in the rest of the wood but are of

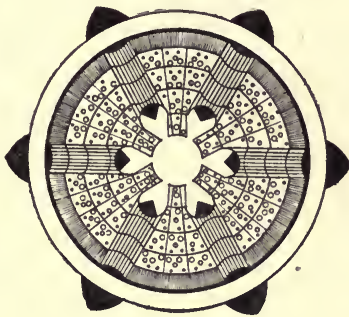

FIG. 62.-Diagrammatic transverse section of the stem in Casuarina. Explanation in the text. greater width. The aggregations of rays related to the leaves shown in the diagram are, in fact, clustered rays of the type exemplified by C. torulosa (Fig. 59) and are, as a consequence, aggregate rays. Since they are in this instance clearly related to leaves, they may at the same time be appropriately designated foliar aggregate rays.

With the exposition of the essential features of organization present in coniferous and dicotyledonous stems, respectively, we are in a position to proceed further with the highly important discussion of the evolution of the radial parenchymatous structures of dicotyledonous woods. Simplicity will be served and ambiguity avoided if in further elaboration we hold to the conception of the rays as they present themselves in a small twig of a few years' growth. Fig. 63 illustrates synoptically the main types of rays in the dicotyledons as seen in small branches. The only essential departure from the conditions in nature is the delineation of the 
three important categories of rays as occurring side by side in the same stem. In the center of the circle representing diagrammatically a dicotyledonous stem is figured a leaf gap and

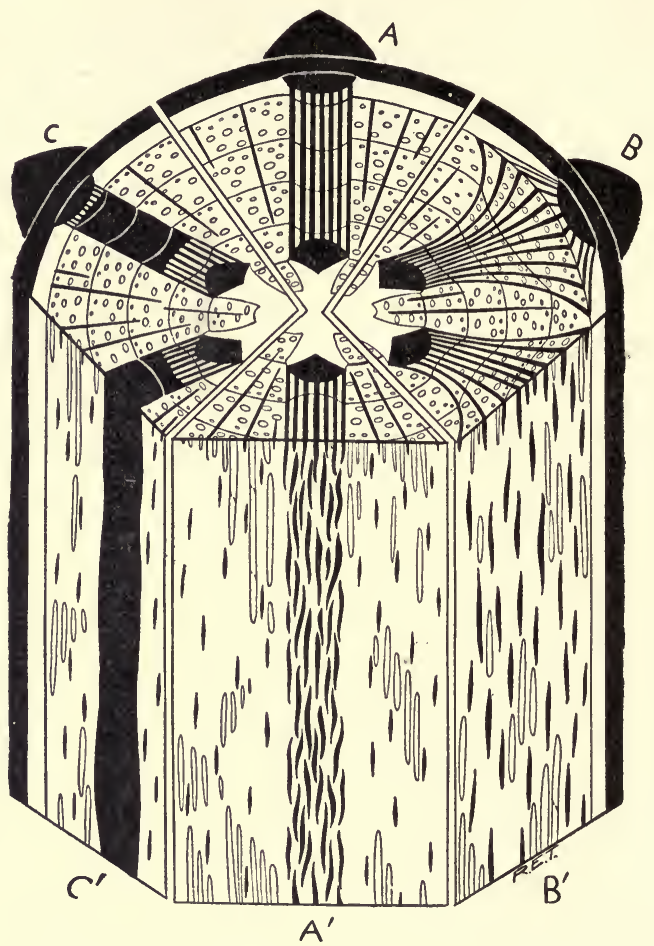

Fig. 63.-Synoptical diagram representing the transverse and longitudinal topography of the rays related to the leaves in species of Casuarina. Explanation in the text.

peripherally its subtending leaf. Between the two lies a corresponding ray of the aggregate type. It is to be noted here that there is a modification of the rate of growth of the annual rings 
in the region of the aggregate ray which results in their being locally depressed.

To the right is to be seen another leaf with its corresponding gap and ray. In this case the ray structure where it is still near the leaf trace (solid black) is the same as in that just described, namely, aggregate. Farther out, however, the components of the aggregate ray, instead of maintaining their original relations to one another, begin to diverge in the tangential plane. At the same time vessels which are conspicuous by their absence while the ray is in the aggregate condition begin to appear in the widening strands of wood which separate the diverging rays. This process continues and becomes more and more marked in successive outer annual rings. The final result is that what was once a congery or aggregation of rays separated from one another by purely fibrous strands becomes a more and more diffuse cluster of rays separated by everwidening vascularized intervals of wood. In this condition the original aggregation of rays not only becomes diffuse in a fanlike fashion in the outer region of the woody cylinder, but the individual rays subdivide, thus accentuating the condition of diffusion. The phenomenon of the subdivision of the rays for the sake of simplicity is omitted in the diagrammatic representation. It is clear that the appearance of the conditions depicted in the ray to the right of the diagram (at $b$ ) in the case of all the foliar rays of a stem would result in a diffusion of rays of a medium breadth throughout the older wood-in other words, to the condition shown in Fig. 60 for the adult wood of Casuarina equisetifolia or an allied species.

Turning now to the left of the diagram (at $c$ ), we observe a foliar or leaf ray of still another type. Here, as in the diffuse condition of the foliar ray represented in $b$, the original state is that of aggregation with the exclusion of vessels, a situation which is permanent in the type diagrammed at $a$. In the later annual rings in this type the aggregation becomes a homogeneous mass of parenchyma by the disappearance of the fibrous strands which separate the components of the aggregation or congery from one another. Where the clusters become fused into large homogeneous bands of storage tissue by the parenchymatous transformation of the original separating fibers of the aggregation, the result is the compound ray 
represented at $c$ in our diagram. This condition is present in the adult structure of the wood of Casuarina Fraseri, as is shown in Fig. 58. The primitive aggregate condition persists, on the other hand, in C. torulosa, as is shown in Fig. 59.

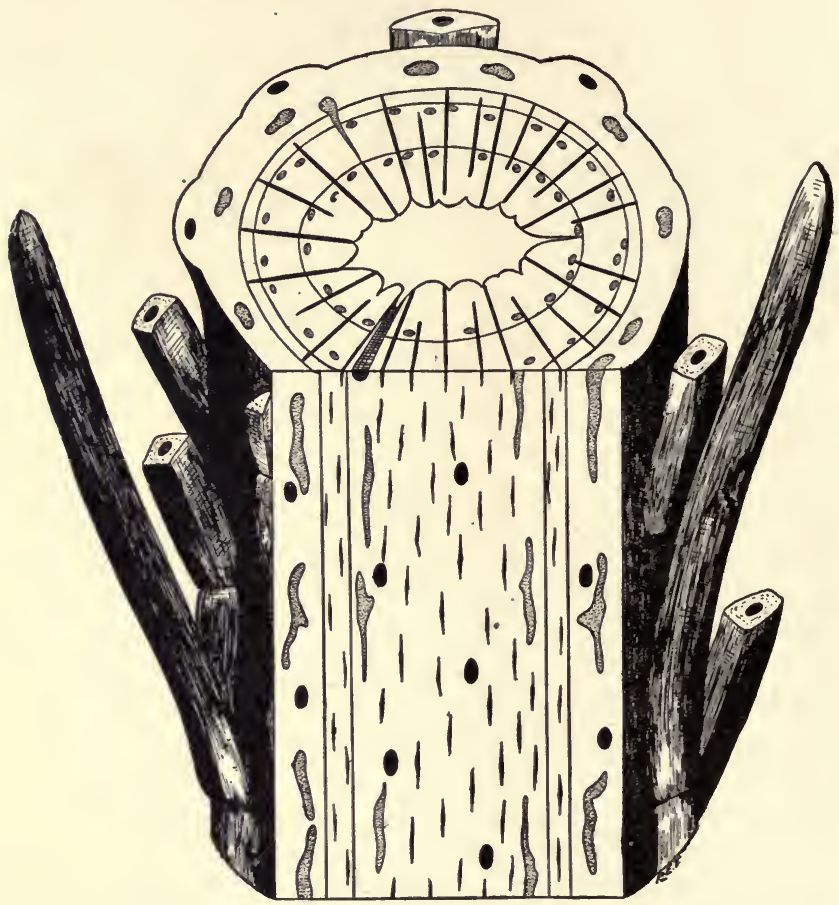

FIG. 64.-Diagrammatic representation of a twig of Picea canadensis. Explanation in the text.

In the longitudinal aspect of the diagram presented to the reader are shown the vertical views of the three types of rays illustrated horizontally in $a, b, c$. Fig. $63 a^{\prime}$ shows the tangential topography of an aggregate ray. The clustering of the masses of radial 
parenchyma and the exclusion of vessels can be readily seen. At $b^{\prime}$ is indicated the tangential projection of the diffuse condition of rays. Here the original cluster has become scattered and vessels are now present among the rays. In $c^{\prime}$ is shown the longitudinal tangential aspect of the compound ray-that is, the condition in which the original aggregation has become fused into a large solid mass of radial parenchyma.

The conditions in a diagram of a coniferous stem in three dimensions may now be discussed. Fig. 64 illustrates the topography of a two-year-old twig of Picea canadensis. In the transverse aspect the pith surrounded by xylem, phloem, and cortex can be dist inguished. Projecting from the surface are the

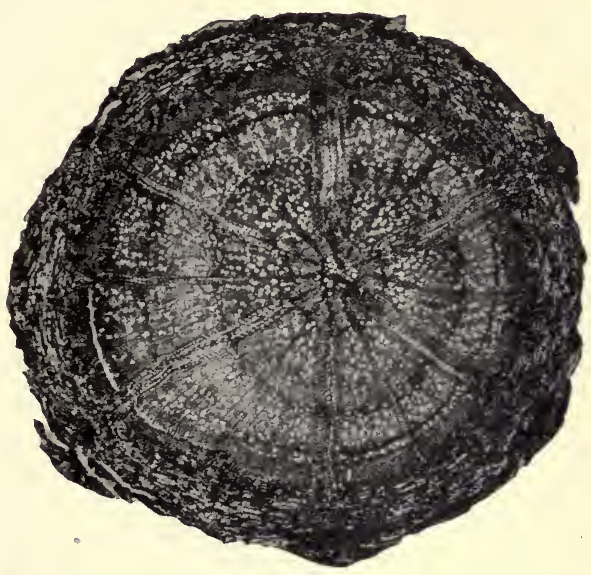

Fig. 65.-Transverse section of a twig of Casuarina Fraseri

leaves or their bases. The outline of the pith is indented by a number of bays, which are the deeper the nearer they are in the vertical plane to the departing trace of a leaf. In relation to one of these on the side of the pith nearer the observer is an actual trace running horizontally in the wood. The transverse aspect of the wood shows the presence of numerous narrow rays. The face of the stem facing the observer is cut away to show the topographical relations of the leaf traces in the wood. It is clear that, contrary to the conditions observed in the stem of Casuarina diagrammatically represented in Fig. 62, there are no modifications in the grouping of the rays or in other features with reference to the foliar traces (appearing as oval dots). 
It will be advantageous as a sequel to the representation of the prominent types of rays in the dicotyledons in diagram to view them in actual photographs in the case of the genus Casuarina. We may profitably begin here, as in the case of the diagrams, with transverse sections. Fig. $6_{5}$ reproduces the cross-section of a small twig of Casuarina Fraseri. The leaves are in the main still present in depressions on the surface of the stem and clearly sub-

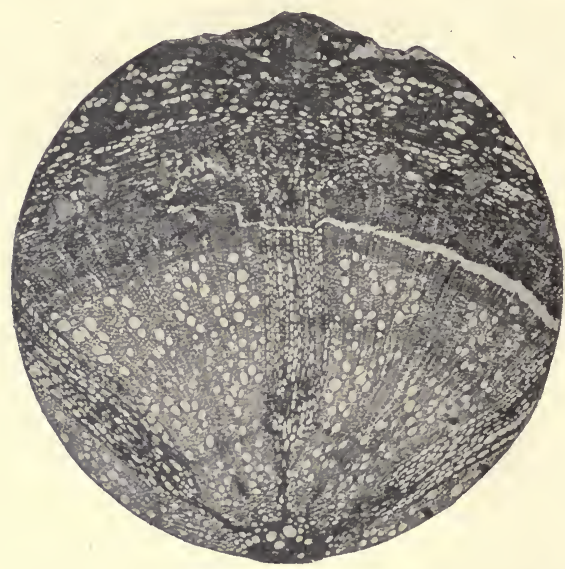

Fig. 66.-Part of transverse section of a twig of Casuarina Fraseri more highly magnified.

tend the foliar rays. Rather narrow leaf gaps penetrate the first annual ring and are twice as numerous as the appendages at a given node (that is, there are twelve gaps, although only six a p pendages ). This duplication of the gaps is due to the fact that the whorls of leaves alternate at different nodes, and the gaps corresponding to these are persistent, with the natural result of gaps twice as numerous as the appendages. Fig. 66 shows part of the foregoing much more highly magnified. At the top is a persistent leaf and in the middle line below lies the pith, which is sending off an extension, the leaf gap; and this, on reaching the second annual ring, undergoes considerable enlargement. Between the wide termination of the leaf gap in the beginning of the second annual ring and in line with and subtending the leaf on the outside of the stem lies the foliar ray. The magnification is not sufficient in the figure to show the organization of the leaf ray; hence a more enlarged representation is introduced in Fig. 67. Here it is clear 
that the structure of the ray in the twig in $C$. Fraseri is different from the adult condition, shown in Figs. 58 and 63 , for in the younger axis the ray is obviously penetrated by fibers, and these are absent in the adult. The truth of this statement will become still more apparent by reference to Fig. 68, which reproduces the tangential aspect of the wood in a somewhat older branch of the same species. The prominent mass of radial storage tissue in the center is the foliar ray. It is distinctly fibrous and is consequently still in the condition of aggregation. As the stem thickens the fibers are gradually transformed in to parenchymatous elements more and more like the cells of the ray. Thus it is that the compound ray of C. Fraseri comes into being. It is evident that its early condition is

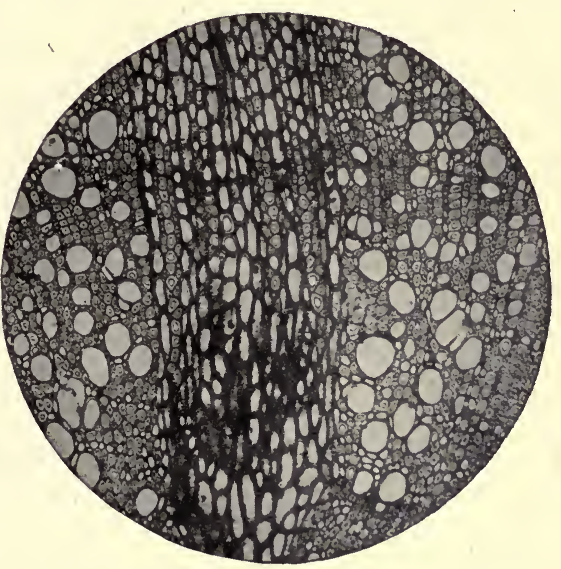

Fig. 67.-Portion of Fig. 66 still more highly magnified to show organization of the foliar ray. Explanation in the text. one of aggregation, and that this is followed by a gradual transformation into the compound state by the fusion of the originally separate members of the aggregation.

The photographic representations lead likewise to conclusions in harmony with the diagrammatic figures in the case of the diffuse condition of the foliar ray. It will not be necessary to introduce a total general and a partial more detailed view of the twig in this instance, since the topographical relations are practically the same as those shown in the case of C. Fraseri. Fig. 69 illustrates the situation in the diffusion of the foliar ray as exemplified by 
C. stricta. It is evident from the photograph that a mass of ray tissues on the left (representing the end nearer the pith), characterized by moderate breadth and the absence of vessels, passes toward the right (outer side topographically) into a continually widening fanlike cluster of rays among which vessels become more and more prominent. Fig. 70 shows a tangential, longitudinal section of the wood in a small branch of $C$. equisetifolia. Here is

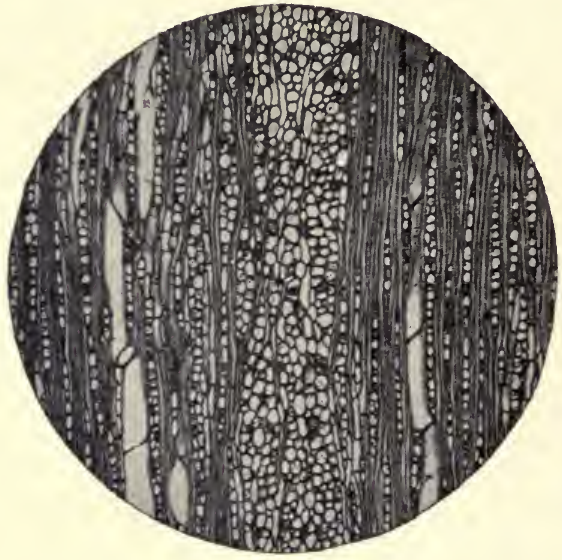

Fig. 68.-Tangential section of a large ray in the younger stem of Casuarina Fraseri, showing aggregate condition.

present an aggregation of rays, foliar in its character, which as yet has scarcely begun to diverge into the diffuse condition and consequently includes no vessels in its substance. C. stricta and $C$. equisetifolia are both species with the diffuse type of ray in the adult wood. C. stricta has been chosen to illustrate the transverse aspect of the diverging rays in the diffuse type only because the larger size of the radial bands in this species make the topographical relations more obvious. The difference in gross appearance between stems with aggregate or compound rays on the one hand and diffuse rays on the other hand, is very striking. This is well illustrated by Fig. 7oa. On the left is seen a polished segment of the trunk of $C$. Fraseri (compound and aggregate rays). On the right appears a polished trunk of $C$. stricta (diffuse rays). Here the rays are at first distinct and later die out. The conspicuous stage is aggregate, while that representing the disappearance 
of noteworthy bands of radial parenchyma marks the diffuse condition.

It has been demonstrated in the case of Casuarina that there are three main types of radial parenchyma in the secondary wood -namely, the aggregate, the diffuse, and the compound. Of these three types the aggregate is manifestly the oldest, and the other two have originated from it by divergence in the diffuse

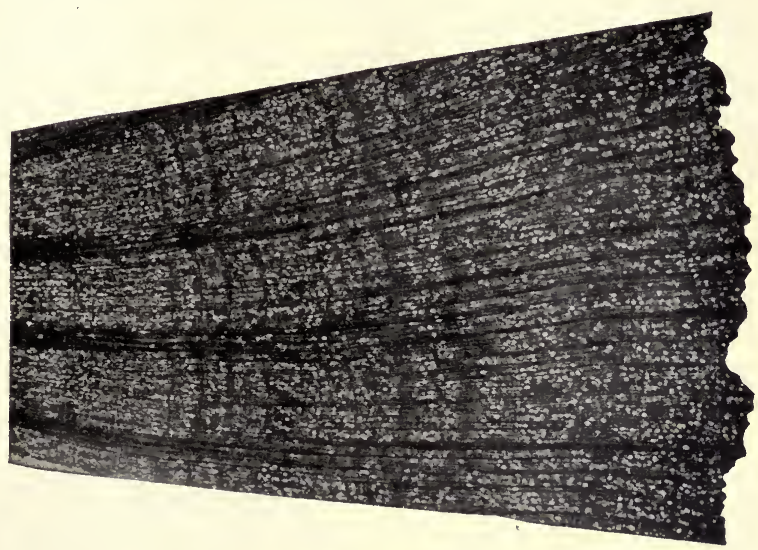

FIG. 69.-Transverse section of aggregate rays diverging into the diffuse condition in Casuarina stricta.

condition and by fusion in that state designated compound. What is diagrammatically clear in Casuarina for the radial parenchymatous structures is much less obvious in most other dicotyledons, and in no case are the relations so well shown as in the genus named. Among arboreal forms the oak is of interest in exhibiting both the aggregate and the compound condition of the rays. In this genus the species occurring in warm regions are ordinarily characterized by the possession of clustered or aggregate large rays in contrast to the uniseriate or linear rays present in the mass of the wood. In species of northern climates the rays are solid or compound in their nature, but even here the condition of aggregation 
is found in the young plant as a passing phase and as frequently persisting for many years in that most conservative of plant organs,

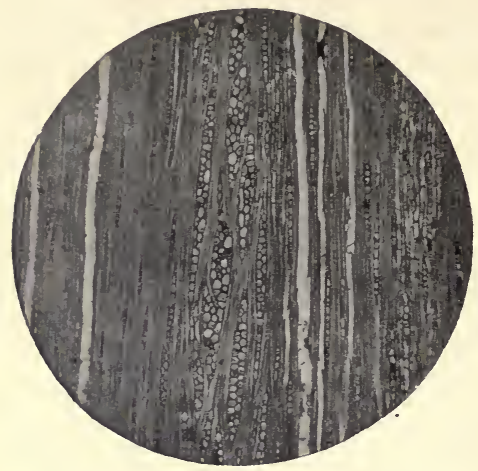

FIG. 70.-Tangential section of young twig of Casuarina equisetifolia, showing an aggregate foliar ray in center flanked on either side by uniseriate rays. the root. In the Betulaceae, by contrast, the diffuse type of wood ray prevails, and it is likewise clearly derived from a primitive state of aggregation. In the birch, for example, diffuse rays more usually distinguish the stem, while the aggregate type is often found present in the case of the root. The general anatomical conditions as well as experimental data in the Fagaceae and Betulaceae justify the conclu-
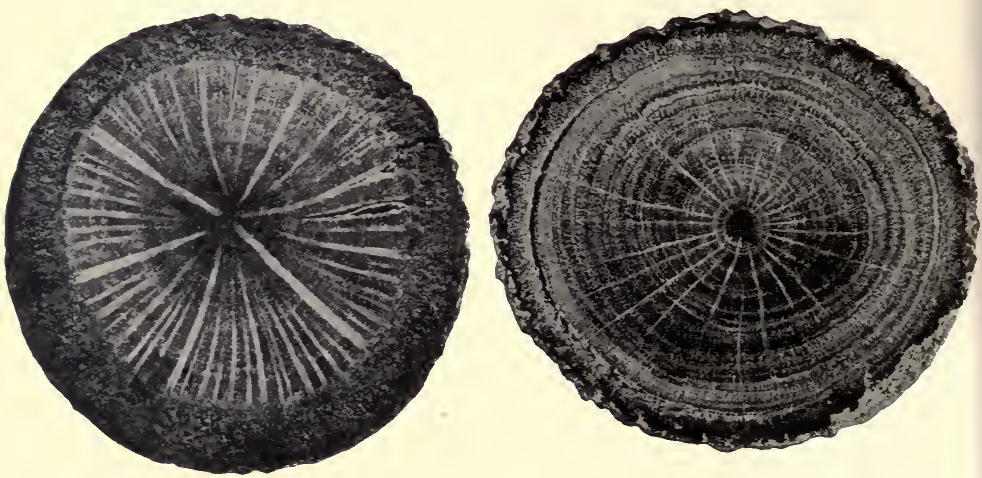

Fig. 70a.-Photographs of transverse views of polished stems of Casuarina stricta (right) and Casuarina Fraseri, showing the general topography of diffuse and compound rays. The diffusing rays of $C$. stricta appear to die out, while the rays of the other species become not only accentuated but also clearly more numerous. 
sion that wherever the compound or diffuse types of rays are present in members of these groups they have been derived from an earlier condition of aggregation. The type of ray structure is not strictly related to any particular organization of the flower, since amentaceous, archichlamydeous, and metachlamydeous families present to an almost equal extent the various categories of rays. In a general way it may, however, be stated that the diffuse condition of the rays is characteristic of arboreal forms, while the compound condition occurs mainly in vines and herbs. 


\section{CHAPTER VII}

THE FIBROVASCULAR TISSUES: SECONDARY WOOD-VESSELS

The vessel is an element of structure which in the higher forms and in the secondary wood is of extremely great evolutionary importance. Before we take up this type of ligneous element in the seed plants it will be well to consider its occurrence in lower forms and in the primary wood. It was pointed out by De Bary many years ago that many of the scalariform tracheids of the bundles in the bracken fern are of the nature of vessels, using that term to apply to elements which are not merely pitted but actually perforated at the ends, thus permitting a much more ready passage of water. The statements of the distinguished anatomist of Strassburg have been confirmed and denied by more recent investigators, but there seems to be no doubt whatever on the basis of our improved technique that he was in every respect correct. Fig. 7 $a$ illustrates the organization of a vessel in Pteris aquilina isolated from the surrounding tracheids by maceration. It is clear that the scalariform bars are separated from one another by wider intervals at the ends of the element and that the bars themselves are considerably more slender in this region and are not distinguished by as pronounced overhanging margins as is the case in the similar structures on the lateral walls. In $b$ is represented a profile view of the terminal inclined wall of the tracheid, indicating plainly the absence of the membrane which characterized the lateral scalariform pits. Further, the margin or border in the case of the terminal open pits is slightly developed in comparison with that of the lateral pores with membranes intact. Conditions similar to those found in the bracken appear in many other representatives of the Filicales and are by no means confined to the Polypodiaceae, which at the present time not only are the largest, but also are considered in many respects the most specialized family of the group. Elements resembling vessels in the presence of terminal perforations have likewise been described for the genus 
Selaginella among the lycopodineous forms. It is thus clear that, so far as the primary structures of the wood are concerned, vessels are present even in the case of representatives of the lower vascular plants.

Although in certain ferns and lycopods structures occur which, physiologically at any rate, represent vessels, these cannot be regarded as quite on the same- morphological and evolutionary footing as the vessels of the highest gymnosperms and the angiosperms. The step from a tracheid to a vessel, where all the tracheids are scalariform, as is the case with the tracheids of ferns and lycopods, is a much shorter one than when the fibrous elements are pitted and not scalariform. We find as a consequence that, although vessels or elements which have been regarded as such occur low in the scale of the Vasculares in the primary structures, they make their appearance in the secondary wood only in the higher representatives of the seed plants.

The secondary wood of the extinct Paleozoic arboreal cryptogams, although often perfectly preserved, in no authentic instance has yet revealed ele-
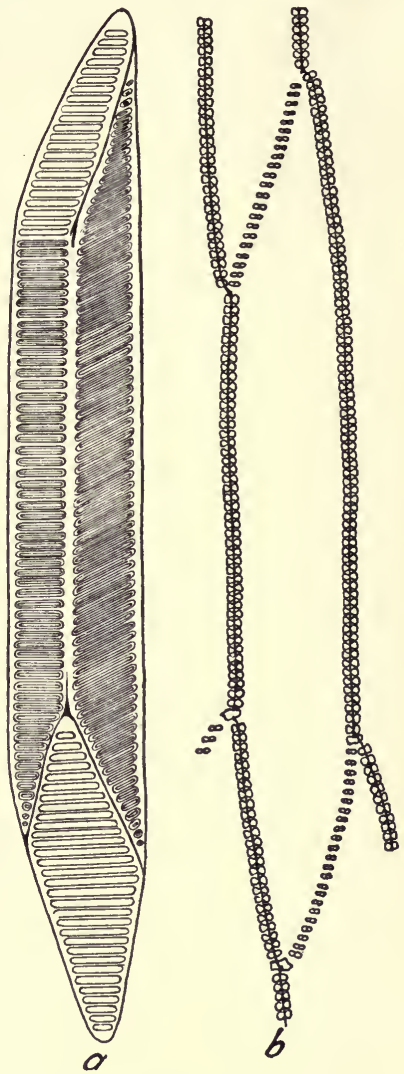

FIG. 7r.-Face and profile views of the type of vessel found in the primary wood of the bracken. ments which may with any degree of propriety be designated as vessels. The same situation obtains in the case of the lower 
gymnosperms and even in the higher representatives of the group as far up as the conifers. It is in the Gnetales, the most advanced

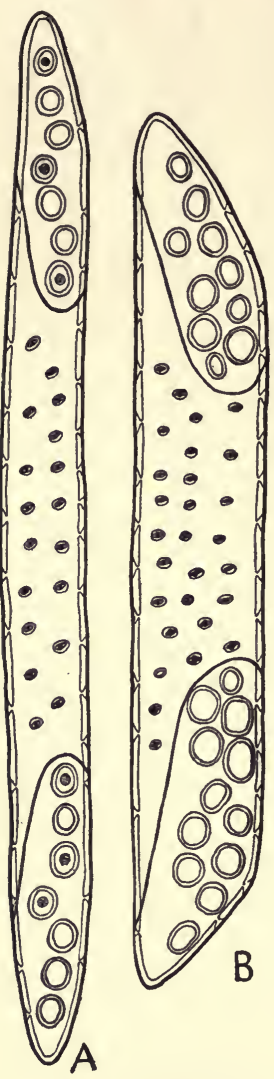

Fig. 72.-Smaller and larger vessels of Ephedra. forms among the naked-seeded Spermophyta, that vessels first make their appearance in the cylinder of the secondary xylem. Fig. $72 a$ represents a smaller element of this type from the wood of the genus Ephedra. The walls terminating the structure under discussion are distinctly at angles to the lateral ones and are remarkable for the extremely large pits which cover their surfaces. The pits of the terminal walls are not only very much larger than the lateral pits, but, with two or three exceptions, they have lost their membranes. In the pores where the membranes still persist a well-marked torus reveals its presence in face view. The pits of the lateral walls of the tracheids in the case of Ephedra are not very much narrower than those of the terminal surfaces, but invariably are closed by membranes thickened centrally by a wellmarked torus. Fig. $72 b$ shows a somewhat larger vascular element from the wood of Ephedra, which has its enlarged terminal pit entirely perforate as a result of the complete disappearance of the membranes. In Fig. 73 is shown a vessel in which, as an exceptional condition for the genus under discussion, there is a tendency to fusion on the part of the enlarged terminal pit. Although in the Gnetales the phenomena of fusion of the end pits of the vessels is rare, it becomes the rule in the angiosperms, as will be shown at a later stage. At $a$ appears a vessel which is transitional from an element of this type to a 
tracheid. It is distinguished by the fact that its end walls do not make a definite angle with the lateral ones but taper gradually. It is further peculiar in the circumstance that its terminal pits are imperforate with one exception and have their membranes marked by the presence of a very distinct torus. In $c$ is figured a tangential view of a vessel in Ephedra, showing the enlarged terminal pores in profile and making it clear that in this case, as in Pteris aquilina, the vessels become patent by the disappearance of the membranes of the pits. The organization of the
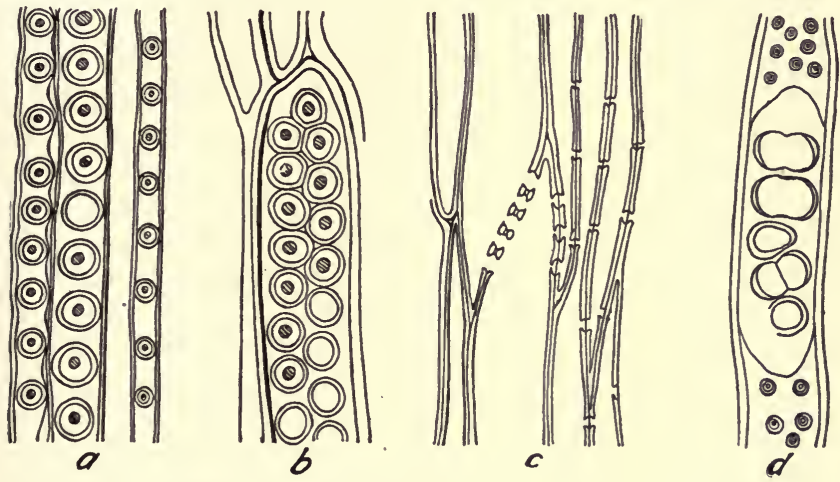

Fig. 73.-Vessels of Ephedra (after Thompson). Explanation in the text

tracheae or vessels in the two remaining genera of the Gnetalesnamely, Welwitchia and Gnetum-is not essentially different from that described for Ephedra, and consequently need not be considered here. The Gnetales as a whole are distinguished, not only by the presence of vessels in their wood, but by the possession of rays of the angiospermous type, as has been indicated in the preceding chapter.

, The angiosperms are characterized throughout by the presence of vessels, generally of a high type, but in some cases showing clear indications of derivation from tracheids. It is only in certain xerophytic genera among the Magnoliaceae and in certain Cactaceae and Crassulaceae that these, characteristically angiospermous 
elements are absent in the dicotyledons. No case is at present known of their default in monocotyledons. There are two main modifications of vascular elements or tracheae in the angiospermsnamely, vessels with scalariform perforations and those with

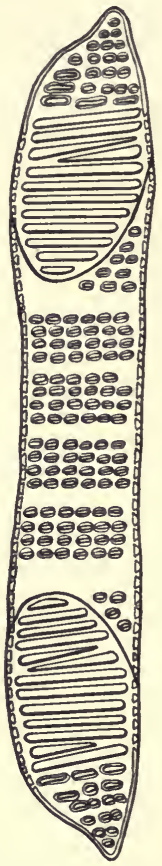

Fig. 74.-Vessel from Alnus, showing the scalariform type of perforation. porous perforations. The term perforation is applied to the actual apertures which make their appearance in the terminal or other walls of typical vessels. The first-named condition of perforation is characteristic of lower groups and lower genera among the dicotyledons, while the second is usually found in the case of the higher orders (the Compositae, for example) of the dicotyledons and seems to be universal for the monocotyledons.

In Fig. 74 is represented a vessel of the lower type - that is, one in which the perforations in the terminal wall are scalariform. This is an element from the root of the alder. The lateral confines of the vessel are covered with small bordered pits which in this particular case are arranged in a somewhat regularly banded fashion as a result of the contact of the conducting element under discussion with the cells of a ray of the wood. If the relation had been with tracheids or other vessels, the arrangement of the pores would not be so regular. Pits are clearly seen in profile on the sides of the vessels, indicating the fact that water-conducting elements belonging to this category do not necessarily have their lateral pitting confined to the radial walls. The ends of the vessel are strongly inclined and are manifestly at definite angles to the lateral walls. The illustration presents the vascular element from the radial view; hence it is obvious in this case that the perforated end walls are radial. This is a situation seldom departed from in the woods of dicotyledonous trees. In other words, a radial section of a dicotyledonous wood 
ordinarily reveals the face view of the characteristically perforated terminal walls of the vessel. We find that it is distinguished by the presence of open slits which are in general without borders and by smaller or larger clearly bordered pits. The latter structures retain their membranes and, where they become extremely elongated, are clearly the result of the fusion of two or more horizontally approximated bordered pits. Fig. 75, which represents part of the terminal wall somewhat diagrammatically and on a larger scale, shows the mode of fusion of pits and also shows that the final result of this process, when a number of horizontally seriate pits are concerned, is the formation of an elongated slit which reveals its primitive nature only by the retention of borders at the ends. The slit as a whole not only has lost its borders, but likewise the membrane of the row of fused pits

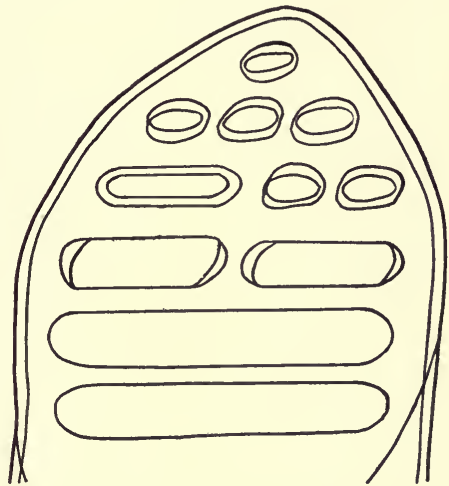

FIG. 75.-Diagram showing the origin of scalariform perforations by the fusion of pits in the end wall of the vessel.

has disappeared. It is thus evident that the slits which occur between the horizontal bars of the lattice work in the terminal walls of a vessel of this type are the result of the fusion of rows of pits, accompanied by a simultaneous loss of pit membranes.

Before we leave the subject of the mode of origin of the perforations which characterize the terminal walls of vessels of the lower type in the angiosperms it will be well to discuss the situation in another group which shares with the true Amentiferae in the minds of students of evolution the claim to be primitive representatives of the dicotyledons. In Fig. $76 a$ is shown a vessel from the root of Liriodendron Tulipifera. In this instance there is no sharp differentiation between terminal and lateral walls, although in general in the genus such a distinction is clearly present. In 
passing from the upper part of the figure downward there is a transition from bordered pits arranged in horizontal rows to perforations of somewhat larger dimensions which are obviously derived from bordered pits by the disappearance of borders and membrane. The truth of this statement can be inferred from the fact that some of the perforations still retain more or less of the original bordered condition, particularly along their lateral margins. Fig. $76 b$ shows another vessel from the root of the same
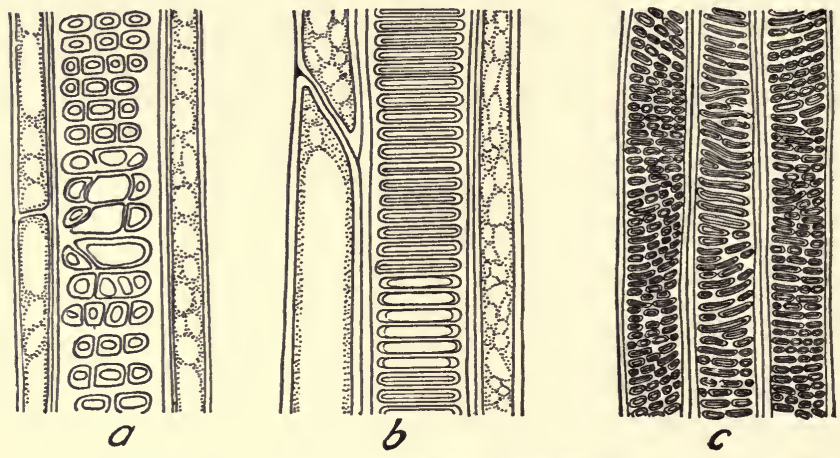

Fig. 76.-Vessels from root of Liriodendron Tulipifera ( $a$ and $b$ ); $c$, vessel-like elements formed after injury in the non-vascular magnoliaceous genus Drimys.

genus in which the situation is somewhat different. Here the pits are scalariform, as in the tracheary elements of the Pteridophyta, but with an important distinction which is not always kept in mind in evolutionary speculations. In the ferns and allied forms scalariform elements are a primitive feature of organization of the wood, while in the secondary wood of the angiosperms the scalariform sculpture of the lateral walls is an exclusive feature of the vessels and is the result of the lateral fusion of horizontal rows of pits and consequently cannot in any way be regarded as a primitive condition of structure as in the lower forms. Proceeding from top to bottom, as in $a$, we find in this case the same loss of borders and membranes leading to the appearance of perforations of the scalariform type. The structure in question, in fact, exactly 
simulates the vessel of Pteris aquilina figured in connection with an earlier paragraph. Although the result is the same, the manner of reaching it is different in the two cases, and a distinction should of course be made in drawing any evolutionary conclusions. It will be clear to the reader from the evidence presented in the present and in a former paragraph that the vascular structures of the lower dicotyledons do not originate as in the Gnetales simply by enlargements of pits and the disappearance of the pit membranes in the terminal regions of the vessels. On the contrary, they typically take their origin as a consequence of the lateral fusion of horizontal rows of pits with a correlated disappearance of the membranes. The vessel in the lower angiosperms is, however, as clearly a derivative of the tracheid as it is in the case of the highest of the gymnosperms. Further, in the angiosperms the vessel or trachea, as a result of its much more complex mode of evolution, is more distinct from the fibrous or tracheary element than it is in any of the lower groups.

The type of vessel characteristic of the higher dicotyledons and the mass of monocotyledons may now profitably occupy our attention. Fig. 77 illustrates three vessels belonging to the category of elements with porous end walls (that is, vessels with so-called porous perforations). In $a$ is shown such an element from the oak in approximately radial aspect. The terminations of the vessel taper and are distinguished by the large aperture or pore. The lateral surfaces are covered with pits, which are of two kinds. The smaller ones, provided with a distinct border, put the vessel in relation with other similar structures and with tracheids. The simple and slightly irregular pits represented in about the middle horizontal region indicate the presence of a small ray in contact with the vascular element. In $b$ is represented a vessel from the wood of the poplar in radial view. The same slanting ends and large terminal pores as are present in the oak similarly characterize the vessel of Populus. The uniform crowded lateral pitting indicates that the face of the vessel presented to the observer was in contact with another vessel. In $c$ is presented the somewhat tangential view of a vessel in the maple. The ends of the vascular element do not differ essentially from those of the oak and poplar. 
In this case the wall to the left has been in contact with a ray, as evidenced by the grouping of the pits. The region of the lateral wall to the right has been in contact with another vessel and is
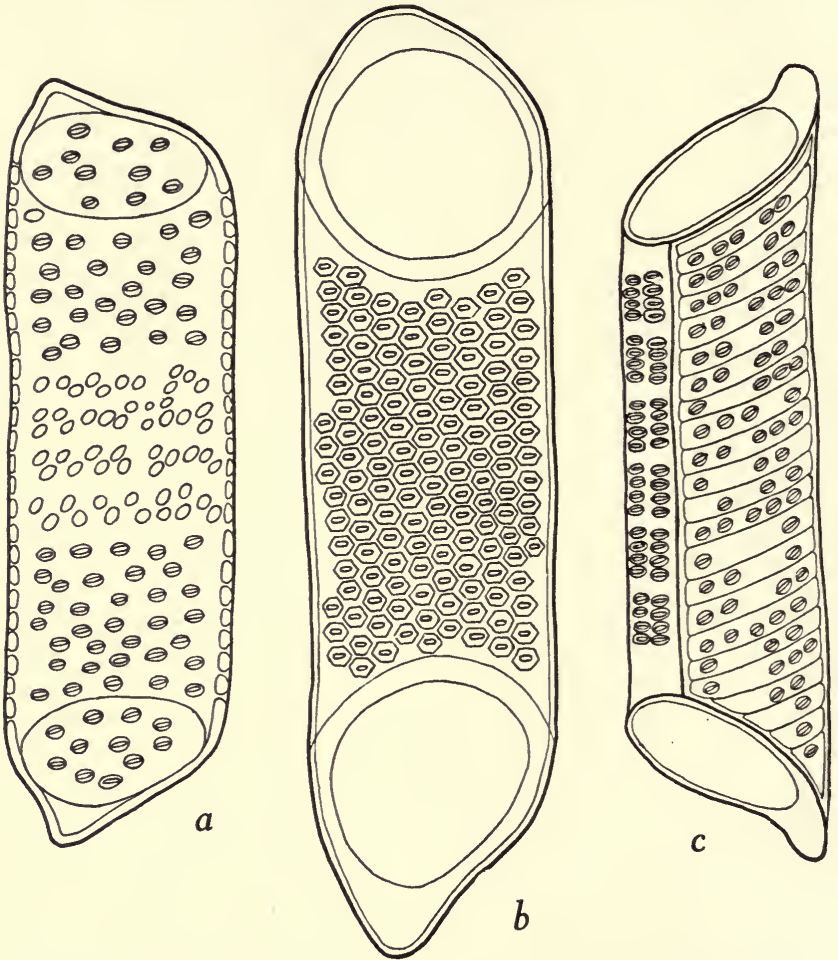

Fig. 77.-Various types of vessels in the dicotyledons. Explanation in the text

conspicuous, not only by reason of the characteristic arrangement of the pits, but also by the presence of a somewhat spiral internal sculpture, the so-called tertiary thickening, which always marks (when present) the contact or "party wall" between vessel and vessel. Those surfaces of the vascular element which are in contact 
with the libriform fibers are devoid of both pits and spiral internal sculpture.

It will be profitable to consider next the type of vessel characteristic of the older wood of the stem of Liriodendron. This category of vessel is covered with pits in horizontal rows, indicating contact with another vascular element. Below and above the sharply sloped end walls are seen; these are neither porous, as in the case of the vessels of the last figure, nor marked by the simultaneous presence of narrow scalariform perforations and bordered pits, as in the rootwood of Magnolia and in the general secondary wood of many of the Betulaceae, etc. The terminal apertures of the vessel, in fact, are crossed by a few remote bars. The type of vascular element characteristically present in the old wood of Liriodendron serves, indeed, as an intermediate stage between the vessel with true scalariform perforations and that with porous terminal apertures.

The truth of this assertion becomes manifest from a consideration of Fig. 79, which represents vascular structures from the wood of the high blueberry, Vaccinium corymbosum. In $a, b$, and $c$ are shown successive transitions from end walls with many pits and few narrow scalariform perforations to fewer pits and more scalariform apertures, and to a third condition where the pits have disappeared and only somewhat wide scalariform openings remain. In $d$ and $e$ appear further stages in which the bars separating the terminal large slits become fewer and more degenerate until finally a simple porous condition is reached. It is thus clear that the vessel with porous perforations is a further elaboration of that with scalariform

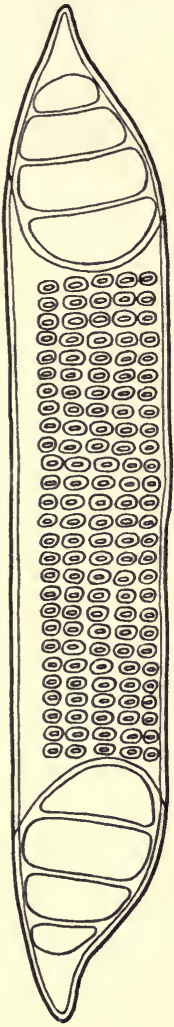

Fig. 78.-Vessel from the stem of Liriodendron. perforations, just as this in turn has taken its origin from a vascular element with pitted perforations of the nature found in the Gnetales. 
The hypothesis that the scalariform vessel is the forerunner of the porous one is also confirmed by a consideration of the first-formed region of the woody cylinder. In the oak, for example, as well as in many other instances, although vessels with porous terminal

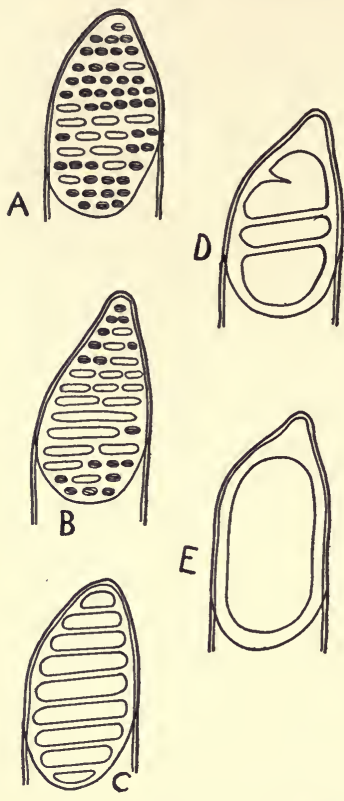

Fig. 79.-Vessel of Vaccinium corymbosum, illustrating the origin of the porous type of perforation from the scalariform. Explanation in the text.

apertures are characteristic of the mature wood, elements of vascular nature with scalariform perforations are commonly present in the first annual ring, particularly in the vicinity of the protoxylem. It is thus evident that the vascular structures in dicotyledonous woods supply a valuable argument for the general validity of the hypothesis of organic evolution.

The lateral walls of the vessels may next receive further consideration. It has been indicated in a former paragraph that the side walls of a vessel have their sculptural features largely determined by the nature of the adjacent cells of the wood. Where vessels are in contact with rays, the communicating pits are characterized by a grouping and seriation corresponding to the outlines and direction, whether transverse or longitudinal, of the ray cells. Where the relation is with other vessels or with true tracheids, the pits are numerous and likewise arranged in a somewhat definite manner. If merely mechanical elements abut on the vascular walls, pitting is quite absent. Further, if so-called tertiary spirals are present on the inner side of the walls of the vascular elements, these are confined to those vertical regions in contact with other vessels or with tracheids. 
Where vessel comes in contact with vessel, we find frequently a mode of pitting characteristic of a genus or even a family. Details in this connection are beyond the range of a work so elementary as the present one, but a few prominent features may be indicated. As has been shown in the figure of the vessel of Liriodendron above (Fig. 76), lateral pits may be in horizontal rows. In other instances, as in the oak shown in Fig. 8oa, the lateral pits are arranged in an alternating fashion. Again, in $b$, representing the lateral pitting of the vessel in the poplar, alternation is accompanied by crowding to such an extent that the pits become angular by mutual contact.
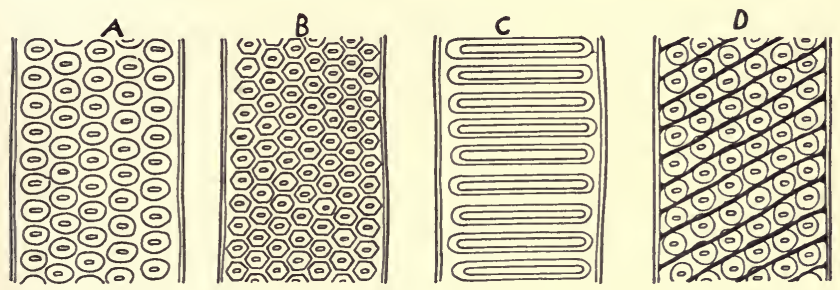

FIG. 80.-Lateral pitting of vessels of dicotyledons. Explanation in the text

In $c$ is shown the characteristic pitting of the vessels of the vine family (Vitaceae). Here the sculpture of the lateral walls consists of elongated slits closed by equally elongated membranes. A reference to the structure of the secondary wood in the region of the pith in this instance makes it clear that the slitlike pits of the Vitaceae are not persistent scalariform pores of the first-formed region of the xylem, but are the result of the lateral fusion of horizontal rows of pits in the side walls of the vessels. In $d$ is represented a condition of structure of the vascular wall which is common in the higher types of dicotyledonous woods. Here the alternating pitting is overlaid internally by spiral structures which it is customary to call tertiary thickenings.

In concluding the statements in regard to vessels it is necessary to refer to the phenomenon of tylosis or occlusion of the lumina or cavities of the vessels by parenchymatous ingrowths. These 
structures originate under various conditions, but are more usually found in connection with those changes which precede the transformation of sapwood, or alburnum, into heartwood, or duramen. As the living cells in proximity to the vessels (whether they belong to the radial or vertical systems of storage tissues appears not to
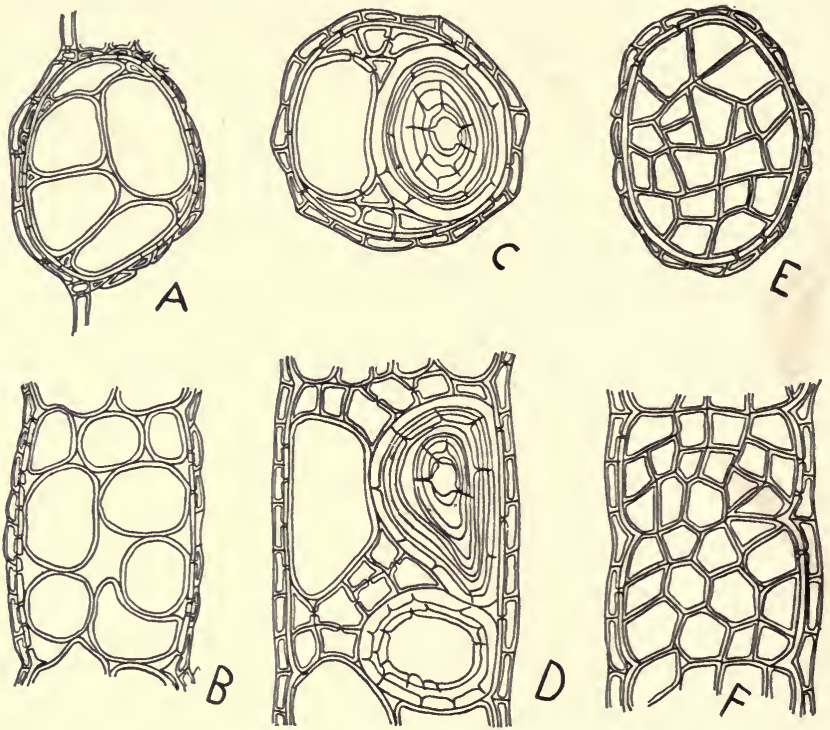

Fig. 8I.-Tyloses in oak and locust. Explanation in the text

be of importance) are about to die, they push processes into the adjacent vessels by the bulging and growth of the cellulose membranes of the pits connecting them with the vascular structures. These ingrowths are occupied by protoplasm and, sometimes at any rate, by nuclei as well. When abundantly developed, they completely close the lumen of the vessel and render impossible the passage of water. Fig. 43, on page 57, has made clear the contrast resulting from this phenomenon in the sapwood and the heartwood 
of the oak. In Figs. $8 \mathrm{r} a$ and $b$ is shown the phenomenon of tylosis in the vessels of the white oak. In this species all the larger vessels are more or less completely occluded by tyloses extending from and only excluding the latest complete annual ring. In $c$ and $d$ a similar condition is represented in the case of Quercus Engelmanni. In this instance the tyloses have developed a very thick and strongly pitted wall, a condition more rarely found. In $e$ and $f$ tylosis as found in the case of the black locust, Robinia Pseudacacia, is represented.

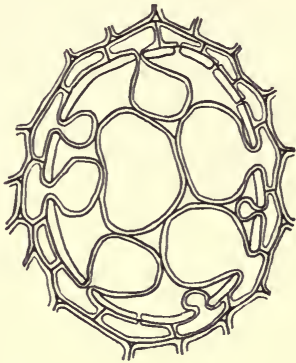
Here the ingrowths derived from adjoining parenchyma cells are very numerous and form a mass completely occluding the cavity of the vessel. In Fig. 82 are seen, both in transverse view, earlier stages of the invasion of a vessel in the oak through the agency of tyloses. The representation in this

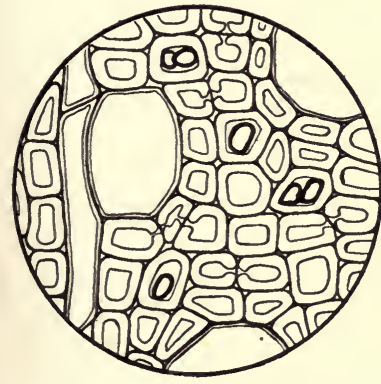

Fig. 83.-Tylosis in tracheids of Liquidambar.

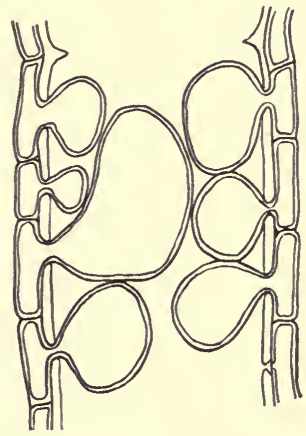

FIG. 82.-Diagrammatic representation of the process of tylosis.

illustration is somewhat diagrammatic to show more clearly the entry of the ingrowths through the medium of the pits in the walls of the vessels.

The phenomenon of tylosis is in all probability an extremely ancient one for the higher plants, as it is found far back into the 
Mesozoic. It is not confined by any means to vessels and even occurs rarely in the tracheids of dicotyledons. Fig. 83 shows the presence of tylosis in the fiber-tracheids of the sweet gum (Liquidambar styraciflua). The heavily outlined bodies in the cavities of certain of the fibrous elements are of the nature of tyloses. Similar conditions have been found in the case of other dicotyledonous woods. It has been made clear in an earlier chapter that ingrowths resembling tyloses occur in the resin canals of the pine in the region of the heartwood. Occlusion by means of parenchymatous invasions is, however, not confined to the resiniferous spaces in the genus mentioned. In the roots and likewise in the cone the tracheids of the wood often show themselves occluded by ingrowing parenchyma cells. Such conditions are not normally found in the vegetative stem of living pines, although they are known to occur in the branches of cretaceous Pityoxyla from the Eastern United States. 


\section{CHAPTER VIII}

\section{THE FIBROVASCULAR TISSUES-PHLOEM}

The ligneous or woody tissues which have been discussed under various headings in previous chapters are of great importance on account of their conservatism. They manifest a high degree of differentiation and are also important by reason of the fact that their relative imperishability has resulted in their being abundantly preserved as fossils from the most ancient times to the present. Woods consequently furnish on the whole the most important historical document in favor of the hypothesis of evolution, and those who are interested in the doctrine of descent as applied to the case of plants cannot afford to neglect the investigation of the ligneous tissues of the vascular plants. The situation for the plants provided with tracheary tissues is, in fact, very different indeed from that presented by the lower vegetable organisms, where evolutionary data are necessarily largely speculative and experimental. Invariably associated with the woody tissues are those of the phloem. The primal function of the woody tissues was apparently to conduct water into the superior or outlying parts of the plant. To this function have been superadded in the long course of geologic time, as pointed out in preceding chapters, the functions of strength and storage, with corresponding and appropriate modifications of structure. Just as the wood in the first instance served the purpose of the movement of water from the soil, so the primal condition of the tissues of the phloem was that of conducting the elaborated foodstuffs manufactured in the leaves to other parts of the plant where they are either utilized in the processes of growth and respiration or stored up as reserves.

The primitive type of phloem, as that of xylem, appears to have existed in the case of the lepidodendrids. Its structure has not been satisfactorily preserved in these primitive vascular plants, and there is some difference of opinion as to interpretation. Some investigators have expressed the opinion that no true phloem is 
present in these ancient organisms, while others have contended for a very simple type of organization in the arboreal club mosses of the Paleozoic age. In view of the differences of opinion and the uncertainty of the data in the case of the phloem of Paleozoic Pteridophyta, it will be well to confine our attention in this instance to the living representatives of the fern alliance.

Fig. 84 shows the detailed organization of one of the fibrovascular strands of the bracken Pteris aquilina. The bundle as a

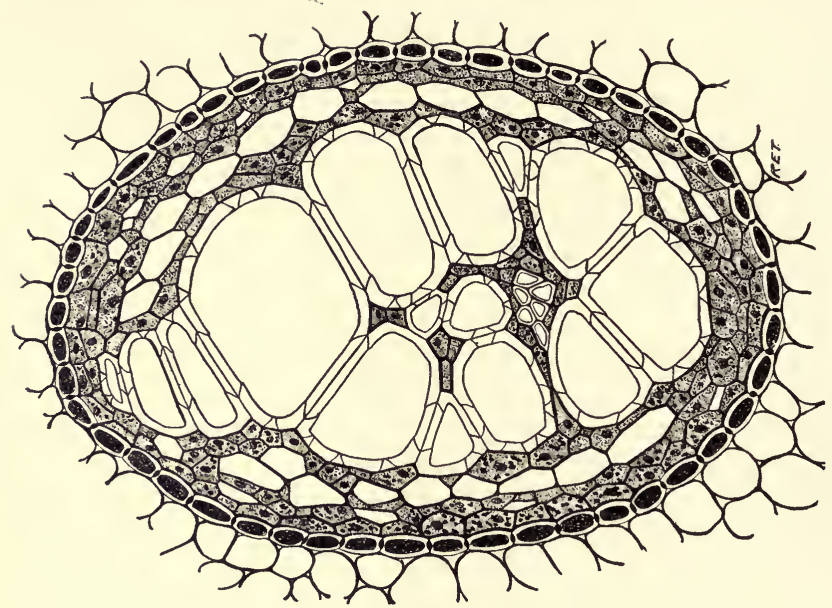

FIg. 84.-Bundle of Pteris aquilina. Explanation in the text

whole is clearly limited by a dark uniseriate layer, the endodermis, which is ordinarily interpreted as the innermost layer of the fundamental tissues in juxtaposition to the fibrovascular system. Within the endodermis is situated a layer one or sometimes two cells in breadth, the pericycle, which constitutes the external boundary of the fibrovascular tissues, just as the endodermis marks the internal limit of the fundamental system. Next to the pericycle are certain extremely minute, apparently empty, cells, the firstformed elements of the phloem, or, as they are technically designated, the protophloem. Next inward lie storage cells, nucleate 
and more or less loaded with starch. These are the parenchyma of the phloem. We are not acquainted with the origin of parenchymatous cells in the phloem for reasons indicated at the outset of the present chapter. Farther inward lie the larger conductive elements of the phloem, known as the metaphloem. These are cells which have no contents but a delicate lining of protoplasm, and this does not appear in the illustration. The elements under discussion as well as those of the protophloem are known, on account of certain structural features to be described later, as sieve tubes. It is important to note in the present connection that sieve tissues do not present the variety of modes of development which characterizes the xylem. Almost invariably in this category of tissues the first-formed elements are external and those of later appearance are laid down in centripetal order. As a consequence the terms exarch, mesarch, and endarch, which are so significant in connection with the development of the primary wood, have little bearing in the case of the sieve tissues. Internal to the larger sieve elements of the metaphloem lies a more or less continuous band of parenchyma, separating the phloem from the xylem. The tracheary elements of the wood are individually more or less completely surrounded with parenchyma. The phloem does not form a continuous band about the xylem, but is interrupted at the two ends of the elongated mass of wood. In certain ferns the tissues of the phloem occur only on one side of the bundle, in which case the strand of fibrovascular tissue is known as collateral. Where the phloem forms a complete jacket about the xylem the condition is known as concentric; and where, as in the bundle appearing in the figure under discussion, the sieve tissues are confined to two opposite sides the condition may be designated as bicollateral.

In Fig. 85 is represented a longitudinal view of part of the same bundle. The sieve tubes now appear as elongated cells with tapering ends and sculptured walls. The relief of the walls consists of somewhat angular crowded areas, although on the whole they are tolerably evenly distributed. These areas are perforated with very small simple pits and are the sieve plates. It is these structures which give to the sieve tube its name. After the characteristic elements of the phloem have reached a certain age 
their pores become more or less completely occluded by slimy plugs known as callus. The details of origin and the significance of this substance are not fully understood and from the standpoint of the present statement are of relatively little importance.

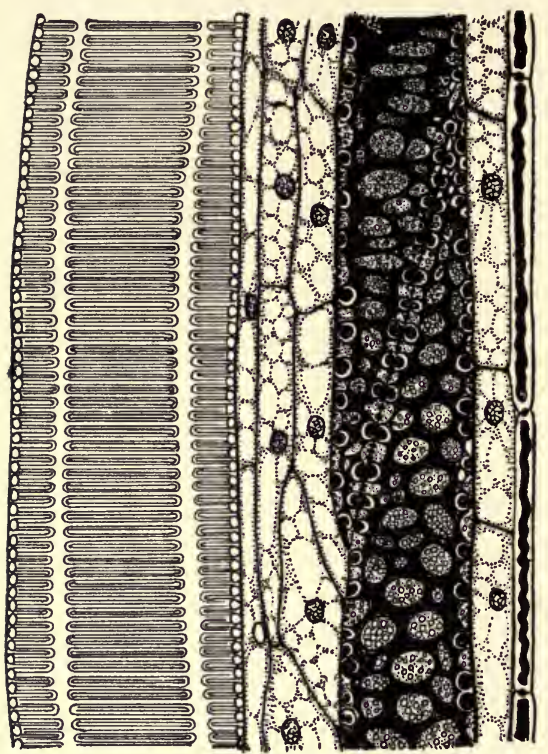

FIg. 85.-Longitudinal view of part of a bundle of Pteris aquilina. On the left is shown a vessel, while toward the right and represented in black are sieve tubes.

In Fig. 86 is reproduced a photograph of the wood and inner bark of the pine. The xylem is clearly very regular in its structure and consists of a series of annual rings, the organization of which has been dealt with in a preceding chapter. In contrast to the persisting regularity of the wood the phloem presents itself as a mass of tissue radially seriate only for a short distance outward from the cambium, or zone of growth. Externally its elements are thrown into more or less meandering lines as a result of the collapse of certain of its constituent cells, which will be considered more in detail in the sequel. The section reproduced in the photograph under discussion was made from material secured in the winter period of rest. The uncollapsed part of the phloem corresponds to a single year's growth, and all that region of the inner bark or phloem characterized by the meandering course of the rays is nonfunctional so far as the most characteristic elements, the sieve 
tubes, are concerned. Fig. 87 shows a highly magnified view of the region immediately outside and inside the zone of growth, or cambium. Internal to the active layer the wood is recognizable by its bordered pits. The initial region, known as the cambium, is distinguishable by the richly protoplasmic character of its cells and their thin tangential walls, which are in contrast with the thickness of the radial boundaries. External to the cambium lies the phloem, composed most characteristically of somewhat rectangular cells, the sieve tubes. In these, unlike the cambial elements, there is no nucleus, and the scanty parietal protoplasm presents likewise a striking difference from the richly protoplasmic cambial region.

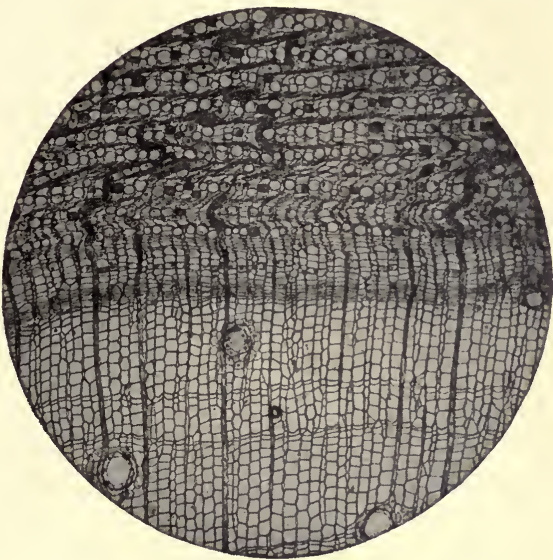

Fig. 86.-Inner bark with cambium and adjacent wood of the pine.

Scattered thinly through the phloem are a few rounded cells which contain protoplasm and grains of starch (represented black as if stained with iodine). The rays cross the longitudinal elements of the phloem, the sieve tubes, and the parenchyma cells at right angles and appear in the figure as single files running nearly straight for a short distance and then pursuing a meandering course outward. The cells of the two rays shown in the figure present a different appearance. In the file of radial parenchyma on the right the elements are filled with protoplasm and show the presence of a nucleus. In addition, a considerable amount of starch is present in the form of grains tinctured black by iodine. It is clear that the cellular elements of the ray to the right continue elements 


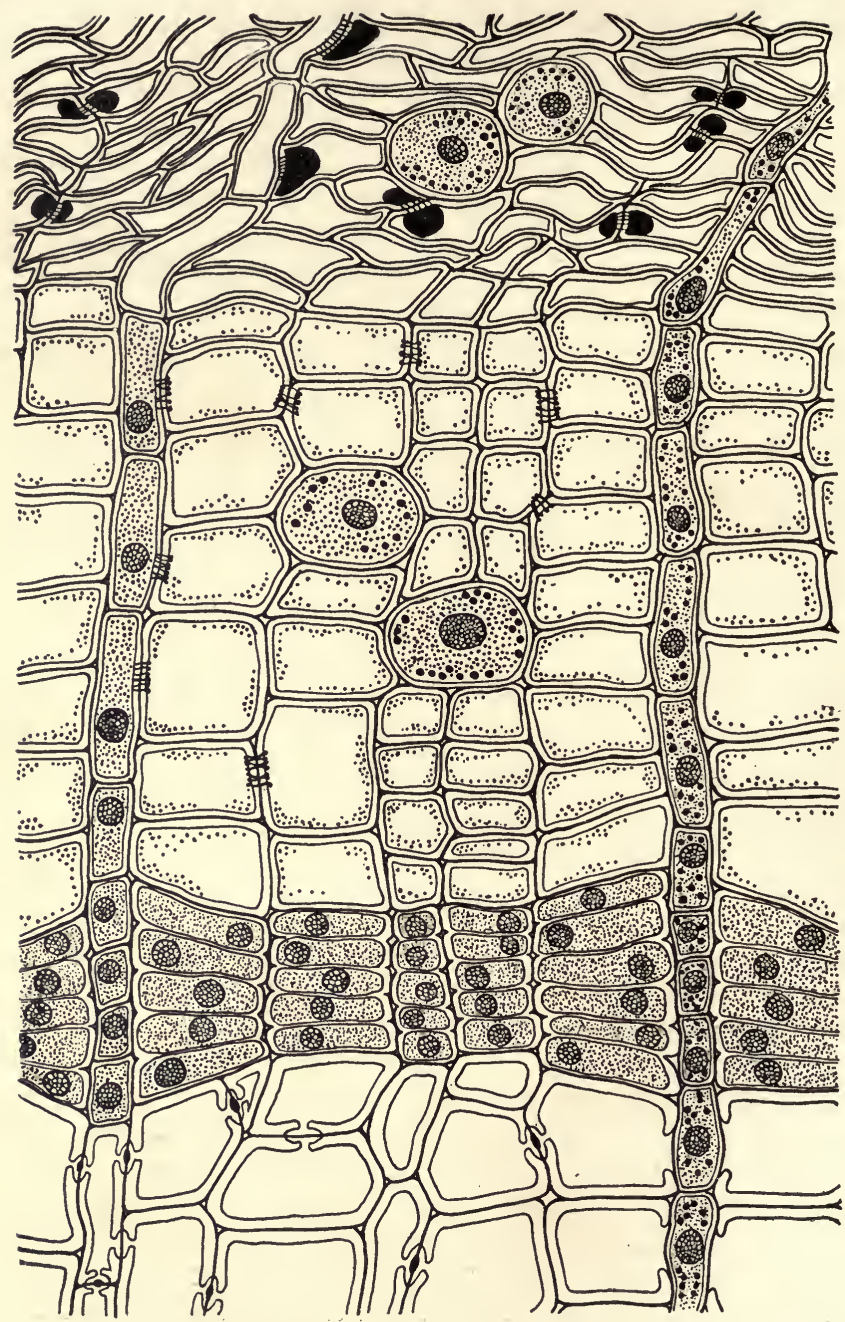

FIG. 87.-Transverse section of xylem, phloem, and cambium of the pine, more highly magnified. Explanation in the text. 
of a similar nature in the portion of the ray contained in the wood. If we now turn our attention to the ray on the left, a somewhat different situation is presented to the eye. Here the cells are entirely without starch, although they still show protoplasm and a nucleus. Internally they are in line with the marginal tracheids of the ray in the wood, which have been described in an earlier chapter. Obviously there are two categories of cells in the rays of the phloem in Pinus, just as has been shown to be the case for the radial parenchyma of the xylem. It will now be convenient to consider the relations of the various elements of the phloem to one another. In this connection we may first discuss the sieve tubes. It will be noticed that there are certain dark lines stained with iodine occurring in groups on the radial walls of the sieve elements. These are the transverse sections of sieve pores. Parenchymatous cells of the phloem have no intimate relation to the tubes, as no pits facilitate interchanges between the two types of elements. The same statement holds in regard to the cells of the ray shown on the right side of the figure. Orr the left, however, the elements of the ray are clearly related to the sieve tubes by means of sieve plates, distinctly differentiated on the side of the sieve tubes, but not well developed on the side of the ray. It is evident that there is a specially intimate connection between certain elements of the ray, which are contrasted with the remaining constituents by the absence of starch, and the conductive elements of the phloem or sieve tubes.

If the organization or, more correctly, the disorganization of the phloem is followed more externally, it will be observed in the illustration that the ray on the right continues to retain its protoplasmic contents and its grains of starch, while that on the left has entirely lost its living contents. A further feature of the phloem as represented in the external region of the figure is the distortion and final collapse of the sieve tubes, preceded by the loss of the delicate protoplasmic lining surrounding the inner walls of these elements. The parenchymatous cells retain their integrity in the collapsed region of the phloem, precisely as is the case with the ray indicated on the right of the figure. Another feature of the disorganization of the phloem is the appearance of large masses or plugs of material, 
appearing black in the figure, which are present exclusively on the radial walls of the elements, although in some instances the collapsed state of the tubes gives rise to the appearance of a tangential position for the bodies under discussion. The plugs mentioned above constitute the callus, a substance which in the conifers and the great majority of seed plants higher in the scale makes its appearance on the sieve plate at the time the tube is in the initial stages of collapse. The callus completely blocks the pores of the sieve plate and, after persisting for a short time, disappears, leaving the pores of the sieve area quite open. It is also apparent that in the case of the ray cells on the left of the figure the callus present is unilateral and occurs only on the side of the sieve tube. Obviously the type of ray element appearing on the left in the illustration is definitely related in its duration to the life of the sieve tube, and both structures cease to be functional at the same time.

In the next figure (Fig. 88) the radial aspect of the phloem is shown. Here the ray naturally appears in longitudinal section. Above and below in the region of the phloem it shows vertically placed cells which contrast in the position of their axis of greatest length to the central elements of the radial parenchyma. These socalled "erect cells" correspond to the elements shown in the ray to the left of Fig. 87 and are similarly characterized by the absence of the starch, which is a feature of the contents of the central or prone cells of the ray. In passing toward the right from the cambium the protoplasmic filling of these elements becomes less abundant, until finally, where the callus presents itself in face view, it disappears altogether, in this respect presenting a contrast to the central cells which retain their protoplasm and starch indefinitely. The sieve tubes now appear in longitudinal view, and nearer the cambium they have a delicate protoplasmic lining often containing some small grains of so-called transitory starch. The sieve areas, or sieve plates, are presented to the observer in face view, and toward the extreme right callus plugs occlude their pores and at the same time the tube has lost its living contents. It is clear in the radial view, as in the transverse, that sieve tube and marginal erect cell cease to exist and develop callus plugs 
simultaneously. A single row of parenchymatous elements is represented as crossing the axis of the ray. To the left of the cambium lies the wood with its tracheids and the continuation of the ray. The radial structures in the xylem show also a differentiation into central and marginal cells, but here ray-tracheids take the place of the erect cells of the ray in the phloem.

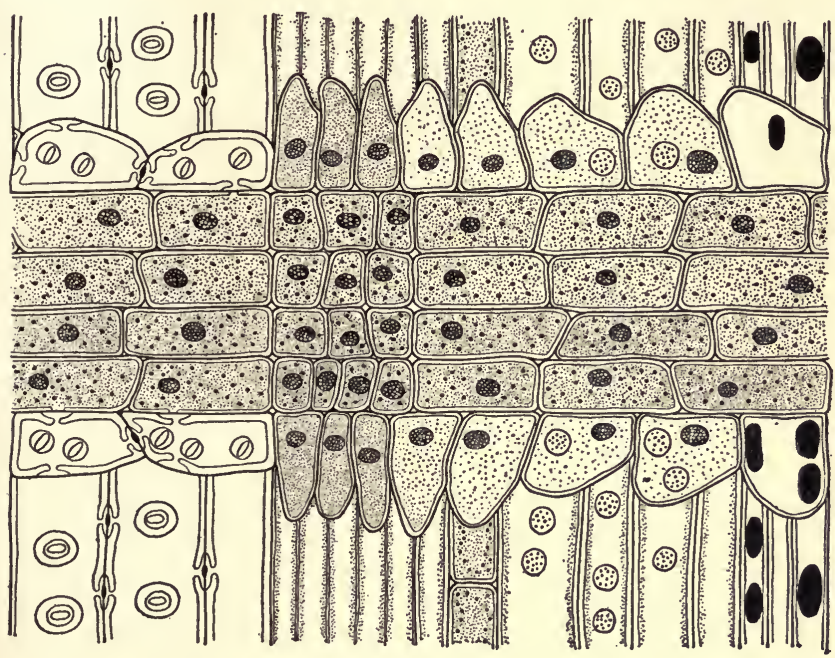

Fig. 88.-Radial section through xylem, cambium, and phloem of the pine. Explanation in the text.

In Fig. 89 appears the tangential section of the tissues of the phloem in Pinus. The plane of incidence of the section is tangentially slightly oblique; hence elements of different ages appear on the opposite sides. Toward the right are represented sieve tubes in which the protoplasmic lining has disappeared and the sieve areas (seen here in profile) are blocked by masses of callus. In the case of the rays which are naturally shown in transverse section the marginal or erect elements are calloused on the side of the tubes. The central cells of the rays still show the presence of 
protoplasm and starch. In the middle region of the figure a file of parenchyma cells is present. To the left of the middle line the sieve elements show the delicate protoplasmic lining which characterizes them in the functional condition. The radial parenchyma is also entirely in a living condition, although the marginal cells are differentiated by the absence of the starch contents found in the
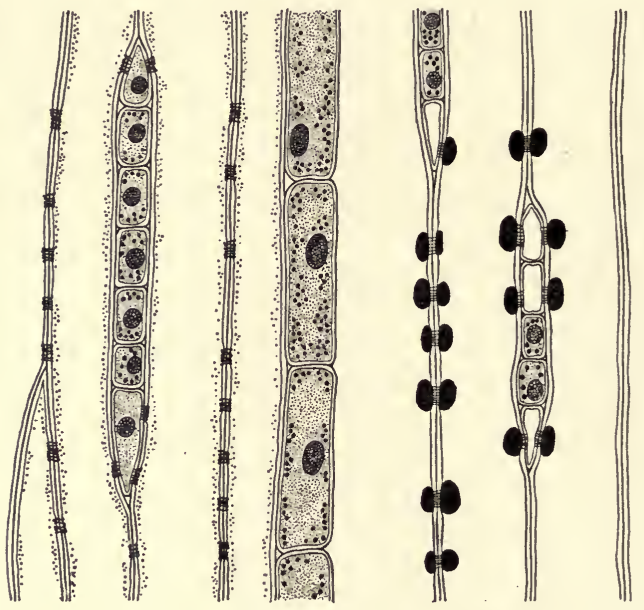

Fig. 89.-Tangential section through the phloem. Explanation in the text

central region of the ray structure. The callus in both sieve tubes and marginal cells is here conspicuous by its absence.

A somewhat complicated condition of the phloem has purposely been chosen in the case of the coniferous gymnosperms, because there is good reason to believe that in this group, which is a decadent one; the more elaborately organized condition is antecedent to that marked by a greater degree of simplicity. On the whole, the pine and its allies represent the most highly differentiated structure of the phloem in the group. In the Cupressineae, Taxodineae, Araucarineae, Podocarpineae, and Taxineae the radial structures of the phloem show a marked degree of simplification as compared with 
the pine family proper. It is not necessary in an elementary work like the present one to enlarge further on the organization of the phloem of the gymnosperms in general or the conifers in particular.

The structure of the phloem in the angiosperms, and especially of arboreal dicotyledons, will next occupy our attention. Here the

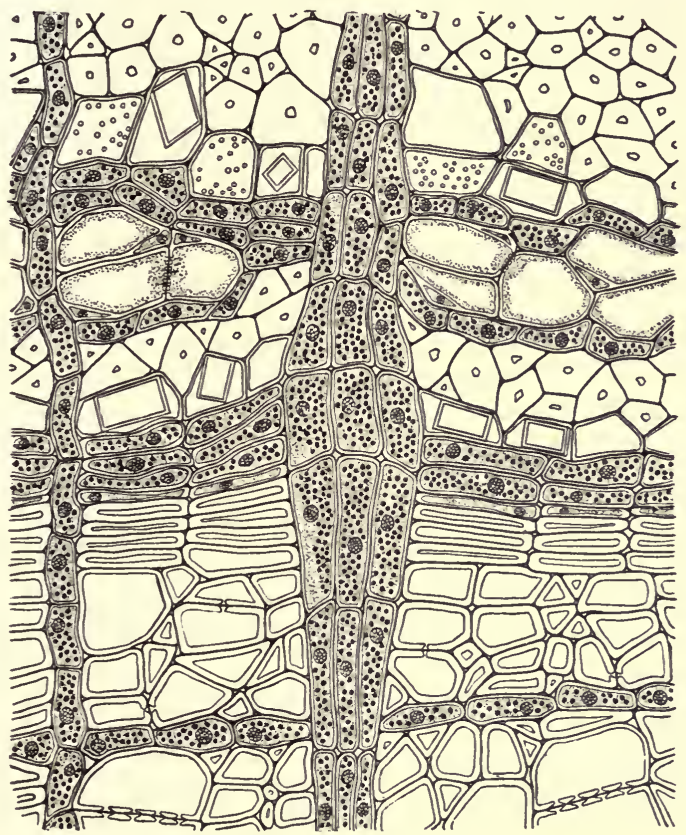

Fig. 90.- Transverse section of the xylem, cambium, and phloem in the American linden. Explanation in the text.

general organization is quite different from that characteristic of gymnospermous groups and accordingly merits detailed consideration. Fig. 90 shows a transverse section of the region immediately internal and external to the cambium in the trunk of Tilia americana, the American linden or basswood. Toward the lower side of the 
illustration appears the xylem, consisting of vessels, libriform fibers, parenchyma, and wood rays. Above the tissue so organized lies the cambium, or zone of growth, which alternately adds elements to the structure of the xylem and that of the phloem. The latter tissue appears above the cambium in the figure and outside it in the trunk of the tree. The preparation which served as the basis of the illustration was made from material secured during the period of winter rest. Next to the cambium lie fibrous and șimilar elements, to the abundant development of which in its inner bark the basswood (literally bastwood or tree useful for binding) owes its name. The fibers of the phloem are often called hard bast. Examination of the figure will show that the fibrous elements are separated from the cambium by a row of cells either quite empty or containing large crystals of calcium oxalate. These are the socalled crystallogenous cells and are very commonly present internal to the zones of fibers or hard bast in the phloem of the form under discussion. External to the hard bast lies a zone distinguished by the presence of cells either richly protoplasmic as to their contents or, in case the protoplasm is more scanty, characterized by relatively thin and unlignified walls. This region is the so-called soft bast and shows considerable complexity of organization. First taking the cells with abundant protoplasm, we see clearly that these can be divided into two categories-namely, those which contain grains of starch (represented in black as if treated with strong iodine solution) and those in which amylaceous substances are absent. The latter are further distinguished by their generally small size and somewhat triangular shape, due to the fact that they are usually accommodated in the angles of large thin-walled elements with a delicate protoplasmic lining. The triangular cells are known as "companion cells" and are a constant feature of structure of phloem of the angiosperms as contrasted with the gymnosperms. The larger elements of the phloem with thin walls and scanty parietal protoplasm are the sieve tubes. In some instances the sieve plate can be seen in the transverse section reproduced in the figure, and it is clear that its position is characteristically radial. Farther outward the densely filled parenchymatous cells of the soft bast give place again to the dead crystallogenous elements, 
which are in turn followed by bast fibers. The rays of the phloem in the basswood and other dicotyledons show no special feature of interest, since they do not in any case manifest the intimate relation to the sieve tubes described above in the pine.

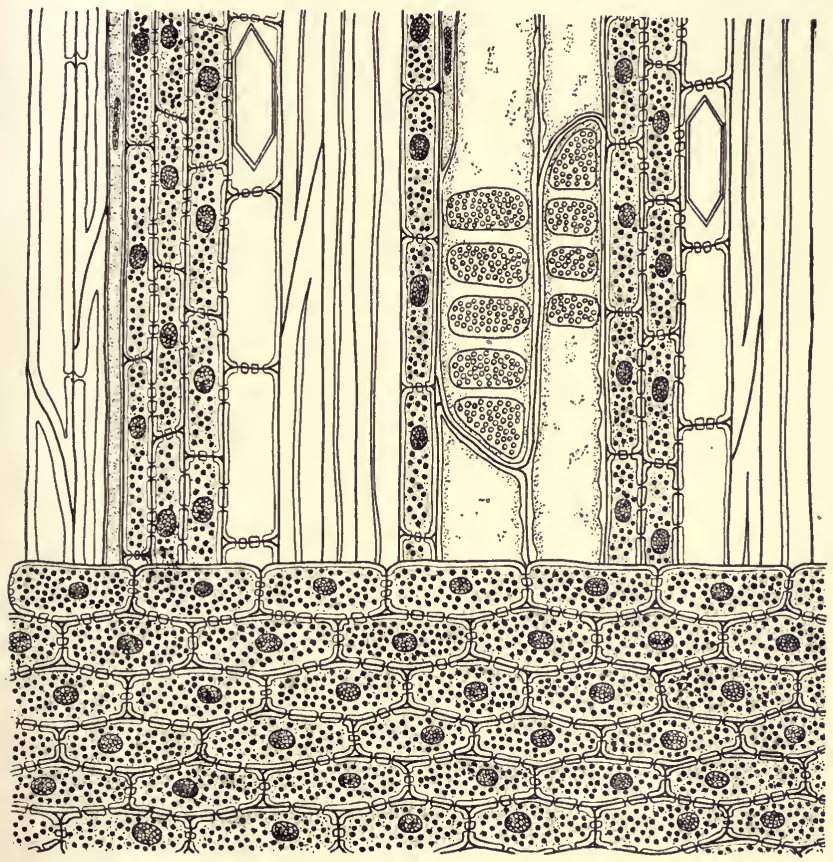

FIg. 91.-Radial section of the xylem, cambium, and phloem of the linden, of basswood. Explanation in the text.

In the next illustration (Fig. 9I) the radial aspect of the phloem in Tilia is shown. Here the rays appear naturally in longitudinal section and are composed of heavily pitted cells containing dense protoplasm and much starch. Above the ray are represented the various elements of the hard and soft bast. On the extreme left 
are to be seen the summer elements of the wood in contact with the cambium. They are followed by parenchyma and by crystallogenous cells, empty and heavily pitted on their horizontal walls. Next, the fibers of hard bast meet the view, and these pass in succession into bast parenchyma, characterized by heavy pitting, abundant starch, and protoplasmic contents, and by sieve tubes of large lumen and scanty parietal protoplasm. Within, in the walls of the sieve elements, lie the companion cells, distinguished by their slender form and the absence of starch in their protoplasm. The sieve tubes in the radial aspect present to the eye strongly inclined end walls which may be compared with the similarly slanting partitions in the vessels of the wood. The sieve element is, in fact, the exact analogue of the vessel, and, just as the vascular elements in angiospermous woods play the main part in the transport of water, so the sieve tubes perform the principal rôle in the movement of the elaborated organic stuffs from the leaves. Another feature of analogy between the sieve tube and the vessel is not only the more or less inclined terminal walls at angles to the lateral ones, but also the function of transport specially provided for in connection with the ends of the element.

In the vessel this condition finds its expression in the development of scalariform or porous perforations and in the case of the sieve tubes by the appearance of particularly extensive and largepored sieve plates on the terminal inclined walls; and these in highly specialized forms, such as herbaceous dicotyledons and monocotyledons, may be the only functional regions. Following the sieve tubes is a zone of bast parenchyma, succeeded in turn by another zone of crystallogenous cells and hard-bast fibers.

In Fig. 92 is shown the tangential longitudinal aspect of the phloem in the basswood. Here the rays naturally appear in transverse section and are clearly of diverse sizes, but are all composed of strongly pitted cells containing abundance of protoplasm and starch. In this aspect the plane of section is purposely slightly oblique so as to exhibit all categories of constituents of the phloem at the same time. To the left lie crystallogenous cells near a large ray. The ray is in contact on the opposite side with parenchyma of the soft bast. Then follow sieve tubes and their related com- 
panion cells. In the tangential aspect the terminal sieve plate, since it is radial in position, is shown in profile. The protoplasm, present more densely in the region of the terminal plates, is purposely omitted in order that the sculpture of the lateral walls may
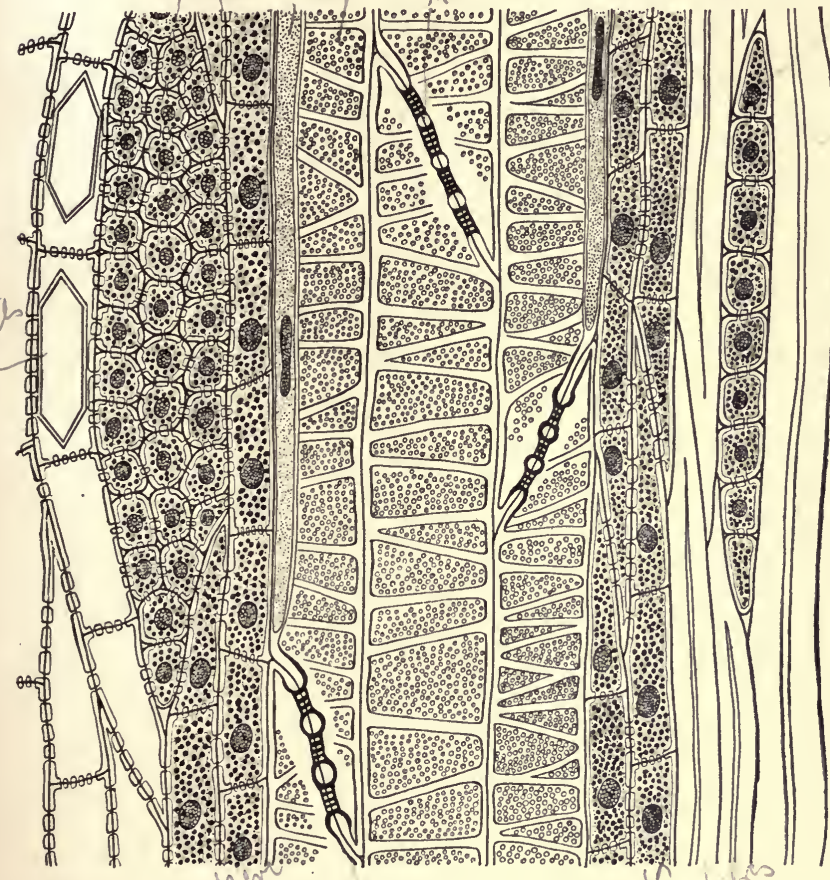

FIG. 92.-Tangential 'section of the phloem of the linden. Explanation in the text.

on tally

$a^{2}$

be apparent. There are manifestly strongly punctuated areas in the side walls of the sieve element. These represent sieve plates which have more or less completely lost their function as a result of degeneracy. In herbaceous types lateral sieve areas frequently have no longer even a morphological expression, the structural 
as well as the functional plates being almost exclusively limited to the terminal walls. Abortive and lateral sieve plates in the angiosperms are known as lattices. In woody dicotyledons the lattices are, in the case of lower types, often well developed and are likewise frequently functional as actual sieve plates. Farther

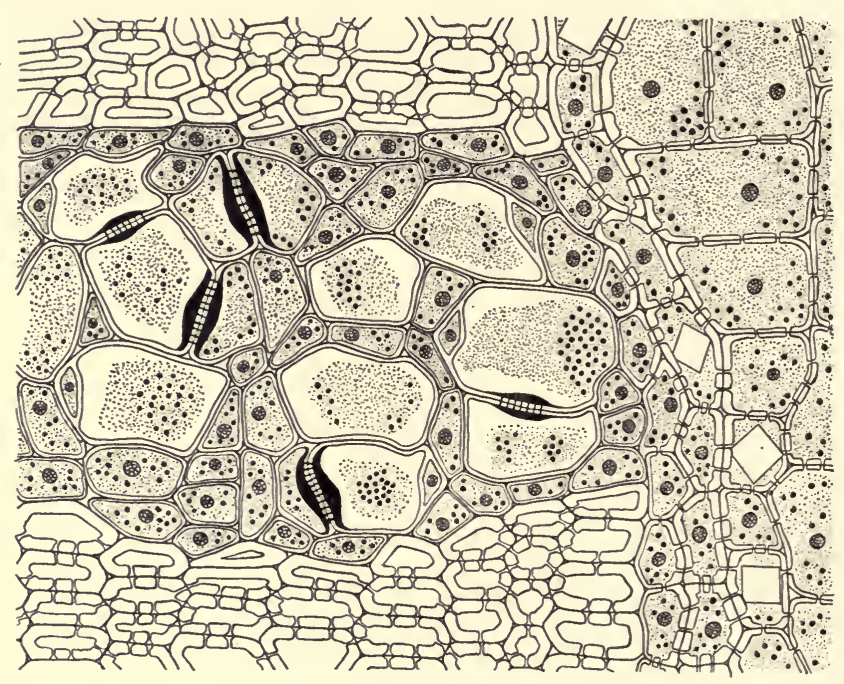

FIG. 93.-Transverse section of the phloem in the grapevine. Explanation in the text.

to the right the figure shows more parenchyma and additional fibers of the hard bast.

For comparison with the basswood is presented in Fig. 93 the transverse view of the phloem in the grapevine (Vitis species). To the right lies a large ray of the compound type. To the left of this is situated the phloem proper, consisting, as is often the case in the dicotyledons, of hard and soft bast. The former tissue is composed of fibers which differ chiefly from the corresponding structures in Tilia by the fact that they are septate when viewed in 
longitudinal section. The soft bast consists of parenchyma, sieve tubes, and their companion cells. Crystallogenous elements are inconspicuous in the bast of the grapevine. The sieve tubes in some cases show their terminal and mainly functional sieve areas in transverse section. The plate in this instance is blocked by a formation of callus which in the vine covers the plate each autumn and disappears again in the following spring when vegetative activity is renewed. This condition is unusual, since. quite generally in the angiosperms, as in the gymnosperms, the appearance of a callus marks the end of the life of the sieve tube. A seasonal callus is likewise found in the barberry and in certain other instances. The final callus, marking the dissolution of the characteristic functional elements of the phloem, is known as the definitive callus. The tubes of the phloem in the grapevine are of interest in showing a considerable amount of so-called transitory starch, indicated in the figure by the larger black granules.

Both the xylem and the phloem of herbaceous dicotyledons and of the monocotyledons show interesting modifications of the conditions characteristic of arboreal and perennial forms. Here the vessel and the sieve tube constitute to an increasing extent, and sometimes exclusively, the functional elements of the two important regions of the fibrovascular bundle and are present in what may be regarded with a strong degree of probability as their highest and most specialized form. The vessel is practically always porous in herbs and presents as a usual peculiarity of organization terminal walls which are nearly or quite horizontal. The sieve tube also tends toward horizontal end walls, and the sieve plates have practically disappeared from the lateral walls, the terminal areas alone being functionally important.

In concluding the statement regarding the organization of the structures in the phloem it will be well to represent side by side the stereoscopic aspects of the several types of sieve tubes in comparison with corresponding varieties presented by tracheids and vessels. In Fig. 94a is shown diagrammatically the organization of a sieve tube in the secondary phloem of a gymnosperm. It is clear that the plates are confined to two opposite walls, which are the radial ones. The cell, further, tapers imperceptibly to a point at 


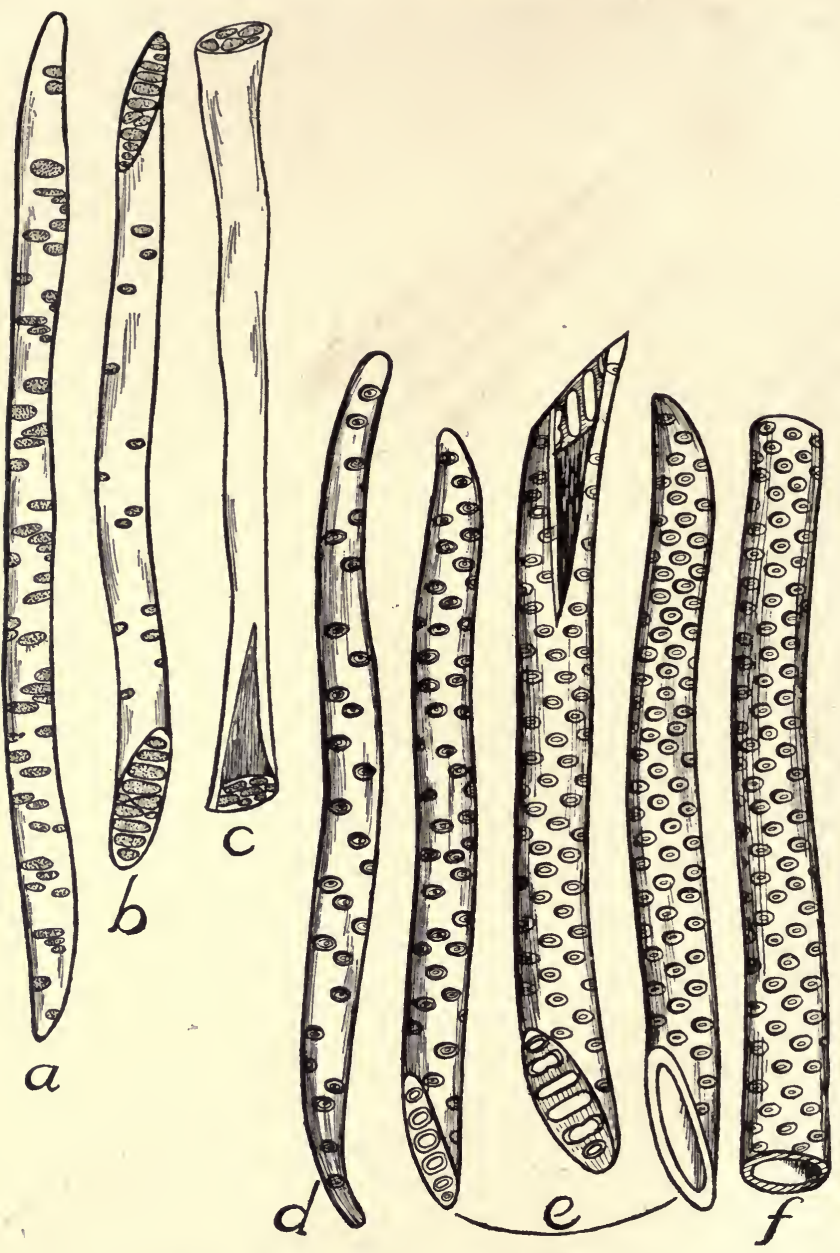

FIG. 94.-Diagram showing types of sieve tubes and vessels in the higher vascular plants. Explanation in the text. 
either end. For comparison with this condition is shown in $b$ the sieve element of an arboreal dicotyledon. Here the terminal walls are distinctly at angles with the lateral ones and bear the bestdeveloped sieve plates. Laterally, on two opposite and usually tangential sides, are situated the degenerate sieve plates, or lattices. The third item, $c$, visualizes the type of sieve tube characteristic of an advanced herbaceous dicotyledon or of a monocotyledon. In this instance the terminal wall of the sieve tube is horizontal, and parietal lattices are conspicuous by their absence. This state represents the highest degree of differentiation of the sieve element. In the following items are delineated corresponding conditions for the water-conducting elements of the xylem. In $d$ appears a tracheid from the spring wood of Pinus, showing tapering or fusiform configuration and exclusively radial pitting. Next, in $e$, appear the main vessel types of Gnetales and perennial dicotyledons, showing inclined terminal walls which in one instance have scalariform perforation of the lower and in the other the porous perforation of the higher perennial types. Finally, in $f$ is depicted the vessel of the monocotyledons and extreme herbaceous dicotyledons. Here the terminal walls are practically horizontal, and the perforation is porous (in the case of the monocotyledons invariably so). 


\section{CHAPTER IX}

\section{THE EPIDERMIS}

In vascular plants the epidermis is ordinarily a single layer of cells, and this fact, together with the external position of the epidermis, makes the latter extremely easy to distinguish. The organization of the epidermis is often strongly influenced by conditions of environment, and as a consequence the integumentary structures have a value from the evolutionary standpoint which can be very easily overestimated. On account of its relatively slight phylogenetic interest the epidermal tissue will receive small attention in the present connection.

In the case of the organs of plants normally exposed to air and not to water or earth we find the integumentary structures consisting of a well-marked layer of cells, which ordinarily remains single and is characterized by the presence of stomata or pores. The cells of the epidermis in lower forms often contain chloroplastids, while in higher forms chlorophyll-containing plastids are usually found only in water plants or in those inhabiting shade. Plastids or masses of organized protoplasm in the epidermal cells of the higher plants are not indeed rare, but usually when found in the superficial layer they lack color and consequently are included in the category of leucoplastids. The chloroplastids of the epidermal structures of Pteridophyta may possibly indicate the primitive presence of these bodies in the integumentary tissues of the higher plants, but they are perhaps equally susceptible of interpretation as a response to a shaded or damp habitat. The presence or absence of chloroplastids in the epidermal elements cannot consequently receive a very high valuation from the standpoint of determination of the relative phylogenetic position of groups of vascular plants. The situation is particularly obscure on account of the lack of bearing of fossil evidence on the question, both because plastids are rarely preserved in the cells of extinct plants and because, even if they were, it would be impossible to distin- 
guish between chloroplastids and leucoplastids in the altered state which necessarily results from the condition of fossilization.

In some instances the epidermis commonly becomes a multiple layer often known as water tissue. This is particularly the case with plants of different systematic affinities which are exposed to extremely arid conditions. Ficus elastica affords an excellent illustration of the multiplication of the epidermal layer in leaves
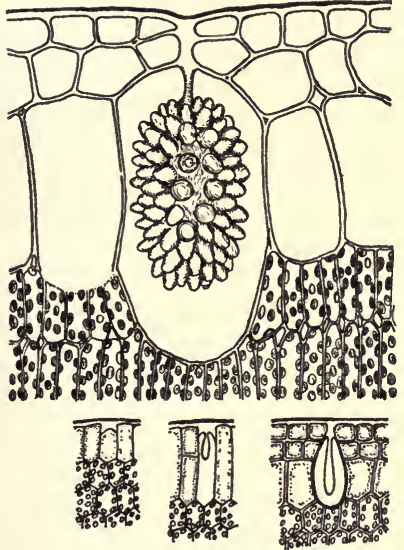

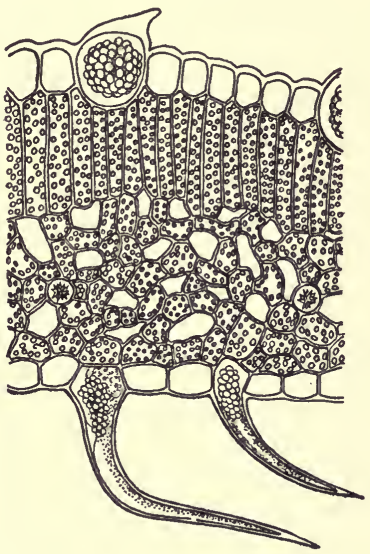

FIG. 95.-Epidermis of the Urticaceae. Explanation in the text

of xerophytic habit. Here the epidermis, which in the young condition consists of a single stratum, as the leaf matures becomes divided into several stories of cells, all characterized, like the epidermis from which they are derived, by the absence of chloroplastids. Another feature which especially distinguishes the epidermal structures of the Urticaceae and Acanthaceae is the presence of deposits of carbonate of lime, generally in the form of acinose masses supported on a peduncle from one side of the cell. In urticaceous leaves which are mesophytic in their environment, such as the nettle or hemp, the structures under discussion, known as cystoliths, are found in the outermost cells of the plant-that is, 
in the elements of the simple epidermis. In Ficus elastica, on the other hand, the multiplication of the epidermal layer has resulted in the imbedding of the cystoliths deeply in the substance of the water tissue. Cystoliths are a valuable diagnostic feature in the case of the Urticaceae and Acanthaceae.

In many gymnosperms the epidermal layer is reinforced, frequently in the leaf and more rarely and only in the most ancient representatives of the group in the stem, by a layer of colorless and generally strongly thickened cells known as the hypoderma. It is not at the present time clear that the hypoderma so called in gymnospermous leaves is derived from the epidermis by the multiplication of its cells, but the structure in question is most conveniently discussed under this head. This layer occurs very distinctly in the leaves of the cycads, Ginkgo, and the conifers, and deepens the strata of colorless cells bounding the surfaces of the leaf. In older types the hypodermal layers tend to become ribbed, and in the leaves of Mesozoic pines and other conifers, as well as of the Cordaitales of the Paleozoic, this condition is often very marked. In the Cordaitales, Cycadofilicales, and associated groups the ribbed condition of the tissues supporting the epidermis is often strongly developed in the stem organs. It is not, however, by any means settled whether the hypodermal layers are of common origin with the epidermis and hence belong to the same histological category.

In plants exposed to extreme drought, physical or physiological, the epidermis responds not only by the frequent multiplication of its layers but also by the development of a well-marked cuticle and by the cuticularization of the outer region of the external walls of its cells. This condition is well developed in plants of desert habit or in those rooted in poisonous soil, even when moist conditions are present. In aquatics, on the other hand, the cuticular structures are poorly differentiated and may be lacking even in the case of the spores, which more constantly than any other structures of the higher plants maintain a cuticularized exine or outer coat. The question of the development of cuticle in plants is of some interest from the standpoint of past climatic conditions on the surface of our earth, although even here it is not conclusive on account of the nearly identical influences of dry and merely poisonous substrata. 
Structurally the value of the cuticle for purposes of identification or as a basis of evolutionary speculations can easily be exaggerated. There is little doubt that it shares with the general external form of plants the shortcoming of unreliability, which results, as in the case of formal characters, from close connection with physiological necessities.

The epidermis is not only a limiting membrane of plants, but is likewise charged with the important office of bringing about regulated interchanges between internal spaces and the outside air. The mechanism which performs the function of facilitating gaseous and other interchanges is the stoma. This structure consists of a pair of guard cells variously organized to meet conditions of environment and bounding a pore, the stoma proper. The guard cells in cases where they are actually functional have their inner and outer walls so thickened that the accumulation of osmotic pressure within the cells leads to their divergence in the middle line with the resultant opening of the pore. No matter what may be the conditions in regard to the presence or absence of chloroplastids in the general epidermal cells, the guard cells are always provided with green corpuscles and even under conditions of starvation retain a modicum of starch after this substance has disappeared in the rest of the cells of the leaf. In plants exposed to a high degree of drought and insolation the guard cells of the stomata become very much thickened and respond to the stimulation of light and moisture only under extreme conditions. A further safeguarding of the epidermal pores or stomata often results from their being depressed below the surface of the leaf or sheltered under a hairy protection. Plainly, under the last-described conditions the loss of water from the stomata will be much less than in the unsheltered condition. The guard mechanism in plants exposed to extreme drought is sometimes throttled, as it were, by a surrounding zone of cells which differ from the ordinary epidermal elements in possessing chloroplastids. Cells of this type are known as accessory cells. The accessory device is variously related to the guard cells, the free movement of which it serves to check. Very frequently the braking mechanism consists of a collar surrounding the guard cells, while in a large 
number of instances the accessory cells lie over the guard cells, so that the latter are nearly or quite shut off from the surface of the leaf.

In many cases the guard cells have walls which differ chemically in different regions. In the pine, for example, the walls of the elements inclosing the pore of the stoma along their inner margins, where they actually abut on the stomatic aperture, and on the opposite sides, where they are in direct contact with the adjacent cells of the epidermis, are in a condition of pectic cellulose and absorb strongly certain of the hematoxylin stains. It has been quite generally observed in physiological investigations that the inner and outer walls of the stomatic guard cells are extremely viable to water-so much so, in fact, that a deficient water supply in plants flourishing under ordinary garden conditions results in the rapid loss of turgescence in these elements.

The epidermal structures are frequently characterized by outgrowths known as hairs. These are most generally composed of comparatively few cells, but in some instances may become very complicated in their organization. When it is clear, in spite of any degree of elaboration and magnitude, that the organs are purely epidermal in their origin, they are properly designated as hairs or trichomes. If tissues of the fundamental or fibrovascular systems, or both together, enter into the structure of the processes, they are known as emergences, provided they do not come more accurately under the caption of modified leaves or lobes of leaves, or, finally, of branches. The trichomes are not usually of a high value in connection with evolutionary anatomy, since they are subject to a very considerable degree of variability in accordance with conditions of environment.

The sporangia of vascular plants are sometimes considered to belong in the category of trichomes, but it is very difficult to bring them consistently under this heading. While it may be maintained with a certain degree of force that the spore sacs of the polypodiaceous ferns are of the nature of hairs, since they are clearly entirely derived from the superficial cells of the leaf, it is difficult to homologize them on this interpretation with the sporeproducing members of the lower and more primitive groups of 
Pteridophyta. It must be confessed that the actual organization of the sporangium is a better indication of its morphological significance than is any relation in origin to any particular tissue system or any combination of tissue systems. Taking the vascular plants as a whole, it seems evident that the sporangium probably antedated the clear differentiation of the tissue systems and is accordingly best regarded as an organ sui generis and as belonging consequently to a distinct anatomical category. It will be shown in what follows that there is good evidence in certain instances that tissue systems other than the epidermis enter into the organization of the sporangial structures of the seed plants.

In conclusion it may be stated that the epidermal structures show an exceptional degree of plasticity to the molding influences of environment, a condition which makes them of less value from the phylogenetic standpoint. This highly variable system constitutes the boundary between the organism and its surroundings, whether fluid, gaseous, or solid. It consists normally of a single layer of cells which in certain drought-resisting plants is multiplied to constitute what is ordinarily called water tissue. The epidermis is perforated by pores known as stomata and produces outgrowths known as trichomes or hairs. The former structures consist of a pair of cells usually highly responsive to the combined presence of light and carbon dioxide and surrounding an occlusible pore. True stomata are confined to the spore-producing generation of the higher plants (from the Bryophyta upward). In spite of erroneous statements to the contrary, in the case of certain badly preserved fossil plants there are never more than two guard cells related to a stomatic opening. Assertions contradicting this are based on the interpretation of accessory cells as guard cells, where the former overlie the elements guarding the stomatic pore. Neither the shape of the epidermal cells nor the organization of the stomata nor the structure of the hairs or trichomes can be regarded as having a very high importance from the standpoint of the anatomical identification of extinct plants in the absence of the confirmatory evidence presented by external form and internal anatomy. 


\section{CHAPTER X}

\section{THE FUNDAMENTAL TISSUES}

It has been indicated above that the epidermal tissues constituting the external boundary of the plant toward the outer medium have relatively slight value from the standpoint of evolutionary anatomy. The same statement may be made (with less emphasis) concerning the category of cellular organization, known as the fundamental tissues. This group is on the whole much more extensively developed and more clearly defined in the lower forms of vascular plants than in the case of those higher in the scale. The more massive presence and the more distinct individualization in the fernlike representatives of vascular plants naturally carry with them a higher degree of histological differentiation. The fundamental system is definitely bounded internally by the endodermis (phloeoterma of Strasburger) in most of the lower groups of plants, while in more advanced vegetable organisms the limits between the fundamental and fibrovascular systems are often obscurely indicated except in that most conservative of all organs, the root. Externally the limits of the fundamental system are usually clear unless the multiplication of the epidermal layer conceals the situation. Occasionally in roots the fundamental system is sharply marked off toward the epidermal system by the presence of a welldeveloped limiting layer, the exodermis, which externally is the counterpart of the endodermis, indicating the internal confines of the cortex.

The accompanying figure (Fig. 96) of the subterranean stem of the bracken fern will illustrate both the definite limits and the considerable degree of specialization and consequent physiological importance of the fundamental tissues. It is clear that within the epidermis and external to the fibrovascular bundles there is present a large amount of cellular substance. This is distinctly differentiated into two categories-namely, sclerotic or supporting tissues and storage parenchyma. The former is present in two 
distinct, more or less continuous, zones: an outer one lying immediately under the epidermis and somewhat interrupted opposite the angular projections on the sides of the rhizome, and an inner one coming between the large medullary and the smaller external series of bundles. The storage cells, which are characterized in the figure by their walls and in nature by the large amount of starch present in their cavities, occupy all the transverse section not taken up by the epidermis, the fibro-

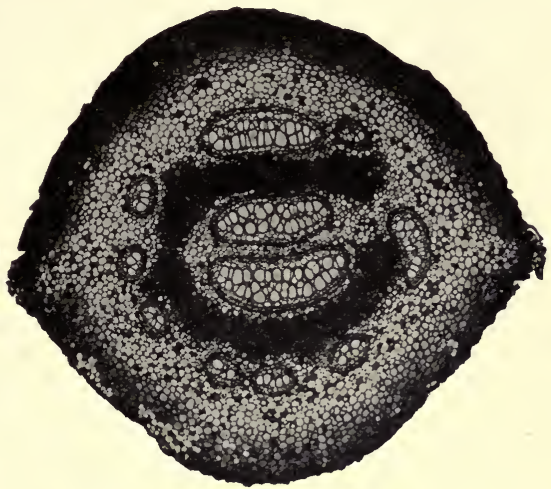

FIG. 96.-Rootstock of the bracken fern

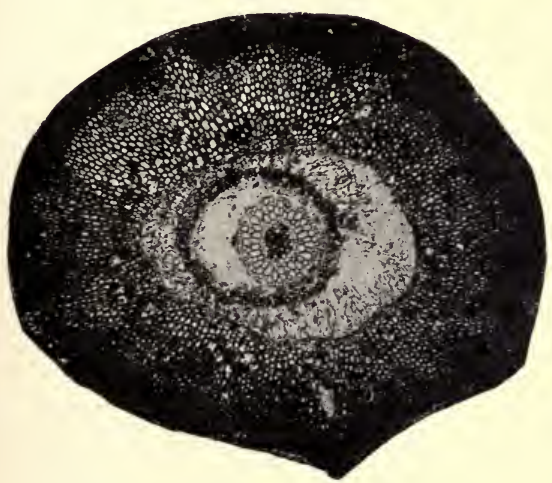

FIG. 97.-Stem of Lepidodendron Spenceri

vascular system, and the sclerotic bands just described. The mechanical requirements of the stems of many lower vascular plants are provided for by skeletal structures present in the fundamental system. This situation is common to both the Pteropsida and the Lycopsida. The truth of this statement is revealed by the figure presented of the stem of a lepidodendrid, a very ancient representative of the group of club mosses or Lycopsida. Here zones of thick-walled skeletal tissues 
belonging to the fundamental system can readily be distinguished. It may be stated in a general way that the higher vascular plants have their skeletal tissues developed from the progressive

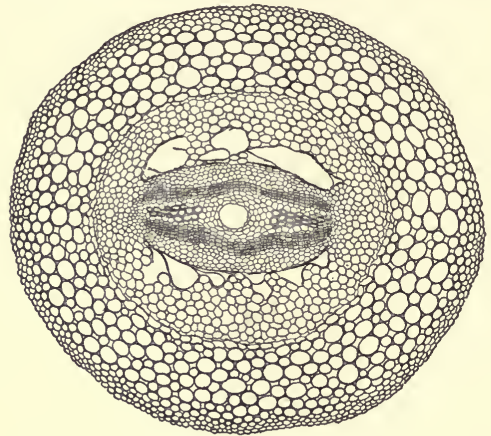

FIG. 98.-Transverse section of young root of the balsam fir. Explanation in the text. mechanical differentiation of the primitively solely water-conducting fibrovascular system, in contrast to the Pteridophyta, in which the mechanical function mainly resides in the fundamental system. In the higher vascular groups not only does the mechanical principle find its best expression in connection with the fibrovascular system,

but the fundamental category of tissues becomes relatively insignificant in amount, particularly in stems with perennial growth. Further, the boundaries between the fibrovascular and fundamental tissues have gen erally disappeared through the degeneracy of the

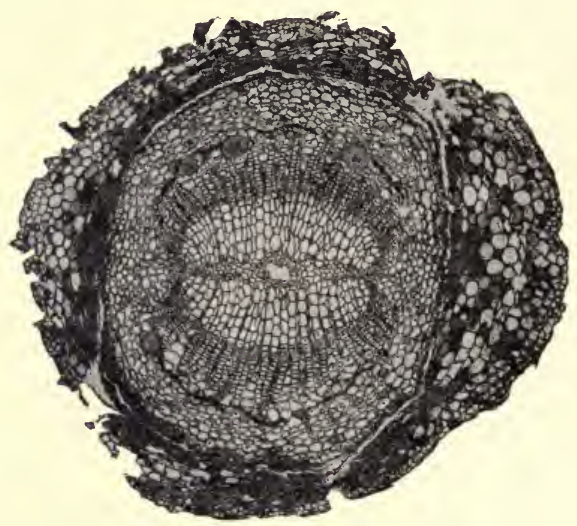

FIG. 99.-Older root of the balsam fir endodermis.

In the case of the root of all vascular plants the limit between the conductive strands and the fundamental system is clearly marked from the lowest forms to those at the very summit of the vascular 
series. This situation is in accordance with the marked conservatism of roots. The clear boundaries between fibrovascular and fundamental structures are, for example, as well marked in the cycads, conifers, and angiosperms as they are in the case of the lycopods, ferns, and Equisetales. In the conifers and many dicotyledons the fundamental group of tissues, although well developed in the young condition of the organ, later entirely disappears. The accompanying illustrations (Figs. 98 and 99) of the root in the balsam fir make the truth of this statement sufficiently clear. In the first of the two figures the organ is shown in a young condition, as is evidenced by the slight degree of development of the woody cylinder. A clear boundary separates the outer region of the root from the tissues belonging to the fibrovascular system. This is the endodermis or innermost layer of the fundamental system. The organization of the second figure shows a further degree of development of the fibrovascular system, while the fundamental tissues, inwardly limited by the endodermis, have begun to shrivel and break away from the surface of the fibrovascular cylinder. The destruction of the jacket of fundamental tissues is the result of the appearance of a corky layer immediately within the endodermis; and this, impervious in its nature, cuts off the supply of nutrition to the external zone. This region of the root consequently dies and flakes off.

In the case of the leaf the fundamental tissues are of considerable importance, since they constitute the green substance or mesophyll of foliar organs. The limits between the stelar or fibrovascular strands in the leaf and the surrounding mesophyll are in general much less well marked than in the root, but on the whole much more clearly indicated than in the stem. In the Pteridophyta in general the endodermis is distinctly developed in the leaf. In the gymnosperms the limits between the fundamental system and the fibrovascular strands are already less obvious, and in the case of the angiosperms a somewhat similar condition is to be observed. The usual situation justifies the summary statement that in foliar organs the fundamental category of tissues is always well developed and is physiologically of great importance, since it subserves the cardinal functions of transpiration and photosynthesis. Further, the morphological limitation of the fundamental system is only less distinct in leaves than it is in roots. 


\section{CHAPTER XI}

\section{DEFINITIONS OF THE ORGANS}

Not uncommonly an organ is defined as the tool of a function. Of course from the evolutionary point of view this conception cannot hold, because the same organ in a plant often at different times subserves very diverse functions. For example, the stem may function as a leaf and the leaf as a root. From the standpoint of the doctrine of descent the value of a particular organ in the course of evolution is assigned, not so much on the basis of what it does, as on that of what it is. In other words, an organ is known mainly by its organization.

In the case of the vascular plants there are a number of distinct structural units to which the name of organ is applied. It is not by any means clear that the different organs of plants were always as distinct from one another as they are at the present time. For example, there is some reason to believe that the root may manifest the primitive structural features of vascular plants, and in the course of time the stem may have become differentiated from the root, arriving finally at that degree of distinctness which at present definitely separates it from the root. It has, moreover, often been suggested in recent years that the leaf of the higher vascular plants was originally of the nature of a branch and that its distinguishing features are the product of later evolution. It would be going beyond the range of an elementary work like the present one to discuss the question of the origin of organs, particularly as the data are extremely meager and not always easy to interpret. Disregarding, consequently, any speculations as to the appearance of the organs of the higher or vascular plants, we may proceed at once to the enumeration of those which are generally accepted by anatomists.

The parts or organs of the higher plants are usually distinguished as three-namely, root, stem, and leaf. To these may be added a fourth, the sporangium or spore sac. Each of the organs named 
has its particular features of organization and can be traced as a definite and distinct structure far into the geological past of our existing plants. Of the organs the root is the one usually distinguished by subserving the function of attachment to the substratum and also that of absorption of nutritive substances in solution from the soil. In the case of the root, also, the direction of growth is normally and primitively downward. The stem has an upwardly growing axis which serves as a support for the other parts of the plant. The leaf is distinguished in turn by its usually flattened form, which particularly qualifies it for its important function of bringing the organism into relation with light and the gases of the atmosphere. The sporangium has the particular office of producing spores and may consequently with a certain degree of appropriateness be designated as the organ of reproduction. The indications supplied above as to the rôles ordinarily played by the respective parts of the higher plants by no means afford reliable definitions of those organs. Very frequently stems, more rarely leaves, and sometimes even sporangia serve in connection with the function of attachment, so that the relation to the substratum cannot be assigned as a fundamental and exclusive feature of the root. Similar objections may be raised in regard to the functional definition of all the parts or organs of.plants. In the present connection it will be well to consider these important categories of structure from the standpoint of organization rather than function, as that procedure is most advantageous from the evolutionary point of view.

\section{THE ROOT}

There is good reason to believe that the root is on the whole the most primitive of plant organs, and it will be shown in subsequent chapters that, even if there be room for doubt as to its primitiveness, there can be none as to its conservative character. There is, in fact, no organ of plants so antique in its organization or so retentive of ancestral traits as is the root. Functionally, as has been indicated above, it serves usually to connect the plant with the substratum. Its unique and distinguishing features, however, are supplied by its internal organization. Roots are characterized by two main structural features-namely, the possession of a root 
cap and a type of fibrovascular organization known as radial. The root cap or pileorhiza clearly differentiates roots from other structures in the plant which may happen under the stress of environment to assume a subterranean mode of existence. Moreover, the root cap persists even in those cases in which the root becomes aërial or aquatic in its habit. We need not devote attention to the mode of origin of the pileorhiza, or protective tip of the root, as studies of this nature are at the present time of doubtful morphological value, and the consideration of them in an elementary work is quite out of the question. All that need be stated is that the cap occupies the tip of the root and is continually renewed from behind, sometimes, but not invariably, by a well-defined active tissue known as the calyptrogen. The protective cap is characteristic of all roots from the lowest to the highest plants. There is only one general condition under which this structure is ordinarily lacking. In the fungus-infected roots or mycorrhizae of humus plants, in which the fungal infection is superficial and does not notably invade the internal tissues of the root, the root cap is degenerate or absent. In the case of the haustoria of certain root parasites, such as the dodder, not only is the root cap absent, but also the general condition of degeneracy is so marked that frequently the only criterion of the morphological value of the structure is its internal or endogenous origin in the axis of the parasite.

A salient feature of the organization of the root is furnished by the fibrovascular tissues. Here the type of fibrovascular system is that known as radial. In this condition the masses of phloem, instead of surrounding the wood or xylem (a state commonly found in the ferns and their allies) or lying just outside of it in the same radius (seed plants) as in the stem and leaf, lie in different and distinct radii. This mode of relative disposition of phloem and xylem is responsible for the term radial as applied to roots, and it is a very important characteristic of their organization. Where the phenomenon of secondary growth in thickness is absent, there is no subsequent modification of the general relations of tissues in roots. In the case of roots with secondary growth, however, the situation changes with the appearance of the secondary xylem and phloem. The secondary tissues begin to form in definite 
relation to the clusters of primary phloem in all probability because the foodstuffs are provided by that tissue. The active layer which makes its appearance here continually adds new elements on the outside to the phloem, and on the inside gives rise to the secondary xylem. As a consequence of this situation the secondary wood, being formed opposite the clusters of primary phloem, naturally alternates with the primary wood. With the beginning of the secondary growth the root therefore abandons the radial type of organization of the primary structures. The lateral roots originating from a given root have their position determined, however, by the topography of the primary structures in the main root and grow out in vertical rectilinear rows corresponding to the primary xylem. But in the monocotyledons an exception to this condition is found, since each lateral root originates in the interval between two angles of the primary xylem.

Although the secondary structures of the root, as has been indicated above, are collaterally organized, the primary plan of the root is radial. The root, in fact, is the only organ of the plant for which a single ground plan will illustrate the conditions found in all groups of plants, living or extinct. The extreme conservatism of the anatomical organization of the root is an outstanding feature and, as will be indicated in succeeding chapters, renders it of the greatest value in working out the evolutionary sequence of the various groups. Extreme conservatism, radial organization of the primary fibrovascular structures, the possession of a root cap or pileorhiza, and an internal origin from the surface of the fibrovascular cylinder are the salient and important criteria of the root.

\section{THE STEM}

In the case of the axis or shoot of plants no such general formula can be arrived at as in the root. Just as the root is the least changeable of all the organs of the plant, so the stem is of all the most variable. In internal organization it varies greatly from the lower and more ancient groups to the higher and more modern. So numerous are the types of stem as regards anatomical structure that they can most profitably be discussed under the particular groups of the vascular plants in later chapters of the volume. In 
the absence of any general and common anatomical characteristics of the stem for vascular plants as a whole, it is necessary to define it by the important criteria supplied by the mode of attachment of the appendages. The axis is characterized by the fact that it is divisible into nodes from which the appendages normally take their origin and into segments more or less elongated separating these from one another and known as internodes. By the possession of nodes and internodes the stem is at once distinguished from the root and the leaf. There are usually at least two kinds of appendages attached to the stem in the region of the nodes-namely, the leaves and secondary axes or branches. If the axis be subterranean, roots may also make their appearance in the region of the nodes and are clearly separated from the other appendages by their internal origin. Both leaf and branch are formed superficially or exogenously on the axis. The leaf and secondary axes or branches are frequently related to one another in a quite definite manner, the young branch appearing in the upper angle or axil of the leaf. Further, the arrangement of the leaves and consequently of their axial related structures, the branches, follows a somewhat definite plan. Where more than one leaf occurs at a node, the foliar structures are said to be opposite or whorled; and where they are attached singly to the nodes, the arrangement of the leaves or phyllotaxy is said to be spiral. The spirals are of different types, according as the number of foliar organs intervening between two vertically opposite leaves is greater or smaller; and the spiral turns about the stem, drawn through the leaf bases, are more or less numerous. In the grasses, for example, the spiral phyllotaxy is expressed by a fraction of which one is the numerator and two the denominator. In the alder the fraction is one over three, and in the oak or willow two over five. The numerator indicates the number of turns about the stem in passing from one leaf to the next one vertically above it. The denominator is supplied by the number of leaves attached to the stem in the interval between two which are vertically opposite.

The stem is not only extremely variable as regards its anatomical structure in the various groups of higher plants, but its external form is greatly modified in correlation to different conditions of 
existence or various needs on the part of the plant. In general, however, the possession of nodes and internodes serves as a suffcient diagnostic character, since this feature is rarely obliterated even by the most extreme conditions to which the organ is exposed.

\section{THE LEAF}

This organ has a double importance, because it not only subserves the vegetative functions present in the case of root and stem, but is also charged with the extremely important office of reproduction. The leaf from the purely vegetative aspect is of considerable evolutionary significance, since, although much less conservative than the root, it is much more retentive of ancestral characteristics than is the stem. Its chief evolutionary value, as will appear in subsequent chapters, is in connection with the unraveling of the relationships of the older and lower gymnosperms. In more modern types the anatomy of the root generally throws more light on the relationships than that furnished by the leaf. Reproductively the foliar organs are of great importance in connection with taxonomic arrangement. In fact, at the present time the angiosperms are almost universally classified on the features presented by their reproductive leaves, and in the case of the higher gymnosperms anatomical features of the vegetative structures are only beginning to receive adequate consideration in connection with investigations as to evolutionary sequence.

As a reproductive structure the leaf is best considered under the particular groups to be discussed in subsequent chapters, as no general statements can be advantageously made at the present stage. As a purely vegetative organ the leaf is readily distinguished from the stem, of which it forms an appendage, by the absence of nodes and internodes. It is not easy to summarize the anatomical structure of the leaf, since it is to a large degree variable in passing from lower to higher groups. A useful criterion is its dorsiventral organization, which is usually present, at least in the region of the expanded part of the leaf or blade. Another outstanding feature of the leaf is the fact that it is an axillating organ, and as a consequence other appendages are commonly axillary to it. The 
general external form of the foliar structure may reveal the presence of a narrow base or stalk known as the petiole, and this is terminated by the broad, generally horizontally placed, region called the blade or lamina. The petiole often has related to it paired and lateral appendages known as stipules. Occasionally an unpaired appendage of the nature of a stipule, often called the ligule, is present.

\section{THE SPORANGIUM}

This is the organ of reproduction. Although ordinary vegetative parts under certain conditions, and particularly in herbaceous forms, may serve to perpetuate the plant, still the sporangium is the special organ of reproduction and has the most important evolutionary significance in this respect. The organ under discussion is very accurately defined by the fact that it produces spores and is, indeed, the only structure in the higher plants set apart for this purpose. In some cases the essential fact of sporogeny is more or less concealed as the result of the deep-seated modifications connected with heterospory and the seed habit; but in such instances a study of internal organization suffices to make the situation clear. Normally the sporangium is a structure which is an appendage of the leaf. Sometimes it appears to be axillary, although there is no reason to believe that this was its primitive relation to the foliar organs. Very frequently the reproductive leaf undergoes profound modification in connection with the functions it has to perform, and these features furnish a generally approved basis of systematic grouping for the vascular plants. They need not be considered in any detail in an elementary work on anatomy and are best discussed in connection with particular groups in appropriate later chapters. Unlike the other organs, the sporangium shows no indications of derivation from another structure. It has, in fact, been suggested that the sporogonium of the lower bryophytes is the ancestral primordial from which all the other organs have been derived. Professor Bower has developed this idea in lis Origin of a Land Flora. 


\section{CHAPTER XII}

\section{THE ROOT}

As has been indicated in the preceding chapter, the root is characterized by the possession of a protective terminal structure known as the root cap, by its radial organization, and by its endogenous or internal mode of origin. The first and the last characteristics are exclusive features of the root, but that of radial organization is often present in stems of the more primitive types. A comparison of the upright stem of the common club moss, Lycopodium clavatum, with the root of the same species makes the truth of this statement particularly apparent. It is clear from Figs. roo and Ior that the radial organization is common to the two organs and that they

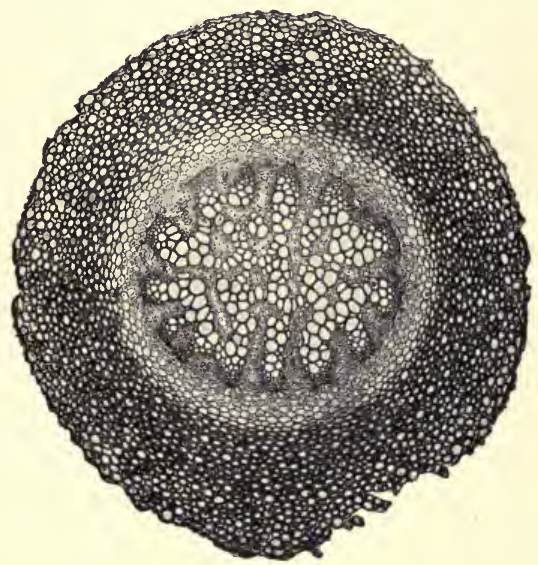

FIG. I0o.-Transverse section of the root of Lycopodium clavatum. differ only in the fact that the stem bears leaves. This resemblance in anatomical structure between stem and root is often present in the lower Lycopodiales and furnishes a strong argument, taken together with their incient occurrence and early decline as a prominent element of the earth's vegetation, for the view that the lycopods are the most primitive plants.

In the great majority of vascular plants the contrast between the organization of the stem and that of the root is very striking. 
In the case of a conifer, for example, the root is clearly distinct from the stem by the possession of a woody cylinder devoid of pith. The primary wood, moreover, in the root is very well developed and forms a lens-shaped mass in the center of the cylinder which is clearly centripetal in its development, since the smaller elements are situated in two or more groups in an external position. The

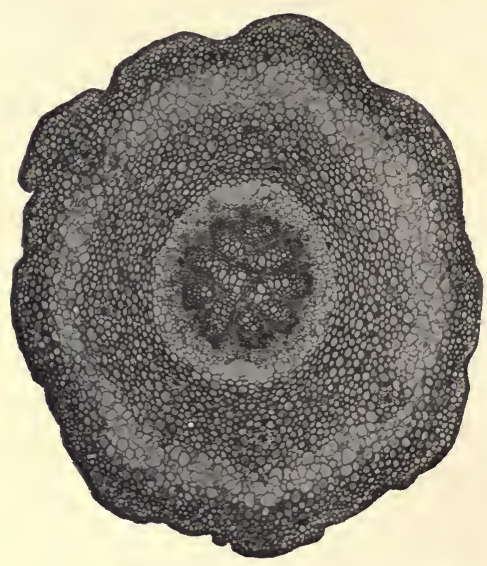

FIG. Ior.-Transverse section of the upright stem of Lycopodium clavahum. primitive organization of the root in contrast to the more progressive organization of the stem is particularly well indicated in connection with the rays. In the cauline woody cylinder these appear to take their origin from the pith; hence arises the commonly employed appellation, medullary rays. The intimate relation between rays and pith which apparently obtains in this case is in reality merely a semblance, owing to the fact that the primary wood has become nearly obsolete in stem organs in the higher gymnosperms and the angiosperms. In the root the primitive situation in which the radial parenchyma has no relation whatever to medullary tissues is very clear. It is obvious that the common term medullary rays is extremely inappropriate as applied to the radial parenchyma of the secondary wood. The true situation is not only revealed by the comparison of stem and root, but it also becomes even more apparent when the stem in older types is compared with that found in existing forms. The more detailed consideration of the contrasts in organization between stem and root is best deferred to later pages, after the anatomy of the various groups has been discussed. 
We may now turn our attention to the particular features of organization presented by the younger and older root in a conifer. In the early stage of development presented in Fig. IO2 the fibrovascular region is sharply separated from the rest of the transverse

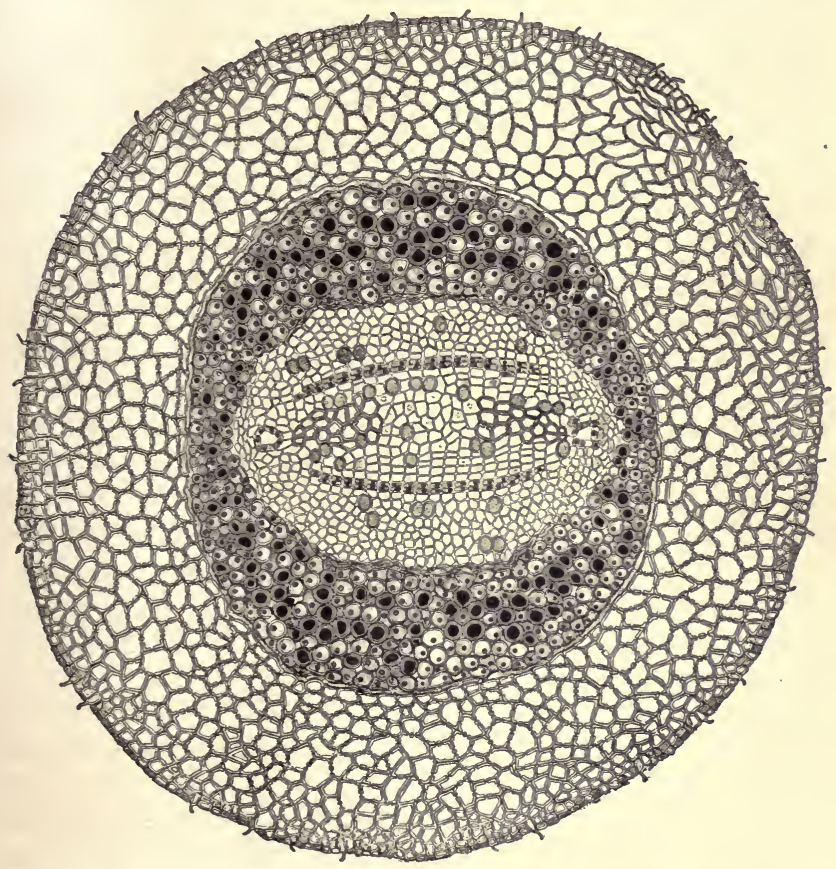

FIG. 102.-Transverse section of a young root of the American larch

section by a well-marked endodermis. Within this layer lies a broad zone, the pericycle, which abuts upon the primary phloem. Inside of the primary phloem lie two bands represented as double rows of cells with protoplasmic contents. This is the cambium, which provides the elements of the secondary xylem and phloem, neither of which can be said as yet to have come into existence. In 
the oval area between the cambial bands lies the primary xylem. This consists of smaller elements at the two external angles, the protoxylem strands. The development of the primary wood is not complete; hence there is a considerable interval in the center which will be later transformed into tracheids of the primary metaxylem. In the first-formed elements of the primary wood the bordered pits of the tracheids can be plainly seen. Subtending each cluster of protoxylem is a resin canal, a common feature of the organization of the root in Picea and allied genera of the Abietineae. It is manifest from the general situation represented in the median region or central cylinder of the root that the primary structures are the only ones conspicuously in evidence, and of these the wood is still incomplete. Further, there is a clear indication of the presence of cambial layers, which will a little later give rise to secondary elements of both wood and bast. Surrounding the central cylinder or stele of the root is the cortex, which is limited internally by the endodermis and externally abuts on the root-hair-producing stratum of the root: this is ordinarily called the piliferous layer. As the section is taken very near the apex of the root, a few rather poorly developed root hairs are seen. It has often been wrongly asserted that there are no root hairs present in coniferous roots. While this condition may be found in the case of those fungusinhabited radical organs known as mycorrhizae, carefully excavated normal coniferous roots nearly always show a band of root hairs in proximity to the root cap or pileorhiza. It will be made clear in what follows that both root hairs and cortex are of short duration in coniferous roots.

In the older root marked differences present themselves both in the region of the central cylinder and in the cortex. Taking first the fibrovascular tissues, we find that the pericycle is as well marked as in the younger condition of the organ, but is now characterized by the formation in its outer region of a zone of regularly arranged cells known as the periderm. This layer has an important influence, as will be indicated later, on the tissues constituting the outer region of the root. It is clear that the primary phloem has more centrally been superseded by a mass of tissue of very regular radial arrangement, the secondary phloem, derived as a result of the 
external activity of the cambium. The primary elements of the phloem constitute a distinct band of collapsed cells on the upper and lower sides of the cylinder, and this is usually very conspicuous in sections of the mature root, provided the organ has not become too old. Within the zones of radially disposed secondary phloem lies the secondary xylem, which has its cells similarly arranged in series. The two masses of secondary wood, facing the corresponding aggregations of secondary phloem, are joined internally with the primary xylem, and they are readily distinguishable from this by the presence of rays and the regular disposition of the tracheary elements. The primary xylem is now complete and no longer shows the hiatus or interruption in the center which is found in the younger root. The two resin canals which lie opposite the groups of small tracheids constituting the protoxylem have not collapsed. The primary wood in the root, in contrast to the primary phloem, does not break down, but persists in maturity. Meanwhile in the exterior or cortical region of the root an important change has taken place in consequence of the formation of a layer of impervious secondary tissue known as periderm. This zone, by reason of its imperviousness, prevents the conveyance of water and nourishment to the outer portion of the root, and the latter consequently dies and is sooner or later exfoliated as a shriveled mass of collapsed cells, as indicated in the diagram (Fig. I03). The result of the loss of the cortex and piliferous layer in the older roots of the conifers is to bring the fibrovascular tissues in immediate relation to the soil. In this stage, however, they no longer serve the function of absorption, but only that of conduction, since the taking up of water and nutritive substances in solution is an activity of the younger root when it is still provided with root hairs.

The subject of the structure and development of the root is so important from the evolutionary standpoint that it is advisable to supplement the description furnished in the two preceding somewhat diagrammatic drawings (Figs. 102 and 103 ) of the organization of the root with actual photographic reproductions of the structures present. In order that the general validity of the principles involved may be made apparent, a different genus of the conifers has been purposely chosen. The diagrams (Figs. I02 and 103) refer 
to the genus Larix, while the actual photomicrograms illustrate the fir (Abies). In the first of these (Fig. ro4) is depicted the root in a younger stage of development. The general situation is clearly identical with that in the larch, since the central region of the root is sharply set off from the cortex by the presence of a well-marked endodermis, a structure, by the way, not found in any organ of the genus but the root. In the central cylinder may be seen the broad

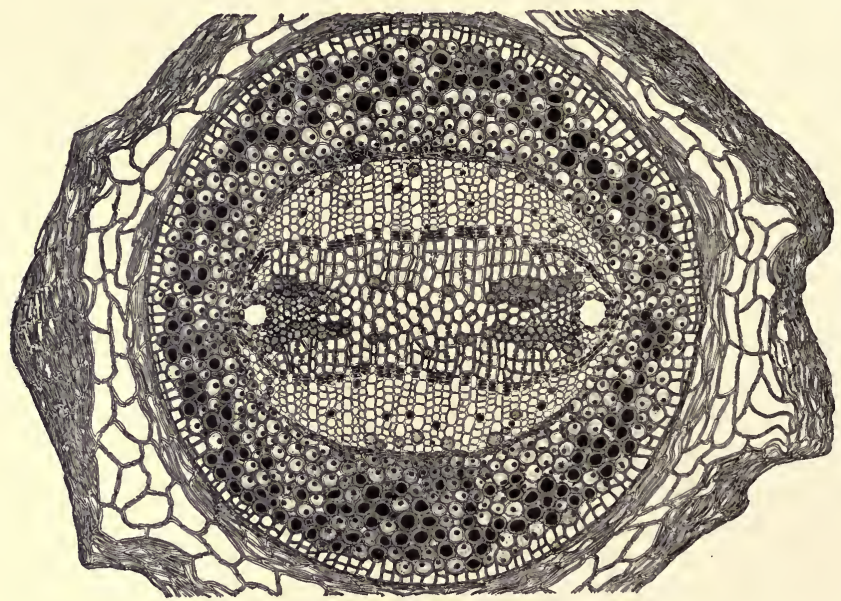

FIg. 103.-Transverse section of an older root of the American larch. Explanation of this and preceding figure in the text.

encircling pericycle, bounding the primary phloem above and below. Within the first-formed phloem lies the mass of primary wood, not as yet completed in the central region, and thus clearly indicating the centripetal order of development of the elements of that portion of the woody structures. In the midst of the primary xylem is situated a resin canal which is not yet fully developed. The singularity of number and the axial position of the secretory canal furnish practically the only features of distinction from the corresponding structures in the case of the larch, where the plural 
resin canals are found exclusively facing the clusters of protoxylem and are never present in an axial position.

In Fig. I05 an older stage of the root of Abies is presented. Here the development of the primary wood is completed, and as a consequence the axial resin canal stands out more distinctly. The secondary activity has proceeded so far that there is now a considerable zone of radially seriate xylem and phloem. The primary phloem has col-

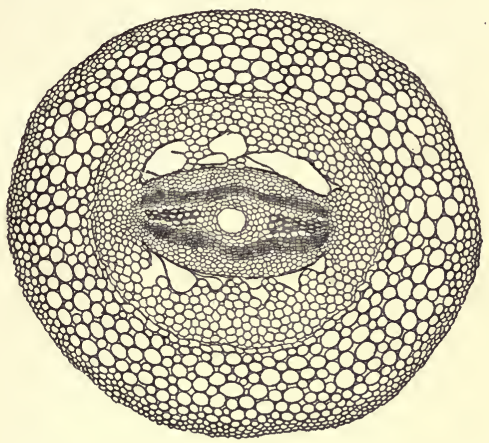

Fig. I04.-Young root of Abies balsamea

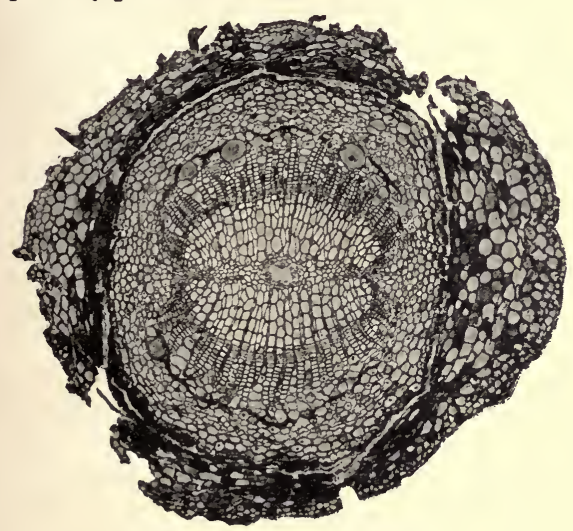

Fig. 105.-Photograph of an older root of Abies balsamea. Description of this and foregoing figure in the text. that the radial parenchyma of the root of the conifers can in no wise be appropriately designated medullary rays, since the structures lapsed. The appearance of a layer of cork or periderm with radially disposed elements is obvious in the region of the endodermis, and the activity in this tissue is the cause of the death and the ultimate shedding of the external or cortical region of the root. It is quite clear, as has, indeed, been pointed out above, 
in question have no relation whatsoever to the pith, which in this case is non-existent. On general evolutionary grounds there are the best of reasons for regarding the type of organization present in the root of the conifers and, in fact, of the gymnosperms in general as more primitive than that which characterizes the stem, and consequently of great value from the standpoint of the doctrine of descent.

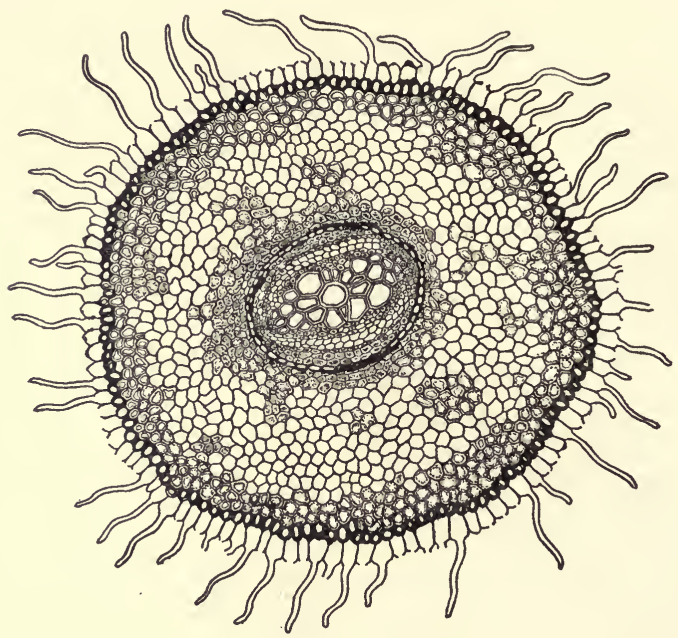

FIG. I06.-Root of Osmunda cinnamomea. Explanation in the text

The root of the ferns and their allies differs from that found in the gymnosperms only by the absence of secondary tissues. In Fig. Io6 is shown the root in Osmunda. Here central cylinder and cortex are clearly delimited by the endodermis, composed of cells with dark contents. Within this boundary lie the two masses of phloem, and in the intervening region is seen the primary xylem. This consists externally of narrow primitive elements which are continued toward the center by the progressively broader tracheids of the metaxylem. No secondary activities subsequently modify 
the topography of the root in any living representative of the fern alliance or of the Pteridophyta in general.

The structures of the root in the dicotyledonous angiosperms are appropriately considered at this stage. Since the early condition of development of the root has been already discussed and figured in detail for the conifers, it will not be necessary to enlarge upon this phase in the present connection, for the underlying principles involved are the same in both conifers and dicotyledons. It will be convenient to begin with a woody or arboreal root.

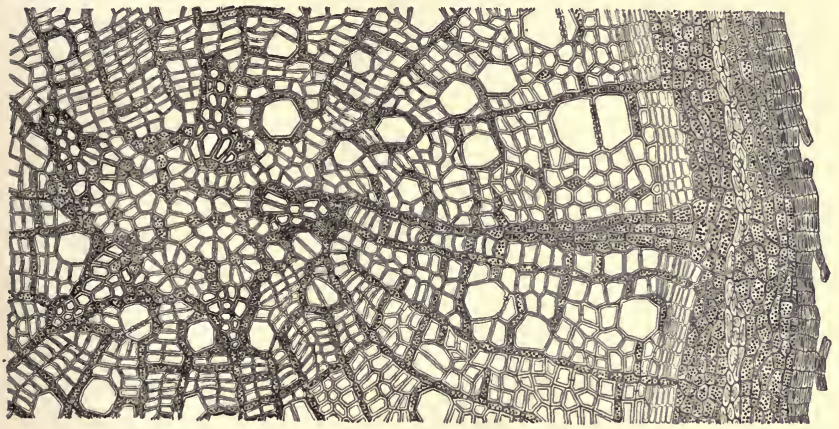

FIG. 107.-Transverse section of root in Almus incana. Explanation in the text.

Fig. I07 illustrates the organization of an older root in Alnus incana. Toward the right appears a layer of periderm bounding the outside of the organ. Within lies the cortex, which terminates at a layer of thick-walled cells, the pericycle. Within the pericyclic layer, marking the outside of the fibrovascular cylinder, lies the phloem, not depicted in detail. Then comes the cambium, followed internally by the secondary wood, in which conspicuous rays are present. Toward the extreme left of the illustration lies the fiveangled star indicating the topography of the primary xylem. The stellate aggregation of elements shows at each angle of its five points groups of cells of smaller size, the clusters of protoxylem consisting of spiral tracheids. The mass of metaxylem into which 
the angles of protoxylem become confluent by continued centripetal development has interspersed with its elements a varying amount of parenchyma. Vessels are present in the primary five-angled region of the wood, but they do not so conspicuously differ from the tracheids in transverse section as is the case with the similar elements of the secondary wood.

Two features are particularly worthy of note in the present

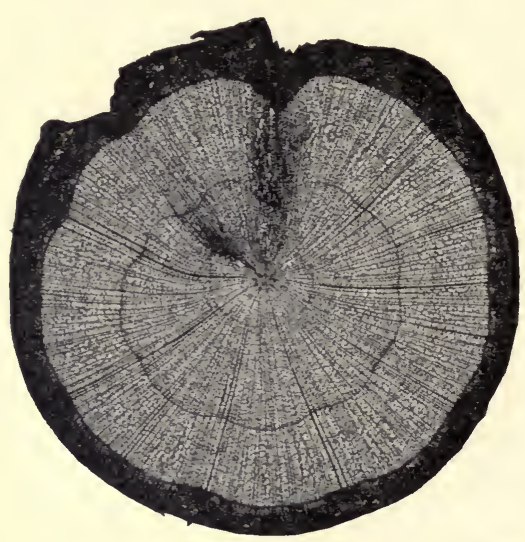

Fig. 108.-Transverse section of root of Alnus japonica. connection. First of all, the layer of periderm does not occupy so deep a position in the root as in the larch and fir shown above. As a consequence the central cylinder does not generally become stripped of its cortical envelope as has been found in the conifers. Another interesting. feature is presented by certain modifications in the rays in relation to the star-shaped mass of primary wood. This situation is more easily understood by reference to the accompanying photographs. The first illustration (Fig. I08) represents a transverse section of a mature root of Alnus japonica. The secondary wood is characterized by certain modifications in the structure of the radii corresponding to the angles of the primary wood. These consist of clusters, more or less pronounced, of rays of greater width than the uniseriate condition found in the wood at large, among which the vessels, characteristic of the organization of the remainder of the xylem, are absent. In other words, aggregate rays are plainly present in the secondary wood and are quite clearly related to the groups of protoxylem marking the angles of the primary xylem. Since 
the secondary roots definitely take their origin from the same clusters of protoxylem which are subtended by aggregate rays in the secondary wood, it follows that the secondary roots are correlated with aggregate rays precisely as the traces of the leaf are imbedded in similar modifications of the radial parenchyma in the case of the stem. Aggregate rays are much more commonly found in the root than they are in the shoot organs, a consequence of the conservatism of the root, which is to be emphasized in a later chapter. The intimacy of the relation between the aggregate ray and the trace of the secondary root can be readily inferred from the inspection of Fig. I09, a photograph of a transverse section of the root of Alnus japonica. It is clear that the trace is related to a mass

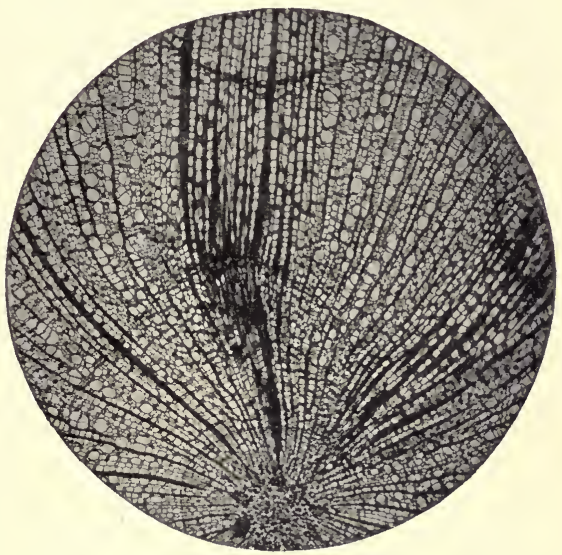

FIG. I09.-Transverse section of part of same, more highly magnified. Description of this and last figure in the text.

of enlarged rays among which vessels are conspicuous by their absence, precisely as in the leaf trace illustrated in Fig. I30, page I77. It thus becomes clear that aggregate rays may be a feature of organization of the root as they are of the stem, and that in both organs the clusters or congeries of rays are most conspicuously developed in relation to the appendages.

The secondary structures of the root are characterized by the same three types of multiseriate rays found in the wood of the stem as figured and diagrammed in chapter vi. In the case of the root, however, the primitive aggregate condition which precedes both the compound and diffuse types tends strongly to persist. 
The truth of this statement can be made apparent by reference to the oak and the birch which represent, respectively, in the species

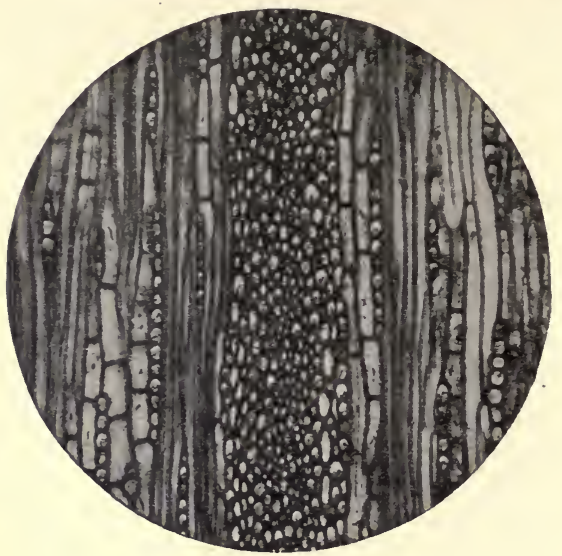

Frg. Iro $a$

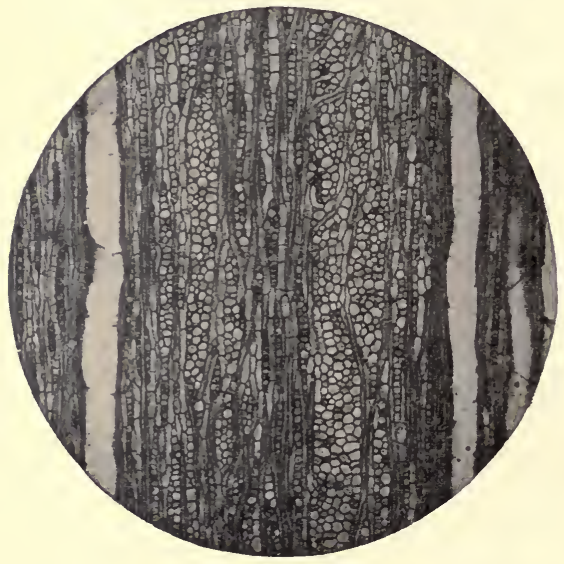

FIG. IIO $b$

FIG. r ro $a$ and $b$.-Tangential sections of large rays in stem and root of Quercus rubra. Explanation in the text. found in the Northern United States and Canada the complete exemplification of the compound and diffuse types of rays. In Fig. I Ioa and Fig. I $10 b$ are shown tangential views of the wood of the stem and the root of Quercus rubra, the red oak. In each appear large rays. Above is the illustration of the condition in the stem, and here the ray is obviously of the type described in an earlier chapter as compound. Below, the condition typical for the root is indicated, and it is here equally clear that the large ray is made up of a congeries of smaller rays separated from one another 
by fibers. The state of the ray figured for the root is the primitive one for the stem and the ancestral one for the genus Quercus, as evidenced by its tropical and subtropical representatives (live or evergreen oaks) which typically manifest the presence of aggregate rays. In the genus Betula the same situation presents itself with reference to the aggregate and diffuse rays. In the stem, as is shown in Fig. I I I $a$, the multiseriate rays are diffused throughout the structure of the wood, while in the root aggregations of such rays are related to the outgoing traces of the secondary roots, Fig. I $1 b$. Similar conditions are of widespread occurrence, and the root clearly furnishes evidence as to the nature of primitive organization
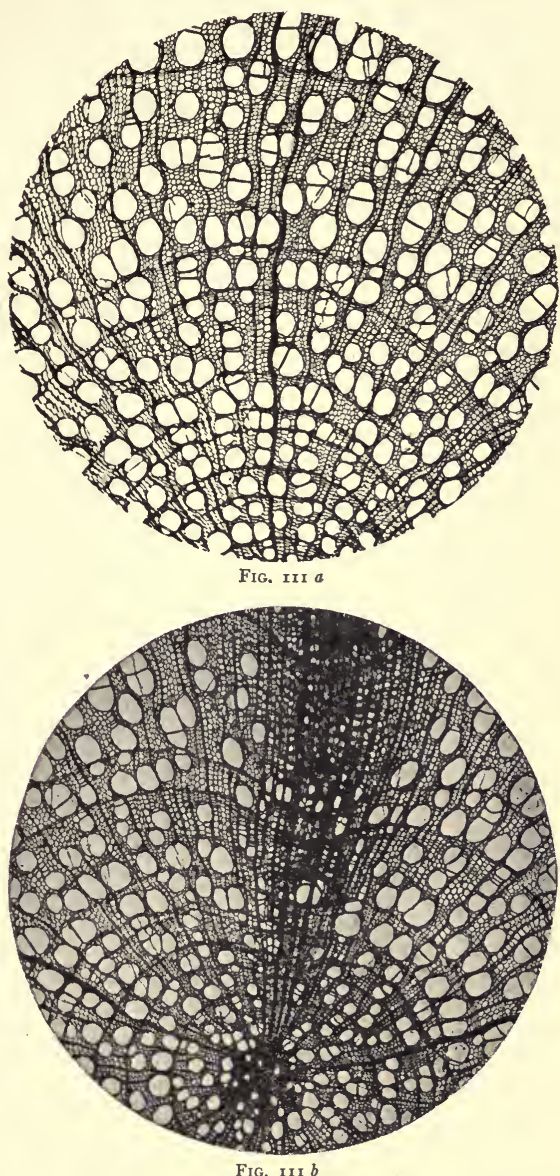

FIG. II I $a$ and $b$.-Transverse section of rays in stem and root in Betula papyrifera. Explanation in text. 
in general in vascular plants. The illustration supplied by the rays serves as an exemplification of the original condition of the root as regards its secondary structure. Additional examples will present themselves repeatedly in the subsequent chapters. In the case of the primary organization the uniformity of the radial structure presented by the root throughout the vascular series, in contrast

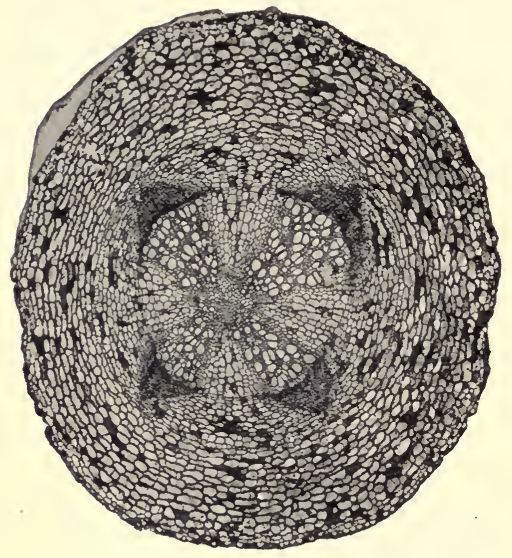

FIG. II 2.-Transverse section of a root of Actaea alba.

to the great variety of types exemplified by the stem both in regard to the arrangement and mode of development of the fibrovascular elements, vouches even more emphatically for the value of the anatomy of this organ in evolutionary investigations which are not entirely speculative in their nature.

It will be convenient and appropriate at this stage to consider the root in the herbaceous dicotyled ons. The Ranunculaceae will serve here as an excellent exemplification of the conditions in plants in which the annual herbaceous habit has brought about at once reduction in the development of the secondary tissues and marked modifications in their topography. Fig. I I 2 illustrates photographically the organization of a root in the case of Actaea alba. The cortical tissues are somewhat clearly separated from those of the fibrovascular system by an endodermal zone. The central region of the root is distinguished by the presence of four aggregates of phloem. A marked feature of the secondary wood is its division into widely separated segments by broad rays of the compound type. This condition is extremely common in roots of forms in which the herbaceous habit has become strongly developed. 
Fig. I 33 shows the central region of the root under a higher degree of magnification, and the arrangements described above now become much more clearly visible. Another structural feature now for the first time presents itself-namely, the presence of groups of smallsized elements of the primary xylem in alternation with the more

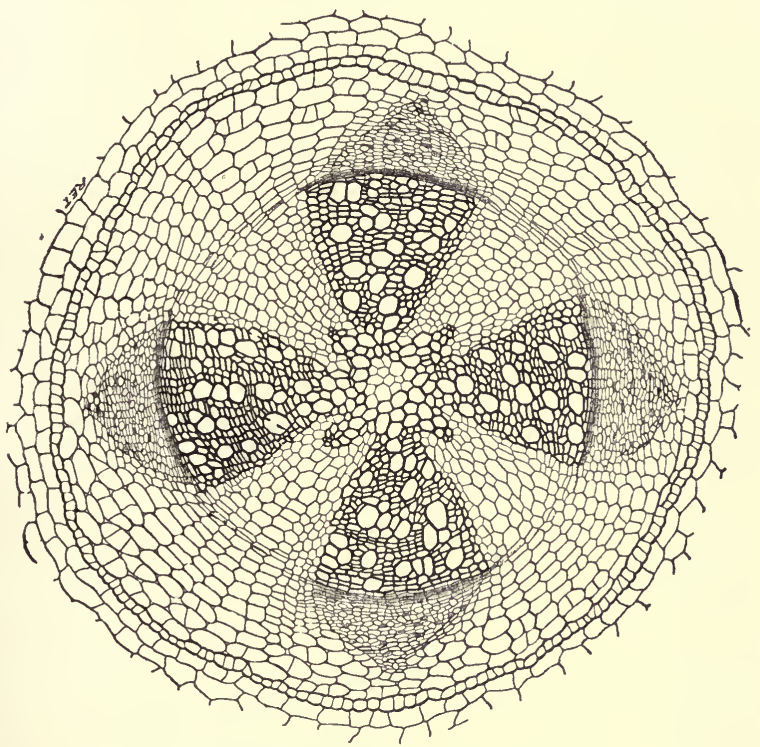

Fig. I 13.-Part of same, more highly magnified

pronounced radial bands of secondary wood and phloem. The protoxylem groups are incompletely united in the center, and a pithlike cluster of parenchymatous cells is consequently found in the midst of the primary wood, a condition quite frequently present both in herbaceous dicotyledons and generally in the monocotyledons. In the woody dicotyledons and in the conifers a structure comparable to the pith of the stem is ordinarily absent in the root. 
In the monocotyledons the root is characterized by the usual absence of any indications of secondary growth. In Fig. II4 is represented the transverse view of the root of the carrion plant, Smilax herbacea. The root hairs are very conspicuous (Fig. II5), and underneath the layer which forms them, the piliferous layer, is seen a zone of cells which, with certain exceptions, possess thickened walls. Next to this structure, known as the exodermis, lies the

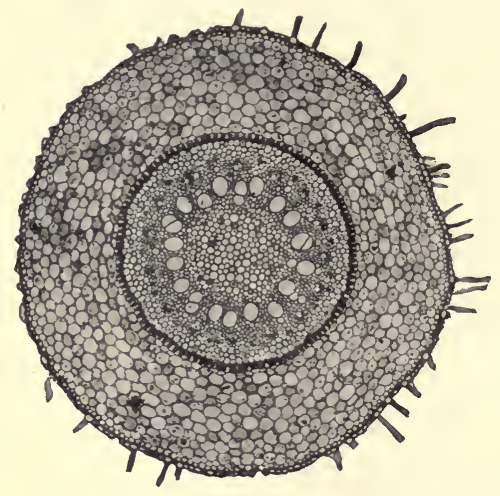

FIG. Ir4.-Root of Smilax herbacea cortex, which terminates with another cellular limiting membrane, the endodermis. The center of the root is occupied by the central cylinder and the pith. The former consists of numerous and distinctly alternating clusters of primary xylem and phloem which, as is usual in roots, occupy different radii. There is no indication of secondary thickening in connection with either the xylem or the phloem. The wood elements are smaller on the outside and become larger as they pass inward. Although the usual gradation in size is found to exist in the elements of the wood in monocotyledonous roots, the order of development of the tracheary cells is not infrequently the reverse of their gradation in diameter. In other words, the internal elements of a tracheary nature are completed before those lying farther outward in the region of the protoxylem. This is one of the many abnormalities which characterize the anatomical structure of this important group of the angiosperms. The central region of the root is occupied by a well-marked pith, a condition of very general occurrence in the group under discussion. A similar situation in regard to the frequent presence of medullary structures is found in the case of herbaceous dicotyledons. It seems 
certain that the presence of a pith in roots is not a primitive feature, but one which has been secondarily acquired.

In the mass of monocotyledonous roots there is very little departure from the state of affairs found in the illustrations shown above. At most, the modification in the central cylinder consists of variations in the number of groups of xylem and phloem. An interesting complication of the piliferous or root-hairforming layer is frequently present in certain orchids and aroids. Here the normally uniseriate piliferous layer becomes multiplied, giving rise to a white spongy substance covering the surface of the root and known as the velamen or veil. This structure is peculiarly characteristic of the aërial roots of tropical epiphytes belonging to the two orders mentioned above, although sometimes found in a condition of imperfect development in

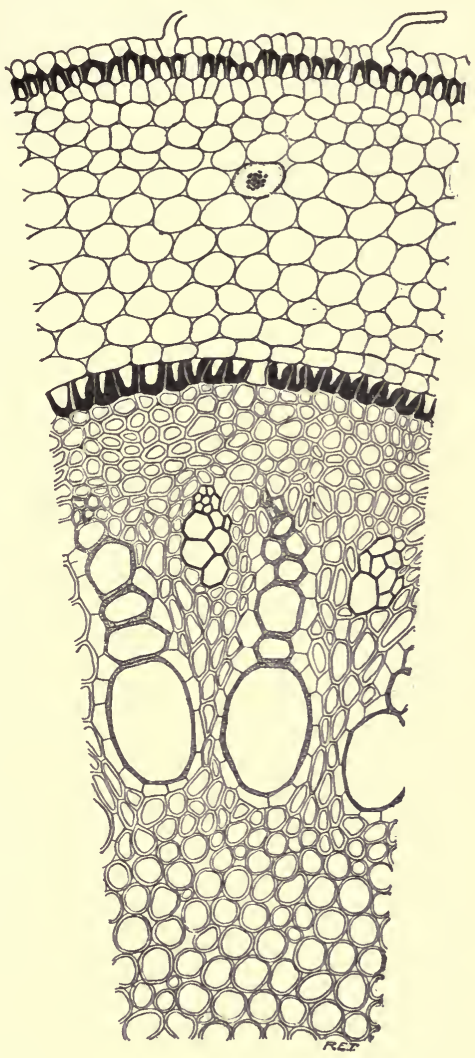

Fig. I I5.-Part of transverse section of root of Smilax herbacea. terrestrial orchids of tem-

perate climates. The general relations presented by the velamen are shown in Fig. I 6 . Here it is clear that the structure under consideration 'lies external to the exodermis and is consequently 
morphologically equivalent to the normally single piliferous layer of terrestrial monocotyledonous roots. The cells of the velum have banded thickenings in their walls which in a general way simulate those found in certain types of tracheids. The elements in question are also sometimes porous, so that water enters readily

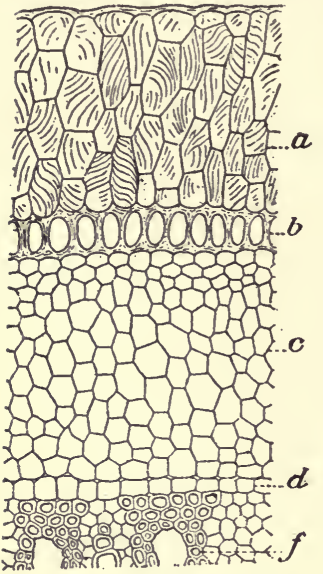

Fig. I 16.-Transverse section of an orchid root, showing multiple piliferous layer developed as a velamen. into their cavities during the rainy season, to be conserved for use in the following period of drought.

In vascular plants in general the secondary roots are related in a definite fashion to the primary structures of the main root. In the greater number of cases the young root appears as a local development of cells on the central cylinder of the main root. This cellular proliferation is opposite one of the clusters of protoxylem, so that the arrangement of the rootlets is predetermined by the organization of the primary structures of the main root. In many monocotyledons the indication of a developing root appears, not opposite one of the protoxylem groups, but in the interval between two of these, thus constituting a departure from the usual topography. It is evident that the secondary roots are formed endogenously and subsequently bore their way outward. This internal mode of origin is characteristic of roots and rootlets, the only exception being found in the case of the primary root of the seedling. Frequently roots are definitely related to other appendages, such as branches and leaves, and in these instances originate at or near the node.

All roots, with the exception of the degenerate ones found in certain types of parasitism, are provided with a protective cover over their tender apex, and this is known as the root cap or pileorhiza. This structure is for the purpose of protecting the root 
as it forces its way through the more or less resistant soil. The tissues of the root usually become stiffened at an early stage by the rapid thickening of the walls of the tracheary elements of the primary wood. Rigidity is so much a necessity in the case of the wood of the root that ringed and spiral tracheids, such as ordinarily characterize the first-formed region of the xylem in the aërial stem, are conspicuous by their absence or at any rate by a scanty degree of development. In Fig. II7 are represented the protoxylem of the stem and root of the balsam fir. The great contrast in the development of the extensible ringed and spiral tracheids is shown
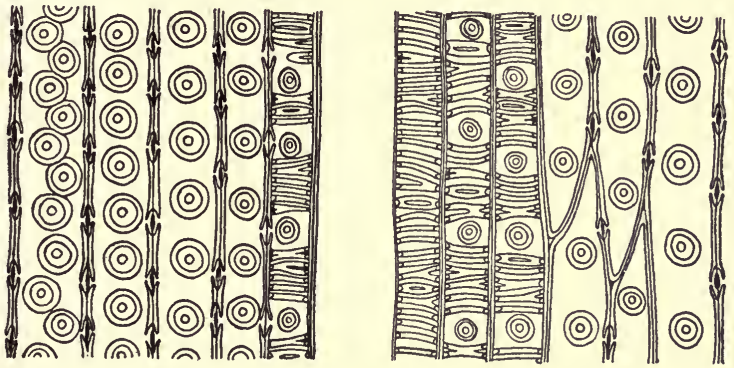

FIG. II 7.-Longitudinal view of the primary tracheids of the root and stem of the balsam fir, showing the different degrees of development of the protoxylem elements in the two organs.

in the diagram. It is obvious that ringed and spiral sculpture alone is not an entirely constant character of the first-formed wood in roots.

A great deal of morphological importance has in the past been attached to the arrangements of cells at the apices of the roots. It is not clear, however, that inferences drawn from such data have a very great evolutionary significance; and certainly in other organs of the plant, such as, for example, the stem, they are of very slight value in view of the extremely contradictory results reached as a consequence of adherence to this criterion. At the present time the organization of the growing point in plant organs is regarded as of less importance than the histological structure of the mature parts in reaching any conclusions as to equivalence and course of evolution. 


\section{CHAPTER XIII}

\section{THE STEM}

In the older types, represented by the vascular cryptogams, the general organization of the stem presents more variety in ground plan than that found in the case of the seed plants. In these earlier forms the fibrovascular tissues are present in two main conditions. In the primitive type of structure the xylem is a solid mass which does not include any medullary substance or pith. This variety is represented in Fig. II8 and is known as protostelic. The protostelic condition is found in many older groups and is of almost universal occurrence in the most conservative of all the organs of the plant, the root. Where the mass of primary xylem is smooth in contour and does not show on its surface any projecting salients of protoxylem, the phloem forms a continuous layer surrounding it, and the whole fibrovascular complex so organized is known as concentric. Usually in concentric protosteles the protoxylem elements are situated in relation to the general organization of the xylem in the manner described in an earlier chapter as mesarch. Here it will be recalled that the first-formed elements of the wood are ultimately completely surrounded by those of later origin. When, by reason of the projecting masses of protoxylem not imbedded in the main mass of the primary wood as in the mesarch type, there are more or less deep furrows on the face of the xylem, the phloem ceases to constitute a continuous layer, but is present in isolated strands alternating with the projecting angles of the protoxylem, and the stelar structure is described as radial in its organization. In this condition the development of the wood is usually entirely centripetal, or toward the center of the organ. This mode of organization is anatomically described as exarch, because the protoxylem elements are external in position to all the later-formed portion of the wood.

Another primitive type of structure presented by the fibrovascular organization of the stem is that known as siphonostelic. 
Here the phloem and xylem, instead of constituting a solid mass without any parenchymatous center or pith, are laid down in the form of a hollow cylinder inclosing a central medulla or pith. This type of organization of the central cylinder or stele is technically called siphonostelic, on account of the tubular condition present. Primitively the siphonostele or tubular central cylinder seems to

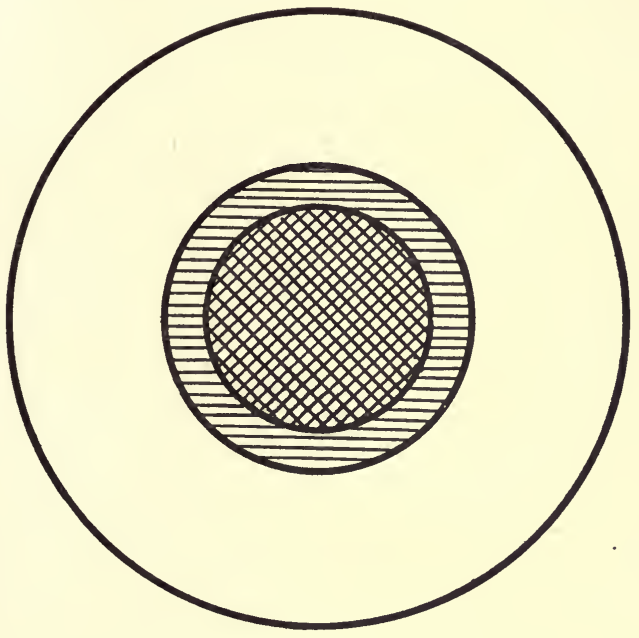

Fig. I18.-Diagram of a protostelic stem

have been organized in such a manner that phloem clothed its inner surface as well as its outer one. Further, both inwardly toward the pith and outwardly toward the cortex the tissues of the central cylinder or stele are clearly delimited from those of the fundamental system by a well-marked endodermal layer. The siphonostele may be either concentric or radial in its organization, precisely as in the protostele, the general situation being identical in the two cases except for the presence of the pith. The essential features of the siphonostele are presented in Fig. IIg. Here may be seen cortex and pith marked by a similarity of 
organization and separated from the fibrovascular category of tissues by the endodermis. To the left in the figure the siphonostele is depicted in a continuous or closed condition, while on the right it is open on one side. The opening corresponds to the giving off of the trace of an appendage, which in this instance is a leaf. A general feature of the tubular or siphonostelic central cylinder in

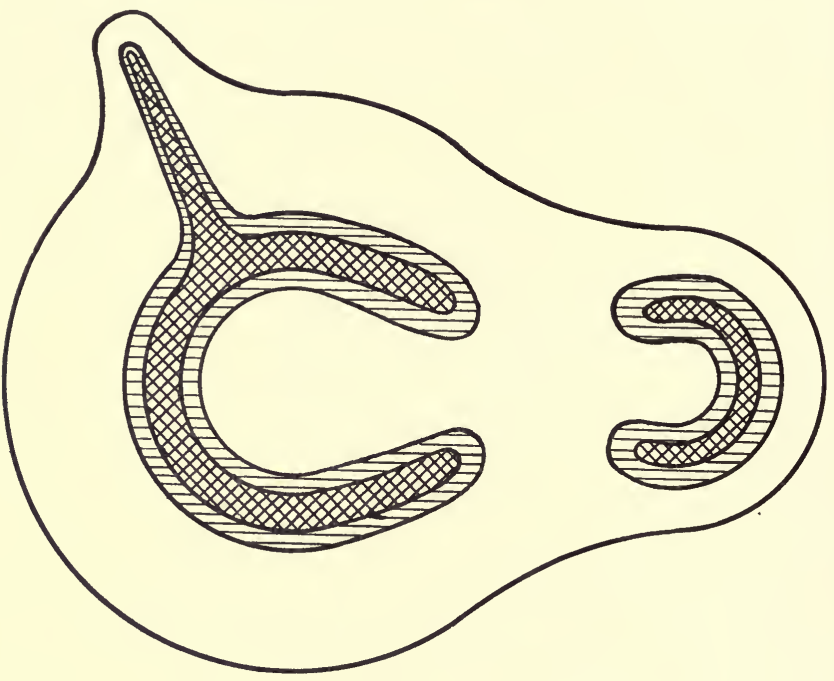

FIG. I I9.-Diagram of a siphonostelic stem

the ferns and their immediate allies is the appearance of openings in the tubular central cylinder which correspond to either leaves or branches. These openings persist for a longer or shorter distance above the point of departure of the fibrovascular supply to the appendage and are known as leaf gaps or branch gaps according to the organ involved. Around the edges of these gaps the endodermis of the inside becomes continuous with that of the outside. Usually, too, the phloem of the inner surface of the tubular stele joins with that situated on the exterior. Naturally, also, the fundamental tissue of the outside, commonly known as the cortex, 
becomes reunited with that situated in the center of the stele, designated in turn as the medulla or pith.

Fig. II depicts a siphonostelic central cylinder. The traces of leaves determine the presence of lacunae, or interruptions in the continuity of the cylinder, for some distance above their points of departure. These are the foliar gaps or, in case they are related to lateral branches, the branch gaps.

The protostelic and siphonostelic conditions of stem are both subject to further modification which may now be discussed. In the protostelic stem, for example, the phloem may more or less completely disappear on one side of the stele, a situation found in the stem and sometimes even in the root of certain ferns. This condition is known as collateral and is extremely rare in protostelic organs. This state of reduction is, however, very common in the siphonostele, particularly in the higher forms where the tubular type of central cylinder has become universal. A proper understanding of the phenomena of reduction presented by the siphonostele is advantageously gained by the consideration of allied forms presenting the stelar conditions both in the normal and in the reduced state. Illustrations of this nature can best be drawn from the lower vascular plants, the ferns and their allies. Fig. I 20 represents on the left a siphonostelic stem in which internal and external phloem are both well developed, and likewise the endodermal layers, which respectively limit these from pith and cortex. In the illustration at $b$ appears a stem in which the internal phloem has disappeared on most of the interior of the stele and is found merely on the margins of the foliar gap. In the case of the channeled leaf trace, subtending the foliar gap, in contrast to the situation presented by the fibrovascular system of the stem, the internal phloem is as well marked as that occurring on the outer or convex surface. This is in accordance with a general principle to be more definitely elaborated at a later stage. The basal region of the foliar fibrovascular strand in vascular plants is found in general to retain the more primitive type of organization. In the particular instance under consideration the retention of internal phloem in the leaf trace is the significant feature in a considerable number of ferns and fern allies. It may be stated that a 
universally accepted procedure in comparative anatomy is to judge the former organization of the walls of the stelar tube from pertinent conditions presented by the leaf traces which take their origin from it. In other words, ancestral conditions which have disappeared in the stele of the highly progressive and easily modified stem may continue almost indefinitely in the fibrovascular
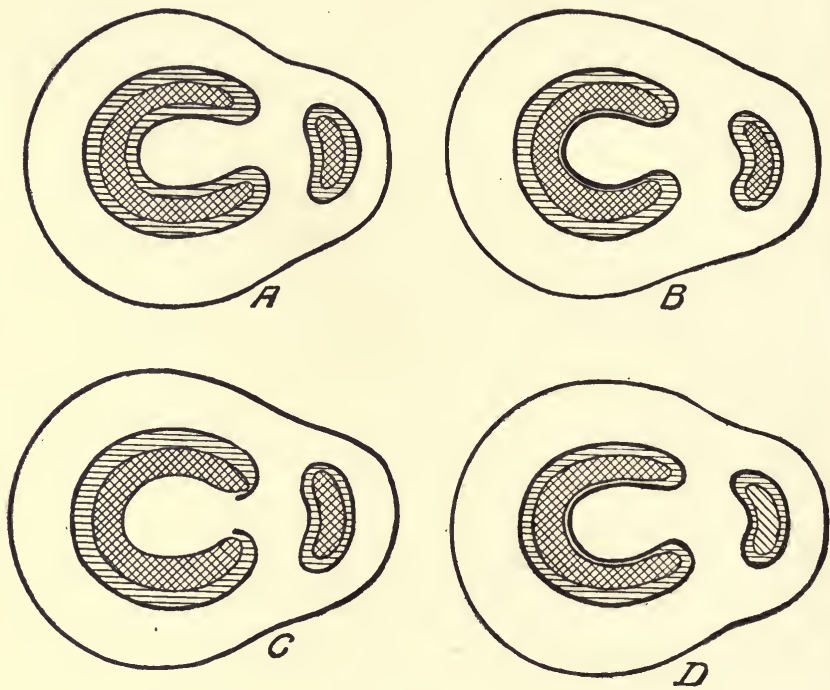

FIG. I20.-Diagram showing degeneracy of internal phloem and endodermis in the siphonostelic central cylinder. Explanation in the text.

structures of the leaf. The great value of this principle is quite generally admitted in the case of the gymnosperms, but its validity for the fernlike forms is unfortunately not so universally conceded in spite of cogent logical considerations in its favor. At $c$ is represented a further condition of reduction. Here not only the internal phloem has disappeared in the stele, but likewise there is no internal endodermis, although both are present in the leaf trace. At $d$ in the same figure is seen the anatomical 
condition presented by the young plant or sporeling. Here the tubular central cylinder is characterized by an internal as well as an external endodermal layer. The general principle applicable to this situation is that young stages frequently perpetuate conditions which have disappeared in the adult. It is apparently clear from commonly accepted canons of comparative anatomy which will be elaborated in a subsequent chapter that leaf and seedling stem frequently enable us to reconstruct the course of evolution in the siphonostelic cylinder.

The presence of internal phloem and endodermis has been described in the former paragraph as a primitive feature of the earlier types with the siphonostelic central cylinder. Other important anatomical structures of lower or cryptogamic vascular plants are exarch and mesarch primary wood. It will be recalled that the term exarch has reference to the fact that the protoxylem elements - that is, the tracheary tissues which are first laid downare external in position, and that the subsequent development of elements of the wood is centrad, or toward the center of the organ. Likewise in the case of mesarch structure the situation is first characterized by the exarch condition, but after a certain progress has been made the formation of new elements of the xylem shifts to the external aspect of the protoxylem, and as a result we find the first-formed elements more or less completely surrounded by those of later origin. Later in geological time and in still higher types the mesarch condition in turn gives place to the endarch by the disappearance of the centrad or centripetal wood. This situation can best be illustrated by a diagram. In Fig. I2I at $a$ is shown the exarch condition of development of the structures of the primary wood. The small-sized elements, normally ringed or spiral, are outside the later-formed scalariform or pitted tracheids. In $b$ is represented the mesarch condition, in which the development is at first centrad, but later becomes peripherad, so that the protoxylem comes to occupy a central position. In $c$ is shown the endarch condition where the seriation from the protoxylem is no longer centrad but has become entirely peripherad or centrifugal. The condition in $a$ characterizes the organization of the primary xylem in the stem of the lycopods and their allies, and is 
found without exception in the roots of all vascular plants. The situation diagrammatically delineated in $b$ prevails in the stem organs of the ferns and their allies, the lower gymnosperms. In the higher gymnosperms and the angiosperms the axial organs exemplify almost exclusively, in living types at any rate, the endarch condition diagrammatically represented in $c$. As might be expected, the primitive occurrence of exarch and mesarch conditions

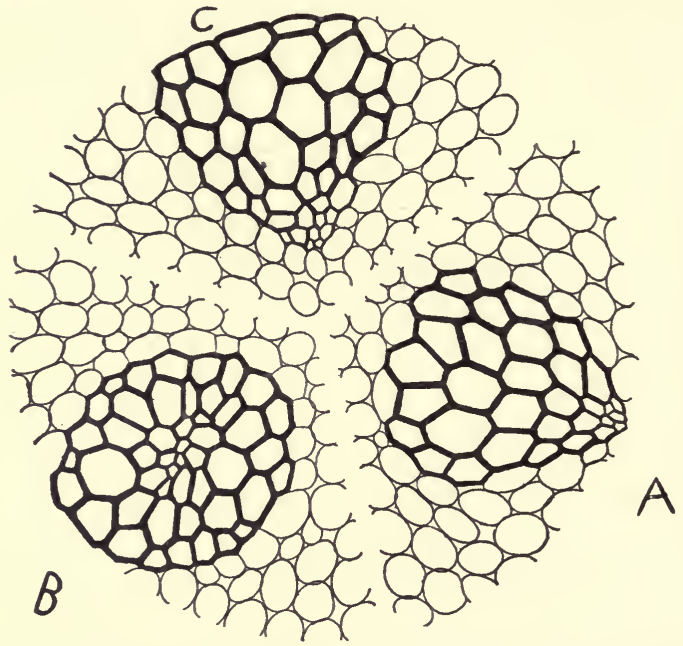

Fig. I21.-Diagram to indicate the relations of protoxylem to metaxylem in vascular plants. Explanation in the text.

in the case of the stem can frequently be inferred from the anatomical situation presented by the leaves. The universal presence of the exarch type of primary wood in the root furnishes good evidence that this was the original type of organization and development of the primary wood in the vascular series.

It has been made evident in an earlier chapter that, in addition to the primary organization of the wood consisting of tracheary and parenchymatous elements arranged in irregular order, there is often present, particularly in arboreal types and perennials, ancient 
and modern, a subsequent ligneous development made up of cells regularly seriate radially and including masses of radial parenchyma. This regularized and later-appearing xylem is known as the secondary wood. In older types the primary wood is distinct from the secondary ligneous structure, and its conspicuousness under these conditions is due largely to the more abundant development of the primary elements and their frequent exarch or mesarch configuration. In the higher gymnosperms and in the angiosperms they can be recognized only with difficulty except in the root. This situation is the result of the slight development of the primary elements and of the fact that they are in series with, and formed in, the same direction as the secondary wood.

It will be apparent from the statements made in the preceding paragraphs that the organization of the stem is characterized by a considerable degree of variety as regards the general topography and microscopic organization of the primary structures. When the special consideration of the ferns and their allies is reached in later chapters, it will be clear that the possible complexities of the gross fibrovascular structures have been by no means exhausted.

It is now convenient to turn our attention to the secondary fibrovascular structures of the stem. In the older vascular plants the secondary wood was extremely simple in its organization and consisted merely of radial parenchyma and longitudinal tracheary elements. The radial parenchyma in the lowest forms was definitely separated from the pith, as is well illustrated in Fig. I22, by the presence of a clearly developed zone of primary wood. With the progress of geological time came a progressive reduction of the primary wood and important modifications in the structure of the secondary woody cylinder. The gradual reduction of the primary structures of the woody cylinder has involved interesting conditions which must now occupy our attention. The progressive reduction of the region of the wood known as primary brought with it certain important topographical changes. The primary wood was at first continuous and in most instances constituted in the stem a siphonostele interrupted only by the passing off of the traces of branches or leaves. The breaks in the continuity of the primary cylinder thus determined are 
known as leaf or branch gaps, as the case may be. In the lower forms, with super-addition of secondary to primary woody structures, the primary cylinder of the siphonostele still maintained to a large degree its individuality. This is, for example, true in

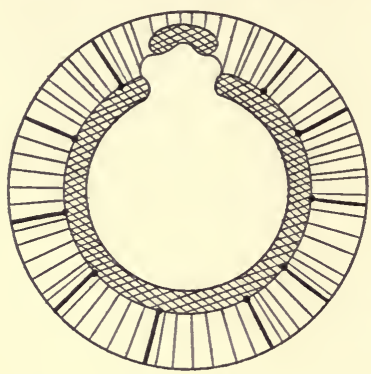

FIG. $122 a$

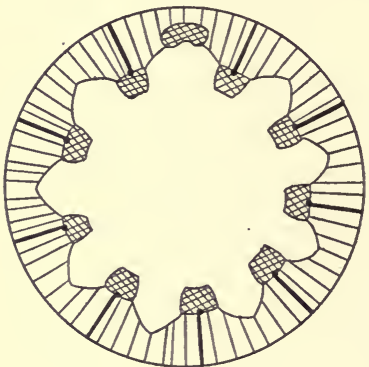

Fig. $122 b$ Fig. I $22 a$ representing the organization of a stem in a lepidodendrid, an arboreal club moss of the Paleozoic age. The lepidodendroid arboreal club mosses include forms referred to the general cognomen of Sigillaria. In lycopods of this type, particularly in the Permian age and toward the end of their term of existence as an element of our earth's flora, the primary wood became much reduced in amount.

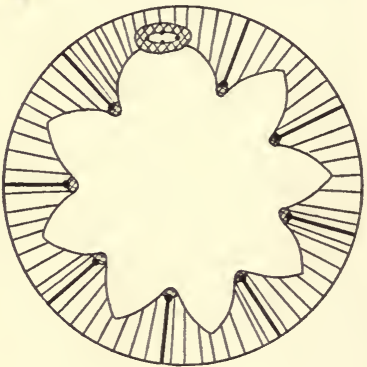

FIG. $122 c$

Fig. I $22 a, b$, and $c$.-Diagrams to illustrate the effect of the degeneracy of the primary wood on the development of the secondary xylęm.

Not only did this situation express itself in the thinning down of the primary woody cylinder, but there was also present a lack of continuity due to the complete elimination of tracheids in certain segments. This phase is revealed in $b$. Here the primary wood constituting a continuous cylinder in $a$ is interrupted by intervals resulting from the scanty development of the primary xylem. The gaps between the resulting slender isolated strands are per- 
petuated in the early development of the secondary organization, since the radially disposed elements make their first appearance opposite the clusters of primary wood, and only as the secondary segments widen out later do they finally bridge over the intervals in the continuity of the cylinder. On the upper side a branch causes a still more prominent hiatus in the inner region of the cylinder. The condition in a calamitean stem may next engage our attention. Here the primary wood is much less well developed even than in the later Sigillariae, and only in the earliest representatives of the genus does it show an indication of the ancestral centripetal type of development. The task of bridging over the primary intervals by secondary growth is correspondingly greater than in the sigillarian stem, and the bays extending into the secondary wood proportionately deeper and wider. In the upper region of the cylinder is figured a siphonostelic branch which is responsible for a wide hiatus in the secondary cylinder. An inspection of $a, b$, and $c$ makes it clear that the progressive degeneracy of the primary wood as well as the departure of traces belonging to the branches causes interruptions in the continuity of the secondary wood. These are filled by soft tissues continuous with the parenchyma of the pith. The secondary cylinder is characterized; as has been demonstrated in the diagrams, by bands of radial parenchyma which are often wrongly called medullary rays. That they cannot receive this appellation with any accuracy will be apparent from a comparison of the conditions presented by $a, b$, and $c$. In $a$ the rays in no case reach the pith. In $b$ the radial parenchyma is clearly continuous with the soft tissues of the pith in the intervals between the isolated segments of primary wood, while in those regions of the secondary cylinder subtended by primary xylem, which of course represent the primitive topographical relation, no such continuity is possible. In $c$, by the still further reduction of the primary structures of the wood, a much larger number of bands of radial parenchyma appear to abut on the pith. It is clear from the conditions outlined in the diagram under discussion that it is entirely inappropriate to describe the radial storage tissues of the secondary wood as medullary rays. A satisfactory appellation for them is simply wood rays, and 
this involves no erroneous hypothesis of their relation to the pith. It is therefore necessary to distinguish carefully between wood rays and gaps or discontinuities in the woody cylinder originating as a result of conditions described above. It seems quite evident that the failure to realize this distinction invalidates investigations on the woody cylinder involving the confusion of thought elucidated

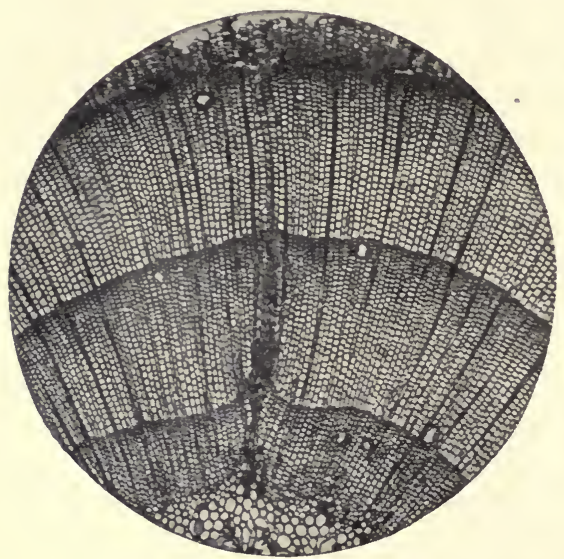

FIg. I23.- Stem of Picea, showing apparent relation of wood rays to pith.

by the items in the accompanying figure.

The last paragraph will have convinced the reader that radial bands of parenchyma, formed as a result of cambial activity, cannot accurately be described as medullary rays. We are now in a position to consider the situation presented by the stem of perennial seed plants of gymnospermous affinities. Fig. I23 reproduces part of a transverse section of a stem of the white spruce, Picea canadensis. The woody cylinder is characterized by numerous radial bands of parenchyma, apparently in every instance taking their origin from the medulla or pith. Further, there are present broad outwardly directed bays from the medullary region which represent the gaps corresponding to outgoing leaves (for in the gymnosperms, in contrast to the lycopods and their allies, the leaves are related to foliar gaps). A superficial examination of the figure would justify the application of the term medullary ray both to the narrow radial bands of parenchyma and to the broader bays extending from the pith. The considerations advanced in the last paragraph make it clear, 
however, that this is an inaccurate interpretation. The justice of the criticism here advanced is, moreover, evidenced by reference to the veryconservative structures of the root. Fig. I 24 illustrates the central region of this organ in the same species of spruce. The primary xylem is pronounced and distinctly centripetal in the order of development of its elements. There is no medulla present, and the rays of the secondary wood consequently not only have no relation to a medullary

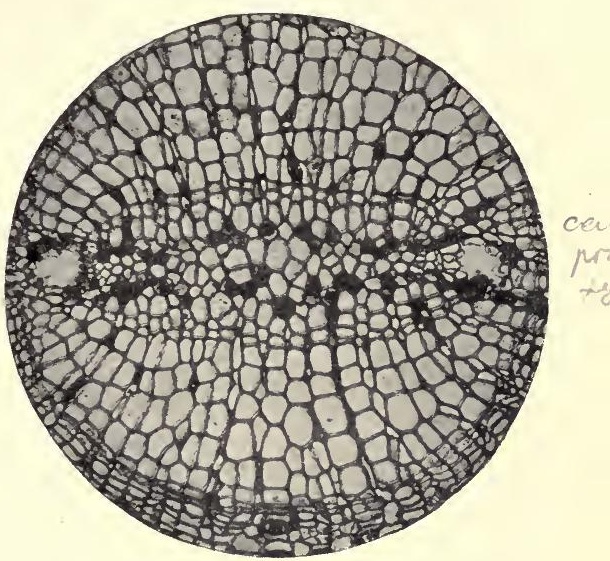

FIG. I24.-Root of Picea, showing primitive condition region, but also end in the vicinity of the primary wood. It is

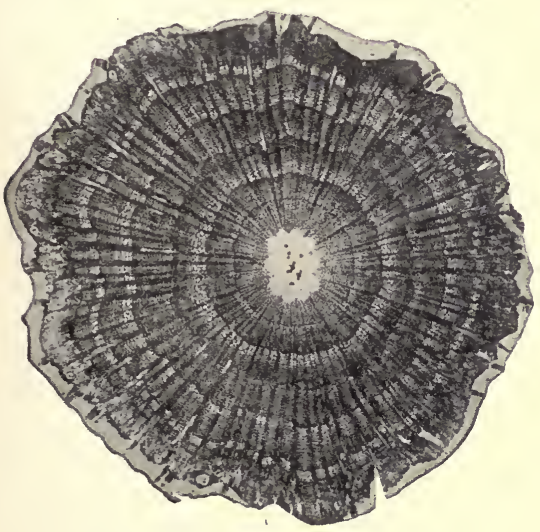

FIG. I 25.-Stem of Ephedra gerardiana clear that the radial parenchyma of the root cannot come under the term medullary ray, and it will now be obvious to the reader that in no case can the radial bands of storage tissue in the secondary wood be accurately called medullary rays. They are properly described as wood rays.

It will not be necessary to consider 
any other lower seed plants in the present connection. The Gnetales and angiosperms present the next significant modification of

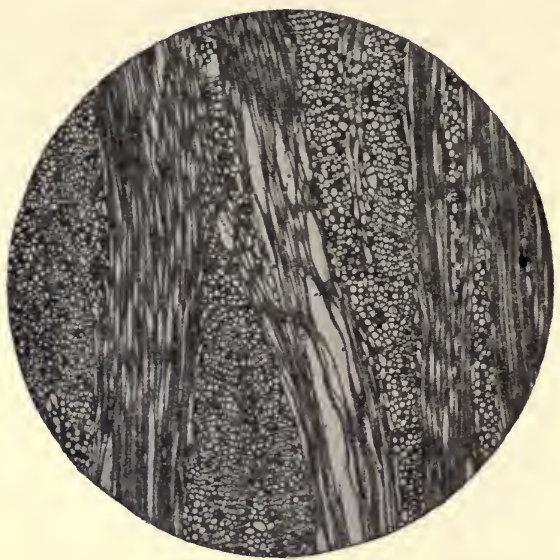

Fig. 126.-Tangential section of wood of Ephedra species, showing presence of large rays. structure in the stem. Fig. I 25 illustrates the organization of the axis of Ephedra gerardiana. Obviously there are numerous broad bands of radial parenchyma present. Another feature of interest is the presence of true vessels in the cylinder of secondary wood. Broad rays of the type found in Ephedra and in the angiosperms are definitely correlated with the appearance of vessels. A tangential sec$\mathrm{t}$ i on (Fig. I 26 ) through the wood of Ephedra reveals the true nature of the rays in the Gnetales. Fig. I 27 illustrates the organization of an individual large ray in the outer region of the woody cylinder. The ray is

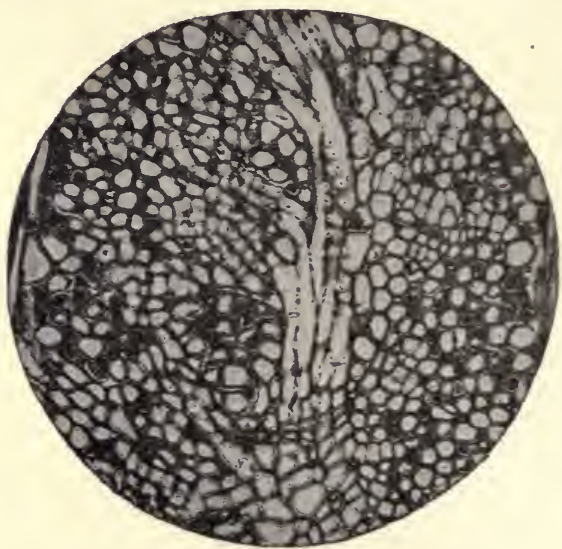

FIG. 127.-Portion of large ray highly magnified, showing presence of tracheary elements in ray substance. 
plainly not of homogeneous organization, but contains in its substance, in addition to true radial parenchyma, large quantities of fibrous elements and even some vessels. The fibrous elements when studied under higher magnification than that of the figure often show a gradual transition to parenchyma. By referring to chapter vi it will be seen that the large ray of Ephedra comes under the heading of aggregate ray, described in that chapter, since it is a composite structure made up partially of true storage cells and partially of longitudinal elements of the wood in process of parenchymatous transformation. Fig. I 28, which shows the ray of Ephedra in its narrower condition nearer the pith, supports the view of the nature of the large ray arrived at as a consequence of an examination

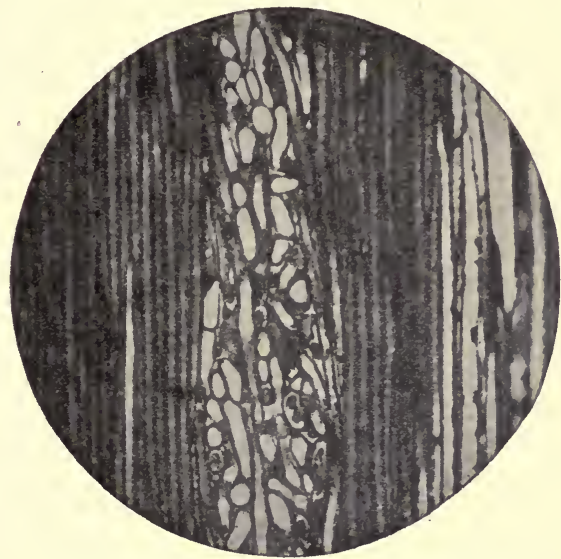

FIG. 128.-Aggregate ray of Ephedra species in region near the pith. of its organization in the outer region of the wood. In its earlier stage of development it contains equally clearly a mixture of storage parenchyma and of fibers more or less completely transformed into cells resembling the ordinary elements of the rays. It is obvious in Ephedra (and this statement holds of the Gnetales generally) that the higher organization of the water-conducting elements of the wood carries with it a more abundant provision for the storage of food products elaborated by the leaves. Of course the stage of evolution here attained is quite impossible in the case of a more primitive type of wood in which longitudinal parenchyma either has not yet made its appearance at all or has progressed to such 
a slight degree that it does not constitute an important feature of organization. With the strong development of longitudinal storage cells, as has been explained in an earlier chapter, owing to the septation of elements originally destined to become tracheids, a co-ordination between these and the earlier evolved radial parenchyma provides possibilities in connection with storage quite adequate to accommodate the output of the more efficient and

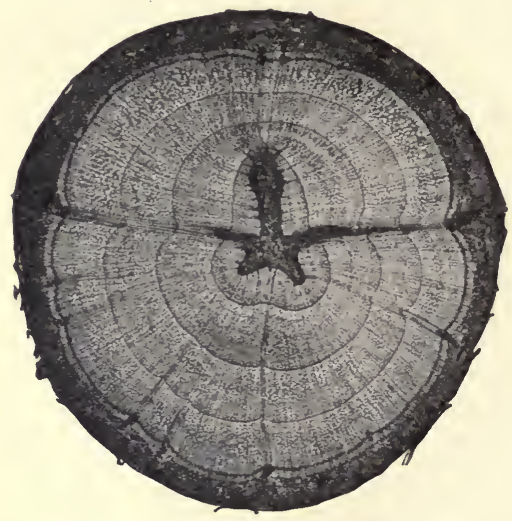

Fig. 129.-Transverse section of the stem of Alnus japonica, showing presence of aggregate rays.

hygrophilous leaf of modern floras. The aggregate ray is evidently the result of the correlation of radial and longitudinal storage devices. Its appearance is a phenomenon of prime biological importance and is intimately related to the origin of the herbaceous type in the angiosperms on the one hand and the evolution of the highest vertebrates, the warmblooded mammals, on the other. In Gnetum, the highest genus of the Gnetales, the condition of aggregation is no longer prominent, but has been superseded by the compound type of ray.

The next significant forms to occupy our attention are the woody dicotyledons. These are best introduced in connection with the alder and the oak. In Fig. I 29 appears a photograph of a branch of Alnus japonica several years old. The central pith is triangular, corresponding to the one-over-three phyllotaxy of the genus. The annual rings which surround the pith are characterized by the presence of conspicuous broad rays, of which the largest and most striking extend from the sides of the longest angle of the triangular pith. These most conspicuous radial structures are related to the 
two lateral traces of a leaf and may consequently be appropriately called leaf or foliar rays. The less conspicuous rays in other radii of the stem are in relation to other traces of other leaves lower or higher in the stem. In the following illustration (Fig. 130) a portion of the stem represented in Fig. I 29 is shown. The greater magnification makes it apparent that the broad radial band related to the outgoing leaf trace is in reality an aggregation of rays and not a simple structure. It is, in fact, largely composed of fibers as well as of radial parenchyma, to the exclusion of the vessels which form a prominent feature of the remaining organization of the wood. In the case of the large ray figured it is clear that conspicuous depressions mark the surface of the annual ring where the broad radial band crosses

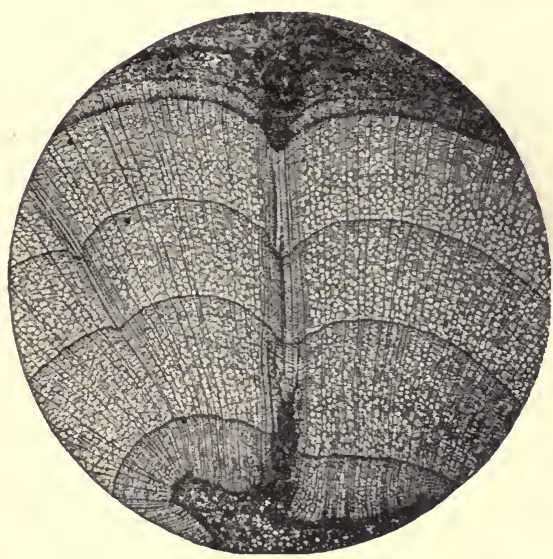

Fig. I30.-Part of transverse section of stem of Alnus japonica, showing aggregate ray in relation to a leaf, more highly magnified.

it. In Fig. I3 I the same ray appears in the vertical section of the wood. It is now evident that the structure in question consists of an aggregation of rather small rays separated from one another by fibers and including in their midst the transverse section of a foliar strand or leaf trace. It will be obvious from the various figures of the stem of the alder that in this genus there are groupings of rays in relation to the foliar traces, and that these storage bands on account of their topographical and physiological relations may appropriately be designated foliar rays.

It will now be convenient to refer to the conditions found in the case of the oak. Fig. I32 illustrates a transverse section of 
the wood of the red oak, Quercus rubra. Large rays are plainly present which differ from those found in the alder by the fact

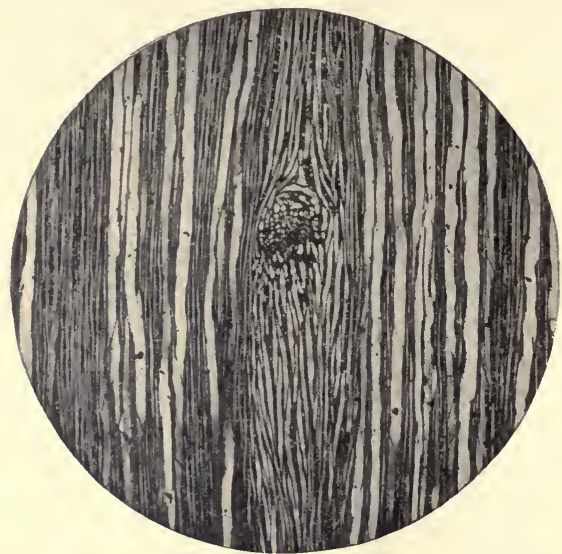

Fig. 13r.-Tangential section of stem of Almus japonica, showing leaf trace imbedded in the aggregate ray to which it is related. that, instead of being composed of a mixture of smaller rays and separating fibers, they are constituted entirely of parenchymatous elements. In spite of this difference in the organization of the broad bands of radial parenchyma they are correlated with the same dips in the annual rings found in the alder as described in the

foregoing paragraph. In Fig. I33, p. I80, is shown a transverse section of a branch of the velvet oak. Here there are five pairs of rays corresponding to the five faces of the fiveangled pith, which by its configuration indicates the phyllotaxy precisely as does the triangular medulla in the alder. In the oak there are

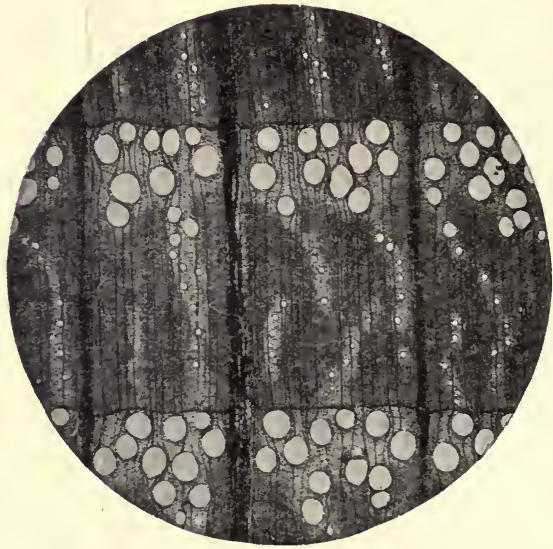

FIG. 132.-Transverse section of the wood of the red oak 
two important lateral traces in relation to each leaf, and less well-developed central ones. The most distinct foliar rays are those formed in relation to the lateral traces; since there are two of these for each leaf and five leaves in a cycle, there are ten conspicuous rays in the woody cylinder of the stem. An inspection of the figure makes it clear that the rays are related in approximated pairs, and that in the narrower intervals intervening between the foliar rays the woody cylinder is depressed. These depressed segments are five in number, corresponding to the five pairs of propinquitous large rays. The natural explanation of the depression is that it is the result of the local effect of the large rays on the rate of growth of the cylinder. It has been made clear above that wherever a large ray crosses an annual ring a depressing influence manifests itself, resulting in a corresponding dip in the surface of the yearly zone of growth. Obviously if two large rays occur close to one another they will be likely to exercise a depressing effect on the region of the annual rings lying between them. That this is the real explanation of the depressed segments of the stem of the oak and the stems of a similar type of organization is shown by the fact that, where the broad rays are equidistant, as, for example, in the grapevine, the segments fail to become depressed. On the other hand, where broad rays happen to be absent for any cause in the branches of the oak the depression is likewise absent. A further elucidation of the situation is furnished by the ranunculaceous genus Clematis. Here in species with approximated broad rays there are depressed intervening segments, while in the few species where the broad rays are equidistant no such depressions occur.

The existence of depressed segments (Fig. I33) in the stems of woody dicotyledons with large rays has been made the basis of an erroneous and historically incongruous hypothesis of the evolution of the woody type. This misconception originated with the Prussian botanist Sanio in the nineteenth century, and, elaborated in clear diagrams in Sachs's classic textbook, has become universal in the pedagogical literature of botany. It is considered that the primordial condition of the woody cylinder is that of numerically varying separate bundles. These strands of fibrovascular 
tissues are imagined to have been originally quite distinct from one another and to have been linked up by the formation of woody commissures organized entirely of secondary xylem and laid down by the activity of the so-called interfascicular cambium. The narrower and more depressed segments are supposed to owe their peculiarities to their late and entirely secondary origin. The five outstanding segments of wood in the oak and similar forms have

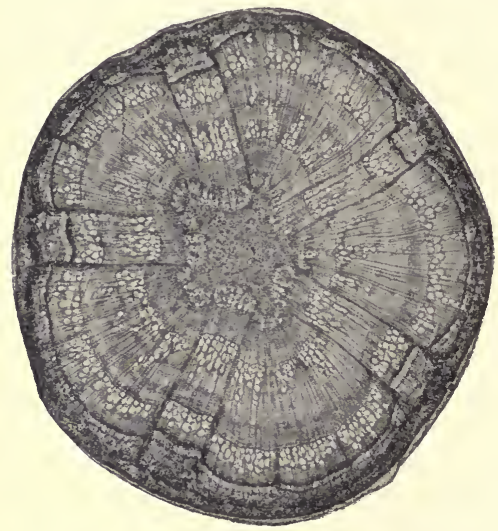

FIG. 133.-Transverse section of a twig of the velvet oak (Quercus velutina) showing five pairs of foliar rays.

been accordingly dubbed the fascicular wood, and the five intervening depressed segments the interfascicular wood. An unfortunate situation often encountered by this hypothesis is the fact that primary wood is frequently as well developed on the inner surface of the depressed segments as on that of the outstanding ones. The depression, as has been pointed out above, is susceptible of an entirely different explanation-namely, as the result of the local inhibiting influence of approximated broad rays on the rate of growth of the annual ring. In effect, moreover, the hypothesis of Sanio and Sachs derives woody from herbaceous forms, a conclusion entirely at variance with the paleontological history of plants. It is clear that the woody forms have preceded herbaceous ones in all the main series of vascular plants. A single illustration will serve in the present connection. Our somewhat herbaceous existing lycopods and Equiseta are certainly known to have come from ancestral forms which possessed so conspicuously the arboreal and perennial habit that for many years a controversy raged as to their affinities. The majority of paleobotanists for a 
long period considered them as belonging to the seed plants, since all the types of the present age which possess marked secondary growth are seed plants of gymnospermous or angiospermous affinities. The situation in regard to the relation of sequence between herbaceous and woody types might be almost endlessly illustrated, but the examples cited will serve for the present.

It has been assumed in the references to the large rays in the alder and the oak that, although differing from one another in organization (in one case aggregate and in the other compound), they have the same morphological explanation. It is clear from the descriptions supplied in the present and a foregoing chapter that they are similarly related to the appendages. It is now apposite to

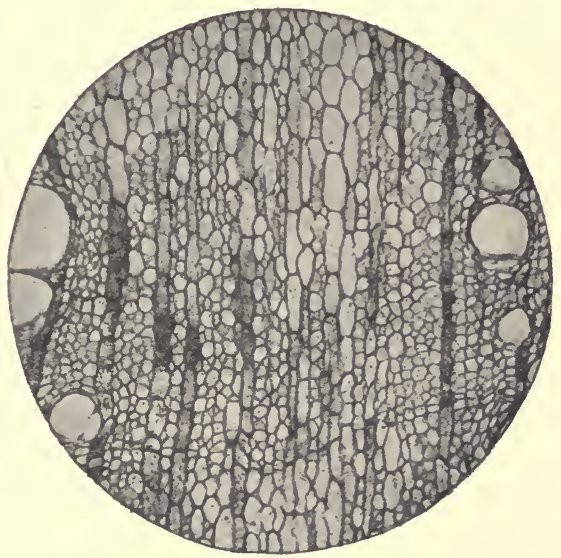

FIG. 134.-Transverse section of large ray in root of Quercus rubra. establish the fact

that the two types are related to one another in the particular forms under discussion in the present connection. Fig. I34 illustrates the organization of the large ray in the wood of the root of the red oak (Quercus rubra) as seen in transverse section. It is quite obvious that the structure differs from that found in the stem as exemplified in Fig. I35 by the fact that the ray is not homogeneous in its organization, but has numbers of fibers intermingled with its parenchymatous elements. In other words, the large mass of radial parenchyma under discussion must be considered as being of aggregate organization and not as belonging to the category of rays defined in an earlier chapter as 
compound. In Fig. ${ }_{3} 6$ the longitudinal view of the root in the red oak is shown. It is now more than ever evident that the compound condition of the rays characteristic of the stem of the oak, at least so far as the red oak is typical of the genus, is not present in the roots, but that in the latter organ the broad bands of radial storage elements belong to the type defined as aggregate. In the case of the root, in fact, one finds for many years of development that

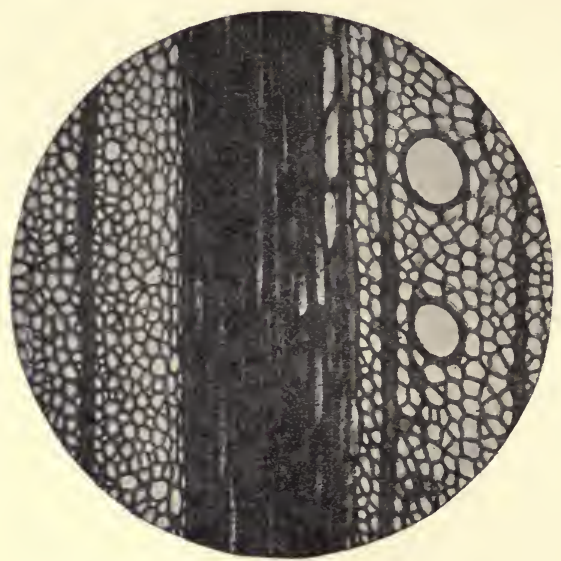

FIG. I35.-Transverse section of large ray in stem of Quercus rubra.

the more prominent radial structures are aggregate, a condition which gives place in old and thick axes to the compound type of organization. In the seedling stem or in branches derived as the result of injuries from the lowest region of the main trunk, the aggregate ray occurs and persists often for a long period. In anticipation of certain general principles of comparative anatomy to be set forth in detail in a later chapter of this work it may be stated that it is apparent in the case of the oak that the aggregate condition of the rays has preceded that defined as compound, and, in fact, that the aggregate condition is the forerunner of the compound ray. It is accordingly clear that as regards both the nature of the large rays and the relationship which they bear to the appendages the alder and the oak are substantially in a similar condition. In other words, the question of large storage rays is one susceptible of elucidation in accordance with well-defined evolutionary and physiological principles. 
In the earlier chapter dealing with the various types of rays, particularly in those paragraphs concerning the radial parenchyma of the higher seed plants, it has been shown that the great mass of arboreal dicotyledons is characterized, not by extremely large rays in marked contrast to radial parenchymatous strands of the uniseriate type, but by rays of more moderate dimensions scattered throughout the wood. Obviously, just as the compound type of radial parenchyma has taken its origin from the fusion of the units of the congeries of rays known as aggregate, so the diffuse condition of rays characteristic of the dicotyledonous forest trees in general has taken its origin by the progressive divergence of the original components of aggregate rays in the outer annual rings of older stems. The phenomenon of diver-

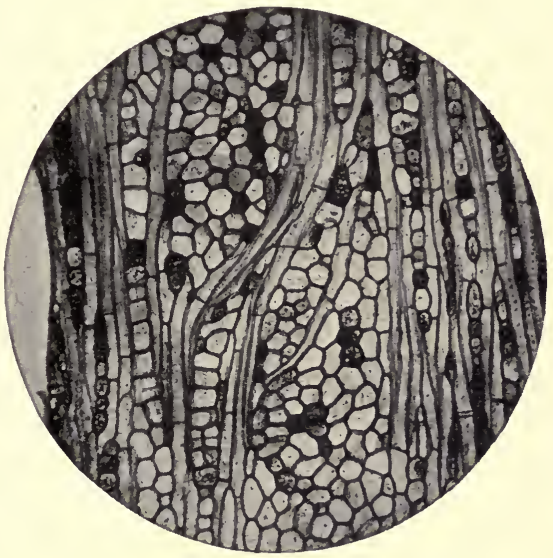

FIG. I36.-Tangential section of large ray in root of Quercus rubra.

gence can actually be seen with clearness in the genus Casuarina, while in other cases rays of the aggregate type persist in the root or may be recalled as the result of injury even when they are not found in the adult stem. It may accordingly be stated for the woody dicotyledons in general that in all there was primitively present the category of ray known as aggregate. As a result of the fusion of the elements of such rays, compound rays have made their appearance. Further, as a consequence of the divergence of the members of the aggregations, the diffuse condition is reached. The former state of rays is extremely rare in forest trees of angiospermous affinities, while the latter 
prevails almost universally in the organization of their woody cylinder.

It will now be advantageous to consider those angiospermous stems which are united in habits under the forms known as vines and herbs. In this connection it will be well to restrict our attention to the typical representatives of the two groups, since plants which are not clearly marked either as herbaceous forms or as

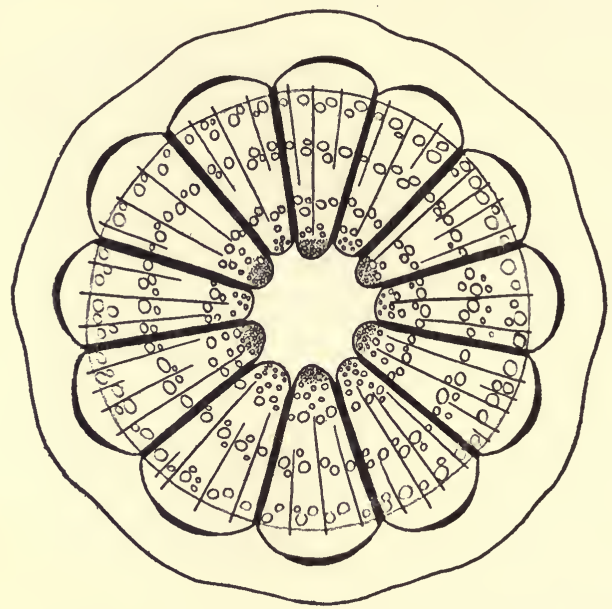

Fig. 137.-Diagram of the organization of the stem in Leea, a shrubby tropical representation of the Vitaceae.

vines are of very little importance in the present connection. A general feature of the forms included under the two headings here discussed is the presence of large rays of the oak type. This situation has long been recognized in the case of the particular modifications of the stem to be elucidated in the present and in a following paragraph. The vine type may well stand first, as it is nearer the ordinary woody perennial condition of organization than that found in herbs. Fig. I37 illustrates the organization of the woody cylinder of Leea, a shrubby tropical genus belonging to the Vitaceae. Here we find very obviously both 
compound and primitive rays. In shrubby representatives of the genus Vitis occurring in the southwest region of the United States there is sometimes present the same mode of organization of the rays as characterizes the stem of Leea figured above. In more northern species of Vitis, however, a very different situation presents itself-namely, that found in Fig. I38. Here the only rays present are of the compound type. An interesting

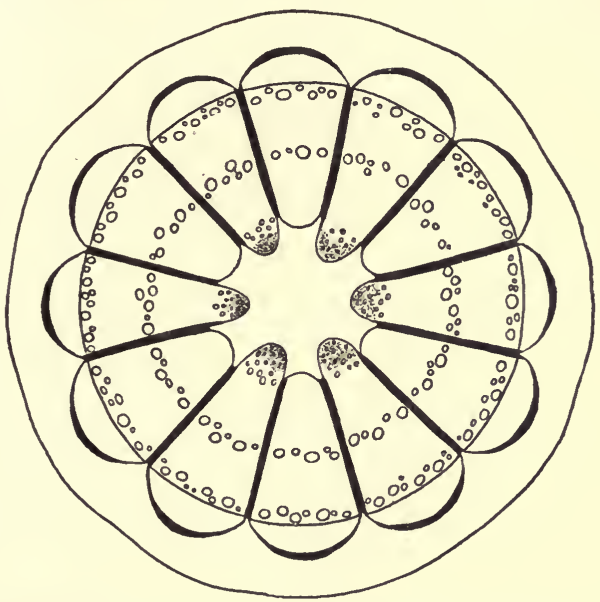

Fig. 138.-Diagram of the organization of the stem in a northern species of Vitis

light is thrown on the situation by the consideration of the seedling in the genus Vitis. Fig. I39 presents the stem of the young individual. There are seen masses of wood representing leaf traces still in position in the woody cylinder of the stem. The segments under consideration are clearly bounded on either side by compound rays, while in the wood are present distinct vestiges of primitive rays. It is evident that, if the anatomy of the seedling has any clear bearing on the problem of the origin of the type of stem presented in our northern species of grapevine, primitive rays were once present in the intervals of wood bounded laterally by the large or compound rays, and that these have subsequently been 
lost. The disappearance of the primitive rays from the bundles of the stem is, in fact, a general feature of organization of the true vine type and is as plainly indicated, for example, by the Ranunculaceae as by the Vitaceae. It is obvious that, as a result of the considerations elucidated above, the typical vine possesses compound rays, but that the primitive rays have usually quite disappeared.

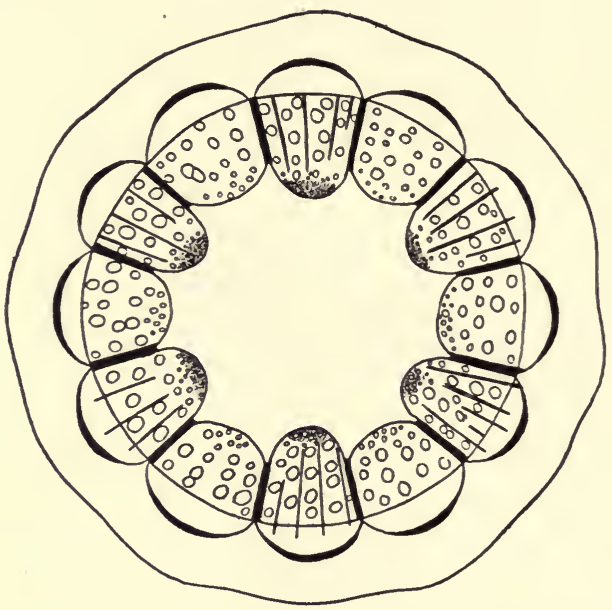

FIG. 139.-Diagram of the organization of the seedling stem of a northern species of Vitis.

Very frequently the vine type passes almost imperceptibly into that of herbs, as, for example, in the genus Clematis among the Ranunculaceae and the genus Aristolochia belonging to an order of the same name. It is convenient, however, to take up the appearance of the herbaceous type in an exemplification where it is not linked in any way with the habit or conditions of organization found in the case of vines. The genus Potentilla will serve admirably in the present connection. To reduce the illustrations to the smallest possible compass, and to make the situation at the same time clearer, a diagrammatic mode of representation will be 
advantageous. In Fig. I $40 a$ is shown the stem of a woody Potentilla (Potentilla palustris). There are five broad segments of the stem, and alternating with these are five narrower ones. The more exiguous segments are related to the leaf traces and are characterized, as are the similar regions of the woody axes of Casuarina and $A \ln u s$ represented in earlier illustrations in this work, by the conspicuous absence of the vessels present in the remaining and broader sectors. Although in the outer region the sectors under discussion show a complete absence of vascular structures, in the region near the pith a few elements of this nature are present. These belong to the leaf trace proper, which lies on the inner sur-

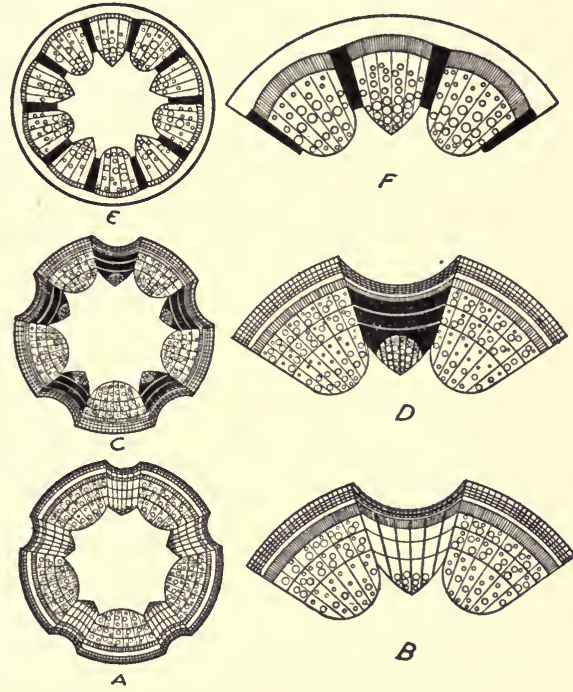

FIG. I40.-Diagrammatic representation of stems of species of Potentilla. Explanation in the text.

face of the narrower segments of the stem. Not only is the leaf trace segment narrower and distinguished by the absence of vascular structures, except in the actual trace itself, but it is depressed below the surface of the adjoining broader sectors. In $b$ these features are indicated on a larger scale, so that the conditions described become the more obvious. It will be noted under the more favorable conditions of greater enlargement that the cluster of vessels lying on the inside of the narrow segment and near the pith is flanked on either hand by a region free from vessels. In $c$ is shown the organization of the perennial region of the stem in another 
species of Potentilla, namely, $P$. intermedia. In this instance the same outstanding and depressed segments are found as in the previous species, but the conditions otherwise differ in important respects. The depressed segments corresponding to leaf traces in $P$. intermedia are represented in solid black, which is the diagrammatic expression of the fact that they are no longer composed of fibrous elements, as is the case with the species discussed above. The substance of the segments is now, in fact, entirely parenchymatous, and they are comparable to the compound type of ray found in the oak and similar forms. On the other hand, the fibrous regions of the cylinder in $P$. palustris supply the equivalent of the aggregate rays of the alder and of types which resemble it. In $d$ is shown a more magnified view of a part of $c$. The cylinder presents three annual rings, which are seen in both outstanding and depressed segments of the stem. In the innermost annual increment of the depressed foliar region is imbedded the leaf trace, marked by the presence of vessels. Obviously it is not only faced by parenchymatous tissue (indicated in black), but is likewise flanked by the same substance precisely as in $b$ the trace is faced and flanked by the fibrous modification of the wood. In $d$ we have the thick cylinder resulting from perennial growth during several years. If the external region of this cylinder were cut away to such a depth as to reach the surface of the leaf trace, we should have the condition which is found in an annual stem of the same plant, such indeed as is shown in $e$. The topographical consequence of the thinning out of the woody cylinder, as a result both of less massive development and of the annual habit, is the increasing of the relative importance of the leaf traces as components of the woody cylinder. This means that the storage cells which originally not only flanked but also subtended the traces are now confined to the sides or flanks of the foliar traces and, in fact, separate these from the adjacent segments of the cylinder. This situation is distinctly shown in $e$ for the cylinder as a whole, and on an enlarged scale for a single foliar trace and the two adjacent segments of the woody cylinder in $f$. It is clear from the diagram. supplied in Fig. I40, which may be profitably compared with that illustrating the topographical and evolutionary features of the 
rays on page 82 , that the herbaceous stem may result from the breaking up of the originally continuous woody cylinder through the formation of special storage devices in connection with traces of the leaves. This process is far more striking in the more delicate annual stems by reason of the slender character of the cylinder. It is apparent that the conditions found in the stem of the oak, etc.,

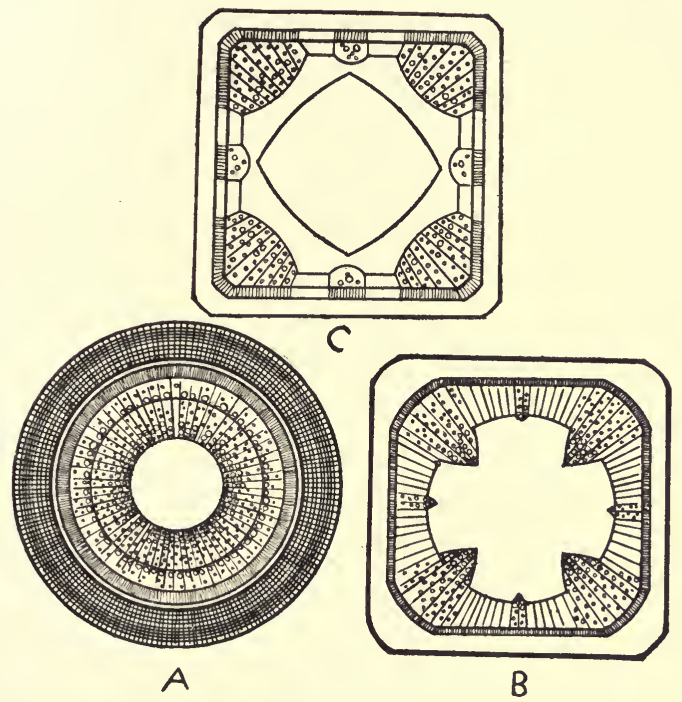

FIG. 14r.-Diagram of stem of Labiatae. Explanation in the text

as regards the presence of large foliar rays, elucidate the origin of the herbaceous type.

The situation illustrated in Fig. I40 is not, however, by any means a universal expression of the topography in herbaceous stems. It will be well to show further possibilities in this direction by reference to a family of dicotyledons much higher in the scale than are the Rosaceae. In Fig. I4 $a$ is shown the perennial stem of the common garden sage, Salvia officinalis. The outside is covered with an armor of periderm within which lies the narrow cortex, 
in turn abutting directly on the fibrovascular cylinder. The latter is circular in outline and shows no variation of structure in different segments. In $b$ an annual branch of the sage is diagrammatically represented so as to indicate the essential features of organization. The stem is now square in outline, as is the usual condition in herbaceous representatives of the Labiatae. It is to the flat surfaces of the quadrilateral stem that the paired leaves are attached. An examination of the fibrovascular cylinder shows that the uniformity of structure characteristic of the round perennial region is no longer present. Opposite the flat surfaces the cylinder is much thinned out, and vessels are conspicuous by their absence, except in small clusters marking the position of the leaf trace, which will pass outward at a higher node. Although the fibrous modification of the structure of the wood in relation to the foliar fibrovascular strands is found here as in the case of Potentilla and Alnus, it seems clear that we have to do with a less primitive phase of the origin of the herbaceous type. In $c$ is presented a diagram of the extremely herbaceous stem of Lamium album. The situation as regards flattening of the stem in connection with the quadrangular structure characteristic of the axes in the labiates is virtually the same as in the annual shoots of the sage, with the difference that rays are no longer seen in the purely fibrous and very narrow portions of the cylinder facing the flat surfaces and flanking the leaf traces on either side. In still softer and more herbaceous stems the fibers in turn may be largely or entirely replaced by parenchyma, so that the cylinder becomes definitely broken up into separate strands. Similar conditions in a general way present themselves in all cases in which the axial organs of dicotyledons become annual and assume the herbaceous texture-for example, the Solanaceae, as illustrated by the woody stem of the bittersweet (Solanum Dulcamara) and the angular soft one of the potato (S. tuberosum) and of the tomato (S. esculentum). In the two lastnamed species the furrows of the stem as well as the fibrous or parenchymatous regions of the fibrovascular cylinder correspond in position to the leaves which are a factor in the evolutionary processes under consideration. The Labiatae and Solanaceae have been chosen to illustrate the development of the herbaceous 
type in higher dicotyledonous orders because the simple foliar trace, which is a feature of the two groups under discussion, makes the topographical conditions more easily understood. The Leguminosae in the case of the garden bean or, better, of the garden pea, illustrate the situation appropriately for forms with plural traces. At the same time the two types just mentioned, as well as the tomato and the sage, exemplify the principle of recapitulation, since, in all, the young stem is round and woody and only later assumes the angular or furrowed configuration of maturity. The hypocotyledonary region of the stem in the bean, for example, and the lower region of the epicotyl in the pea both present a circular and complete woody cylinder. The developmental evidence, as also that derived from comparative anatomy, thus clearly points to the derivation of herbaceous forms from woody ones and not of arboreal perennials from annuals, logically following from the account of the origin of the structures of the stem in dicotyledons originated by Sanio and disseminated by Sachs and De Bary.

Another feature of organization of herbaceous stems which often, although not invariably, appears is the degeneracy of the rays other than those broad masses of storage parenchyma which, as has been set forth in the foregoing paragraphs, flank the foliar traces in their vertical course in the stem. This situation is well illustrated among the ranunculaceous representatives of the Ranales, for example, the buttercup, the meadow rue, and the clematis. It has already been discussed in sufficient detail in connection with the vine type of stem in a former paragraph and therefore need not be referred to here.

The discussion of the herbaceous dicotyledons brings us to the consideration of the stem in the monocotyledons. This important group of plants, which in physiological efficiency excels all the other large divisions of vascular plants, is practically entirely herbaceous in its structure; and even in those of arboreal habit the internal organization is that of herbs. The monocotyledons on account of the relative simplicity of their fibrovascular strands have been referred by many to affinities with the ferns or, on the basis of their habit, to relationship with the older gymnosperms. These attributions of relationship are, however, little supported by either 
anatomical evidence or reliable data derived from the study of the remains of plants in the geological strata.

The stem in this large and important group is distinguished both by the structure of the fibrovascular strands and by their mode of arrangement in a transverse section of the stem (Fig. 142). The continuous woody cylinder of the older dicotyledons in the herbaceous types more adapted to modern climatic conditions in temperate

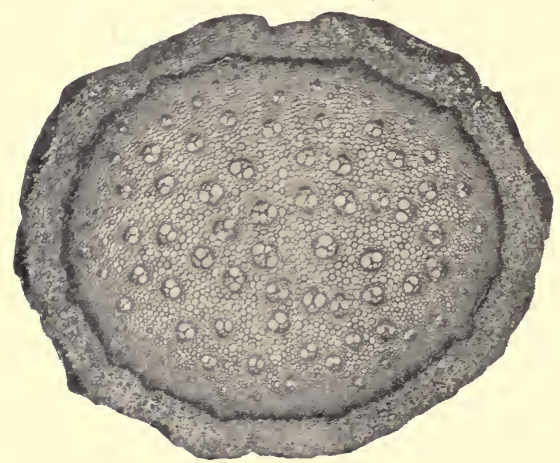

FIG. I42.-Stem of Smilax regions has already given place to a condition of discontinuity. In the monocotyledons the process of disintegration of continuity has gone much farther than even in the herblike dicotyledons. For here the separate strands abandon, under the further influence of the necessities con.

nected with the leaves, the original circular arrangement for a scattered disposition through the transverse section of the stem. The high efficiency of the monocotyledons in the elaboration of foodstuffs naturally has correlated with it an ample provision of numerous fibrovascular strands for conducting the assimilates into the stem or axis. This situation brings it about that many traces enter the stem at a node instead of the three or, at most, several traces which pass into the nodal region of the axis in the mass of dicotyledons. This multiplication of the conducting strands brings with it complications of arrangement in the stem, for the number is too large to be accommodated on the periphery as is the rule even among herbaceous dicotyledons. As a consequence of the necessities which have thus arisen, the leaf traces are displaced from the margin of the central cylinder into the pith or medulla. It is thus clear that, just as the woody stem has given 
place to the herbaceous one under the influence of necessities connected with the greater elaborative efficiency of the leaves, so a still further accentuation of foliar efficiency, correlated with a corresponding multiplication of foliar traces, has led to the further modification of the cylinder of the stem, resulting from the necessity of accommodating a very much larger number of foliar traces in the transverse area of the cylinder. This is usually effected by moving the foliar traces from a peripheral position to the medullary region and more rarely by the running of the foliar traces for one or more internodes in the cortex. The multiplication of the conductive strands from the leaves has a further effect in the organization of the bundles which will be discussed in a later paragraph.

Not only in the arrangement of the fibrovascular bundles do the monocotyledons differ from the dicotyledons. The bundles themselves differ in the mass of monocotyledonous forms from the great majority of the dicotyledonous angiosperms in the fact that cambial activity is absent. Fig. I 43 shows the general topography of the bundle in Smilax herbacea, the carrion flower. The fibrovascular strand is surrounded by a well-marked sclerenchymatous sheath in all probability corresponding to the pericycle in lower forms. It is only rarely that in the group under discussion endodermal structures are well developed about the strands in the stem. Generally a continuous external endodermal sheath surrounds the bundles in common and no internal structures of this nature can be made out. That the parenchymatous tissue lying between the bundles in the stem belongs to the fundamental system can often clearly be inferred from its histological character where its elements strongly resemble those of the cortex. The internal organization of the bundle is distinguished by the presence of phloem and xylem collaterally disposed in relation to one another. The xylem consists for the most part of vessels and parenchyma, and the vessels are of the porous type. The elements of the wood farthest away from the phloem are typical spiral and ringed protoxylem. No cambium or zone of growth ordinarily is interposed between the wood and the phloem, and this condition has gained for the fibrovascular strands of the monocotyledons the appellation of closed bundles. It has often been supposed that the closed 
character of the bundles in the group is an indication of affinity with the pteridophytes, but this view seems to have little value when it is realized that the earlier vascular cryptogams usually

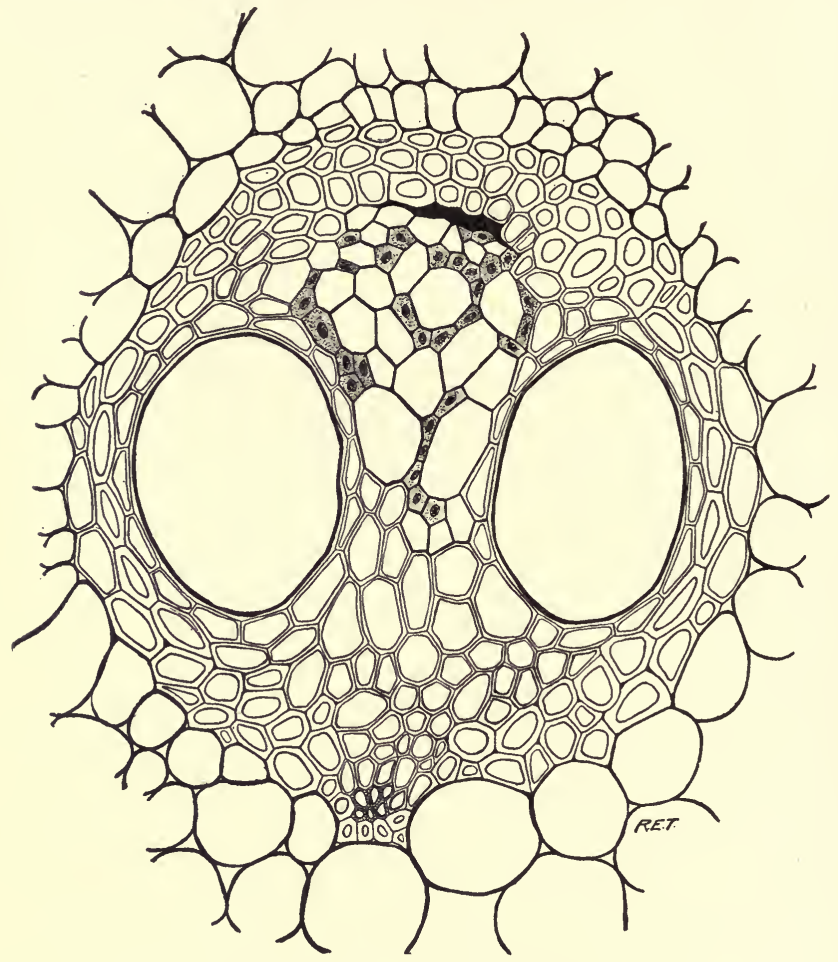

Fig. I43.-Bundle of Smilax herbacea

possessed well-marked secondary growth. Toward the periphery of the stem in each fibrovascular strand lies the phloem, composed mostly of sieve tubes and their companion cells. The small elements of the phloem lying on the very outside and close to the bundle sheath represent the protophloem and are often in a some- 
what swollen condition. The sieve tubes in the monocotyledons are of a high type and are characterized by terminal walls which are generally horizontal. The lateral abortive sieve plates, known as lattices, present on the side walls of the sieve tubes in woody dicotyledons and many herbaceous ones as well, are usually absent in the group. Both phloem and xylem consequently represent a high condition of organization and indicate an advanced phylogenetic position for the monocotyledons.

In certain monocotyledonous families cambial activity has been detected in some instances. This phenomenon is quite generally present in the grasses, as has been pointed out by Chrysler. In the Liliaceae and aroids as well as in the Cyperaceae the presence of a cambium has also been recorded. Fig. I44 shows the presence of secondary growth in the region of the node in Erianthus Ravennae.

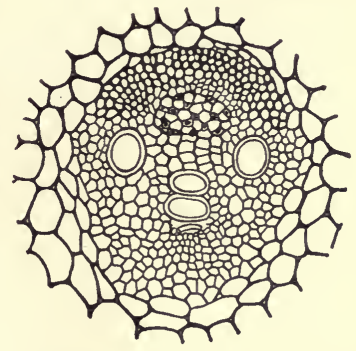

FIG. 144.-Bundle of Erianthus Ravennae, showing presence of cambial activity (after Chrysler).

Frequently the bundles of the stem in monocotyledons are of the closed collateral character, but in some cases, particularly in rootstocks with closely approximated nodes, they present a different type, a concentric condition which differs from that found in the Pteridophyta by the fact that the xylem surrounds the phloem instead of the phloem forming a continuous girdle about the xylem. This modification of the bundle is known as amphivasal to distinguish it from the amphicribral condition characteristic of fern allies. A strand of this nature is shown in Fig. I45. The phloem occupies the center of the figure and is surrounded completely by the tissues of the xylem. Bundles of this type are apparently the result of the crowding and fusion of the foliar strands at the nodes, for it is in the nodal region that they are best developed. Where the nodes are remote from one another, the amphivasal condition may entirely disappear in the internode to reappear at the next node. With the approximation of nodes, characteristic of many creeping monocotyledonous rootstocks with 
tufted leaves, the amphivasal condition becomes continuous. Fibrovascular strands of this type are, in fact, a common feature of organization of the subterranean regions of many monocotyledons, 'even when they are absent in the stem. This situation is well exemplified by the orchids and Iridaceae. In Gramineae and Cyperaceae, on the other hand, amphivasal strands are present in the somewhat remote nodes of the annual flowering stems, but

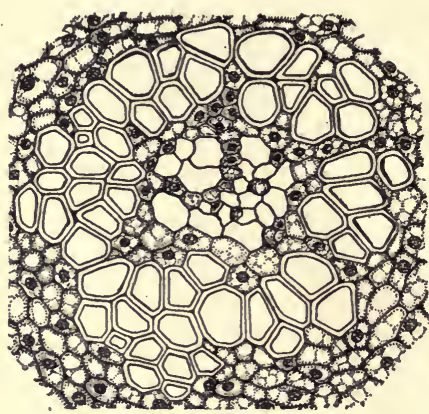

FIG. 145.-Amphivasal concentric bundle of rootstock of Smilax. disappear in the internodes, and a similar situation is present in the perennial subterranean stem in case the nodes are not crowded. In the latter condition the amphivasal state becomes continuous. In the true palms (Principes) and in many Scitamineae the amphivasal bundle seems to have entirely disappeared, although this is a matter for further investigation. There seems to be little doubt that the formation of amphivasal bundles in the region of the nodes is an original feature of organization of the monocotyledons, and that it persists very strongly in the perennial subterranean stem or rootstock, but may entirely disappear in the annual stem. The amphivasal condition of the bundles seems to be definitely correlated with the numerous leaf traces passing into the stem at the node in the monocotyledonous angiosperms. This hypothesis of its origin is supported by the fact that in herbaceous dicotyledons with numerous leaf traces the occurrence of amphivasal bundles at the node can frequently be observed. The Umbelliferae may be cited as an excellent example of this condition.

The consideration of the stem has now been completed, and it will be advantageous in conclusion to indicate the main tendencies manifested in its course of evolution from lower to higher forms. It has been noted that the first important phase in the evolution is connected with the primary structures of the fibrovascular region. 
In the stems of lower types these present themselves under two general conditions: namely, the protostelic and the siphonostelic. In the former the xylem constitutes a solid mass without any central pith, while in the latter the fibrovascular complex is thrown into a hollow cylinder in which gaps are frequently present. These are generally related to certain of the appendages, but may not have any topographical connection with the lateral parts. In the siphonostelic condition internal phloem is often seen, particularly in the lower forms, and the stele or central cylinder is clearly separated from the central pith or medulla by the presence of an internal endodermis. In higher types the internal surface of the tubular stele tends to degenerate, with the resulting disappearance of internal phloem and endodermis. The original state of the wall of the hollow cylindrical stele can often be inferred from the character of the foliar strands which, in accordance with a general principle to be enlarged upon in a later chapter, often perpetuate a situation which has disappeared in the stem. Where the foliar traces are concentric and separated from the fundamental tissues by a well-marked endodermis, it may be assumed that a similar condition was formerly present in the organization of the central cylinder of the axis.

A further interesting phase in the evolutionary development of the vascular cylinder is to be noted in connection with the progressive degeneracy of the primary wood. In the lower forms this region of the xylem is well developed, and both by its massive character and by the lack of seriation of its elements it is clearly distinguishable from the secondary cylinder which surrounds it. The stem of higher groups presents the primary structures in an obsolete condition, which results in the bringing of the rays of the secondary wood into apparent contact with the pith or medulla. This advanced state of modification of the central cylinder is responsible for the extremely inappropriate name medullary rays, which is applied to the radial parenchyma of the secondary wood. These structures are best called wood rays or simply rays, since primitively in the stem and always in the root they are quite divorced from any confection with the pith. 
The next important modification in the case of the fibrovascular structures of the stem is the appearance of aggregations of rays by reason of the increased efficiency of the secondary fibrovascular cylinder in regard both to its conducting and to its storage elements. The clusters or congeries of rays originally diffused throughout the woody structure tend in higher groups to become somewhat definitely related to the appendages. By further progress in the evolution of radial parenchyma we have the compound and diffuse types of rays making their appearance, the former particularly characteristic of herbaceous types, and the latter of the mass of dicotyledonous forest trees. The appearance of large rays, interrupting the continuity of the central cylinder and bringing about the differentiation of separate fibrovascular strands, is a particular feature of the herbaceous type representative of later geologic time and of cooler climatic conditions.

The woody cylinder broken up into fibrovascular bundles as the result of the evolution of storage devices in connection with the appendages in the herbaceous dicotyledons, in the monocotyledons undergoes further modification in relation to decrease in size and increase in number of the bundles in correlation with the greater efficiency and consequently more numerous conducting strands of the leaves. The decrease in size of the bundles is definitely related to the loss of the cambial activity which characterized the fibrovascular structures of the monocotyledonous angiosperms as a whole. The increase in number of the cauline bundles, resulting from the entry of very numerous leaf traces into the stem at the nodes, brings with it the distribution of the fibrovascular strands throughout the transverse section of the stem. The monocotyledons may, in a sense not altogether figurative, be said to represent the second childhood of the vascular plants, just as the Pteridophyta constitute its true infantile phase of development. It is necessary, however, to distinguish very clearly between the primary structures of the monocotyledonous angiosperms which are the result of the loss of secondary growth, and the primary structures of the Paleozoic forms which, so far as we know, are a primitive feature of organization. 


\section{CHAPTER XIV}

\section{THE LEAF}

A general definition of the leaf or foliar organ has been supplied on an earlier page. The leaf is important, not only on account of its features of structure as an organ of vegetation, but also because of the primitive and intimate relation between it and the organs of reproduction. The latter parts will be discussed in two chapters following the present one, while the main features of organization of the leaf itself will occupy attention in the present connection. The anatomy of the foliar organs of the cryptogamic forms, living or extinct, need not be considered at this stage, as any references to them which are neces-

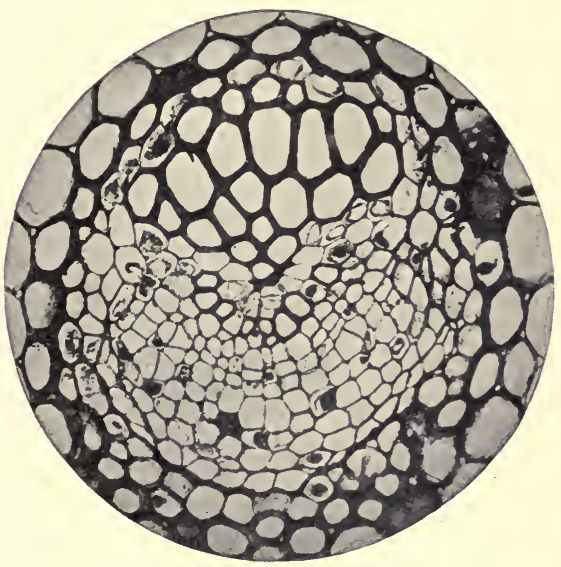

Fig. 146.-Leaf bundle of Cycas revoluta. Explanation in the text. sary will appear in later chapters dealing with particular groups.

We may conveniently begin the discussion of the leaf with the most primitive type of living gymnosperms, the Cycadales. This group, much reduced in numbers under modern conditions, has a type of organization in the leaf which is of great interest from the evolutionary standpoint. Fig. 146 presents the transverse view of one of the bundles of the main axis or rachis of the leaf in Cycas 
revoluta. Toward the lower side of the figure is seen the phloem, composed of elements which in the main are arranged in regular radial rows indicating an origin from cambial activity. The upper region of the bundle is composed of xylem, consisting of very large elements which become progressively smaller and fewer in number in the downward direction. The lower point of this aggregation of cells is the protoxylem. This region is surrounded inferiorly by a few rows of parenchyma which give place in turn to more

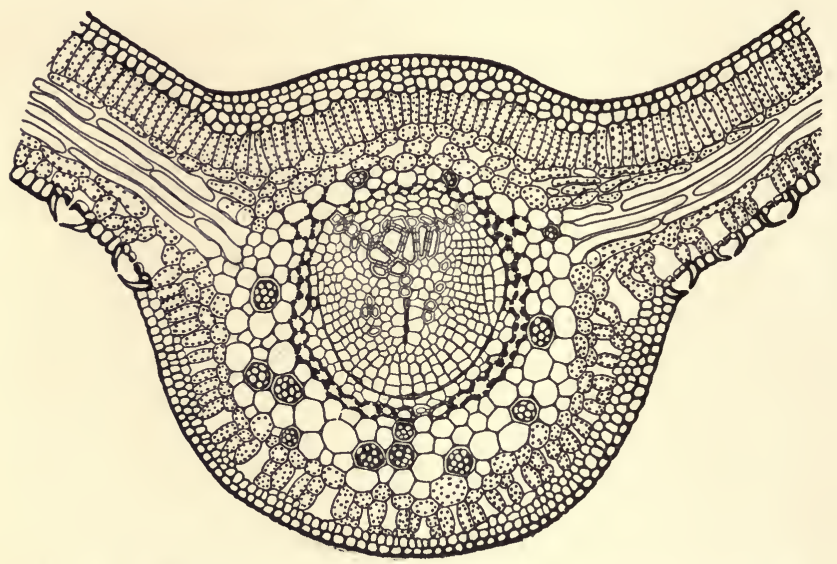

Fig. 147.-Transverse section of leaf of Cycas revoluta. Explanation in the text

thick-walled elements of the xylem, in contact with the regular radial files of the cambium. The lower region of the xylem is the so-called centrifugal xylem; the upper portion broadening from the narrow protoxylem is the centripetal or cryptogamic xylem. The first-mentioned group of elements of the xylem are pitted in their character, as will be made clear in a subsequent longitudinal illustration. The latter, the so-called cryptogamic wood, is of importance from the evolutionary standpoint because it indicates at once a clear relationship on the part of the Cycadales to the vascular cryptogams and to the lower and extinct gymnosperms. In the next figure (Fig. I47) is represented a transverse section of one of the 
divisions of the leaf. The cryptogamic wood is here relatively better differentiated than in the axis of the leaf, and it is obvious that the seriation of its elements is toward the upper surface of the foliar organ. The centrifugal wood is very slightly developed. In the next figure (Fig. I48) appears a foliar bundle of Cycas revoluta in its course in the cortex of the stem. There is a mass of irregularly arranged primary wood in the center, surrounded by the radially disposed secondary wood, which in turn is followed by the tissues of the phloem. The phloem entirely surrounds the bundle, and this is consequently of the concentric type often found in the living ferns and their allies. The contrast in organization of the bundles of the leaf in different parts is very striking. The lower concentric region, in

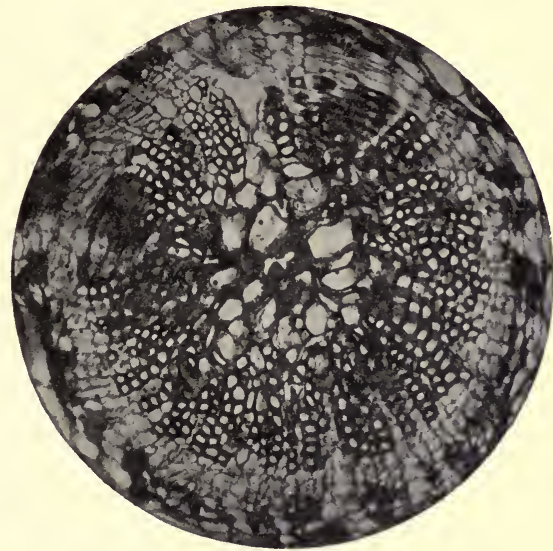

FIG. 148.-Concentric foliar bundle from the cortex of Cycas revoluta. accordance with

generally accepted principles of comparative anatomy to be elucidated later in a special chapter, may be regarded as supplying evidence of the former concentric character of the woody cylinder of the stem in the Cycadales, a hypothesis entirely justified by the existence of a remarkable group of fernlike seed plants in the Paleozoic, known as the Medulloseae, from which on both anatomical and reproductive evidence the living group seems to have been derived. In these forms the bundles of the stem were, as will be subsequently shown, often concentric in their organization. In the higher region of the leaf trace in the cycads the concentric character is lost, but the centripetal development of 
part of the wood still sufficiently vouches for the cryptogamic affinities of the lowest living gymnosperms. In Fig. I49 is shown the longitudinal view of the leaf trace in the rachis of the leaf. To the right appears the phloem made up of elongated elements, the sieve tubes. To the left of this region lie certain pitted tracheids belonging to the centrifugal or modern wood. Then intervene a few parenchymatous cells, followed by the spiral elements of the centripetal or cryptogamic wood. These pass farther to the left

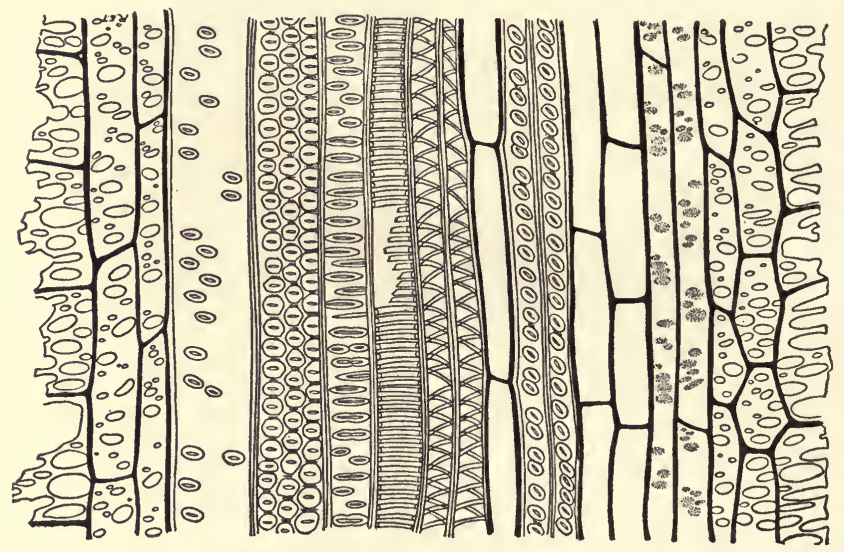

FIG. 149.-Longitudinal section of a foliar bundle in Cycas revoluta

into ringed, scalariform, and, finally, pitted tracheids. The structure of the leaf in the Cycadales is clear evidence of their relationship with the ancient fernlike gymnosperms, which in turn were doubtless in filiation with actual ferns in the remote geologic past.

The Cycadales have their nearest affinities in the past with a group of gymnosperms, the Cycadofilicales or the Pteridospermeae, which were so strikingly fernlike in their habit that until the very end of the last century they were regarded as ferns. In contrast to the Cycadales the mass of surviving gymnosperms have taken their origin from forms which are known as Cordaitales. These had nothing in their habit which recalls the ferns, but often 
present a strong superficial resemblance, particularly in the conformation and venation of their leaves, to the monocotyledons. The Cordaitales had leaves varying in width, but always characterized by few or numerous parallel veins. The structure of the fibrovascular bundles constituting these veins, however, was entirely different from the features of organization found in the nerves of the leaves of living monocotyledons. Fig. I50 illustrates the general organization of the leaf in Cordaites principalis. The veins are numerous and separated from one another by intervals of the soft mesophyll of the leaf. The epidermis is

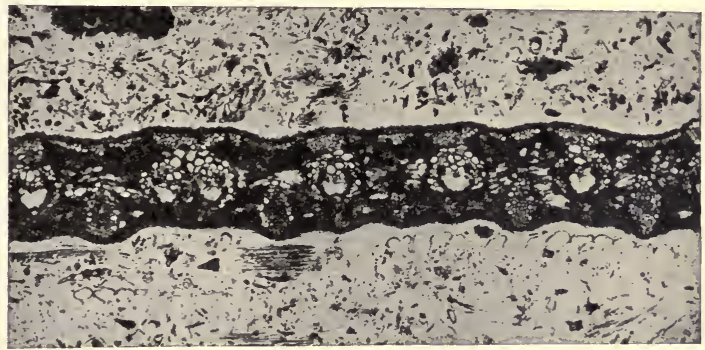

FIG. 150.-Transverse section of the leaf of Cordaites principalis

strengthened, particularly in the region of the fibrovascular bundles, by hypodermal bands of thick-walled cells. In Fig. I5I is shown an enlarged transverse section of one of the foliar bundles of the species under consideration. The wood was entirely centripetal in its development and ended on either flank in a band of thick-walled elements with narrow central cavities. The zone of thick-walled elements was in contact externally with a second zone made up of cells with thinner walls and much larger lumina. Both the zones just described inclose the phloem, which lies below the mass of centripetal wood. The longitudinal view (Fig. I 52) throws additional light on the organization of the bundle. The double sheath connected with the flanks of the xylem and forming a complete circle about the phloem is composed of elongated elements with bordered pits on their walls. These are known as transfusion cells and are 
an important and phylogenetically interesting component of the foliar fibrovascular strands in many gymnosperms. They are not a salient, and certainly not a primitive, feature of organization of

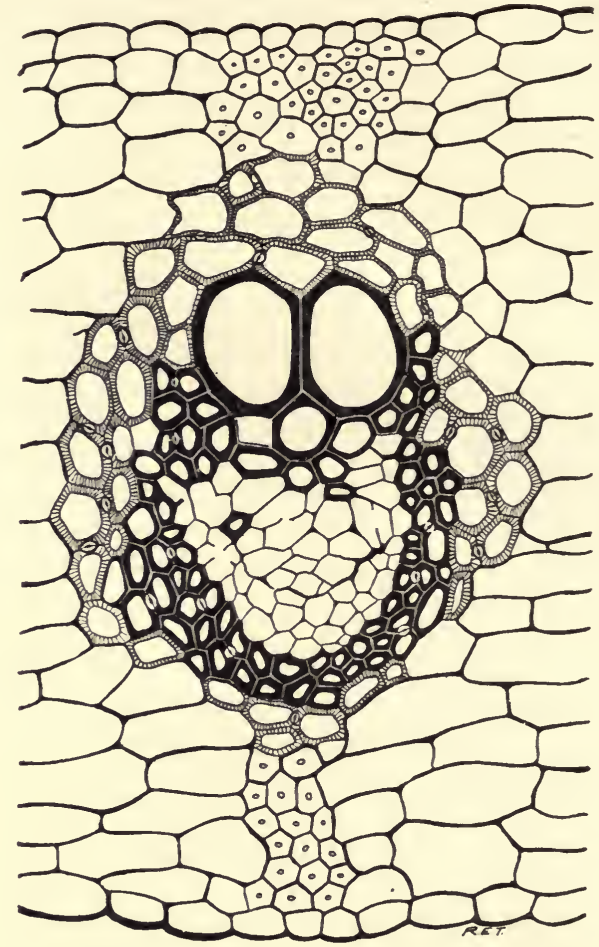

FIG. 151.-Transverse section of the leaf bundle in Cordaites principalis

the leaf bundles of the living Cycadales and their allies, the Paleozoic Medulloseae. The transfusion sheath of the foliar bundles in the Cordaitales did not always manifest the complexity that it shows in the case of $C$. principalis figured above. In most instances only the broader and shorter thin-walled elements were present, the 
elongated and thicker-walled inner sheath being absent. The intimate connection between the centripetal or cryptogamic wood and the transfusion tissue is observed in all cases. It is clear accordingly in the Cordaitales that the transfusion tissue which, as will be subsequently shown, plays an interesting rôle in the evolutionary history of higher gymnospermous groups is primarily related to the centripetal or cryptogamic wood.
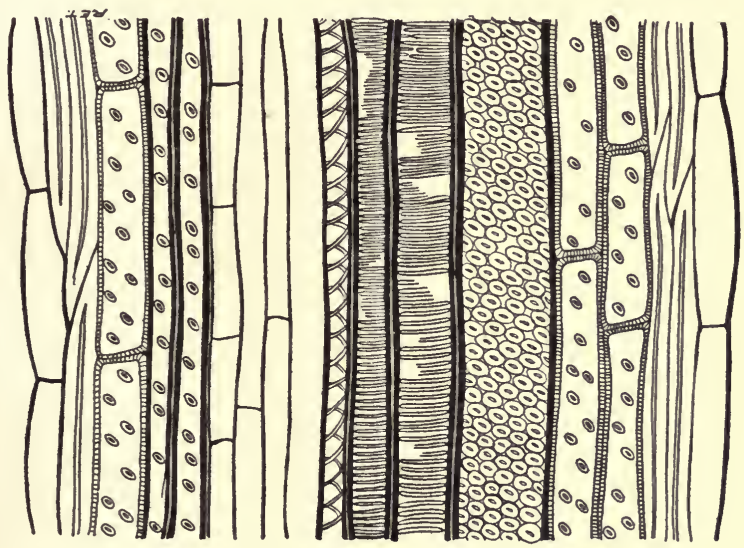

FIG. 152.-Longitudinal section of a leaf bundle in Cordaites principalis

The organization of the leaf in the conifers will next occupy our attention, since the present chapter deals with the leaf only in the features which are of general evolutionary importance. Fig. I53 shows an enlarged view of the"whole leaf of Pinus strobus, the white pine. The epidermis, reinforced by a hypoderma, surrounds the median green part of the leaf, the mesophyll, in which lie resin canals and a single fibrovascular bundle. The latter, exceptionally for the conifers, is marked off from the surrounding fundamental tissues by a well-defined circular endodermis. The fibrovascular strand consists of xylem entirely centrifugal and mostly secondary in its origin, and this meets with the phloem on its lower border. Surrounding the conducting strand of the leaf is a mixture of ordinary 
parenchymatous elements containing protoplasmic structures and short empty cells with bordered pits. These are the transfusion elements, and it is easy to observe that they are most abundant on the flanks of the xylem and, in fact, take their origin from this general region. As in the case of the Cordaitales, the cells of the transfusion tissue surround the phloem as well as the xylem. An important difference, however, is the absence of intermingled

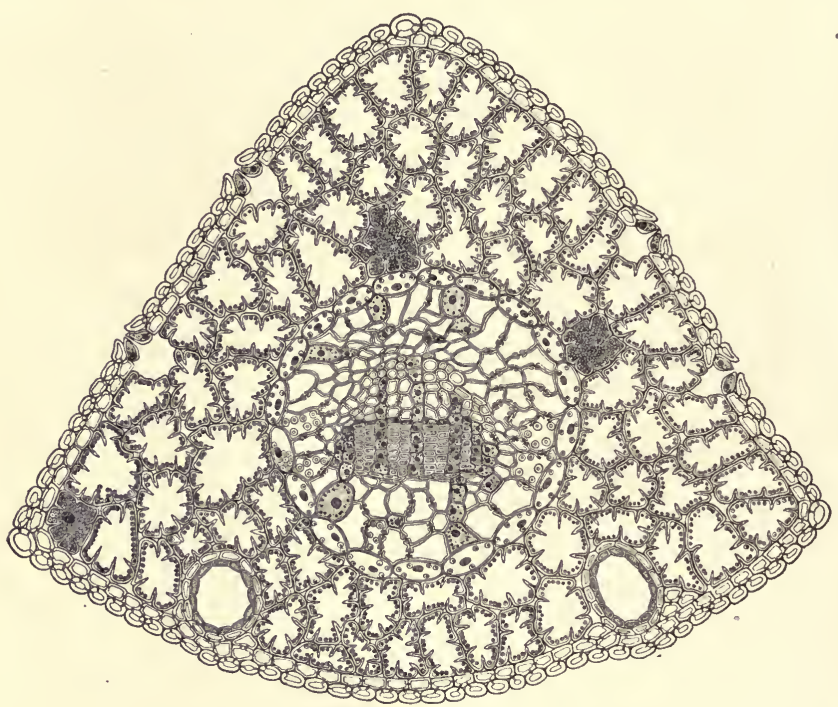

Fig.. 153.-Transverse section of leaf of Pinus Strobus

parenchymatous elements in the girdle of transfusion tissue of the older group. Transfusion tissue is better developed in the leaf of the pine than in any other of the subtribes of conifers. In most of the other subgroups of the Coniferales the transfusion tissue is mainly confined to the flanks of the fibrovascular bundle, and the conducting strand as a whole is not sharply separated from the remaining tissues of the leaf on account of the absence of an endodermis. 
A link between the conditions found in the case of Pinus and the anatomical organization of the Cordaitales is presented by a mesozoic fossil form allied to Pinus to which the name Prepinus has been given. Fig. I54 illustrates the organization of the leaf in this species. It is bounded by flat surfaces as a result of contact with surrounding leaves of the many-leaved fascicle. The hypodermal structures are strongly developed as in the Cordaitales and constitute the same strengthening ribs as are found in the leaves of

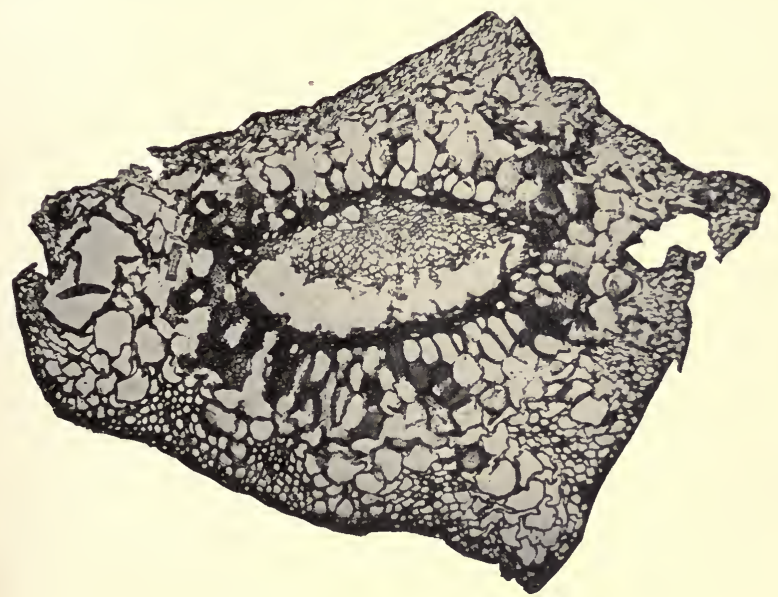

FIG. 154.-Transverse section of leaf of Prepinus statenensis

that genus. On account of the changes resulting from fossilization the endodermis is not so distinct as in living pines, and the mesophyll or soft substance of the leaf is very poorly developed. The bundle is surrounded by a very dense transfusion sheath in which all the cells are empty and provided with bordered pits. Not only is the transfusion sheath massive in Prepinus and entirely composed of transfusion tracheids to the exclusion of parenchymatous elements, but it is further complicated by the presence of an internal thick-walled zone comparable with the similar structure in $C$. principalis figured above. In the comparatively 
low magnification shown in the photomicrograph the inner zone of the transfusion sheath appears merely as a dark boundary surrounding the fibrovascular bundle proper. The conducting strand of the leaf is represented by the xylem alone, the phloem having disappeared during fossilization. In Fig. I 55 the central region of the foregoing is represented under a higher degree of magnification.

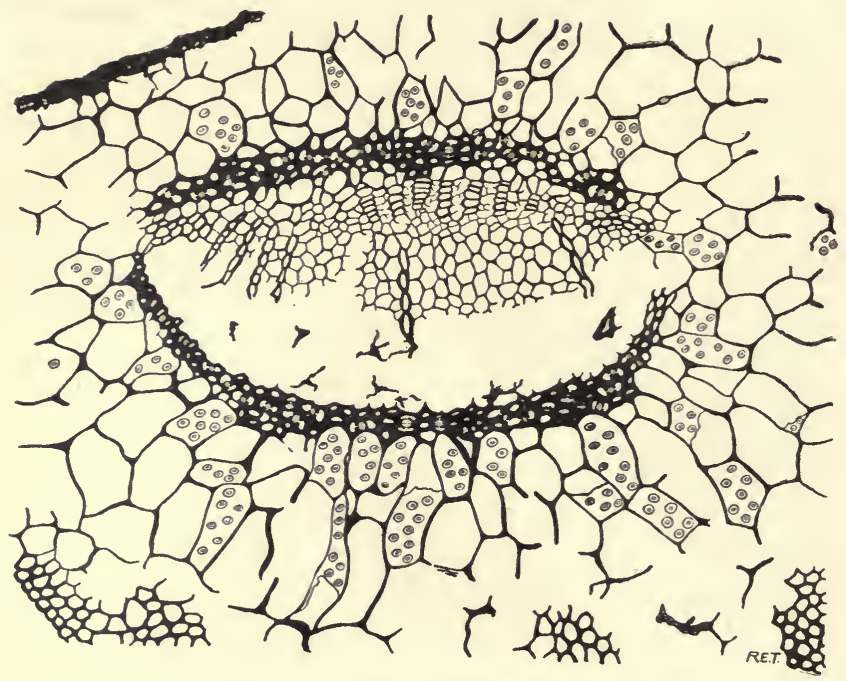

Fig. 155.-Portion of leaf of Prepinus statenensis, more highly magnified

The tracheary character of the transfusion sheath can now clearly be discerned, as well as the fact that it contains no cells of the nature of parenchyma. The narrowness of the cells constituting the inner transfusion sheath is also now quite apparent. In Fig. ${ }_{5} 6$ are shown the various structures of the fibrovascular bundles longitudinally and on a still higher scale of magnification. The outer transfusion sheath is composed of elements with distinct and rather large bordered pits which abut inwardly on the narrow thick-walled cells of the inner transfusion sheath, in turn connected 
with the xylem of the fibrovascular bundle. Before we consider the nature of this relation it will be well to examine more carefully the bundle itself. The conductive strand is represented by its xylem only; this consists, interestingly enough, of two regionsa lower one in contact with the empty space once occupied by the phloem, and an upper, consisting of distinct rows separated by thinner-walled elements. The latter is the true centripetal or

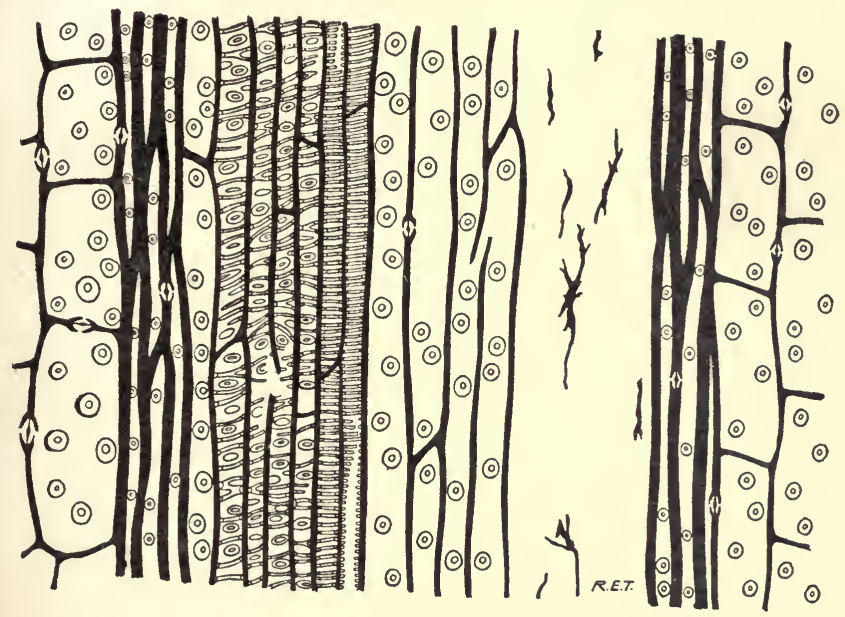

Fig. 156.-Longitudinal section of the leaf of Prepinus statenensis. Explanation in the text.

cryptogamic wood which in Prepinus alone among the conifers is present in a typical form. The lower region of the xylem is centrifugal and corresponds to the whole of the xylem in the leaf of the modern Pinus: In the modern or centrifugal region of the wood, rays are distinctly present, and these pass from its substance into the cavity once filled by the phloem. The centrifugal region of the wood provided with rays is related to the inner transfusion sheath on its flanks alone. This relationship corresponds in fact to that observed in the case of living pines, as is shown above in 
Fig. I53. The most significant relation of the xylem in the leaf trace is by means of the series of centripetal or cryptogamic elements. These are numerous and serve to bring about a very intimate and copious connection between the bundle proper and the transfusion tissues. There is good reason on the basis of the anatomy of Prepinus and the Cordaitales to regard the transfusion tissue which characterizes the foliar organization of all but the very lowest of

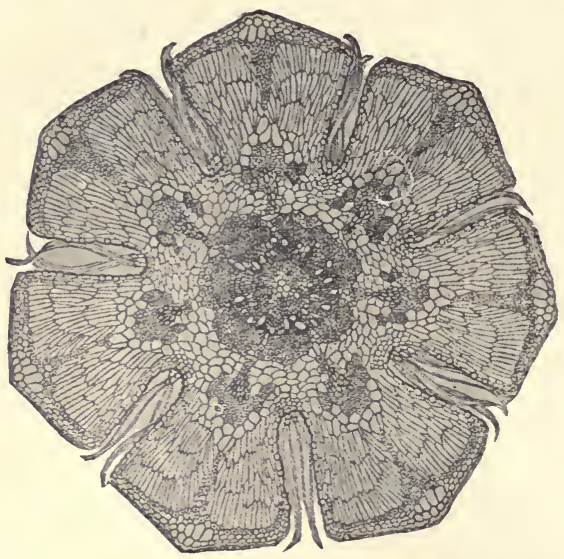

FrG. 157.-Leafy twig of Casuarina equisetifolia (after Solerderer).

appeared, the relation between the bundle and the transfusion tissues occurs on the flanks of the centrifugal wood. It will be obvious to the reader that the tissues surrounding the fibrovascular bundle in the conifers and their allies are of considerable interest from the evolutionary standpoint. The transfusion tissue at the present time has a significance in the vegetative leaves of all but the lowest gymnosperms in connection with the storage of water and the conducting of it to the green cells of the mesophyll. In the reproductive leaves it has taken on another but equally interesting function, as will be indicated in the next chapter.

Transfusion elements are found in the leaves of all seed plants from (and excluding) the cycads upward. In the conifers they are 
well developed, but are distinctly degenerate in the higher subtribes of the group and are less well developed in any living conifers than they are in Prepinus and Cretaceous species of Pinus. The Gnetales often show the transfusion tissues in a high degree of development. The small leaves of Ephedra naturally show them less distinctly than the large persistent foliar organs of Welwitschia, where they constitute a very conspicuous feature of the organization of the leaf. In Gnetum, again, in accordance with its higher

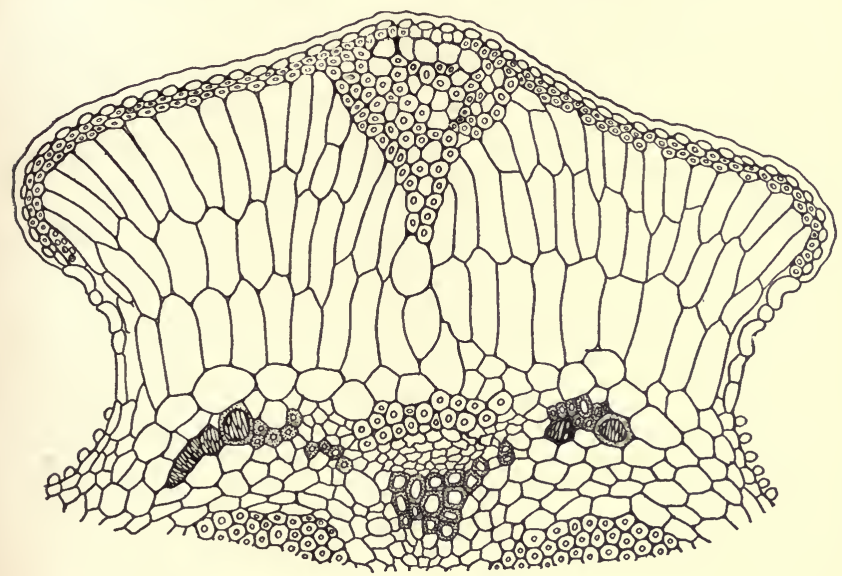

Fic. 158.-Base of leaf of Casuarina equisetifolia, showing transfusion tissue

systematic position, the transfusion sheath is less conspicuous. Among the angiosperms transfusion tissues are present in the dicotyledons, but present themselves in the condition typical for the higher gymnosperms only in the genus Casuarina. Here, as is shown in Fig. I57 illustrating the organization of a leafy twig of Casuarina equisetifolia, there are clusters of thick-walled empty cells flanking the leaf traces. This relation to the foliar strands strikingly resembles that found in the higher gymnosperms and appears to be good evidence of the primitive position of this interesting genus. In Fig. I 58 is shown one of the foliar bundles of the 
genus under discussion much more highly magnified. The flanking relation of the transfusion elements to the strand of xylem is now very distinct and recalls that found in the leaves of the Cupressineae, Taxineae, etc. Less typical manifestations of the development of transfusion tissues in the dicotyledons are provided by those forms in which bundles related to stomata exuding fluid water, and consequently known as water-stomata, terminate below

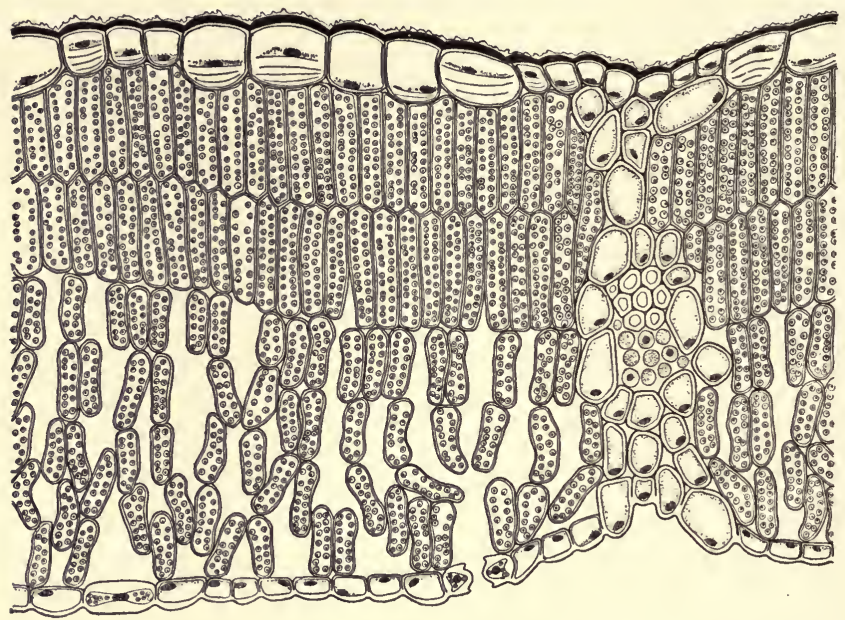

Fig. 159.-Transverse section of leaf of Alnus incana

the stomatic pores in a mass of wide, short, tracheary elements. This condition, although doubtless derived from ancestral gymnospermous structure, has departed so far from the original transfusion tissue that it can scarcely be included in the same morphological category.

Transfusion tissue, as will be apparent from the last paragraph, has become a feature of very subordinate importance in the organization of the leaf in the mass of the dicotyledons. The general structure of the foliar organs in the group may profitably occupy attention at this stage. Fig. I59 reproduces somewhat diagram- 
matically the anatomical features of the leaf of Alnus incana. Above is a layer of cells containing only protoplasm and a nucleus. A similar situation is presented by the lower surface of the leaf, with the exception that the continuity of the epidermis is locally perforated by stomatic openings. The elements related to these, the guard cells, are distinguished from the rest of the epidermal layer by the presence of chloroplastids. The upper epidermis is contrasted with the lower, not only by the absence of stomata, but also by the presence of a rough impervious covering, the cuticle. Another feature of interest in the superior epidermal cells is the mucilaginous modification of their inner walls, and this is expressed in the illustration by a thick layering. The central portion of the leaf is occupied by the mesophyll, composed of the palisade (upper) region and the spongy (lower) region. The development of palisade parenchyma in foliar organs is definitely related to the amount of insolation or exposure to light, while the spongy layer is less well developed when the leaf is strongly illuminated, and becomes much more accentuated in foliar organs exposed to shade and a damp atmosphere. The leaf of the monocotyledons supplies little in a general way which is of interest from the standpoint of evolutionary anatomy, except the occasional persistence of cambial activity. 


\section{CHAPTER XV}

\section{THE MICROSPORANGIUM}

The microsporangium of the vascular plants is considered first, because there can be no question that it is the primitive type of sporangial structure for the long series of forms which are characterized by the possession of water-conducting tracheary tissues. In the lower representatives of the Vasculares the microsporangium is the only type present, and in the heterosporous cryptogams and the seed plants it keeps its place, with little change of its original condition of organization, side by side with the highly modified megasporangium and seed. The relative constancy of microsporangial structures makes them in many respects of the greatest value from the evolutionary standpoint.

If the liverworts are correctly regarded as the forms nearest to the Pteridophyta in the series of the bryophytes, there can be little doubt that the sporangium in its primitive form of sporogonium is the forerunner of the sporophyte of the vascular series. Professor Bower has brought forward an impressive aggregation of evidence in favor of the hypothesis that the sporophyte is the result of progressive sterilization of sporogenous tissue. Although the definite mode by which the simple sporogonium of the thallose liverworts gave rise to the sporophyte of the Pteridophyta, so complicated in its internal structure and external organization, is highly speculative, it will serve a useful purpose to indicate the main probabilities in this connection based for the most part on the investigations of Leitgeb. In certain liverworts, such as, for example, Corsinia and Boschia, the spore sac gives rise to sterile cells as well as spores. There is clear evidence in these and in similar cases that the sterile cells are modified or, as Professor Bower expresses it, sterilized potential sporogenous cells. In many liverworts the sterile cells are useful in distributing the spores. In this instance they are much elongated and have their walls spirally thickened. The spirals recoil when the spores are ripe, 
giving them an impetus which scatters them over the surface of the ground. These spore-distributing mechanical cells are known as elaters. In certain liverworts, such as Pellia and Aneura, the elaters, in addition to occurring loosely among the spores which they serve to scatter, are aggregated in a compact elongated mass at one end or the other of the spore sac or theca. This longitudinal cluster of elaters may be regarded with some degree of probability as the prototype of the fibrovascular bundle of the Pteridophyta and higher groups of vascular plants. In Anthoceros and allied forms the cluster of elaters, known as the columella, becomes a much more important structure and traverses the sporangium from end to end. Laterally at intervals it gives off transverse ramifications which divide the mass of spores into separate clusters, and these may perhaps be regarded as the prototypes of the sporangia found in the vascular series. The situation in the horned liverwort Anthoceros, in which there is an extensive columella with lateral ramifications, gives some support for the hypothesis of the derivation of the sporophyte from the sporogonium by the sterilization of potential sporogenous tissues. The mode in which the organs, leaf, stem, and root arose from such a primitive condition of organization is much disputed, since none of the hypothetical transitional forms between the moss capsule and the sporophyte of the higher plants have yet been discovered. There can be little doubt, however, in a general way that the sporogonium is the forerunner of the sporophyte and that the elater is the prototype of the tracheid in vascular plants.

It will be clear from the foregoing statement that the sporangium is so intimately involved in the primitive organization of the most ancient spore-producing members that it is entirely proper to consider it a definite organ of higher plants on a footing of equality with the root, stem, and leaf. If this view of the matter is sound, obviously no very useful purpose can be served by the examination of the development of particular cells of the foliar organs which are in some way or other related to the origin of sporangia. It is likely, moreover, that the sporangia of the Pteridophyta give us on the whole a less accurate picture of the original type of sporangium than those of the lowest seed plants. It is therefore appropriate 
in the present connection to begin the discussion of the sporangium with the consideration of the situation presented by the lowest living gymnosperms, the Cycadales. Fig. I60 shows the organization of a microsporangium in Zamia muricata. The structure in question is covered on the outside by an envelope of thick-walled cells which in the condition of maturity determine its dehiscence. The mechanical structure is known as the annulus and is of great

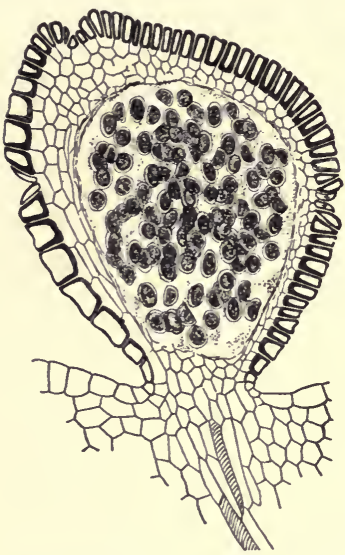

FIG. r60.-Sporangium of Zamia muricata. importance in bringing about the distribution of the spores, particularly in the lower Vasculares, in which it takes the place of the elaters found in many of the liverworts. The annulus is plainly an epidermal structure, both because it is actually the external layer of the sporangium and because its continuity is interrupted by the presence of stomata. These can be seen in the figure in profile view.

The situation in regard to the annulus in the Pteridophyta may be briefly summarized. In lower forms the thick-walled epidermal cells which serve as the mechanism for the opening of the sporangium are massive in their development, while in the higher forms of the vascular cryptogams the amount of mechanical tissue tends to become more and more restricted. Fig. I6 $a$ shows the structure of the sporangium and its annulus in Selaginella. The mechanical layer in this case is extensive and is almost coextensive with the surface of the spore sac. In contrast to the conditions shown in Selaginella are those presented by many of the ferns. In Fig. I6 16 is reproduced the organization of the sporangium of Polypodium vulgare as an illustration of the higher type of annulus in the Pteridophyta. The opening mechanism here constitutes an incomplete vertical ring and in consequence literally merits the name of annulus. The further consideration of the types of annulus 
presented by those forms included under the general heading of Pteridophyta need not occupy our attention in the present connection, important as these structures are from the standpoint of taxonomy and the evolution of particular groups.

Returning to the seed plants, we find that the Cycadales are the only living forms in which the organization of the mechanical tissues of the sporangium corresponds with that generally existing

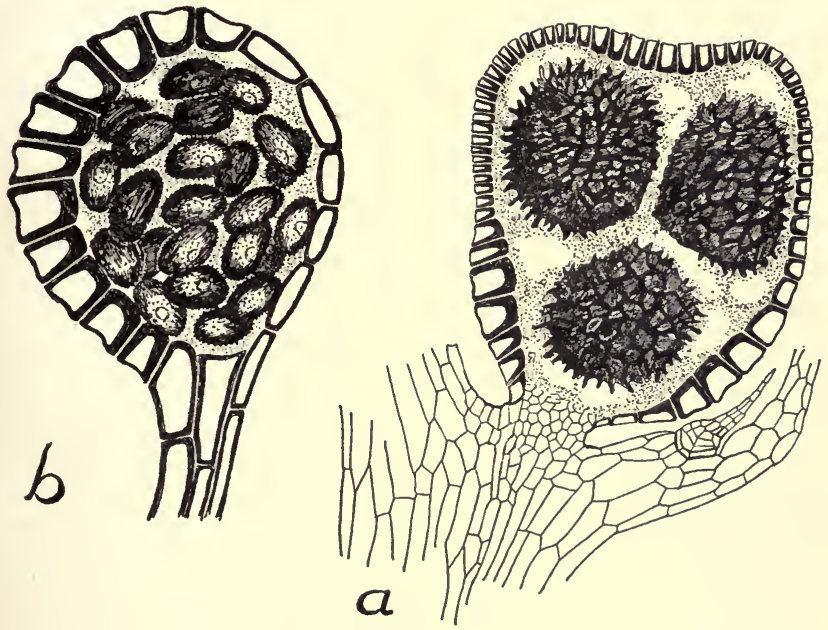

FIG. 161.-Sporangium of Selaginella spc. and of Polypodium vulgare

in the Pteridophyta; and in accordance with this general situation it will be made clear that the epidermis has not an important relation to the distribution of the microspores of plants producing seeds. The interesting genus Ginkgo will serve to illustrate advantageously the situation for the lower living seed plants. Fig. I62 shows the organization of one of the two sporangia of the microsporophyll of this genus as viewed in longitudinal vertical section. Clearly the cells of the epidermis are thin-walled and can perform no important office in the openings of the spore cavities. Beneath the epidermis is found a broad zone of cells provided with barred 
thickenings in their walls, resembling, in fact, short tracheids with reticulate thickenings. By following the mass of reticulate mechanical cells to the proximal end of the sporangium we find that they are continuous with, and pass by imperceptible transitions into, transfusion elements related to the fibrovascular bundles of the sporophyll. In Ginkgo it is evident that the opening mechanism of the sporangium is a derivative of the fibrovascular system, and

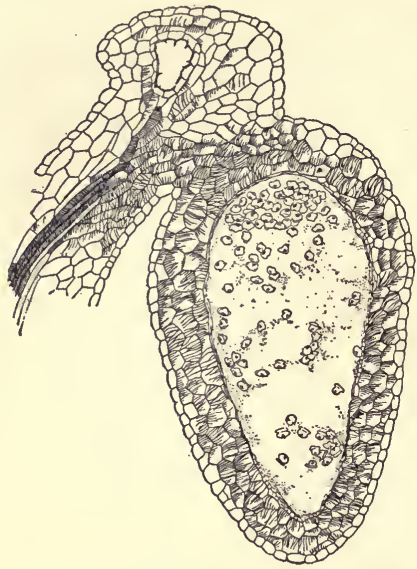

FIG. 162.-Sporangium of Ginkgo biloba does not take its origin from the cells of the epidermis, as is the case with the annulus of the Cycadales, and forms lower in the scale of vascular plants. The situation is so important in this respect that it is worthy of being given a special nomenclature. In those types in which the dehiscence depends on epidermal mechanisms, including the Pteridophyta and the very lowest seed plants, the term ectokinetic may be applied to the sporangium. On the other hand, in the long series of forms beginning with Ginkgo and ending with the monocotyledons, in which the opening mechanism is of internal origin and related to the fibrovascular system, the designation endokinetic definitely indicates the origin of the apparatus involved.

It is necessary to examine more in detail the conditions found in the walls of the sporangia of the forms above Ginkgo. In Fig. I63 is represented a longitudinal vertical section of the microsporangium in Pseudolarix Kaempferi, a representative of the Abietineae or pinelike conifers. Here the situation resembles in a general way that found in the case of Ginkgo, for the opening of the sporangial sac is due to the presence of reticulately thickened cells which are likewise related to the fibrovascular system of the trace of the 
microsporophyll. As in Ginkgo, the transition from the mechanical tissue to the tracheids of the fibrovascular bundle is effected by transfusion elements. An interesting difference between the situation found in Ginkgo and that illustrated in the Abietineae and other conifers is the fact that the mechanical elements invade the epidermis in Pseudolarix, but fail to do so in the more primitive genus. In other members of the Abietineae, particularly where the wall of the sporangium is very thin, the mechanical tissue becomes correspondingly reduced in amount and no longer shows any clear relation to the fibrovascular system. In the subtribes of Coniferales above the Abietineae this condition, in fact, becomes the rule, and so abortive does the mechanical

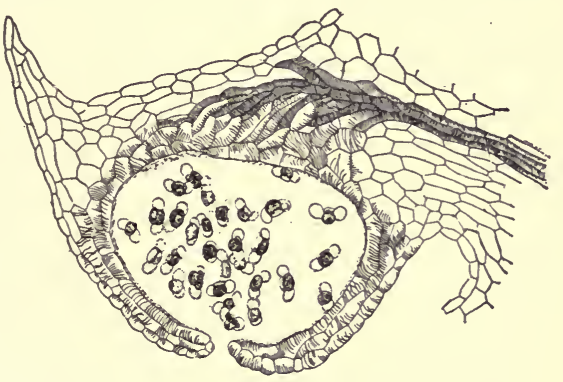

FIG. 163.-Sporangium of Pseudolarix Kaempferi layer become that it is represented by the reticulately thickened cells of the epidermis alone. It thus results that the opening of the sporangial cavity is once more effected by superficial cells, but the situation here represented should be carefully distinguished from that in the Pteridophyta and Cycadales, since it is the result of the invasion of the epidermis by mechanical tissues of fibrovascular origin. Subsequently, when the dehiscing mechanism was reduced, the epidermis once more became secondarily the seat of the opening device. It is not without significance in this connection that the araucarian conifers, which are often regarded as the lowest, present the sporangial arrangements of the mass of conifers in which the dehiscing mechanism is reduced to its lowest terms, and not that luxuriant and apparently primitive condition exemplified by Ginkgo and the Abietineae.

In the Gnetales the small sporangia are not characterized by the presence of a very well-developed opening device. The situation 
in the case of the angiosperms, however, is different. In Fig. 164, showing transverse sections of the anthers of a tulip and a honeysuckle, the mechanical tissues are clearly differentiated and occupy an entirely internal position precisely as in Ginkgo. There is a very important difference between the situation presented by the angiosperms, whether dicotyledons or monocotyledons, and Ginkgo and the Abietineae. In the higher group the mechanical tissues, constituting the so-called fiber layer of the anther
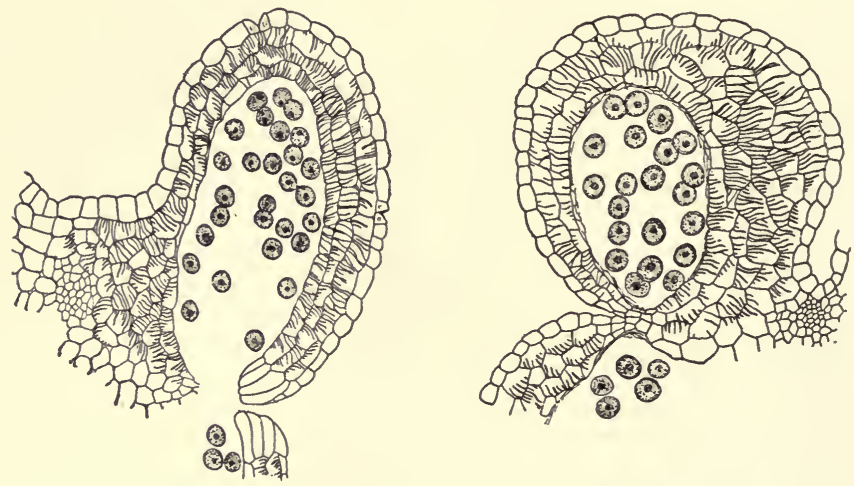

Fig. I64.-Sporangia of tulip and honeysuckle

wall, have no longer any relation to the fibrovascular bundles of the filament. In the case of the angiosperms the relation once existing between the fibrovascular system and the opening mechanism has apparently been lost. The dehiscing apparatus is, however, still in a good state of development and in this respect contrasts to the situation presented by the Gnetales and the higher Coniferales.

The structure of the walls of the microsporangia of the vascular plants from Ginkgo upward is highly interesting from the standpoint of the doctrine of descent. In the lower members of this series the opening device of the sporangium is clearly in relation to the transfusion tissue connected with the fibrovascular bundles of the reproductive leaves. In the Cycadales, the lowest living seed 
plants, we find a complete absence of typical transfusion tissue in the leaves, although its presence has been erroneously described for the group. The cycadean gymnosperms in the absence of foliar transfusion tissue resemble the true ferns, which are also characterized by the exclusion of tracheary tissues belonging to this category from their foliar organs, whether vegetative or reproductive. It is highly significant that an epidermal sporangial mechanism and the absence of transfusion tissue are features which alike mark the Pteridophyta and the seed plants most nearly allied to these. Beginning with the Ginkgoales and proceeding upward, we find transfusion tissue progressively taking the place of the centripetal or cryptogamic wood in the vegetative leaves; and in the reproductive leaves the transfusion tissues or structures definitely associated with them assume the function of opening the sporangium at the time of the ripening of the spores. The correctness of this interpretation of the situation is best seen in Ginkgo, in which in the lower region of the sporophyll the transfusion tissues are developed very much after the manner in which they present themselves in the case of the vegetative leaves. In the upper region of the sporophylls, bearing the microsporangia, the transfusion elements grade imperceptibly into the reticulately thickened mechanical tissues of the sporangial walls. In the subtribe of the conifers which is beginning to assume importance as a candidate for the most primitive phylogenetic position in the group (namely, the Abietineae), we find the transfusion zone not only well developed in the vegetative leaves of both living and fossil representatives, but likewise occurring under highly significant conditions in relation to the sporangial mechanisms. In the remaining gymnosperms the mechanical tissue shows a strong tendency to become reduced in amount and loses all direct relationship to the fibrovascular tissues proper. In the angiosperms, as has been pointed out above, the fiber layer characteristically present in the anther wall is well developed, but no longer has any relation whatsoever to the fibrovascular system.

In conclusion, it may be stated that the opening mechanism of the sporangia of the Pteridophyta and of the lowest gymnosperms is epidermal in its origin, while that of the seed plants from Ginkgo 
upward is clearly derived from transfusion tissue. This category of tissue is the final stage of persistence of the protean centripetal or cryptogamic wood of the lowest vascular plants. In the angiosperms the mechanical tissues in the walls of the anthers exemplify the highest level of survival of the old centripetal wood of the Pteridophyta and the lowest gymnosperms. In fact, the socalled fiber layer of the anther in the case of the angiosperms supplies the clearest instance of the persistence of this ancestral structure outside of that most conservative of all organs, the root, in which, as has been made clear in an earlier chapter, it still maintains its pristine development in the primary organization. The two mechanisms correlated with sporangial dehiscence previously described may appropriately be designated as ectokinetic and endokinetic. 


\section{CHAPTER XVI}

\section{THE MEGASPORANGIUM AND SEED}

In the Pteridophyta the phenomenon of heterospory has developed in many different groups. The result of the realization of this condition has been the appearance of smaller sporangia producing numerous small spores known as microspores and of larger ones giving rise to a few large spores designated as megaspores or macrospores. In the case of the sporangia which give rise to megaspores, or the megasporangia, the conditions connected with opening are the same as those exhibited by the mass of Pteridophyta; in other words, the spore sacs are ectokinetic and owe their dehiscence to the activity of a mechanical layer derived from the epidermis. As this situation has been sufficiently discussed in the previous chapter, it will not be profitable to return to the matter here. Megasporangia in the proper sense of the word, wherever they occur, are ectokinetic.

It has been recognized since the times of the great German morphologist Hofmeister that seeds represent modified megasporangia. This view of the origin of seeds is justified, not only by their anatomical structure and by the cytological conditions observed in the development of the endosperm, but also by the actual persistence of the megaspore membrane in the seeds of many of the lower gymnosperms. If any remaining doubt existed as to the origin of seeds from megasporangia, it would be removed by the discovery of certain interesting structures in the case of Paleozoic lycopods which present at the same time many of the distinctive characteristics of megasporangia and seeds. So strikingly does one of these (described by Scott) resemble a seed in its external appearance that it was for a time actually regarded as one, until its internal organization revealed its anomalous character. Fig. I65 illustrates the vertical section of Lepidocarpon Lomaxi, the seedlike fructification of a lepidodendrid. Internally is shown a mass of cells, the gametophyte, surrounded by a heavy dark line, 
the section of the megaspore membrane. In addition to the germinated megaspore shown in the figure, three other abortive megaspores make their appearance at an earlier stage, and these have thicker walls than the functional spore. These are not shown in the late stage of development appearing in the figure. Not only does the megaspore retain its thick membrane in the fructification of Lepidocarpon, but, contrary to the conditions found in typical

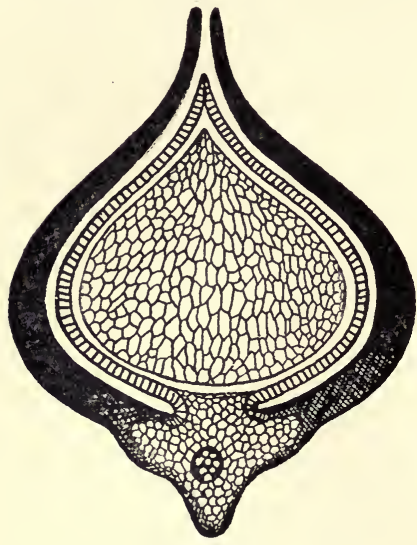

Fig. I65.-Seedlike sporangium of Lepidocarpon (after Scott). seeds, the mechanical layer of the megasporangium likewise is well developed and was probably capable of dehiscence. The integument with which true seeds are provided is represented in this foreshadowing of seminal structure by the upfolded edges of the sporophyll. The absence of an apparatus for receiving the microspores or pollen likewise differentiates the fructification under consideration from the seeds of even the lowest of the seed plants.

In Fig. 166 is shown the longitudinal view of another seedlike structure from the Carboniferous of England known as Miadesmia. Here the resemblance to the real seed is much more marked than in Lepidocarpon. The sporophyll so completely involves the megasporangium that only a small aperture is left which corresponds physiologically, although not morphologically, to the micropyle of the seed in the true seed plants. Within the "integument" is inclosed, not only the megasporangium, but also the ligule. The megasporangium is much less typical than that of Lepidocarpon, for its mechanical layer fails to develop and it produces only one spore in contrast to the four that come into existence in the case of the Lepidocarpon. There is no good reason, however, to regard the structure here described as representing a 
true seed any more than that delineated in connection with the last paragraph.

An interesting condition has been described by Miss Lyon in the American species of Selaginella, S. apus, and S. rupestris. Here the spores germinate and are fertilized within the megasporangia, a condition favored by the fact that the microsporangia are situated in the upper region of the cones. The microspores undergo development unshed, and on a wet day the antherozoids to which they give rise are able to make their way to the lower region of the strobilus, where the germinated megaspores present their archegonia for fertilization. The development of the embryo takes place after the union of the sexual ele-

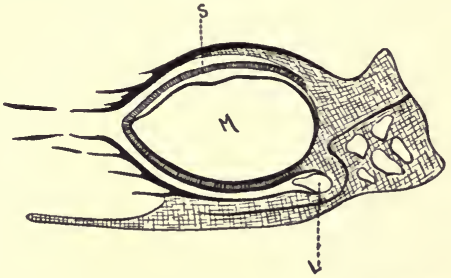

Fig. 166 - Seedlike sporangium of Miadesmia (from Seward, after Scott). ments, and the sporelings later grow out among the leaves of the cone. The situation represented by the two species mentioned appears to be somewhat general for the genus and throws an interesting light on the conditions which were probably present in Lepidocarpon and Miadesmia.

The most ancient types of seeds known to us have an organization differing in important particulars from the seedlike structures described in the two preceding paragraphs. In the first place, the most antique seeds are provided with a true integument and are not merely wrapped in the sporophyll as a whole. Secondly, they present a very important feature in the presence of a so-called "pollen chamber" which receives the microspores and provides a fluid in which they may undergo germination and later effect fertilization.

A primitive type of seed is diagrammatically represented in Fig. 167. The megasporangium appears within the integument which covers it almost completely, so that communication with the outer world is only by a narrow canal at the apex known as the micropyle. The megasporangium is without any mechanical layer 
such as appears in the seedlike structures described above for the lycopods. Such a layer was doubtless originally present, but has ceased to be necessary as a protection on account of the shelter afforded by the integument; moreover, it could no longer be func-

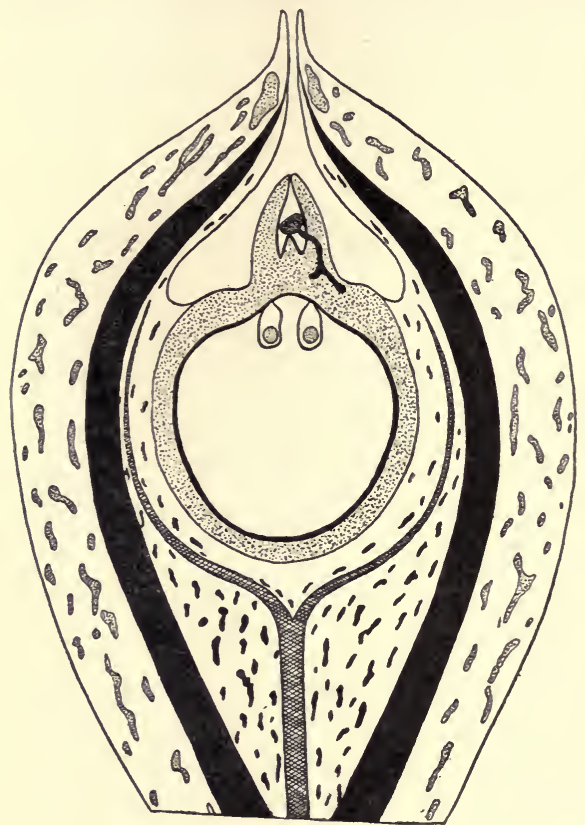

FIG. 167.-Diagram of seed of a cycad tionally useful in opening the sporangium since the megaspores in the case of true seeds are permanently inclosed. The elimination of the ectokinetic mechanical layer of the older seeds must lie far in the geological past, because no evidence of its presence has been observed in the oldest seeds with the structure of which we are acquainted. The pointed apex of the megasporangium is occupied by a cavity, the pollen chamber, in which the pollen grains or microspores come to rest before germination. This cavity has its capacity much reduced by the presence of a central column known as the columella. Below the pollen chamber lies the germinated megaspore with its somewhat thickened megaspore membrane. The membrane incloses the gametophyte, bearing the archegonia in its upper region. Surrounding the part of the seed corresponding to the megasporangium and fused with it, except in the upper region, is the integument, and this consists 
of a hard inner layer known as the sclerotesta and a softer outer one which contains mucilage canals and to which the name of sarcotesta is applied. Both sclerotesta and sarcotesta are provided with a system of fibrovascular strands, but tracheary elements of any kind are absent in the region of the nucellus or megasporangium.

The type of seed delineated in connection with the foregoing paragraph is not the only one characteristic of more ancient plants. In Fig. I68 is shown another category of seed which, although presenting the general features of the Paleozoic type, is characterized by certain interesting and important peculiarities. The integument in the diagram is repre-

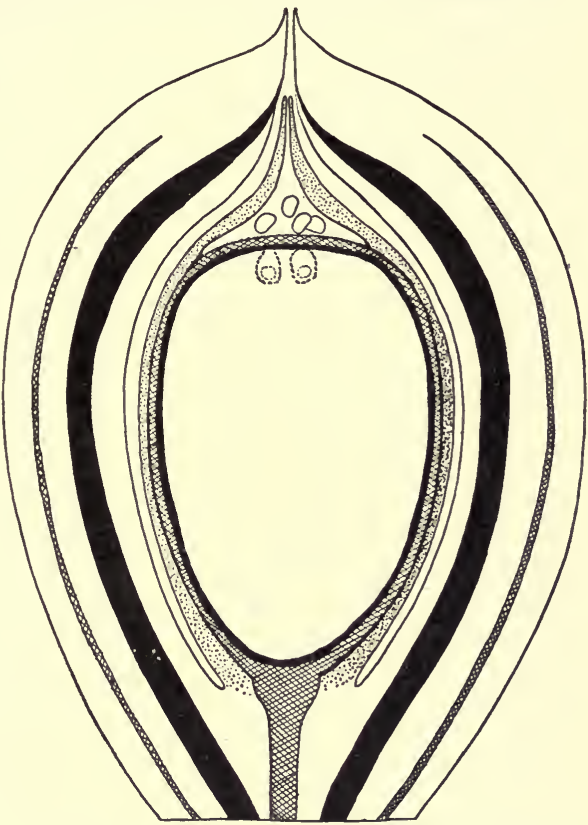

FIG. 168.-Diagram of an ancient type of seed with tracheary mantle surrounding the gametophyte (modified after Oliver).

sented as consisting of an outer softer sarcotesta and an inner resistant sclerotesta. It incloses, as in the other type, the megasporangium or nucellus, and this is likewise provided with a pollen chamber. The only important difference between the seed under discussion and that described in the preceding paragraph is the distribution of the fibrovascular bundles. In the seeds of the first type the tracheary strands are present in both 
sarcotesta and sclerotesta, but are lacking in the nucellus or megasporangium. In the seed now under consideration a fibrovascular envelope surrounds the megaspore and ends upwardly in the pollen chamber. It is clear in the present instance that the tracheary tissues invade the megasporangium precisely as they do the microsporangium of Ginkgo and seed plants higher in the evolutionary scale. The function of the tracheary tissue which ends under the pollen chamber doubtless was that of supplying water to facilitate the germination of the microspores, and the fertilizing movements of the antherozoids originated from these. It seems clear that we have in the type of seed 'figured in connection with the present paragraph a counterpart to microsporangia with the endokinetic mode of dehiscence. A number of ancient seeds of the anatomical organization indicated here have been investigated, but unfortunately they have not been connected with absolute certainty with any definite vegetative types.

The seed of the living Ginkgo throws no light upon the question of the affinities of the second type of Paleozoic seeds, for, although tracheary tissues are abundant in the base of the seed, they do not penetrate into the megasporangium proper. It is likely that degeneracy of the fibrovascular structures has here obscured the real situation, since from the organization of the microsporangium in the genus we should expect to discover tracheary elements in the walls of the nucellus or megasporangium.

In the seeds of the Cycadales we have realized the general features of organization depicted in Fig. I67, which is, in fact, modeled from the young seed of Cycas revoluta. The organization of the microsporangium in the cycads does not support the hypothesis that nucellar tracheids were once present in the seed of the group and have disappeared in its modern representatives. The conservative tendencies of microsporangial structures as contrasted to those of the megasporangium or seed which is considerably more rapidly influenced by the course of evolution constitute a valuable situation from the standpoint of comparative morphology.

The general anatomical features of the seed in the higher gymnosperms may next be considered. In Fig. $6_{9}$ is illustrated the more 
important structures of the seed in one of the Abietineae or pine family. It is provided with an integument in which a small pore known as the micropyle is present at the apex of the seed. Within the integument is accommodated the nucellus or megasporangial portion of the seed. This is not characterized, as is that of the older and fernlike gymnosperms, by the presence of a pollen chamber. The pollen grains or microspores are received, in fact, on the smooth apex of the nucellus and very soon send out pollen tubes which bore their way through the tissues of the nucellus in order to reach the archegonia, situated on the apex of the gametophyte. The absence of the pollen chamber and the presence of functional pollen tubes are features which most clearly distinguish the seeds of the modern gymnosperms from those of the lower largely extinct types included under the heading of the Archigymnospermae. The nucellus is not provided with a tracheary mantle, such as is found in the ovular structures of certain extinct gymnosperms. The soft sarcotesta often presented by more ancient types of seeds is

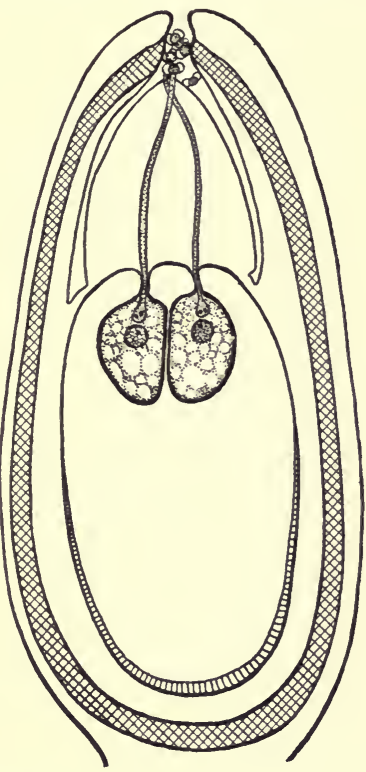

FIG. I69.-Diagram of seed of a conifer likewise conspicuous by its absence in the case of the more typical representatives of the higher gymnosperms.

In the angiosperms the situation in regard to the seed is still further modified by the fact that the pollen is no longer received on the apex of the ovule or young seed, but comes to rest on a special region of the closed sporophyll known as the stigma (Fig. I70). The microspores or pollen in germinating send out pollen tubes, of greater or less length, which penetrate first the tissues of the 
closed reproductive leaf or megasporophyll, and later enter the ovule, either through the micropyle (in porogamous forms) or through the chalaza or breech of the seed (a condition found in the so-called chalazogamous dicotyledons). Not only do inclosure of the ovules

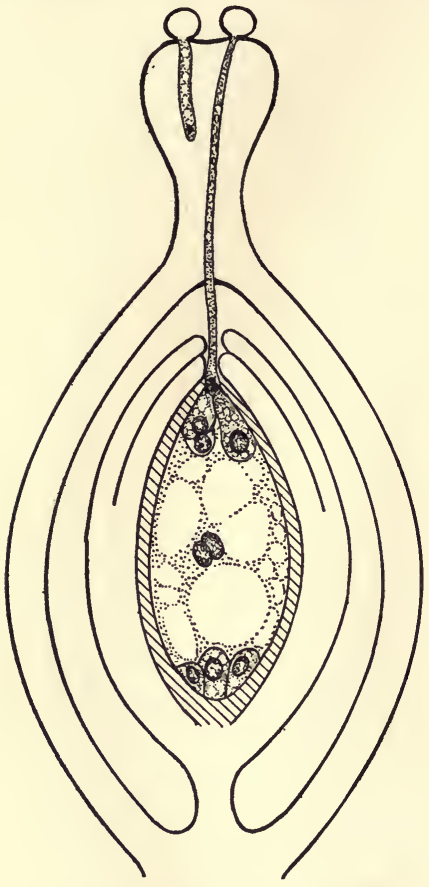

Fig. 170.-Diagram of a porogamous dicotyledon.

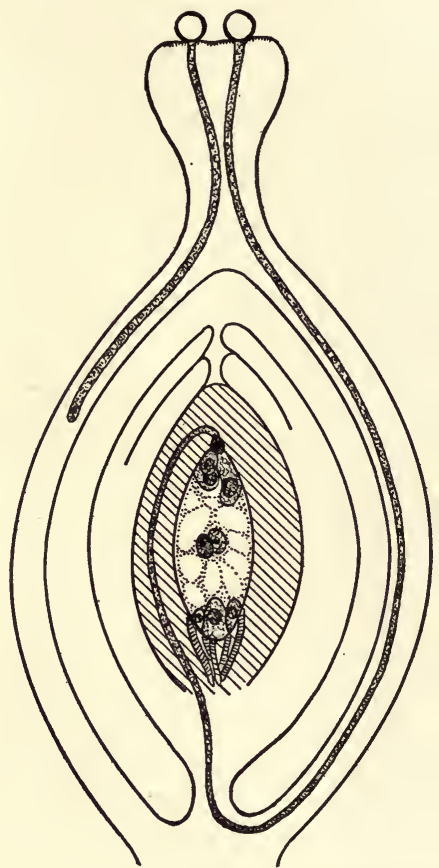

FIG. I71.-Diagram of a chalazogamous dicotyledon.

in an ovary and the consequent exclusion of the pollen from direct access distinguish the angiospermous seed from that found in lower forms, but likewise the very considerable reduction in the amount of gametophytic tissue. In this large group of seed plants the prothallial portion of the young seed contains typically eight 
nuclei, which by the fusion of two become seven. Of these, one nucleus surrounds itself with a protoplasmic body to become the egg, while two others are related to the so-called synergidae. Of the remaining four nuclear structures, three belong to the antipodals, a group of cells present in the base of the embryo sac or prothallus, while the fourth, the product of the fusion of two nuclei as mentioned above, becomes the so-called endosperm nucleus, which later develops the endosperm or food substance of the ripened seed. In certain of the chalazogamous angiosperms (Fig. I7I), notably Casuarina and the hazel (Corylus), tracheids are found present in the nucellus. The most natural interpretation of this condition is in connection with the tracheary apparatus in certain extinct seeds described in a foregoing paragraph. If the presence of tracheary tissues in the substance of the megasporangium or nucellus in certain angiosperms is to be interpreted as the persistence of an ancestral character, it would indicate a relatively primitive position for the chalazogamous forms in which it occurs.

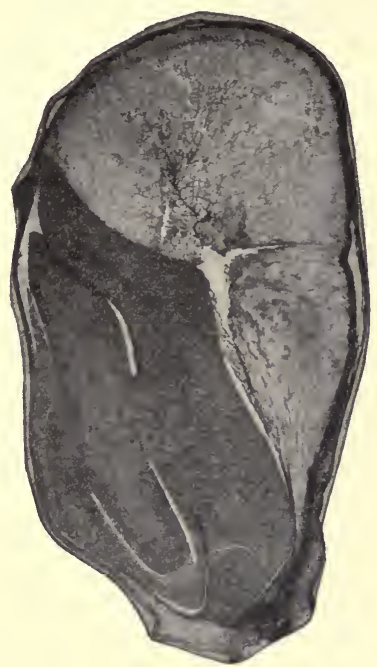

Fig. 172.-Seed of Zea mais

The structure of the mature seed in the angiosperms naturally claims a greater interest in a work devoted to anatomy. We may first take the cases of dicotyledons and monocotyledons. Fig. I72 illustrates the organization of the seminal organ of the Indian corn $(Z e a)$. To one side lies the embryo, which is provided with a single seminal leaf or cotyledon. This is very large in size and has its inner surface applied broadly to the food substance of the seed or endosperm. The embryo proper lies to the outside of the cotyledon and is characterized by the presence of the primary shoot, or plumule, and the primary root, or radicle. These are inclosed 
in protective sheaths respectively known as coleoptyle and coleorhiza. The endosperm and embryo are inclosed by a covering composed of the fused wall of the ovary and the integuments of the ovule. On the flatter side of the seed toward which the embryo

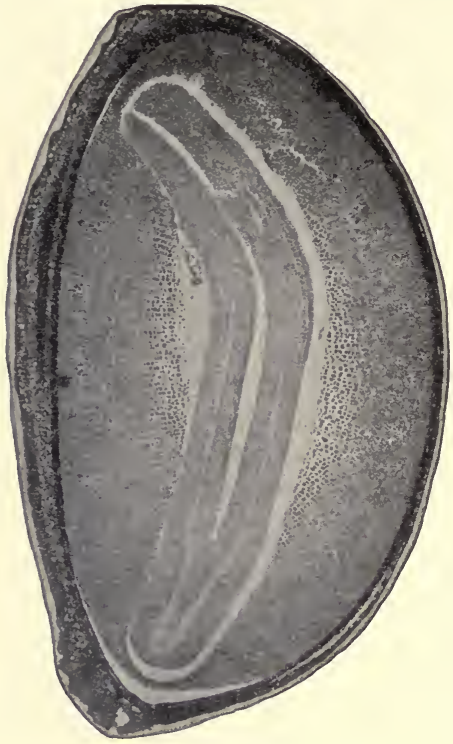

Fig. 173.-Seed of Celastrus scandens

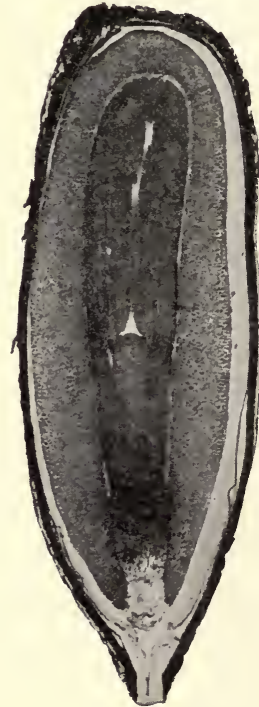

Fig. I74.-Seed of Pinus palustris.

is placed a more or less prominent elevation is seen which is the base of the style.

Fig. I73 is a photomicrograph of a dicotyledonous seed. Here the embryo occupies a median position and is provided with two cotyledons instead of a single one. The endosperm or food substance, as a result of the position of the embryo, entirely surrounds the rudiment of the young plant. In the seed illustrated the coat consists of the hardened integument, and the wall of the ovary is not involved in the formation of the seminal covering. 
The typical condition, of course, for both monocotyledons and dicotyledons so far as the coat or coats of the seed are concerned is that shown in Fig. I73, since only rarely does the wall of the ovary participate in the formation of the protective envelope.

Certain other varieties are presented by the organization of the seeds of angiosperms. For example, the endosperm or food substance may be absent altogether, a condition illustrated by the legumes and the Compositae among the dicotyledons and by the Orchidaceae among the monocotyledons. Again, the nucellar or megasporangial substance, usually absorbed as the development of the endosperm and embryo proceeds, sometimes persists and is then known as perisperm. Another variation which may present itself is the development of a supernumerary integument, often brightly colored, after the seed has been fertilized. This subsidiary coat is known as the arillus and is frequently found in families not nearly related systematically.

The organization of the seed in the pine is presented in Fig. 174 for comparison with the angiospermous conditions illustrated in Fig. I73. The integument is clearly distinguished as a hard investment surrounding the abundant endosperm. The food substance in the seeds of the gymnosperms is derived directly from the transformation of the gametophyte and is not a new structure, as is the case with the endosperm of angiospermous seeds. Within the substance of the endosperm lies the embryo, with its narrower end toward the micropylar region of the integument. The more slender portion of the embryo or young sporophyte is the primary root, which is capped with more or less lax tissues. These are the remains of the suspensors which in the developing seminal organ forced the young embryo down into the midst of the endosperm. The larger end of the embryonic sporophyte owes its breadth to the presence of numerous cotyledons or seed leaves, and these distinguish the embryo of the pine from those of the angiosperms. 


\section{CHAPTER XVII}

\section{THE CANONS OF COMPARATIVE ANATOMY}

With the completion of the consideration of the various tissues and organs we are in a position to take up the relation of anatomical structure to evolutionary sequence in the various groups of vascular plants. Before we proceed to this phase of the subject, however, it will be necessary to consider the general principles or canons of comparative anatomy. It may be pointed out in this connection that anatomy, in common with other branches of the sciences, is based on inductive reasoning. The general principles are consequently arrived at as the result of the consideration of large numbers of facts with particular regard to the conclusions which may be drawn from them. The anatomy of plants has made great progress in recent years and in direct proportion to our increasing knowledge of fossil forms. The most interesting and valuable results from the evolutionary standpoint have been reached in connection with the anatomical investigation of extinct organisms of earlier geological ages. Naturally those of the great coal-producing period, the Paleozoic, first received attention on account of the importance of the study of plants of that age in connection with the search for productive coal seams. In more recent years the Mesozoic, which is of the greatest interest in relation to the appearance of our modern types, has begun to be studied. The results bearing on the advancement of our knowledge of the general principles of the evolution of plants exemplified in their anatomical structure cannot be too highly estimated. It will be the aim of the present chapter to set forth comprehensively the main conclusions of anatomical paleobotany in their relation to the interpretation of the affinities of the main groups of vascular plants now in existence on the earth.

THE DOCTRINE OF RECAPITULATION

An important general doctrine developed in connection with the evolutionary study of living beings is the hypothesis of recapitu- 
lation. It is assumed in connection with this doctrine that the young of any species may in the course of its individual development pass through the phases present in ancestral forms. As examples of this principle we may take extreme types of vegetation, such as the almost leafless cacti, forms with phylloclads, or those characterized by the presence of short-shoots. In the mass of Cactaceae the leaves are abortive and are represented at most by spines. In the seedlings, in contrast to the adult, the foliar organs are distinctly present and are clearly recognizable as such. In coniferous species belonging to the genus Phyllocladus the branches in the mature state form flattened expansions known as phylloclads. If a seedling of any species of Phyllocladus be examined, it becomes clear that a normal round and leafy axis is present such as ordinarily characterizes the conifer (Fig. I75). Likewise in the pine the seedling shows the primary leaves arranged on the stem in the usual fashion for
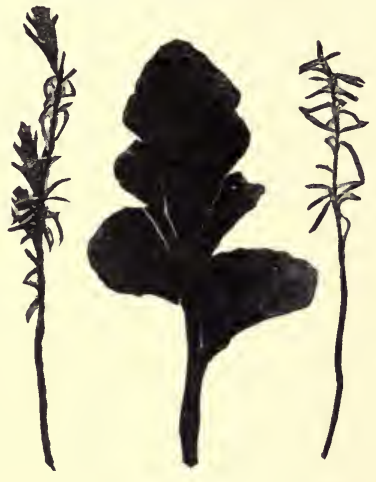

FIG. 175.-Seedlings and mature branch of Phyllocladus species. coniferous gymnosperms and not clustered on short-shoots or brachyblasts as in the adult branches of the genus. Further, in a conifer like the larch, which is differentiated in habit from the mass of the group by its deciduous foliage, we find in the seedling that the leaves persist for several years, thus revealing the probable ancestral condition for the genus. An additional example among the dicotyledons is supplied by the oak. The adult in northern oaks is characterized by deciduous leaves. Oak seedlings and saplings, however, even in the case of typically northern species, retain their leaves during the winter, thus recalling a situation characteristic of the live oaks of warmer latitudes which have evergreen foliage and represent anatomically the primitive type of organization. 
The phenomenon of recapitulation is not confined, however, to external features of organization, for it is often equally well exemplified by internal anatomical structure. A good illustration of the principle of recapitulation is presented by the seedling of the araucarian conifers. The adult stem of the kauri, or of any other araucarian conifer, is characterized by two unique features. One of these is the persistence of the traces belonging to the leaves long after the foliar organs have fallen. The foliar fibrovascular strands are continued for many years, amounting in some cases to centuries, through the activity of the cambium, even when the surface of the trunk has long ceased to show even the scars of the leaves of which they were once the fibrovascular supply. Further, in araucarian woods there is present a peculiar variety of tracheary structure which clearly differentiates their ligneous organization from that of all other living subtribes of conifers. The tracheids in the Araucariineae have their bordered pits arranged in an alternating manner and not disposed in an opposite fashion, as is the characteristic condition in the rest of the living conifers. In araucarian woods of the Mesozoic belonging to the genus Brachyoxylon the leaf traces persist only for a short time and are no longer formed through the instrumentality of the cambium after the leaves to which they belong have fallen from the stem. Again, the pits do not manifest the alternating and crowded condition presented by the wood of the living genera. In the seedling of both Agathis and Araucaria the leaf trace persists only so long as it is related to a functional leaf, and does not continue to develop for many years after the fall of the foliar organs, as is the case in the older trunk. Also in the araucarian seedling the pitting is like that found in the Cretaceous araucarian genus Brachyoxylon. In this instance we have a striking exemplification of the principle of recapitulation.

The law or principle under consideration has many illustrations in the vascular plants, but on the whole it cannot be said to have so great a validity as among the higher animals. It is further necessary to note in the present connection that the absence of a given structure in young individuals is by no means evidence of its absence in the ancestral forms from which they have been derived. For example, there is good evidence that the cycadean 
gymnosperms have come from ancestors possessing concentric bundles and centripetal wood, yet the seedlings of cycads in general do not support this conclusion by their anatomical organization. The doctrine of recapitulation is of value, accordingly, when it presents positive evidence from the seedling for the ancestral occurrence of a given feature of organization; but negative testimony from this standpoint must be estimated as having little or no value. A failure to realize this situation is responsible for much fallacious biological reasoning.

A very important exemplification of recapitulation is frequently supplied by the first annual ring of the older stem of arboreal forms. Often in groups which have suffered considerable reduction, such as, for example, the gymnosperms in general, the phenomenon of recapitulation, although absent in the seedling, may be clearly illustrated by the first annual increment of woody growth in the older regions of the stem. An illustration of this principle is supplied by the living araucarian conifers. Taking as an example the genus Agathis, the kauri of Australasia and the East Indian region, we find in the first annual ring an organization distinctly different from that in the subsequent annual increments of the wood. More or less abundant wood parenchyma is present, although longitudinal storage elements are conspicuous by their absence in the adult wood of the stem. This situation is of great interest in view of the fact that the fossil wood of the kauri from American Cretaceous deposits is characterized by the presence of parenchymatous cells, not only in the first annual ring, but in all subsequent zones of ligneous growth. The persistence of the structure of Mesozoic forms in the first annual ring of living species of the genus Agathis is a feature most appropriately falling under the principle of recapitulation. The situation here indicated is of great value and wide validity, not only for the gymnosperms, but also for the dicotyledons. It might readily be much more abundantly exemplified in the present connection, but the instance supplied above will serve to make the situation clear. Many other cases will present themselves in later chapters in connection with the discussion of the evolution of the different groups as inferred from their anatomical organization. 


\section{THE DOCTRINE OF CONSERVATIVE ORGANS}

This doctrine has received a great impetus from the study of Mesozoic conifers, but was first put forward, naturally enough, in connection with comparisons between the older existing gymnosperms and their Paleozoic ancestors. The leaf first came into prominence in relation to the hypothesis of conservative organs. It has been known for many years, particularly since the investigations of Mettenius, that the foliar organs of the Cycadales present remarkable features of anatomical structure. Here the fibrovascular bundles of the leaves are distinguished by the presence of centripetal or cryptogamic wood, a detail of organization conspicuously absent in the stem of the genera of the living Cycadales. In the Cycadofilicales of the Paleozoic the bundles of the stem were always characterized by the presence of centripetal xylem and sometimes by concentric organization as well. The Cycadofilicales are by common and competent consent regarded as the ancestral types from which the living cycads have been derived. The clear and universal presence of centripetal wood in the foliar fibrovascular bundles of living genera of the Cycadales is good evidence at once of the relationship of these gymnosperms to the Cycadofilicales and of the validity of the doctrine of conservative organs so far as it applies to the anatomy of leaves. Many other illustrations of the prevalence of this principle might be supplied in foliar organs, but the one described above will serve appropriately to elucidate the situation and is particularly apposite in the present connection because it is probably the first case to be cited in evolutionary anatomy.

The foliar organs are not, however, the only parts of the adult plant which present illustrations of the principle of conservative organs. The stem in that region devoted to the function of reproduction has also figured strikingly in this connection. To Scott belongs the credit of having drawn attention to the fact that the peduncle or base of the cone in certain Cycadales furnishes a clear example of the persistence of an ancestral structure in the reproductive axis which has quite disappeared in the ordinary vegetative stem. In Stangeria, Zamia, and other genera of the Cycadales he noted that the fibrovascular strands of the axes 
of the cones frequently manifested the presence of vestiges of centripetal wood, although xylem belonging to this category has wholly disappeared in the vegetative axis. The value of this generalization can scarcely be overestimated. During the interval of nearly twenty years dating from Scott's brilliant discovery, much additional evidence has been supplied in support of the conservatism of the anatomical structure of the reproductive axes. For example, it has been shown by comparison of the vegetative organization of Mesozoic conifers with the anatomical structures found in the ovuliferous cones of living forms that the latter frequently perpetuate features which have vanished in the vegetative parts. This is manifestly the case in the abietineous and araucarian conifers which compete with each other for the claim of being the most ancient representative of the coniferous stock. The cone in both Pinus and Agathis presents numerous Mesozoic features which have disappeared elsewhere in the stem organs of existing species belonging to these genera.

The value of the anatomy of reproductive axes cannot be estimated so highly in the case of the angiosperms, since the relatively slight development of fibrovascular structures in flowers and inflorescences leaves less scope for the appearance of phylogenetically significant structures. This situation needs to be particularly emphasized in view of some recent highly fallacious attempts to utilize the anatomy of reproductive axes in working out evolutionary sequences in the woody dicotyledons. Clearly in this instance only structures are significant which find adequate development in the somewhat slender annual woody cylinder of the flowering parts of perennial dicotyledons. In the monocotyledons the restrictions on interpretation are still greater on account of the usual absence of secondary growth in this great division of the angiosperms. In the application of the doctrine of conservative parts to the reproductive axes of the angiosperms it must be recognized that a little knowledge is a dangerous thing.

It has been made clear in the preceding paragraphs that the doctrine of conservative parts is well exemplified by the leaf and is manifested in the stem by the more archaic axes connected with reproduction. The situation in the organs cited has for a long time 
been apparent. Of more recent origin is the realization that, in modern plants at any rate, the root is the most valuable of all organs from the standpoint of evolutionary anatomy. In the case of comparisons between Paleozoic and Mesozoic groups the root, in fact, has proved to be in general too conservative to furnish significant examples of the retention of ancestral characters. The search for centripetal or cryptogamic wood has been in the foreground in investigations bearing on the relationship between Paleozoic and Mesozoic groups. Since in all forms of every geological age the root has centripetal primary wood, in regard to this crucial feature of more ancient types it obviously does not supply distinctive evidence in connection with the doctrine of descent. In other respects, however, and particularly with reference to the organization of the tissues of the secondary wood, the root has proved itself to be of greater significance than any other organ of the higher plants. Of course the most striking evidence of conservatism in the root has resulted from the comparison of Mesozoic and modern forms. It has, for example, been shown that the root in modern conifers clearly and persistently perpetuates the features of organization which characterized the structure of the stem in the Cretaceous and earlier periods of the Mesozoic age. In Pinus and Agathis the root presents in many of its earlier annual rings the distinctive organization found in the stem of these types in the Mesozoic. Nor is the inherent conservatism of the root of value in the study of the gymnosperms alone. Although our knowledge of the anatomical structures of the angiosperms in Mesozoic times is as yet extremely inadequate, we are able in many instances by the application of the doctrine of the conservatism of the anatomical organization of the root to infer the ancestral type of stem in the highest vascular plants.

It will be evident from the foregoing paragraphs that conservatism is particularly inherent in the leaf and root of vascular plants and that the highly progressive stem presents only features which are of interest from the standpoint of the doctrine of descent, either in its first annual ring or in axes specially allocated to the function of reproduction. The sporangium is a fourth structure recognized as a primitive organ of vascular plants in an earlier chapter of the 
present work. Here the lack of complexity of organization rather militates against the presence of phylogenetically important structures. It must nevertheless be noted that the sporangium is an extremely conservative organ and, as far as the relative simplicity of its organization supplies points of comparison, is of very great significance for the doctrine of evolution. It has been demonstrated in an earlier chapter that the sporangium perpetuates the protean centripetal xylem in the form of its opening mechanism to a higher point than any other organ but the root. The value of the spore sac in phylogeny, although limited by the relative simplicity of the organ, must consequently be estimated as great.

The doctrine of conservative organs is of the greatest significance in connection with the study of the evolutionary history of plants, because of the abundance and reliability of the evidence which the various parts furnish in this connection. Obviously, if leaf, reproductive axis, root, and sporangium all supply consonant and harmonious testimony in the same direction, a sound conclusion must inevitably be reached. There can be little question that the doctrine of conservative organs is the most important one which modern inductive anatomy has supplied as a tool of evolutionary investigation. In fact, the general principles included under this head are of such great significance that the present volume may be considered as written only for those whose anatomical training has progressed to such a point that they are able to appreciate the universal value and validity of the doctrine here discussed.

\section{THE DOCTRINE OF REVERSION}

This doctrine is well shown in the case of plants with a considerable amount of secondary growth-namely, the gymnosperms and the dicotyledonous angiosperms. It is of little value in herbaceous forms, whether cryptogamic or phanerogamic, since in these the effects of injury are usually in the direction of degeneracy only. In plants with secondary growth and consequently more massive organs the effect of injuries frequently is to recall ancestral features of organization. This phenomenon is called reversion. Only in certain conifers can we observe the effect of injury in recalling in living forms features which are known to have been 
present in their Mesozoic forebears. For example, in the araucarian conifers, cited earlier in another connection, the Mesozoic organization of the wood is recalled in the stem of the existing species as a consequence of injury. The wood formed subsequently to the infliction of the injury shows a type of structure characteristic of the group in an earlier geological epoch. Although only in rare instances can structures which are actually known to have been present in the past be recalled by injuries, in many other cases we find the infliction of wounds followed by the formation of features which are normally present in the conservative organs. Consequently the doctrine of reversion finds support, not only in the facts of paleobotanical anatomy, but also in the much more richly exemplified doctrine of conservative parts. It is further clear that we cannot interpret all structural peculiarities which result from injury as reversions to a former condition of organization, but only those which are definitely paralleled by known conditions in fossil forms or are illustrated in the anatomy of the conservative organs - the leaf, reproductive axis, and stem, or at least some of these.

Carefully as the doctrines of recapitulation and of conservative parts must be applied to elucidate the course of evolution, the principle of reversion must be invoked in phylogenetic studies with even greater precautions. A wide knowledge of fossil forms as well as an extensive acquaintance with the facts of comparative anatomy are necessary for a successful application of this doctrine to the data derived from the investigation of injuries. In this connection it is further necessary to distinguish clearly between the phenomena of hypertrophy and those of actual reversion. For example, when the stem of a dicotyledon or of a conifer is injured, a local damming of food substances results as a consequence of the elimination of the conducting tissues of the phloem in the immediate vicinity of the wound. Overdevelopment consequent on overnutrition accordingly is locally most conspicuous. Very often true reversionary changes resulting from the impulse supplied by the injury present themselves only at some distance from the wound. This situation is found in the conifers in connection with reversionary conditions following injuries in the case of the rays 
which ordinarily manifest themselves, not in the actual wound cap or hypertrophied mass of wood formed after injury, but on the opposite side of the stem. Among the dicotyledons the birch exemplifies the same condition in regard to the formation of aggregate rays. The diffuse structure of radial parenchyma which characterizes the normal organization of the wood of the genus Betula gives way to a condition of aggregation following injury, not on the edges of the wound (that is, in the actual wound cap), but in the region of the axis diametrically opposite to the injury. In accordance with the general reversionary principles here stated, the wound cap as often as not presents structures in an advanced or accelerated, and not in a reversionary, condition. Some recent publications on the anatomy of the dicotyledons reveal a failure to realize this fundamental principle, and therefore it is well that it should be emphasized in the present connection.

The foregoing paragraphs elucidate the most important canons or principles of evolutionary anatomy. It eannot be too strongly urged that all the evidence available under the various principles here described and exemplified should .be brought into consideration, as a failure to check up one kind of evidence against another often results in a fallacious and ephemeral deduction. The most cogent testimony to the validity of any evolutionary conclusion is naturally supplied by the conditions actually realized in ancestral fossil forms. Since, however, by reason of the incompleteness of the geological record and our consequent ignorance of the organization of older forms we are frequently not in the position to avail ourselves of the actual past history of given plant types, we must of necessity have recourse to the valuable aid furnished by the important general principles described in the earlier paragraphs of the present chapter. Where the canons of evolutionary anatomy are judiciously employed, the result is usually so clear and convincing as to commend itself to the unprejudiced mind. 


\section{CHAPTER XVIII}

\section{THE LYCOPSIDA AND PTEROPSIDA}

The discussion of tissues and organs and the elucidation of the general principles applicable to these in the earlier chapters of the present work bring us to the point where the higher plants may profitably be discussed in regard to their general anatomy and evolutionary affinities. Obviously, if it is possible to compass a general grouping which will at the outset indicate the main lines of evolution, the consideration of the particular groups will be much facilitated. Some years ago the writer put forward a general classification of vascular plants, based on the cardinal features of the reproductive structures and the salient anatomical characters. This attempt to group the higher plants in accordance with their more important reproductive and anatomical features has met with approval among competent judges and as a consequence may be conveniently utilized in the present connection.

A general survey of vascular plants, existing and extinct, reveals the fact that there are certain features correlated in a significant way. For example, a large number are characterized by the possession of ventral or adaxial sporangia together with usually small leaves. Another large assemblage presents relatively large leaves and more numerous sporangia which are dorsal or abaxial in position. The former group is characteristically represented by the lycopods or club mosses and their allies, while the latter includes the ferns and the forms more nearly related to them, the gymnosperms, and the angiosperms. The first aggregation of types, known as the Lycopsida, is now practically extinct, but played a large rôle in the Paleozoic and furnished a considerable proportion of the raw material of that extremely important combustible called coal, together with its derivative products, petroleum and natural gas. The second alliance, to which the appellation Pteropsida is appropriately given, although abundantly present in the remote past, still prevails and has given rise to the seed plants 
and to the forests of Mesozoic and Cenozoic times, as well as to the herbaceous types which under modern conditions more and more predominate in the plant population of our earth.

A more detailed characterization of the Lycopsida is now desirable. Fig. I 76 represents diagrammatically the organization of an axis of the lycopsid type together with its appendages. The latter consist of leaf, branch, and root. Root and branch are intimately associated, the former usually proceeding from the base of the latter. The upper end of the main axis is represented in transverse section to show the anatomical relations of the organs. The fibrovascular cylinder in the particular case illustrated is siphonostelic, although it might equally well be protostelic, especially in the lower representatives of the group. The tubular stele or central cylinder constituted by the fibrovascular system is interrupted only at one point-where the fibrovascular supply of a branch takes its origin from the main cylinder. The gap thus appearing is known as the branch gap or ramular lacuna. On the margin of the stem appear certain projections, the leaf bases, within which are included the fibrovascular strands destined for the leaves or foliar traces. These subtend projections on the surface of the central cylinder occupied by groups of protoxylem. The projections in question are the starting-points of the foliar traces, and it is clear that in no instance is there an interruption in the continuity of the central cylinder corresponding to the departing leaf traces. The central cylinder of the Lycopsida is said on this account to be without foliar gaps. The absence of leaf gaps or foliar lacunae is characteristic of all the Lycopsida and is a diagnostic feature of importance for the great group or phylum which they represent. Even in those Lycopsida in which the leaves are, superficially at least, relatively large (for example, the Sigillariae, in which the foliar organs were sometimes over a meter in length) there were still no foliar gaps. The absence of foliar gaps is an anciently inherited or palingenetic feature of the Lycopsida, and they may therefore be technically characterized as palingenetically microphyllous (small-leaved).

Not only has this large group universally small leaves, but it also possesses another important, salient, and constant general 


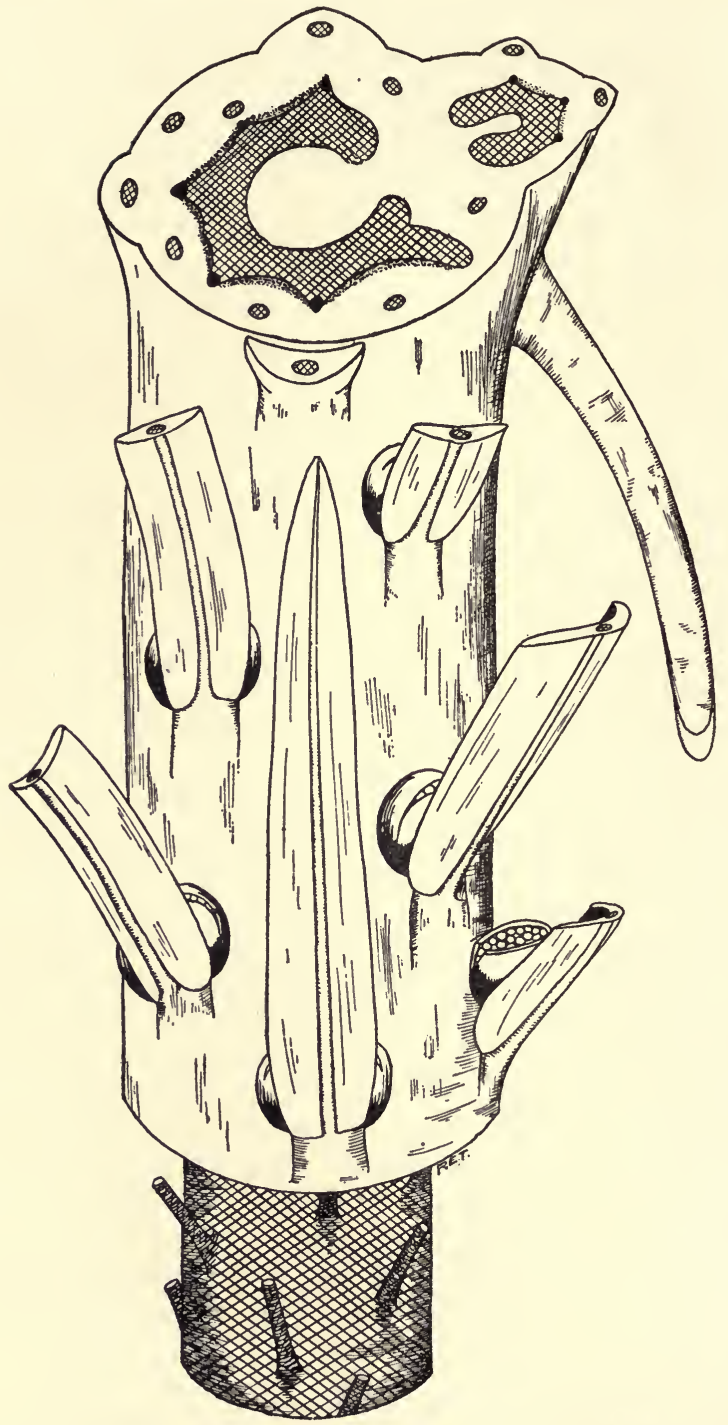

Fig. I 76.-Diagrammatic representation of the Lycopsida 
feature of organization-in this instance related to reproduction. In the phylum under discussion the sporangia or spore sacs are invariably on the upper or adaxial (ventral) surface of the sporophyll or reproductive leaf. The sporangia are single or at most relatively few in number and are invariably ectokinetic in their mode of dehiscence. True seeds seem never to have made their appearance in the Lycopsida, although, as has been pointed out in an earlier chapter, structures somewhat simulating seeds have been found in certain of the Paleozoic representatives of the group. The organs in question, however, lacked a true integument and, so far as is knewn, were without arrangements for receiving the microspores or pollen grains, a universal equipment in the case of pteridospermous and gymnospermous seeds. Possibly the failure to achieve true seminal structures was the cause of the decline of the Lycopsida, which at the present time constitute an insignificant proportion of the vegetation of the world. The group is very ancient and goes back to the beginning of the geological record. The primitive forms representing the Lycopsida were often arboreal in their habit, and the alliance reached its culmination in the carboniferous forests of the Paleozoic. It became largely reduced in the Mesozoic, and the Cenozoic saw its virtual extinction.

In the Pteropsida we have to do with forms in which the leaves are relatively large in comparison to the stem and often extremely complicated in structure. As is shown in the diagram (Fig. 177), the transverse section of the stem reveals a central cylinder which, when siphonostelic, as in the illustration, is characterized by gaps corresponding to the traces departing to the leaves. This feature of its anatomy is in marked contrast to that found universally in the Lycopsida, in which the leaves are not related to foliar gaps. The traces of the branches in the Pteropsida likewise subtend gaps in the central cylinder (which may be designated as ramular gaps). The fibrovascular supply of the root makes its exit from the stele or fibrovascular cylinder without causing any gap or interruption. The roots are often, but not invariably, related to the bases of the leaves. The foliar organs, usually much larger in size than in the Lycopsida and often of great dimensions and lobed in a complicated manner, are to be regarded as palin- 


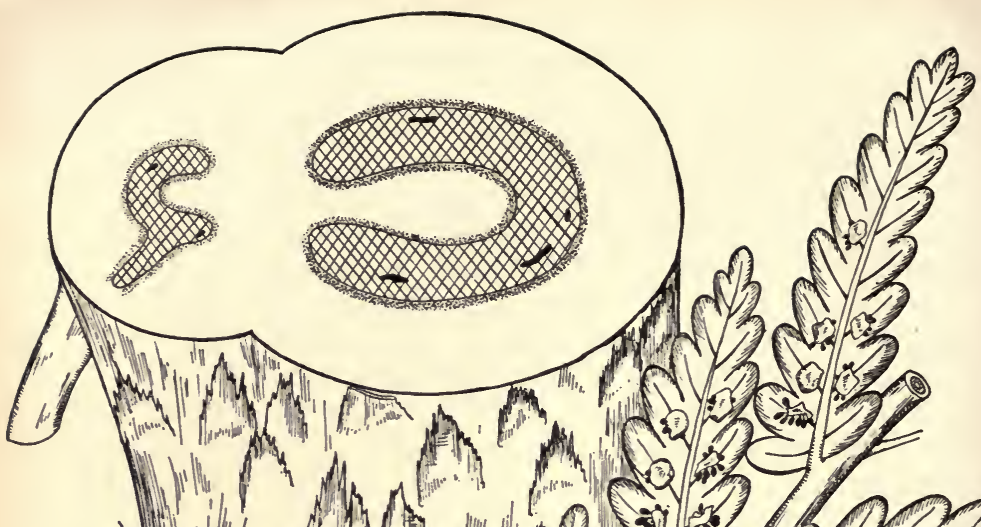
Whin 1 . (m) M.1. M $\rightarrow$ D (1)
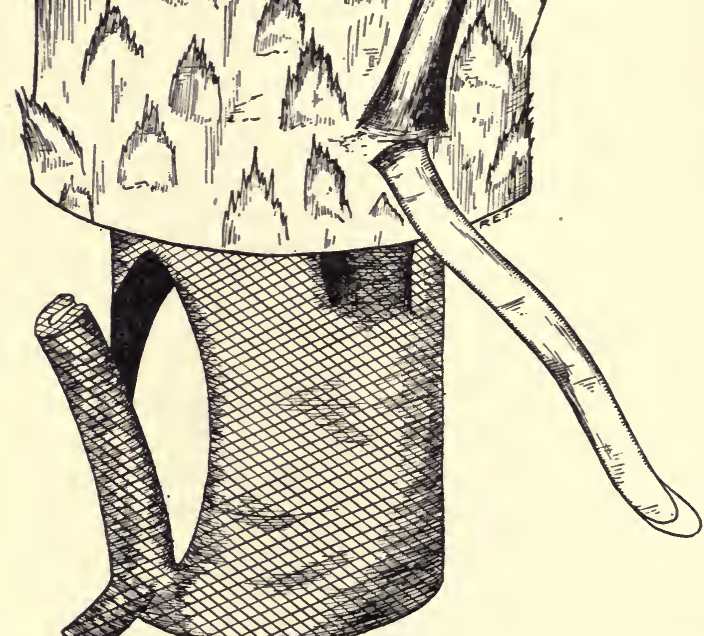

FIc. 177.-Diagrammatic representation of the Pteropsida 
genetically megaphyllous, since their anatomical relations are • always characterized by the presence of foliar gaps. The reproductive organs or sporangia in the case of the Pteropsida are on the lower or abaxial (dorsal) surface of the leaf, and are often numerous and complicated in structure. In the lower forms the dehiscence of the spore sacs is ectokinetic, but in the higher representatives of the phylum the opening mechanism is derived from modified transfusion tissue (in turn derived from the centripetal wood of the ectokinetic and lower forms). Those Pteropsida characterized by an internal reproductive mechanism derived from transfusion tissue are appropriately designated as endokinetic. The higher members of the series have developed true seeds provided with an integument and equipped with an apparatus either related to the seed itself or to the seed leaf (or megasporophyll) for the reception of microspores or pollen. Although developed in very early geological times in forms resembling ferns, the Pteropsida are still in full vigor; and in their highest manifestation, the angiosperms, they constitute an overwhelmingly large proportion of the present vegetable population of the earth. They have reached their zenith of efficiency in the herbaceous angiosperms, which in all probability will supplant arboreal angiospermous types in the not very remote future. The gymnosperms, and in particular the conifers, were the prevailing Pteropsida of the Mesozoic, while in the Paleozoic age pteridosperms (Cycadofilicales) and other lower gymnosperms and fern allies represented the group.

The Pteropsida and Lycopsida are distinct as far back as the geological record can be perused, and there seems to be little doubt that they constitute two primitive stocks of vascular plants. They are so clearly diverse even in their earliest manifestations that it is difficult to picture how they may have been formerly connected. It is obvious, however, that the lycopsid type has not been able to cope with the changing conditions of environment and, comparatively early in the periods recorded in the rocks, was relegated to a position of relative inferiority. Whether this situation was the result of the failure to achieve true seeds or is to be explained as the basis of some fundamental defect of internal organization incapacitating the Lycopsida to succeed in competition 
with the large-leaved Pteropsida must, for the present at any rate, be left an open question. Although the Lycopsida were the predominant constituent of the Paleozoic forests, the Pteropsida in many cases have entered largely into the composition of the more ancient coals and can often be clearly recognized, particularly in the carboniferous coals of the Middle Western states (Illinois, Ohio, etc.), as charred remains of the axes and even as pinnae or smaller segments of the leaves in the form of so-called "Mother of Coal."

After having described the general characteristics of the Lycopsida and Pteropsida, we find it in order to indicate the main groups which come under these two great divisions of the vascular plants. The Lycopsida include two principal subdivisions-the Lycopodiales and Equisetales. The Lycopodiales are characterized by the alternating nature of their foliage, while the Equisetales have their leaves disposed in whorls on a stem presenting marked ridges and furrows. The Lycopodiales are again subdivided into isosporous and heterosporous families. Of the former there are twothe Lycopodiaceae and the Psilotaceae. The Lycopodiaceae are characterized by the possession of well-developed roots and undivided sporangia, while in the Psilotaceae the sporangial structures are septate, and organs of the nature of roots are entirely absent. The heterosporous Lycopodiales have in common a foliar appendage, known as a ligule, which is present on both vegetative and reproductive leaves, and in the case of the latter occurs just above the insertion of the sporangium. Of the three families presenting the phenomenon of heterospory the first, the Selaginellaceae, are terrestrial forms included under a single genus, Selaginella, in which the megasporangia never produce more than four spores. In the second and usually aquatic family, the Isoetaceae, there is likewise a single genus, but the sporangia are provided with transverse processes known as trabeculae, and the megaspores are numerous in each sack. Lastly, the Lepidodendraceae, including the Sigillariae and their allies, are terrestrial extinct forms often of arboreal habit and of somewhat diverse megasporangial structures.

The Equisetales, as has been indicated at the beginning of the foregoing paragraph, are distinguished by the whorled arrangement of their leaves. Another feature which they possess in common is 
the usual exhibition of a high degree of multiplication of the sporangia which are often disposed on common stalks known as sporangiophores. The branches instead of being truly axillary are borne alternately with the leaves at the nodes. The Equisetales may conveniently be divided into three families- the Sphenophyllaceae, the Calamitaceae, and the Equisetaceae - which are in all probability related to one another in the order indicated by their enumeration. The Sphenophyllaceae were forms in which the central cylinder of the stem was protostelic. The cones consisted of sporophylls presenting various degrees of complication. In the simplest forms the sporangia were inserted singly on the stalks or sporangiophores and were numerous for each sporophyll. In more advanced types the sporangia became two or more for each sporangiophore. The Calamitaceae, like the Sphenophyllaceae, are organisms entirely extinct. They usually possessed the arboreal habit and were invariably characterized by a siphonostelic central cylinder. The sporangiophores bore four sporangia and were variously related to the sporophylls. In the Equisetaceae are included a number of genera, living and extinct, of herbaceous habit and possessing so far as is known a simplified type of cone in which sporophylls are represented by the sporangiophores alone.

In the Pteropsida, marked by the general features enumerated in earlier paragraphs of the present chapter, there are three large subdivisions - the Filicales, the Gymnospermae, and the Angiospermae. The first of these, as the name indicates, include the fernlike forms - that is, those in which the reproduction takes place through unicellular bodies known as spores. In the Gymnospermae true seeds are present which in every case are equipped to receive the microspores; and these after germination effect fertilization either by means of antherozoids (Archigymnospermae) or through the agency of pollen tubes (Metagymnospermae). In the last and (in the present epoch) most important subdivision of the Pteropsida, the Angiospermae, the pollen is received on the apex of the closed megasporophyll and no longer falls upon the seed. Fertilization is invariably by means of a pollen tube. The habit of the angiosperms is either arboreal or herbaceous, and the fibrovascular tissues show a high degree of specialization. 


\section{CHAPTER XIX}

\section{THE LYCOPODIALES}

This group, as has been indicated in the last chapter, has spiral phyllotaxy. It includes both isosporous and heterosporous families. The latter are distinguished by the presence of a ligule, while in the former this structure is lacking. In the genus Lycopodium the central cylinder of the stem is radial and protostelic. As a consequence of the radial organization of the stele, the masses of phloem lie in the intervals between the generally radially directed bands of xylem. The sieve tubes are separated from the tracheids by several rows of parenchyma on either side. In the vertically directed reproductive axes of Lycopodium the organization of the fibrovascular tissues is typically radial, while in the creeping stems or rootstocks the arrangement of the xylem and phloem is somewhat dorsiventral. The upright axes of Lycopodium, aside from the fact that they bear leaves, are scarcely distinguishable in structure from the roots. This resemblance in organization between root and shoot is an indication of the antiquity of the lycopodineous stock, since in the higher groups the differentiation between the axial and radical organs becomes more and more marked.

The monotypic genus Phylloglossum possesses a siphonostelic stem. In this form the lower region of the axis is tuberous and contains the most massive development of the fibrovascular system. In the tuber also, as is shown in Fig. I78, there is both internal phloem and internal endodermis. As the stele of the inferior region passes upward, it gives off a branch into the peduncle of the tuber which is to perpetuate the plant in a subsequent season. The trace of this appendage in departing from the central cylinder leaves a well-marked branch gap. The foliar organs indicated as swellings on the outline of the section cause no interruptions in the continuity of the central cylinder by their departure from its surface. In this respect Phyllcglossum shows itself, to be a veritable representative of the Lycopsida. In the higher and aërial region of the 
axis the fibrovascular tissues become so much reduced that internal phloem is no longer developed, and the continuity of the cylinder is interrupted by gaps which are not related to organs, but merely indicate the incomplete development of the xylem. Where a trace is given off, as is shown in Fig. I79, it takes its origin opposite a strand and does not subtendan interval between the bundles, clearly showing
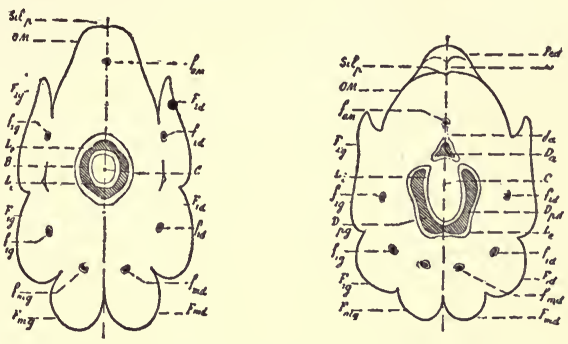

FIG. I78.-Diagram of the lower region of the stem in Phylloglossum (after Bertrand). the lycopsid condition, even in the state of stelar reduction pre-

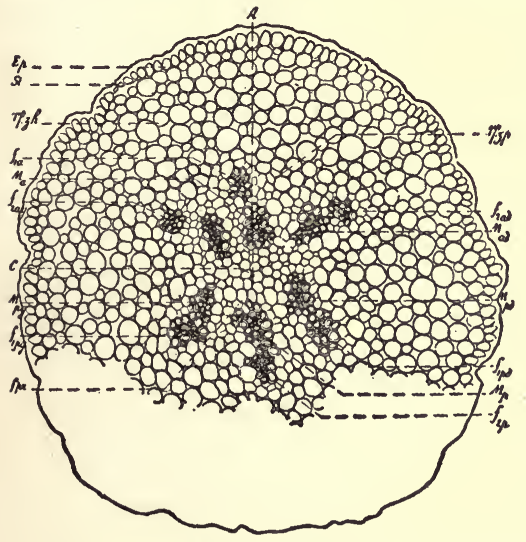

FIG. I79.-Diagram of exit of leaf traces in the aërial stem of Phylloglossum (after Bertrand). sented in the evanescent aërial axis.

The Psilotaceae are anatomically distinguished from the Lycopodiaceae by the absence of true roots. Here the aërial stem, unless it be of very small size, is siphonostelic in its organization. A thick-walled medulla is often present, but no internal phloem has been observed. The organization of the conducting tissues is radial and exarch; the leaf traces, as in Lycopodium, take their origin from the angles of the stele. In smaller aërial shoots and in the subterranean ones the central cylinder is usually protostelic. Sometimes gaps are present in the walls of the tubular cylinder of 
the larger stems, but these in no case are related to outgoing foliar traces. The general topography of a stem of the type found in the Psilotaceae is presented in Fig. r8o.

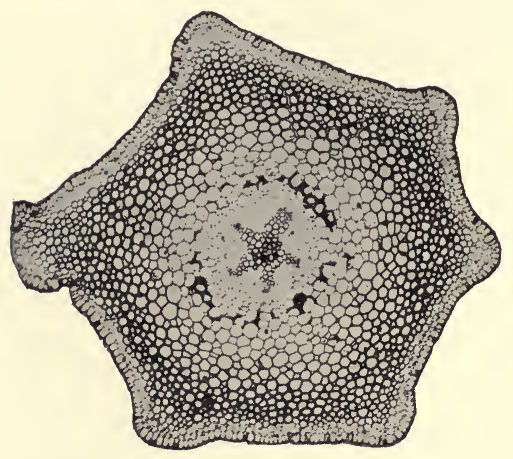

FIG. 180.-Transverse section of the stem of

In the Lycopodiaceae in general the leaf traces are ordinarily mesarch in their organization, a condition more or less characteristic of the Lycopsida as a whole. An endodermis can usually be distinguished about the foliar strands, although this limiting layer is ordinarily conspicuous by its absence in the stem in most species of Lycopodium. In Psilotum.

other representatives of the two families under discussion an external endodermis is usually found in the stem, and, as has been shown above, an internal endodermal zone is seen in the tuberous subterranean stem of Phylloglossum.

In the genus Selaginella the fibrovascular tissues of the axis are distinguished by considerable variety in topography. In some species the stele is a single mass, separated from the

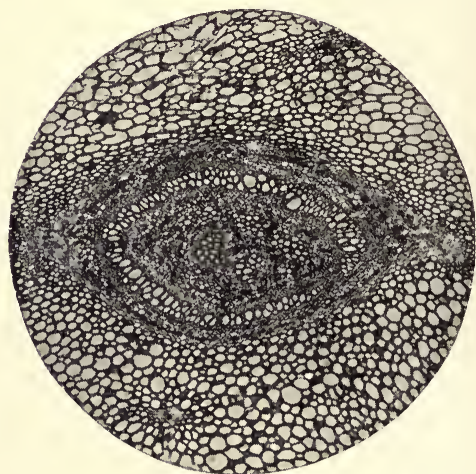

FIG. I8r.- Transverse section of stem of Selaginella laevigata, showing a siphonostelic central cylinder. cortical tissues by an air-containing region representing the endodermis. In other species the fibrovascular system becomes divided, and the protostelic condition as a result passes into that known as 
polystelic. In still another modification, presented by $S$. laevigata from Madagascar (Fig. I8I), a true siphonostele is exemplified which is complicated by the presence of medullary strands joining up with the walls of the tube in the regions where branches are given off. In the species under discussion the traces of the leaves illustrate the condition typical for the Lycopsida and pass off from the cylinder without causing any gaps in its continuity.

Isoetes has a protostelic stem which is remarkable among existing Lycopsida in manifesting well-marked secondary growth (Fig. I82). The external product of cambial activity is a radially disposed storage parenchyma, while internally the dividing layer originates additions to the fibrovascular tissues which are most commonly interpreted as consisting of alternate zones of xylem and phloem. The situation here, however, is disputed, and uncertainty persists as a result of the indifferent development of the tissues resulting from the characteristically aquatic habit of the plant.

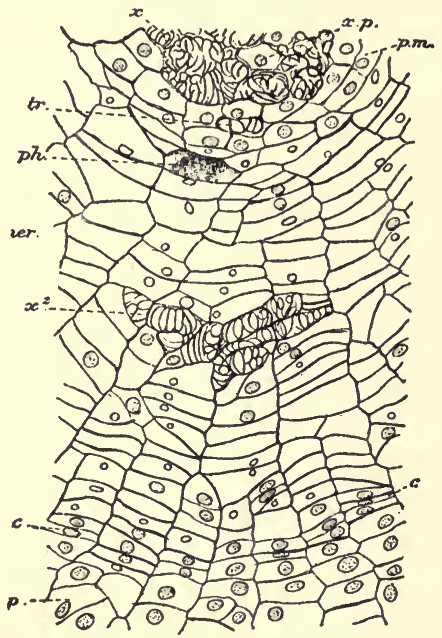

Fig. 182.-Cambial activity in Isoetes guished, in common with the smaller radical organs of a number of the lower Lycopsida, by the fact that they develop a single mass of xylem in proximity to a single strand of phloem. The leaf is not worthy of special note.

In the Lepidodendraceae the stem manifests great diversity of structure, as would naturally be expected in a group which in Paleozoic times displayed numerous types with generally marked secondary growth. The primary structures of the stem were either protostelic or siphonostelic. In the former condition a considerable amount of parenchymatous tissue was developed 
among the tracheids, particularly toward the central region of the stele. This peculiar organization of the median area of the stele in protostelic lepidodendrids is responsible for a hypothesis as to the origin of the medulla or pith. Quite generally it is considered that by continuing the process of transformation of tracheids, first into short tracheary elements and then into parenchymatous cells, there is formed in the center of the stele a pith

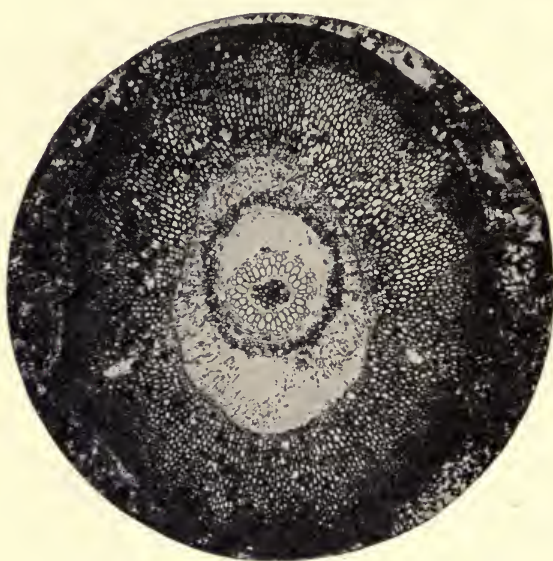

FIG. 183.-Transverse section of the stem of Lepidodendron Spenceri. of stelar origin. In accordance with this view the central region of the stele in many protostelic lepidodendrids is called a "partial pith." There is no conclusive evidence, however, that the socalled "partial pith" in reality gives rise to the true medulla in those lepidodendroid types which possess it. Moreover, the evidence in the case of the Pteropsida, which are very much better displayed in the period of time which we are able to investigate, is distinctly against the validity of the stelar origin of the pith, since the medulla in the large-leaved vascular cryptogams shows very marked indications of derivation from the fundamental system. It seems on the whole more likely that the medulla in the lepidodendrids, where such a structure is found, is an inclusion of fundamental tissues on the part of the stele. This conclusion is particularly favored by conditions found in lepidodendroid stems in which there is no indication of secondary growth, as, for example, in Lepidodendron Spenceri, shown in Figs. 183 and 184 . Here the medulla is largely occupied by dark-brown sclerenchymatous tissues similar 
to those appearing in the cortical region. It may accordingly be stated that even the imperfect evidence supplied by the stem of the lepidodendrids in a condition of obvious degeneracy of the primary stelar tissues when they are first presented on the pages of the geological record does not definitely justify the conclusion that the pith is of stelar origin. In a later chapter it will be made clear that the evidence supplied by the lower Pteropsida, which is at once more abundant and more decisive, distinctly vouches for the extra-stelar derivation of the medulla.

The degeneracy of the tracheary elements which has already been noted in the case of the protostelic type of cylinder in the le pidodendrids makes itself particularly obvious in the higher

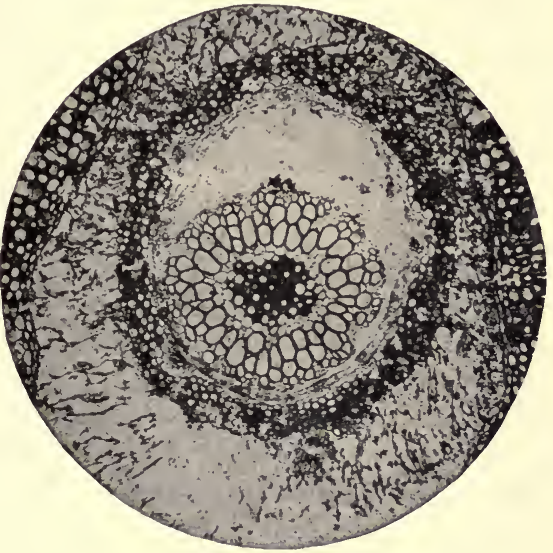

FIG. 184.-Part of the same, more highly magnified

siphonostelic representatives of the group and especially in the stems included under the Sigillariaceae. The reduction in amount of the primary tissues results in the appearance of gaps in the wall of the stelar tube which are not related to appendages. The central cylinder consequently becomes discontinuous, as has been shown in Fig. I22, page r70. Since the later-developed secondary tissues naturally first appear opposite the framework outlined in the primary wood, the secondary xylem is likewise at the beginning of its formation discontinuous, and continuity appears only after cambial activity has resulted in the formation of a woody cylinder of some thickness. Where the primary structure of the xylem has undergone the extreme degree of reduction 
found in the stem of many of the Equisetales as well as of all but the very lowest seed plants, the initial discontinuity of the secondary wood often becomes very marked. The situation presented by these extreme types unco-ordinated with the elucidative anatomical features presented by more primitive forms has been the cause of serious misunderstanding as regards the origin of the so-called medullary rays. The case of the siphonostelic lepidodendrids and Sigillariae, as diagrammatically represented in Fig. I 22, page I 70, seems to indicate definitely that parenchymatous interruptions in the secondary cylinder resulting from the discontinuity of the primary wood cannot be interpreted as true rays any more than are the gaps related to the departing traces of appendages to be brought into the category of rays. Much confusion of definition has resulted from the failure to interpret the conditions found in higher forms in terms of the structures presented in earlier and more primitive types.

It has been made clear in an earlier chapter that the lepidodendrids proper, which beyond question represent the more primitive state of organization for the Lepidodendraceae as a whole, supply evidence for the derivation of radial parenchyma as the resuit of the transformation partial or complete of radial tracheary strands into storage parenchyma. The older representatives of the Lepidodendraceae show themselves in this respect the most archaic of all the vascular plants with secondary growth. Not only is the origin of radial storage cells in the secondary xylem elucidated by the lepidodendrids, but, as has been pointed out in an earlier chapter, the parenchymatous elements of the primary wood have their origin illustrated in the conditions found in the primary region of the stem in this ancient group of vascular plants. It has been indicated in Figs. 29 and 30 that the living cells occurring in the wood of the primary region of the axis in protostelic lepidodendrids have been derived by septation from elements belonging to the category of tracheids. Some of the resultant elements persist as short tracheids with thickly reticulated walls, while others maintain a thinner wall and in all probability in life were occupied by living protoplasm. In the siphonostelic Lepidodendraceae and in the sigillarian forms as a whole there is no evi- 
dence as to the origin of parenchymatous elements in the primary xylem. In the later (Permian) representatives of the Sigillariae the primary cylinder became so much reduced that it was no longer continuous. This topographical condition of the primary wood was responsible, as indicated above, for a resultant discontinuity of the secondary xylem. The processes of the pith extending between the primary bundles and a short distance into the secondary cylinder are in a certain sense medullary rays, since they take their origin from the medulla; but they have nothing in common with the radial masses of storage tissue resulting from cambial activity which characterize the organization of the secondary cylinder. Further, they should not in any way be confused with foliar gaps, since in the Lycopsida interruptions of this nature in the fibrovascular cylinder do not occur. In the particular case under consideration the leaf traces originate opposite the strands of primary xylem and do not subtend the intervals between them. It is clear that the Lepidodendraceae, although entirely extinct, furnish extremely valuable data for the elucidation of the origin of the parenchyma in the primary wood and for that of the radial storage devices of the secondary xylem. Further, they throw a very clear light on the general morphology of the secondary woody cylinder in vascular plants, since the comparative study of their stems from lower to higher geological levels makes it obvious that the radial parenchymatous bands of the secondary wood cannot appropriately be called medullary rays. They should be called wood rays, as the inward relation to the medulla is neither a primitive nor an essential condition.

Not only in regard to the parenchymatous structures of their primary and secondary wood, as well as by their great geological age and early culmination, do the lepidodendrids in the large sense show themselves to be primitive representatives of vascular plants, but also by the organization of the fibrous elements of the secondary wood. It has been pointed out in an earlier chapter that a typical element of the primary wood in all plants is the scalariform tracheid. In the secondary xylem of plants in general the scalariform element has given place to the pitted tracheid, which is universal for the various groups of gymnosperms and for the angiosperms with 
secondary growth. In the Lepidodendraceae in the narrower sense the secondary wood is distinguished from the primary structures only by the presence, exclusively, of radial parenchyma and by the radial seriation of its tracheary elements (Fig. I85). The organization of the tracheids of the secondary wood is, in fact, identical with that found in the primary region. In the higher lepidodendroid forms assembled under the appellation Sigillariae

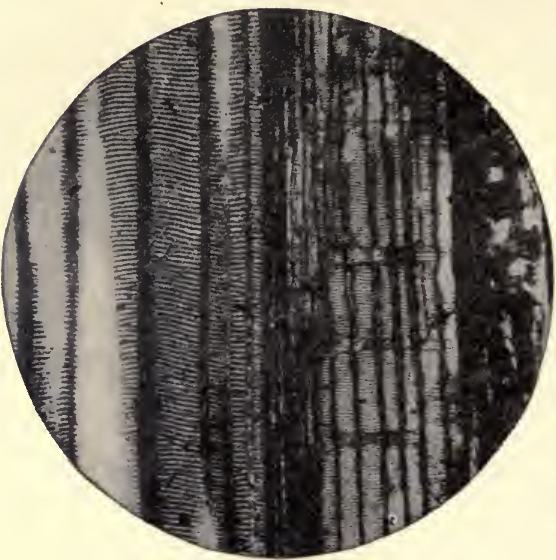

FIG. 185.-Longitudinal section through primary and secondary wood of Lepidodendron species. very frequently, particularly in the region of the secondary wood more remote from the pith, the tracheids cease to be scalariform and assume the pitted type characteristic of the gymnosperms and other higher representatives of the Vasculares.

The vascular strands of leaves in the lepidodendrids are characterized, as are those of the Lycopodiales in general, by mesarch organization. This condition is clearly shown in Fig. r86. It is apparent that the foliar trace is surrounded by secondary wood. It has been stated by Scott that true transfusion tissue is present in the leaf of the lepidodendrids, but this statement, in view of the situation present in the Lycopodiales in general and in the Lepidodendraceae in particular, seems open to some question, and certainly the subject seems to require further investigation. The foliar organs of the lepidodendroid stock were characterized by the presence of two aërating strands on either side of the foliar trace; these were in communication below with the external air through the agency 
of stomata, ordinarily accommodated in furrows on the lower surface of the leaf. The aëriferous structures of the blades of the leaves were continuous with air-containing radial structures in the outer and inner bark known as parichni. These are a noteworthy feature of structure in the lepidodendrids and have attracted a large amount of attention from students of the group. It should be pointed out, however, that they are by no means a unique structure, since special aërating devices are likewise found present in relation to the leaves of the Coniferales.

The roots of the lepidodendrids have been the subject of much discussion. Their ultimate divisions, the so-called stigmarian rootlets (Fig. 187), are characterized by a very simple organization, since only a single group of protoxylem is present. The rootlets of this order divided dichotomously, as is often

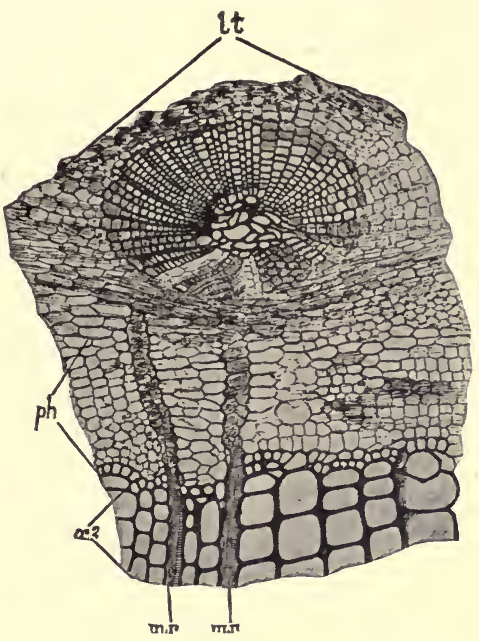

Fig. 186.-Leaf trace of a lepidodendrid (after Scott).

the case with those of the living Isoetes and Lycopodium. The main roots of the lepidodendroid forms are in all probability only partially known to us and present a curious type of structure. To begin with, there is a large pithlike mass around which is developed an extremely small amount of primary wood, at times so rudimentary as to be scarcely recognizable. The small degree of development of the primary structures and the quincuncial arrangement of the lateral rootlets of Stigmaria have led to a great deal of doubt as to their morphological nature. They have often been regarded as creeping stems or rootstocks, and this view of their nature is found even in recent literature on the subject. The mass of anatomical 
opinion now, however, is united in favor of the view that they represent the larger or main roots of lepidodendroid forms. They are, in fact, to some extent comparable to the anomalous roots found in the genus Selaginella known as rhizophores. It seems highly improbable that the type of organization presented by Stigmaria could have belonged to lepidodendrids with protostelic central cylinders. In general, the subterranean organs of plants are less frequently preserved with structural organization, since

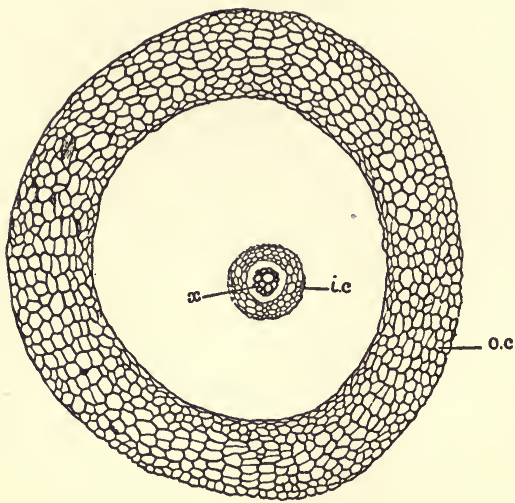

Fig. 187.-Rootlet of Stigmaria (after Scott) they are from the nature of things less likely to find their resting-place in open bodies of water, and this condition must usually be realized in order to insure petrifaction. It is accordingly probable that the genus Stigmaria, as at present defined, represents only to a limited extent the main radical organs of lepidodendroid forms.

The Lycopodiales as a whole are a group which reached its culmination in the Paleozoic age and from the richness of its display in earlier geological times must be regarded as extremely ancient. Although the group is almost extinct, its interest from the evolutionary standpoint is great by reason of its antiquity, which supplies valuable data for the elucidation of some of the most important problems of primitive organization in vascular plants. Clearly the group as a whole displays a reduction series in which the few types which survive under modern conditions represent, not primitive states, but the final results of a process of simplification extending through almost countless ages. Consequently it is highly inadvisable, in attempting to arrive at a conception of the evolutionary significance of the group, to turn exclusive attention to modern simple forms, 
such as Lycopodium or Selaginella. On the contrary, the most valuable results from the standpoint of the doctrine of descent can be derived from the study of the complicated arboreal extinct types known as lepidodendrids and sigillarians. It has been made clear in the preceding paragraphs that study of the last-mentioned types throws extremely important light on the origin of storage devices in the primary and secondary wood and makes clear the status of the so-called medullary rays. The Lycopodiales, although largely extinct, cannot accordingly be neglected by students of the data of evolution, and they supply valuable evidence, if any were needed, for the necessity of a knowledge of extinct forms as an indispensable basis for the understanding of organisms now living. 


\section{CHAPTER XX}

\section{THE EQUISETALES (INCLUDING SPHENOPHYLLALES)}

The forms to be discussed in the present chapter are contrasted with the Lycopodiales by the general fact that the appendages of the stem are arranged in a whorled or verticillate manner instead of in the spiral fashion characteristic of the group considered in the preceding chapter. Not only, however, are they characterized by the verticillate arrangement of the appendages, but also by the longitudinal ridges and furrows which mark the surface of their stems. Contrary to the conditions found in the furrowed or angular stems of certain woody dicotyledons, the traces or fibrovascular strands of the leaves correspond in position to the salient regions of the stem and not to its depressions. The branches, moreover, are not truly axillary as is characteristic of the higher forms, but occur at the node in alternation with the foliar organs. This situation is very striking and characteristic. The older forms in the Equisetales usually possessed dichotomously divided leaves or at least foliar organs in which the veins repeatedly forked. Another important characteristic of the older representatives of the Equisetales was the continuity of the ridges and furrows at the nodes. In more modern types this condition gives place to distinct and finally universal alternation of the ridges and furrows in the nodal region.

The Equisetales may be conveniently divided into three groups - the Sphenophyllaceae, the Calamitaceae, and the Equisetaceae. Of these the first-named may now be discussed. The Sphenophyllaceae are Paleozoic forms with slender stems marked by the presence of a relatively small amount of secondary growth. Their slender conformation has led to the suggestion that they were either vines or aquatics. There is, however, no convincing evidence of the correctness of either of these views in regard to their habit. Fig. I88 illustrates the organization of the stem in Sphenophyllum insigne. The center of the cylinder is occupied by the primary 
wood, which is triangular in configuration, with the small elements of the protoxylem at the angles. The primary wood presents no features of special interest as viewed in transverse section beyond the fact that it is protostelic in its organization and consequently lacks a parenchymatous medulla or pith. The secondary wood which surrounds the primary structure is characterized by the radial seriation of its elements and by the presence of medullary rays. There are no other parenchymatous structures in the secondary wood except the rays. The radial parenchyma of the Sphenophyllaceae is peculiar in the fact that its cells, instead of being strictly elongated in the radial direction and at right angles to the longer axis of the tracheids, frequently have their greatest dimensions in the vertical plane. This situation leads

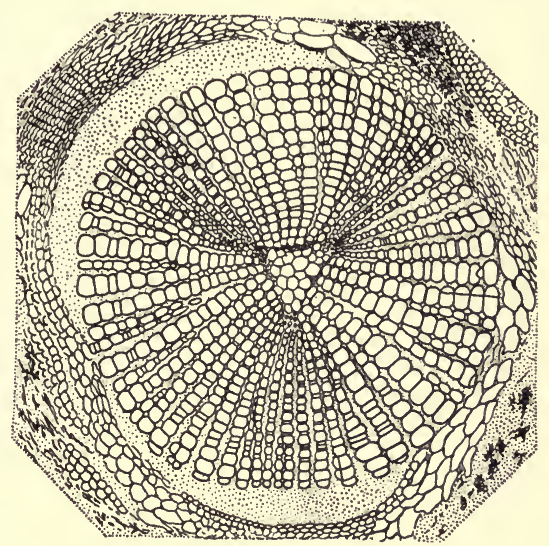

FIG. I88.-Transverse section of the stem of Sphenophyllum insigne.

to the extension of the cells of the rays along the edges upward and downward among the tracheids in a manner simulating true wood parenchyma. This is, however, merely an appearance, for longitudinal storage cells of the type ordinarily known as wood parenchyma have not yet been found in any Paleozoic wood of secondary origin. Wood parenchyma, indeed, as has been indicated in an earlier chapter, was primitively intimately associated with the phenomenon of annual rings which appeared for the first time in the Mesozoic age. It is clear from the description of the wood of the Sphenophyllaceae supplied in the present connection that it shows, as indeed might be expected, a general resemblance to that of the more ancient representatives of the Lycopodiales. The tracheids were somewhat 
scalariform in their sculpture, but tend, like those of the arboreal Lycopodiales, to develop the pitted condition. The pits, whether scalariform or rounded, were found equally on radial and tangential surfaces of the elements of the secondary wood, a condition paralleled in the ancient treelike representatives of the Lycopodiales. The outer region of the figure shows the soft tissues in a condition of relative disorganization, which does not make their discussion profitable.

The most interesting general features presented by the anatomy

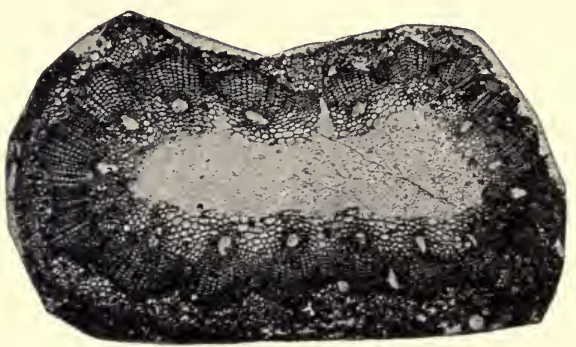

Fig. 189.- Transverse section of a small stem of Calamites. of the stem in Sphenophyllum are its essentially protostelic character, the peculiar organization of the rays, and the tangential as well as radial pitting of the tracheids. The leaves and roots are not well known as to their anatomical organization and in the actual state of our ignorance manifest no features of unusual interest.

The Calamitaceae are distinguished from the Sphenophyllaceae anatomically by the siphonostelic organization of their central cylinder. In the more ancient types of Calamites the ridges and furrows of the stem were continuous at the nodes, precisely as is the case in Sphenophyllum. Moreover, in the Archaeocalamitaceae the leaves divided dichotomously. In more modern calamitean types the alternation of the ridges and furrows in the nodal region began to become a marked feature of organization except in the cones or reproductive axes, which adhered to the more ancient topography with non-alternation at the nodes.

Fig. 189 illustrates the organization of a younger stem in a calamite. The outer region of the axis has generally disappeared as a result of fossilization, but the woody and medullary regions are 
clearly shown. The wood is apparently entirely secondary in its origin, but it is distinguished by certain lacunae or cavities which occupy the apices of the wedgelike masses constituting the rather slender cylinder under discussion. These cavities indicate the position of the poorly differentiated and evanescent primary wood. So far as is known, the primary xylem of the calamites of the later Paleozoic was entirely centrifugal or peripheral in its development. The great reduction in the primary structures of the calamites has led to a discontinuity of the cylinder comparable to that found in the higher and more modern members of the Sigillariae described in the preceding chapter. The lack of continuous development in the cylinder of primary wood brings with it a corresponding organization of the secondary xylem. The general result of the situation portrayed is the organization of the secondary cylinder in the first instance as separate wedges which finally become united by their increasing breadth. In the group under consideration we have one of numerous examples of an interrupted secondary cylinder resulting from the meager and sporadic development of the primary wood when the latter presents an extreme degree of reduction. The pointed, outward excursions of the pith in calamitean forms are in marked contrast to the true medullary rays, which in this case are narrow structures. The rays in Calamites are characterized by the often vertical elongation of their elements, a situation which parallels that described above in Sphenophyllum.

The slight development and the entirely centrifugal origin of the primary xylem in the true calamitean forms is in marked contrast to the conditions presented by the genus Sphenophyllum, where the primary structure is not only massive, but also entirely centrad or centripetal in its development. There is, of course, a very wide gap between the organization of the axis in Sphenophyllum and that found in Calamites. This gap is for the most part still unbridged by the discovery of intermediate forms, but an interesting condition is found in a stem from the lower Carboniferous which is described by Scott. In Fig. Igo is reproduced a somewhat oblique section of the primary region of one of the woody wedges of a calamitean stem. The lacuna or cavity representing the position of the ephemeral primary wood has 
tracheary elements, not only on the side which lies toward the secondary wood, but also on that in juxtaposition to the medulla

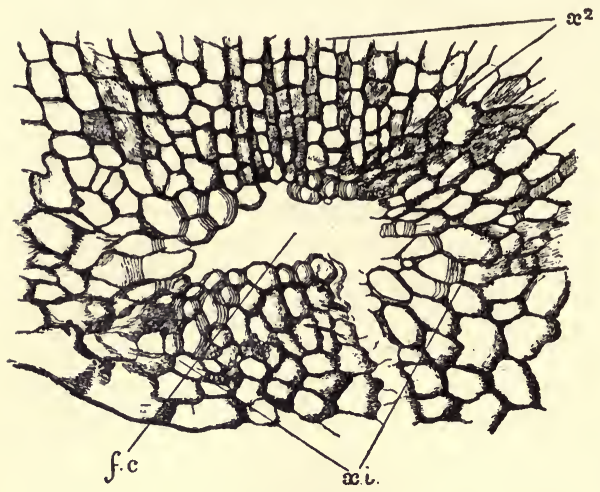

Fig. I90.-Transverse section through the primary wood of Protocalamites (after Scott). or pith. In other words, this calamitean stem, to which its discoverer has applied the name Protocalamites, somewhat clearly presents xylem of the centrad or centripetal type.

The secondary wood of calamitean forms was in its early organization largely composed of scalariform ele-

ments which in the later development gave place more or less completely to the pitted tracheids. Fig. I9I illustrates the structure of the stem of a calamite in proximity to the primary region. It is clear that the tracheids are still very largely scalariform. The pitting of the tracheary elements in Calamites, whether

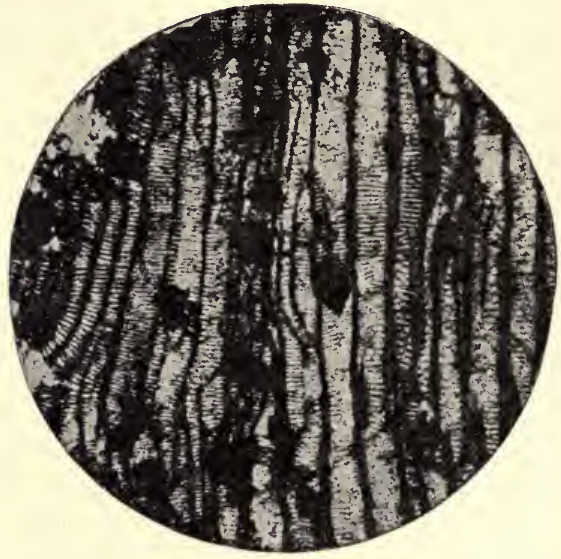

FIG. I9r.-Longitudinal section of the wood of Calamites scalariform or rounded, was confined to the radial walls of the elements as in the lower gymnosperms, and was not present on the 
tangential aspects. True wood parenchyma did not occur in the secondary wood of Calamites. Although the primary xylem of calamitean forms has departed far from the primitive condition, the secondary ligneous organization of the group is characterized by features which are only less primitive than those presented by the lepidodendrids.

The organization of the leaf in Calamites is not well known as regards those features which are of interest from the comparative anatomical standpoint, but this situation is fortunately relieved by certain data observed in the case of the foliar organs of the living genus Equisetum, which will be described in a subsequent paragraph. The root of calamitean forms was not recognized at first as belonging to the forms

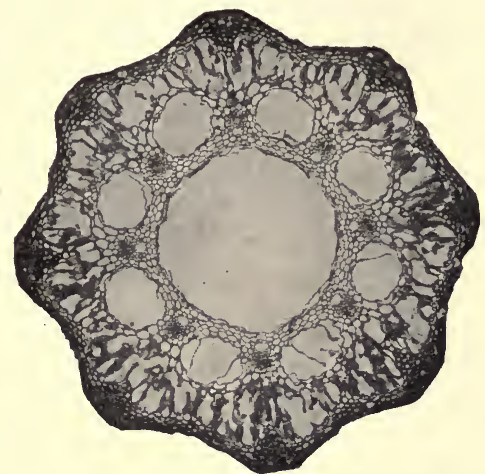

FIG. I92.- Transverse section of stem of Equisetum variegatum var. Jesupi. included under this appellation and was called Astromyelon. Its organization can best be aiscussed in connection with that of the root of the living genus Equisetum.

In Fig. 192 is portrayed the structure of a transverse section of the stem of the genus Equisetum. It is evident that the center of the figure is occupied by a large air space shared by the calamitean forms and indicative both for the Equisetaceae and their fossil forebears, the Calamitaceae, of a primitively amphibious habitat. This central cavity is often called the medullary fistula and in the case of the ancient representatives of the Equisetales was often molded in stone as pith casts resulting from mud entering the central spaces of the fallen trunks rotting in the shallow waters of Paleozoic lakes. Surrounding the large medullary space are the fibrovascular bundles, which are of small size and somewhat remote from one another. The strands are marked by cavities in their 
inner region which from their topographical relation to the ridges

FIg. I93.-Transverse section of bundle of rootstock of Equisetum arvense.

true scalariform or reticulate elements which are laid down after the elongation of the internodes has come to an end. The two masses of metaxylem inclose between them the tissues of the phloem, consisting of larger sieve tubes and smaller parenchymatous cells. In Fig. I94 is shown a longitudinal view of the fibrovascular bundles taken a little to one side of the central region. To the left may be seen the carinal cavity containing remains of ringed and spiral protoxylem. To the right appear the reticulate elements of the metaxylem which in the transverse view flank the phloem.

The organization of the pith and the distribution of the endodermal structures in the genus Equisetum are of considerable tracheids which constitute the metaxylem. These are

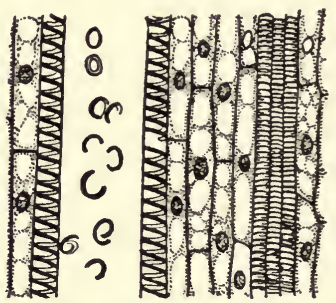

FIG. 194.-Longitudinal section of bundle of rootstock of

Equisetum silvaticum.

To the right appear the
in the transverse view of the stem are known as carinal air spaces. In alternation with these are larger spaces in the cortex situated beneath the furrows of the stem and designated consequently as vallecular lacunae.

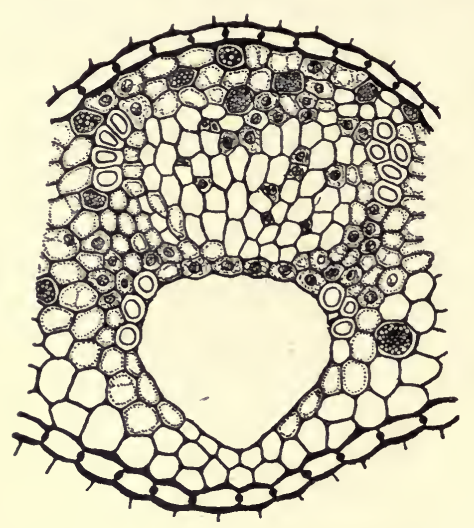

The cortex is largely composed in life of green cells and performs the assimilative and transpiratory functions inadequately subserved by the minute leaves.

The fibrovascular bundle must now receive further consideration (Fig I93). The tracheary elements are scantily present on the margins of the so-called carinal lacunae. This region is the protoxylem. Outwardly on either flank is seen a row of

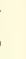


endodermis except in the region of the nodes where continuous internal and external endodermal layers are seen. The pith of the genus Equisetum, particularly in the region of the nodes, is frequently characterized by the presence of nests of dark-brown sclerotic cells, resembling similar structures found in the cortical tissues of both stem and leaf. On the grounds of comparative anatomy we shall accordingly be compelled to regard the pith of Equisetum as of cortical origin.

The arrangement of the fibrovascular strands at the nodes in living and fossil representatives of the Equisetales (Equisetaceae and Calamitaceae) must now be considered. The situation present is best revealed by means of diagrams (Fig. I96). In $A$ is depicted the arrangement of the fibrovascular structures at the node in the vegetative stem of the living Equisetum. Across the center of the diagram passes a heavy transverse band, the so-called nodal wood. In this the strands of the upper and lower internodes end in such a manner that they alternate with one another. The traces of the leaves originate from the strands of the lower internode and thus subtend the intervals between the strands which are joined with the nodal wood from above. A superficial view of the topographical conditions represented here would result in the conclusion that the Equisetaceae are provided with foliar gaps precisely as is the case in the Pteropsida. A consideration of $B$ makes this view of the matter difficult to sustain. In the figure the foliar strand is represented in radial aspect as it comes off from the fibrovascular tissues of the axis. It is clear that the trace of the leaf takes its origin below the so-called nodal wood and passes out without showing any foliar gap above it. It is true that, as is indicated in both $A$ and $B$, a gap is present subtending the foliar trace above the continuous zone of the wood at the node; but a consideration of the historical and comparative anatomical data makes it difficult indeed to regard the gap in question as a foliar one. In $C$ is shown the arrangement of the strands of the primary wood in an ancient calamitean form (Archaeocalamites). It is evident in this case that the strands of the upper and lower internodes, instead of meeting the nodal wood in alternation as they typically do in Equisetum, exactly coincide with one another and are not subject 
to alternation. As a result of this condition the leaf traces, which here, as in the modern type, originate from the lower internodal strand, subtend, not a gap above the nodal wood, but the strand of the upper internode. It is thus clear that the older condition
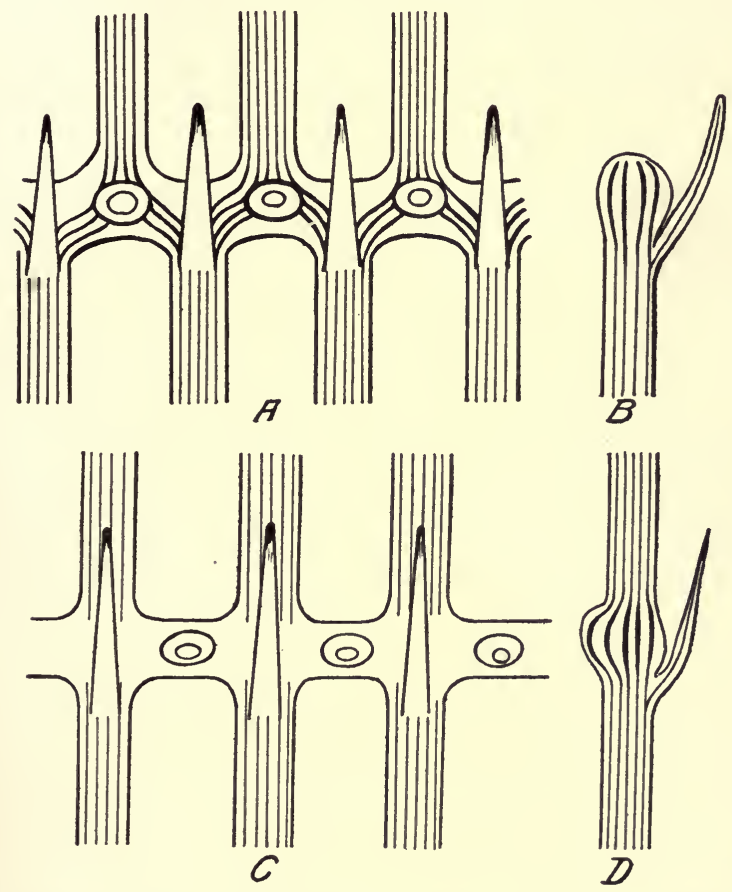

Fig. I96 $a, b, c$, and d.-Diagrams illustrating the relations of the bundles in Equisetum and Archeocalamites. Explanation in the text.

for the Equisetales is one in which there cannot possibly be a gap corresponding to a leaf, even above the so-called nodal wood. A radial view of the situation in Archaeocalamites makes the topography still more clear, and this is furnished in $D$. Obviously on historical grounds the Equisetales are without foliar gaps and 
hence are to be regarded as Lycopsida. The comparative anatomical evidence on this point is equally unequivocal. In the reproductive axes or cones of both Calamitaceae and Equisetaceae the strands typically fail to alternate at the nodes, and the traces of the sporophylls are consequently quite without corresponding gaps. There apparently can be no question on anatomical grounds that the Equisetales are justly included in the Lycopsida.

If the evidence as to the

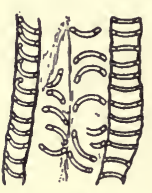

$a$

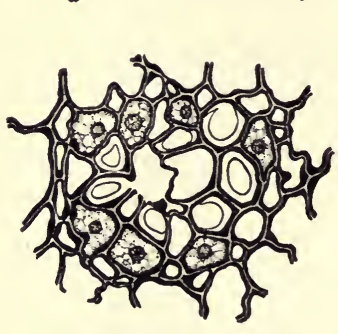

$c$

$c$

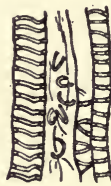

$b$ relationship of the Equisetales to the phylum Lycopsida is clear on anatomical grounds, it is equally definite from a consideration of the features of organizationof the

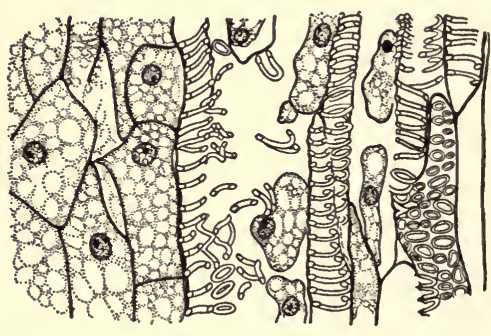

$d$

FIG. 197.-Longitudinal section of bundle in vegetative and reproductive leaves of Equisetum (after Eames).

cones. In the Sphenophyllaceae and Calamitaceae the sporangia or sporangiophores are known to be ventral appendages of the sporophylls and thus present the condition characteristic of the Lycopsida. On the basis of both reproductive and anatomical features the group under discussion clearly belongs under the large heading of Lycopsida.

The leaf in the genus Equisetum is of considerable interest in view of the fact that it displays the presence of centripetal or cryptogamic xylem which has entirely disappeared in the stem. Fig. I97a illustrates the organization of the trace of the vegetative leaf of E. maximum. It is obvious that the xylem includes a central 
spiral protoxylem region flanked inwardly and outwardly by reticulate metaxylem. The condition present is, in fact, mesarch and strikingly resembles the common anatomical situation in the leaf of the Lycopodiales as described in an earlier chapter. In the sporophyll of the living representatives of the Equisetales the mesarch structure of the trace is even more conspicuous than in the vegetative leaf. The situation in this respect is shown for $E$. palustre (b). But the reproductive leaf not only manifests centripetal wood in its trace, but it also presents an equally significant condition in the relation of the phloem to the xylem. In $c$ is shown a transverse section of the trace of the sporophyll in 'E. hiemale. Sieve tubes can plainly be seen surrounding the xylem elements, while the latter have in their midst a more or less obvious lacuna representing the evanescent protoxylem. On the grounds of comparative anatomy it is clear that the Equisetaceae once possessed

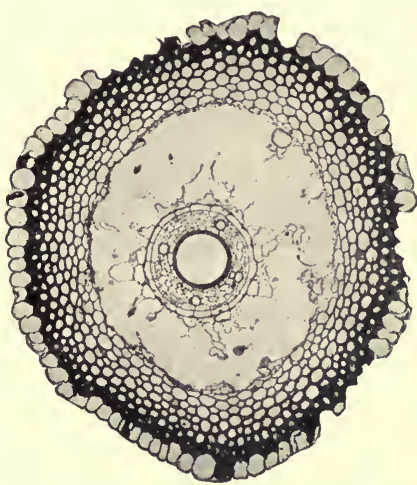

FIG. 198.-Transverse section of the root of Equisetum hiemale. centripetal wood in the stem.

That this was the former situation for the stock as a whole is clearly indicated by the anatomy of Protocalamites shown in Fig. I90. It is further rendered highly probable by the concentric as well as the mesarch organization of the trace of the sporophyll in the living genus that the bundles of the axis were formerly concentric in their organization. This condition must, however, have been realized in the extremely remote past, as no indication of concentric structure has been found in the stems of even the most ancient anatomically investigated remains of calamitean forms.

The root in the genus Equisetum (Fig. I98) shows the presence of four protoxylem groups alternating with as many clusters of elements of the phloem. The metaxylem consists ordinarily of a 
single large central tracheid in which the four masses of protoxylem unite. In the smaller roots of Calamites the same general organization is found as presents itself in Equisetum. The radical organs of this extinct group of the Equisetales are known as Astromyelon, a name given before their connection with calamitean stems was known.

In the Equisetales as a whole, represented by the Sphenophyllaceae, Calamitaceae, and Equisetaceae, very marked features of organization are present. In fact, the group shows characteristics which may well be denominated unique. It is clear that the group is of very ancient origin, since when it first comes into view it is distinctly set off from the other large alliance of the Lycopsida, the Lycopodiales, by the whorled character of its appendages, the ridges and furrows of the stem, and the sporangiophoric manner of reproduction. Scott has regarded these features as sufficiently distinctive to warrant the establishment of a third great phylum of vascular plants, the Sphenopsida. This group he considers as on the whole more nearly allied to the Pteropsida than to the Lycopsida. In view of the absence of foliar gaps in the series under discussion in the present chapter, particularly clear when both fossil and living forms are brought into consideration, there does not seem to be any adequate anatomical evidence to support the separation of the Equisetales under the heading of Sphenopsida. The reproductive characters of the equisetal series are likewise most easily reconciled with an affinity to the Lycopsida in general and to the Lycopodiales in particular. Scott regards the Psilotaceae as more nearly related to the Sphenophyllaceae than to the lycopodineous forms. The evidence in favor of this view does not seem, however, to be of a compelling character. 


\section{CHAPTER XXI}

\section{THE FILICALES}

This group of vascular plants presents the features of the Pteropsida in their most primitive and least modified condition. Large leaves are consequently the rule, and these normally, when functioning as sporophylls, bear numerous sporangia on the lower or abaxial (dorsal) surface. When the central cylinder of the stem is siphonostelic, as is most frequently the case, the traces of the leaves take their departure from the wall of the stelar tube with the formation of foliar gaps subtending the departing strands. In the Filicales reproduction is always by means of spores, which are in general isosporous, but which in a few instances represent the heterosporous condition. The Filicales constitute a remarkably clearly defined group, at least so far as their modern representatives are concerned; and the only family which has had its affinities with the Pteropsida brought into question is the Ophioglossaceae, regarded in some quarters as derived from lycopsid ancestry. This attribution of affinity, however, is not now considered justified. The Filicales constitute the largest element composed of vascular cryptogams in the existing flora of our earth and are on that account of great importance from the evolutionary standpoint. Anatomical problems which, in the Lycopsida, are difficult of elucidation by reason of the large degree to which the group has suffered extinction in the existing flora are much more advantageously approached, in the group under consideration, as a consequence of the large number of forms which are offered for study by the existing plant population of the earth. The value of the filicinean Pteropsida is particularly great in respect to the fibrovascular structures, and an attempt will be made in the present connection to utilize these to the full. Naturally, in a group surviving in relatively large numbers only the more salient and significant facts can be brought into prominence in an elementary treatise like this. 
The stem as the most plastic of the organs in vascular plants presents the greatest variety of structure in the Filicales. The leaf, and particularly the root, offer little diversity of organization and may consequently be dismissed with relatively slight consideration. Anatomically the stem presents itself in the case of the Filicales under two main conditions: the protostelic, in which there is no medulla present in the fibrovascular system,

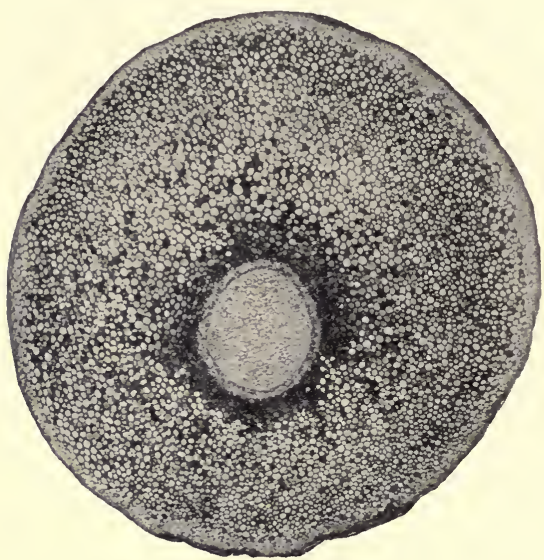

Fig. I99.-Transverse section of the stem of Gleichenia species. and the siphonostelic, characterized by the existence of a central mass of parenchyma known as the medulla or pith. The first condition is represented in Fig. 199, portraying the transverse section of the stem of a species of Gleichenia. The siphonostelic modification is delineated in Fig. 200, reproducing the transverse aspect of the stem of the maidenhair fern, Adiantum pedatum. In the second figure we find the fibrovascular tissues organized in the form of a tube, limited both internally and externally by an endodermal boundary which becomes continuous around the margins of the gaps caused by the exit of the traces of the lateral branches and leaves. Not only is the tubular central cylinder bounded continuously by an endodermal layer, but it is likewise characterized in the particular case under discussion by an inner and outer lining of phloem. In the walls of the stelar tube so organized there are gaps formed in connection with the exit of the fibrovascular strands leading to both leaves and lateral branches. In addition to these there may be interruptions in the continuity of the fibrovascular hollow cylinder which are not related to 
departing strands of any organs. The root is not responsible for the appearance of a gap in the wall of the stelar tube unless it happens, as is sometimes the case, to be closely related to a foliar organ. Under these conditions the apparent gap is naturally foliar and not radical.

Before passing to the discussion of modifications of the siphonostelic central cylinder as presented by the stem in the Filicales it will be well to consider the general topography of the fibrovascular system in the various organs. The account of the stelar system

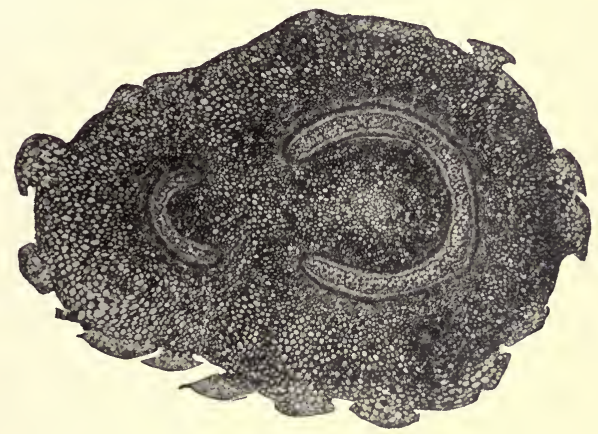

FIG. 200.-Transverse section of the stem of Adiantum pedatum

of the stem outlined in the preceding paragraph will suffice for the cauline organ, so that it is possible to turn at once to the discussion of the leaf and the root. As has been indicated above, the foliar trace departs from the tubular central cylinder of the axis in topographical relation to a lacuna in the stelar wall which is known as the foliar gap. The outgoing foliar trace in the lower region where it runs in the stipe or rachis or even sometimes in the subdivisions in. the main veins of the flattened region or lamina, is concentric or bicollateral in its organization. This characterization means that the xylem is either completely surrounded by phloem or at least has phloem on its two opposite sides. As the fibrovascular strands which innervate the blade of the leaf become more finely divided they lose 
their original concentric or bicollateral structure and develop a collateral organization. This condition of the finer fibrovascular structure of the foliar organ is clearly correlated with the dorsiventral structure of the leaf as a whole, and favors its functioning in relation to photosynthesis and transpiration. The upper region of the foliar strands is consequently largely under the sway of physiological conditions, while in their lower course, and especially before they have suffered much in magnitude as a result of subdivision, the ancestral conditions may as a rule be more readily observed. Further, the more aberrant the anatomical structures are in any given case the less will be the degree of development of the ancestral conditions in the foliar traces. In argument regarding the interpretation of the fibrovascular structures in stems the organization of the foliar trace after it has left the stele of the leaf is of great importance. In the root of such relatively low types as the Filicales little evidence is supplied which is of value in the interpretation of the primitive organization of the tubular stele of the stem. This statement holds, not only for the true ferns, but also for those lower and ancient gymnosperms which have the most marked filicinean affinities. It will be apparent, however, when the discussion of the anatomical organization of the axis in higher gymnosperms and angiosperms is reached, that the root assumes an evolutionary significance which is not observed in the lower groups of the Vasculares. With these preliminary remarks it is possible to pass with advantage to the consideration of the organization and evolution of the tubular central cylinder of the axis.

First will be discussed modifications of the tubular stele which are in the direction of greater complexity. In many ferns the central cylinder or stele in the adult stem is represented by a complex grouping of strands. In the bracken fern (Pteris aquilina), as is shown in Fig. 20I, the older stem presents in transverse section two series of bundles-two large central ones and a ring of usually much smaller ones forming a circle outside these. The significance of the conditions present in the stem of this most commonly studied fern have been very generally misunderstood. It has been maintained by the distinguished French anatomist 
Van Tieghem that the vascular system of the bracken arises by the continued forking of an originally simple (protostelic) strand. According to his view the two large central bundles are the first to appear as the result of the process of forking, and the smaller circle of strands lying outside these is formed later. On account of the supposed origin of the strands in the stem of Pteris aquilina by repeated division, the name polystelic was given by Van Tieghem to this and similar conditions of anatomical organization in the ferns and their allies. As a matter of fact the situation is very different indeed from that indicated by the term polystelic. In Fig. $202 a$ is shown the transverse section of a very young stem of the bracken when it is still in the upright condition

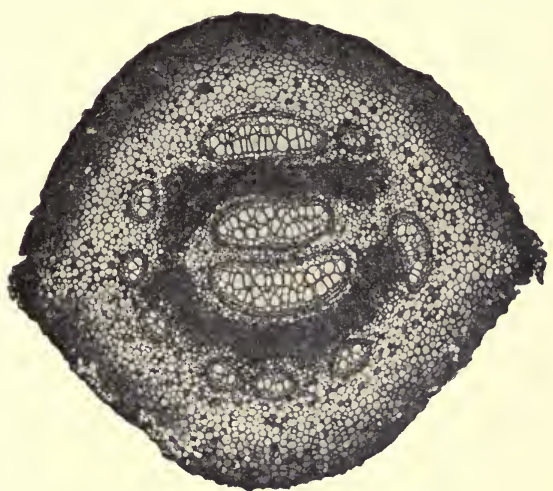

Fig. 20r.-Complicated bundle system of Pteris aquilina and has not given rise to the subterranean horizontal branches which come into existence at a comparatively early stage. The stem at this age is obviously siphonostelic and is marked by a gap correlated to an outgoing leaf trace. In $b$ a later stage of development, characteristic of the young horizontal stem, is shown. Here the tubular central cylinder is giving off internally and toward the center a medullary strand. Later the single medullary strand becomes double and the condition attained in the adult is reached. It is clear from an examination of the actual course of development in the stem of the bracken fern that the medullary strands are not formed first, as is assumed by Van Tieghem, nor does the stelar system of the adult result from repeated bifurcation of an originally single strand. On the contrary, the course of development presents first a protostelic 
condition in which no medullary tissues are present, a phase followed by the siphonostelic one, which in turn develops medullary strands from the inner surface of its walls. The outer series of bundles is consequently antecedent to the medullary bundles, a situation which may be readily inferred without the study of the young plant from the fact that the roots are attached to the external strands. Not only does the bracken manifest the general
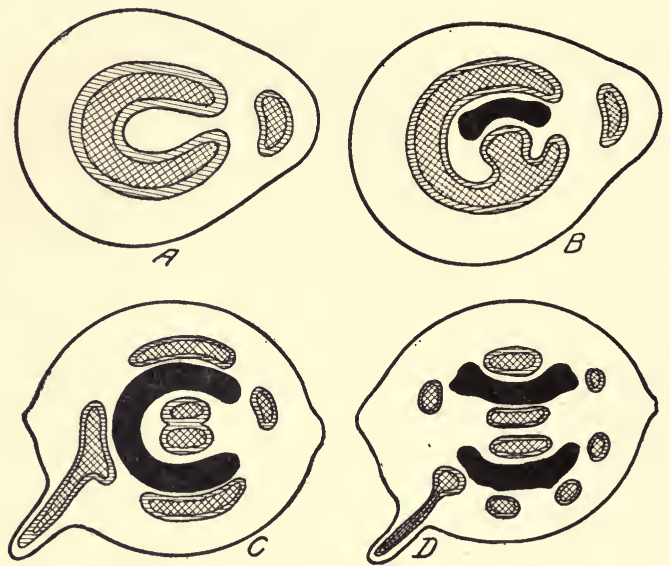

FIG. 202.-Diagram to illustrate the development of the stem in Pteris aquilina. Explanation in the text.

conditions described above, but all ferns with complicated arrangements of conducting strands in the stem can readily be included under the same general statement. This, for example, is true of the complex stem of the Cyathaceae or tree-ferns and also of that of the large tropical ferns known as the Marattiaceae. It is apparent from the account supplied in the present connection that the tubular condition is both typical and primitive for the ferns in general, with the sole exception of those forms in which the organization of the stele maintains the original protostelic condition. This type of structure is, however, comparatively rare in existing ferns, although it has been found, as would 
naturally be expected, much more generally in Paleozoic types belonging to the Filicales.

Before proceeding further with the question of the organization of the tubular or siphonostelic type of central cylinder, it will be well to discuss the important problem of the origin of the pith or medulla in the Filicales. The situation in this group is much more favorable than it is in the Lycopsida, living and extinct, because the facts at our disposal are much fuller and as a consequence put the whole subject on a more satisfactory footing. There are two views in regard to the origin of the medulla in stems of the type here designated siphonostelic. One hypothesis considers it as originating from the central region of the xylem of the stele by the transformation of tracheary elements into parenchyma. It has been shown in earlier chapters that there can be no question that parenchymatous elements may be derived from tracheids in the primary wood, and the evidence in this direction clearly points to a possible origin of the medullary tissues from transformed tracheids. Indeed, in the case of the lepidodendrids the facts which favor the tracheary origin of the medulla are not without importance. Therefore, if we are to regard the medulla as derived from tracheary tissue on the basis of the existence of transitional stages between tracheids and parenchyma, it will be only fair on the other side to assume that where elements clearly of a cortical nature occur in the region of the pith they afford evidence for the other hypothesis of the origin of medullary parenchyma, namely, its derivation from the included cortex. It is, of course, illogical to admit evidence for a derivation of medullary structures from tracheids and at the same time to reject equally cogent data as to the origin of the pith from cortical tissues. Even in the lepidodendrids the evidence for the tracheary derivation of the medullary structures is not complete, for transitions from tracheids to parenchymatous cells are presented only by protostelic stems and are entirely absent in tubular cylinders. Indeed, in cases where secondary growth is not characteristic of the siphonostele in this group, the medullary region of the stele is frequently occupied by sclerenchymatous elements similar to those which occur in the cortex. The hypothesis 
of the tracheary origin of the pith is also open to question in other representatives of the Lycopsida. In Phylloglossum the medulla in the region of the tuberous portion of the stem is actually surrounded by an endodermal layer, a structure peculiar to the cortex. In Selaginella laevigata, also, the tubular stele is bounded internally as well as externally by a well-defined endodermal layer, and the tissues of the pith, further, clearly resemble those of the cortex. In both Psilotum and Tmesipteris the medullary tissues are frequently composed to a large extent of brown sclerenchymatous elements resembling similar structures found in the external fundamental tissues. In the genus Equisetum dark-brown sclerenchyma is present in the medulla and the cortex. It is clear on the basis of data derived from resemblances between the tissues of the pith and the cortex, on the one hand, and the tracheary tissue, on the other, that the preponderance of evidence weighs heavily on the side of the fundamental or cortical origin of the medulla. And heavily as the scale seems to incline on the side of the fundamental derivation of the pith in the Lycopsida, it seems entirely overwhelming in the case of the Filicales.

There are two general arguments which have been invoked against the origin of the pith from the fundamental tissues in the Filicales. One is the denial that it is possible for the stele to include the tissues of the cortex. This reasoning cannot be given very serious weight in view of the fact that in certain filicinean steles-for example, that of the polypodiaceous genus Onoclea and the schizeaceous genus Anemia-the possibility of the inclusion of fundamental tissues within the tubular stele must apparently be granted, because the epidermis and chaffy ramentum as well as the outside air are actually included within the medullary region of the central cylinder. Moreover, the phloem, a tissue primitively occurring on the outer surface of the central cylinder, is frequently included in its interior. If any further evidence were needed as to the possibility of the xylem including tissues of another morphological category, it is furnished by the case of certain filicinean foliar traces within which it is universally admitted, even by the most convinced adherents of the hypothesis of the tracheary origin of the pith, fundamental tissues may not 
only be included, but in some instances may be entirely shut off from the similar tissues outside. Good illustrations of this condition are provided by species of the Gleicheniaceae, Polypodiaceae (notably the common bracken fern), and Marattiaceae. The possibility, then, of the inclusion of the pith within the stele must apparently be granted.

The second argument against the cortical origin of the medulla is directed against the evidence based on the similar histological nature of pith and cortex in many cases, a resemblance which is the more marked the more the medullary tissues are in histological continuity with those lying outside the tubular stele. It is assumed by the advocates of the tracheary origin of the pith that the striking histological similarity which often exists between the medulla and the cortex is merely "physiological." This argument would be worth more if its exponents did not admit all histological evidence in favor of the tracheary origin of the medullary tissues while denying that indicating their derivation from the fundamental system. Such argument is clearly fallacious, and the logical procedure is to admit to equal consideration evidence for the tracheary hypothesis of the appearance of the medulla on the one hand and that for its derivation by the inclusion of cortical or fundamental tissues on the other. The advocates of the cortical origin of the central parenchyma of the tubular stele are apparently advantageously situated in this respect, for they can equally well emphasize the extremely abundant data for the origin of the pith from the fundamental system and at the same time explain away the meager evidence for the tracheary derivation of the medullary tissues. So far as the plentiful evidence in the case of the Filicales is concerned, it seems beyond reasonable doubt that the median parenchyma of the tubular or siphonostelic central cylinder has come from the outside and is not the result of internal differentiation within the stele.

The question of the origin of the pith is a very important one from the phylogenetic standpoint, since correct views in regard to this matter are necessary for the interpretation of the evolution of the tubular cylinder from lower to higher forms. It would be going beyond the range of the present somewhat elementary 
treatise upon the anatomy of the vascular plants to elaborate at any length the question of the phylogeny of the tubular central cylinder, important as this is for the proper understanding of the higher plants in the light of the doctrine of descent. The whole situation may conveniently be illustrated by reference to the Osmundaceae. In discussing the evolutionary principles involved in the development of the siphonostele in this group, we

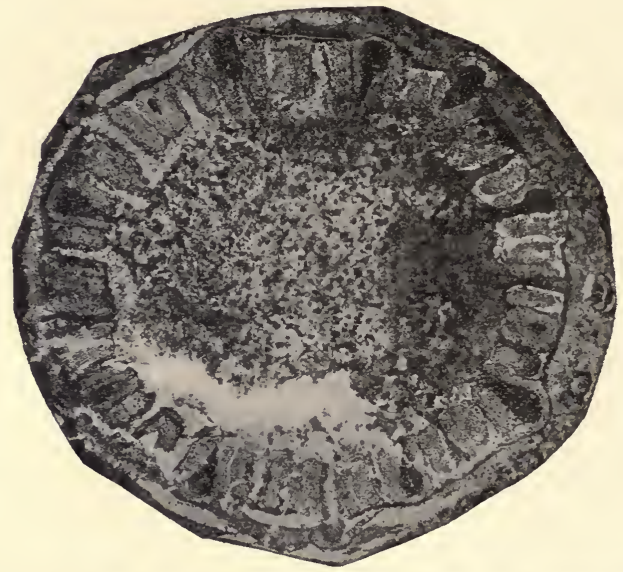

FIG. 203.-Transverse section of the stem of Osmundites skidegatensis

shall appropriately start with types from earlier geological periods. In Fig. 203 is shown a photograph of the central cylinder of a fossil osmundaceous stem from the Lower Cretaceous of Western Canada. In the stem under discussion the magnitude of the stelar system is much greater than that found in any living species belonging to the tribe and, in fact, more clearly resembles the conditions presented in a large stem of a fern of the ordinary type. The pith as well as the cortex is occupied by numerous dark masses of brown sclerenchyma - a condition closely resembling the state found in siphonostelic stems of ordinary ferns. An inspection of the periphery of the stele shows the presence of numerous 
foliar gaps, through some of which the cortex and the pith are actually continuous, as is made clear by Fig. 204, representing a small segment of the fibrovascular cylinder. In Fig. 205 is shown a still more magnified view of the marginal region of the stele, and it is here apparent that phloem is present both on the inside and on the outside of the stelar tube. The situation revealed by the three figures of the stem of Osmundites skidegatensis s how $n$ above makes it evident that in this ancient representative of the group a condition of organization more nearly resembling that found in our ordinary polypodiaceous ferns was present. The only important difference is furnished by the extremely numerous foliar gaps corresponding to the more

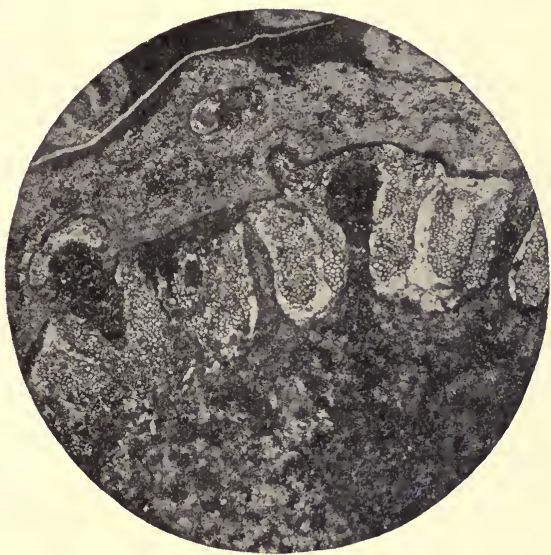

Fig. 204.-Part of the last, more highly magnified to show the leaf gaps. crowded condition of the leaves. This, of course, is not a morphologically important distinction.

After the description of an osmundaceous fern from the Mesozoic, the stele of living species of the Osmundaceae may conveniently be considered. Fig. 206 illustrates the organization of the central cylinder of Osmunda cinnamomea, the cinnamon fern. The fibrovascular tissues in this case are characterized by the same numerous foliar gaps as are found in $O$. skidegatensis, but the cortex and the pith do not communicate through them as in the Mesozoic type. Further, the phloem in the specimen under discussion, unlike that present in the cretaceous species, 
is confined to the outside of the xylem. Endodermal layers, both internal and external, are present, and the pith often contains patches of brown sclerotic cells similar to those found in the cortex. In spite of the fact that the bundles of the central cylinder of the stem are collateral, those of the leaves are concentric in organization. Applying the principles of comparative anatomy to the situation, we find that in $O$. cinnamomea the pith and cortex

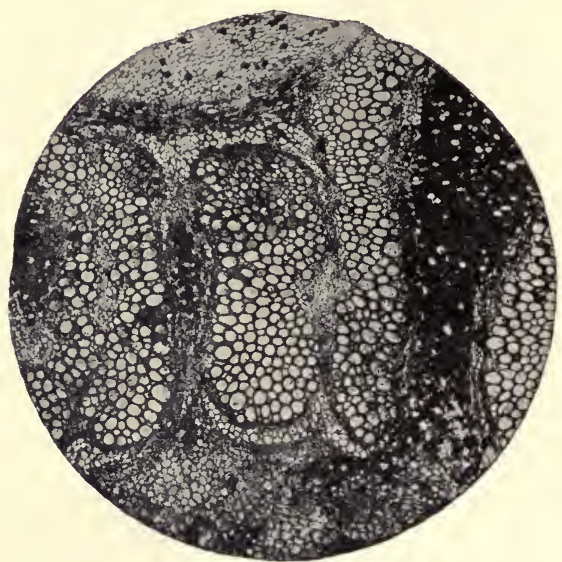

FIG. 205.-Part of the stem of $O$. skidegatensis, still more highly magnified to show the presence of both internal and external phloem.

are of common origin and that the bundles of the stele, on the evidence furnished by the foliar strands, were formerly concentric in structure. The conclusion which is reached from the consideration of the stem of the species under discussion, in the light of universally valid principles of comparative anatomy, is that its tubular stele was formerly concentric and that the foliar gaps were once large enough to permit of the joining of cortical and medullary tissues with one another. The inferences drawn from the data indicated above are, moreover, entirely justified by the consideration of the anatomy of the Lower Cretaceous species described in the preceding paragraph. In particularly vigorous specimens of the stem in O. cinnamomea the ancestral condition, moreover, frequently returns, for both open foliar gaps and internal phloem are often seen in such axes. In O. regalis among living representatives of the Osmundaceae medullary brown sclerenchyma is occasionally found in the pith, although an internal endo- 
the paleontological facts, but is likewise at variance with the fundamental principles of comparative anatomy. It presents the further serious difficulty of supposing that the simpler type of stele characteristic of the more modern representatives of the Osmundaceae, although primitive, has nevertheless come from a more complicated condition in the past. Finally, the hypothesis of the greater primitiveness of tubular steles without internal phloem supplies no valid explanation of the frequent occurrence of internal phloem, internal endodermis, and cortical sclerenchyma in the medullary region of various existing species of the Osmundaceae. The view that such structures where they occur are "physiological" has little to commend it and is, moreover, a hypothesis which cuts both ways; for if we are to interpret cortical structures occurring in the medulla as merely physiological, we must likewise consider the possibility of a similar explanation of the rare and quite exceptional occurrence of a so-called "mixed pith," consisting partially of tracheary tissue and partially of parenchyma.

The Osmundaceae on the whole present the evidence in regard to the evolution of the tubular stele in later geological times more clearly than does any other group of ferns. It should, however, be emphasized that in practically all cases where the siphonostelic central cylinder without internal phloem or endodermis is present there are found elements in the region of the medulla which present the characteristics of cortical tissues. It seems illogical to interpret these structures as possessing in every case merely a physiological significance, particularly since, in view of their imperfect and sporadic development, it is difficult to attribute to them any functional importance whatever. The structures in question possess, in fact, all the criteria of vestigial features persisting from an earlier more complicated condition of organization. Our knowledge of groups which have their climax of development in the past justifies the view that evolution in decadent series proceeds by simplification. A final general objection to the tracheary hypothesis of the origin of the pith is the outstanding fact that the more modern groups of plants, and especially the seed plants, are the ones which particularly and universally represent 
the condition which the supporters of this hypothesis regard as primitive - that of the tubular stele without internal phloem and endodermis. On the other hand, the Filicales, in which the siphonostele commonly incloses a pith strikingly resembling the tissues of the cortex and quite generally lined inwardly with endodermis and phloem, must be regarded as more modern. The improbability of this general hypothesis seems very great.

It may be assumed on the basis of the considerations advanced in earlier paragraphs that the siphonostelic condition in the Filicales is susceptible of complication through the development of medullary strands on the one hand, and, on the other, of simplification through the loss of internal phloem and endodermis and the progressive narrowing of the leaf gaps resulting in sequestration of the pith. The view that the latter simpler condition is more primitive not only runs counter to the conditions shown in the general sequence of types in geological time, but is also at variance with the general principles of comparative anatomy detailed in a former chapter of this work. The hypothesis that the simpler condition of the tubular stele is more primitive than the more complex marks, moreover, an evolutionary attitude which is becoming generally obsolete as our knowledge of the actual organization of extinct forms, particularly of those types which manifested their greatest luxuriance in the past, becomes fuller and more complete. It is now clear from evidence of this kind that the lower gymnosperms have come from the Filicales as a result of the simplification and reduction of the primary structures of the stele of the stem on the one hand, accompanied by the marked development of secondary fibrovascular tissues on the other. In the gymnospermous Pteropsida this anatomical progress has been associated with the attainment of the seed habit. In the Lycopsida, by contrast, a similar anatomical progress culminated in much earlier geological times and was not accompanied by the evolution of true seeds. 


\section{CHAPTER XXII}

\section{THE ARCHIGYMNOSPERMAE: CYCADOFILICALES}

\section{AND CYCADALES}

The earlier seed plants of Paleozoic and Mesozoic time were characterized by the possession of naked seeds upon which the microspores or pollen grains were directly deposited. The free exposure of the seminal structures to the air has gained for the types marked by this feature the appellation of gymnosperms. In the large group thus characterized there are two main modifications. In the earlier and more primitive gymnosperms the pollen grains or microspores were accommodated in a chamber in the apex of the megasporangium known as the pollen chamber. This cavity, filled with fluid derived either from the surrounding parenchymatous cells or in some cases from a special fibrovascular system present in the walls of the megasporangium, provided for the germination of the microspores and the subsequent fertilization, effected in every instance by swimming sperms. These forms in general present a marked resemblance to the Filicales, often in external habit and always in internal andtomical structure. In contrast to them are the higher gymnosperms, in which there is no true pollen chamber. Here the act of fertilization is effected, not by swimming sperms, but by means of a pollen tube developed as an outgrowth of the microspore and directed toward the egg. The external habit of these gymnosperms is never fernlike, and their anatomical organization shows only the slightest cryptogamic features in living forms. The types possessing pollen chamber, swimming sperms, frequent filicinean habit, and cryptogamic organization of the fibrovascular structures are conveniently designated as the Archigymnospermae or more primitive gymnosperms. Those forms which are contrasted to these by the absence of pollen chamber and the presence of pollen tube in connection with fertilization, as well as by the disappearance of filicinean habit and anatomical characteristics, may be, on the other hand, designated as the Metagymnospermae or higher gymnosperms. 
The present and the following chapter will deal with the representatives of the Archigymnospermae.

Our knowledge of the Archigymnospermae, from the fact that many of the group are extinct, is necessarily incomplete. It will accordingly be convenient in the present chapter to focus attention on the best-known fossil group and the nearly related family which possesses living representatives. The extinct aggregation of forms which here present the strongest claim to attention are the Cycad ofilicales, characterized by a habit so closely resembling that of the true ferns that it is only since the very end of the last century that they have been recognized as seed plants. The type of seed connected with the vegetative structures of the Cycadofilicales

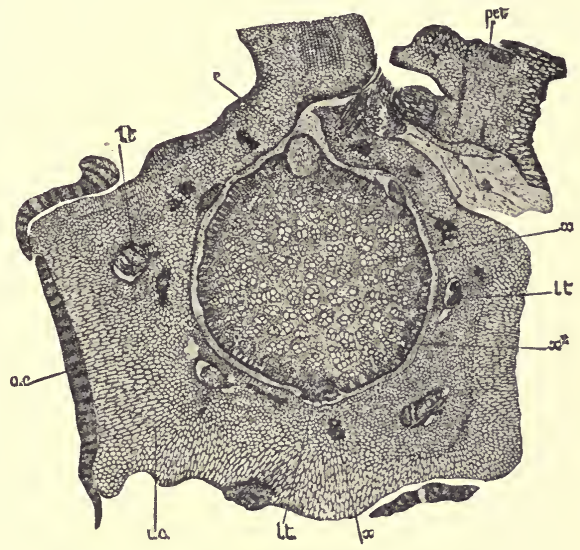

FIG. 207.-Transverse section of the stem of Heterangium (after Scott).

has been diagrammatically indicated in an earlier chapter, to which the reader is here referred. In the present connection only anatomical features of the group need be discussed. The antiquity of the Cycadofilicales is vouched for, not only by their Paleozoic occurrence, but also by the occasional presence of protostelic stems, axes of this type not being known for any other group of seed plants living or extinct. Fig. 207 illustrates the organization of the protostelic axis of the genus Heterangium from the English Carboniferous. The transverse section shows the presence of an external ribbing of sclerenchymatous strands, a feature common to many older representatives of the Gymnospermae. The fibrovascular apparatus consists of protostelic primary wood surrounded by a thin layer of secondary xylem. 
We may now turn our attention to more complicated stems belonging to the general group of the Cycadofilicales. The genus Medullosa is of particular importance, since it possesses a type of stelar organization presented by several or many concentric strands which are best considered as belonging to a siphonostelic cylinder. The general organization of the axis in the genus under discussion is well shown in Fig. 208, which portrays in a somewhat diagrammatic fashion the structures involved. There are three large concentric fibrovascular strands present which consist of a central core of primary wood indicated by cross-hatching, surrounded

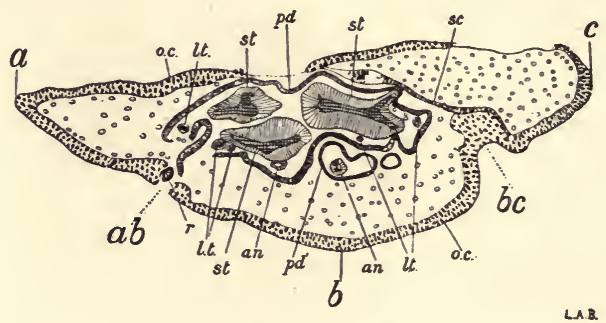

Fig. 208.-Transverse section of the stem of Medullosa anglica (after Scott)

with a cordon of secondary xylem, represented by radiating lines. The outline of the stem is irregular, the salients being due to the presence of the bases of large leaves. The surface is covered with the reticulated sclerenchymatous strands already described in the case of Heterangium. The fundamental tissues are occupied by secretory canals, which probably contained mucilage, as do those of the living Cycadales. In the cortical region are accommodated larger and smaller strands ordinarily lacking secondary growth. The bundles of greater dimensions are mostly the larger foliar bundles and possess concentric organization. The small strands which are found characteristically in the actual leaf bases are collateral in their organization and exarch in the structure of their xylem. Fig. 209 illustrates two of the smaller strands together with fundamental tissues and contained mucilage canals. The general situation in the genus Medullosa is complicated frequently 
by the presence of more numerous large concentric fibrovascular strands than those shown in Fig. 208. In such instances the concentric strands often show a more or less complete degeneracy of the secondary xylem on their inner surfaces. This situation has been regarded with reason as of significance in foreshadowing the type of organization found in the axes of the Cycadales, living and extinct. It will be seen from the account of Medullosa here supplied that the genus presents some marked features of anatomical resemblance to ferns, the main contrasts in organization being due to the appearance of secondary growth in connection with the strands belonging to the stem proper. In accordance with the canons of anatomy formulated in an earlier chapter of the present work, the secondary activity has not yet penetrated in to the traces which pass out into the more con-

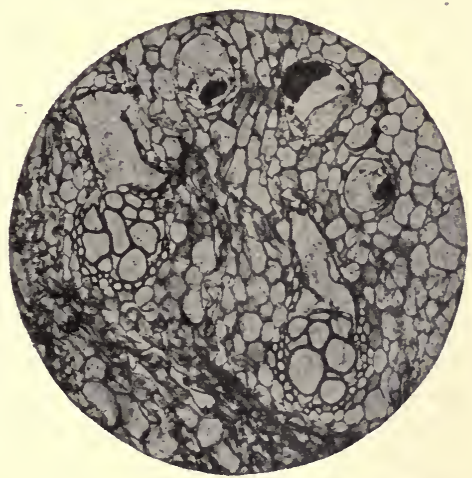

FiG. 209.-Foliar bundles of Medullosa anglica servative foliar structures.

The Medulloseae are undoubtedly of great interest from the standpoint of the evolution of cycadean forms, and there can be little question that these types so common as charred remains in the Carboniferous coals of Europe and America came very near to being the actual ancestors of our living Cycadales.

As a third illustration of the Cycadofilicales we may take the genus Lyginodendron, the organization of which has been so admirably described by English anatomists. Fig. 210 illustrates the structure of the stem in this genus. The same sclerified ribbing is observed as in the other two genera discussed above. The central cylinder, however, presents a marked contrast to that of either Heterangium or Medullosa, for it consists of collateral strands arranged in a closed circle. The pith is occupied by sclerotic 
nests of cells which are duplicated by similar structures in the cortex. The inner surface of the cylinder of xylem shows clusters of primary wood which sharply contrast with the secondary region by the irregularity of the arrangement of the elements and the absence of rays. Fig. 2I I illustrates a portion of the cylinder more highly magnified, and with the greater enlargement the distinct and mesarch character of the primary region becomes apparent. The secondary wood is characterized both by its regularly radial arrangement and by the presence of rays which

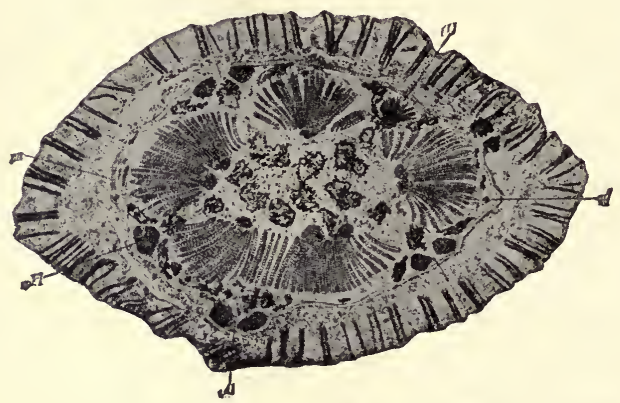

Fig. 2ro.-Transverse section of the stem of Lyginodendron oldhamium (after Scott).

are often two or three cells in width. The tracheids of secondary origin are marked by crowded pits which alternate in position and are often angular from mutual contact. These pores are confined strictly to the radial walls of the elements, no tangential pitting occurring in the secondary tracheids of any Paleozoic gymnosperms with which we are at the present time acquainted. The foliar traces in Lyginodendron are at first single, but bifurcate shortly after leaving the primary region of the stem, and lose their secondary xylem, becoming concentric instead of collateral in their organization. It is evident on the basis of the general canons earlier elucidated that the bundles of the stem in the case of Lyginodendron must formerly have been concentric in their structure, since this condition persists in the traces of the leaves. Further, the presence of secondary wood in the stem 
and its absence in the foliar traces show that secondary growth is a comparatively recently acquired feature in the genus under discussion. The sclerotic nests in the pith appear to vouch for the extra-stelar origin of the medullary region. The situation as a whole is like that found in the case of the Osmundaceae, except for the complication introduced by the presence of the secondary growth. In the genus Botrychium among the Ophioglossaceae secondary tissues, however, are often well developed, and it has long been realized that the occurrence of secondary activity in the fibrovascular tissues is not an extremely important criterion from the evolutionary standpoint. It was at one time thought that the genus Lyginodendron was the prototype of the Cycadales, but this opinion has been given up in favor of a derivation of

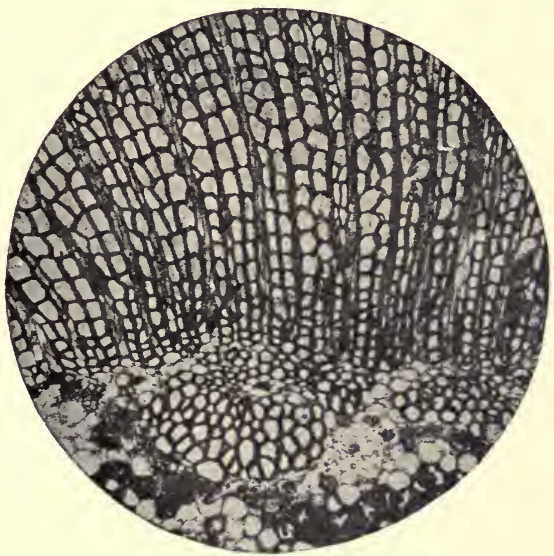

FIG. 2II.-Transverse section of primary and secondary wood in Lyginodendron.

the cycadean type from forms like the Medulloseae, for which the evidence is extremely strong.

We may now turn our attention to the anatomical organization of that group of gymnosperms which still persists as the Cycadales. This family was well developed in Mesozoic times, but there is no evidence that it was represented in the Paleozoic age. The stem of the cycadean gymnosperms, in its modern representatives at any rate, is soft and parenchymatous and is characterized by a very large medulla. Both cortex and medulla contain numerous mucilage canals. The woody cylinder is ordinarily very thin 
and in the vegetative axes does not manifest the presence of cryptogamic or centripetal wood. In certain genera of the Cycadales, both living and extinct, the woody cylinder often shows a curious reduplication, resulting from the appearance of new circles of fibrovascular tissue in the pericycle. The explanation of these structures is somewhat difficult. One view is that they represent a persistence of the numerous cycles of fibrovascular strands in certain types of Medulloseae. This view, however, is rendered difficult by a situation which often presents itself in the Cycadales. In the region of the stem where a cone is attached the medulla is frequently occupied by numerous strands which are absent elsewhere. It is natural to regard such structures as representing a return of the medullosan organization in relation to the attachment of conservative reproductive axes. If this explanation be adopted for the medullary bundles, the interpretation of the supernumerary zones of fibrovascular tissue formed successively in the pericycle of the original cylinder cannot be accepted. A more probable elucidation of the situation is furnished by the habit of certain Mesozoic representatives of the group which are contrasted to modern forms by their slender and freely branching habit. This condition, for example, is found in the genus Anomozamites. It is not improbable that the genus mentioned was a climbing plant. The formation of supernumerary zones of fibrovascular tissue is a common feature of organization of the stem in climbing plants of widely diverse gymnospermous and dicotyledonous affinities. It is consequently not unlikely that the Mesozoic forebears of living Cycadales developed successive zones of fibrovascular tissues in their stems as a result of a climbing habit. After this feature had become thoroughly fixed by the lapse of long geological time, a desert habit was superimposed on it with a resulting anatomical organization such as is found in the living representatives of the group. A similar hypothesis has to be invoked, as will be shown in a subsequent chapter, to explain the remarkable anatomical resemblance between the desert-inhabiting gnetalian genus Welwitschia and the vinelike Gnetum.

Although there is no instance of the presence of centripetal or cryptogamic wood in the vegetative axis of the Cycadales, 
not a few examples of the existence of xylem of this type have been described by Scott in the reproductive axes or cones. In Fig. 2 I 2 is shown a transverse section of part of the peduncle of the female cone of Stangeria paradoxa. Although the wood as a whole is strongly centrifugal or peripheral in its development, a

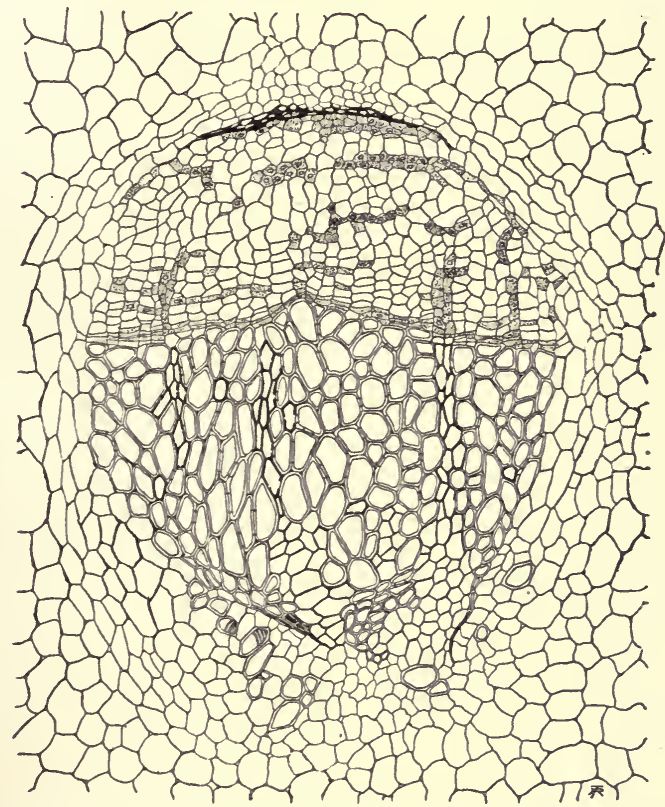

Fig. 21 2. -Transverse section of one of the strands of the peduncle of the cone in Stangeria paradoxa.

small amount is formed over against the medullary region. This condition is a persistence of the centripetal wood of the Paleozoic Cycadofilicales, from which the Cycadales have in all probability been derived. The anatomical principle here involved is one of great importance and was first clearly emphasized by Scott. Subsequent to his observations on the Cycadales, it has been 
discovered to be exemplified in a remarkable manner by the observation of the wood in the axes of members of the Coniferales, as will be noted at a later stage.

The leaves of the Cycadales are of great importance from the standpoint of evolutionary anatomy. Here the centripetal wood, which is poorly developed at best in reproductive axes, is abundant, well defined, and universal in its occurrence. Fig. 213 illustrates the transverse section of a leaflet of $C y c a s$ revoluta. It is apparent

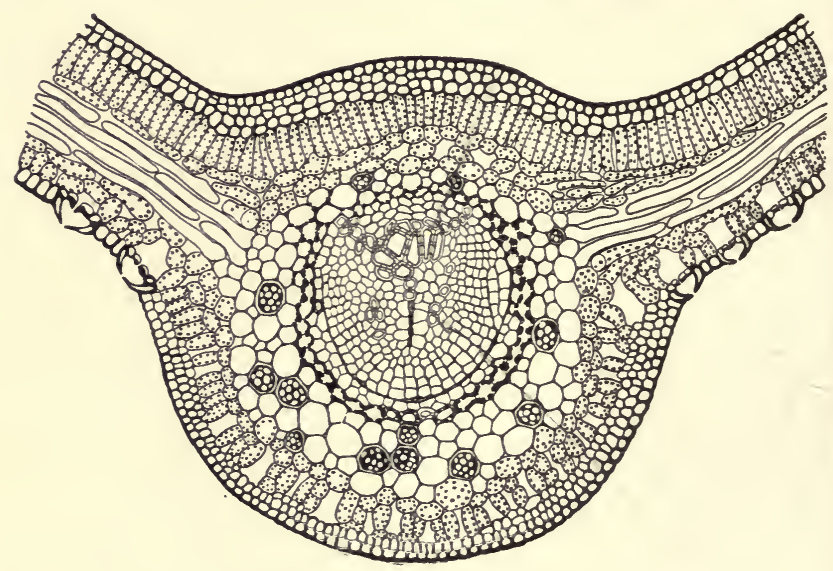

FIG. 213.-Transverse section of a foliar strand in Cycas revoluta

that the mass of the xylem is of primary origin and spreads out in a fanlike fashion toward the upper surface of the leaf. It is therefore centripetal in its development. Below the mass of centripetal xylem lies a much more scanty development in the region of the regularly arranged phloem. This is the centrifugal xylem, which is partially primary and partially secondary in its origin. Fig. 2I4 illustrates the longitudinal section of a leaf trace in Cycas. The phloem, easily recognized by its characteristic sieve tubes, is seen toward the right. To the left is situated the centripetal wood, composed from left to right of pitted, scalariform, reticulate, and spiral elements, the latter constituting the protoxy- 
lem. After a narrow parenchymatous interval the pitted elements of the centrifugal metaxylem are seen in close proximity to the phloem. The general situation in the transverse and longitudinal sections of the foliar strands shown in the two figures is universal for the Cycadales, any minor divergences which have been observed being mainly the result of the smaller size of the strands in the genera other than Cycas.

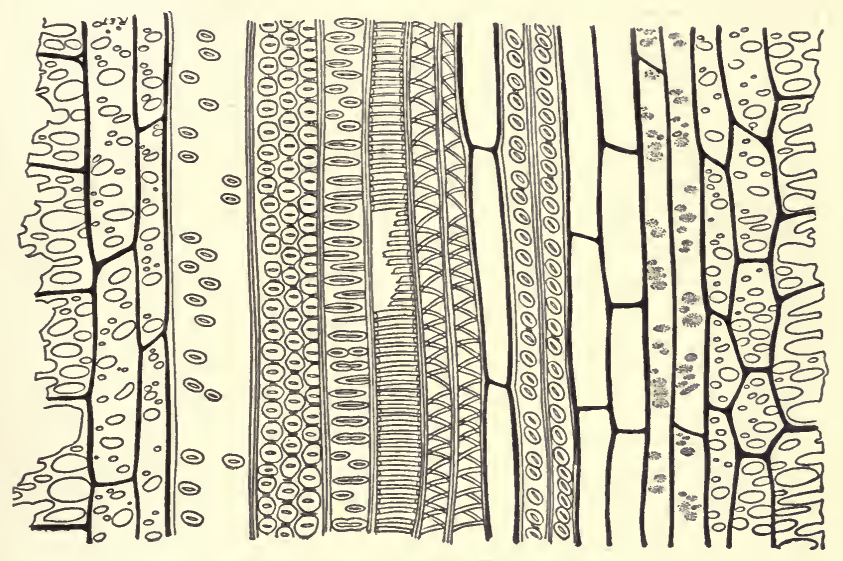

FIG. 214,-Longitudinal section of a foliar strand in Cycas revoluta

The foliar traces in the genus under consideration show other interesting features of organization in the lower part of their course, where they pass from the base of the petiole of the leaf into the cortex of the axis. These features have to do both with the arrangement of the traces and with their anatomical organization. In the vegetative axes of living genera of the Cycadales the foliar strands, instead of passing directly outward to their respective leaves, pursue a meandering course through the cortex and are known as "girdles." This condition is confined to the modern genera, for in the Mesozoic representatives of the group united under the caption of Bennettitales the leaf traces pass directly to their corresponding leaves. Interestingly enough this 
condition is paralleled in the reproductive axes of the cones of the living Cycadales and in the seedling of their most primitive genus, Cycas.

As regards anatomical organization the foliar traces in their cortical course, and even sometimes in the bases of the petioles, show an interesting resemblance to the bundles of the stem in Medullosa. Fig. 2I5 illustrates the structure of one of the leaf

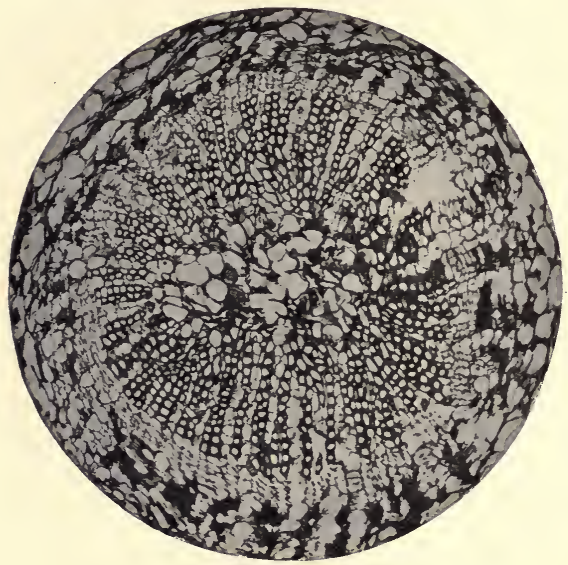

Fic. 21 5.-Cortical strand of Cycas revoluta traces of Cycas revoluta as it passes through the cortex. The bundle is clearly concentric and consists of a central core of primary wood surrounded by regularly seriate secondary xylem and traversed by wood rays. If the figure under discussion be compared with that of a stem bundle of Medullosa (Fig. 208), it

becomes evident that the only striking difference is presented by the much smaller size of the strand of the living genus.

The root in the Cycadales presents little that is of interest, since the feature of the presence of centripetal wood is equally exemplified by all roots as a characteristic connected with their extremely conservative organization. It is only between Mesozoic and modern types in which the evolutionary importance of the centripetal wood has passed into the background that the anatomical structure of the root comes into the phylogenetic foreground in connection with the doctrine of descent. A feature not of evolutionary interest presented by the cycadean root is the frequent presence of root tubercles. 
The Mesozoic Cycadales are ordinarily grouped under the heading Bennettitales on account of the remarkable features presented by their reproductive structures, which are very different from those exemplified by any living Cycadales. The uniting of both microsporophylls and megasporophylls in the same strobilus is a frequent feature of this group and has been considered by some to indicate an affinity with the angiosperms, particularly as the ovuliferous sporophylls are shut in by a panoply provided by the swollen apices of so-called interseminal scales-sterile structures interposed among the fertile ones. The question of the possible descent of the angiosperms from the Bennettitales is one which can scarcely be considered seriously from the anatomical standpoint, since there is practically nothing in common in the anatomical organization of the two groups, either in the reproductive or in the vegetative features. The fructifications of the Bennettitales before their real affinities were known were attributed by the distinguished French paleobotanist Saporta to a group which he designated as proangiosperms. Now that their actual relationships are known, there seems scarcely any reason for regarding them as allied to the angiosperms. The Bennettitales supply an excellent illustration of the relative conservatism of anatomical structures, for, although they present marked differences in reproductive features from the Cycadales, their vegetative anatomy does not differ in any important particular from that of the modern group.

If the anatomical situation for the Cycadales in the large sense be summarized, it becomes clear on the basis of the general canons of comparative anatomy elucidated in earlier pages of the present volume that they exhibit strong filicinean features. The anatomical examination of the foliar strands reveals cryptogamic or centripetal wood and in some cases concentric organization. The evidence derived from the consideration of the anatomy of the leaf is confirmed in a number of instances by the organization of the strands of xylem in the reproductive axis. The testimony of the conservative parts leads to the assumption of the former presence of centripetal xylem and concentric fibrovascular strands in the vegetative stem. The general result reached on 
the basis of comparative anatomy receives strong support from the organization of certain Paleozoic Cycadofilicales, notably the Medulloseae. There seems to be little doubt that the genera of Cycadales in the broadest sense of the term have been derived from ancestors closely resembling vegetatively the Medullosa type. It follows that so far as their primary fibrovascular structures.are concerned they present a condition of reduction from the anatomical organization found in the oldest and most fernlike gymnosperms. 


\section{CHAPTER XXIII}

\section{THE ARCHIGYMNOSPERMAE: CORDAITALES AND GINKGOALES}

The two tribes of gymnosperms discussed in the present chapter no longer possess the fernlike habit of the Archigymnospermae which have just been considered, but still maintain the pollen chamber and the cryptogamic wood so characteristic of the Cycadofilicales and Cycadales. Of the two groups here discussed the Cordaitales became entirely extinct at the end of the Paleozoic or at the beginning of the Mesozoic and have a geological range extending far into the past. On the other hand, the Ginkgoales, although present somewhat doubtfully in the Paleozoic, had their greatest development in the Mesozoic and persist at the present time in a monotypic genus of Eastern Asia.

The Cordaitales were freely branched trees resembling in general habit our existing conifers, to which they are usually considered to have given origin. The trunks often reached a considerable size, and the woody cylinder included a large pith, in some instances septate and occupied by air spaces, which, however, unlike the medullary fistulae of Calamites, were much more numerous than the internodes. The cavernous character of the pith as well as its large size is responsible for the existence of medullary casts in the case of the Cordaitales, and these are assembled under the form-genus Artisia or Sternbergia. The surface of the stem was often provided with the sclerenchymatous armor which has been noted as a feature of organization of the axes of the Cycadofilicales. Fig. 2I6 illustrates the general topography of a segment of the stem in a cordaitean form. The woody cylinder was without annual rings, except in the later Paleozoic and in higher latitudes. This situation is very well shown by Figs. 21 7 and 218, illustrating the structure of cordaitean woods from Northern England (Lancashire) and Southern Canada (Prince Edward Island). In the first specimen annual increments can be somewhat clearly distinguished, while in the second they 
are conspicuous by their absence.. Although some indication of the existence of annual diversities of temperature is supplied by certain cordaitean stems, the organization of the annual ring in such cases is extremely simple, and even the differentiation involved in the presence of terminal tangential pitting is not seen, as may be ascertained by reference to Fig. 219, representing a specimen from Lancashire, England, in stereoscopic view under a high magnification. It is apparent from the figures that the

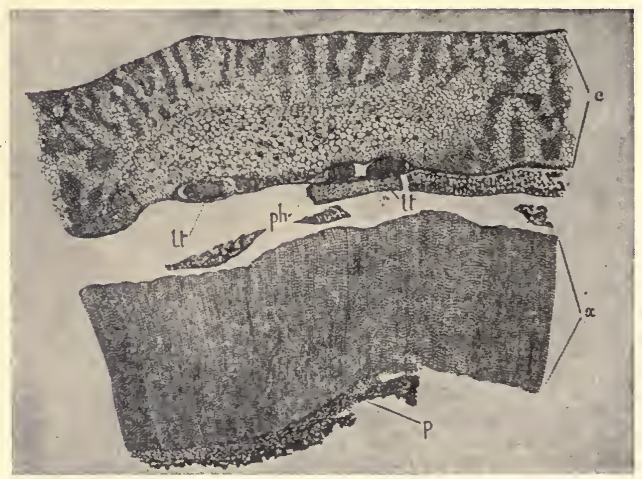

Fig. 216.-Part of a transverse section of a cordaitean stem (after Scott)

secondary xylem of Cordaitales was of very simple structure and that the rays, unlike those of the two tribes considered in a previous chapter, were usually a single row of cells in width. This narrowness of the strands of radial parenchyma is shared by the Cordaitales with the Ginkgoales and the conifers. The wood, like that of other Paleozoic gymnosperms, was entirely without longitudinal parenchymatous elements, and the bordered pits were strictly confined to the radial walls of the tracheids.

Another interesting feature of the organization of the xylem in cordaitean forms was the extremely long region of transition between the spiral elements of the protoxylem and the first pitted 
elements of the secondary wood. This is well shown in Fig. 220. Among the living conifers this condition is most nearly paralleled by the Abietineae.

The foliar trace is of course of great importance in this as in other ancient $\mathrm{g}$ y m nos perms. Fig. 22 I illustrates the organization of one of the bundles of a broad cordaitean leaf (Cordaites principalis) in both transverse and longitudinal section. In $a$ is shown the transverse topography of the strand,

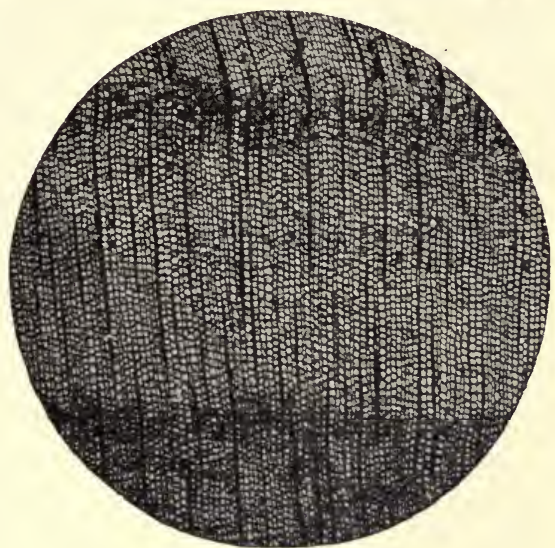

FIG. 217.-Cordaitean wood from England and it is clear that the centripetal wood is well developed, ending

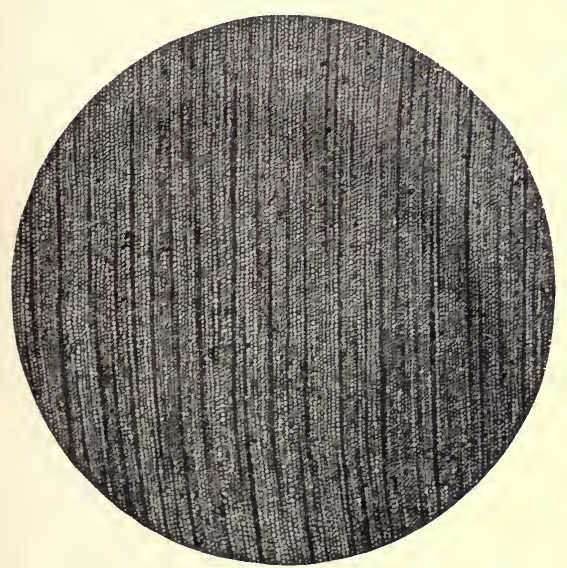

FIG. 218.-Cordaitean wood from Prince Edward Island upwardly in large elements which are pitted in their character. In the particular instance figured there happens to be no development of the centrifugal xylem, so that the phloem abuts immediately on the protoxylem. With the flanks of the metaxylem is connected a zone of narrow thick-walled elements which form 
a cordon completely around the phloem. Outside the cells of narrow lumen is found a second zone, composed of elements larger in diameter. Both outer and inner zones consist of tracheary elements

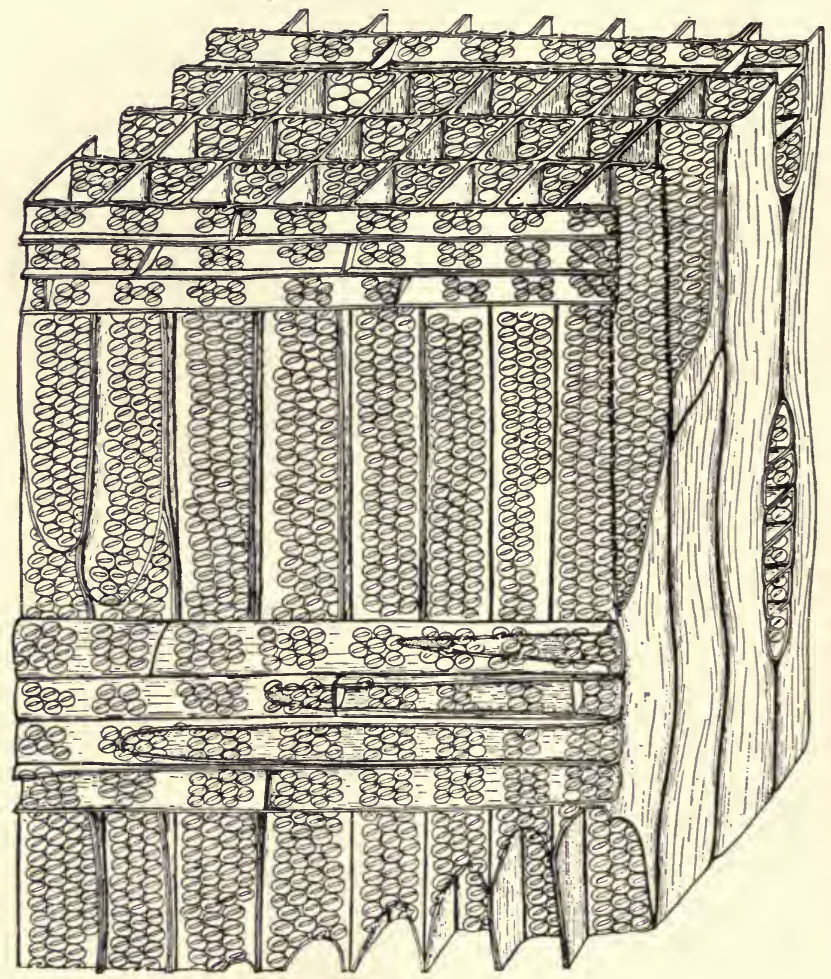

FIG. 219.-Stereoscopic view of a cordaitean wood from Lancashire, England

and constitute the so-called double transfusion sheath. In $b$, the longitudinal view, the various elements of the bundle are shown in the median section. The protoxylem is continuous with tracheids which by the usual transitions gradually pass into the 
pitted elements of the last-formed metaxylem. Below the protoxylem lies the phloem, and still farther down the more elongated and narrower elements of the inner transfusion sheath, which in turn abut on the short, broad tracheary elements of the outer transfusion sheath. At the very top lie other short transfusion cells, and the inner elongated sheath in this region is absent as a result of conditions which can readily be inferred from the inspection of the transverse view in $a$. There is some variety in the development of the foliar bundles of the Cordaitales, but all are characterized by the presence of well-marked centripetal wood and a cordon of short tracheary elements, known as trans-

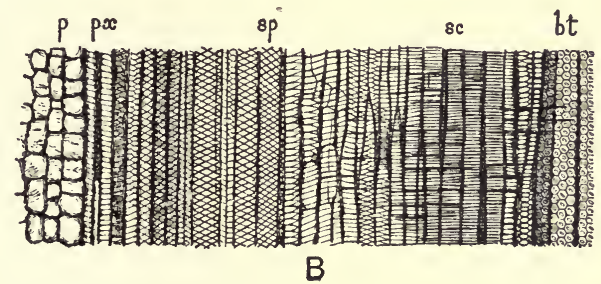

FIG. 220.-Longitudinal view of cordaitean wood near the pith (after Scott)

fusion cells, which are closely related to the centripetal or cryptogamic wood.

The root in cordaitean forms, for reasons applying equally to all Paleozoic gymnosperms, presents no features of special interest beyond illustrating the general cordaitean organization modified to the needs of root organs.

It will be obvious from the statements made in the foregoing paragraphs that there is clear evidence in the organization of the foliar structures in the Cordaitales for their close affinity with the Filicales, although naturally the degree of relationship is much less intimate than that which characterizes the Cycadofilicales and even the Cycadales. Concerning the organization of the microsporangia and seeds of the Cordaitales our knowledge is unfortunately somewhat meager. The evidence in regard to the microsporangium is not sufficiently definite to warrant an opinion as to whether it was ectokinetic or endokinetic in its 
mode of dehiscence; but, in view of the strong development of transfusion tissue in the foliar organs of the group under discussion, a clear feature of distinction from the Cycadales and Cycadofilicales (in both of which the microsporangia are ectokinetic), it is somewhat probable that the pollen sacs owed their dehiscence to a layer of tracheary origin. The seeds of Cordaites have been

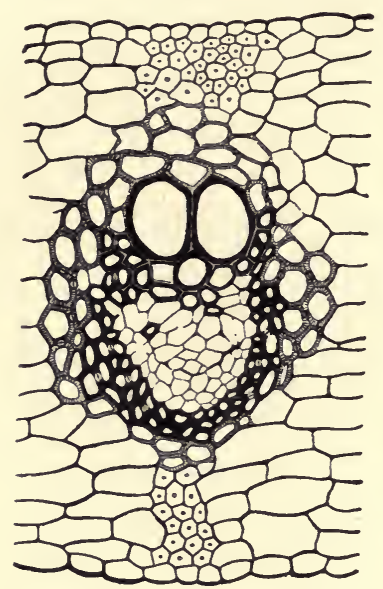

$a$

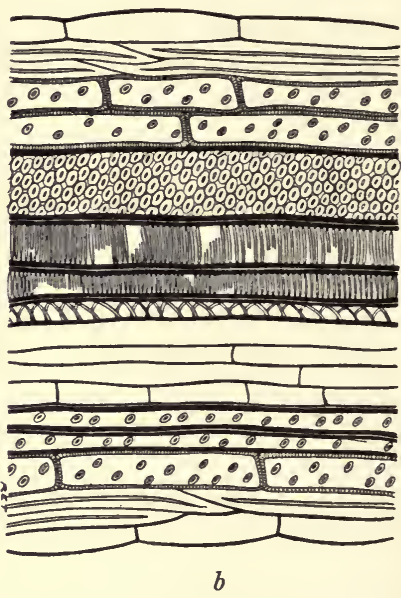

$b$

Fig. 221.-Transverse and longitudinal sections of a leaf bundle in Cordaites principalis.

anatomically investigated by Renault and they possessed a wellmarked pollen chamber. A more complete knowledge of the reproductive structures of cordaitean forms and of the types which connected them in the more remote Paleozoic with filicinean ancestors is much to be desired.

The Ginkgoales are represented by a single living genus, but were extremely abundant in the Mesozoic and are thought to have been continued into the Paleozoic by the somewhat problematical genus Whittleseya. Unfortunately our anatomical knowledge of the group beyond that supplied by the investigation of the 
living genus is extremely meager and in fact is confined to the structure of woods which have been referred to the group.

The stem in Ginkgo is characterized by the presence of clear annual rings which terminate with tracheids provided with tangential pits and in this respect reveal a marked contrast to the tracheary elements constituting the remainder of the annual increment. It is obvious that as regards the organization of the annual ring the group under discussion is distinct from those rare cordaitean stems in which yearly zones of growth can be distinguished, by the presence of tangential terminal pitting. In other respects, however, the structure of the wood is clearly archaic, for there are no parenchymatous elements present other than those related to the rays. The pith and cortex in the group possess secretory canals which are comparable to those found in certain Abietineae.

The longitudinal aspect of the secondary xylem in Ginkgo is very different from that of the Cordaitales. In the more ancient group the radial pits are often extremely numerous and they are then angular by mutual contact. In Ginkgo the pores of the tracheids are not so abundant as to be described as crowded and, moreover, instead of being alternating and angular as in the older tribe are round and opposite. Another equally striking feature offers itself in the presence of transverse bars of pectic cellulose in the walls of the tracheids between the pairs of opposite pits. These may conveniently be designated bars of Sanio, to distinguish them from the trabeculae of Sanio, structures which are found not uncommonly in all woods of secondary origin from (and including) those of the Cycadales to those of the dicotyledons. The latter structures consist of ligneous processes crossing the cavity of the tracheid, possibly due to the activity of parasitic fungi, while the true bars of Sanio are concealed in the wall itself and consist of pectic cellulose. Bars of Sanio are found only in the walls of tracheids of secondary origin, and statements as to their occurrence in any elements of the primary wood are erroneous.

An interesting condition appears in the organization of the secondary wood of the peduncle of the seed. In Fig. 222 is shown the transition region in the xylem. It will be observed 
that the pitted tracheids nearest to the primary wood are entirely without bars of Sanio, which make their appearance only at an interval from the protoxylem. The pitting to a large extent is alternate. It is very generally admitted by competent judges at the present time that the Ginkgoales are derived from cordaitean ancestry, and it is accordingly highly interesting to find in the
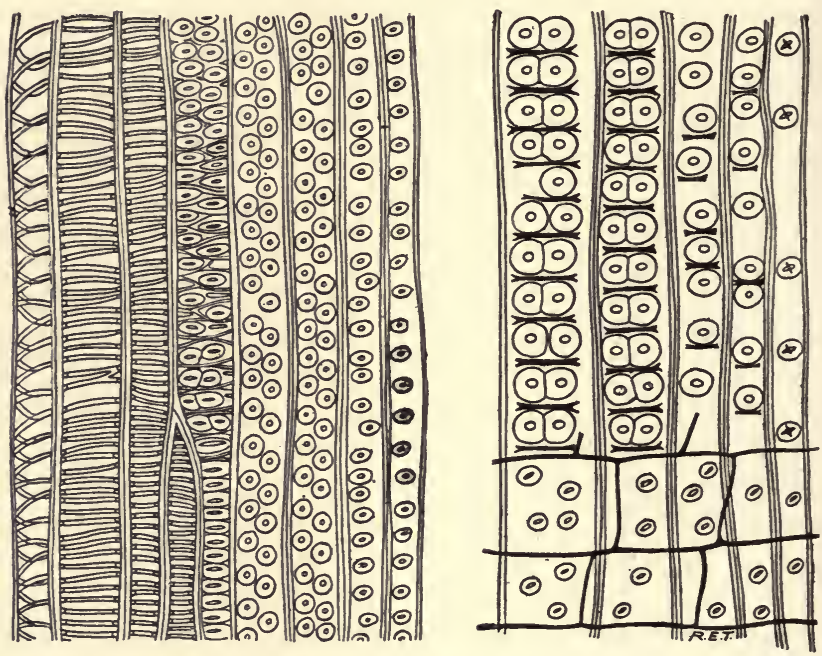

FIG. 222. - Longitudinal view of the tracheids in the peduncle of a seed in Ginkgo. To the right is shown the arrangement of the tracheids in the mature wood.

organization of vegetative and reproductive axes evidence based on the pitting and distribution of the bars of Sanio favorable to such an opinion. Farther away from the primary wood the secondary tracheids quickly develop the opposite pitting and bars of Sanio characteristic of the mature wood. It is clear from the figure, moreover, that the tracheids of the primary wood are quite devoid of structures of the nature of the bars of Sanio. It is well to emphasize the conditions found in the organization of the root and reproductive axis of Ginkgo, because there prevails 
at the present time an almost inexcusable ignorance in regard to the nature and distribution of the structures here designated bars of Sanio. They are clearly correlated with opposite pitting and are a feature of the secondary wood, not appearing in the organization of the tracheids of the primary xylem. Evidently the structures in question are of considerable value in the identification of gymnospermous woods and consequently must rank high as a diagnostic criterion among competent anatomists. The mature vegetative leaf in Ginkgo supplies very little evidence

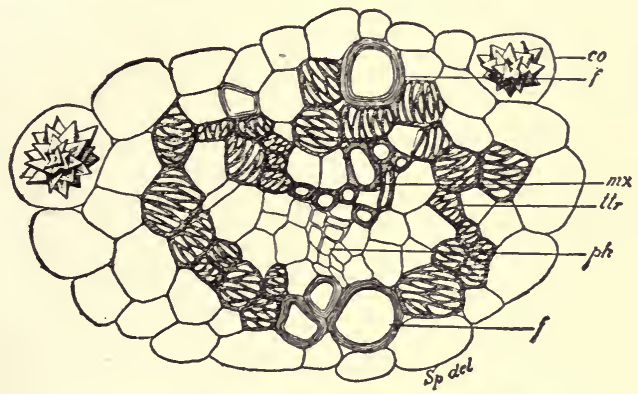

Fic. 223.-Foliar bundle of Ginkgo, showing transfusion tissue (after Sprecher)

of the presence of centripetal elements in the strict sense of the term. In the terminal region of the blade of the leaf a wellmarked zone of transfusion tissue manifests itself, as is shown in Fig. 223; but typical centripetal tracheids are usually conspicuous by their absence. In the cotyledon, however, the centripetal or cryptogamic wood is present in a much clearer manner in accordance with the principle of recapitulation discussed in an earlier chapter. The reproductive leaves, both ovuliferous and staminate, also show the centripetal elements in a good condition of development, although even here they more nearly resemble transfusion tissue. In the stalk which supports the pair of ovules centripetal elements and ordinary transfusion cells are seen in the upper region in great abundance and are likewise found in the collar surrounding the base of the seeds. The situation presented by the 
microsporophyll is, however, of greater interest in the present connection. In the petiole of the bisporangiate microsporophyll tracheary elements of a centripetal character occur on the upper side of the protoxylem (Fig. 224). These elements can scarcely

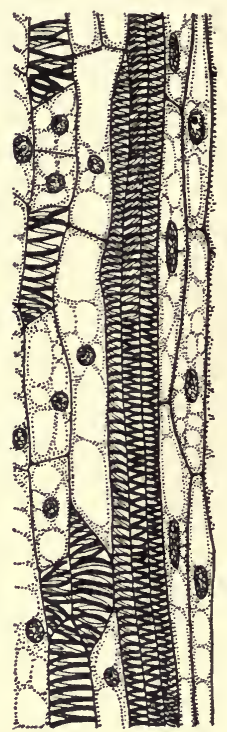

$a$ be said to constitute typical centripetal tracheids, since they are often of wide lumen and are correspondingly abbreviated in length. As the foliar traces ascend into proximity to the sporangia, they separate from one another and the xylem of each rotates so as to occupy a position near the middle line of the

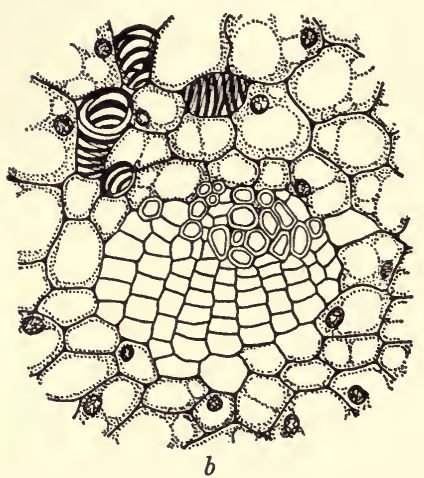

FIG. 224. - (a) longitudinal, (b) transverse, section of wood of bundle in microsporophyll of Ginkgo.

sporophyll, while the strands of phloem turn outward, to end in the bases of the sporangia. Meanwhile the transfusion elements occurring on the upper side of the tracheary strands in their upward course pass imperceptibly into the fibrously thickened mechanical elements which are responsible for the dehiscence of the sporangium. Further, the apex of the tracheary strands passes gradually by means of short transfusion tracheids into the mechanical elements which lie along the median sides of the sporangia. In this fashion there is established an intimate relation between the tracheary tissues of the bundles of the reticulate cells which constitute the 
opening mechanism of the microsporangia. The microsporophyll of Ginkgo accordingly has a double interest from the evolutionary standpoint, for it not only shows the centripetal or cryptogamic wood more clearly than it is exhibited by the vegetative leaves, but at the same time manifests its transition by imperceptible gradations into the mechanical tissues of the sporangium wall. As has been indicated in an earlier chapter, Ginkgo is the lowest type in which the dehiscence of the microsporangium no longer depends on an annulus derived from the epidermis but is effected by an internal mechanism derived from the old centripetal or cryptogamic wood of the fibrovascular bundle.

In the stalk of the ovule centripetal elements and transfusion tissue are also well developed, but they apparently do not at any time penetrate into the substance of the megasporangium. It is not unlikely that tracheary tissues of a transfusionary nature were formerly present in the megasporangial structures of the Ginkgoales, but that in the course of time they have suffered abortion. The organization of a number of seeds of Paleozoic age of unascertained affinities is good evidence in favor of the probability of this view. Moreover, in one of these, Stephanospermum, characterized by a tracheary mantle in the wall of the nucellus ending in the pollen chamber, pollen grains are present, winged all around and strongly resembling those of Ginkgo. It is accordingly not impossible that Stephanospermum was the seed of some Paleozoic representative of the Ginkgoales.

The importance of the sole surviving and monotypic genus Ginkgo from the standpoint of the evolutionary transition from the ancient to the modern gymnosperms cannot be overestimated. It constitutes virtually a link between the Archigymnospermae and the Metagymnospermae, since it presents to so large a degree the characteristics of both. Its affinities on the lower side are clearly with the Cordaitales, as has been recognized by all competent investigators in recent years. Its relationship with the Abietineae among the Coniferales is equally well indicated by comparative anatomical and paleobotanical data, as will be shown in the following chapter.

The indications of relationship with the Cordaitales are presented in connection with the organization of the wood in primitive 
organs and regions. It has been pointed out in the foregoing paragraphs that, although centripetal wood of the cryptogamic type is represented almost exclusively by transfusion tissues in the mature vegetative leaf of Ginkgo, it is present in a clearly recognizable form in the cotyledon, in the microsporophylls, and in the peduncle of the ovuliferous apparatus. In the case of the microsporophyll the xylem, and more particularly the vestigial centripetal xylem and transfusion tissue, are in clear relation to the reticulately thickened opening mechanism of the microsporangia. This feature is of value, not only as indicating the filiation of the Ginkgoales with lower groups, but also as indicating the morphological nature of the arrangements for dehiscence of the spore sac in the seed plants above the Cycadales. The absence of longitudinal parenchyma in the secondary wood is another criterion of the relationship of the Ginkgoales with lower groups, while the presence of tangential pitting in the terminal region of the summer wood clearly relates the group with modern gymnospermous types. The radial pitting of the tracheids and associated structures is also of importance as indicating the phylogenetic position of the genus. As has been shown above, the radial pits of Ginkgo are opposite in the mature wood, and often in the intervals between them, particularly toward the ends of the tracheids, have transverse bars of pectic cellulose imbedded in the tracheary wall, and these are conveniently designated bars of Sanio. The opposite pitting and the occurrence of bars of Sanio are features which clearly co-ordinate the wood of the Ginkgoales with that of the higher gymnosperms. However, in the primitive regions and organs of the living Ginkgo we find both the pitting of the Cordaitales and the absence of bars of Sanio which are universally characteristic of the older gymnosperms from the Cycadales downward. It seems quite obvious that Ginkgo is a genus of the utmost importance from the standpoint of evolutionary anatomy, since it summarizes in such a remarkable manner the anatomical characteristics of both Archigymnospermae and Metagymnospermae. Its significance in the direction indicated will be fully realized only after the next tribe, the Coniferales, have been anatomically considered in the following chapter. 


\section{CHAPTER XXIV}

\section{THE METAGYMNOSPERMAE: CONIFERALES}

As has been indicated at an earlier stage, the gymnosperms are somewhat clearly divisible into two large groups: the Archigymnospermae, which are often fernlike in habit and always cryptogamic in the anatomical organization of their primary wood and in their mode of fertilization by antherozoids, and the Metagymnospermae, which present no external resemblance to the members of the fern series, and in which the centripetal or cryptogamic primary xylem has given place, in living forms at any rate, to transfusion tissue, and in which, also, fertilization by means of a pollen tube is a universal feature. The Coniferales are the largest and the most important group under the Metagymnospermae. Their significance from the evolutionary standpoint can scarcely be overestimated, not only because they are more abundantly represented in the living plant population of the earth than are any other gymnosperms, but also because they are copiously preserved as fossils as far back as the Paleozoic age. They thus supply the most important document for the inductive study of general principles of evolution presented by any group of living organisms, vegetable or animal, living or extinct. The paleobotanical and anatomical investigation of the Coniferales has greatly changed our views in regard to their phylogenetic sequence in recent years. The older students of the group restricted to a knowledge of living forms naturally assumed that the conifers, which are the simplest in organization of their vegetative and reproductive parts, are most primitive. By those entertaining this view the Taxineae are considered the most ancient conifers, and with them have been connected, not only the Cordaitales, but also the living genus Ginkgo. In the most recent systematic treatment of the coniferous tribe as a whole we find this attitude maintained, for Ginkgo and Taxus are regarded as closely related. It is needless to state that there 
is nothing in common between the anatomical structures of vegetative and reproductive parts in Ginkgo and the Taxineae. A later, but apparently equally erroneous, tendency is to interpret the evolutionary sequence of the Coniferales entirely in the light of the data derived from the study of Paleozoic gymnosperms. This attitude is of course found strongly in evidence in those countries which have contributed notably to the elucidation of the organization of the seed plants of the Paleozoic. By those who are affected by the Paleozoic bias the araucarian subtribe of the Coniferales is considered the oldest representative of the group. There are those again who attempt to reconcile the taxinean and araucarian hypotheses of derivation by the assumption that the Araucariineae have come from the Cordaitales, while the remaining coniferous subtribes have been derived from the Taxineae. The hypothesis of a lycopodineous origin of the group has been put forward at various times, but need only be mentioned here, as it has few advocates and does not appear to derive any support from paleobotanical or anatomical facts. The views in regard to the phylogeny and evolution of the Coniferales adopted in the present work represent an attempt to interpret this large and important group of gymnosperms in the light supplied by the anatomy of Mesozoic forms as compared with representatives of the group still living. The method of treatment adopted will be, so far as the limits of space in an elementary textbook permit, purely inductive. As has been emphasized in an earlier paragraph, the conifers, on account of their abundant presence in the floras past and present, supply a most valuable document for the interpretation of the fundamental principles of evolution.

Since the araucarian conifers are quite generally regarded at the present time as the primitive representatives of the group, it will serve a useful purpose to consider these first. A transverse section of the wood of the stem in this subtribe (Fig. 225) generally reveals the presence of annual rings, unless the particular species under investigation happens to be of lowland tropical origin. The autumnal tracheids are marked by tangential pitting, a general feature of organization of the more modern gymnosperms. The 
wood normally shows the presence of only radial parenchyma, longitudinal storage elements being absent. The longitudinal radial section of the wood (Fig. 226 ) shows a condition of pitting resembling that found in the Cordaitales, namely, one which is alternating and characterized by the absence of the bars of Sanio. The absence of wood parenchyma and the alternating character of the pitting are features which at first sight would seem to justify the assumption of a

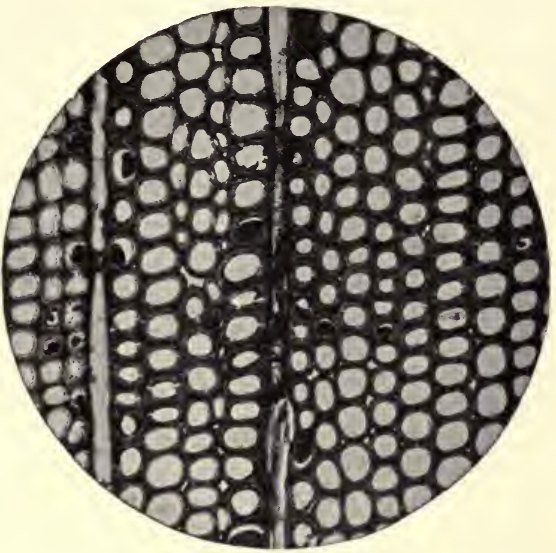

Fig. 225.-Transverse section of the wood of $A$ gathis ausiralis.

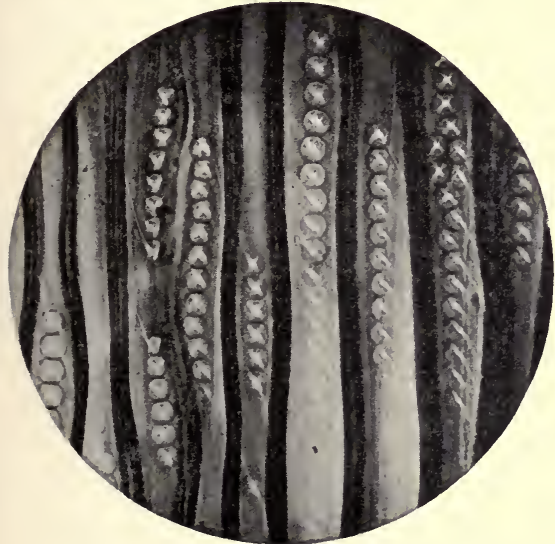

FIg. 226.-Longitudinal section of the wood of $\mathrm{Aga}$ this australis. close degree of relationship between the araucarian conifers and the Cordaitales, and this view of their affinities has been the one almost universally adopted. Before we inquire as to its validity it is necessary to examine the organization of Mesozoic representatives of the group. In Fig. 227 is shown in transverse section the 
structure of a Cretaceous araucarian wood of the type designated Araucarioxylon. The annual rings are much less clearly developed

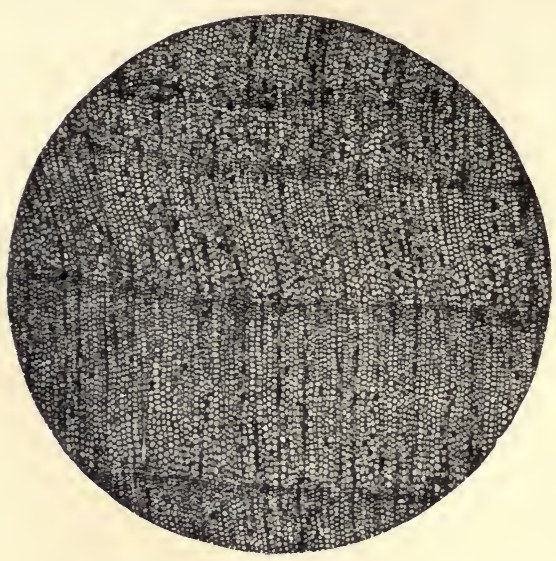

Fig. 227.-Transverse section of A raucarioxylon from the Cretaceous of the eastern United States.

than in the wood of Agathis. The rays are uniseriate, as in the living type, but a marked contrast is presented by the presence of wood parenchyma in the fossil. The longitudinal section portrayed in Fig. 228 shows both alternating pitting and the presence of parenchyma. Certain of the tracheids are also filled with dark contents derived as an exudation from the rays. This is a condition often present in both living and extinct woods of araucarian affinities. We may now turn our attention to another araucarian type commonly present in the Mesozoicthe genus Brachyoxylon. In this type the wood in transverse section shows annual rings and the

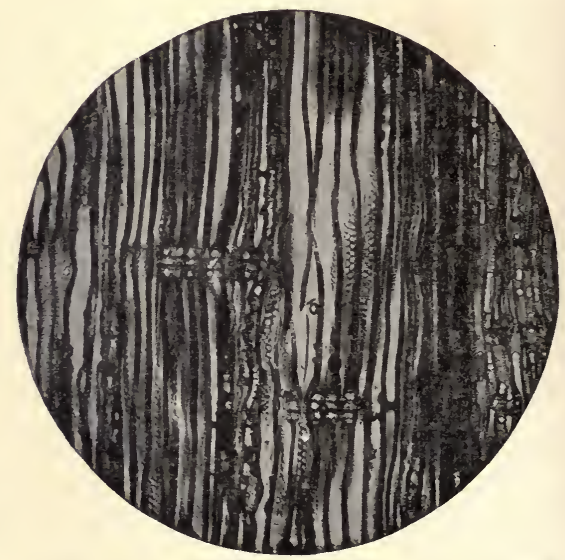

FIG. 228.-Longitudinal section of the same Araucarioxylon. 
absence of longitudinal parenchyma. In longitudinal radial aspect it manifests a kind of pitting which is only partially araucarian, for more often than not the pores are separated by considerable intervals and fail to alternate (Fig. 229). It is only occasionally that the typical araucarian crowding and alternation are present. Moreover, in woods of the Brachyoxylon type wounding brings about the formation of traumatic resin canals (Fig. 230) such as

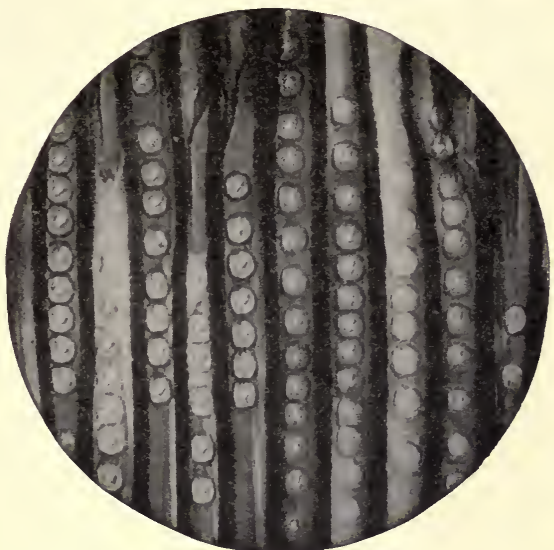

Frg. 229.-Longitudinal section of the wood of Brachyoxylon.

appear after injury in certain of the Abietineae and in the genus Sequoia.

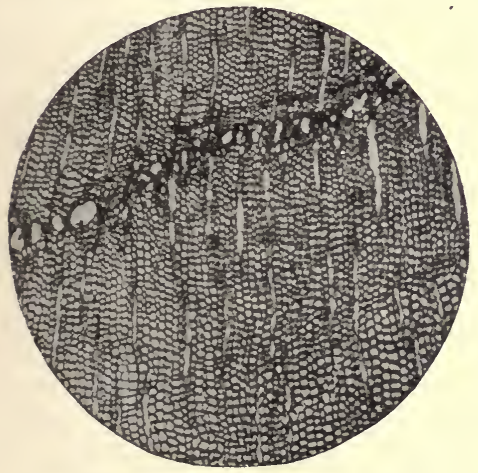

FIG. 230.-Transverse section of the wood of Brachyoxylon formed after wounding.
After the consideration of the conditions in the mature wood of living and extinct representatives of the araucarian conifers, we may now turn our attention to the organization of the xylem in the conservative organs of the existing araucarian conifers. Fig. 23I illustrates the structure of the wood in the root of $A$ gathis australis in a region not very remote from the primary wood. 
It is evident that, in the secondary xylem of the root, parenchyma, conspicuous by its absence in the mature wood of the stem, is abundantly present. Not only is this the case with the root, but the same situation is found in the first annual ring of the vegetative stem and also very strikingly in the woody axis of the ovuliferous cone. The facts here mentioned are of particular significance when correlated with the organization of the Cretace-

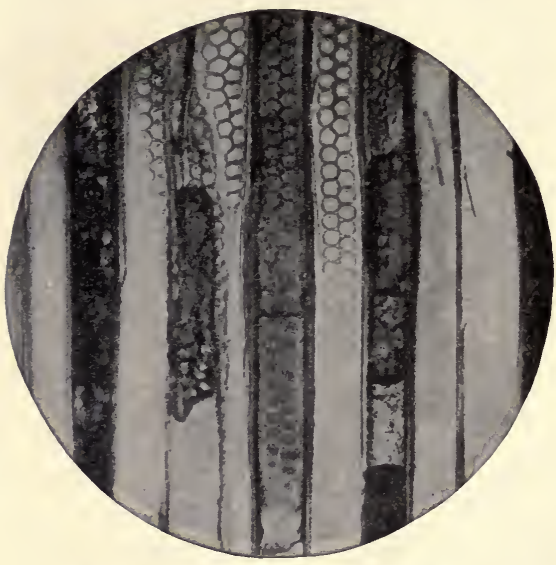

FIG. 231.-Longitudinal section of the wood of the root in Agathis australis.

ous Araucarioxylon shown in Fig. 227. Obviously the parenchyma present in the older type of araucarian wood is perpetuated in those regions of the living form which we have learned in an earlier chapter to regard as conservative. It may accordingly be logically assumed that woods of the type of the living araucarian conifers formerly possessed longitudinal parenchyma and in this respect are at variance in organization with the ligneous structure of the Paleozoic Cordaitales. This conclusion is reinforced by an examination of the effects of injury in the living genera, for the infliction of wounds results frequently in the recall of the lost parenchyma even in the adult axis.

We may now pass to the consideration of other features which are supposed to indicate a close degree of relationship between the Araucariineae and the Cordaitales. The most important of these are the crowded pitting and the absence of bars of Sanio. In Fig. 232 is shown the organization of the wood in the stem of the seedling of $A$ gathis australis as viewed in longitudinal 
radial section. The pits are neither crowded nor alternating as in the wood of the adult. An examination of the organization of the seedling in the living representatives of the araucarian conifers therefore justifies the view that the ancestral forms did not possess crowded pitting. Precisely similar conditions are found in the cone, for here the pits in the tracheids nearer the primary wood lack the crowded and alternating disposition of the mature vegetative wood of the genus. But a still more important feature is presented by the organization of the wood of the ovuliferous cones of the living Agathis and Araucaria. Fig. 233 shows a longitudinal radial view of the secondary wood of Araucaria Bidwillii in the vicinity of the protoxylem. The

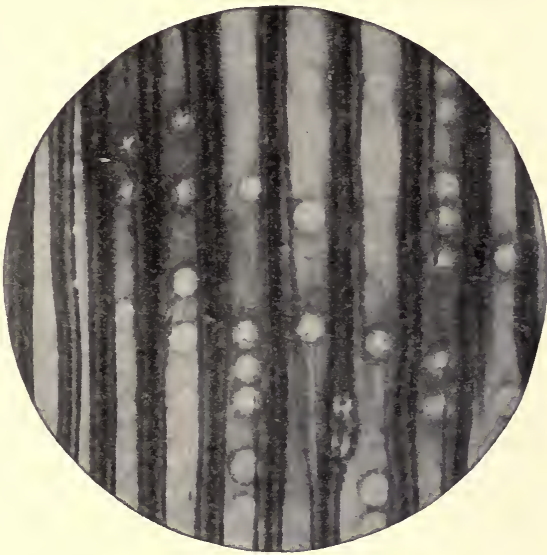

FIG. 232.-Longitudinal section of the wood of the seedling in Agathis australis.

pits show a very strong tendency to opposition in arrangement, and are certainly not angular by mutual contact, as is often the case in cordaitean woods. The most interesting feature shown by the figure, however, is the presence of bars of Sanio such as are entirely lacking in the adult vegetative wood of existing species of the araucarian conifers. As a consequence of the situation revealed in the conservative reproductive axis of the araucarian conifers, we are justified in assuming that the absence of bars of Sanio and the presence of alternating pitting are not primitive features of the organization of the wood of the subtribe, and consequently cannot be brought into court to prove its cordaitean affinities. The evidence, in fact, must be read in exactly 
the opposite sense from that in the Ginkgoales; for, as has been shown in the preceding chapter, the anatomical facts there justify the assumption of the original presence of cordaitean structure, characterized by alternation of pitting and absence of bars of Sanio. In the case of the Araucariineae, on the contrary, we must assume on the basis of the structure of primitive regions that bars of Sanio and opposite pitting were an older feature of

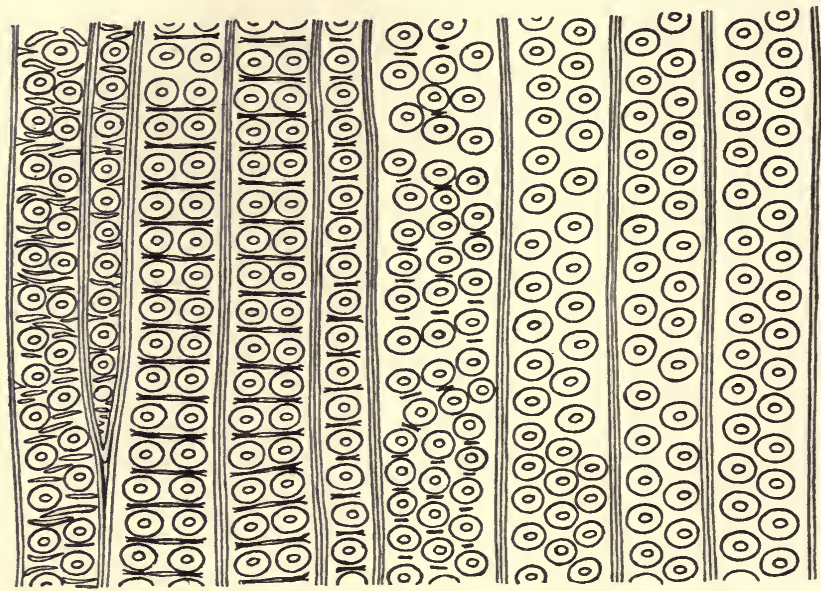

FIG. 233.-Longitudinal section of the wood of the cone axis in Araucaria Bidwillii in the region of the pith.

organization of the wood and that they are as a consequence not nearly related to the Cordaitales.

It becomes clear, when we consider the arguments derived from comparative anatomical data and those furnished by the study of extinct forms, that the organization of the mature wood in the living Araucariineae cannot be accepted as sufficient evidence of their relationship with the Cordaitales. The persistent foliar traces of the two living araucarian genera have been regarded in some quarters as an important indication of their primitive character. Here again the comparative anatomical situation 
as well as the conditions found in allied fossil conifers do not justify the conclusion reached. The seedlings of both Araucaria and Agathis show the earlier leaf traces as evanescent structures which cease to be formed by the cambium after the leaves to which they belonged have disappeared. It is only in the older trunk that the formation of foliar strands is perpetuated for many years, amounting even to centuries, after their corresponding leaves have disappeared. The persistent leaf traces which constitute so remarkable a feature of the organization of the mature trunk of the existing Araucariineae cannot therefore be regarded as anything but a bizarre and freakish feature which has no evolutionary importance. If any remaining doubt can be considered to exist on the subject, it is set aside by the organization of the Mesozoic araucarian woods, which (with the exception of the type known as Araucarioxylon), are distinguished by the absence of persistent foliar traces. Comparative anatomy lends very little support to the inference of cordaitean affinities for the araucarian conifers, and the evidence against this widely cherished view becomes quite overwhelming when the anatomical situation in the other subtribes of the Coniferales is considered. The further estimation of the claims of the Araucariineae to the pre-eminence of being the oldest conifers may appropriately be delayed until the anatomical features of other important subtribes have been examined.

The Abietineae have in recent years made progress as claimants to the primitive position among Coniferales and to that of nearest proximity to the Cordaitales. At first sight the extremely complicated organization of both vegetative and reproductive structures in the abietineous conifers appears to stand in the way of any such conclusion; but the results of the comparative investigation of the living and fossil representatives of groups which have passed the zenith of their development has taught us not to consider complexity of organization as necessarily a criterion of modernity. It will be shown in subsequent paragraphs that the Abietineae are in a very strong position as regards primitiveness, both because they are apparently related, on good anatomical evidence, to such ancient groups as the Cordaitales and Ginkgoales and because 
they are clearly antecedent to the mass of other living coniferous subtribes.

The mature wood of Pinus, illustrated in Fig. 234, is characterized in transverse section by the presence of resin canals and by rays of complex organization. The longitudinal structure of the wood, as is shown in Fig. 235, is characterized by the presence

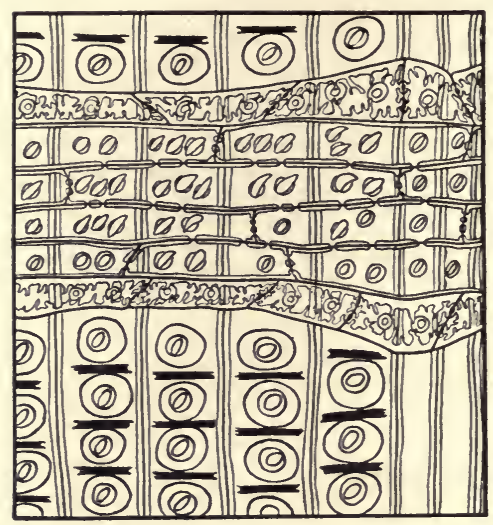

FIG. 234.-Longitudinal view of the wood in Pinus resinosa. of bars of Sanio. The rays are of complicated structure, even when uniseriate, and are composed of central storage cells and marginal elements resembling tracheids. If a primitive region such as that provided by the wood of the cone axis or root be investigated, it becomes clear that the bars of Sanio are not an ancestral feature of organization of the wood, since they are absent in the inner region of the wood of the cone and are also often lacking in the tracheary elements of the root, especially in proximity to the primary wood. This situation is portrayed for the cone of the Italian nut pine (Pinus pinea) in Fig. 236. It is obvious that the tracheids retain for some time the spiral markings of the primitive region. The walls of the tracheary elements show not the slightest indication of the presence of bars of Sanio until a region remote from the pith has been reached. The rays also are without the marginal tracheids which manifest themselves at an early stage in the organization of the wood of the vegetative branches. Clearly, so far as the structure of the wood in the reproductive axis is concerned, the Abietineae as represented by Pinus are derived from ancestors possessing the structure of the wood of the Cordaitales. Another interesting 
feature of resemblance to cordaitean forms is the absence of a torus in the membranes of the bordered pits of the tracheids lying nearer to the primary wood. The situation in this respect is the exact opposite of that found in the case of the araucarian conifers,

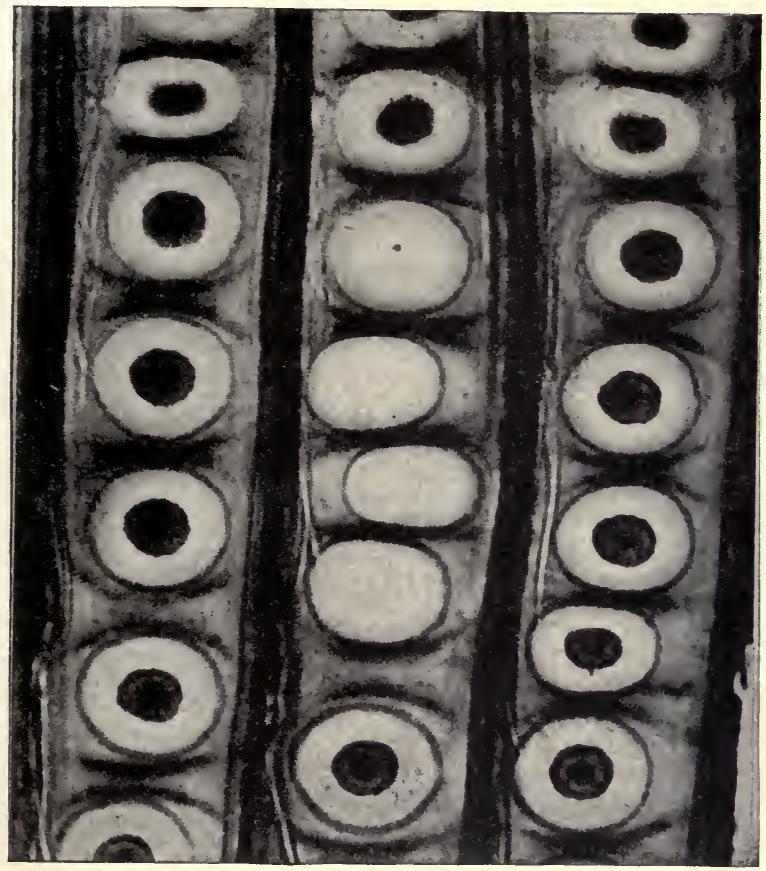

FIG. 235.-Highly magnified view of the tracheids of a species of pine, showing the bars of Sanio (after Gerry).

in which the torus is sometimes present in the region near the primary xylem of the wood of the cone axis and is entirely absent elsewhere. The organization of the wood alone in primitive organs and regions justifies the conclusion of a filiation between the Abietineae and the Cordaitales rather than between the Araucariineae and the Cordaitales. 
We may now turn to the brief consideration of evidence derived from the organization of the wood of fossil forms and bearing on the respective antiquity of the Abietineae and the Araucariineae. The investigations of recent years have brought to light in the Jurassic and Cretaceous numerous coniferous woods which to a large degree possess characteristics intermediate between those
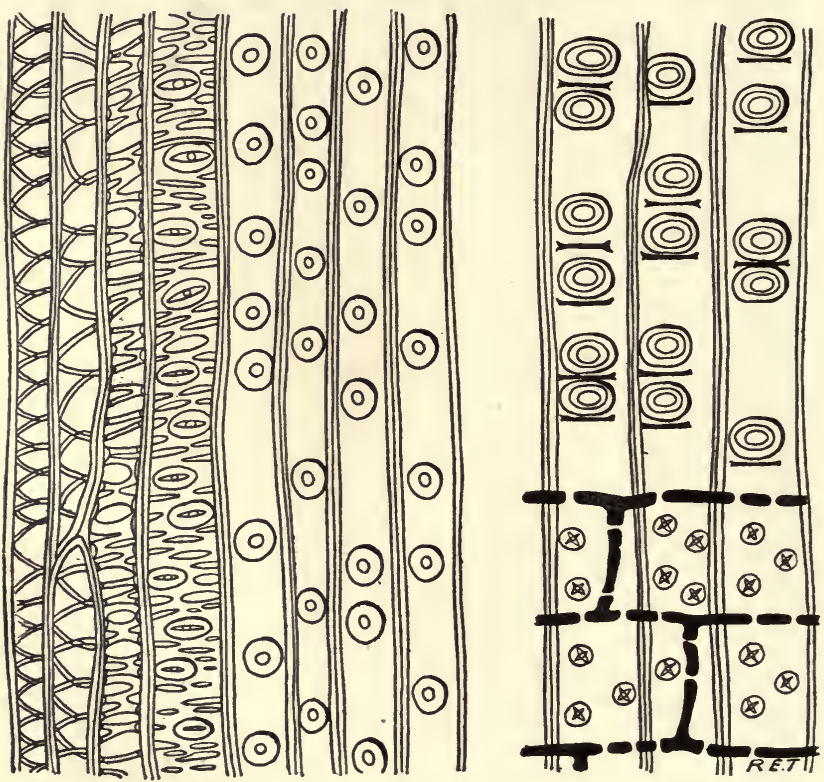

FIG. 236. -Transitional region from the xylem of the cone of Pinus pinea

of the Abietineae and Araucariineae. The conclusion naturally follows that the two subtribes were less remote from one another in Mesozoic time than they are in the present epoch. The question of interpretation is strongly debated in the case of these woods. The mass of paleobotanists, obsessed by the araucarian hypothesis of the derivation of the Coniferales from their cordaitean ancestors and little concerned with the fundamental principles of comparative 
anatomy, have assumed that the transitional woods in question are those of Abietineae which are losing their primitive araucarian characters. A fatal objection to this point of view, however, is the fact that none of these transitional woods shows the presence of bars of Sanio. In other words, they must clearly be diagnosed as belonging to the araucarian side on the basis of the most reliable of diagnostics of coniferous woods-bars of Sanio. Attempts in the direction of proving the woods in question abietineous rather than araucarian have chiefly taken the form of discussions as to the value of ray structure in the diagnosis of coniferous woods. It is beyond the range of the present volume to discuss details of the organization of the radial structures in the Coniferales; but it may be stated in a general way and on the basis of comparative anatomy that no

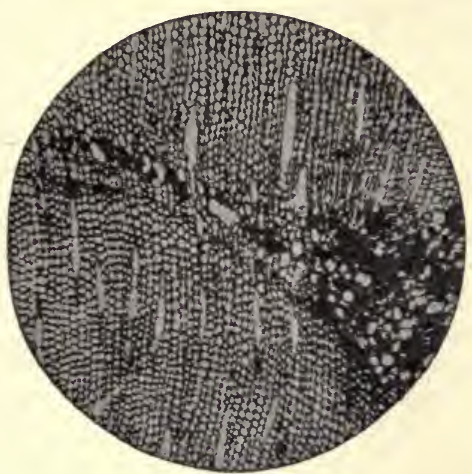

Fig. 237.-Wood of Brachyoxylon formed after wounding.

feature is more subject to variability within the limits of a single subtribe and hence is less available for comprehensive conclusions in regard to evolutionary sequence. A final argument against the araucarian descent of the Coniferales from the Cordaitales is supplied by the extremely abundant Mesozoic araucarian type of wood known as Brachyoxylon. In wounded specimens of wood of this genus traumatic resin canals are formed (Fig. 237), resembling those of the normal wood of the pinelike conifers. The occurrence of resin canals as a consequence of injury in Brachyoxylon, in view of the fact that this genus is admitted by competent paleobotanists to be of unquestionable araucarian affinities, is of great significance. This being the case, we are justified in interpreting the canals formed after wounding as a reversionary phenomenon, indicating relationship to the pinelike 
Abietineae. This interpretation of the situation is vindicated by the recent discovery of normal resin canals in the wood of the axis of the ovuliferous cone of a Javanese species of Agathis, A. Bidwillii (Fig. 238).

Having discussed, so far as the limits of the present volume permit, the organization of the wood in conservative axes and in fossil forms, we must now turn to the discussion of that extremely important organ, the leaf. It has been made clear in an earlier

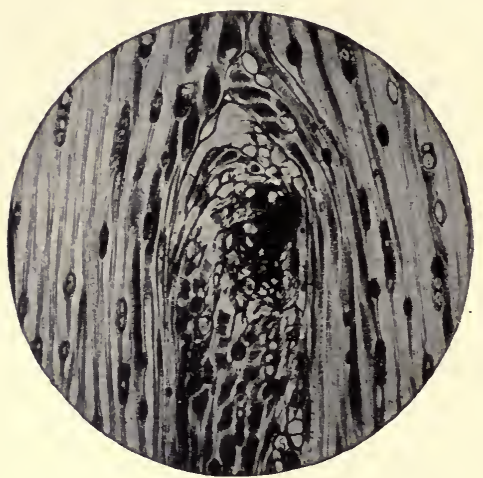

FIG. 238.- Resin canal in the trace of the seed scale of Agathis Bidwillii. chapter that the foliar organ of Pinus is characterized by the remarkable structure of its fibrovascular tissues. In the genus under discussion and to a less extent in allied genera the foliar conducting strand is surrounded by a cordon of transfusion tissue. The situation in this respect may be clearly ascertained by reference to Fig. 239. The transfusion elements are distinguished by the absence of protoplasmic contents and by the occurrence of bordered pits in their walls. It is obvious that the tissues of this nature become joined with the xylem of the foliar bundles on its flanks. The transfusion tissue in modern representatives of the genus Pinus is not a continuous mass of tracheary cells, but has interspersed throughout its substance a considerable number of living cells provided with protoplasm and a nucleus. The investigation of the Cretaceous deposits at Kreischerville, Staten Island, has provided us with extremely valuable data for the determination of the organization of the leaf in Pinus and allied forms in the later Mesozoic. In some of the numerous species of Pinus which flourished in the American Cretaceous, transfusion tissue was present in large amount and contained little or no admixture 
of parenchymatous elements. In still other species the short tracheary elements ordinarily called transfusion cells were much less well developed. It is the remarkable genus Prepinus, however, which provides the most important evidence for estimating the bearing of the anatomical organization of the leaf on the problem of evolution of the genus Pinus in particular and that of the Abietineae in general. In Fig. 240 is shown the transverse section

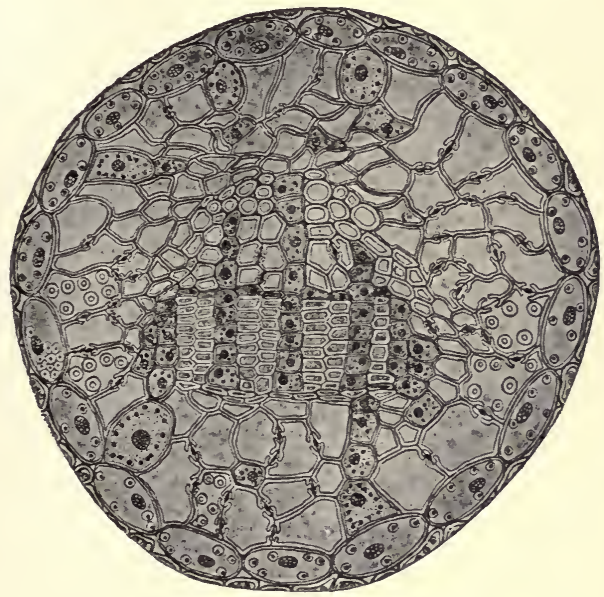

FIG. 239.-Leaf bundle of Pinus Strobus

of the leaf in $P$. statenensis. The outline is angular because of mutual contact with other and surrounding leaves of the fascicle. In Prepinus the growing point of the short-shoots persisted as it does in the living Ginkgo, and the fascicular leaves, instead of being few and definite in their number, were indefinitely numerous. It is interesting to note in this connection, however, that, although in the true Pinus of the Cretaceous the number of leaves in the fascicle was few and fixed as in modern forms, nevertheless the growing point of the short-shoot persisted indefinitely and did not disappear at an early stage, as in the living representative of 
the genus. Within the angular outline of the leaf in Prepinus is seen, beneath the epidermis, the ribbed hypodermal tissues, recalling those of the older gymnosperms. The cortical region of the leaf terminates in a not very clearly marked endodermis which doubtless owes its loss of definiteness to the changes resulting from fossilization. Within the endodermal boundary lies the entirely tracheary and strongly pitted transfusion tissue of the

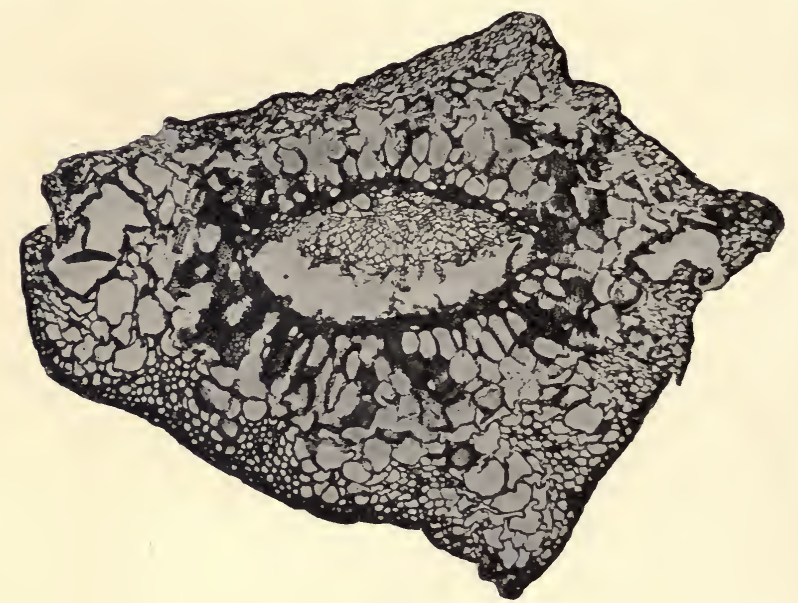

Fic. 240.-Leaf of Prepinus statenensis

leaf. The elements of this category are differentiated into two zones-an outer one composed of short, broad, pitted elements, and an inner one consisting of thick-walled cells of narrow lumen. The former are known as the outer transfusion sheath and the latter as the inner transfusion sheath. Both structures have their counterpart in the leaf of certain of the Cordaitales. The double transfusion sheath was also frequently present in the foliar organs of true pines of the American Cretaceous. Another most interesting feature of the organization of Prepinus was the structure of the xylem. As may be seen from Fig. 24I, the wood presents two regions, an upper and a lower. In the former the 
elements are arranged in radial rows and usually increase in size toward the upper surface of the leaf. In the xylem directed downward are seen indications of wood rays, and the inspection of the longitudinal aspect reveals the fact that it is made up largely of tracheids with bordered pits. This is the centrifugal xylem and corresponds to the mass of wood in the foliar trace of the fascicular

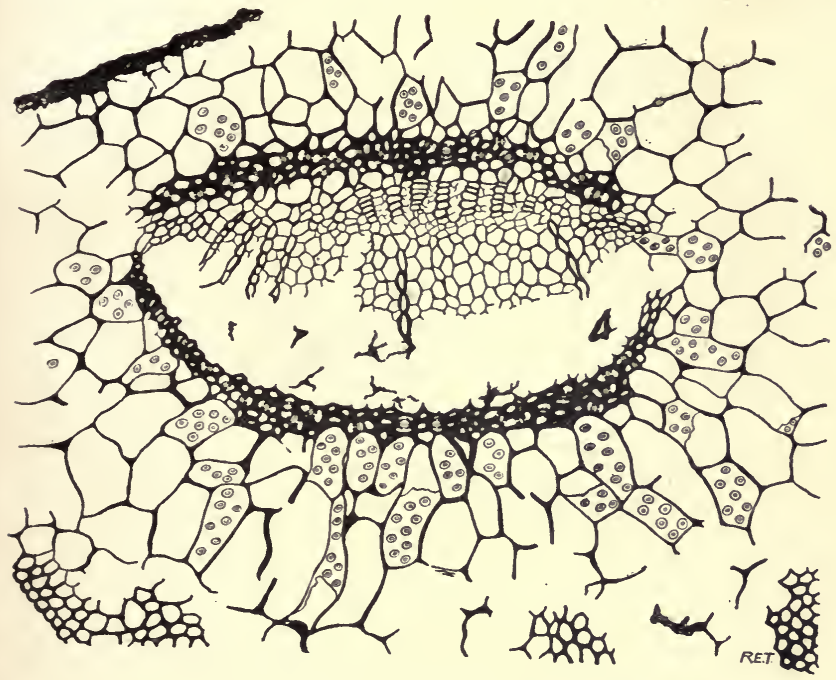

FIg. 24I.-Portion of leaf of Prepinus statenensis

leaves of living pines. The upwardly developing wood is the cryptogamic xylem and confirms the conclusion as to the affinity of Prepinus, already suggested by the organization of its transfusion sheath, namely, that the genus is allied to the Cordaitales. It is thus apparent that the details of organization of the leaf in Prepinus, which in turn is clearly the ancestor of Pinus, justify an attribution of cordaitean ancestry to the Abietineae. This conclusion as to relationship is supported by the primitively cordaitean character of the pitting which so strikingly indicates a 
relationship of the Abietineae rather than the Araucariineae with the Paleozoic gymnosperms known as Cordaitales.

Not only do the Abietineae as a result of their anatomical organization and paleobotanical history present a strong claim to direct relationship with the older gymnosperms, but they supply equally compelling evidence that they are ancestral to other prominent coniferous subtribes. It will be well in this

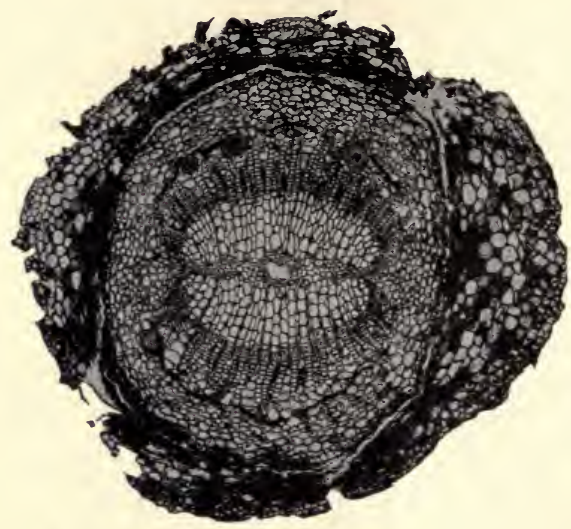

FIG. 242.-Transverse section of the root of Abies balsamea, showing the presence of a resin canal in the primary wood.

planes. In contrast to these, in the second series the ligneous resin canals of the secondary wood are notably absent. It is only in regions recognized as conservative that they make their appearance-in the primary structures of the xylem of the root (Fig. 242), in the secondary wood of the axis of the ovuliferous cone, and sometimes in the first annual ring of the vegetative branches. Further, resin canals are found in the wood of the Abieteae as a result of injury. Both comparative anatomical and experimental evidence, as a consequence, vouch for the derivation of the Abieteae from ancestral forms possessing well-developed ligneous resin canals. It is of significance to note in this connection that the genus Cedrus, for the great antiquity of which the geological record supplies clear 
testimony, not only from American, but also from European deposits, is strikingly distinguished by the fact that it produces both horizontal and vertical resin canals resulting from injury (Fig. 243). This condition is in contrast to that manifested by the other genera of the Abieteae, in which only vertical resin canals make their appearance in the secondary wood after wounding. It is now generally admitted by competent anatomists that there is strong evidence for the derivation of the Abieteae from the Pineae as a result of reductionary modification. This conclusion is reached, not only on the testimony supplied by the resin canals as described above, but also from the comparative anatomical consideration of the organization of the rays and the parenchyma of the secondary wood. It is apparent in regard to these particular structures that the Pineae are

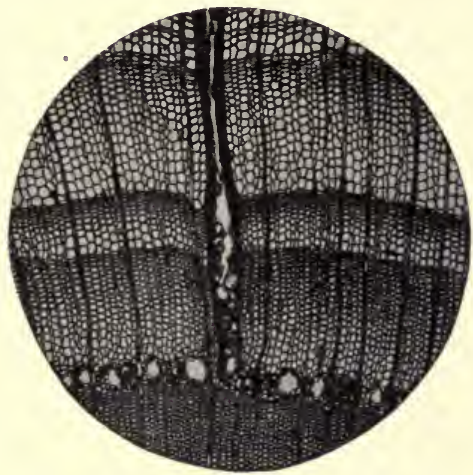

FIG. 243.-Transverse section of the wood of Cedrus deodara formed after injury, showing reversionary appearance of resin canals in both vertical and horizontal planes.

more primitive than are the Abieteae. The ray of the Abieteae is often characterized by the loss of the marginal tracheids so distinctively developed in the radial parenchymatous strands of Pinus and its living allies.

The internal conditions in the Pineae may now claim our attention. Here we find a striking separation between Pinus on the one hand and Picea, Larix, and Pseudotsuga on the other, resulting from a consideration of the lining of the resin canals in the wood. In the first-named genus the secretory canals are lined by thin-walled parenchyma which, in the transformation of heartwood into sapwood, develops processes known as tyloses, which more. or less completely occlude the resin canals. In the 
three remaining genera of the Pineae the lining of the secretory space is composed mostly of thick-walled more or less lignified cells. The resin canals in these forms do not accordingly contain well-developed tyloses in the heartwood. Another important distinction between Pinus and allied genera is the complete absence of wood parenchyma in the former. It has been shown in an earlier chapter that longitudinal storage parenchyma is formed in the secondary wood as the result of the modification of elements

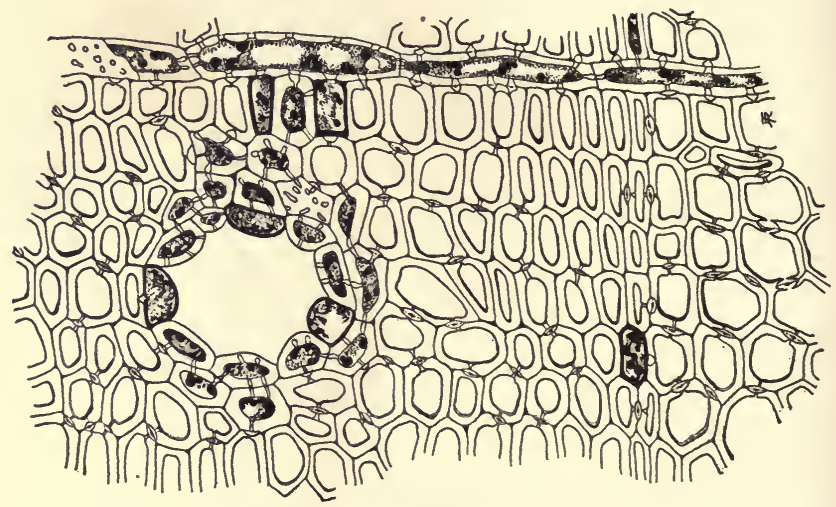

Fig. 244.-Wood of Picea canadensis, showing terminal parenchyma

destined to be tracheids. In Picea, Larix, and Pseudotsuga storage parenchyma is present, but at the end of the annual ring only (Fig. 244). In this position, particularly in the case of the root, it manifests convincing evidence as to its derivation by the occurrence of transitional stages between merely septate tracheids and rows of parenchymatous elements, resembling in their general configuration tracheary elements. In Pinus, therefore, there is no true parenchyma of the wood, since such storage cells are found only in the three other genera of the Pineae. Where wood parenchyma is present, moreover, it is confined to the end of the annual ring and is clearly in a condition of derivation from tracheids, a state found normally in no other living representative of the Coniferales. It 
has been demonstrated in earlier pages that Paleozoic gymnosperms are characterized by the complete absence of parenchymatous elements in the wood and at the same time by the general absence of annual rings in the stem. Pinus, as regards the organization of the storage devices of the wood, is therefore clearly allied with Paleozoic types such as the Cordaitales.

There are other conditions, however, which indicate for Pinus a primitive position among the Abietineae. First of all there is the possession of short-shoots. Pinus in this feature of organization presents a marked resemblance to the Ginkgoales, which

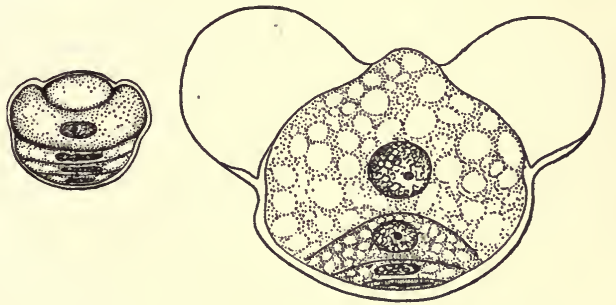

Fig. 245.-Microspores of Ginkgo and Abies

also bear their foliar organs on special spurs or short-shoots. Nor is the common possession of short-shoots unparalleled by other significant characteristics. Ginkgo and the Abietineae strongly resemble one another in the possession of bisporangiate sporophylls. In the two groups there are two microsporangia and two megasporangia or seeds on the reproductive foliar organs. The view sometimes advanced that the ovuliferous scales in the Abietineae consist of a fused pair of foliar structures has apparently no evidence in its favor. It is as clearly a single leaf as is the microsporophyll. The microspores in the Abietineae and in the Ginkgoales also present striking points of resemblance which have only recently been completely realized. In the monotypic Ginkgo the pollen is winged as in the more primitive Abietineae and resembles in its internal organization the structures found in the microspores of that subtribe of conifers. Fig. 245 illustrates the numerous features of internal and external resemblance between 
the pollen of the Abietineae and that of the Ginkgoales. Nor is the similarity confined to the structure of the microspores. It has been pointed out in an earlier chapter that the organization of the wall of the sporangium in relation to the opening mechanism and to its derivation from the fibrovascular structures is practically identical in the Ginkgoales and Abietineae. Finally, the organization of the tracheids of the wood is similar in the case of the two groups under consideration. Pinus seems beyond question, by the possession of short-shoots, the number and organization of its microsporangia and megasporangia, as well as by the structure of its microspores, the general organization of the wood, and, finally, by the absence of true wood parenchyma, clearly allied to the sole surviving genus, Ginkgo.

Pinus, lastly, presents a very strong claim to primitiveness among the Coniferales by reason of the general presence of shortshoots, such as are usually regarded as the prototypes of the ovuliferous scales of the female cone of the Coniferales as a whole. It follows, on the general principles of anatomy laid down in an earlier chapter, that the genus which still shows vegetatively the structures known as short-shoots is in an excellent position to claim a primitive position among the Coniferales. The absence of short-shoots in the seedling of Pinus is obviously no ground for an argument of any significance against the primitive presence of short-shoots in the genus. Negative evidence furnished by seedlings is of no value, since only positive testimony in connection with the hypothesis of recapitulation can be accepted as valid in evolutionary argument. We may therefore assume that the presence of short-shoots in Pinus and Ginkgo, as well as many other features of resemblance between the two genera, is an unmistakable indication of affinity. Further, since short-shoots are very generally assumed to have been the prototype of the ovuliferous scale in the female cones of the Coniferales throughout, we may infer that the coniferous genus which has manifested these structures as a normal vegetative feature from remote geological times must be a very ancient representative of the Coniferales.

But we are not by any means limited to a consideration of the general organization of the female cone in inferences regarding 
the relationship of Pinus to the other Coniferales. An excellent illustration of the value of anatomical evidence in the case of this problem is furnished by the interesting taxodineous genus Sequoia. The Taxodineae as well as the nearly allied Cupressineae are characterized anatomically by the organization of the female cone and the structure of the wood. The scales of the cone are superficially single, but in section they show the presence of a double series of oppositely orientated fibrovascular bundles, thus indicating the origin of the seed scales from the externally

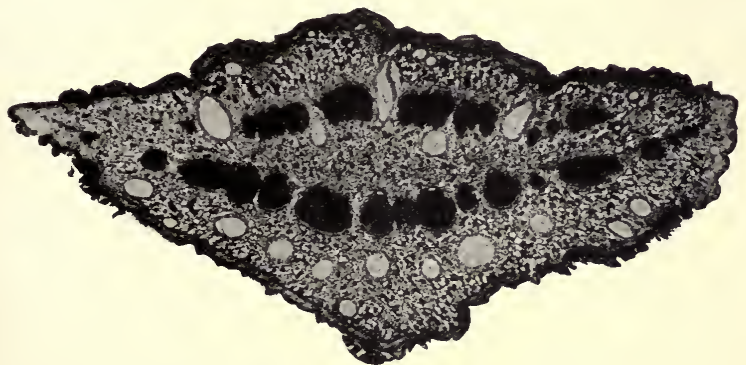

Fig. 246.-Transverse section of the cone scale of Sequoia gigantea, showing a double system of bundles with opposite orientation.

double structures of the ovuliferous cone of the Abietineae (Fig. 246). In the organization of their wood the Taxodineae differ from the Abietineae in the absence of resin canals. There is, however, a resiniferous secretion produced by scattered parenchymatous elements of the wood. In the structure of the radial parenchyma a condition of simplicity contrasting with that found in the Abietineae is manifested, for the marginal tracheids of the rays of the Abietineae are conspicuously absent in the normal wood of the Taxodineae in general and of Sequoia in particular. If we consider the genus Sequoia in the light of the canons of anatomy formulated above, very interesting results are reached. First, if a transverse section of the axis of the cone or of the ovuliferous scale of Sequoia gigantea be examined, resin canals reveal themselves in the wood in proximity to the primary xylem (Fig. 247). 
Further, an investigation of the first annual ring of the stem in trees which have attained such vigor of development as to pro-

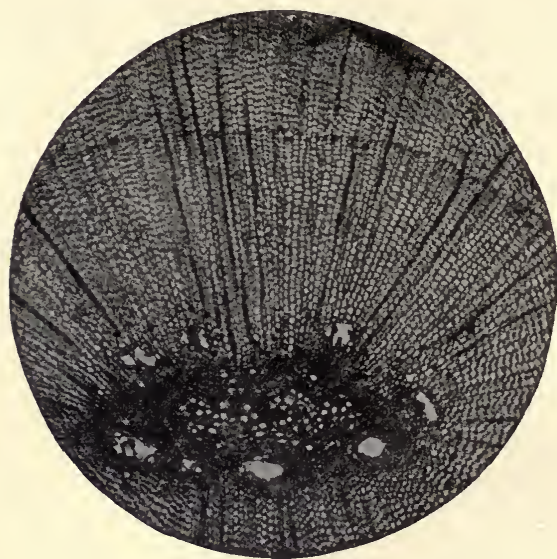

FIG. 247.-Transverse section of reproductive axis of Sequoia gigantea, showing resin canals in the wood. duce seed often shows the presence of resin canals such as are not normally found in the subsequent annual increments of growth (Fig. 248). Finally, the fibrovascular strand of the leaf frequently contains in the region of the xylem a single resin canal. The occurrence of ligneous resin canals in the various primitive regions indicated above is good evidence of the original presence of such structures in the woody tissues of Sequoia. Additional information on this subject is furnished by the wound reactions of the genus. In either of the two species, $S$. gigantea or $S$. sempervirens, the infliction of wounds may be

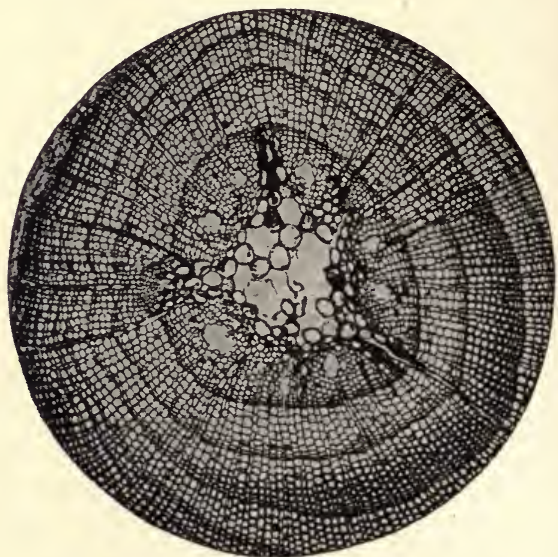

FIg. 248.-Twig of Sequoia gigantea, showing presence of resin canals in the first annual ring. 
followed by the appearance of traumatic or wound resin canals as a reversionary feature. In $S$. sempervirens, the redwood, this is the only mode of occurrence of these structures. It may be added in this connection that the normal seedling of Sequoia (either species) shows no resin canals in the wood. On the fallacious logic that structures absent in the seedling are not ancestral it could be argued that resin canals in the secondary wood are not an ancestral feature of the genus Sequoia. This genus is the only one in any coniferous subtribe, other than the Abietineae, characterized in living species by the formation of traumatic resin canals. In Sequoia a sound inference based on the principle of conservative organs is that its ancestral forns were provided with resin canals throughout their structure.

Although only the genus considered in the foregoing paragraph presents the abietineous feature of resin canals in the secondary wood as a result of injury, many genera of both taxodineous and cupressineous affinities revert to the abietineous type of ray as a response to wounding. This is notably the situation in Sequoia and is illustrated in Fig. 53. In another figure (Fig. 52) the occurrence of marginal tracheids is indicated for the cupressineous species Chamaecyparis nootkatensis. The investigations of Miss Holden have made it clear that the presence of traumatically recalled ray-tracheids is a common feature of the Cupressineae and the Taxodineae. In neither of these tribes are such structures known to occur normally in conservative organs; hence their former presence is revealed only by reversionary phenomena. It is worth while to note that the recurrence of marginal tracheids as the result of injury is usually exemplified, not in the immediate region of the wound where hypertrophy alone prevails, but in a region of the stem more or less remote from the actual injury. This situation is of interest because it is paralleled by conditions found in connection with certain other wound reactions, notably those presented in the case of the rays in certain angiosperms. It is obvious from what has been stated above that ray-tracheids, although of much wider occurrence among the Cupressineae and Taxodineae, probably appear only as the consequence of experimental conditions and are no longer a normal feature of structure. 
Normal and traumatic resin canals and traumatic ray-tracheids are abietineous structures occurring in certain Taxodineae and Cupressineae, facts which, in accordance with the general principles of comparative anatomy already elucidated, may be regarded as indicating the abietineous origin of the two coniferous subtribes in which they occur. This conclusion is now somewhat generally accepted by those whose anatomical knowledge of the conifers makes their opinion of weight.

Before we take leave of the two subtribes considered in the foregoing paragraphs it will be well to direct attention to the distribution and origin of wood parenchyma in woods of this type. The secondary xylem is characterized by the presence of usually abundant wood parenchyma, not confined to the end of the annual rings, but scattered throughout. The parenchymatous elements secrete a generally highly antiseptic essential oil. As a result of the presence of essential oils, and sometimes also by the infiltration of the tracheary walls with tannin, woods of taxodineous and cupressineous origin are frequently resistant to decay. The oil-secreting cells in the subtribes under discussion do not under normal conditions betray their derivation from tracheids except by the fact that they are grouped in series which have the fusiform shape of tracheary elements. In injured woods it is often possible to observe transitions between septate tracheids and rows of parenchymatous elements. It may accordingly be assumed, independently of the evidence of abietineous affinities supplied in the previous paragraphs, that the storage elements in the woods of the Cupressineae and Taxodineae are of tracheary origin. Another feature must be considered in this connection. In the minds of those who regard the Coniferales as an ascending series and not one of reduction, the resin canals which characterize the wood structure of the older Abietineae owe their origin to the clustering of the resin cells of the cupressineous or taxodineous type. This view of the origin of secretory canals in the coniferous series has been particularly emphasized by Penhallow. It meets with numerous difficulties, the chief of which is that the cells which surround the resin canals are not resin cells. The latter possess dark-brown contents and produce their secretion in an 
intracellular manner. The epithelial cells of the resin canals in the abietineous conifers, on the contrary, do not manifest the dark-brown (so-called) "resin," but pour their secretion at once into the secretory space. The secretory canals found in the case of Sequoia gigantea are surrounded by cells which correspond only to a very limited extent to the resin cells of cupressineous. woods. Most of the secretory elements are devoid of the so-called "resin" or dark-brown contents, and those containing this substance are present in about the same proportion near the canal as they are in the structure of the adjacent wood. This situation is clearly revealed in Figs. 249 and 250 .

In addition to the Taxineae, Araucariineae, Abietineae, $\mathrm{Cu}$ pressineae, and Taxodineaesubtribes of the Coniferales which have been discussed to a greater or less extent in the earlier paragraphs of the present chapter-there remain the Podocarpineae, a group which in the present period is confined almost entirely to the Southern Hemisphere. The podocarpineous forms are

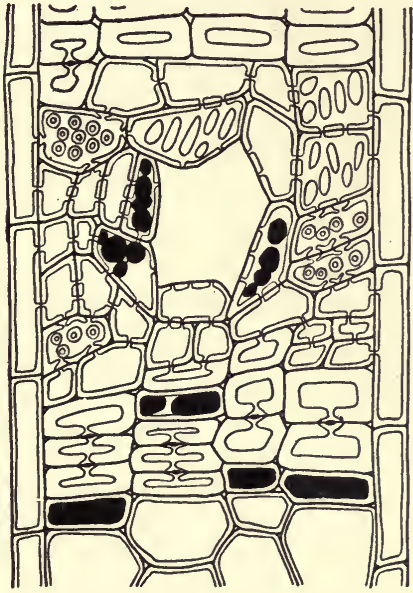

FIG. 249.-Transverse section of a traumatic or wound resin canal of Sequoia sempervirens. generally regarded, and with a strong degree of probability, as somewhat closely allied to the Taxineae or yews, which have their main distribution in the Northern Hemisphere. They are characterized, however, by a less degree of simplification in their ovuliferous cones and frequently by the possession of winged pollen of the abietineous type, produced always in bisporangiate microsporophylls. The organization of the female cone in the Podocarpineae as a whole, and particularly in the genus Podocarpus, recalls by the presence of a bract and a subtending ovuliferous 
scale the conditions found in the double scales of the ovuliferous strobilus of the Abietineae. The reproductive features consequently supply some evidence for the association of the podocarps
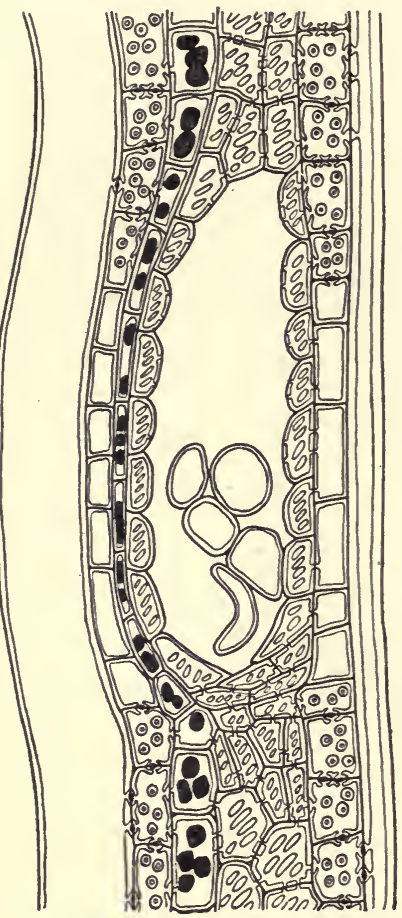

FIG. 250.-Longitudinal section of a traumatic resin canal in the same species.

with the abietineous conifers. The organization of the wood in the group under consideration is very similar to that found in the Cupressineae and Taxodineae. The tracheids possess opposite pitting and bars of Sanio. The parenchyma is abundant and scattered throughout the annual ring. A clear difference from the Cupressineae and Taxodineae is supplied, however, by experimental evidence, since neither traumatic resin canals nor marginal ray-tracheids have been found as yet in any of the genera of the Podocarpineae. We shall probably not make further advance in the final determination of the phylogenetic or evolutionary position of this subtribe of the Coniferales until our present extremely meager knowledge of the fossil conifers of the Southern Hemisphere has been notably increased. It seems highly probable, on the basis of the organization of the scales of the female cone and of the sporophylls and spores of the male cone, that the Podocarpineae have abietineous affinities. Their final position will ultimately be determined by a better knowledge of the anatomy of extinct forms.

Of the coniferous subtribes enumerated in the preceding pages the Taxineae have not as yet been considered anatomically. 
The reproductive structures in this group are extremely simple, and the ovuliferous apparatus produces in maturity a single ovule. In the genus Cephalotaxus of the Eastern Hemisphere an ovuliferous strobilus is present in the early stage of development; this is composed of scales each producing a pair of ovules-a condition comparable with that found in the Abietineae. As the development of the seed progresses all but a single seminal structure abort, so that in the end no vestige of the presence of a true female cone can ordinarily be observed. In accordance with the principle of recapitulation, we must regard the young cone of Cephalotaxus as indicating the more primitive condition for the ovuliferous apparatus in the genus. Taxus presents a marked contrast to that genus by the fact that at no

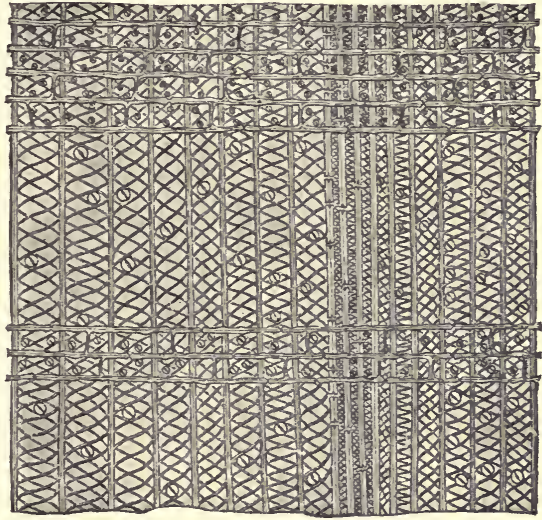

FIG. 251.-Semidiagrammatic view of the longitudinal organization of the wood in the Taxineae.

time is the presence of a female or ovuliferous cone indicated. From the first there is but a single ovule, and this is not related at any time to a visible ovuliferous scale. The organization of the male cones in the Taxineae is naturally less reduced than that of the female, in accordance with the general principle of the conservatism of the microsporangial apparatus in heterosporous groups. In the case of the Taxineae the reproductive structures furnish convincing evidence for the origin of the subtribe as the result of a process of reduction.

The structure of the wood in the Taxineae is quite characteristic. The tracheids are marked by internal spiral bands which are of late origin and are frequently for that reason designated as tertiary thickenings (Fig. 25I). Opposite pitting is present when the pores are 
numerous, and bars of Sanio are clearly developed. The statement is often made that the ligneous structure of the Taxineae is free from parenchymatous elements (Fig. 252). This is certainly true of the mature wood of the stem in both Taxus and Torreya. If, however, the roots in the two genera be examined, a varying amount of storage parenchyma is discovered which is ordinarily better developed in proximity to the region of the primary wood (Fig. 253). In species common to Europe and America, namely,

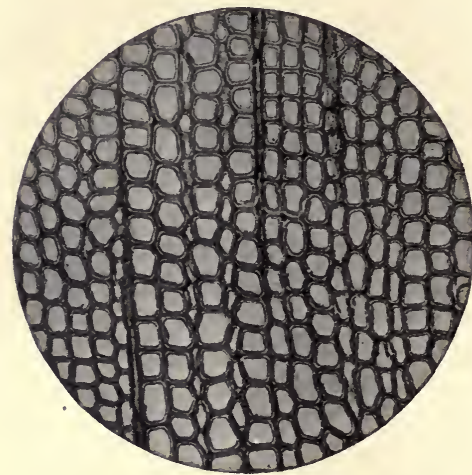

FIG. 252.-Transverse section of the wood of the stem in Taxus cuspidala.

Taxus baccata and its varieties, very little parenchyma is found even in the root; but in oriental species of the genus storage elements are somewhat abundant in this organ. The young stem, and particularly the root, of the genus Torreya show clearly developed longitudinal parenchyma in the wood. In Cephalotaxus, which by reason of the presence of a well-developed female cone at an early stage must be regarded as a primitive genus of the subtribe, parenchyma is markedly abundant in the organization of the wood. Injuries, also, frequently result in the recall of parenchyma in those taxineous woods which are normally without it. The generally uniseriate rays of the subtribe do not show the presence of traumatic raytracheids as a result of injury, and in this respect they present a marked resemblance to the woods of the Podocarpineae. Resin canals are conspicuously absent in the ligneous structures of the Taxineae, and not the slightest evidence of their former occurrence can be produced by experimental data. The genus Taxus is entirely without resin canals, even in its leaves, thus providing the only example of a conifer completely lacking these structures. An interesting parallel is presented by the hemlock (Tsuga) among 
the Abietineae. Here resin canals are normally absent, not only in the structure of the wood, but also in the other tissues of the stem. In the root of the genus they occur in the primary wood; and in the cone and leaves, in the tissues of the cortex. This distribution of resin canals is of course entirely in accord with the general principles of evolutionary anatomy. Tsuga, however, differs markedly from Taxus in the degree of obliteration of resin canals, since these structures here persist in conservative regions, while in the latter genus they have entirely disappeared. In Cephalotaxus resin canals are well developed except in the wood, but are apparently not susceptible of reversionary recall in ligneous structures.

It will be obvious from the description of the reproductive and vegetative organization supplied in the two preceding paragraphs that so simple a type as the genus Taxus

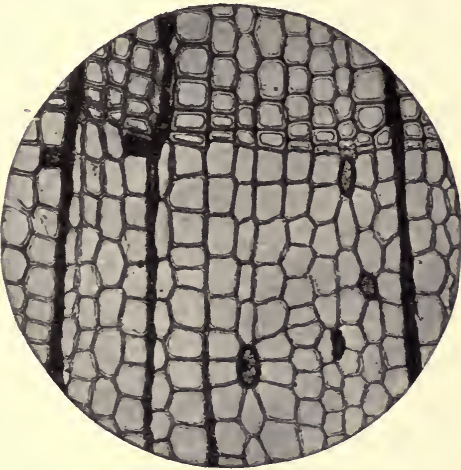

FIG. 253.-Transverse section of the wood of the root in Taxus cuspidata (after Bliss). cannot, according to the well-established principles of anatomy, be regarded as a primitive form. Its acceptance in this capacity by the earlier and philosophical taxonomy is shown on inductive evidence to be entirely unjustified. It appears on the basis of the facts at present available that the Taxineae are a reduction series in which the oriental Cephalotaxus among existing genera occupies the lowest position, while Taxus represents the summit. It is further obvious that the absence of wood parenchyma is not a primitive feature of organization of the group. If it be admitted that longitudinal parenchymatous elements primitively characterized the organization of the wood, a derivation from the same stock as the Podocarpineae is plainly indicated. This conclusion is justified by the consideration of the young ovuliferous cone in 
the genus Cephalotaxus. The anatomical evidence points very clearly to the Taxineae as a reduction series, taking its origin from the same general group or plexus as the Podocarpineae. The somewhat meager fossil data at our disposal do not warrant us in assuming a very ancient occurrence for the subtribe Taxineae, since woods of this type are not found earlier than the Tertiary or Cenozoic. It is true that cones and leafy twigs from various levels of the Mesozoic have from time to time been referred to taxineous affinities, but there is no anatomical evidence that they belonged here. So far as any data derived from anatomical structure are concerned, we are not justified in attributing to the Taxineae a great geological age. The external habit as a diagnostic criterion in the Coniferales has been shown in recent years to be almost as misleading as it has proved to be in the Cycadofilicales and other Paleozoic groups.

Having considered the anatomical organization of the various subtribes of the Coniferales in such detail as comports with an elementary work like the present, before summing up the situation from the evolutionary standpoint we may well make some reference to the anatomical characteristics of coniferous woods which are utilized by paleobotanical investigators. The genera of fossil woods logically increase in number as our knowledge of the ligneous organization of extinct plants becomes fuller and more complete. For the present purpose only a few of the more important genera of fossil woods need be considered. By reason of its relative resistance to decay, wood naturally often becomes isolated from its accompanying tissues and frequently is the sole surviving evidence of the former existence of gymnospermous groups. The situation thus presented is a difficult one, and the earlier characterizations of fossil wood were naturally largely empirical and made without reference to the facts or principles of comparative anatomy.

In deference to the prevailing view that the araucarian conifers are the most ancient and form the connecting link between the Coniferales and the Cordaitales, we may consider them first. It has been indicated in a previous paragraph that the mature secondary wood of the two living genera of the Araucariineae is 
characterized by alternating crowded pitting and the absence of bars of Sanio as well as of parenchymatous storage elements. To this type of wood occurring as a fossil the name Araucarioxylon is given. A second genus of fossil woods is diagnosed by the presence of spirals on the inner walls of the tracheids and by the absence of wood parenchyma, under the generic designation Taxoxylon. Those woods characterized by the possession of ligneous resin canals in the horizontal and vertical planes are included under the generic appellation Pityoxylon. In coniferous wood without either resin canals or conspicuous storage parenchyma, the name Cedroxylon is adopted. Woods which, on the contrary, are provided with abundant storage parenchyma are designated Cupressinoxylon. In all genera of fossil woods except the first the pitting is characteristically opposite. In the Araucarioxylon type the pitting is frequently crowded and alternation and bars of Sanio, universally present in other existing coniferous woods, are conspicuous by their absence.

Clearly the use of even the small number of genera of fossil woods indicated in the preceding paragraph in connection with evolutionary inferences must, in the light of the conditions described for the various living conifers in the earlier part of the present chapter, be a matter of great difficulty. This arises from the fact that the organization of the mature wood in a given conifer is by no means necessarily an indication of its true systematic position. The interpretation of the significance of wood structure in fossil and existing conifers can be successfully attacked only with a knowledge of the general principles of comparative anatomy. A failure to realize this situation has led to very many erroneous interpretations, both anatomical and paleobotanical. For example, it is quite clear from the data supplied at an earlier stage that the primitive condition of both the Araucarioxylon and Taxoxylon type was a Cupressinoxylon, since abundant wood parenchyma diffused throughout the annual ring was formerly a feature of organization of these ligneous types, as is shown by a consideration of conservative organs, experimental results, and fossil evidence. Further, in some instances the Cupressinoxylon type has been clearly derived from the complicated structure 
found in Pityoxylon. This is, for example, true in the case of the genus Sequoia, which, on the basis of general anatomical principles, obviously formerly possessed both the ligneous resin canals and the marginal ray-tracheids of the older Abietineae. Examples might be indefinitely multiplied to show that the use of the mature structure of the wood in the Coniferales, without recourse to comparative anatomical and experimental data, is almost certain to lead in a given case to fallacious conclusions.

It is now possible to sum up the situation from the standpoint of evolutionary anatomy for the phylogenetic sequence. It will be convenient to indicate preliminarily that the most generally accepted hypothesis of the morphological nature of the ovuliferous cone in the Coniferales furnishes a prima facie argument in favor of the primitive position of the Abietineae. In this subtribe the female cone consists of pairs of scales, the upper of which is ovuliferous and the lower sterile. The ovuliferous scale is with a strong degree of probability to be regarded as a persistent single sporophyll bearing two seeds or megasporangia on its morphologically lower but physically upper surface. The megasporophyll is in relation to the abortive axis of the reproductive short-shoot. Aside from the morphological interpretation of the structures concerned, however, the fact remains that the units of structure in the ovuliferous cone of the Abietineae are double and separate in their nature. In the female cones of the remaining coniferous subtribes there is clear evidence from comparative anatomy of the presence of fused pairs of scales in the ovuliferous structures. In accordance with general principles of morphology and without necessarily accepting the hypothesis of the short-shoot nature of the vertically paired scales of the abietineous cones, it is probable that the Abietineae are an older group than are the remaining coniferous subtribes.

An additional argument for the antiquity of the Abietineae is furnished by their obviously close relationship to the Ginkgoales, which are admitted on every hand to be a primitive group of gymnosperms. The affinity with the Ginkgoales shows itself in the common possession of vegetative short-shoots by both the sole surviving Ginkgo and by the ancient but still prolific genus 
Pinus. Further, both megasporophylls and microsporophylls in the two cases produce paired sporangia. The microsporangia of Ginkgo present a common difference with those of the Abietineae from lower forms in owing their dehiscence to a mechanical layer derived, not from the epidermal, but from the fibrovascular, tissues. An additional feature of affinity is supplied by the winged character and the internal organization of the microspores in the two groups, for they are practically identical. The structure of the tracheids of the wood in the Abietineae and Ginkgoales is significantly similar in the presence of opposite pitting and bars of Sanio, a common feature which distinguishes them from the Cordaitales and other older "gymnospermous groups. Further, although the pitting and other details of organization of the tracheids in the two groups is of the modern gymnospermous type, the structure of the xylem in primitive regions clearly shows a filiation with the conditions characteristically presented by the Paleozoic gymnosperms.

If a strong argument for the primitive position of the Abietineae is supplied by a comparison with the structural features of the Ginkgoales, an even more cogent one is furnished by their resemblance in important anatomical characteristics to the Cordaitales. It is the leaf of the extinct genus Prepinus which manifests, as has been indicated in earlier pages, the most categorical and distinct similarity to the foliar organization of certain Cordaitales. Not only is Prepinus the only known representative of the Coniferales to show distinct and unmistakable centripetal wood as distinguished from transfusion tissue, but it manifests its affinity to cordaitean forms by the presence of a double transfusion sheath in relation to the centripetal wood of the foliar bundle. It is further impossible to deny for Prepinus a close degree of relationship with Cretaceous species of Pinus which, like Prepinus, possess a well-marked double transfusion sheath and differ from the more primitive genus only in the absence of true centripetal wood. The agreement in foliar organization between Prepinus and cretaceous species of Pinus on the one hand and the Cordaitales on the other cannot be overlooked in any discussion of the evolution and affinities of coniferous subtribes. Although the wood 
of Pinus and Prepinus resembles that of Ginkgo in possessing opposite pitting and bars of Sanio, this organization contrasting with the tracheary structure of older types like the Cordaitales and the Cycadales in reality presents no difficulty. For in primitive regions and organs the alternating pitting without bars of Sanio characteristic of the Cordaitales is present in the Abietineae (particularly in Pinus and Prepinus), and passes by gradual transitions into the opposite pitting with bars of Sanio exemplified in the structure of the mature wood both in Ginkgo and in the Abietineae. The reproductive structures of the Cordaitales are too little known to us to supply appropriate points of comparison. It may be stated in summary that the Abietineae present as good anatomical evidence for derivation from the Cordaitales as do the Ginkgoales; and, moreover, they have an additional claim to such affinity by their clear relationship with the Ginkgoales.

But it is not only by reason of resemblances to the Ginkgoales and the Cordaitales, neither few nor unimportant, that the Abietineae show themselves to be a primitive subtribe of the Coniferales. When the anatomical organization of the remaining coniferous subtribes is investigated in the light of the general principles so often emphasized in the present volume, it supplies in many cases distinct evidence that the Abietineae are the primitive stock from which all have taken their origin. This situation may be illustrated first in the case of the Araucariineae, which are somewhat generally regarded as the primitive conifers and those most nearly connected with the Cordaitales. The strongest evidence for this claim is supplied by the organization of the tracheids, presenting the same alternating pitting and the absence of bars of Sanio as are manifested in the cordaitean and cycadalian gymnosperms. If alternating pitting alone were a sufficient criterion of relationship, many angiosperms by the possession of this feature could establish a claim to relationship with that ancient group of gymnosperms. The evidence here as in other cases should be read in the light of the general principles of comparative anatomy. The longitudinal radial section of the cone axis, and to a lesser extent of the leaf trace, of either Araucaria or Agathis at once reveals the true situation. In these cases one always finds bars of Sanio 
in the region of the secondary wood near the primary xylem, and, where the pitting is abundant, frequent opposition of the pores. In other words, a situation presents itself which is the exact converse of that found, for example, in the Ginkgoales, in which the primitive region of the secondary wood shows alternating pitting and no bars of Sanio. In the later-formed wood the pits begin to appear in opposition and are separated by bars of Sanio. If we are justified in regarding the type of tracheid found in Ginkgo as originating from that typical of Cordaitales and other ancient gymnosperms, we are similarly warranted on the same evidence in viewing the alternation of pitting and the absence of bars of Sanio in the adult wood of the existing araucarian conifers as derived from a state in which both opposition of pitting and bars of Sanio were present. The evidence supplied by fossil forms, moreover, justifies the derivation of the Araucariineae from abietineous forbears, since many distinctly araucarian woods have been discovered in recent years in the Mesozoic which clearly present either normal or traumatic features uniting them with the Abietineae. In some instances, for example, thick-walled, heavily pitted ray cells, similar to those commonly characteristic of radial parenchyma in living and fossil Abietineae, are found in Mesozoic araucarian woods. This feature is significantly paralleled in the rays of the cones of living araucarians. Traumatic resin canals are also found commonly in woods which are transitional from the abietineous to the araucarian type. This is notably the case in Brachyoxylon, a very common Mesozoic type of wood, and also in the much rarer Araucariopitys. Normal resin canals are, moreover, found in the wood of the ovuliferous cone of the living Agathis Bidwillii, from Java. It has been stated in earlier pages that there is distinct evidence from the organization of actual fossils and from the structure of conservative regions in the existing Araucariineae that abundant wood parenchyma was an original feature of organization of the araucarian as contrasted with the abietineous Coniferales and the Ginkgoales, in which tangential storage elements are conspicuously absent.

Not only, however, have abietineous forms obviously given rise to the araucarian stock on the basis of the general principles of 
comparative anatomy and the organization of fossil forms, but the same derivation is likewise indicated by two other series-the Taxodineae-Cupressineae on the one hand and the PodocarpineaeTaxineae on the other. The first series, on the basis of comparative anatomical evidence, formerly possessed the ligneous resin canals and the marginal ray-tracheids of the later Mesozoic Abietineae. The supposed species of Sequoia of the Mesozoic are not representative of the living genus, but have the organization of araucarian conifers, as has been shown by recent anatomical investigation of the forms from the American Cretaceous. They accordingly have no bearing on the question of the origin of the Taxodineae-Cupressineae. In the case of the series which has been described above as the Podocarpineae-Taxineae, the evidence from fossil forms is practically non-existent in the present state of our ignorance in regard to the organization of the Mesozoic conifers of the Southern Hemisphere. The situation must therefore be judged on the basis of the living forms. The reproductive structures of the Podocarpineae, particularly those of the genus Podocarpus, are strikingly abietineous and sufficiently clearly indicate the affinities of the subtribe. The primitive Taxineae, obviously in accordance with the established principles of comparative anatomy, formerly possessed the abundant wood parenchyma of the Podocarpineae, and their systematic position is therefore elucidated. If the statements in the present paragraph are well founded, evidently both the Taxodineae-Cupressineae and the Podocarpineae-Taxineae are of abietineous origin.

The time has now come to summarize the phylogenetic affinities of the Coniferales, both as regards the general relationship of the tribe and as regards the affinities of its particular subtribes with one another. It seems clear that the Abietineae have on all counts the strongest claim to be considered as primitive representatives of the Coniferales. These may be summarized as follows: filiation with the Cordaitales and co-ordination with the Ginkgoales; precedence to the Araucariineae, to the TaxodineaeCupressineae, and to the Podocarpineae-Taxineae. The evidence for the ancestral character of the abietineous conifers may in the future be fuller, but scarcely any stronger, than it is at the 
present time. The accompanying genealogical tree (Fig. 254) will make clear the views as to sequence and affinities developed in the previous paragraphs of the present chapter. The Cordaitales of the Paleozoic serve as the starting-point, and from them were derived two cognate stocks-the Coniferales and the Ginkgoales. The latter have suffered much extinction and end in the present epoch in the sole surviving genus, Ginkgo. The Abietineae in earlier Mesozoic time gave rise to the Araucariineae, which flourished greatly in the later epochs of that period, to become almost extinct in the glaciation which ushered in the Tertiary. The araucarian conifers distinctly took their origin from the abietineous stock after it had developed ligneous resin canals, but before marginal ray-tracheids had

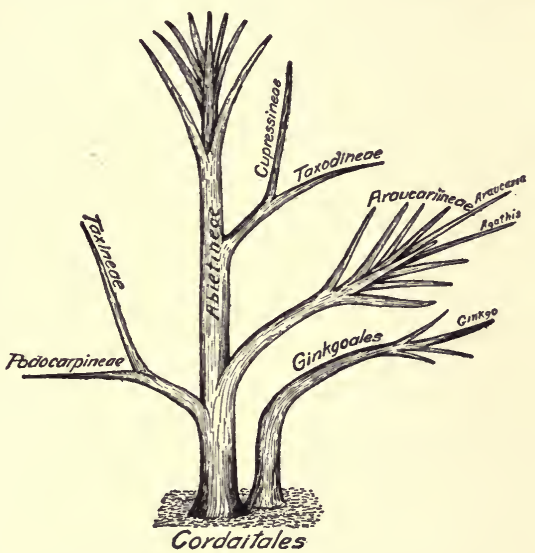

Fig. 254.-Genealogical tree of the Coniferales, showing their proximity to the Ginkgoales.

made their appearance. The Podocarpineae-Taxineae may have originated at a still earlier epoch and before the abietineous stock had developed the resin canals or the marginal ray-tracheids which distinguish it from the Cretaceous onward. Unlike the Araucariineae the Podocarps and their allies, the yews, did not lose the opposite pitting and bars of Sanio common to the parent stock of both Abietineae and Ginkgoales. The Taxodineae are a still later offshoot of the strong abietineous line and come into existence subsequent to the appearance of marginal ray-tracheids and ligneous resin canals. Certain conifers of the earlier and later Mesozoic, such as Voltzia, Brachyphyllum, Geinitzia (sometimes erroneously designated Sequoia), etc., which have been referred to taxodineous 
affinities, in reality have nothing to do with that group, but are araucarian or pre-araucarian in their relationships. There is at the present time no trustworthy evidence that the Taxodineae were in existence before Tertiary times, although it is quite possible in view of the general situation that they made their appearance in the later Cretaceous. The Cupressineae must be regarded as a continuation of the taxodineous line and as having a similar relation to the abietineous ancestral forms.

In conclusion, it may be remarked that, whatever may be the differences of opinion in regard to the reading of the evolutionary document supplied by the Coniferales as they now present themselves to our gaze or are preserved for us as fossils from earlier geological epochs, there can be no doubt that they constitute the most important of all documents for the development of general evolutionary principles as the result of inductive reasoning. The treatment of the group in the present and preceding chapters is intended to clear the way for their further study by the development of general situations in relation to particular anatomical and paleobotanical facts. A continued investigation of the group, for which our American Mesozoic deposits have already yielded so much material of crucial importance, is likely to result in the firm establishment of extremely valuable general principles for that type of biological research which bases its conclusions on inductive reasoning rather than on any purely philosophical attitude, either mechanistic or vitalistic. 


\section{CHAPTER XXV}

THE METAGYMNOSPERMAE: GNETALES

The aggregation of forms included in the present chapter is very small, but is of great importance from the phylogenetic standpoint. The Gnetales are represented in the existing flora of our earth by three genera. Of these the genus Ephedra occurs throughout the Northern Hemisphere, being somewhat abundant in the American and Asiatic continents and rare in Europe. Gnetum is a characteristically vinelike form occurring in the eastern and western tropics. The third genus, Welwitschia, is monotypic and is confined to the southwestern region of the African continent. Of the three generic types enumerated, Ephedra must be considered on account of both its reproductive and its vegetative features as on the whole the most primitive, although naturally its desert habitat has exerted more or less influence on its internal organization. Gnetum in general represents the highest stage of development attained in the group, and this statement is particularly true of the features presented by the organization of its gametophytes. Anatomically, however, Gnetum seems to occupy a somewhat less specialized position than does the extremely xerophytic Welwitschia. The South African genus just named in its gametophytic organization is in a general way intermediate between Ephedra and Gnetum. The consideration of the reproductive structures proper, using that designation to cover both the floral organization and the gametophytes, need not be covered more than incidentally in the present volume, since the morphology of the parts related to sex has recently been fully discussed in Coulter and Chamberlain's Morphology of Gymnosperms.

A transverse section of a stem of Ephedra (Fig. 255) reveals an anatomical situation not found in any of the Coniferales. Here the woody cylinder is characterized by rays which become very broad in the outer region of the wood but are quite narrow in the vicinity 
of the pith. Not only is the structure of the wood in the genus under discussion contrasted in the nature of its radial parenchyma with that of the Coniferales, but it also presents a striking resemblance to the dicotyledons in the possession of true vessels, albeit of a primitive type. The correlation of large rays and true vessels in the organization of the wood in both Gnetales and dicotyledons is a feature which is clearly not without evolutionary significance, as will be shown in subsequent pages. The pith is of moderate

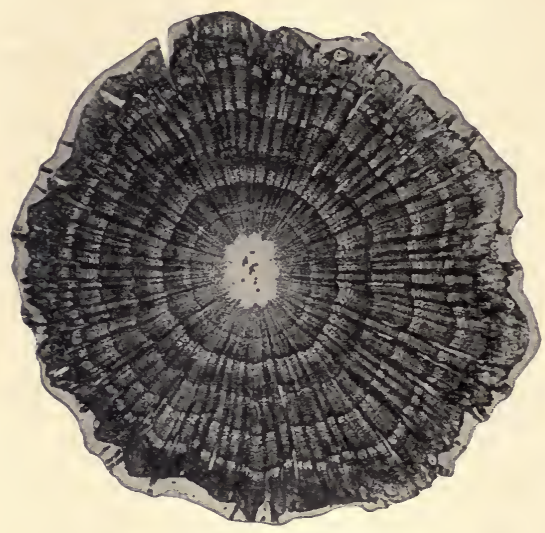

Fig. 255.-Transverse section of the stem of Ephedra gerardiana. size and the phloem and cortex constitute a rather thin layer on the surface of the strongly convoluted woody cylinder. The depressions on the face of the wood plainly correspond to the position of the large rays, as is the case with identical conditions in certain dicotyledons.

In Fig. 256 is shown a transverse view of the structure of the wood somewhat highly magnified. The features presented by the vessels and rays stand out very clearly. It is obvious that annual rings are present, although not conspicuously developed. The parenchyma is of diffuse distribution as in the higher conifers. The large rays, however, illustrate interesting and phylogenetically important features. Even in the transverse view they are clearly composite structures and do not consist homogeneously of parenchyma, as in the corresponding radial bands in our northern oaks and in the genus Gnetum. Vessels are seen intimately incorporated in the organization of the ray. The vascular are not the only elements of the longitudinal structure of the wood 
incorporated in the large ray, for fibers are also present, although they cannot be so well recognized in the transverse section. The constitution and origin of the large rays in Ephedra are of great significance, not only for the Gnetales themselves, but also in connection with the problem of the evolution of the higher forms known as dicotyledonous angiosperms. It will accordingly be considered somewhat in detail.

Fig. 257 illustrates the organiza- species.

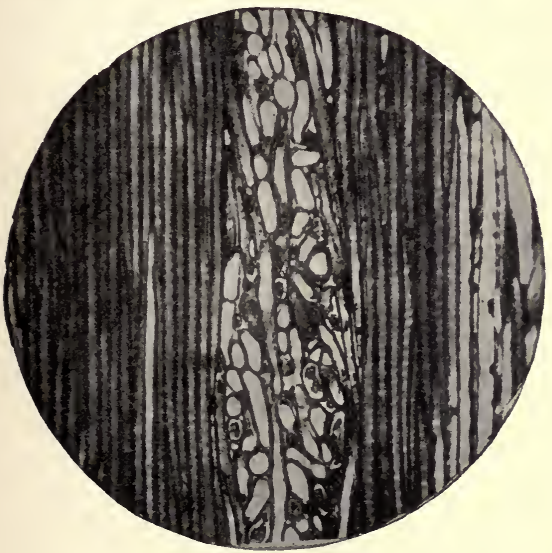

Fig. 257.-Longitudinal section of a large ray of Ephedra in proximity to the pith.

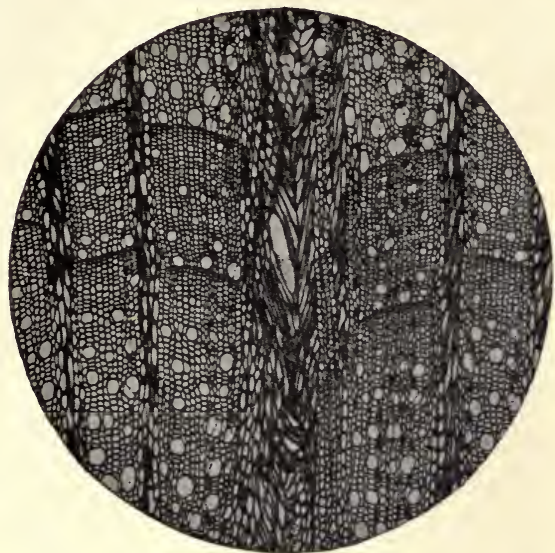

Fig. 256.-Transverse section of the wood of Ephedra tion of the large ray in an early stage of development when it is still rather narrow and close to the medullary region of the stem. An inspection of the illustration makes it clear that the ray is by no means a homogeneous structure composed entirely of storage parenchyma. Fibrous elements necessarily enter 
largely into its composition, and these are often in the condition of septation. The ray, in fact, is a composite structure, organized only partially from true radial parenchyma and also consisting largely of transformed longitudinal fibrous elements of the wood. These first become septate, and, particularly in the more external regions of the wood, their divisions become progressively more and more like the ordinary storage elements of the ray. Fig. $25^{8}$ por-

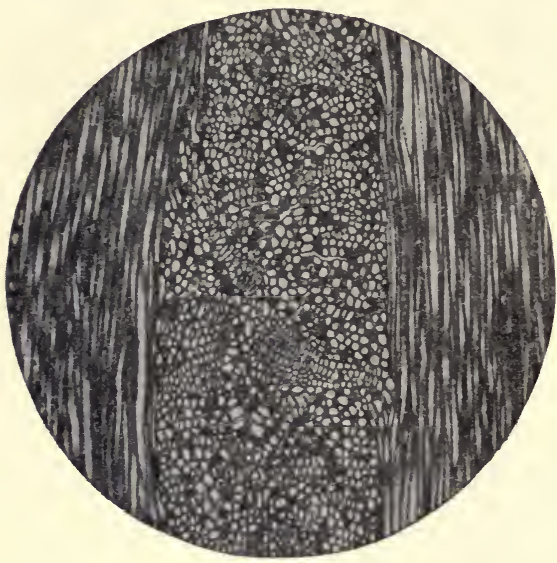

FIG. 258.-Tangential view of a large ray of Ephedra in its external region.

trays the longitudinal organization of the wood in the outer region of a rather thick stem of Ephedracalifornica. The large rays are here conspicuous and numerous, but not of equal size. In general, those of greater dimensions have originated in the region of the medulla, while those less conspicuous by their size have come into existence more recently. The small degree of magnification employed in the figure does not make it possible to discern clearly the composite character of the radial parenchyma. The next illustration, Fig. I29, which reproduces one of the smaller radial masses under a higher magnification, makes the organization of these structures apparent. Obviously not only ordinary radial parenchyma is concerned in the constitution of the rays, but also numerous fibers and even vessels. It may here be stated, although that situation is not clearly shown in the illustration, that fibers are seen in such rays in all conditions of transformation into elements resembling the ordinary radial parenchyma. In the genus Ephedra we have the wedding, as 
it were, of radial and longitudinal storage devices to constitute a new and, from the evolutionary standpoint, an extremely important type of radial organization. A fact not without significance in the present connection is the correlation of vessels with the more abundant storage devices present in the wood of the Gnetales. The large ray in the Gnetales is evidently a composite derived from the fusion in certain radii of the wood of the original radial storage parenchyma with parenchymatous elements derived from the copious transformation of the longitudinal tracheary elements of the wood by septation. As has been indicated in earlier pages, wood parenchyma made its appearance in the earlier and lower Coniferales first in relation to the end of the annual ring

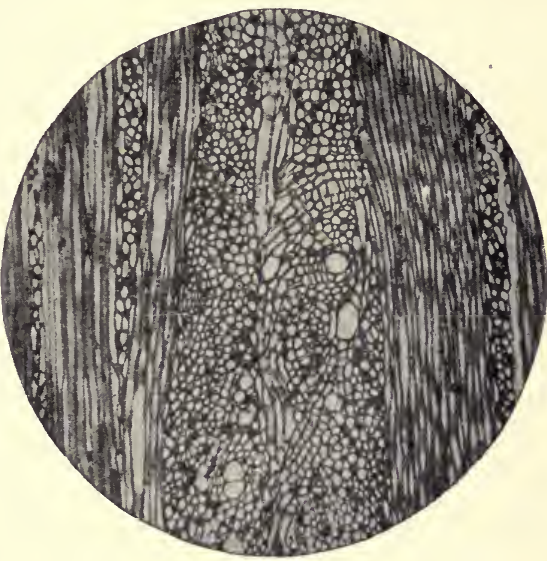

FIG. 259.-Another view of the same and in Mesozoic times, when well-marked annual periods of vegetative inactivity had become established. Subsequently the wood parenchyma, in its inception clearly derived from the septation of tracheids, became diffused throughout the annual ring. When it had become abundant and well established in this condition, a situation favorable to the appearance of large rays was attained. The final impetus to the appearance of these structures was supplied by the origin of vessels which, by providing a greater supply of food substances and water from the soil, rendered possible larger and more efficient leaves. These in turn produced larger quantities of assimilates which by the appearance of the large rays (co-ordinate in their origin with vessels and 
diffuse wood parenchyma) found storage in the woody tissues of the axis.

It will be obvious from the facts brought forward in the preceding paragraph that diffuse and abundant wood parenchyma, vessels, and large rays are features which are intimately correlated in the organization of the woods of the Gnetales and the dicotyledons. That the situation portrayed is correct from the

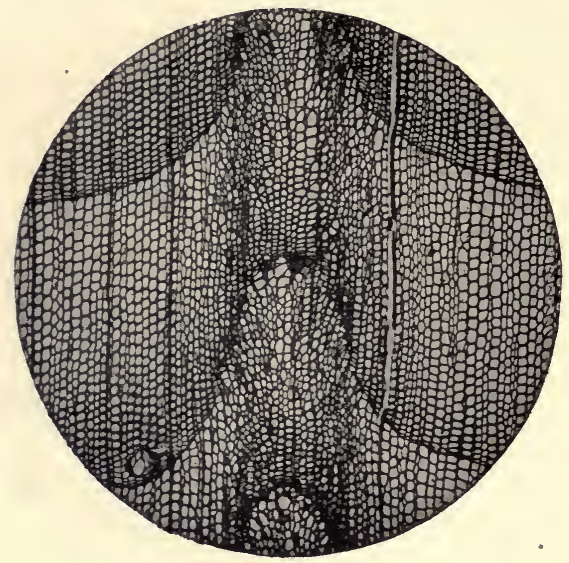

FIG. 260.-Aggregate ray from Pinus Strobus evolutionary standpoint is rendered clear by a condition presented not infrequently in the wood of our living species of Pinus. Fig. 260 illustrates an interesting abnormal feature which is often found in the wood of the white pine. The annual rings are strongly depressed locally, and in these regions the rays of the wood are unusually abundant, or, in other words, are clustered or aggregated. Since in the genus Pinus wood parenchyma is absent-a feature, as has been indicated in earlier pages, definitely correlated with its primitive phylogenetic position - the clustering of the rays, particularly in the absence of vessels, is of no evolutionary significance. This, of course, is primarily the result of the absence of the later acquired capacity of producing the longitudinal parenchyma so necessary for the fusion of the aggregation of rays into large and homogeneous storage units. It may further be remarked in a general way that there is no evidence to show that the large masses of storage parenchyma which are so striking a feature of the organization of the woody cylinder of both the Gnetales 
and the dicotyledons were in the first instance related to the appendages, whether branch, leaf, or root. While, however, the prominent masses of radial storage parenchyma which distinguish the groups under consideration are not primarily related to the appendages, they become somewhat definitely limited to this position in many forms, and even in the Gnetales themselves are more strongly developed in connection with lateral organs.

We may next turn to the anatomical organization of the genus Gnetum. Fig. 26I illustrates the general structure of the stem in this genus as exemplified by a young stem of Gnetum scandens. The appearance is very similar to that of a dicotyledonous vine like Clematis or Vitis. Extremely prominent large rays separate the woody cylinder into distinct fibrovascular

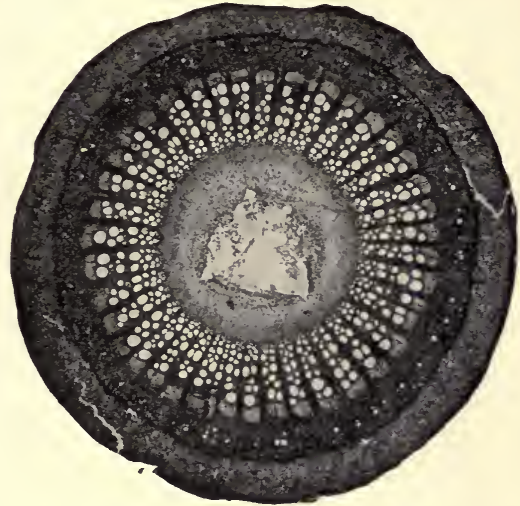

FIG. 26r.-Transverse section of young stem of Gnetum scandens.

strands. The large rays of Gnetum are distinguished from the corresponding features of organization in Ephedra by two important details. In the first place, like those of the dicotyledonous climbers cited above, they extend broadly to the pith and do not appear first as narrow rudiments which are widened as they pass toward the outside of the woody cylinder. Secondly, the broad rays of Gnetum are in the stem usually entirely homogeneous; that is, they contain no distinct vestiges of fibrous and vascular structures such as appear in the broad radial storage bands of Ephedra, although in types like $G$. scandens the broad rays of the stem are not obviously derived from compounding of aggregations of rays and longitudinal elements of the wood. Investigations on the part of Professor W. P. Thompson as yet unpublished seem to make it clear, how- 
ever, on the basis of the organization of primitive organs and regions that the condition of aggregation presented by the genus Ephedra was once present in Gnetum. We may therefore regard the large rays of the genus as comparable with the similar structures in the mature wood of the stem of our northern oaks and derived in a similar manner as the result of aggregation and fusion. The minute organization of the secondary xylem in Gnetum reveals

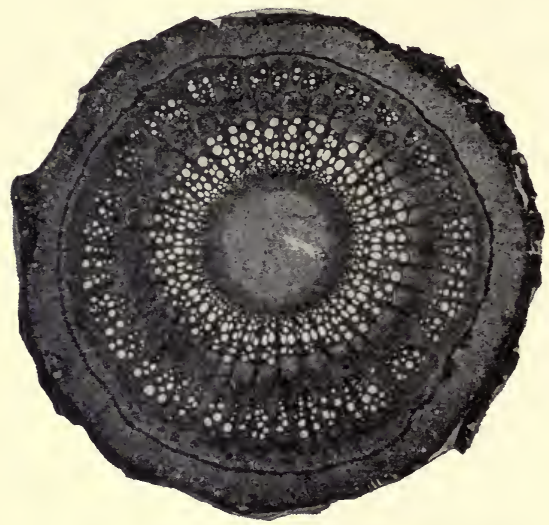

FIG. 262.-Transverse section of an older stem of the same.

condition. Last and by no means least, vessels which in most species of the genus present the large caliber characteristic of climbers are present. These will be considered later in a special paragraph.

In Fig. 262 is shown an older stem of G. scandens. Here the woody cylinder instead of consisting of a single circle of bundles has become polydesmic. This condition cannot be regarded as having any large evolutionary significance, as it is commonly found in the stems of climbers of remote systematic affinities. Its chief significance is in connection with the origin of the type of cylinder presented by the genus Welwitschia and that found in certain cycadean types, living and extinct. Gnetum shows, not only the polydesmic stem often found in woody climbers, 
but also the extreme condition found in strap-shaped lianae. Fig. $26_{3}$ portrays this condition in the stem of $G$. latifolium. The woody strands of the polycyclic cylinder fail to develop on two opposite sides of the axis, and this organization is correlated with the flattened transverse section in the stem. It is obvious that the genus under discussion has advanced to a condition of organization such as is paralleled by perennial climbers among the dicotyledons, and it must therefore be considered as having reached a high evolutionary position.

The broad short axis of the remarkable genus Welwitschia may now claim our attention. Here the stem never attains a height

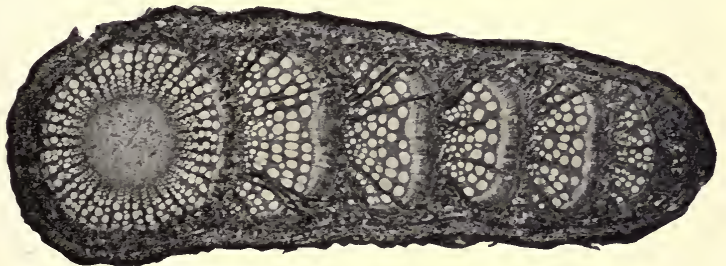

FIG. 263.-Transverse section of the flattened stem of Gnetum latifolium

of more than a half-meter and bears two large perennial leaves which, according to the investigations of Bower, are not the persistent cotyledons, but a subsequent pair of foliar organs. The perennial leaves of Welwitschia are inclosed at their bases in hollow spaces resulting from the outgrowth of the stem. Within these cavities, which function as moist chambers, are situated the basal growing regions of the leaves; they are thus preserved from fatal desiccation under the extremely arid conditions connected with the desert habitat of the genus. Professor W. P. Thompson has been able, through the co-operation of the Sheldon Foundation of Harvard University, to secure an abundant supply of material illustrating the anatomical organization of Welwitschia. When the results of his investigations have been published, our knowledge of this interesting and aberrant South African genus will be largely increased beyond that supplied in Hooker's well-known memoir. The general organization of the axis in the genus is well illustrated 
by the accompanying figure (Fig. 264) of the stem of a seedling collected by Professor Thompson in Southwest Africa. It is clear that the same polydesmic organization of the axis is present as is found in the older stem of species of Gnetum. In the South African genus, however, the polydesmic condition extends to the roots and is accordingly to be regarded as a more innate feature of organization than in Gnetum. The structure of Welwitschia

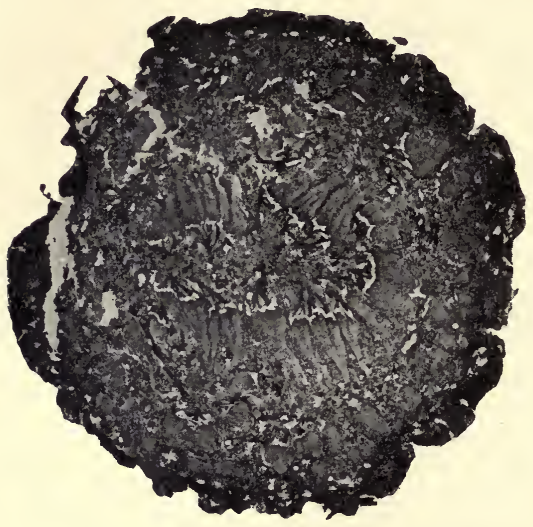

FIG. 264.-Transverse section of young stem of Welwitschia mirabilis. suggests a climbing ancestry. Although we do not know the explanation of the polydesmic condition in climbers, it is clearly co-ordinated with the vine habit. We may suppose that the forbears of the genus were originally forest climbers and that the surviving strongly truncated desert species has persisted in its present habitat with the retention of the ancestral polydesmy. A similar suggestion has been made in an earlier chapter as a possible explanation of the phenomenon of polydesmy in the cycadean forms, living and extinct.

Having considered the general topography of the stem in the Gnetales with particular reference to the presence of large rays of the dicotyledonous type and the phenomenon of polydesmy, we may now profitably turn our attention to the more minute organization of the wood. The ligneous tissues consist of rays and longitudinal elements. The former have already been sufficiently discussed in previous paragraphs. The longitudinal structures of the xylem consist of tracheids, vessels, and storage parenchyma. The tracheids do not need any extended reference, as they have 
been described in an earlier page of the present work. They possess clearly marked bordered pits larger than those of the dicotyledons and provided with a distinct torus. The vessels of the tribe are of considerable interest from the standpoint of the doctrine of descent, as they clearly indicate the derivation of vascular structures from tracheids. This takes place by the modification of the terminal regions of the incipient vessel from gradually tapering to distinctly inclined walls at angles with the sides of the element. These differentiated terminal aspects of the vessels are provided with very much larger pits than are found in the lateral walls. These pits, however, generally in Ephedra, and apparently universally in the two higher genera, lose their membranes at an early stage, and free intercommunication is thus established. In the higher genera there is a marked tendency for the terminal walls of the vessel to develop a single huge bordered pit in which the membrane is lacking. In Ephedra, on the other hand, the terminal pits are numerous, and in a few cases are found to fuse with one another horizontally with the resultant appearance of slits comparable to those in the vessels of the lower dicotyledons. The type of vessel found in Ephedra has been recently stated to persist in the reproductive axes of the genus Gnetum. The parenchymatous structures of the Gnetales need no extended reference, for on the whole they resemble those of the higher Coniferales, both in their distribution in the annual ring (and this is diffuse) and in their configuration. Sometimes structures occur in the woods of the Gnetales more or less resembling substitute fibers, since with pointed elongated configuration they unite a persistence of protoplasmic contents. It is clear from the brief summary of the organization of the wood here supplied that the Gnetales are of great importance from the evolutionary standpoint, particularly in connection with the important problems presented by the evolution of large rays and vessels. They furnish a valuable criterion for the estimation of primitive anatomical characteristics in the organization of the wood in that huge heterogeneous aggregation of forms assembled under the caption of dicotyledons. Their value in this respect will appear at a later stage. Although the Gnetales clearly indicate conditions of anatomical organization 
which are of a primitive nature, we are unfortunately even less well informed as to their fossil representatives than in the case of the angiosperms. There are, in fact, no well-authenticated gnetalian remains, even from the later period of the Mesozoic, in which the dicotyledons had become well established as an important component of the plant population of the earth. The wide geographical distribution of the three living genera may perhaps be regarded, in conjunction with their anatomical organization, as a definite indication of their earlier more abundant occurrence.

The root in the Gnetales needs no special consideration in the present connection. In the genus Gnetum it furnishes some evidence as to the original organization of the large rays, but in Welwitschia it is polydesmic like the stem and is of little value from the standpoint of evolutionary anatomy. The structure of the root in Ephedra closely resembles that in the stem, except as regards those general features which distinguish root from axis.

The foliar organs of the group which forms the subject of the present chapter are distinctly gymnospermous in their anatomy and frequently exhibit the copious development of transfusion tissues of the type characteristic of the leaves of the Ginkgoales and Coniferales. Centripetal wood is conspicuously absent in the leaves of the Gnetales, unless it be assumed that the transfusion elements are actually representatives and not merely derivatives of the old centripetal or cryptogamic wood. On account of the small size of the leaves in Ephedra the transfusion tissues are relatively poorly developed. In Gnetum a higher systematic position is strongly vouched for by the organization of the leaf, in which transfusion elements are not particularly well developed. The foliar organ of Welwitschia supplies us with transfusionary features most strongly manifested. The truth of this statement may be verified by reference to Fig. $26_{5}$, which illustrates a part of a transverse section of the leaf of the South African genus. Transfusion elements originating on the flanks of the xylem extend, as in certain Coniferales, above and below outside the sclerenchymatous sheath which surrounds the fibrovascular bundles of the leaves. 
The anatomy of the Gnetales is of particular importance at the present time when they have come to the front once more, either as a cognate stock with the dicotyledons or as their actual ancestors. The study of the internal organization of the group in comparison with the dicotyledonous angiosperms reveals many features of marked resemblance. Both are provided with large rays which are clearly fusion products; and in both the wood shows conducting elements belonging to the category of vessels. The rays apparently supply a very cogent argument for the close affinity of the Gnetales and the angiosperms. In the case of the vascular structures, however, it is not so clear that a morphological identity of the elements present in

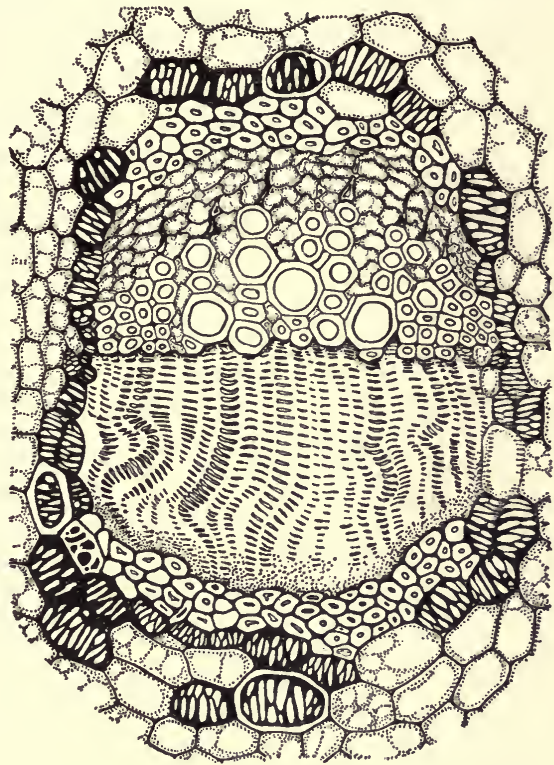

FIG. 265.-Transverse section of a leaf bundle in Welwitschia mirabilis. the two groups can be successfully maintained. In the dicotyledons the pits present on the terminal walls of the vascular elements are not larger than those which appear laterally, and the perforation of the vessel takes place as the result of fusions of opposite pits, as has been shown in an earlier chapter. A very different situation manifests itself, as indicated above, in the genera of the Gnetales. Here the vascular elements have exceedingly large pits on their terminal walls, and in Ephedra these are usually without membranes, 
while in the other two genera they are invariably so characterized. The phenomenon of fusion of the pits to form the scalariform perforations present in the lowest type of vessel of the dicotyledons is rare in the woods of the Gnetales. The general anatomical organization of the fibrovascular tissues in the Gnetales, however, may be said distinctly to favor the hypothesis of dicotyledonous affinities, or, at any rate, to be more in harmony with such relationship than with that of any other group of seed plants. The affinities of the Gnetales on the gymnospermous side are much more difficult to discover. This situation is due no doubt in large part to the fact that as yet no properly authenticated fossil representatives of the group have been found. In recent years there has been a tendency to associate this group with the Cycadales, but it is extremely difficult to discover any valid reasons from the anatomical side to justify such a relationship. There seem, in fact, to be no anatomical features of wide validity which can be invoked in favor of an affinity between the highest living gymnosperms and the lowest. It seems on the whole more probable that the Gnetales are cognate with, or derived from, the Coniferales, since there are a number of features which make such a connection likely. The transfusion tissue of the highest gymnosperms is clearly of the coniferous type, and this feature must count strongly against any close relationship with the Cycadales, in which, owing to the strong persistence of the centripetal wood, true transfusion tissues have not yet made their appearance. The uniseriate rays of Ephedra and Gnetum also point to coniferous affinities and not toward a connection with the Cycadales, in which the rays are universally multiseriate. The presence of bars of Sanio in the vessels of Ephedra supplies likewise an argument for the coniferous rather than the cycadalian relationship.

The main support for the cycadean origin of the gymnospermous group which forms the subject of the present chapter has been derived from the reproductive structures of the living and extinct representatives of the cycadean stock. The investigations of Wieland have brought to our knowledge the complete organization of the reproductive parts in the Bennettitales. These consist 
of cones involved in the young condition in rather large sheltering bracts. Immediately above the series of bracts microsporophylls (usually of large size and pinnate structure) are attached to the axis of the strobilus. The main part of the cone is, however, made up of the seminal organs, consisting of pedicels to which single seeds are attached in an orthotropous manner. The seeds are somewhat sheltered by the swollen ends of sterile appendages inserted among the seminal organs on the axis. The protected condition of the seeds has given rise to the suggestion of angiospermous organization. This apparently cannot be regarded as more than the merest analogy, since the seeds are not sheltered within a closed megasporophyll and the pollen is deposited directly upon them after the typical gymnospermous manner. Further, the cycadean fertilization is not even siphonogamous, as would be expected of a group presenting valid claims to be regarded as ancestral to the highest seed plants. Nor is the analogy with the flower of the angiosperms to be given greater weight, for the arrangement alone and not the intimate organization of the reproductive parts shows any real resemblance to the conditions found in the floral structures of the angiosperms. An androgynous cone such as is frequently present in the Coniferales supplies an equally good basis for comparison; for here the bracts below correspond to the floral envelopes of the flower, while the lower whorls of microsporophylls simulate the anthers, and a plausible resemblance to the carpellary whorls is presented by the ovuliferous scales of the upper region of the modified cone. The most striking objection and the same as occurs in the case of the Bennettitales is the fact that the ovuliferous scales are not angiospermous. The siphonogamic fertilization of the Araucarian conifers, on the other hand, reveals a nearer degree of resemblance to the conditions found in the flower of the angiosperms than does the zoidogamy of the archigymnospermous Bennettitales. In the present state of our ignorance of the fossil ancestors of the Angiospermae it seems impossible to fix on any group of gymnosperms, living or extinct, except the Gnetales, which can be regarded with any degree of probability as having been either ancestral to the highest of the seed plants or even cognate with them. 
In accordance with the hypothesis of the derivation of the angiosperms from the Bennettitales, a relationship between this cycadean group and the Gnetales has been suggested. There seem to be very slight grounds for this assumption of affinities. The so-called "pollen chamber" of Ephedra is not morphologically equivalent to the pollen chamber of the lower gymnosperms which is derived by the breaking down of cells in the lysigenous manner, and in this respect is in marked contrast to the depression on the apex of the nucellus of Ephedra, which is continuously covered with the epidermis. Moreover, Ephedra and the other Gnetales are siphonogamous in contrast to the zoidogamous condition of fertilization in the Cycadales. The details of organization of such a flower-like structure as is found in Welwitschia apparently supplies no adequate basis for comparison with the bisporangiate strobilus of the Bennettitales. The original error of Saporta in designating the impressions of the fructifications of bennettitean types as belonging to hypothetical proangiosperms or primitive angiosperms has manifested a considerable degree of vitality in the rather long interval since it was first advanced; but there seems little reason to accept it at the present time, in view of our increased knowledge of the organization of both vegetative and reproductive parts in the various groups of seed plants, living or extinct.

The Gnetales are clearly gymnosperms which in certain features of anatomical structure and reproductive organization have made a marked advance in the direction of the characteristics of the angiosperms, and it is not improbable that they are at least a cognate group. They present, however, no valid resemblances in either their reproductive or their vegetative parts which justify an assumption of relationship, even remote, with the extinct bennettitean Cycadales. It therefore appears highly unlikely that either the angiosperms or the Gnetales have any close degree of relationship with any archigymnospermous group, although it seems not at all improbable that they are somewhat closely related to one another. 


\section{CHAPTER XXVI}

\section{THE ANGIOSPERMS}

The angiosperms as a whole possess features which separate them sharply both reproductively and vegetatively from gymnospermous groups. The first of these in importance is the phenomenon of angiospermy itself. In the group the microspores or pollen grains no longer reach the micropylar canal at the apex of the ovule, but are accommodated on the tip of the megasporophyll, which becomes modified as the receptive prominence or stigma. The reception of the microspores on the terminal region of the carpel or sporophyll is not, however, an exclusive characteristic of the angiosperms, since the same feature is presented by the surviving araucarian conifers. The megasporophylls in this largest and most important group of the seed plants are either folded upwardly upon themselves in such a manner as to inclose the young seeds or ovules, or undergo protective fusions with other similar structures in the same flower. The angiosperms are characterized anatomically throughout by the possession of vessels, these structures being absent only in certain aberrant representatives of the Cactaceae, Crassulaceae, and Trochodendraceae. In all the exceptional cases mentioned there is clear evidence on comparative anatomical and experimental grounds that vessels were formerly present. The possession of histological structures in the wood known as vessels may accordingly be set down as a primitive feature of organization of the angiosperms. Associated with the vascular structures just indicated, there is a general improvement in the storage devices of the wood which, in an extreme form, leads to the appearance of the herbaceous type. The gametophytes, particularly the male gametophytes, of the angiosperms present a very marked degree of uniformity in the two great divisions of the group. The microspore or pollen grain undergoes part of its germination in the microsporangium and develops normally a tube nucleus and a generative cell. The 
latter, unlike the similar structure in the microspores of the higher Coniferales, does not give rise to stalk and body cells, but originates directly by division the two generative nuclei which function in fertilization. The microspores are sheltered in sporangia, which are typically four in number, on each sporophyll. The sporangia owe their dehiscence to a mechanical layer within the wall, resembling in structure that found in Ginkgo and certain Coniferales, but no longer related, as in these, to the fibrovascular system of the sporophyll. The megasporangium is much modified and, as in other known seed plants, is without any opening mechanism. In certain of the lower and amentiferous dicotyledons, however, tracheary structures have been discovered in the inferior region of the megasporangium (Casuarina, Corylus, Castanea). The germinated megaspore normally gives rise to a gametophyte containing originally eight naked nuclei which result from three successive divisions. Of these, six become organized as cells by the development of inclosing protoplasmic bodies, while the two remaining nuclei, one from each pole of the elongated sac, unite to constitute the endosperm nucleus, a structure characteristic for the angiosperms and not occurring in any lower forms. Three cells in the micropylar region of the gametophyte become organized as the single egg and the two synergidae. Three others constitute the antipodals in the base of the gametophyte. After the egg has been fertilized by one of the sperm nuclei of the pollen tube and the endosperm nucleus has contracted a union with the remaining male element, the egg develops as the embryo and the endosperm nucleus gives rise to a mass of tissue which usually quite supplants the original gametophyte or embryo sac as nourishing substance for the developing embryo. The seed of the angiosperms consequently typically contains, in addition to the gametophyte and sporophyte of lower seed plants, a third generation. This is known as the trophophyte or endosperm, and it is distinguished from gametophyte and sporophyte, not only by its peculiar mode of origin, but also by the fact that in its nuclear divisions three times as many chromosomes are present as in the gametophyte and one-half more than in the sporophyte. This cytological condition is doubtless due to the three nuclei which are fused to 
constitute the original endosperm nucleus from which the cells of the endosperm or trophophyte take their origin. The high degree of constancy in the essential features of organization of both sporophyte and gametophyte in the angiosperms mark them as a monophyletic group in which the two great divisions, dicotyledons and monocotyledons, have had a common origin.

There seems to be no reasonable doubt that both divisions of the angiosperms - the dicotyledons and monocotyledons-have originated from gymnospermous ancestors and not directly from any existing or extinct group of vascular cryptogams, since they entirely lack cryptogamic features of organization in all their organs with the sole exception of the root. The radical organ, however, is without significance as indicating cryptogamic derivation, for cryptogamic or centripetal primary wood is present in all roots without exception. It further seems obvious that the angiosperms in neither of their two divisions can have originated from the Archigymnospermae, since they present the siphonogamous mode of fertilization in contrast to the zoidogamy or fertilization by antherozoids present in the lower gymnospermous tribes which are more nearly related to the Filicales.

The general features of the angiosperms indicated above characterize the two great divisions, dicotyledons and monocotyledons, in common; and it is now necessary to specify the distinguishing structures which separate these from one another. Since the dicotyledons are with the greater probability the older and more primitive of the two main groups, they will first be considered. In the dicotyledonous angiosperms the seed is distinguished by an embryo possessing paired cotyledons or seed leaves. This feature is perhaps the most constant characteristic of the group. In the fibrovascular structures the wood is distinguished by wellmarked secondary growth which becomes feeble only in forms in which the herbaceous habit has become distinctly developed. The tissues belonging to the conductive category are, moreover, typically arranged in the form of a cylinder which is continuous in woody forms but becomes broken up into separate strands in stems with herbaceous texture. When the fibrovascular organization consists of isolated bundles, these are ordinarily arranged in 
a circular fashion, but occasionally in stems with large leaves provided with numerous foliar traces the periphery of the cylinder is no longer capable of accommodating the bundles, so they have to be disposed of in a position either medullary or cortical. The leaves of the dicotyledons are usually distinguished by skeletal -structures or veins ending freely toward the margins. The main veins may be arranged either in a radiating or in a palmate fashion, or may take their origin in an alternating manner from opposite sides of a main vein or midrib, in which case the venation is said to be pinnate. The free venation of dicotyledonous angiosperms gives them a considerable advantage over the monocotyledons in the possibility of submerged existence or a shaded habitat, when their leaves of ten become finely dissected. The root of dicotyledons presents no special features worthy of note in a general statement. The floral parts are ordinarily present in multiples of five, and the floral envelopes show themselves on the whole less likely to vary from the pentamerous condition than do the essential or strictly reproductive whorls, the stamens and pistils. Pollination is sometimes effected through the agency of currents in the air, but more commonly in the higher families by insects. Fertilization results from the penetration of the pollen tube from the stigma to the micropyle of the ovule. The course of the pollen tube after it leaves the region of the style may either be direct through the cavity of the ovary to the micropyle or, avoiding the leap across the ovarial air space, it may make its way round through the basal or chalazal region of the ovule. The last method of fertilization is characteristic of the genus Casuarina, the Betulaceae, the Juglandaceae, certain Urticaceae, etc., and is known as breech fertilization or chalazogamy. It has been suggested by Treub and Nawaschin that this is a primitive method of penetration for the angiosperms and marks a transition from the siphonogamous and higher gymnosperms in which the pollen, being deposited directly upon the ovule, has not become accustomed to leaping an air space. In the lower representatives of the dicotyledons the pollen, although no longer deposited on the micropyle, still maintains its primitive inability to cross an air space. There is much to be said for the hypothesis of the primitive 
significance of chalazogamy from the standpoint of fibrovascular anatomy. It seems, however, a feature too likely to be modified somewhat rapidly by conditions to rank as a criterion of the first order for the distinguishing of the most primitive dicotyledons.

The monocotyledons are distinguished, as their name indicates, by a seed containing an embryo with a single cotyledon or seed leaf. This feature is very constant, but there are indications of the presence of a second cotyledon in certain of the grasses, such as Zizania, Avena, etc. The fibrovascular strands of this group are ordinarily closed; that is, they do not possess the capacity to increase in thickness through the activity of a cambial layer. The arrangement of the strands in the monocotyledonous angiosperms is also distinctive, since the bundles, instead of being disposed in a circular fashion as in the mass of herbaceous dicotyledons, are scattered throughout the transverse section of the cylinder and sometimes even occur in the cortex. This peculiar disposition is, beyond any reasonable doubt, the result of the entrance of numerous leaf traces into the axis at each node, a consequence of the high assimilative efficiency of the foliar organs. The veins of monocotyledonous leaves are distinguished primitively by a closed arrangement; that is, starting out at the base of the leaf as a closed system, they reunite at the apex of the leaf. This disposition of the skeletal tissues of the leaf makes it immune from tearing action. The lateral veins, in consequence of this situation, are largely abortive. In many palms, aroids, and Scitamineae the venation of the leaf becomes open as a result of changes in the apex. In such cases the venation of the earlyformed leaves of seedlings is closed, showing that this condition is the primitive one for the group. The root in monocotyledons is distinguished, as is the stem, by the absence of secondary growth. The bundles are usually distributed in a circular and radial fashion, but in certain palms and orchids they may be scattered throughout the transverse section of the organ as they are in the stem. The floral parts occur in multiples of three, and the floral envelopes, as in the dicotyledons, show less numerical variability than do the essential whorls consisting of stamens and carpels. Pollination is effected usually through the agency of insects, but may be 
brought about by currents of air in some of the probably more primitive groups. Fertilization is always porogamous (through the micropyle), and chalazogamy is at the present time quite unknown in the monocotyledons. The members of this group are extremely important as food plants on account of their high efficiency in the elaboration of assimilates. The proportion of seed produced by some of the cereals in a vegetation period of three or four months is often over 30 per cent of the total weighta relative productiveness seldom realized in other plants. 


\section{CHAPTER XXVII}

\section{THE WOODY DICOTYLEDONS}

As a matter of convenience the anatomical organization of the woody dicotyledons will be considered in the present chapter. It must not be supposed, however, that such a mode of treatment implies that the woody texture of the stem has any value from the phylogenetic or taxonornic standpoint. Perennial dicotyledons are distinguished by the possession of a thick woody cylinder resulting from the activity of a cambial layer situated between phloem and xylem; this adds largely to the wood and less copiously to the inner bark during the periods of growth. The woods of the dicotyledons offer a great range of structural organization, and their identification on the basis of anatomical features is correspondingly difficult.

In all except a few instances dicotyledonous woods are provided with vessels. These belong to two main types, namely, those with scalariform and those with porous perforations. It has been made clear in an earlier chapter dealing with the structure and organization of the vessel that the type with scalariform perforation of the inclined terminal walls results from the fusion of rows of opposite pits which gives rise to elongated horizontal pores from which the membranes quickly disappear. This process repeated in successive rows of pits results in the appearance of lattice-like or scalariform perforations in the ends of the vessels, and these permit a ready passage of water. On general evolutionary principles the vessel with scalariform perforations is to be regarded as more primitive than the porous type presently to be discussed. It is not surprising for that reason that it is a characteristic feature of groups which are considered on good grounds to be low in the dicotyledonous scale. The vessel with the porous type of perforation is clearly derived, as has been demonstrated in an earlier chapter, from the scalariform condition, in the first instance at any rate, by the loss of the bars of 
lattice-work through mucilaginous degeneration. The vessel of the second type is found in the woods of the higher groups and indicates a more advanced condition of evolution, other things being equal, than does the vascular type with scalariform terminal perforations. Frequently woods with the porously perforated type of vessel in their mature structure show the scalariform condition in the region of the primary wood, thus providing confirmation of the conclusion that the latticed terminal wall of the vessel is phylogenetically older than that in which large pores are present.

In many instances vessels in dicotyledonous woods become more or less degenerate and are then easily recognized by their inclined end walls and in any case by a lateral pitting and internal sculpture which clearly distinguishes them from tracheids or fibers. Such vessels are often present in woods in which the fibrous elements are of the nature of libriform, substitute, or septate fibers, and are frequently inappropriately designated as tracheids. True tracheids have always tapering or fusiform ends and are not provided with the lateral pitting and sculpture of vessels. It is important to diagnose degenerate vessels as such, because a failure to make this distinction may result in quite erroneous views as to the relationship of woody dicotyledonous forms. In some cases typical vessels may disappear altogether from the mature structure, either generally or locally. Cases of the general disappearance of vascular elements are supplied by certain Cactaceae and Crassulaceae. In the genus Drimys among the Magnoliaceae and in certain genera of the allied Trochodendraceae vessels have also entirely disappeared from the normal wood. In Drimys, interestingly enough, wounds, especially wounds of the root, recall elements which have the lateral sculpture of normal vessels of the Magnoliaceae, without manifesting, however, their characteristic scalariform perforations. This defect is easily explained as a result of the comparatively small size of the reversionary elements simulating vessels. Local absence of vessels is frequently found in connection with the development of the large rays in dicotyledonous woods. This is particularly well illustrated in the wood of Alnus and in the root-wood of certain of our northern oaks. In the region of the aggregate ray, as has been indicated 
in earlier pages, the organization includes only tracheary elements. It is thus apparent that vessels, a characteristic feature of structure in dicotyledonous woods, may in certain instances be absent. There is no reason based on the general principles of comparative anatomy for regarding their absence as a primitive feature. In woods of temperate climates the vessels in the spring wood are often much larger than in the later growth, and the organization in such cases is described as ring-porous. The ring-porous condition is not, however, universal in trees of higher latitudes and is not ordinarily found in tropical woods which in general have their annual zones indistinctly marked.

The tracheary elements in dicotyledonous wood, as has been indicated previously, undergo very numerous modifications. In the lower forms they resemble the similar structures in the gymnosperms, but quite generally they lose to a large extent their water-conducting function and become mechanical or storage elements. The least advanced condition in the mechanical direction is designated the fiber-tracheid, distinguished by the reduction in size and decrease in number of the bordered pits as well as by increase in length and by thickening of the walls. The libriform fiber, a further modification, has lost or nearly lost the bordered pits, these being replaced by simple pores. In the substitute fiber, which retains its protoplasmic contents, and in the septate fiber, which is divided by delicate partitions of pectic cellulose into a number of separate units, are seen storage modifications of the tracheids present in the higher types of dicotyledonous woods.

The parenchymatous elements of dicotyledons are primitively scattered throughout the annual ring. Although rarely and only under experimental conditions revealing by actual transitions derivation from tracheids, the storage parenchyma is generally grouped in longitudinal fusiform or pointed series with robust and lignified partitions which clearly indicate its origin. In the systematically higher dicotyledons the parenchyma is characteristically grouped in clusters around the vessels. This condition is known as vasicentric and is a common feature of woods in which the tracheary elements have become partially or entirely mechanical. 
It must not be supposed, however, that the relation between mechanical fibrous elements and vasicentric parenchyma is absolute, for in groups characterized by this mode of parenchymatous distribution it is present even in genera with tracheary mechanical cells. In other words, the grouping of parenchyma about the vessels has a deeper significance than that of mere convenience to water supply. In not a few instances the storage cells may be confined to the end of the annual ring. This is, for example, the situation found in the Salicales, and it also frequently characterizes genera of the Magnoliaceae occurring in temperate climates. In these instances an examination of the primitive regions, together with experimental investigation, reveals as the original condition either the vasicentric or the diffuse distribution of parenchyma. It may be stated summarily that diffuse storage elements constitute the primitive conditions in the woods of the dicotyledons, and that a later modification is the vasicentric. By reduction either of the two types mentioned may give rise to the terminal condition. Terminal parenchyma is accordingly a phenomenon of reduction in the dicotyledonous series, while in the Coniferales, as has been elucidated at an earlier stage, it represents the primitive state in which all transitions between tracheary and storage elements are frequently and normally found.

It is in the organization of their wood rays or radial storage tissues that the dicotyledons manifest the most distinct differences from the mass of the gymnosperms. It has been made clear in previous pages that the primitive type of ray organization for the group was the linear or uniseriate ray. In the earliest conditions presented to our investigation, however, that is accompanied by the aggregate ray, consisting of more or less fused congeries or clusters of rays separated by fibrous elements. This type we must regard as an original one for rays other than uniseriate in the dicotyledons, because it is clearly found in Ephedra, by common consent the most primitive representative of the Gnetales, and because it is extremely persistent in primitive organs and regions in the dicotyledons themselves. The facility with which fibrous elements are transformed into storage cells in the group under consideration has led to the metamorphosis 
of the aggregate ray, described above, into large homogeneous masses of radial parenchyma as a consequence of the parenchymatous modification of the separating fibers. This condition, known as the compound ray, is found in relatively few dicotyledonous woods, and these are ordinarily regarded as low in the systematic scale. In woody types, moreover, it very readily passes into the antecedent aggregate condition. A third and the commonest condition of organization of the radial parenchyma in dicotyledonous woods is presented by the diffuse condition. Here the original aggregation, instead of retaining its identity or passing into the compound state last described, spreads out in the manner of a fan. This procedure results in the diffusion of the original aggregations of rays evenly throughout the structure of the wood. As a consequence of this phenomenon the organization of the wood in the mass of dicotyledons is characterized by the presence of abundance of rays which are of mediocre width. Sometimes the rays of this type are nearly equal in size, but very generally they range from extremely small to moderately large. Now and then, however, as, for example, in the wood of beeches of the Northern Hemisphere and in the genus Platanus, extremely large rays are found, readily distinguishable from those of the oak type by the fact that they grade almost imperceptibly into radial parenchymatous bands of mediocre dimensions. In the typical compound ray such as occurs in certain species of Quercus, Casuarina, and the Ericaceae the large ray is in sharpest contrast to the primitive uniseriate condition.

In the foregoing paragraphs a general account has been supplied of the various anatomical features of dicotyledonous woods, and an attempt has been made to indicate the primitive condition in connection with each category of structures. It must not, however, be supposed that a primitive condition of organization in regard to any one of the categories described in the preceding pages necessarily indicates for a given group a low position in the evolutionary scale. Taken altogether, nevertheless, they supply extremely valuable testimony from the standpoint of the doctrine of descent and on the whole the best available in the present state of our ignorance regarding fossil ancestors of the angiosperms. 
It will accordingly be of interest in the present connection to summarize the evidence in regard to the primitive type of dicotyledon supplied by anatomical data.

A tendency of long standing is to consider the amentiferous forms as representing a low condition among the dicotyledonous angiosperms. In discussions in this connection it is well to distinguish the true Amentiferae from types which simulate them. The Salicales, for example, on the grounds both of their anatomical organization and of important details of floral structure, cannot be regarded as allied in any but the remotest way with types like the alder and the oak. Further, on anatomical ground types with more or less well-organized floral structure must be admitted to affinity with the Betulaceae, Fagaceae, etc. This is true of the Casuarinaceae and Ericaceae. On the basis of the co-ordinate occurrence of vessels with scalariform perforation, tracheary fibrous elements, diffuse wood parenchyma, and aggregate rays we must accord to the genus Casuarina a primitive position among the dicotyledons. This designation of affinity on the basis of the organization of the wood is supported by the fact that it alone among the dicotyledons possesses transfusion tissue of the flanking gymnospermous type. A further indication of its primitive position is furnished by the presence within the ovules of exceptionally large amounts of sporogenous tissue and also of tracheary elements. Finally, we have the phenomenon of chalazogamy, the significance of which is still much disputed. On anatomical grounds there could scarcely be stronger reasons for regarding Casuarina as the most primitive representative of the dicotyledons. The only objection that has been seriously urged against this position is the fusion of parts in the ovuliferous floral structures. This objection, however, must weigh lightly in the balance in view of our knowledge of the extreme conservatism of anatomical structures in the case of living and extinct gymnosperms. It has, for example, been pointed out that the living cycads are practically identical in anatomical organization with the extinct bennettitean forms, although their reproductive structures differ very widely. Fusion of floral parts does not furnish a sufficient argument for a high systematic position, as on that ground the genus Welwitschia 
would be put in a higher taxonomic position than Ranunculus, because its reproductive structures present a condition of cohesion not found in the flower of the buttercup. The suggestion that Casuarina owes its anatomical organization to its xerophytic habit must be definitely rejected because of the extremely generalized type presented by the organization of its woody structures. In the single genus under consideration all the types of rays found in dicotyledons, as has been shown in an earlier chapter, are presented in the different species. Since all the species are equally xerophytic, it is quite impossible to connect any type of radial parenchymatous organization with the xerophilous habit. Physiological or ecological explanations of anatomical facts are always to be welcomed when they have any logical probability, but when they fail in this respect they only obscure the evolutionary situation. The general anatomical evidence in the case of the interesting genus Casuarina seems, in the present state of our knowledge at any rate, entirely to justify the primitive position assigned to it in the great systematic work of Engler and Prantl, in which it is placed at the very base of the dicotyledons.

If on anatomical and other grounds Casuarina must be regarded as a primitive representative of the dicotyledonous angiosperms, it is equally clear on the same evidence that with it must be joined the Betulaceae and Fagaceae and in all probability the Ericaceae. The usual fusion of parts in the flowers of the groups enumerated cannot apparently, in view of the overwhelming anatomical evidence for their primitive position, be regarded as a very important systematic criterion. Wind pollination and the general absence of herbaceous forms further supply striking features of conformity to the conditions found in the higher gymnosperms from which it is very likely the dicotyledons have taken their origin.

Another assemblage of forms which has been pushed into the foreground, particularly in recent years, as the primitive representatives of the dicotyledonous angiosperms are the Ranales. Without discussing whether the families included under this general heading all properly belong here, we may point out that on anatomical grounds the claims of that group to affinity with 
the Gnetales and other gymnospermous types must be regarded as somewhat doubtful. The general anatomical organization of the Ranales presents some range of variety; but they do not strikingly exhibit either vessels, fibers, parenchymatous distribution, or organization of the rays such as would be expected from the study of the higher gymnospermous types and the investigation of primitive regions and organs in the dicotyledons themselves in primitive representatives of the angiosperms. The only strong argument which can be advanced for the low systematic position of the Ranales is based on their floral organization. The fact that this has been deliberately disregarded in the Natürliche Pfanzenfamilien of Engler and Prantl shows that from the systematic standpoint it is a criterion which cannot be considered as of overwhelming importance. With the ranalian origin of the angiosperms is tied up the whole hypothesis of their derivation from the Bennettitales. It has been pointed out that, from the point of view of anatomy, there seems to be little reason to entertain the hypothesis that the proangiosperms were of cycadean origin, even if the earlier discovered impressions of the reproductive remains of Mesozoic cycadean forms were originally referred to angiospermous affinities by so distinguished a paleobotanist as Saporta. Anatomically speaking, there seems accordingly to be equally slight grounds for the derivation of the angiosperms from zoidogamous gymnosperms as from ranalian dicotyledons. The whole question, however, must await final and satisfactory solution until we are actually acquainted with the earlier angiospermous types which will doubtless be sooner or later brought to light from the Jurassic or from even earlier epochs of the Mesozoic. In the meantime such knowledge of the general anatomical principles as has been gained from the comparison of extinct and living gymnosperms appears to point toward types like Casuarina and its allies as representing, at any rate anatomically, the most primitive conditions. 


\section{CHAPTER XXVIII}

\section{THE HERBACEOUS DICOTYLEDONS}

The herbaceous type in the dicotyledons, although not of any definite systematic value, has a considerable significance from the anatomical and evolutionary standpoints. It has been pointed out in earlier pages that discontinuity of the woody cylinder in siphonostelic forms sinulating that found in herbaceous dicotyledons is frequently present in older groups. In such cases, however, the situation has a very different anatomical explanation and a diverse evolutionary interpretation from that presented by the dicotyledonous angiosperms. In many of the lower groups of plants belonging to both Lycopsida and Pteropsida we find the surviving representatives distinguished from those characteristic of the period of greatest luxuriance by a herbaceous, as opposed to an arboreal, habit. Such herbaceous types owe their origin to degenerative changes and present a distinct contrast to the situation exemplified in the herbaceous dicotyledons, where the modification is the result of differentiation and specialization and not the consequence of mere degeneracy.

It is necessary to illustrate clearly the mode of appearance of the herbaceous type in the older groups before passing on to the consideration of the situation manifest in the herbaceous angiosperms. Fig. 266a illustrates the organization of a siphonostelic stem in a lepidodendrid. Here the primary wood is well developed and constitutes a continuous cylinder. The secondary xylem, which is the result of cambial activity on the inside of the primary phloem, forms a continuous layer interrupted only in the region of exit of a strand destined to a branch, which causes a gap in the primary cylinder that is naturally perpetuated in the earlier organization of the secondary cylinder. In $b$ is shown the condition presented by a sigillarian of Permian age. In this instance the siphonostelic primary cylinder has become degenerate and consequently is only well developed in the regions facing departing 
foliar traces. As a direct result of this discontinuity of the primary cylinder, the secondary cylinder becomes united only by the gradual broadening of the originally separate secondary segments. In $c$ is portrayed the condition found in the stem of a third representative of the Lycopsida-namely, a calamite. Here the primary wood is extremely degenerate, and the secondary segments as a consequence are very narrow and quite widely separated.

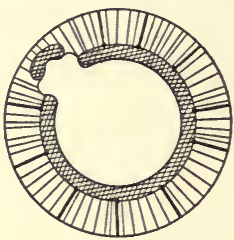

a

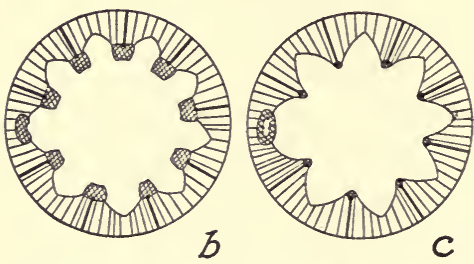

FIG. 266.-Diagram showing the effect of degeneracy of the primary wood on the development of the secondary cylinder. Explanation in the text.

of comprehension by the presence of the often numerous foliar gaps which characterize the anatomical organization of the siphonostelic central cylinder of that phylum of vascular plants. The principles involved are, however, the same. It may accordingly be stated that discontinuity in the primary siphonostelic central cylinder is due either to gaps related to the exits of traces supplying the appendages or to the local degeneracy of the cylinder itself. These interruptions are perpetuated in the early organization of the secondary cylinder. If the secondary tissues are also degenerate, as in the existing survivors of cryptogamic groups, a pronounced herbaceous condition is the result. This is, however, 
pre-eminently the consequence of degeneracy and has no dynamic evolutionary significance in contrast to herbaceous modifications presented by the stems of dicotyledonous angiosperms.

With the foregoing preliminary statement in regard to the appearance of the herbaceous type in primary and secondary cylinders (or in the secondary cylinder alone) in lower forms we are in a position to attack the origin of the stems of herbaceous texture in the highest seed plants. It has been noted in the preceding paragraphs that the degenerate herb is derived from ancestral forms characterized by woody stems. The same general condition is found in dynamic herbaceous types among dicotyledons; and it is extremely important to keep this situation clearly in view, as a failure to do so results in an anatomical fallacy. It cannot accordingly be too strongly emphasized that a proper understanding of herbaceous axes in the angiosperms can be reached only by the comparison of nearly related stems of woody and herbaceous texture. Any other procedure leads to erroneous results.

In former chapters the various types of rays in the Gnetales and the dicotyledons have been discussed. In the present connection only one of these need seriously be considered - the compound ray. The large or compound type of ray is characteristic of only a few arboreal types of probably primitive antecedents, but occurs in a more or less modified form in many herbaceous and climbing stems. It will be convenient to discuss first the conditions present in vines, as these are usually more woody in their character and consequently serve as an appropriate transition to axes of soft or herbaceous texture. As a preliminary to the consideration of the anatomy of the vine, it will be well to devote some attention to the organization of an exotic member of the Vitaceae from the tropics. Fig. 267 reproduces the woody cylinder of the shrubby genus Leea from the eastern tropics. To the right are to be seen a number of large rays which clearly belong to the category designated in an earlier chapter as compound. Above and below, and particularly to the left of the illustration, the compound rays become much smaller in size. Those which are of greater dimensions are in relation to leaf traces passing out in the 
region of the node. Fig. $268 a$ shows one of these on a higher scale of magnification. In the large ray and toward the bottom of the figure lies the leaf trace, which is both subtended and flanked by storage parenchyma. The wood on either side of the large ray shows the presence of the primitive rays, which, as has been pointed out on an earlier page, may be considered in a general way as equivalent to the narrow rays of the conifers and similar

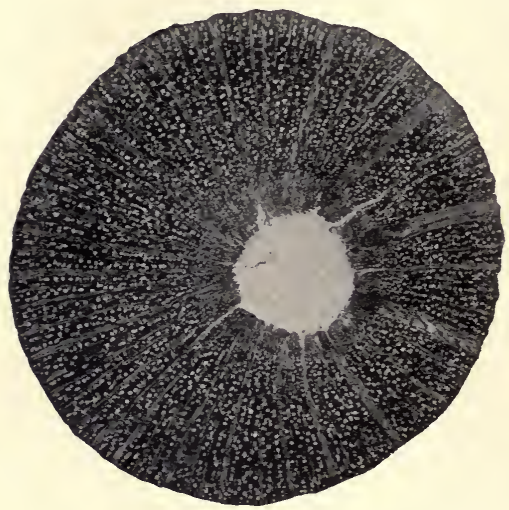

FIG. 267.-Transverse section of woody cylinder of Leea. Explanation in the text. forms. In Fig. $268 b$ is seen a vertical tangential section through one of the large leaf rays of the same genus. It is evident that the foliar trace runs in a much enlarged ray and is surrounded on all sides in its horizontal course by ray parenchyma. It is very clear from the consideration of the genus Leea that there are present large rays in storage relation to the leaf traces, precisely as in the oak, Casuarina, and similar forms. We may now pass to the situation in Vitis, where the conditions are not so manifest, but are quite intelligible by comparison with the shrubby member of the Vitaceae just described. Fig. 269 shows the general topography of the stem of the Concord grape in the region of the node. Seven leaf traces are to be seen as darker masses. Of these only one is passing into the cortex and the rest are still contained within the woody cylinder. Fig. $270 a$ illustrates one of the foliar segments of the stem with a higher degree of magnification. The segment in question is separated on either hand from the rest of the woody cylinder by broad radial bands of parenchyma. In the leaf trace itself may be found radial dark stripes, the primitive rays. These are absent in the segments of the cylinder which 
lie on either side of the trace. The leaf trace thus illustrates in the genus Vitis the conservative character which has been asserted for it on earlier pages. The most interesting feature presented by Fig. $270 a$ is, however, the fact that the leaf trace is subtended externally by a broad mass of parenchyma which on its flanks passes inwardly into the broad rays, separating the foliar segment from its neighbors on either hand. The situation in a general
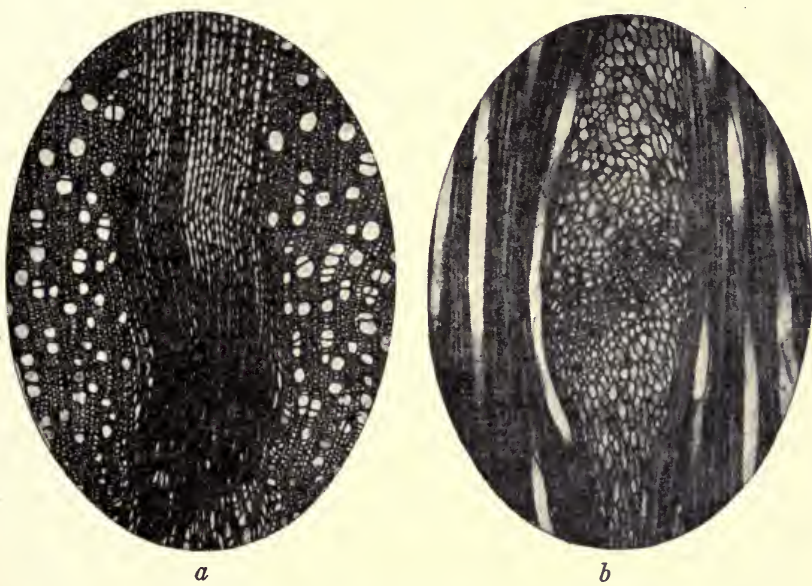

FIG. 268.- $a$, transverse section of leaf ray in Leea; $b$, vertical section of leaf ray in Leea.

way, in fact, duplicates that found in the case of Leea, except that primitive rays are confined to the traces proper in Vitis and the broad ray subtending the trace is very much shortened in its radial dimension. Fig. $270 b$ shows the same trace in a lower section or, in other words, considerably below the node. Here the broad ray facing or confronting the leaf trace has disappeared, having been gradually replaced by typical woody tissues, consisting of septate fibers and vessels. As a consequence of this situation the flanking rays are now separately continuous to the outside of the cylinder and are not united by a broad tangential 
mass of storage parenchyma, as is the case in the region shown in Fig. 270a. Vertical tangential sections make the situation more apparent. Fig. $27 \mathrm{I} a$ shows a plane of section near the surface of the cylinder. The leaf trace (seen as a dark mass) is completely surrounded by storage parenchyma, which, however, is less well developed below than on the upper side of the strand. It is particularly obvious in this plane that the trace runs in a mass of

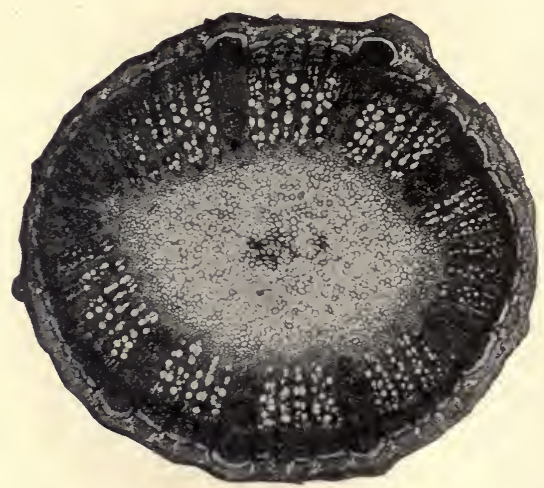

FIG. 269.-Transverse section of the node of the Concord grape. Explanation in the text. storage parenchyma precisely as in Leea, described above: The tangential sections taken nearer the center of the cylinder present a very different appearance. In this region the trace will still be pursuing its vertical course in the stem and as a consequence will be flanked by storage parenchyma laterally. Above the trace lies the parenchyma of the foliar gap. This statement may be verified by reference to Fig. $27 \mathrm{I} b$. The trace distinctly contains numerous primitive rays, conspicuously absent in the segments on either side, the segment on the right showing the presence of some vasicentric parenchyma, such as is characteristic of the Vitaceae in general. It will now be convenient to consider the topography of the foliar trace in its relation to the woody cylinder of the stem in a slender upper node of the vine. Fig. 272 reproduces the general relations exhibited by such a thin axis. Obviously, as the cylinder of the wood becomes more attenuated, the trace of the leaf will increase in size relatively to surrounding parts. This situation is clearly shown in the illustration, which represents a plane of section immediately below the node and corresponding in 
level to that in Fig. 270a. It is thus evident that, if the woody cylinder is thin enough, the leaf trace will correspond to it in thickness, and that as a result the subtending or confronting parenchyma, so prominent a feature of the topography of the leaf trace in the cylinder of Leea, Casuarina, the oak, and other woody stems with compound rays, is plainly absent. The comparison of very slender and thicker annual stems of the vine with those
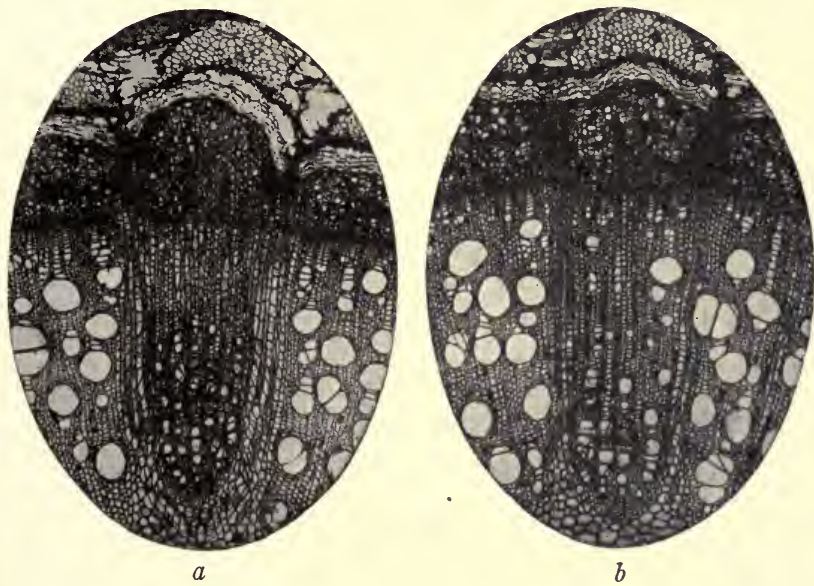

FIG. 270.- $a$, transverse section of foliar ray in Vitis in the region of the node, $b$, transverse section of foliar ray below the node.

of the shrubby Leea makes it clear that an extreme thinning of the axis brings with it a condition of organization in which the storage parenchyma no longer surrounds the foliar trace, but merely flanks it on either hand. In climbing or herbaceous axes, in which this slenderness has become a fixed feature, some compensation for the loss of subtending or confronting storage tissues is provided by the great lengthening of the flanking rays, which often extend, as was pointed out by Strasburger over a quarter of a century ago, through one or more internodes. It will be obvious from the facts introduced in the present paragraph that 
woody axes with the compound type of foliar ray, if they become sufficiently slender, as in stems of herbaceous texture, give rise to a condition in which the storage parenchyma is entirely lateral to the leaf traces.

The subject of the organization of rays in typical vines will be made clearer by diagrammatic representation. Fig. 273 illustrates the appearance of decorticated stems of Leea and Vitis in three
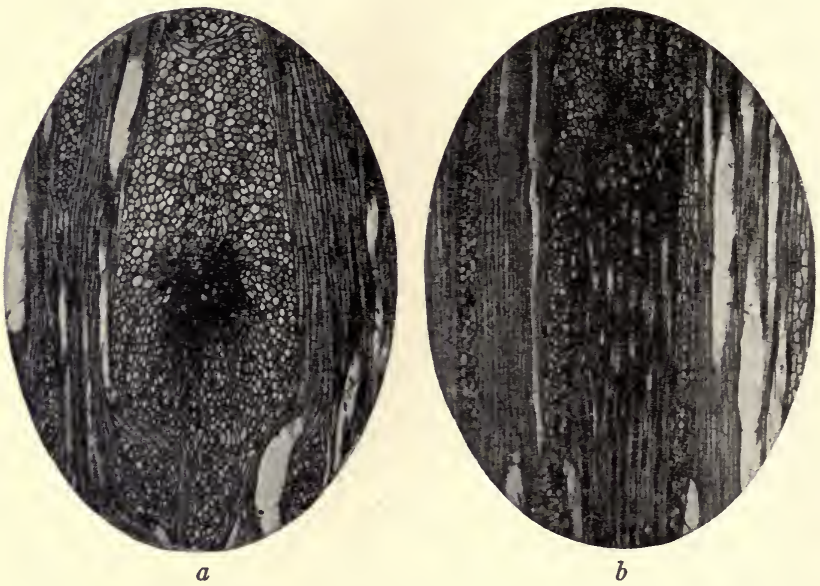

Fig. 27r.- $a$, tangential section showing leaf trace surrounded by storage parenchyma; $b$, section of leaf trace and ray near the pith. Explanation in the text.

dimensions. In $a$ is seen a total view of the nodal region of Leea. The traces originate from the axis along a crescentic line and are shown in face view on the surface of the cylinder and in section on its cut end. Obviously the leaf strands are imbedded in foliar rays when viewed from either the superficial or the transverse aspect. In addition to the foliar rays, which in this case are both broad and shallow, there are other narrower rays, which are usually of greater depth. There are also uniseriate rays, diffused throughout the structure of the wood and presenting a rather marked contrast to the two sorts of broad rays. In $b$ is shown a 
magnified image of a part of the transverse aspect of the stem, making clearer the relations of the leaf trace to the large foliar ray. It is apparent that the ray is similar to that found in oaks in cooler climates and that it has a like relation to the leaf trace. In $c$ is shown a diagram of Vitis, illustrating the relations of large rays and foliar traces. The cylinder in this instance shows only a single annual ring, and the rays subtending the leaf trace in transverse section are consequently less deep in the radial direction than those of Leea. Further, in the facial aspect of the cylinder the leaf rays are seen to be much elongated below and separated by a median process of wood into pairs of rays. This situation is strikingly unlike that in the shallow foliar rays of Leea. Still another contrast to the exotic

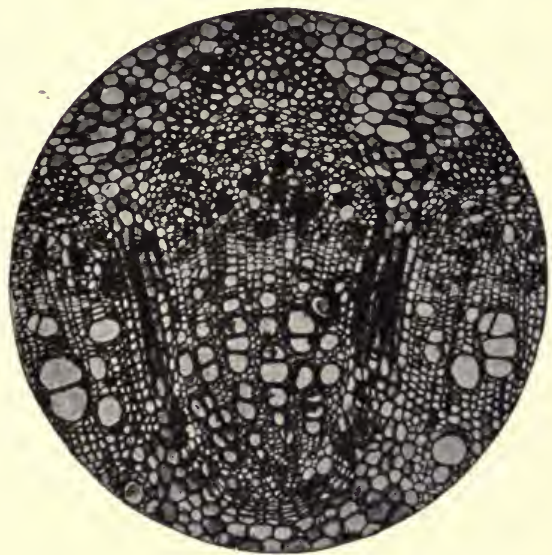

FIG. 272.-Transverse section of part of a thin axis of Vitis. Explanation in the text. genus is offered by the absence of uniseriate rays except in the actual leaf trace, an interesting exemplification of the persistence of primitive characters in foliar organs. A more highly magnified view (d) of a segment of the transverse aspect of the cylinder makes clear the presence of uniseriate rays in the leaf trace and their absence in the adjoining parts.

We may now pass conveniently to the diagrammatic comparison of the topographical relations presented by thicker and thinner annual stems of Vitis. In Fig. $274 a$ is shown a view of a thicker axis from the surface of attachment of a leaf at the median node. The upper part of the figure shows a transverse view of the stem 
just below the next superior node. In the latter the foliar traces, seven in number, are clearly subtended by a greater or less depth of storage parenchyma, precisely as is the case in the oak. In $b$ the foliar trace is shown in its radial and tangential aspects. In the radial view the trace is confronted in its upper region by storage parenchyma (black), while below it is subtended by ordinary wood. Above the trace lies the storage tissue of the leaf gap. In the tangential figure the trace appears as a light spot in a mass

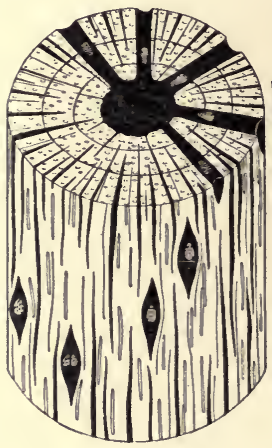

$a$

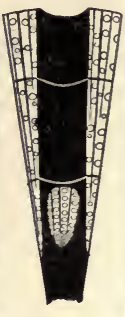

$b$

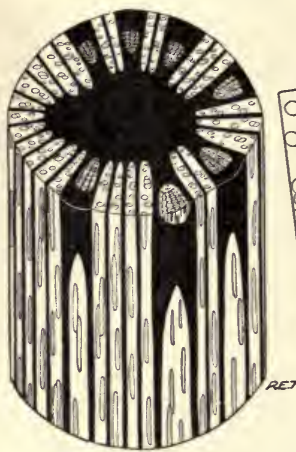

c

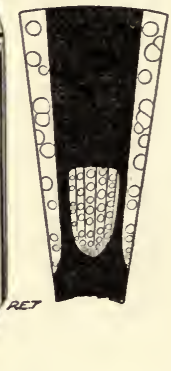

$d$

Fig. 273.-Diagram of decorticated stems of Leea and Vitis in three dimensions. Explanation in the text.

of darkly rendered storage parenchyma. The latter, farther down, is divided into two by a median mass of unmodified wood. In $c$ is shown a view of a slender stem. In this instance, for variety, the opposite or tendril side of the stem is exposed to observation. On account of the shallow diameter of the woody cylinder the trace is of a radial dimension so great that it equals that of the cylinder from which it is derived. As a consequence there is no parenchyma facing the leaf trace, and storage tissue is entirely flanking in its distribution. This is a natural and necessary geometrical result of the thinning of the cylinder of wood in the more slender axes of the vine type. In $d$ are shown the radial and tangential relations of the foliar traces and the adjacent storage 
tissues. It is clear in the radial view that there is in thin axes no parenchyma subtending the leaf traces immediately below the node. This situation necessitates the tangential aspect shown below, where the leaf trace appears flanked by storage tissues on either side. Plainly, therefore, by reason of the slenderness of the woody cylinder and the great lengthening of the rays related to the leaf traces, which may be regarded as in some measure a

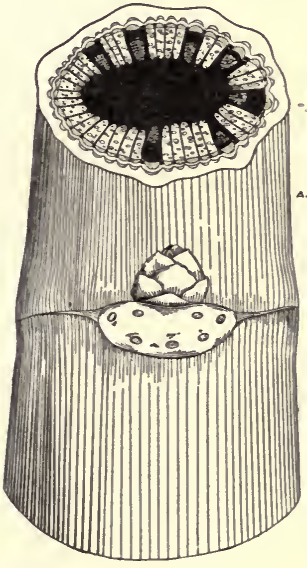

$a$
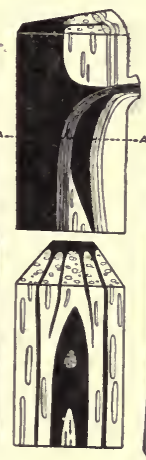

b

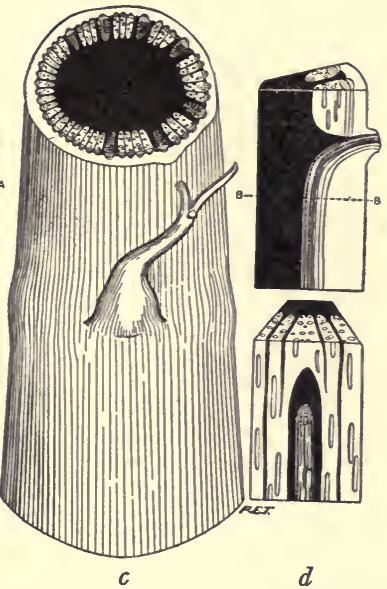

$d$

Fig. 274.-Diagram of thicker and thinner axes of Vitis. Explanation in the text.

compensation for their small radial dimension, axes of the vine type approximate very closely those found in herbs.

The discussion of typical herbs may now be advantageously taken up; and it may here be remarked that, although necessary brevity makes it impossible to consider more than a few instances of the origin of the herbaceous stem, a wide investigation of the situation in many groups of herbaceous dicotyledons has made it clear that the same general conditions are present in every instance. Fig. 275 reproduces part of a transverse section through the upper slender region of the stem of the common stinging 
nettle, chosen on account of its chalazogamous affinities for comparison with Casuarina, Betula, Quercus, etc. In the illustration a leaf trace with its flanking broad rays is shown, as well as the adjacent segments of the cylinder. No primitive rays are to be seen in the trace, which is composed of vessels and wood fibers. In order properly to interpret the slender axis of the nettle, the stouter and more woody regions of the stem should be examined.

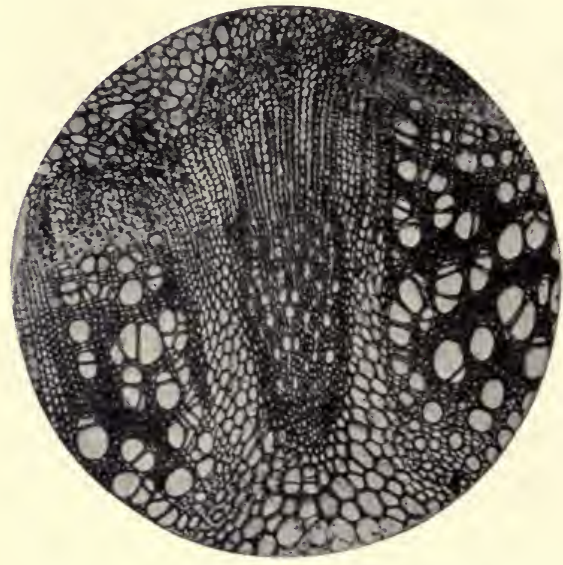

Fig. 275.-Transverse section through part of a slender stem of the nettle. Explanation in the text.

Fig. $276 a$ reproduces the appearance of a part of a section through the thick stem of the species under consideration, taken immediately below the node. The trace appears as a highly vascularized radially directed mass on the margin of the pith. On either side of it are radial bands of parenchyma, which correspond to those shown lateral to the leaf traces in Fig. 275. In addition to flanking storage tissue the woody region of the stem of Urtica shows a very massive band of radial storage tissue confronting the leaf trace, comparable to the similar structures found in the case of the oak and Leea. The banded appearance of the broad foliar ray in this instance is due to the presence of alternating stripes of true parenchyma and substitute fibers. A region of the axis farther below the node may now be considered. Fig. $276 b$ shows a part of a transverse section of the stem some distance below the node. The mass of confronting storage tissue in the region of the node at the lower level has become transformed centrally 
into typical wood, precisely as has been shown above to occur in the vine. A careless study of the facts and, above all, a neglect to examine the conditions present in the region of the node, might lead to the conclusion that the leaf trace in the more primitive and lower woody portion of the stem in Urtica is flanked but not confronted by storage parenchyma.

Although the Urticaceae have been chosen to illustrate the herbaceous condition in a comparatively low order of dicotyledons,
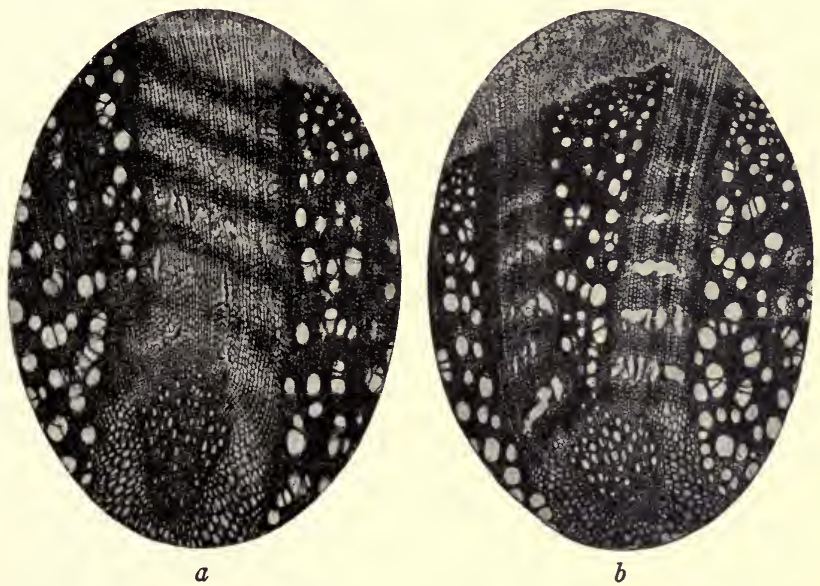

Frg. 276.- $a$, part of the thick stem of the nettle immediately below the node; $b$, section of the same some distance below the node. Explanation in the text.

the Ranunculaceae, as represented by the woody and herbaceous species of Clematis or the more slender and thicker region of the same woody species, yield like results. A group in an approximately intermediate position systematically is the Rosaceae. Herbaceous representatives when compared with woody forms of allied organization yield similar conclusions. For example, in Rubus we find well-marked compound rays, which both flank and subtend the foliar traces. In many herbaceous types of the genus Potentilla broad rays like those of the oak are found in the 
lower regions of the aërial axis, while in the upper parts the confronting parenchyma is eliminated from the cylinder as a result of its progressive reduction in thickness, precisely as in Vitis and Uritica.

The Compositae, so generally conceded a very high systematic position among the dicotyledons, exemplify exactly the same principles as have been noted above. Fig. 277 represents a low

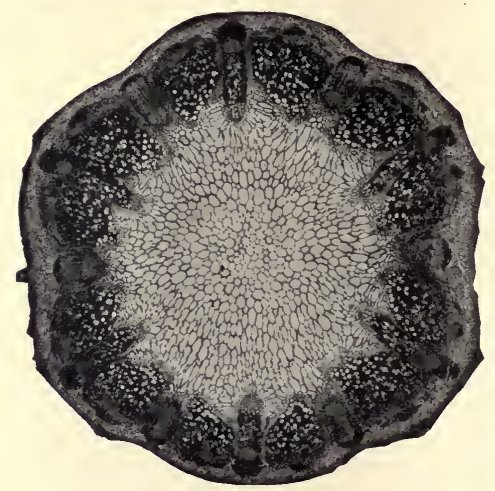

Fig. 277.-Thick stem of Helianthus hirsutus region of a somewhat woody axis of Helianthus hirsutus. The leaves, opposite as in Urtica, have three traces each. The foliar strands correspond to depressed segments of the stem, a situation which, as. has been noted in an earlier chapter, is commonly found in the vicinity of large storage rays and which results from the greater amount of vegetable substance present in such segments and a consequent retarded rate of growth as compared with the more woody regions of the cylinder. In H.tuberosus the fluting of the lower part of the stem in the region of the storage rays related to the leaves becomes extremely marked. As there are six leaf traces at a given node, it follows that there are six corresponding furrows in the internode below. Further, since the traces of successive internodes alternate, the furrows of necessity show a similar alternation. The narrow bundles of greater diameter in the figure represent the foliar traces of the next higher node, and the masses of sclerenchyma subtending the median region of the phloem of the broadest bundles correspond to the now fused traces of a still higher node of the axis. Fig. 278 reproduces a high and herbaceous region of the aërial axis of $H$. hirsutus. The figure is equivalent in nodal relations to the fore- 
going, but the topography is strikingly different. Here the foliar traces, instead of merely standing flush with the surface of the cylinder, as in the upper region of the more woody herbaceous types, are actually outstanding, or salient. As a consequence of this situation the leaf traces no longer correspond to depressions of the stem, but actually underlie ridges on its surface. This condition is, in fact, highly characteristic of extreme herbs, in which

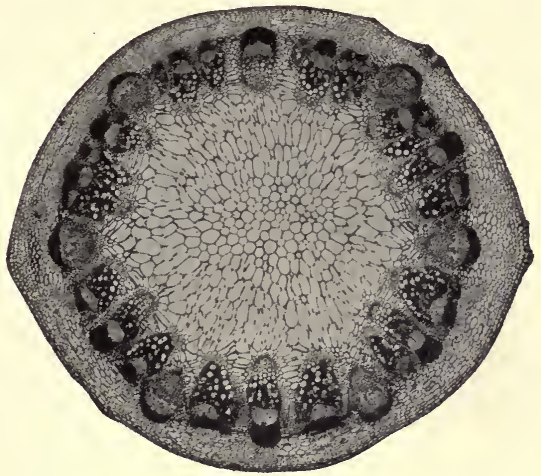

FIG. 278.-Slender upper region of the stem of H. hirsutus.

the leaf trace has become the dominant factor in the organization of the cylinder of the

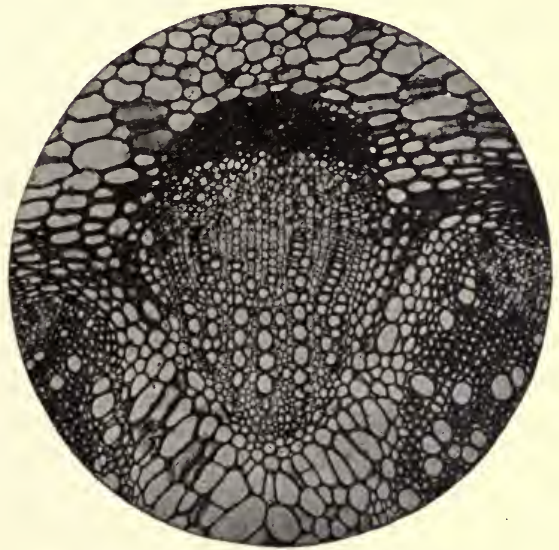

FIG. 279.-Section showing the leaf trace in the herbaceous region of Helianthus. axis. This anatomical situation corresponds to a very high degree of assimilative efficiency on the part of foliar organs, resulting in a large a mount of food storage or seed production, as the case may be.

In Fig. 279 is shown the trace of the herbaceous region more highly magnified. The 
foliar strand projects beyond the surface of the cylinder and is merely flanked and not subtended by storage parenchyma, as in similar regions in other herbaceous stems of the most varied affinities. In contrast, in Fig. 280, which is from a lower and more woody node, a large amount of confronting storage tissue is seen, as well as that present on the flanks of the trace.

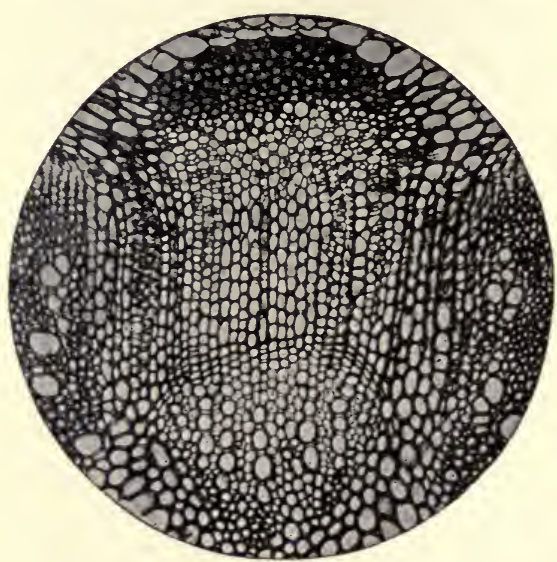

FIG. 280.-Section of trace in thick woody region of Helianthus.

The topographical conditions in the stem of Helianthus, as representing a high and typical herbaceous condition, may now advantageously be depicted in stereodiagram. Fig. 281 $a$ reproduces the lower portion of the stem of Helianthus. The scar of the leaf of one node faces the observer, while the region just below the next higher node is shown in transverse section. The six traces of the two opposite leaves are clearly seen, and it can be readily observed that they are both flanked and faced by storage tissue (black). The very narrow deep bundles in the cross-section represent leaf traces of the next higher node, while the light masses on the periphery of the broad remaining strands indicate the position of the fused foliar traces of a still higher node. In $b$ is shown (above) a radial and (below) a tangential view of the topographical relations of the leaf traces in these planes. In the upper figure of $b$ there is obviously much storage tissue confronting the trace. In the lower item the trace in tangential section is seen entirely surrounded by storage tissue. Fig. 28I $\mathrm{C}$ is a picture of the solid relations of a higher part of the stem in 
the sunflower. In this region the foliar traces, instead of being depressed below the level of the woody cylinder, are outstanding, a condition very commonly found in extreme herbs, which have largely lost their woody texture. In the case of the vine the radial diameter of the trace in the slender region of the stem merely equals that of the cylinder, while in strongly herbaceous types the leaf trace is outstanding or salient. In the high part of the sunflower stem the trace is only flanked by storage tissue in contrast

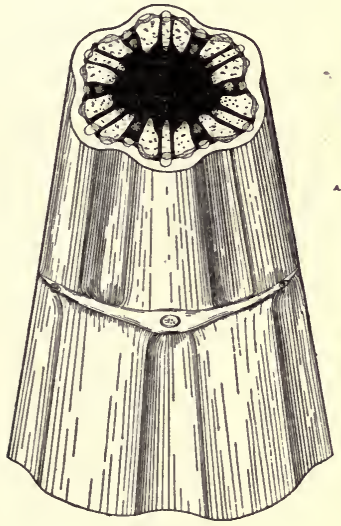

$a$

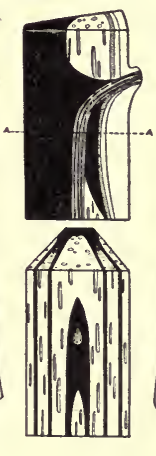

$b$

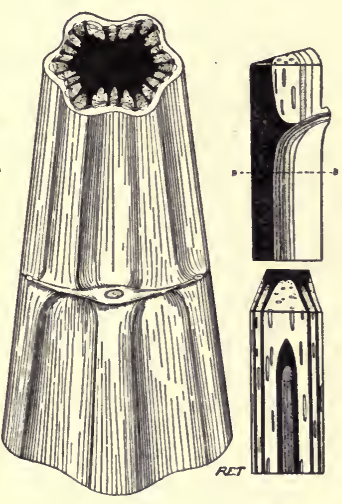

c

$d$

Frg. 28r.-a, diagram of lower region of stem of Helianthus hirsutus; $b$, radial and tangential view of the topographical relations of leaf traces in $a$; $c$, diagram of the upper region of the stem in the sunflower; $d$, topography of the trace in radial and tangential aspects. Full explanation in the text.

to the both flanking and facing topography of the storage parenchyma in the lower and more woody part of the axis. It will be noted that in the high portion of the axis the foliar traces correspond to elevations or ridges on the surface of the stem, while in the regions lower down they subtend longitudinal furrows. In Fig. $28 \mathrm{I} d$ the topography of the foliar trace is represented in radial and tangential aspect. In the upper figure the absence of subtending parenchyma can be clearly discerned, while in the lower one the flanking disposition of the storage tissues is apparent. 
It will be advantageous at this stage to compare the woody region of the herbaceous stem of Helianthus with the axis of $\mathrm{Ca}$ suarina. Fig. 282a represents diagrammatically a Casuarina with compound foliar rays. The best developed of the rays are directly related to leaf traces; while the intervening less pronounced ones belong to the foliar strands of another node. In order that the vertical relations of the foliar bundles to storage rays may
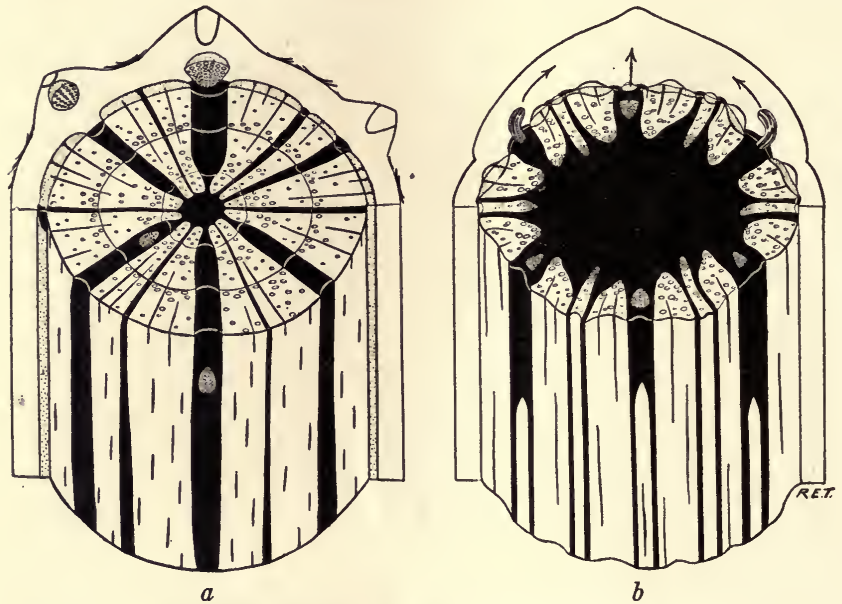

FIG. 282.-Stems of Casuarina and Helianthus. Explanation in the text

be seen, the bark of the stem is removed on the side facing the reader. The distant aspect still retains its bark as well as its leaf bases, which are represented as pyramidal elevations on the surface. In Fig. $282 b$ is seen a corresponding diagram of the lower region of a stem in Helianthus. The topographical conditions are virtually the same, but for the fact that there are three leaf traces to each leaf in the sunflower, instead of a single one, as in Casuarina.

In the oak the longitudinal depressions of the woody cylinder do not correspond to rays belonging to a single leaf trace, but 
to pairs of these related to the foliar strands of diverse leaves at different nodes. This phenomenon is rather rare in the herbaceous type, but is occasionally found in the genera Aster and Solidago, among the Compositae. Fig. 283 represents an axis of this type in Aster multiflorus. There are five depressed segments corresponding to five pairs of foliar traces, to be seen in the woody cylinder. Fig. $284 a$ is a diagram of the topography of the axis in the oak. The bark is represented as removed on the side toward the reader and as still present on the opposite side. To the right on the distant aspect of the stem is shown a leaf base. Into this run three traces. Since the median one passes out at a different level from the other two and exercises no important influence on the topography of the stem, it is represented by a dotted outline. It is to be noted that the lateral traces take their origin

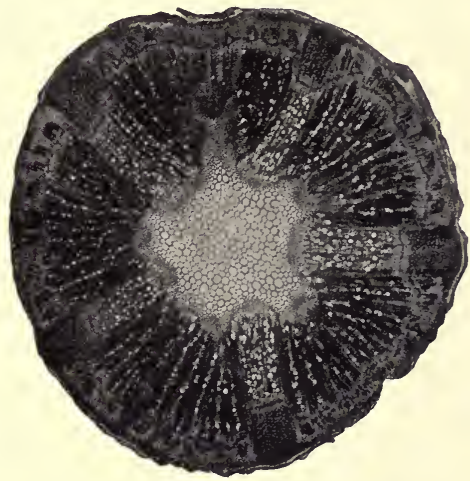

FIG. 283.-Transverse section of the stem of Aster multiflorus.

from the foliar rays which are most remote from the median trace. On the left of the posterior aspect of the axis is shown by a dotted outline a leaf base of a succeeding node. The traces of this leaf are plotted in by broken outlines, so as to indicate their relation to the foliar rays of the stem. It is clear from the illustration that the depressed segment lying uppermost in the diagram is flanked by two foliar rays which belong to the lateral traces of two successive leaves. The depressed segment of the woody cylinder in this instance owes its position to the retarding effect on growth of the two closely approximated foliar rays which bound it on either side. In the case of stems in which the large rays are equidistant there are no depressions, showing clearly that the depressed segment is the result of growth mechanics. This is 
clearly exemplified in certain species of Clematis. In the numerous species in which the large rays are approximated in pairs there are depressed segments in the stem; but in Clematis paniculata, in which the large compound rays are equidistant, the segments of the woody cylinder are all on a level and there are no depressed regions. It is not possible to follow this subject further in the present connection, but it is enough to point out that the facts
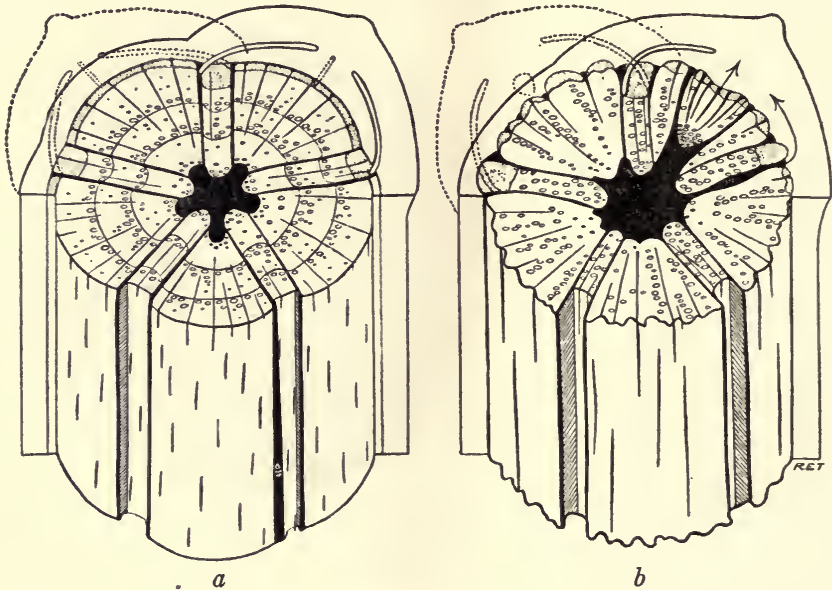

FIG. 284.-Diagram of the topography of the cylinder in Quercus and Aster. Explanation in the text.

entirely invalidate the interpretation of depressed segments advanced by Sanio, Sachs, and De Bary and now classic in botanical textbooks. The view of these authors is that the depressed segments, where they occur, owe their topography to a later formation than the rest of the cylinder. This is distinctly not the case. The classic view just mentioned further involves the equally erroneous hypothesis that the continuous cylinder of woody forms has been derived from the discontinuous one of herbaceous types, a conclusion which, on the evidence furnished in the present and previous chapters, is exactly opposite to the true situation. In 
Fig. $284 b$ is shown a diagrammatic view of the stem in the genus Aster. Obviously exactly the same general conditions are found as in the axis of the oak.

The result of all the evidence considered in the previous paragraphs of the present chapter is to show that the herbaceous type of stem is distinctly derived from the woody one in the dicotyledons. Its earlier expression is in the rather thick stem of herbaceous texture, in which the storage parenchyma especially related to the foliar traces both subtends and flanks the fibrovascular bundles of the leaf in that part of their course which lies within the cylinder of the stem. With subseqquent thinning of the cylinder, clearly connected with greater efficiency in the production of seed and coupled with a more strictly annual duration, the subtending parenchyma is wiped out, and only the flanking storage tissues continue to exist. Obviously a very high potency in the production of seed, correlated with high assimilative power, ultimately makes for a type of annual stem in which storage is effected mainly in the seeds and no longer in the axis itself. Under these circumstances we have the herbaceous type reaching its extreme simplification. The situation just pictured involves a final marked reduction in the organization of the fibrovascular strands which is expressed in its completest form in the bundle system of the axes of the monocotyledons. This group, however, will be considered in the following chapter.

The result of the appearance of the herbaceous type in the angiosperms has been momentous for the development of higher organisms. It is a fact of very obvious significance that the highest vertebrates and the highest seed plants have had a nearly contemporaneous existence. The warm-blooded mammal is in reality rendered possible by the appearance of the herbaceous type in the angiosperms, which directly or indirectly supply the most important part of the food of all the higher animals. It should further be emphasized in the present connection that the dicotyledonous herbs are very different indeed in their mode of origin from those exemplified by lower types and in particular by the vascular cryptogams. Here the herbaceous condition is the result of mere degeneracy in the tissues of the xylem. In 
the herbaceous dicotyledons, on the contrary, we find the origin of herbaceous texture closely associated with special modifications in the organization of the secondary cylinder, and this involves a high degree of specialization on the part of the plants in which it is manifested. The local transformation of the tissues of the woody cylinder, in relation to improved storage in proximity to the leaf trace, had its early expression in the phenomenon of aggregation of rays in proximity to this in its course through the woody cylinder. The phenomenon of aggregation is succeeded in turn by that of compounding, the direct consequence of the facility with which the longitudinal elements of the xylem become transformed into storage elements in the case of the dicotyledons. The final result achieved in the compound ray is the wedding of longitudinal and radial parenchyma in the complex, which becomes of such marked significance in the herbaceous dicotyledons. 


\section{CHAPTER XXIX}

\section{THE MONOCOTYLEDONS}

This group of plants, although only about one-fifth as numerous as the dicotyledons, is nevertheless of great importance on account of its significance in supplying extremely valuable food plants and also by reason of its remarkable anatomical organization, which has been the "cause of much speculation and dispute. In this large group of the angiosperms we have exemplified a practically complete absence of secondary growth coupled with a complicated arrangement of the numerous though slender fibrovascular strands. The absence of secondary growth has led to the association of the monocotyledonous angiosperms with the ferns and their allies. This view of their affinities has been reinforced at various times by the discovery of leaves and even inflorescences in Paleozoic strata which have been referred to monocotyledonous affinities. The foliar and reproductive parts from Paleozoic deposits have, however, in more recent times been clearly recognized as the parts either of cryptogamous or of gymnospermous types not related even remotely to the angiosperms. There is accordingly no reason based either on the possession of cryptogamic characters or on very ancient occurrence as fossils which justifies the view that the monocotyledons are the more primitive group of the angiosperms. The evidence, in fact, when considered in the light of the general principles of comparative anatomy, points in quite the opposite direction and seems to indicate that the group under consideration is derived from herbaceous representatives of the dicotyledons.

A characteristic feature of organization of the axis in the monocotyledons is the scattered distribution of the fibrovascular bundles. These, instead of being arranged in the circular fashion which usually distinguishes the structure of the herbaceous stem in the dicotyledons, are disposed through the transverse section of the organ. An examination of the seedlings and reproductive 
axes in the group supplies convincing evidence that the peculiar arrangement of the fibrovascular bundles in the monocotyledons is not a primitive one. The original manner of distribution of the conducting strands of the stem was in all probability that found in the dicotyledons, characterized by a prevailing circular arrangement of the bundles.

The root in monocotyledons has the usual radial organization, and is distinguished from that of the mass of dicotyledons by the absence of secondary growth. Another feature which is often present in monocotyledonous roots is the origin of lateral roots, not opposite the groups of protoxylem, as is the general situation in the roots of the remaining vascular plants, but in the interval between two protoxylem clusters. This peculiarity has gained for such roots the not very appropriate designation of "double roots." Another feature which has been described in the roots of the group under consideration is found in the abnormal order of development of the elements of the xylem. In vascular plants in general the protoxylem occupying the outside of the xylem star of the root is differentiated first and the successively more central elements in later order. In a number of monocotyledonous roots a remarkable exception to this well-nigh universal seriation of development has been observed, for the more central elements belonging to the metaxylem are differentiated first and the tracheids of the protoxylem are the last to manifest the sculptural features of maturity.

The leaf in the monocotyledons is characterized in general by the closed disposition of the nerves or fibrovascular strands. These usually come together at the tip of the foliar organ and sometimes in this region are in relation to rifts or pores in the epidermis, which allow fluid water to escape in the form of drops of dew. In certain tropical plants with large leaves-such, for example, as the Agave - the loss of water during the night through the rifts in the tips of the leaves where the foliar bundles converge is so great that a constant dripping is heard, often so pronounced as to disturb slumber. As a consequence of the closed disposition of the fibrovascular bundles in monocotyledonous leaves, only the longitudinal veins are, as a rule, well developed, and the 
lateral ones are weak and degenerate. The fibrovascular bundles of the leaf in monocotyledons are ordinarily very numerous and consequently enter the stem in large numbers at the nodes. The large number of foliar bundles passing from the base of the monocotyledonous leaf into the stem is correlated with a high degree of assimilative efficiency which finds expression in a proportion of seed production which has scarcely ever been reached in herbaceous dicotyledons. In many of the cereals, for example, the relative weight of the seed to that of the whole plant very frequently reaches over 30 per cent. The high efficiency of the group, both from the standpoint of production of assimilates and from that of the formation of seeds, naturally puts it in a unique position in supplying important food plants.

In many cases, particularly in the grasses and sedges, cambial activity, absent in the stem and root, is often retained in the basal or sheath region of the

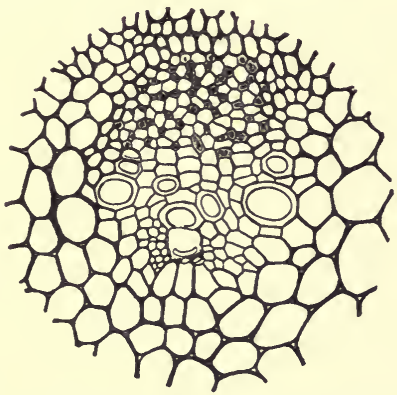

FIG. 285.-Transverse section of a bundle of Avena barbata, showing cambial activity (after Chrysler). leaf or sometimes in relation to the node in the stem. The capacity which grasses manifest for erecting their stems after "lodging" is to some extent the result of the presence of a persistent cambium in the nodal region, either in the base of the leaf or in relation to the stem itself. Fig. 285 illustrates such cambial activity in the case of Avena barbata. It is permissible to view this cambial activity as a persistence of an ancestral character, particularly as it is often found to be present in monocotyledonous seedlings in the lower region of the epicotyl or primitive stem.

The organization of the closed fibrovascular bundles of the monocotyledons is in many cases collateral and, as the descriptive term implies, exhibits no indication of cambial activity. The collateral type of fibrovascular strand is characteristic of the leaf, since that organ, here as elsewhere, is conservative in its structure. 
In the stem, however, particularly in the subterranean axis, the collateral type gives place to a concentric condition in which phloem is completely surrounded by xylem. This modification is known as amphivasal, to distinguish it from the amphicribral concentric strands of the Filicales and certain lower gymnosperms. The concentric strands of the monocotyledons present themselves in a very interesting fashion in the grasses and sedges. Here, in the reproductive axes, amphivasal bundles are very numerous in the nodal regions, where the entering of many foliar traces produces a marked degree of crowding and disturbance. The amphivasal bundle, in fact, seems to have originated as a consequence of the multiplication of foliar traces in the nodal regions of monocotyledonous stems. This hypothesis of the origin of the amphivasal strand is justified by a consideration of parallel conditions exemplified in the organization of the axis in certain dicotyledons. In many instances where the foliar traces are numerous in the nodal region of herbaceous dicotyledons, these become amphivasal in their structure. This organization; for example, is frequently seen in the Araliaceae and Umbelliferae. In the annual stems of the mass of monocotyledons, whether leafy or scapose, amphivasal bundles are ordinarily absent. When the anatomical structure of the perennial subterranean axis which is often found in the monocotyledons is examined, it very generally presents amphivasal bundles in great abundance and not by any means confined to the nodal regions, even when-as less rarely happens - the nodes are not closely approximated. The common occurrence of amphivasal strands in the rhizomes of monocotyledonous stems is no doubt primarily related to the crowded and tufted character of the leaves which results in the strong approximation of the nodes with the consequent multiplication of amphivasal strands.

It is highly probable that the glumaceous representatives of the monocotyledons represent somewhat primitive conditions in the stock, for here both reproductive and anatomical data seem to harmonize in indicating a low systematic position for both grasses and sedges. It is, moreover, probable that the Juncaceae, which in anatomical organization agree very closely with the 
glumaceous monocotyledons, are nearly related to these forms. A striking contrast is presented in the anatomy of the true palms and the Scitamineae. In these groups amphivasal strands seem to be entirely lacking. In the Principes, or true palms, we find a marked difference in anatomical structure from the western tropical Cyclanthaceae, which have on floral grounds often been considered to form a systematic link between the palmlike monocotyledons and the aroids. The anatomical structure of Carludovica and allied Cyclanthaceae is rather that of the aroids than of the true palms, since amphivasal strands are conspicuously present. Our knowledge of the development and comparative anatomy of the groups which appear to lack amphivasal bundles is still too meager to warrant any hypothetical conclusions as to the phylogenetic significance of the apparent lack of amphivasal fibrovascular strands in the conspicuously large-leaved forms, which are united systematically under the headings of Principes and Scitamineae. It may well be that the two large groups above indicated, by the anatomical peculiarities revealed as a result of an examination which is as yet only preliminary, occupy a position high among the monocotyledonous orders.

It will be convenient to elucidate in a general way by means of diagrams the main anatomical conditions presented by the monocotyledons. Fig. $286 a$ illustrates the distribution of amphivasal regions in the stem of a sedge. In the reproductive axis the amphivasal segments are remote and are clearly in the nodal regions. In proximity to the substratum the nodes become more approximated, and in the subterranean axis they are frequently so closely disposed that amphivasal organization is often continuous. This condition is well illustrated by the sedges and rushes and less distinctly by the grasses, since the last have well-spaced nodes. The next diagram $(b)$ shows the situation commonest among the monocotyledons. In this type of anatomical organizations the amphivasal structures are found exclusively in the perennial subterranean axis and do not appear in the annual stem. In the last type (c), which portrays the conditions apparently characteristic of the true palms and the Scitamineae (bananas, cannas, ginger, etc.), amphivasal regions are absent both in the 
subterranean and in the aërial stem. Only continued investigation will show the real significance of the anatomical situation exemplified in these cohorts or orders.

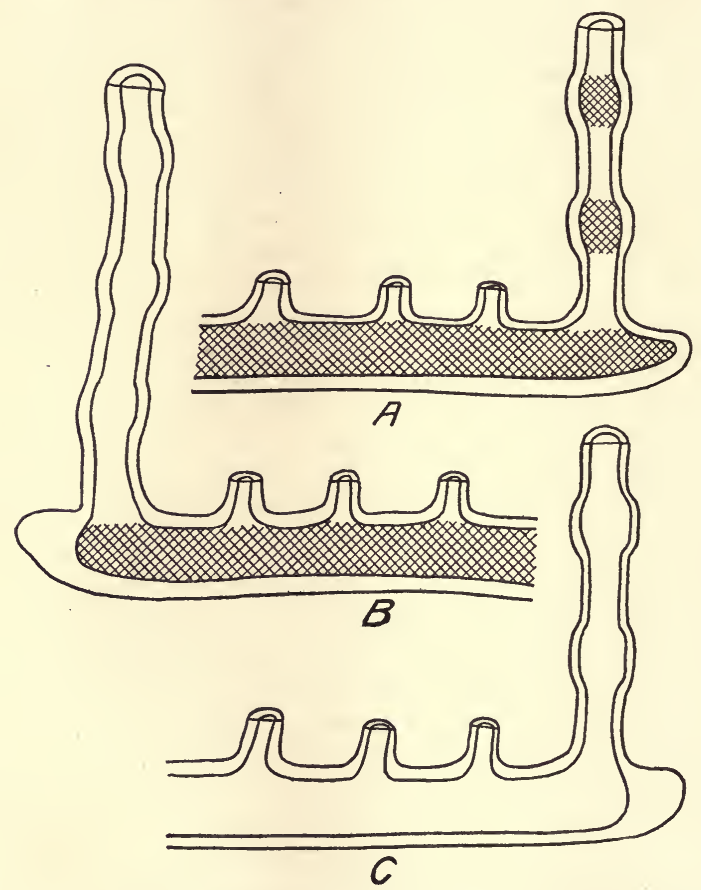

Fig. 286.-Diagrams to illustrate the distribution of amphivasal bundles in the monocotyledons.

The general anatomical configuration of the monocotyledons in the present condition of our knowledge warrants the conclusion that this important class or division of the angiosperms formerly possessed bundles arranged in a circular fashion and characterized by secondary growth. This condition is indicated by the study of conservative organs and regions and seems to justify the inference 
that the monocotyledonous angiosperms have been derived from a dicotyledonous ancestry. The justice of this hypothesis, however, will be finally established only when we shall have at our disposal for anatomical investigation remains of monocotyledons from Mesozoic deposits. Whether or not it is ultimately established that the large group at present under discussion has actually been derived from the dicotyledons, it will doubtless in any case be clear that they cannot in any way be regarded as primitive representatives of the angiosperms. The monocotyledons in fact represent the herbaceous type in its extremest form. In the group the fibrovascular tissues are released from the rigid confinement of the tubular stele in the ancestral forms with secondary growth by the development of large parenchymatous storage devices in relation to the foliar traces. Correlated with this release is the possibility of accommodation of the numerous foliar strands which characterize the basal regions of monocotyledonous leaves throughout the transverse section of the stem. There is doubtless some correlation also between the extreme multiplication and consequent displacement of the strands in the stem of monocotyledons and the disappearance of secondary growth. Possibly an aquatic or amphibious habitat long maintained may likewise have acted as a contributory cause in bringing about the obliteration of cambial activity, since in the dicotyledonous Nymphaceae, in which there is diffuse distribution of the bundles and also the absence of cambial activity, we find these features correlated with an aquatic habitat. It has, indeed, often been suggested that the Nymphaceae or Ranunculaceae are the dicotyledonous ancestors of the monocotyledons. The interesting investigations of Sargent on the fusion of the cotyledonary structures in Ranunculus ficaria, etc., are of importance in indicating how monocotyledony may have arisen as a result of the union of two originally separate seed leaves. Another possibility, of course, is the origin of the monocotyledonous embryo as a consequence of the abortion of one of the two original cotyledons, and this view is perhaps supported by the conditions found in certain grasses, such as Zizania, Avena, etc., in which the vestige of a second cotyledon is considered to be present. The problem of the origin 
of the monocotyledons cannot yet be regarded as by any means settled, and a much fuller knowledge of the anatomy of extinct and living representatives of this extremely important group is necessary before any final results can be reached. If we attempt to picture to ourselves the probable future course of evolution in the angiosperms, it is difficult to concede to the monocotyledons a prominent position. This group seems to have reached such an extreme degree of specialization that it will probably in the long run entirely disappear and be replaced by new derivatives of the still plastic dicotyledons. 


\section{CHAPTER XXX}

\section{ANATOMICAL STRUCTURE AND CLIMATIC EVOLUTION}

General views in regard to the ancient climatic conditions must first occupy attention in the present connection. It is commonly conceded, on the basis both of the nature of ancient organisms and of the evidence supplied by geologic strata, that the earth was formerly much warmer than it is in the present epoch. It is further clear that, other things being equal, the greatest degree of warmth existed in the most remote past. This general situation, however, does not exclude the recurrence at long intervals of periods of refrigeration or glaciation. An age of ice is known to have occurred, not only at the end of the Cenozoic as originally established by Agassiz, but glacial periods also terminated both the Mesozoic and the Paleozoic as ordinarily defined. Evidence of still earlier glacial epochs which exercised a devastating influence on the most ancient animal and plant populations of our earth is not lacking.

Evidence in regard to glaciation in former epochs is both direct and indirect. Direct testimony concerning former ages of ice is supplied by the comparative study of deposits formed in connection with the existing glaciers of high latitudes or of high altitudes. Intimately connected with glacial phenomena are the formations of clays, till, and coarse morainal matter resulting from the movement and melting of ice. Indications of the kinds just enumerated in earlier geological strata supply direct testimony as to former glacial action. Indirect information in the same direction is often furnished by the wholesale extinction of important groups of plants and animals. For example, in the Permian glaciation which marked the close of the Paleozoic the treelike cryptogams, which have contributed so largely to the formation of the older deposits of combustible minerals, disappeared entirely as an important constituent of the plant population of our earth. Glacial epochs are, however, not of direct importance in relation 
to the evolution of plants in response to climatic influences, since their action is mainly negative. It is true, however, that by bringing about the obliteration of important groups of plants or animals greater opportunity is supplied for the surviving and more adaptable forms to develop in the following warmer epochs.

It is the more gradual and not the spasmodic refrigeration which has produced perhaps the greatest effect on the organization of the successive plant populations of the earth. As a preliminary to the discussion of the anatomical modifications which are more or less definitely correlated with climatic changes in the successive geological ages, the evidence furnished by plants in regard to progressive climatic cooling must be considered. This evidence is of two kinds. Perhaps the most important and trustworthy is derived from the organization of the secondary woods in trees of the various geological periods. This testimony can hardly be estimated too highly in arriving at any conclusions in regard to plants as reliable indicators of climatic change. Another kind of evidence is afforded by the character of the plants themselves. At the present time there are large groups of plants which are of more or less definite tropical occurrence and others which prevail characteristically in cooler regions. The advance of tropical types toward the poles or the progress of polar plants in the direction of the equator in earlier geological eras must, other things being equal, indicate variations in climatic conditions in the direction either of greater or of less warmth, as the case may be. Unffortunately, in earlier geologic times the differentiation between polar and equatorial types was not nearly so marked as it is at present, and this general situation militates more or less strongly against reliable conclusions in regard to climate in all but the latest geological eras. Since the present work deals with anatomy, the subject of plant geography in relation to the climatic changes which have marked the successive ages of the earth is obviously of less importance, aside from the limitations indicated above.

As has been already shown, the organization of the secondary wood in extinct plants furnishes the most reliable evidence as to the climatic conditions which prevailed in earlier geological 
epochs. The information derived from this source is not only on the whole the most trustworthy, but it is likewise present in sufficient quantity from different geological periods and diverse geographic areas to justify more or less comprehensive general conclusions. In the Paleozoic trunks which are supplied by the geological formations of Southern C a n a d a th e organization of the wood shows great uniformity, and there are no

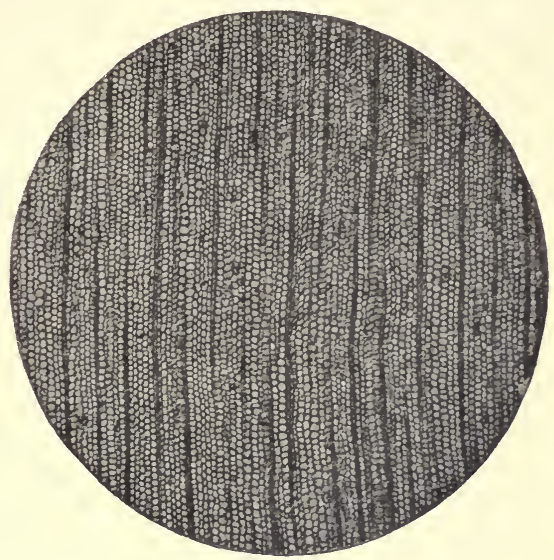

Fig. 287.-Transverse section of the wood of Cordaites from Prince Edward Island.

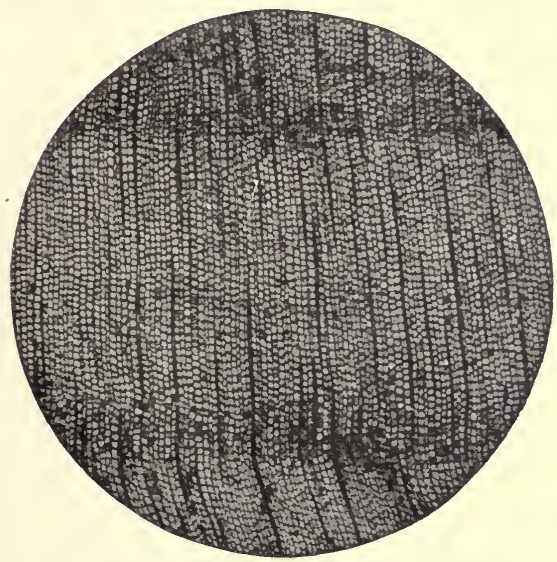

Fig. 288.-Transverse section of the wood of Cordaites from the north of England. modifications of structure which indicate any periodicity in annual conditions of growth. The truth of this statement is well illustrated by the accompanying photograph (Fig. 287) of the wood of a cordaitean form from the Permo-Carboniferous of Hampton, Prince Edward Island. The presence of clear zones 
of periodic growth is, however, frequently found in regions of higher latitude than that portrayed in Fig. 288. The next illustration shows the organization of a Carboniferous cordaitean wood (Mesoxylon) from the northern part of England and consequently of consider-

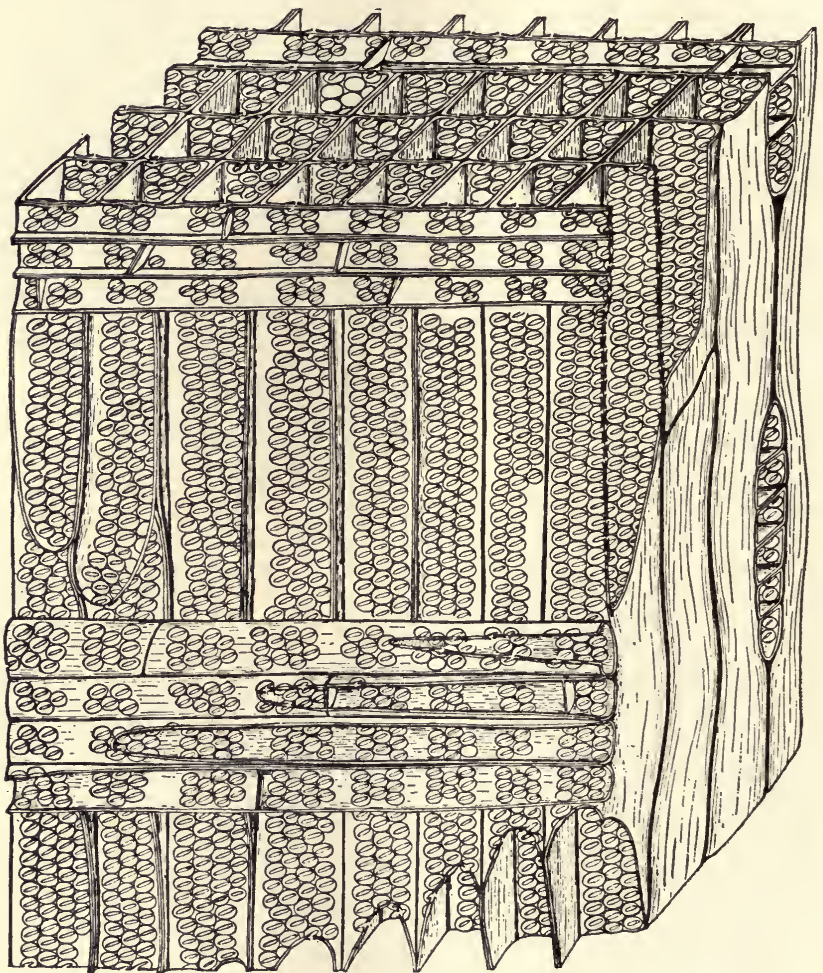

Fig. 289.-Details of wood structure of Mesoxylon (Cordaites) with annual rings. Tangential pitting is absent.

ably higher latitude $\left(54^{\circ} \mathrm{N}\right.$. in contrast to the $46^{\circ} \mathrm{N}$., the latitude of Prince Edward Island). The annual rings in the wood from the English Carboniferous are clearly marked. The next illustration (Fig. 289) reproduces the minute organization of the same wood 
under a sufficient degree of magnification to show the essential features of structure. The tracheids are so long that only a small portion of them can be shown in the figure. Their walls are covered with crowded pits arranged in an alternating fashion with no intervening bars of Sanio. The only parenchyma present is radial, and the end of the annual ring is marked by the presence of tracheids which are slightly narrower in radial dimensions but do not otherwise differ from those found in the rest of the annual ring.

For comparison with the situation revealed by the cordaitean wood from Northern England, a trunk from the Triassic of the southwest region of the United States is shown. In Fig. 290 appears a transverse section of the wood of a tree from the Triassic forest

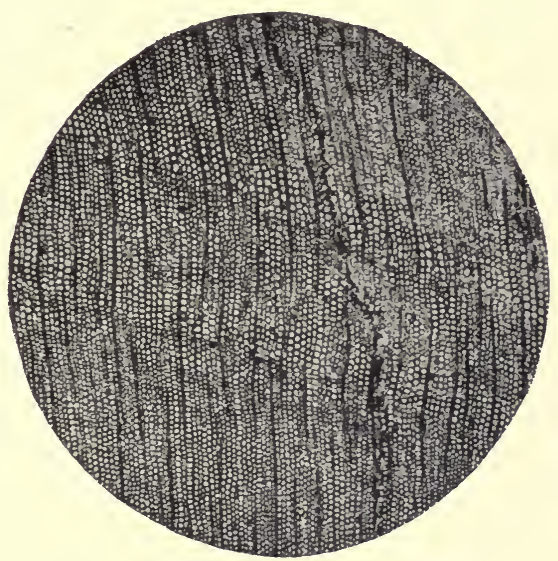

Fig. 290.-Transverse section of a coniferous wood from the Trias of Arizona. of Arizona. The annual rings are not so distinct in the photomicrograph as they appear on the weathered end of the actual petrified specimen. It will be clear from the information supplied in this case that as far south as Arizona in the Triassic annual rings were more or less clearly marked. A noteworthy variation in the annual temperature in that somewhat remote epoch is thus indicated. This situation presents an interesting contrast to the climatic conditions which prevailed in the region of Prince Edward Island toward the end of the Paleozoic. If the situation be summarized, it is clear that in the later Paleozoic the difference between $46^{\circ} \mathrm{N}$. and $54^{\circ} \mathrm{N}$. means the presence in the 
higher latitude of annual rings and their absence in the lower one. On the other hand, in the beginning of the Mesozoic (the Triassic) even at a distance of ro degrees south of the latitude of Prince Edward Island annual rings were quite clearly developed.

Without considering the Jurassic we may pass at once to the end of the Mesozoic period, namely, the Upper Cretaceous. Fig. 29I reproduces the organization of the wood of a Cretaceous

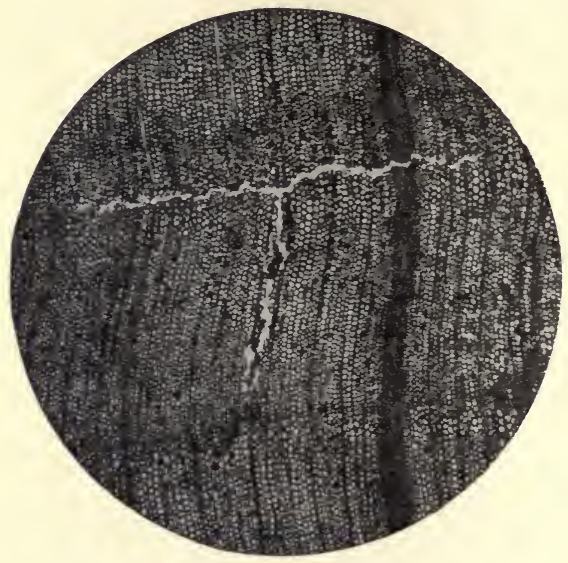

FIG. 29r.-Transverse section of an Araucarioxylon from the Cretaceous of Staten Island. araucarian conifer closely allied to, if not identical with, the living kauri (Agathis). A persistent leaf trace appears running through the figure transversely. The wood is considerably over half a century (as recorded in annual rings) from the center of the stem, and the foliar strand still persists in the manner characteristic of related living araucarian genera. The yearly increments of growth are distinctly indicated, although not very strongly marked. For comparison with the illustration above a photograph of the wood of Agathis australis from New Zealand is shown in Fig. 292. Here annual rings are clearly apparent, although the latitude in the Southern Hemisphere is nearly the same as the place of origin of the Cretaceous araucarian wood above, namely, 40 degrees. It is thus apparent that the Cretaceous of Staten Island was marked by a much less distinct annual variation of temperature than is the South Island of New Zealand of today, although the two localities are of almost identical latitude. Without pursuing the 
subject further we may point out that the organization of the trunks of trees in succeeding geological epochs indicates a gradual cooling of terrestrial climates which, when it became accentuated, gave rise to a marked variation in annual temperature, recorded ever more clearly in the progressively greater definition of the annual rings in later geological times.

It is now necessary to correlate the climatic changes more in detail with the internal organization of the plastic stem organs of vascular plants. It has been shown in Fig. 289, representing the wood of the cordaitean Mesoxylon of Carboniferous age from the north of England, that the only differentiation which marked annual increments of growth in this early age was a

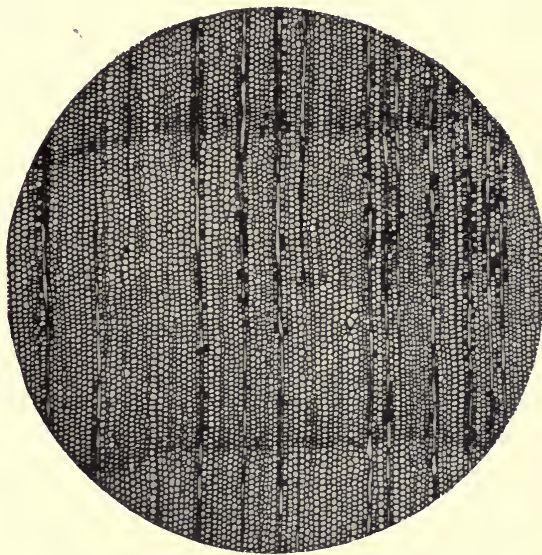

Fic. 292.-Transverse section of the wood of Agathis australis from New Zealand. slight narrowing in radial diameter of the tracheids formed toward the end of the period of growth. No other structural feature related to the phenomenon of annual rings has been recorded as yet from either the late Paleozoic or from the early Mesozoic (Triassic). In the Jurassic, however, and practically universally from this epoch onward, a marked modification in the organization of the annual rings presents itself. This is well shown in the structure of the wood of Ginkgo (Fig. 293), a survivor of a stock which attained its zenith of development in the Mesozoic age. Clearly the tracheids which mark the termination of the annual increment differ from those previously formed in the character of their pitting. The 
pores of the tracheary elements in the first-formed region of the annual ring are exclusively radial. The terminal tracheids in contrast are distinguished by the presence of tangential pitting. It is in the gymnosperms of the middle and later Mesozoic that this

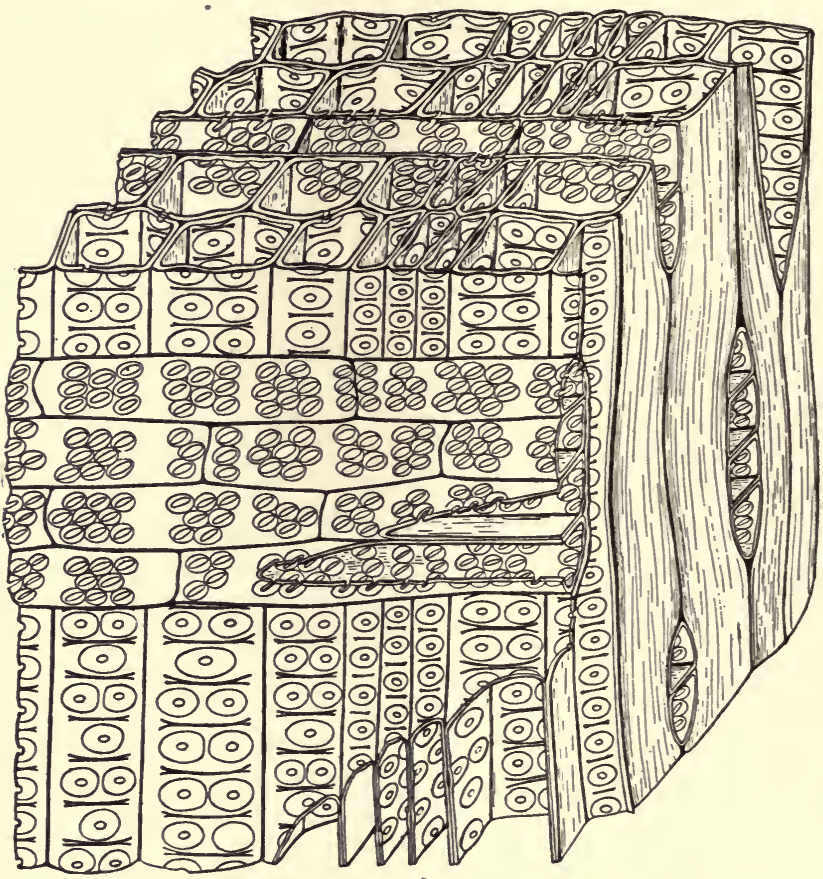

Frg. 293.-Details of organization of wood of Ginkgo. Tangential pitting appears at the end of the annual rings.

feature is first clearly expressed. It has been suggested by Strasburger that tangential pitting is a device for supplying water rapidly and abundantly in the new period of growth to the reawakening cambium. This seems to be a highly probable explanation of the anatomical conditions present and may be accepted in the 
absence of any evidence against its validity. The gymnosperms of the Paleozoic had just begun to develop annual rings toward the end of the epoch, and the phenomenon of tangential pitting had not yet made its appearance. As has been illustrated above, this feature did not become marked until the Mesozoic was well advanced.

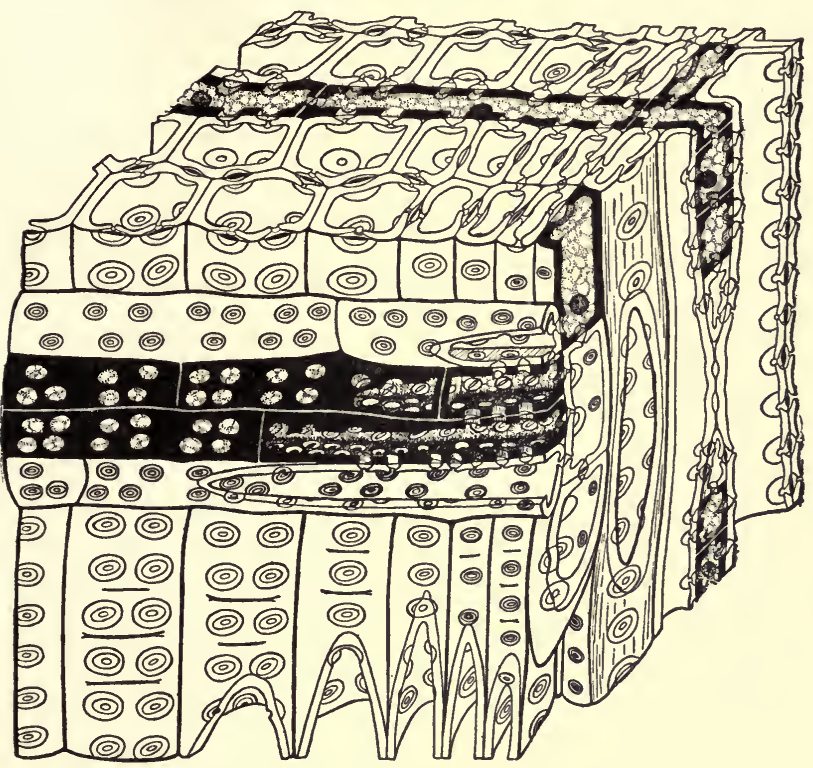

Fig. 294.-Details of organization of the wood in Picea canadensis

The situation in gymnospermous woods did not, however, rest with the appearance of tangential pitting at the end of the annual ring. Not long after this feature had become well established it was accompanied by another even more important anatomical phenomenon. In Fig. 294 is shown a slightly diagrammatic view of the wood of the root of Picea canadensis in three dimensions. The illustration includes the transition from one annual ring to the next, and the terminal tracheids manifest the tangential 
pitting referred to above. Not only is the end of the annual increment marked by tangential pitting, but it is also distinguished by the presence of longitudinal tangential storage elements known as wood parenchyma. In the figure these living cells are strictly confined to the face or termination of the annual ring. They are further present as derivatives from tracheary structures, for stages between them and transversely divided tracheids are plainly seen. Both the mode of occurrence and the manner of origin of the first parenchymatous elements in coniferous woods are of equal interest from the climatological and evolutionary standpoints. The position of the tangential storage elements at the end of the annual ring corresponds in an apparently highly significant manner with the appearance of tangential pitting in those woods of later geological time which have developed the phenomenon of annual rings. It has been suggested with a strong degree of probability that tangential pitting in the gymnosperms is an adaptation for supplying abundance of water to the cambium when it renews its activity after the annual period of rest. It is extremely likely that the terminal parenchyma which, so far as our present knowledge goes, first made its appearance in the Jurassic is likewise a device favoring the activity of the cambium in a new period of growth. If it is probable that the tangential pitting of the terminal tracheids is for the purpose of supplying the cambium with water, it seems not less likely that terminal parenchyma provides a convenient supply of food for the initial cells of the cambial layer. The conditions present strongly suggest that the first appearance of tangential storage elements was in relation to the nutritive demands of the cambial layer. Whether that conclusion is accepted or not, there can be no doubt of the correlation of the phenomenon of annual rings and the first appearance of longitudinal parenchyma in the tissues of the wood. This view of the matter is strongly supported by the conditions observed in Paleozoic woods, for these are equally characterized by the absence of annual rings (except of course at the end of the period and in higher latitudes) and of storage elements belonging to the category of wood parenchyma.

Although at first terminal in position in gymnospermous woods, parenchyma did not long remain restricted to this situation. In 
the Jurassic and Cretaceous, storage elements are already found distributed throughout the annual ring. Among the living coniferous subtribes all but the Abietineae have or formerly possessed diffuse parenchyma or storage tissue scattered throughout the annual ring. This condition, for example, actually holds for the Taxodineae, Cupressineae, and Podocarpineae, and there is good evidence either from fossil forms or conservative organs or from both together that the Taxineae and Araucariineae formerly possessed abundant, diffuse storage parenchyma. In the Abietineae a very interesting condition exists which is quite in harmony with the ancestral position assigned to them in an earlier chapter. In the group Pineae parenchymatous elements are absent in the ancient Pinus and Prepinus; while in Picea, Larix, and Pseudotsuga they occur in an exclusively terminal position and present, particularly in Picea (notably in the root of the various species), all transitionary stages of derivation from septate tracheids. It may be assumed that in the Pineae we have the most primitive conditions found in the conifers as regards the presence, position, and mode of origin of parenchymatous elements. In the Abieteae, comprising Abies, Tsuga, Cedrus, and Pseudolarix, the longitudinal storage parenchyma is characteristically terminal, but no longer shows normal evidence of derivation from tracheary elements. In wood formed after injury, however, transitions from tracheids to parenchyma cells can readily be observed. The genera Abies and Tsuga are of particular interest among the Abieteae on account of the fact that some of their species show a transition toward the diffuse condition of distribution of wood parenchyma. In $A$. webbiana and $A$. cephalonica storage elements scattered throughout the annual ring have been observed, and a similar statement holds for the mature wood of $T$. mertensiana and the branches of $T$. canadensis. In the remaining subtribes of conifers, as shown above, the parenchyma is or has been diffuse in its mode of occurrence. Further, it presents no normal transitions from tracheary elements, although such conditions can frequently be observed in injured material.

The conifers have been chosen to illustrate the correlation between the organization of wood and climatic conditions, because their great geological age and the abundance of their 
structurally preserved fossil woods make them a particularly significant document in this connection. In the higher living gymnosperms and in the dicotyledonous angiosperms the diffuse distribution of the longitudinal storage elements is that typically present. It is reasonable to suppose, in the absence of the abundant evidence presented in the case of the Coniferales, that in the remaining living seed plants wood parenchyma originally appeared much in the same manner as in the coniferous gymnosperms.

It has been noted in a previous chapter that, although in the mass of conifers tangential pitting is characteristic of the terminal tracheids of the annual ring alone, in the higher groups of seed plants it is diffused throughout the annual ring precisely as is the case with the wood parenchyma. It is thus apparent that the phenomenon of annual rings correlated to the recurrence of yearly periods unfavorable to vegetative activity is in turn correlated to the appearance of tangential pitting and wood parenchyma in the series presented by the more modern seed plants. It thus becomes evident that climatic conditions, so far as these two extremely important features of organization of the wood are concerned, have had a potent influence on the organization of structure.

The next impetus to the upward evolution is provided by the appearance of vessels. It is highly improbable that these important structural features of the wood in higher plants owe their origin in any way to climatic conditions. Their appearance, although a phenomenon of the highest importance from the standpoint of the doctrine of descent, is not obviously connected with any known causal conditions. There is no question, however, that the histological element known as the vessel has come into existence as a consequence of the modification of tracheids of the secondary wood. This is the situation in the case of the Gnetales and is only less distinctly manifested by the lower dicotyledons. Although the appearance of vascular elements has no relation to climatic conditions, vessels once established as a feature of ligneous organization have played an important part in the evolutionary history of the higher vascular plants in rela- 
tion to conditions of temperature. The secondary wood of the higher plants is much more efficient in conducting water when vessels are present. It has been estimated by Pfeffer, for example, that the woody cylinder of the birch has about twenty times the water-conducting capacity of that of the pine, and the secondary xylem of the Betulaceae is probably less viable to water, on account of the universal presence of vessels, with scalariform perforations, than are many dicotyledonous woods of higher organization with porously perforated vessels. The greater capacity for conducting water which characterizes the secondary wood of seed plants from the Gnetales upward provides, other things being equal, for a much greater leaf surface. The greater superficial development of foliar organs naturally results in a much increased assimilative capacity. The greater accumulation of the products of photosynthesis directly consequent on increase of foliar surface, and indirectly on the appearance of vessels as an important feature of structure in the wood, naturally involves an increase of storage capacity. Since the secondary wood is the most important storage tissue of the higher plants, increased accommodation for reserve materials appropriately originates as a result of modification of its structure. As has been pointed out in earlier chapters, the Gnetales present, not only the lowest occurrence of vessels in secondary wood, but at the same time the first appearance of the typical aggregate ray. This type of radial storage device results from the clustering and partial fusion of the original narrow rays. Aggregate rays were at first not specially related to the appendages, but in higher types tend to become somewhat definitely connected with leaves, roots, and other lateral organs. The aggregate ray passes by further changes into the compound ray, characterized by a homogeneous organization and no longer including fibers and other features of longitudinal structure of the wood. As an alternative to the compound ray which is the result of fusion, we have the diffuse condition of radial parenchyma resulting from the divergence of the original clusters or aggregations of rays in the outer regions of the woody cylinder. We have accordingly to do with two derivatives of the aggregate ray: the compound condition resulting from fusion and the diffuse modification which 
is the consequence of the separation of the original components of the congeries or aggregation of rays.

The diffuse type of radial organization appears to have been more in accordance with the needs of arboreal dicotyledons in later geological times in which climatic refrigeration, annual or secular, has become more and more marked. It is practically of universal occurrence in the forest trees of the present epoch. The compound type of ray is limited to a very few species of trees, of which the oak is a notable example.

Although large rays of the compound type are usually entirely absent in arboreal forms, they are extremely common in herbaceous stems belonging to diverse and not nearly allied families of dicotyledons. The accumulation of large quantities of storage tissues about the foliar traces, a disadvantageous condition in deciduous trees, is very frequent in typical herbs and vines of herbaceous texture. There is an interesting correlation between climatic conditions and the presence of herbaceous types which is not only geographical, but likewise geological. Taking the geographical or climatological conditions first, we know that it is a notable fact that arboreal forms are much more prevalent within the tropics than they are in temperate regions. This statement naturally applies to the higher rather than to the lower families of the dicotyledons. In the true amentiferous types, such as the Betulaceae, Fagaceae, Juglandaceae, etc., there are no herbaceous representatives at all, although these families are characteristically distributed in temperate climates. The absence of herbaceous forms in this instance presents an interesting resemblance to the conditions found among the Coniferales, which are also without species of herbaceous texture, even in the extreme polar limits of their range. The facts in the two parallel cases seem to have a common and significant explanation, which is that both are relatively primitive groups. It is in the higher orders, such as the Compositae, Solanaceae, Leguminosae, etc., that the principle of the occurrence of herbaceous types in temperate, and of arboreal forms in tropical, climates is well illustrated. There are, for example, very few leguminous trees in north-temperate regions, while herbaceous forms are extremely common. Contrariwise, 
tropical arboreal Leguminosae are common, while herbs belonging to this family are much less abundant in equatorial latitudes.

The geological conditions so far as they are clearly displayed seem to correspond closely with those indicated geographically in the present era. For example, the Páleozoic, which was a period of average high temperature compared with later geological eras, was characterized by the prevalence of arboreal cryptogams belonging to the groups whose survivors are practically entirely herbaceous. In the cooler but still warm Mesozoic the prevailing type of seed plant vegetation was gymnospermous and in particular coniferous, and was consequently arboreal and not herbaceous in habit. Concerning the proportion of herbs and trees in the angiospermous vegetation of the later Mesozoic and of the warmer part of the Tertiary we are unfortunately not well informed. It is certain from still unpublished investigations that herbaceous types were present, although not abundant, in the Upper Cretaceous of the Eastern United States. As the data in this case are supplied by remains preserved from obliteration by charring and subsequent sweeping into open water, it is not unlikely that they indicate, approximately at any rate, the proportion of herbaceous and woody forms in the floras of the later Cretaceous.

The general evidence points conclusively toward the herb as a product of cooler climatic conditions and of later geological times. It should not be forgotten in this connection that the appearance of wood parenchyma, with which in the last analysis the evolution of herbaceous angiosperms is clearly linked, is definitely related to the phenomenon of annual rings, in turn the result of climatic refrigeration in later geological epochs. The impulse toward the formation of tangential parenchyma was thus obviously supplied by climatic cooling. Tangential parenchyma united with radial storage tissues furnishes the explanation of the broad or compound ray as exemplified by the oak on the one hand and by vines and herbs on the other. It will be apparent from the conditions elucidated above that climate has had a paramount influence in molding the organization of plants and that refrigeration has always favored the appearance of herbaceous types. A careful distinction must, however, be made between degenerate herbs 
and dynamic ones. Exemplifications of the former are supplied by the few surviving vascular cryptogams. The latter are represented by the huge aggregation of angiospermous herbs, both dicotyledonous and monocotyledonous, which constitute such a preponderant and aggressive element in the present plant population of our earth. It seems not unlikely that should present climatic conditions remain for a long period either unchanged or even accentuated, the dynamic herbaceous type will definitely supplant the arboreal, at least in temperate regions. If this view is correct, we must regard the strikingly herbaceous flora of existing prairies, steppes, and pampas of higher latitudes as a forerunner of a condition which will ultimately become universal. In any case the appearance of the dynamic herb of angiospermous affinities, which is primarily the result of the differentiation and not the degeneracy of the woody cylinder, is an evolutionary phenomenon of the first order. 


\section{CHAPTER XXXI}

\section{EVOLUTIONARY PRINCIPLES EXHIBITED BY THE COMPOSITAE}

In earlier pages the principles derived from the comparative anatomical study of existing and extinct plants, particularly vascular cryptogams and gymnosperms, have been emphasized. In the present chapter it will be shown that these principles are as applicable to forms the past of which is unknown as to those historically recorded. It will be advantageous in this connection to consider a very high group among the dicotyledons, namely, the Compositae. This family is commonly divided into two sections, the Tubuliflorae and the Liguliflorae. The former are characterized by the fact that the axial florets of the heads are tubular and not ligulate in their organization. Anatomically they are distinguished by the presence of oil canals, which usually occur in the more conservative organs even when they are lacking elsewhere. In the Liguliflorae, on the other hand, the axial flowers of the inflorescence are invariably ligulate. Histologically this group is characterized by a milky juice which is present in the pericycle and the phloem. Indeed, it often happens that the laticiferous ducts are actual sieve tubes or are at least continuous with elements of this nature. It is an interesting fact that in the higher representatives of the Compositae, marked by the presence of a milky or laticiferous secretory system, frequently oil canals such as occur normally in the Tubuliflorae are present under the same conditions as the ancestral features surviving in vascular cryptogams and gymnosperms. This situation is of great general interest as illustrating the wide validity of the general principles elucidated in chapter xvii. Since the Compositae are readily obtainable by reason of their great abundance in the present flora, either in a wild or in a cultivated state, they may serve advantageously to illustrate pedagogically the fundamental conceptions of comparative anatomy.

In Fig. 295 is shown a transverse section of part of the stem of the Jerusalem artichoke (Helianthus tuberosus) moderately 


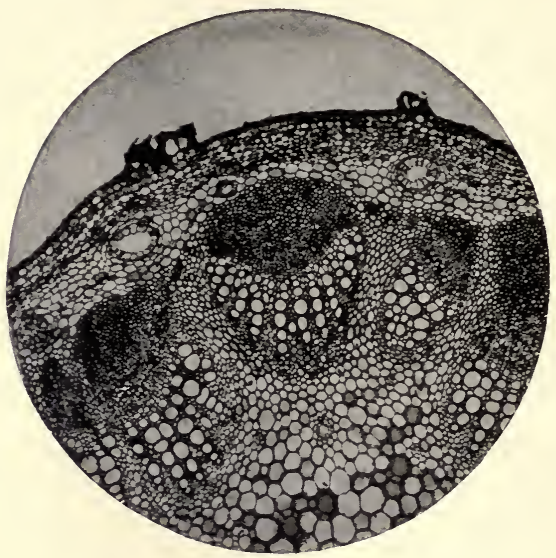

FIG. 295. - Transverse section of part of stem of the Jerusalem artichoke

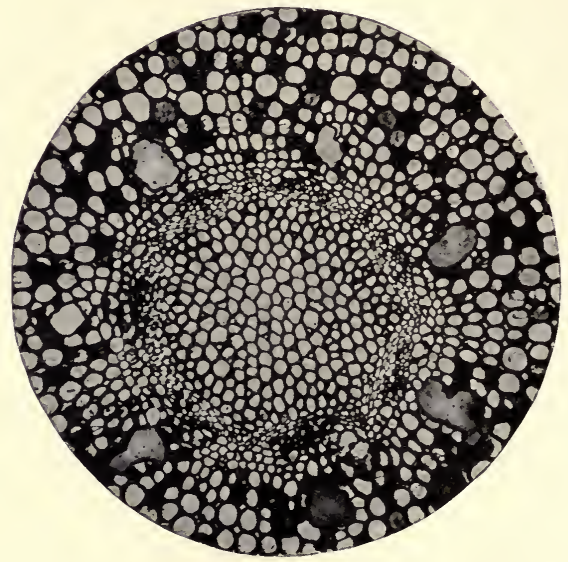

FIG. 296.-Section of part of the root of a species of Aster 
belongs. The circular zones of darker elements mentioned above represent not only the distribution of the laticiferous tissue, but that of the sieve tubes as well, which cannot be clearly distinguished from the latter. The subject of the relation between characteristic elements producing latex and sieve tubes needs further investigation with the improved methods now in vogue. Former statements that the development of the milk system is in inverse proportion to that of the sieve tubes, which have been both denied and affirmed, may advantageously be controlled by examination of more perfect sections. In Fig. 298 is seen a magnification of the laticiferous system of the black salsify (Scorzonera).. It is here obvious that the secretory system with dark contents no longer shows the identity of its originally separate elements, so completely has cellular fusion taken place.

A consideration of the subfamily Cynareae of the Tubuliflorae will next occupy our attention. Here we have to do with a group which is transitional anatomically from the Tubuliflorae to the Liguliflorae, since it possesses partially the oil canals of the lower Compositae and likewise the laticiferous system which is a feature of organization of the higher members of this important group. Fig. 299 illustrates a portion of a transverse section of a root of the so-called French artichoke, Cynara Scolymus. Three oil canals are to be seen in the upper region of the section. We may now pass advantageously to a consideration of the common burdock, Arctium minor. In this species the root resembles that of the artichoke, figured above, in the possession of oil canals. The stem of the burdock in its lower and first-formed region presents some interesting features of organization, which are illustrated in Fig. 300, showing part of a transverse section through the axis. In the lower region of the figure appear the outer parts of a number of fibrovascular bundles belonging to the stem. It is clear that none of these shows the presence of any oil canals. In the phloem may be seen dark dots, which indicate laticiferous elements. Above the zone of stem bundles lies a single leaf trace in the cortex. Along the outer margin of this appears a row of secretory canals. The nature of these is more clearly seen in Fig. 3or, representing the transverse view of the foliar trace under 


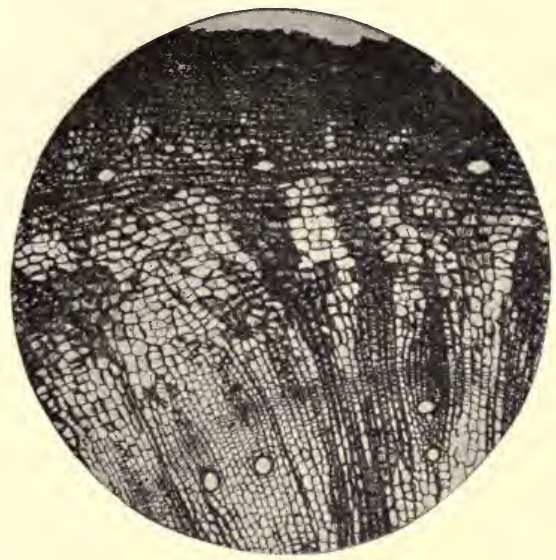

FIG. 299.- Part of a transverse section of the root of Cynara Scolymus

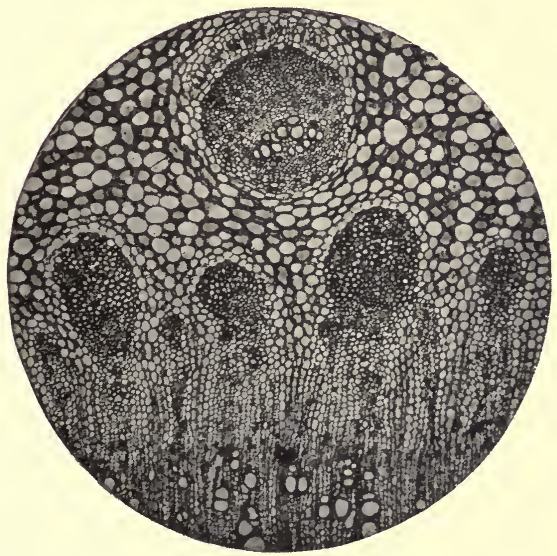

FIG. 300.-Transverse section of the axis of Arctium 


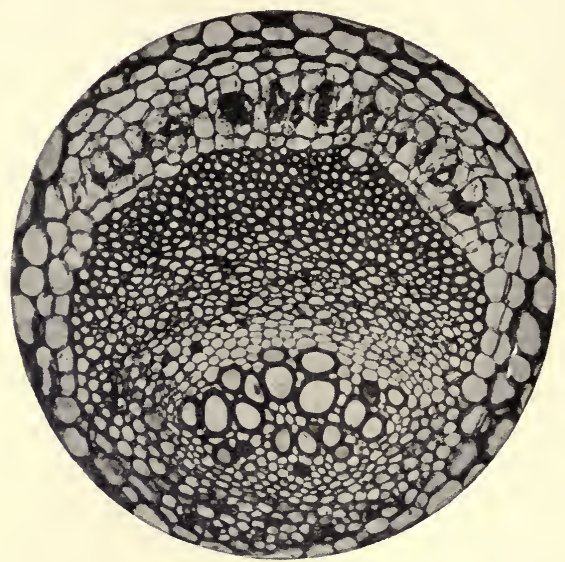

FIg. 30I.-Transverse section of the foliar trace of Arctium

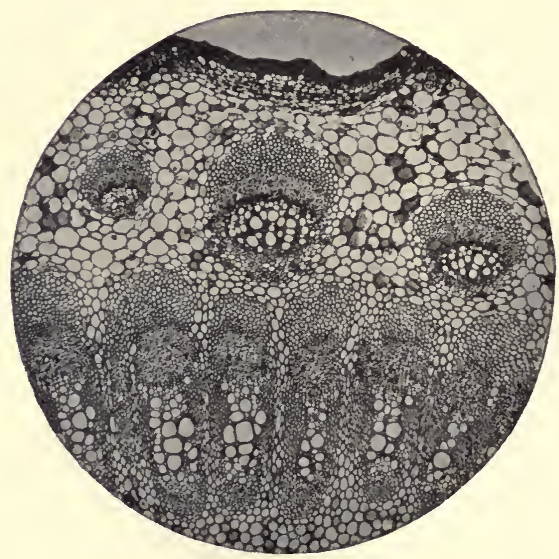

FIG. 302.-Section through the upper aërial stem of Arctium 
a higher degree of magnification. External to the fibrovascular tissues, on the upper side, is a row of somewhat imperfect and vestigial oil canals. The interesting fact in the present connection is that the oil canals appear in relation to the leaf trace, although absent in the ordinary bundles of the stem. Sections lower down toward the hypocotyledonary region show the secretory canals somewhat better developed. In no case do they occur in bundles which belong distinctly to the stem, although they may run a short distance down on the foliar traces as they pass into the cylinder of the axis. In the upper regions of the stem a different situation presents itself. Fig. 302 shows a section through the aërial stem of the burdock in transverse section. Here neither the numerous bundles of the axis nor any of the three foliar strands show the presence of oil canals, although small dark dots in the region of the phloem sufficiently vouch for the presence of laticiferous elements. Fig. 303 reproduces a magnified view of one of the leaf traces, demonstrating the entire absence of oil canals in the foliar strands given off from the upper part of the axis. If the branches are followed to their tips, where the flowering burs are produced, anatomical investigation reveals the continued absence of oil canals.

Similar observations may readily be made on the Scotch thistle, Onopordon, or on the Canada thistle, Cirsium, as well as on a number of other representatives of the Cynareae. The burdock is on the whole much more favorable than any of the common thistles and has accordingly been chosen for illustration. It will serve a useful purpose to return now to Cynara Scolymus, the French artichoke. As is well known, the vegetable so designated is a variety of the common Mediterranean thistle in which the scales of the involucre surrounding the head have become hypertrophied at the expense of the floral organs proper. This overgrown involucral covering is eaten as a prized vegetable in Southern Europe. Fig. 304 shows a section through the lower region of the flowering axis of Cynara Scolymus. The bundles on the lower side of the figure either belong to the axis or have very recently taken their departure from the region of the pith. Those toward the upper side are passing into the basal portion of the 


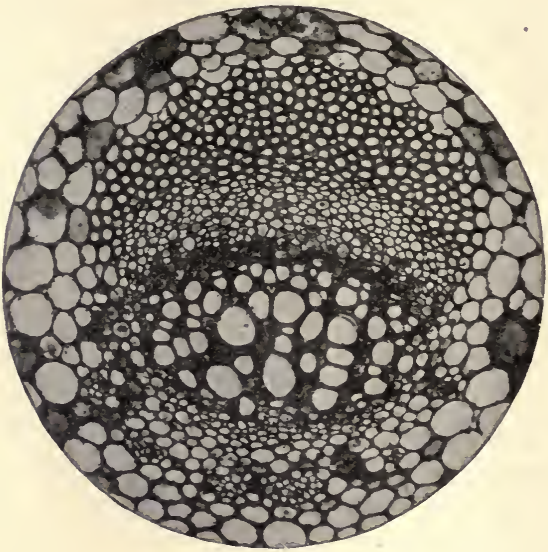

FIG. 303.- Highly magnified view of the leaf trace in Arctium

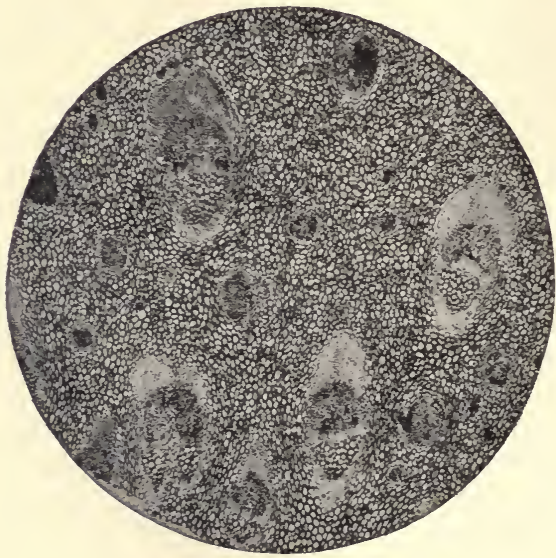

FIG. 304.- Section through the lower region of the flowering axis of Cynara Scolymus. 
involucral scales. Certain dark dots on the superior margin of the upper bundles indicate the presence of oil canals. Structures of this nature are entirely lacking in the axial bundles and in the traces which have only recently left the cylinder of the stem. Fig. 305 will serve to demonstrate the truth of the statement just made. In $a$ is shown a strand near the axial region which obviously is not characterized by the presence of oil canals. In $b$, on the contrary, representing a trace destined to an involucral

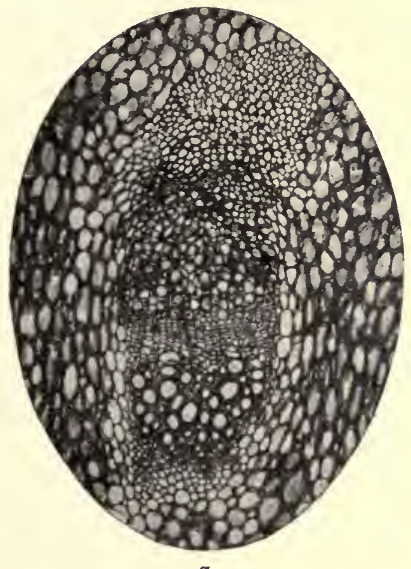

$a$

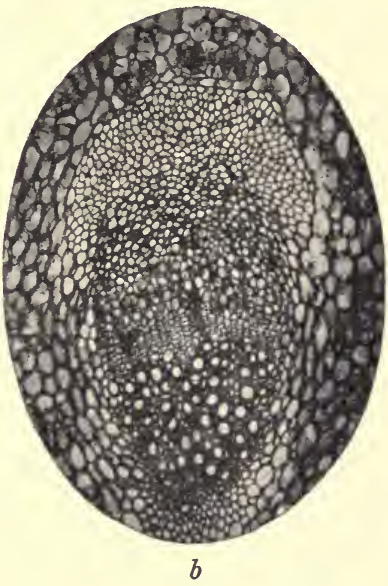

$b$

Fig. 305.- $a$, axial strand of infloresence of $C$. Scolymus; $b$, a strand of an involucral scale in the same.

scale, a series of somewhat imperfectly developed oil canals appears along the upper margin.

The Cynareae represent a transition anatomically from the Tubuliflorae to the Liguliflorae. In the Cichoriae, on the other hand, we have to do with very typical representatives of the Liguliflorae, since in this subfamily all the florets are strap-shaped and the secretion is milky in its nature and contained in the region of the phloem. Interestingly enough in the oyster plant (Tragopogon) and in the salsifies (Scorzonera and Scolymus) vestiges of 
an oil-secreting system are occasionally found in the root only. Even in the chicory itself some slight indications of oil canals are sometimes discovered in the roots, although these are never functional as in the salsifies.

It will be apparent from the various illustrations and statements of the foregoing paragraphs that in the Compositae the oil canals of the lower forms tend to perpetuate themselves in a vestigial fashion in certain conservative parts and organs of the higher types belonging to the Cynareae and the Cichorieae. The general situation can best be visualized by means of a diagram which is made in accordance with the data supplied by the French artichoke, Cynara Scolymus, since this form on the whole reveals the anatomical situation in the fullest manner. In the center of Fig. 306 appears a somewhat conventionalized external view of the species under discussion. The regions which are of critical importance are indicated by dotted lines, marked by letters from $A$ to $D$. At the sides are shown diagrammatic transverse sections of the regions indicated by the letters. In $A$ is seen a view of the root, conforming to the general structure of such organs and at the same time showing a series of oil canals just external to an outer circle indicating the endodermis. In $B$ appears a view of the lower region of the axis showing leaf traces, either present in the cylinder of the stem or lying externally in the cortex. In every case the foliar strand is accompanied by oil canals, which are entirely absent in the bundles of the stem. In $C$ is represented a section through the high aërial portion of the stem. Here the leaf traces, whether still within the cylinder or passing outward in the cortex, are conspicuously without accompanying oil canals, as are also those of the axis proper. In $D$, which illustrates a region of section through the base of the reproductive axis, the oil canals appear again in relation to the traces of the involucral appendages, but are as clearly absent in the axial bundles as they are in the lower parts of the stem. It is accordingly obvious that the various canons of comparative anatomy stated in chapter xvii are as well illustrated by that very high dicotyledonous group, the Compositae, as they are by the conifers and calamites. Moreover, while it is true that in the case of the high dicotyledonous 
group which forms the subject of the present chapter the only data for regarding the Tubuliflorae and the Liguliflorae as respec-

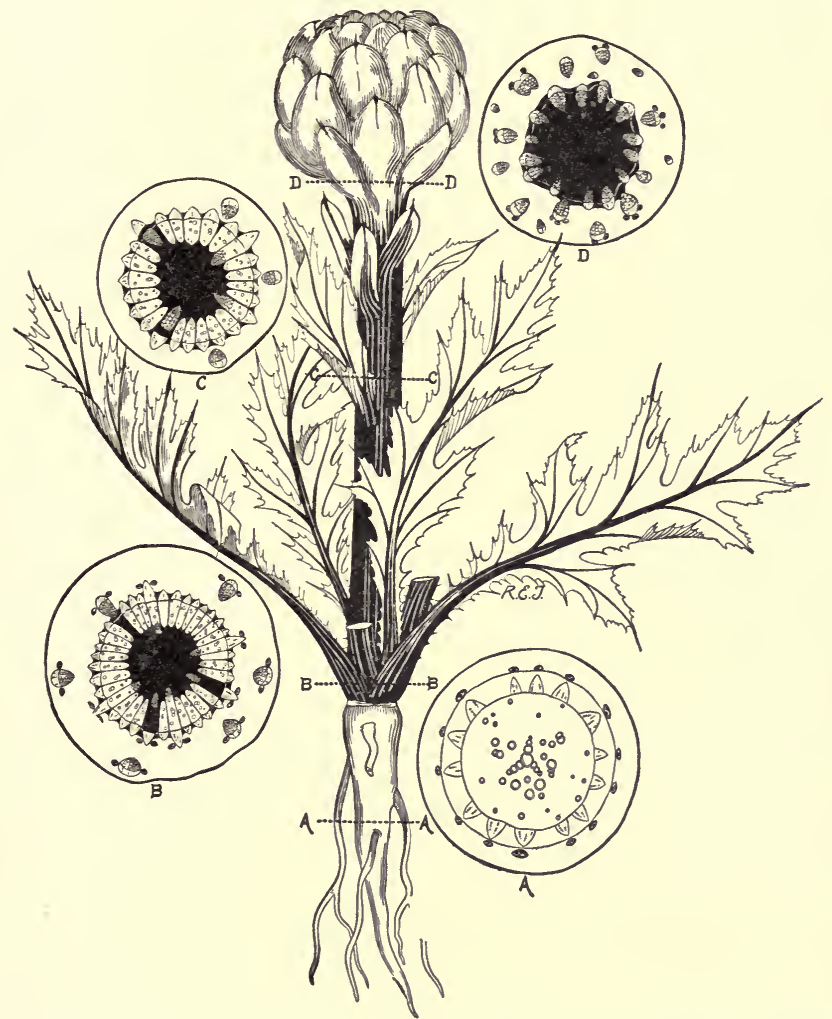

FIg. 306.-Diagram of external habit and distribution of oil canals in the artichoke (C. Scolymus).

tively lower and higher are morphological in their nature, the lines of evidence in this connection are so numerous and so generally admitted that there can be no reasonable doubt as to their validity. 


\section{CHAPTER XXXII}

\section{ANATOMICAL TECHNIQUE}

As a result of the fact that it has been mainly the soft and reproductive parts of plants which in the past have been investigated in connection with the hypothesis of evolution, the methods of examining tissues belonging to these categories have naturally made greater progress than those applicable to the study of harder and vegetative structures. The reawakened interest in fossil plants, which is an important feature of the present phase of the development of morphology, has, however, brought into prominence methods for the anatomical investigation of the hard tissues, since in general it is only the harder and consequently more resistant parts of extinct plants which furnish a basis for comparison with modern types. This general situation is, moreover, a somewhat fortunate one, for it is precisely the enduring woody structures which combine a greater immunity to decay with marked conservatism of organization. Methods of investigating hard tissues in plants must obviously, from the conditions outlined above, include, not only those applicable to relatively unaltered living plants, but also those which may be employed with success in the case of carbonized or petrified vegetable remains. Since the present volume deals with anatomy alone, it will be possible to confine the description of technique to those processes which are of value in the investigation of the hard parts of plants.

\section{THE PRESERVATION OF MATERIAL}

In many cases the preserving of material of hard structures in plants does not present a problem of difficulty. For example, petrified or carbonized stems which have survived the destroying influences of often extended time need not be preserved in order to be studied successfully. In such cases the problem of the preparation of the tissues rather than of their preservation is of importance. A similar situation presents itself in the study of woods. 
Here the material available is frequently in a dried condition; and although its protoplasmic structures have been obliterated, it is still by reason of the durability of its essential organization eminently worthy of investigation. Preservation of hard structures, although not in general as necessary as in the case of the softer tissues, is often, however, a matter of prime importance.

The skeletal structures of plants consist of more or less thickwalled cells united by means of a cement substance which is pectic in its nature. The process of preservation must be such as will not bring about maceration of the hard elements by the dissolution of the bonding material or middle lamella. Further, preservatives which increase the resistance of the thick cell wall to sectioning are in general undesirable. As a result of the conditions just indicated, chromic acid and the salts of chromium cannot in general be advantageously employed for the fixation of hard tissues in plants, valuable as these reagents are in the study of protoplasmic structures. Where as perfect a preservation of the protoplasmic organization as possible is essential, and at the same time the avoidance of undesirable changes in the thick cell wall and the cement substance are sought, alcoholic fluids are to be chosen. Excellent reagents in this connection are solutions of corrosive sublimate or picric acid in alcohol of from 100 to 30 per cent strength. The strength of the alcohol will depend on the nature of the material. In general, those parts which by reason of the presence of a large proportion of water are subject to shrinkage in strong alcohol should be fixed in solutions containing a lower percentage, while harder and more impenetrable tissues may be treated with a higher grade of alcohol. In many instances it is advisable to use the alcohol without any added reagent. This is quite generally the case with wood; for the addition of corrosive sublimate, for example, is not of sufficient advantage in connection with fixation to warrant its employment. Corrosive sublimate, picric acid, or whatever killing reagent may be employed in conjunction with the alcohol can conveniently be used in saturated solution in whatever grade of alcohol is found most advantageous. Where material is fixed in alcoholic fluids it must be washed in alcohol of corresponding or greater strength to remove the excess of reagent. Corrosive sublimate forms a black precipitate 
in the protoplasm of the cells which is to be removed, after the material has been kept in strong alcohol for some time, by the addition of crystals of metallic iodine until the alcohol ceases to lose the brown color imparted by the iodine. In all cases the preserved material should finally be brought into strong alcohol as a preliminary to further treatment. Where the parts are delicate, such as small roots, slender herbaceous stems, or the organs of aquatics, alcohol of full strength should be reached by gradual stages, through $3 \circ, 5 \circ, 70$, and $9 \circ$ per cent grades.

\section{THE MACERATION OF MATERIAL}

It is often important, particularly in the case of the more resistant tissues, to separate the cells from one another by the dissolution of the cement substance or middle lamella. This end is sometimes attained by the use of nitric acid and a chlorate. Such a procedure, however, is unnecessarily violent and causes a considerable amount of injury to the cells themselves. A better method is to use chromic acid in conjunction with nitric acid, and to warm in a paraffin bath, if it is desirable to hasten the process. Maceration in the cold, however, often gives much better results. The strength of chromic acid varies according to the material. From 5 to ro per cent strength of the two acids ordinarily suffices to bring about the necessary degree of maceration. The material after soaking for some time in the macerating fluid may be washed in repeated changes of water and then gently scraped with the point of a needle or scalpel. As a result of this procedure masses of elements may be removed which are readily teased apart with needles or separated by tapping on the cover glass.

In the case of carbonized material, such as, for example, charred wood in coal, commonly but not very aptly designated "mother of coal," boiling in nitric acid often yields quantities of isolated tracheids and other elements of the wood showing all the details of structure. The action of the hot nitric acid in this case is a double one, since it not only affects the isolation of the elements, but also bleaches the black hue of the coal to a light brown. In this manner details of structure are made apparent. Spores are likewise isolated from coal by this method. A more powerful action 
is naturally reached by the use of aqua regia (nitro-hydrochloric acid) or of nitric acid and a chlorate.

\section{THE SOFTENING OF MATERIAL}

In the case of the hard tissues of plants it is usually necessary to employ some method of softening in order to facilitate the subsequent cutting of sections. It has been discovered that the hardness of the wall of the cell in plants, other things being equal, depends upon the amount of mineral matter present. Lime, aluminum, and silicon are elements often found in the wall, particularly where it is considerably thickened. The removal of these substances depends, of course, upon the use of an appropriate acid, and hydrofluoric acid has proved most useful in this connection. This acid has the advantage of not attacking the middle lamella - an advantage which is of considerable importance where good sections have to be secured. For most purposes strong, commercial hydrofluoric acid answers sufficiently well, but in cases where the tissues are delicate the chemically pure reagent is best employed. Hydrofluoric acid is best purchased in gallon leads containing about ten pounds in weight. The leads should be massive, as thin ones are sometimes eaten through, with disastrous results, before the acid is used up. The material is prepared for treatment in different ways, depending on its nature. In the case of the shell of a hickory nut or a piece of ebony or live oak, the dry material is first boiled for some time in water. The boiling should be continued after the sinking of the material so as to insure the driving out of all air and the complete imbibition of the membranes. It is then allowed to cool and is transferred to hydrofluoric acid of full strength. After sojourn in the reagent for a week or two a piece is washed for a short time in running water and is then tested with a knife to discover if it cuts readily. If not, a longer stay in acid is indicated, and in case of very hard substances the time of sojourn may be as long as five or six weeks with occasional renewal of the reagent in extreme instances. The hardest and most refractory tissues of existing plants may be mastered in this manner. Wood is usually cut into cubes, which may advantageously be about one centimeter in each of the three dimensions 
and should have their faces corresponding with the transverse, radial, and tangential planes of structure of the wood.

When the wood is softer, after the preliminary boiling, which should not be shortened, the pieces of larger size, on account of the greater ease of manipulation, are transferred to acid which has either been diluted with water or been used once before on harder material. In this they are left for a shorter time than is necessary to effect the softening of very hard tissues, such as heavy tropical woods or the shells of nuts. For poplar or fir wood a week is quite long enough to bring the tissues into good condition for sectioning.

When complete organs are to be sectioned, such as stems, roots, leaves, etc., a preliminary fixation in some preserving fluid is necessary. After the material has been freed of the fixing agent, in case this is any fluid other than alcohol, it is run up into strong alcohol and then freed of air by means of a good air pump. The air must be removed as completely as possible so that the pieces will sink even in water. After the pumping has been completed, the specimens are transferred to water and left until they sink. If the parts are rather delicate, it is well to carry on the process of demineralization in weak alcohol instead of water to avoid maceration. In most cases, however, if the material has been well fixed in a suitable reagent, no appreciable injury is caused either to the cell wall or to the protoplasm by the use of hydrofluoric acid.

Naturally the demineralization of plant tissues of whatever category through the agency of hydrofluoric acid must be carried on in receptacles made of wax (either hard paraffin or beeswax will serve); or, in case glass bottles are used, these must be coated both internally and externally with wax. This is necessary to avoid the destruction of the container. Gutta-percha or hardrubber bottles are sometimes sold for the purposes indicated, but they are not so resistant to acid as wax and are unnecessarily expensive. Further, they cannot, like wax, be used with chlorates in certain procedures to be later described. Whatever be the nature of the material to be softened, it must, after remaining sufficiently long in hydrofluoric acid, be washed entirely free of this reagent in running water. The washing is best effected in the case 
of small pieces in a vessel covered with gauze or cheesecloth to prevent the loss of material. Larger pieces of wood, particularly when they are heavy, can be washed in open bottles.

The next stage in the process of preparation for sectioning depends on the nature of the material. If the objects are very small, and especially if they lack homogeneity on account of the presence of soft as well as hard tissues, they must be subjected to imbedding in nitrocellulose or paraffin, and the former process is ordinarily preferable. In the case of larger objects one of two conditions may present itself. Either the structure is quite homogeneous, as in peachstones, cubes of oak wood, etc., or else it consists of tissues both hard and soft, as, for example, segments of stems or roots. In blocks of uniform texture, after washing is complete, a transfer is effected to a fluid consisting of equal parts of alcohol of 30 per cent strength and glycerin. Immersion for some days in this reagent fits the material for cutting. When the objects are of some size and are not of homogeneous organization they are run up through alcohols after washing to a strong or 95 per cent solution. This is changed twice and the material is then placed in equal parts of strong alcohol (95 per cent) and glycerin. A week or more is needed under these circumstances to bring about a consistency suitable for sectioning.

\section{IMBEDDING IN NITROCELLULOSE}

When the material is unhomogeneous and is in small pieces, it must be imbedded in nitrocellulose. There are a number of different types of nitrocelluloses which may be employed. On the whole, the best for the purpose is Schering's celloidin. This is a special preparation of particular toughness, solubility, and purity. When extreme transparency is a desideratum, the nitrobody known as photoxylin may be used. In general, however, a nitrocellulose which is entirely transparent is quite unnecessary, since all are sufficiently translucent to permit the ready orientation of the object in cutting. The cheapest and most readily procurable nitrocellulose is photographic guncotton prepared for making the so-called "wet-plates" used in certain photomechanical processes. This is inexpensive and may be purchased at any 
photographic supply store. Whatever nitrocellulose is used, it must be first washed in clean water and then carefully dried. The stock should always be kept under water both to delay changes in chemical composition which result in insolubility and to avoid the risk of explosion. The dry nitrocellulose is dissolved in a mixture of equal parts of good ether and absolute methyl or ethyl alcohol. The first is more advantageous because it forms a better solvent of the nitro-body. A number of solutions grading from 2 to 16 per cent must ordinarily be prepared and kept in labeled bottles with wide mouths and good corks. As a preliminary to imbedding, the material is freed of all gases which may have gathered in its intercellular spaces as the result of the treatment with hydrofluoric acid by means of the air pump. It is then freed of water by two or three successive changes of absolute alcohol. This reagent is best purchased in gallon bottles from a reliable manufacturer, since in containers of one pound capacity it is extremely expensive in the United States. The dehydrated material is now ready for infiltration with nitrocellulose. Small strong bottles are prepared by twisting tough wire about their necks in such a manner as to provide two short and diametrically opposite loops. The wire used for this purpose should be tenacious and not too slender. The bottles must have rather wide mouths and be stopped with corks of the very best quality. One of the bottles prepared as described is partially filled with nitrocellulose of the 2 per cent strength, and the objects are dropped quickly into it from the absolute alcohol so as not to absorb either air or moisture. There should be some space intervening between the cork and the solution of nitrocellulose to allow for expansion in heating. The cork is finally forced in and a piece of soft tough brass or copper wire is passed through the loops on the sides of the neck of the bottle described above. The wire is then drawn tight with the fingers or a pair of pliers and twisted over the top of the cork so as to be held securely in place even when the bottle is exposed to the heat of the paraffin bath. After the material has become thoroughly heated the containers are examined for the purpose of detecting any leak through the cork. Additional security against escape is provided by keeping the bottles in the warm bath either upside down or on their 
sides. It is usually best to allow the material to remain for twentyfour hours in the first grade of collodion or celloidin. The bottle is then cooled in the air or more quickly by cold water. The fluid is thrown away because of the accumulation of extractives from the material. Four per cent of celloidin is then poured on, and the bottle wired again for a twelve-hour immersion in the warm bath. The second solution is returned after cooling to its proper stock bottle, and the successive grades of dissolved nitrocellulose are used respectively until 16 per cent is reached. At this stage chips of nitrocellulose previously dissolved and then dried are used at intervals and in a small amount at a time further to thicken the highest grade of solution. The bottle is returned to the bath with each addition of dry nitrocellulose. If the process has been carried on with proper precautions, the material has becone infiltrated without shrinkage. In the case of soft material which is at the same time rather impervious it is often an advantage thoroughly to prick the pieces with a very fine needle. The pricks are not usually near enough to injure the appearance of the sections which are cut later, if all are made in the same plane, naturally not that of greatest importance from the structural standpoint.

After a sufficient degree of thickening has been attained in the manner described in the foregoing paragraph, the objects, each surrounded by a coating of nitrocellulose, are removed to chloroform for hardening. The chloroform should be liberal in amount to insure thorough induration of the nitrocellulose, and the material should be kept in the reagent for at least twelve hours. Subsequently it is transferred to a mixture of equal parts of alcohol and glycerin, in which it may remain until needed for cutting. Objects stored in this way seem to maintain their properties indefinitely and are certainly still useful after as long an interval as twenty years. The alcohol should be prevented from evaporating by occasional renewals.

\section{SECTIONING OF MATERIAL}

Only in the rarest instances is it desirable to cut material freehand with a sectioning razor. If uniformly good results are sought, the microtome should be used. There are many types of microtomes, but those with the simplest mechanisms and the greatest 
degree of rigidity are most useful for the sectioning of hard materials of vegetable origin. Microtomes in general which provide for the raising of the object by a vertical screw and elaborate ratchet devices should be avoided, as they are very difficult to keep in order when the sections are cut wet in alcohol, as is invariably the case with the materials under consideration. A slicing instrument which has proved itself to be of the greatest value for the purposes here enumerated is the Thoma microtome, manufactured by the Jung firm of Heidelberg. The so-called Naples model is the best size and type and has the advantage of being immune from rust by reason of its phosphor-bronze construction. In this microtome the object is raised by sliding up an inclined plane through the action of a micrometer screw which is accurately gauged by means of an adjustable clicking ratchet device. The horizontal position of this mechanism and its manipulation by hand are great advantages, since by reason of these features it escapes the corrosion which rapidly.impairs vertical screws for regulating the thickness of the sections. For cutting small objects the carrier provided with the instrument which can be orientated in two planes is suitable. The object-carrier is provided with small cylinders which are primarily intended for use with paraffin. The hollow of the cylinder, instead of being filled with paraffin as in its ordinary mode of employment, is blocked by a well-seasoned and accurately rounded piece of wood. This should be dicotyledonous and rather coarse-grained. The smooth upper surface of the block is varnished thoroughly (it is generally best to dip the end of the block into the solution) with a 4 per cent solution of nitrocellulose. If the blocks are being used for the first time, they should receive a second coat of celloidin (or collodion) after the first has had time to dry. The objects imbedded in celloidin (or collodion) are now removed and examined with a lens to ascertain the plane in which they are to be sectioned. When decision is reached on this point, the surface of the hardened nitrocellulose is sliced with a sharp knife in a plane parallel to that of the desired sections. The smoothed surface should be perfectly flat. After wiping, this surface, which should never actually expose the object, is dipped into a 4 or 6 per cent solution of nitrocellulose and then 
firmly pressed for a moment on the prepared surface of one of the wood-blocked cylinders. After resting for ten or fifteen minutes in a warm place it becomes securely fixed and is ready for cutting.

The knives for cutting hard vegetable materials should not be too thinly ground on the edges. The type of edge supplied by the Jung firm under the designation of $c$ answers very well for the purpose. The knives are best not too large in size and should not exceed eight inches in length. They are sharpened by the aid of a cylindrical appliance slipped over the back, which gives the edge a less acute angle to the sharpening stone, with a corresponding advantage in saving of time. The stone or hone for putting the edge of the knife in condition should be preferably a yellow Belgian one, such as is ordinarily employed by barbers, but of considerably larger size and of as fine texture as can be procured. It is better, in fact, to have two stones, one coarser for preliminary use and a finer one for finishing. Carborundum hones, although often supplied for the purpose of sharpening microtome knives, are not advantageous. In renewing the edge of the knife care should at first be taken to remove all hacks or gaps by grinding on a coarse hone. In the sharpening process the edge of the knife should be pushed forward on the stone and not backward, as the latter procedure results in a so-called "wire edge." After the removal of the nicks the edge is finished on a finer hone. If, as a result of frequent sharpening, the knife has been ground away to a very thick edge and consequently sharpens very slowly, it is necessary to grind it on an alundum or carborundum wheel of very fine texture. The knife is held on a support known as a knife-grinder and brought against the revolving wheel at such an angle that the edge is slightly hollow-ground. It requires a little skill to grind a knife on the wheel, but this is quickly acquired and the frequent sending of the knives to the cutler is thus avoided. A further advantage of grinding the knives in the laboratory is the avoidance of drawing of the temper, which is often the unfortunate result of sending them to a professional grinder. The wheel should be revolved at a high rate of speed and the edge of the knife held gently against it. In this manner overheating is avoided. The grinding machines furnished by the Carborundum Company of Niagara Falls and the 
Luther Company of Milwaukee are excellent, particularly the latter.

After the edges have been sharpened on the grinding wheel and hone the knives are finished by means of a strop. The best type has four sides and a solid wooden center. The four sides are covered with leather surfaced in various manners. The first face provides a coarse polish and the two following ones successively finer degrees. The last face is of smooth leather and imparts the final smoothness to the edge. The knife should be carefully wiped with a cloth after being passed on the successive surfaces to avoid the impairment of the finer ones by material from the coarser grades. In stropping, the edge should be drawn backward and not pushed forward as in honing, since failure to observe this precaution results in injury to the leather surfaces. Strops consisting of unsupported leather are not desirable for use with microtome knives.

The knives are held in place on the microtome by the knifeholder, which in turn is fastened to a heavy block of phosphorbronze running in a channel on the right side of the microtome. The holder may be altered in position on the block, forward and backward, inward and outward, by means of appropriate screw holes. It may be raised by plates provided for insertion beneath its shaft. The best type of holder is provided with a tilting mechanism which makes it possible to vary the horizontal angle of the knife to the object. In cutting all hard tissues but coal the microtome knife should be in an oblique position to the object, and in general the inclination should be somewhat acute. In the case of large objects and of those which are likely to curl in cutting, a more oblique position is sometimes advantageous. In making sections the surface of the knife should be kept wet with alcohol of 95 per cent strength. This is ordinarily effected by means of a camel's-hair brush. This is also used for preventing the curling of the section as it comes up on the knife. The proper touch for flattening the section without either rolling or dragging it is acquired by experience only. Sections may be cut from two or three micra to many times that thickness, depending on the particular conditions involved. If the sections curl after being placed in alcohol 
subsequent to removal from the edge of the knife, this disadvantage may be largely obviated by allowing them nearly to dry on the flat surface of the knife at some distance from the part of the edge which is actually being used for cutting.

In the case of hard and homogeneous material, such as wood, pieces of nutshell, coal, etc., a special type of object-holder must be used, in which the material is firmly held in a rigid clamp. Two varieties of these have been devised for the Thoma microtome, recommended for the purposes here described. One, contrived by Professor R. B. Thomson, is made in Toronto, and particulars in regard to it may be obtained by writing to the Botanical Department of the University of Toronto. This is an admirable device and is particularly useful in cutting large pieces of wood which have not been sufficiently softened. A second type of holder for hard objects has been devised by the writer and is manufactured to order by the Jung Company of Heidelberg. In these holders the position of the object can be varied so as to obtain the proper inclination to the edge of the knife. For example, in cutting radial sections of wood the slices obviously must be accurately parallel to the rays, or else a very confused condition is presented under the microscope. The object-carriers in both these holders are very heavy in order to insure the necessary rigidity and inertia. A complete Thoma microtome with the additional holder for cutting hard objects costs about one hundred dollars free of American duty. With the Thomson devices the cost is considerably greater.

As has been pointed out in an earlier paragraph, wood and similar tissues need not be infiltrated with nitrocellulose to secure the best results. If, however, the woody tissues for any reason have become unduly softened, either from too prolonged immersion in hydrofluoric acid or through the ravages of fungi (this is, of course, particularly the case where diseased or rotten wood is being studied), imbedding in celloidin or collodion is necessary. As will be shown in a later paragraph, this procedure is absolutely necessary with most coals. The cutting of sections of very hard tissues involves the same principles as exemplified in the technique of smaller and less homogeneous objects, with the exception only 
of coal, which demands a transversely placed instead of an oblique knife. In cutting more resistant plant substances the edge of the knife must be very smooth and sharp and must be much more irequently renewed than in the case of softer materials.

It is also possible in certain cases to section unhomogeneous materials, consisting of pieces of root, stem, etc., by adopting the ollowing procedure: When the sections are transverse, the pieces nay be clamped directly in the jaws of the wood-holder described above. In the case of longitudinal sections, either tangential or adial, the objects are removed from the mixture of equal parts of strong alcohol and glycerin and accurately smoothed on one surface by means of a sharp knife. The plane surface is dried carefully with a cloth and then painted with a 6 per cent solution of celloidin. After the film of nitrocellulose has thoroughly dried he object is quickly dipped in 4 per cent solution and applied to the surface of a block of wood which has previously been varnished with nitrocellulose in the manner described in a former paragraph. By clamping the block in the wood-holder it is possible to secure extremely thin longitudinal sections without the labor involved n imbedding in nitrocellulose. It should be emphasized, however, that this method is not available in the case of organs which how a considerable diversity of texture. For example, the oranches of Ginkgo and Tsuga must be imbedded to secure successiul sections, while those of Pinus and Quercus provide good transverse preparations without previous infiltration.

Other vegetable materials can best be prepared as thin sections without any previous treatment whatever. This is true of periderm, as illustrated by common bottle cork and "birch bark." These tissues need merely to be clamped in the microtome to furnish sections as good as may be secured by the most elaborate processes of softening and imbedding. Fresh leaves also provide very thin sections when treated in the following manner: Rather thick leaves are best in the case of the dicotyledons and monocotyledons, for example, in Rhododendron and Yucca. These are held between two smooth pieces of pine wood in the jaws of the wood-holder. Sections are made until the knife has cut down sufficiently near to the pieces of pine to secure rigidity in the leaf 
tissues. The sections are then cut thin by appropriate manipulation of the micrometer screw and floated instantaneously on the edge of the knife in alcohol. Thence they are transferred to a dish of water and show all their natural organization and color practically unchanged. Sections as thin as five and ten micra are easily obtained in this manner and are very striking. The leaves of gymnosperms such as cycads and conifers lend themselves to the same treatment.

\section{METHODS OF STAINING}

The thin sections prepared by the methods above indicated are frequently so tenuous as to present insufficient detail on microscopic examination. This inconvenience is ordinarily overcome by the use of stains to bring out contrasts in structure. The subject of staining has been so recently and admirably discussed for American students in Professor Chamberlain's Methods in Plant Histology (3d ed., The University of Chicago Press, 1915) that it need only be elucidated in the present connection in regard to the special conditions prescnted by the hard tissues in plants.

A few stains give the best results in most anatomical investigations, since the protoplasmic structures are of less importance and the cellulosic and lignified conditions of the cell wall are of the greatest significance. In most instances staining with hematoxylin and counterstaining with the anilin dye known as safranin give the best results. In the case of hematoxylin the Haidenhain method on the whole answers best. The sections are washed in distilled water and then immersed in a 3 per cent solution of ammonia-iron alum. The alum should be bluish in color and free from efflorescence. Ten to fifteen minutes in the alum solution are sufficient. Careful washing in distilled water follows. The first change of washing water should remain only a short time and is followed by a second and a third change, so as to remove all alum which has not become fixed in the sections. In material containing a great deal of tannin special precautions must be taken to insure thorough washing by repeated changes of distilled water. When the sections are cleared of excess of alum, they are ready for the hematoxylin solution, which in some cases is employed in 
the strength of one-half of I per cent in distilled water. In some instances this is too great a concentration and leads to overstaining. This may be remedied by the transfer of the sections to distilled water with a few drops of iron-alum solution in which bleaching slowly takes place. When a sufficient degree of decoloration has been attained, the sections must be washed in repeated changes of distilled water as in the first procedure, otherwise the blue coloration will fade in a short time. A better method is to stain more gradually, using only a few drops of the solution of hematoxylin described above. Slow staining in general gives better results than rapid. The blue-tinted sections when properly colored are transferred to distilled water and thence to distilled water to which a drop or two of safranin has been added. This stain is prepared by adding equal parts of water-soluble and alcohol-soluble safranin to strong alcohol until a saturated solution has been reached. The coloration with safranin is best carried on slowly and in dilute solution, as in this way both clear detail and strong contrast are best secured. Very often the best results follow from leaving the sections overnight in the dilute safranin. In this instance, however, precaution should be taken to wash the sections very carefully and repeatedly after the use of iron alum and hematoxylin, as otherwise disagreeable precipitates are likely to make their appearance.

Some plant tissues and remains show to best advantage without staining. This is, for example, frequently true of darkly colored heartwood and of fossil plants and coal. In the case of the two latter it is often expedient to reduce the color by bleaching agents. The most convenient method in this connection is supplied by the use of chlorine water. Hydrogen peroxide, so often recommended for bleaching vegetable tissues, is of no value in the case of carbonized and fossil plant remains. When the dark coloration is very intense, a more vigorous bleaching action results from the use of nascent chlorine, which can be conveniently secured by dissolving a few crystals of a chlorate in distilled water, with the subsequent addition of a small quantity of nitric acid. This combination gives sufficiently vigorous bleaching action without accompanying maceration. 


\section{METHODS OF MOUNTING}

In the case of the great mass of vegetable tissues it is frequently an advantage to bring about the sharpest possible accentuation of the color contrasts resulting from staining by mounting in media of high refractive index. For this purpose the sections must be usually entirely freed from water. This end is effected by means of absolute alcohol. The sections are transferred on a sectionlifter from the solution of safranin to a watch-glass of absolute alcohol, excess of fluid having been removed by touching the lifter to a piece of blotter or filter paper. From the first absolute alcohol they are lifted to a second, which effects the final removal of water. Too long immersion in the first absolute alcohol is likely to extract too much of the safranin stain, but a longer stay is advantageous if the sections have received an excessive coloration in red. In case the sections have been cut from material infiltrated with nitrocellulose, the latter may often be retained with advantage. This object is attained by adding a little chloroform to the two changes of absolute alcohol, this procedure having the effect of preventing the softening of the nitrocellulose. Should it appear desirable to remove the infiltrating substance completely, the sections are transferred to ether after the second absolute alcohol. In any case, after dehydration is effected in any of the ways described, the sections are finally cleared with a reagent of high refractive index. The most convenient of these for vegetable preparations is chemically pure and anhydrous benzene or benzole. After clarification the preparations are ready for mounting. This is effected in hard Canada balsam, dissolved in whatever agent has been used to clear the sections. Benzole, xylol, chloroform, etc., are employed for this purpose.

The solution of balsam should not be too dilute, as the evaporation of the solvent may under this condition result in the disastrous desiccation of the sections. Even where the section is not actually left bare by the drying out of the mounting balsam, the access of air is likely to promote a rapid fading of the colors. It is usually necessary to flatten the sections because the balsam sets by the evaporation of the solvent. This procedure is essential 
in cases where the structures present are to be reproduced by photographic methods. After the preparations have dried for a day or two in a horizontal position at laboratory temperature, they are weighted with lead in the form of rolls. For photomicrographic purposes the final degree of flattening is secured by the pressure of clip clothespins which are prevented from injuring the thin covers by the interposition of thin slices of cork. In the processes of flattening described above, the preparations are subjected to continually increasing heat. This is conveniently secured by laying the slides upon a board on top of a steam radiator. The end of the board nearer the supply pipe of the radiator is naturally much hotter than that nearer the return. The final flattening and setting of the sections is best carried on in the interior of the paraffin bath, which is an adjunct of every botanical laboratory. It is unnecessary, of course, to resort to such extreme measures to secure flatness unless the preparation is to be reproduced by photomicrographic methods.

In a number of instances, especially when the sculpture of the cell wall in elements of the wood is a particular feature of interest, mounting in Canada balsam brings about too great a degree of homogeneity. This inconvenience can be avoided by using other media for mounting. Gum dammar dissolved in chloroform supplies a mountant which presents many of the advantages of Canada balsam without the extreme degree of clarification which makes that reagent in some cases undesirable. Glycerin jelly is an even better mounting medium, but has the disadvantage of tending to extract the safranin. This can be largely overcome, however, by transferring the sections after staining to glycerin containing a large amount of safranin. Under these circumstances the safranin is not extracted from the sections. In mounting, the sections are placed upon the slide and warmed over a burner to make the glycerin quite fluid. This is then allowed to drain off as much as possible, and any remaining trace is removed by wiping round the section with a piece of clean cotton fabric. A drop of melted glycerin jelly is then applied and the cover is placed in position. Weighting is advisable during setting and cooling of the jelly to secure flattening of the sections. 
METHODS OF SECTIONING COAL

This mineral is now universally recognized to be of vegetable origin. Its practical importance and the interesting remains which it contains make it an object not without interest both from the general botanical and from the anatomical standpoints. All except the most highly carbonized coals may be prepared for sectioning on the microtome without great difficulty in accordance with the summary method here described. The mineral is split in conformity to the layers by means of a stout knife and a hammer. The thin slabs thus secured are broken transversely by the aid of pliers or a chisel into pieces which vary in size according to the resistance of the coal. The fragments are put into melted phenol or carbolic acid and kept in corked bottles in the warm bath for about a week. They are then transferred after washing in warm water to strong hydrofluoric acid for a second week. Except in the case of certain lignitic coals further treatment with phenol after washing with water is necessary. This is followed by a second immersion in hydrofluoric acid and subsequent washing. After a third return to phenol (if this is necessary) the material is washed in water and then run up into absolute alcohol which must be changed two or three times During the process of dehydration the coal is kept in or on the paraffin bath. Imbedding in nitrocellulose follows. In this process only 2, 4 and 6 per cent solutions of celloidin are used, and the last of these is followed at once by nitrocellulose of 16 per cent strength. It is an advantage in imbedding coal, not only to pump all air out of its substance, but also, when in the 6 per cent celloidin, to subject to a positive pressure of between two and three hundred pounds. This is easily effected by inclosing in a metal cylinder and raising the internal pressure by means of an automobile pump. After a rapid course of thickening rendered possible by the resistant character of the material, the pieces are dropped into chloroform in the usual manner and later transferred to glycerin and strong alcohol. In sectioning, the fragments of coal are clamped securely in the wood-carrier of the microtome and cut with a transversely placed knife. Only sections which are five micra or less in thickness are of value in the case of this extremely opaque material. The 
utilization of botanical technique in the study of coal has apparently made it necessary to revise the accepted views as to the origin of this invaluable mineral. The hypothesis of the derivation of coals from peat formed in place, as in the northern bogs of our epoch, must be given up in favor of the conception of an accumulation in open water by transport either aërial or aquatic. Coal from its internal organization obviously is comparable to the muck in the bottoms of modern lakes rather than to the surface accumulations of peat which often surround them. The technique of mounting sections of coal is the same as for imbedded material in which the matrix of nitrocellulose is retained. No staining is necessary.

\section{PHOTOMICROGRAPHIC METHODS}

Photography with the microscope has become in recent years an extremely important aid in microscopical investigations of the structure of plants. To such perfection have photographic lenses and photographic plates attained that it is possible to reproduce photographically most of the structural features of tissues and cells in plants. The advantage of photomicrographic reproduction is that it is at once less laborious and more accurate than representation by drawings.

The lenses used for photomicrographic purposes do not differ, except in special cases, from those employed in general microscopic investigation. For very low magnifications such as are ordinarily not available with the compound microscope special lenses of great perfection are now manufactured by the leading makers of microscopical appliances. These are used without eyepieces and can be applied as a rule only to special photomicrographic stands. For moderate and higher magnifications, however, the same optical outfit is used as for observation with the eye. The better grades of lenses naturally give better photographic results than those which are of inferior quality. In the case of very high magnifications special condensers, insuring a great degree of concentration of light and freedom from chromatic aberration, are employed fcr illumination.

Microscopic lenses have certain general defects which militate against their employment for photographic purposes. The most 
serious of these is the discrepancy between the chemical and visual focus. As a result of this shortcoming, a picture sharply focused to the eye appears dull and indistinct upon the photographic plate. In the case of apochromatic objectives and compensation oculars this optical defect is less apparent than in ordinary so-called achromatic lenses and Huygenian eyepieces. The lack of correspondence of visual and chemical foci becomes practically negligible where certain color screens are used in securing the photographic image. In some instances, particularly in the photography of unstained material, lenses of rock crystal are of value, since they shut out less actinic light than do lenses of glass. The great cost, however, of lenses of this construction renders them unavailable for most laboratories, and in any case their value is by no means proportionate to their price.

Since microscopic sections are generally colored either naturally or by means of special stains, photographic plates which are sensitive to color are necessary in photomicrography. There are various types of such plates which are available for different purposes. It has been found that certain chemical substances, particularly anilin dyes, possess the valuable property of greatly increasing the sensitiveness of the photographic plate to colors. The photographic negative is ordinarily produced on a gelatin surface mounted on glass. The gelatin covering is known as the emulsion and contains bromide of silver precipitated in its substance. The silver bromide is rendered more or less sensitive to light by boiling in the presence of ammonia, on the one hand, or by adding an excess of bromide of potash, on the other. It is most sensitive to the radiations of the violet and ultra-violet region of the spectrum. Consequently the common photographic plate will not give good results in the case of sections stained with red, green, or even very dark blue dyes. This original defect of the photographic plate has been almost entirely removed in recent years by the use of anilin sensitizers. For example, erythrosin possesses the property of rendering plates soaked in a weak solution sensitive to greens. Plates which are so treated are ordinarily known as isochromatic. The appellation is, however, obviously a misnomer, since they are only in a very slight degree, if at all, sensitive 
to reds. The most valuable type of plate for general photomicrographic purposes is that treated with a blue anilin dye, isocyanin. This treatment makes the emulsion sensitive to the red end of the spectrum to a very large degree. Such plates are called "panchromatic" or "spectrum" plates and are of the greatest value in the photomicrographic reproduction of extremely difficult microscopic objects, such as coal, highly tanniferous stems, roots, etc.

Further aids to the microscope in the objective reproduction of microscopic images are the so-called color screens. These are of various hues, but need not be considered in detail, since only a few of them are practically useful for photomicrographic purposes. When a yellow screen is employed, for example, it shuts off to a large extent the violet and ultra-violet radiations and as a consequence gives the photographic plate greater efficiency in the reproduction of colors other than light blue. A further extremely valuable result following the use of a yellow screen is the elimination of the contrast between chemical and visual foci mentioned above as a disadvantageous feature distinguishing microscopic lenses from those employed in ordinary photographic cameras. This is a property of the utmost practical importance and makes available lenses which would otherwise be quite useless. The general effect of a yellow screen, then, is to make a plate relatively more sensitive to radiations other than the violet ones and at the same time to favor a more brilliant microscopic image as a result of the elimination of the ordinary chemical or actinic rays. A green screen is in certain cases highly useful, but the numerous other hues which distinguish screens made for the appropriate rendering of color are in general of less value in the practice of photomicrography.

The photomicrographic apparatus may advantageously consist of an ordinary camera with long bellows, supported in a vertical position on a counterpoised sliding back. The back may be fixed at any height by means of window fasteners. The camera is connected with the microscope by a collar which engages with a corresponding collar on the tube of the microscope in such a manner as to break the path of the rays of light. Reflections are prevented by the blackening of the collar rings with matt drop black. 
A shutter which fits on the front of the photographic camera is most convenient, and an arrangement which will admit of exposures of from three seconds to a hundredth part of a second is desirable. The Thornton-Pickard shutter, of English manufacture, but used a great deal in the United States, has proved itself of value in this connection. When a color screen is used, it may be laid horizontally just above the shutter in view of the vertical position of the camera. A special photomicrographic microscope is desirable, although not absolutely necessary, as any ordinary microscope of good size and weight with adequate optical equipment may be employed for photomicrographic work. The microscope is supported on a bench provided with three legs so that it may be always steady. The upright stand to which the camera is attached should likewise have three brass knobs screwed into its base to insure stability. The best source of light is an arc lamp with hand feed. The ordinary projection lantern answers very well for this purpose if it is not too cumbersome. A condenser should be placed in front of the arc to collect the light, and a water chamber should likewise be interposed between the condenser and the microscope to eliminate heat rays. The use of alum in the water chamber to accentuate the exclusion of the heat radiations is unnecessary, and it is inadvisable on account of the evil effects produced on the apparatus by alum. The lantern should be so mounted that it can readily be lowered and raised, and also inclined at any angle. A fixed lantern may be employed, but its utility is much restricted. The carbons are best inclined at an angle of ninety degrees. The light is taken from the end of the positive carbon, which should be in a horizontal position. The current should be direct and not alternating, although the latter may be used with some degree of success. The advantage of the direct current is the freedom from noise and the intense light emanating from the positive carbon. The amperage should be controlled by a rheostat which will permit the passage of from seven or eight to fifteen amperes of current. The high amperages are used in the case of dark-colored or optically opaque objects. In the case of photographs under low magnification a ground-glass screen may often be inserted with advantage between the mirror of the microscope and the water chamber. 
The photographic dark room should be of moderate size and provided if possible with electric lights. In addition to a free light there may be one shaded by a ground-glass cover for examining negatives and exposing lantern slides. Two safe lights for developing are likewise necessary. One of these should be screened with orange and deep-ruby glasses, one behind the other. This light is available for the development of the less sensitive chromatic plates, known as iso-plates, and for all ordinary photographic plates. A second safe light is necessary for use with plates sensitized by isocyanin (so-called spectrum and panchromatic plates), which must ordinarily be developed in total darkness. The Wratten \& Wainwright Company, of Croyden, England, has patented a light which may be used for this purpose, and this is on sale in the better photographic supply houses in the larger American cities. It is indispensable for all highly color-sensitive plates. A sink of good length and with two taps should be provided. To the left there should be a gently sloping draining-board with shelves beneath and above for developing- and fixing-trays of hard rubber, as well as developers and glassware. Ventilation of the dark room is desirable, and is absolutely essential in warm weather. It is best effected by means of an ordinary electric fan placed near the door of the room in such a position as to cause strong currents into and out of the dark room. It may, of course, be set in a special light-tight chamber communicating with the outside, but this fixed position considerably restricts its usefulness.

The plates employed for making negatives depend on the particular needs in special cases. For very exacting work in which the object is either extremely dark-colored or opaque the use of panchromatic or spectrum plates is indicated. In case the contrasts are very slight, and particularly in weakly stained material or objects which present only a slight natural coloration, the socalled iso-process plate is valuable. For the mass of colored preparations the ordinary isochromatic plate answers every purpose. A common need in connection with teaching is the copying of illustrations. When these consist of line or half-tone engravings, process plates giving a high degree of contrast are employed. It was formerly necessary to import color-sensitive plates from 
Europe, but these are now made in good variety and of excellen quality by the Cramer Dry Plate Company, of St. Louis, Missour All the types of plates described above may be purchased from th Cramer Company. Since ordinary photographic supply house often do not carry in stock the special plates needed for photc micrographic and other scientific purposes, the Cramer Compan undertakes to furnish these direct from its factory in St. Louis.

The making of lantern slides is an activity which may often b pursued profitably in larger botanical establishments in unive sities. The most convenient method of making such slides is $b$ placing the lantern plate directly behind a negative of suitab! size, namely, $3 \frac{1}{4}$ by $4 \frac{1}{4}$ inches. A few seconds' exposure to a ligh sheltered by ground glass in the dark room (the time dependin on the character of the negative) suffices. Development of lar tern slides is effected by means of a special developer to be mer tioned in a subsequent paragraph. The slides must be covere with a mask which can be made by cutting out black paper $b$ means of a wheel paper-cutter and appropriate metal forms, obtai able at photographic supply stores. Masks may also be purchase with the various forms and sizes of opening necessitated by th different kinds of pictures. After the mask is applied the lanter slide is protected by a thin cover-glass held in position by blac paper binding strips which may be procured in any photograph establishment. American and Continental European lantern slid are $3^{\frac{1}{4}}$ by 4 inches in dimension. The English slide is $3^{\frac{1}{4}}$ inch square. The storing of lantern slides is always a problem whe their number becomes large, and many filing cabinets for this pu pose are on the market. The essential thing, however, is to hav numbers on the slides and a catalogue, so that they can readil be selected for use and easily returned to their places. The con plexity of the catalogue will of course depend upon the tastes an needs of the individual and the extent of his budget.

The development of the various plates described in the for going paragraph is effected by reagents which reduce to metall silver the parts of the plate exposed to the light. Developers a legion, but two or three seem to answer every practical purpos For making negatives pyrogallol developer is on the whole mo 
advantageous. This is made by dissolving one ounce of Mallinckrodt or Schering pyrogallol in 150 cubic centimeters of tap water, acidulated beforehand with 20 drops of nitric acid. This solution should be kept in the dark room and is known as "stock pyro." A second solution is made by adding to tap water ro per cent of carbonate of soda and ro per cent of sulphite of soda, together with a quarter of I per cent of bromide of potash. In this solution the carbonate of soda is used (with the pyrogallol) for the purpose of reducing the silver in the exposed plate. The sulphite of soda prevents staining, and the bromide of potash restrains a too rapid development and insures a clear image. To make the developing solution, dilute one volume of the "stock pyro" with nine parts of water. This is solution No. I. Solution No. II is the one described above containing carbonate, sulphite, and bromide. Of No. I and No. II take equal parts. The developer should be poured quickly and evenly over the plate and air bubbles should be avoided. The time of development depends upon the nature of the plate, but ordinarily the image should begin to appear in from thirty to sixty seconds. Development is continued until the image begins to disappear. Lantern slides and bromide prints are best developed with metol, which is an organic developer of German origin used almost exclusively in the manufacture of moving-picture films. Its employment in this connection is sufficient proof of its value. The developer is made by adding together in the proportion of one to three the solutions described below under the denominations A and B. Solution A is made by adding Io per cent of caustic soda or caustic potash to distilled water. Solution B consists of Io per cent sulphite of soda, I per cent metol (Metol-Hauff), and a quarter of I per cent bromide of potash in distilled water. After the two solutions are mixed they may be diluted with advantage by adding a third- to a half-volume of tap water. Development of lantern plates and bromide paper is ordinarily complete in from fifty to sixty seconds. The lantern plates are best exposed in the dark room, while it is often advantageous from the standpoint of time-saving to expose printing papers to daylight. Velox papers, and in particular Glossy Velox (ordinary or special depending on the vigor of the negative), may 
be used for making prints for reproduction in scientific journals. The smooth, glossy surface renders this type of paper more suitable for purposes of reproduction than the more artistic papers with matt or rough surfaces.

Photomicrography is an art which can be learned only in part from books, and the beginner is advised either to visit some laboratory where it is successfully carried on, or, failing that, to seek direction from a professional photographer, having regard in that case to the special conditions involved in photomicrographic technique. The account given above is from limitation of space necessarily incomplete and has been written only to supply some answer to numerous inquiries as to the methods pursued in the laboratories of plant morphology in Harvard University. 

INDEX 



\section{INDEX}

A

Abies, root of, 135, 149, 334

Abieteae, 334

Abietineae, 325 ; antiquity of, 325 ; bars of Sanio in, 326 ; cone axis of, 328 ; relation of, to Ginkgoales, 337; relation of, to other Coniferales, 334; resin canals of, 334; wood of, 326

Adiantum, stem of, 279

Agathis: leaf trace of, 324; seedling of, 323; wood of, 319; A. Bidwillii, 330, 353

Air spaces, 3

Alburnum or sap wood, 55, 56, 57

Alcohol, 445

Alnus: leaf of, 212; rays in, 153,177

Amphivasal bundles, distribution of, in monocotyledons, 4I4

Anatomical technique, 444

Angiosperms: general characters of, 373; reproductive structures of, 374 ; vessels of, 95 ; wood of, 373

Annual rings, 15, 16, 42 I

Annulus in Pteridophyta, 216

Araucaria Bidwillii, 323

Araucariineae, 318 ; alternating pitting in, 319; bars of Sanio in, 323; persistent leaf trace of, 324; wood of, 318 Araucariopitys, 353

Araucarioxylon, 349; pitting of, 320; structure of, 320

Archaeocalamitaceae, 266

Archigymnospermae, 292, 305

Arctium, distribution of oil canals in, 437

Arrangement of bundles: in Equisetales, 273 ; in monocotyledons, 192

Aster: oil canals of, 434; resemblance of stem to that of the oak, 406; root of, 434; stem of, 405

Astromyelon, 276

Avena, bundle of, $4 \mathrm{II}$

Barberry, substitute fibers of, 34

Bars of Sanio, 68; diagnostic value of, in Coniferales, 323
Bast fibers, II 8

Bast, hard and soft, II8

Bennettitales, 303; bisporangiate cone of, 303; course of leaf traces in, 301

Betula, wood rays of, 155

Bleaching, 446

Bocoa provacensis, tracheids of, 33

Brachyoxylon, wood of, 320 ; wound reactions of, 32I, 329

Bundle in monocotyledons, 194, 195

C

Calamitaceae, $25 \mathrm{I}$; course of bundles in, 273

Calamites, 266; pith casts of, 269; primary wood of, 266, 268; secondary wood in, 268; young stem in, 266

Callus: definitive, 123; in phloem, 110, II4; seasonal, I 23

Cambial activity in monocotyledons, 195, 4II

Camera, photomicrographic, 464

Canada balsam, 459

Carbonized material, bleaching of, 446

Casuarina: leafy twig of, 211 ; rays of, $77,82,404$; C. equiselifolia, rays of, 78,88 , 90; C. Fraseri, rays of, 77,86 ; C. torulosa, rays of, 77 ; systematic position of, 384 ; transfusion tissue of, 384

Cedrus, resin canals of, 74,335

Cedroxylon, structure of, 349

Cell, I-5

Celloidin, 449

Centripetal wood in Equisetum, 274

Chalazogamy in Amentiferae, 230

Chamaecyparis, marginal tracheids of, 72

Chicory, degenerate oil canals in root of, $44^{2}$

Chlorates, $446,45^{8}$

Chlorine, $45^{8}$

Chloroplastids, 2

Chromic acid, 445, 446

Cirsium, oil canals of, 439

Clematis, wood rays of, 179 , I9I 
Climate in relation to evolution of plants, 417,423

Coal, sectioning of, $46 \mathrm{r}$

Collateral protostele, 165

Collateral siphonostele, 165

Collodion, 449

Color screens, 464

Color-sensitive plates, 463

Companion cells in angiosperms, 118

Comparative anatomy, canons of, 234

Compositae: illustrative of general principles of anatomy, 433; organization of stem in, $400,402,404$

Compound ray, origin of, 82,181

Concentric bundles in monocotyledons, 195

Concentric protostele, 162

Concentric siphonostele, 163

Coniferales, 317; genealogical tree of, 355 ; subtribes of, 355

Conifers, phylogeny of, 355

Conservative organs, 238 ; leaf, 238 ; reproductive axis, 238 ; root, 240

Cordaitales, 305; leaf in, 203, 309; rays of, 66; stem in, 306; transfusion tissue of, 208,308 ; transition region in, 309 ; wood of, $15,28,66,305,308,419$

Corrosive sublimate, 445

Cortex, 10

Crystallogenous cells, II 8

Cupressineae, 339; marginal tracheids of, 34I

Cupressinoxylon, 349

Cuticle, 128

Cycadales, 297; centripetal wood of leaf, 300 , of cone axis, 299; supernumerary zones in, 298

Cycadofilicales, 292

Cycas: cortical bundles in, 20r; leaf bundle of, 199; leaflet of, 200

Cynara Scolymus, distribution of oil canals in, 436, 439, 443

Cystoliths, 127

\section{D}

Dammar, 460

Dandelion, root of, 435

Depressed segments: in Aster, 405; in Clematis, 179; in Quercus, 180

Developing reagents, 468

Diagram of distribution of oil canals in artichoke, 443
Dicotyledons: characteristics of, 375 ; degenerate vessels of, 373,380 ; modifications of tracheids in, $38 \mathrm{r}$; parenchymatous elements of, $38 \mathrm{r}$; phylogeny of, 384 ; wood rays of, 382

Diffuse ray, origin of, 82,89

Distribution of amphivasal bundles in monocotyledons, 41 2

Duramen or heartwood, $56,57,5^{8}$

E

Ectokinetic sporangium, 2I6

Electrical current for photomicrography, 465

Endarch, 23, 167

Endodermis, Io, I I, I 2

Endokinetic sporangium, 217

Ephedra, 357; rays of, 174,358 ; stem of, 173,358 ; tracheids of, $3 \mathrm{r}$; wood of, 94

Epidermis, I26; multiple, I27; water tissue, 127

Equisetaceae, 269; course of bundles in, 273

Equisetales, 250, 264; stem of, 272

Equisetum, 269; bundles of, 270; cone of, 274 ; leaf trace of, 274 ; medulla of, 272 ; primary wood of, 270 ; root of, 275 ; stem of, 269; types of stem in, $27 \mathrm{I}$

Erianthus, bundle of, 195

Ersatzfasern, 34

Exarch, 23, 167

\section{F}

Fagus, tracheids of, 32

Ferns, 277; root of, 150 ; stem of, 278

Fiber tracheids, $3 \mathrm{I}$; tylosis of, 106

Fibers: libriform origin of, 32 ; mucilaginous, 33 ; septate, 34 ; substitute, 34

Filicales, 277; leaf of, 280 ; stem of, 278 ; root of, 20,150

Fixing reagents, 445

Fundamental tissues, ${ }_{132}$

Fusiform rays: in Cedrus, 74; in Pinus, etc., 69

Geinitzia, 355

Ginkgo: absence of parenchyma in, 424 ; alternate pitting in, $3 \mathrm{I}_{2}$; leaf of, $3 \mathrm{I}_{3}$; opposite pitting in, $3 \mathrm{II}$; secondary wood of, $3 \mathrm{Ir}$; sporangia and sporophylls of, $3 \mathrm{I} 4$; tangential pitting of, 423

Ginkgoales, 3ro; relation of, to Abietineae, 337 
Gleichenia, stem of, 278

Gnetales, 357; and Bennettitales, 370; parenchyma in, $35^{8}$; vessels in, $35^{8}$; vine habit in, 364

Gnetum, 363 ; stem of, 363 ; vessels of, 367

Grape vine, septate fibers of, 34

Grinding wheels, 453

Guard cells, I29; number of, I3r

$\mathrm{H}$

Hairs, I30

Hard tissues, preparation of, for sectioning, 447

Heartwood: condition of parenchyma in, 56,57 ; heartwood or duramen, 55

Helianthus: compared with Casuarina, 404; diagram to illustrate herbaceous type, $403 ; H$. hirsutus, ray situation in, $400,402,404 ; H$. tuberosus, oil canals of, 434

Hematoxylin, 457

Herbaceous and arboreal types in relation to climatic conditions, 430

Herbaceous dicotyledons, 186

Herbaceous type: geographical distribution of, 430; in geological time, 431; origin of, in cryptogams, 387 , in the dicotyledons, 186, 387 , in Helianthus, $400,402,404$, in Potentilla, 187, in Salvia, I89, in Urtica, 397

Herbs, substitute fibers of, 35

Heterangium, stem of, 293

Hones, types of, 453

Hydrofluoric acid, uses of, 447

Hypoderma, I 28

I

Imbedding in nitrocellulose, $45^{\circ}$

Inclusion of pith: within leaf traces, 285 ; within stele of stem, 284

Intercellular spaces, 2

Iodine, uses of, 446

Iris, pericarp of, 2

Iron alum, 457

Isoetes, 255

K

Kinds of plates useful for photomicrography, 463, 466

Knives, 453; position of, 453

L

Labiates, origin of herbaceous type in, 189

Lamium, origin of herbaceous type in, 190
Lantern slides, 467

Larch, root of, 22, 145

Large storage rays in relation to climatic conditions, 430

Laticiferous system in the Compositae, 435

Lattices, 122

Leaf: centripetal wood of, in Prepinus, 208; concentric bundles of, in Cycas, 201, 302; cortical bundles of, in Cycas, 201, 302; of Cycadales, 199, 300; definition of, 14I; gap of, in Casuarina, $8 \mathrm{I}$, in conifers, 80 , in Cordaitales, 203 ; organization of, in $A l m u s, 2 \mathrm{r}_{3}$, in Casuarina, 211 , in dicotyledons, 213 , in Gnetales, 211 , in monocotyledons, 213, in Pinus, 205, 330, in Prepinus, 207, 33I; rachis of Cycadales, r99; transfusion tissue in Casuarina, $3 \mathrm{II}$, in Cordaitales, 203, in Pimus, 206, in Prepinus, 207; vegetative, 199

Leea, organization of stem in, 184, 389, 396 ; origin of woody cylinder of, 184 , 389

Leguminosae: fibers and tracheids of, 33; origin of herbaceous type in, I9I

Lenses, photomicrographic, 462

Lepidocarpon, 224

Lepidodendraceae, 250, 255; secondary wood of, 16,260

Lepidodendron, 256; primary wood of, I 70 ; rays in, 258 ; septate tracheids of, 38

Libriform fibers, 32

Lights for photomicrographic purposes, 466

Ligulifloreae, laticiferous system of, 435

Liquidambar, fibers of, Io6

Liriodendron, parenchyma of, 51; vessels of, 97,100

Lycopodiales, 250, 252

Lycopodium, 19, 252; root and stem of, 143

Lycopods, wood of, 252

Lycopsida, geological range of, 244 ; microphylly in, 245

Lyginodendron, 296

M

Maceration, 446

Magnolia, fiber tracheids of, 32; parenchyma of, $5^{1}$

Marginal tracheids: of Cupressineae, 73, 34I; of Taxodineae, 72, 339 
Medulla: cortical origin of, 283 ; in Osmundaceae, 286; origin of, in the Filicales, 284, in Lycopsida, 283

Medullary rays: so called in gymnosperms, 172 ; in Lycopsida, 170

Medullosa, 294; origin of cycads from, 295

Megasporangium: in Pteridophyta, 223; in Selaginella, 216

Mesarch, 23, 167

Mesophyll, 213

Metagymnospermae, 317, 357

Metaxylem, 19

Methods of sectioning coal, $46 \mathrm{I}$

Miadesmia, 225

Microscope in photomicrography, 465

Microscopic lenses, defects of, 462

Microsporangium: in Conifers, 219; in Cycadales, 216; in Ginkgoales, 218 ; in Gnetales, 219; in liverworts, 214 ; in Lycopsida, 216; in phanerogams, 220; in Pteropsida, 216

Microtome, 452

Middle lamella, 3, 445

Monocotyledons, 409; amphivasal bundles of, I96; arrangement of bundles In, 192; bundle of, 193; cambial activity in, 195, 4II; characteristics of, 377; geological age of, 409; leaf structure in, 213, 410; phylogeny of, 412 ; stem of, I92, $4 \mathrm{II}$; root of, I58, $4 \mathrm{IO}$

Mounting of sections, 459

Mucilaginous fibers, 33

Mycorrhiza, 138

Nitrocellulose, 449

Nothofagus, parenchyma of, 53

Nucleus, 2

Oak, wood of, 14, 30, 55, 57

Objects, clamping of, 455

Oil canals of Compositae, 433, 436

Onopordon, canals of, 439

Organs, definitions of, 136

Origin of the herbaceous type: in Helianthus, 400, 402, 404; in Potentilla, 187; in Salvia, 189; in Solanum, 190; in Urtica; 398

Origin of the woody cylinder in Vitis, 39r, 395

Osmunda cinnamomea, 287, 289; O. claytoniana, 289; O. regalis, 288
Osmundaceae: internal phloem in, 287; medulla in, 286; root of, 20,150

Osmundites skidegatensis, 286

\section{$\mathrm{P}$}

Palms, anatomy of, 196,413

Parenchyma. 37; absence of, in secondary woods of the Paleozoic, 40, in Pinus, $4 \mathrm{I}$; diffuse in conifers, 48 , in dicotyledons, 50; distribution of, in Abies and $T$ suga, 47, in dicotyledons, 5०, 52, in primary wood, $37,38,39$; origin of, at end of annual ring, 42, 425; origin of, in Alder, 54, in dicotyledons, 54, in Liquidambar, 54, in Mesozoic, 40, in Picea, 42, 425, in primary wood, 37 , 39, in Prumus, 54, in secondary wood, $42,54,425$; origin of, from tracheids, $38,42,425$; relation of, to annual ring, 45,425 , to climatic evolution, 4I, 425; terminal in dicotyledons, 52 ; transition to, from short tracheids, 43; vasicentric in dicotyledons, 52

Parichni, 26r

Peach stone, 4

Pericycle in Pteris, 108

Phloem: central ray cells in, II3, II5; companion cells of, in angiosperms, Ir8; in Pinus, 1ro; in Pteris, 108; in Tilia, II7; marginal cells in, II3; parenchyma in, II4; rays in, II4; sieve tubes in, Ir4; in Vitis, I 22

Phloeoterma, Io, I2

Photographic dark room, 466

Photographic plates, 463,467

Photomicrography, 462

Phylloglossum, 252

Picea: stem of, 85 ; terminal parenchyma of, 336,425 ; wood of, 42, 44, 425

Picric acid, 445

Pileorhiza, 138

Piliferous layer, I46, I50, 158

Pineae, 335

Pinus: absence of parenchyma in, 336; aggregate rays in, 362; leaf of, II, 33०; needle of, II; phloem in, IIO; short shoots of, 337; structure of wood in cone of, 326 ; tracheids of, 26; tyloses of, 106; wood of, 24, 326

Pinus and Ginkgo: microspores of, 337; short shoots of, 337 ; tracheids of, 338

Pinus Strobus, rays in phloem, 110

Pit membrane, 3, 6

Pith, origin of, in the Filicales, 283 
Pits: absence of tangential, in Cordaitales and other ancient gymnosperms, 27; bordered, 6, 7; condition of, in heartwood, 56 ; half bordered, 7 ; radial, 27 ; simple, $3,5,6,7$; tangential, 27

Pitting, distribution of, 27,428

Pityoxylon, structure of, 349

Plastid, 2

Plates, photographic, 463,466

Podocarpineae, 343

Pollen chamber, 226, 227

Polydesmy in Cycadales and Gnetales, 364

Porous perforation in vessels, origin of, ror

Potentilla, origin of herbaceous type in, 187

Prepinus: leaf of, 207, 331; relation of, to Cordaitales, 332

Preservation of material, 444

Primary wood, degeneracy of, 169

Principes, anatomy of, 196,413

Proangiosperms, 303

Protocalamites, 268

Protoplasm, I

Protostele, 162,278 ; collateral, 165 ; concentric, 162 ; in Filicales, 278

Protoxylem, 18, 19, 20, 2I

Psilotum, 253

Pteris: bundle of, 21, 108; development of stem in, 280, 282; phloem of, to9; rhizome of, 9, 10, 132; vessels of, 92

Pteropsida, 244, 247; geological range of, 249; megaphylly of, 249

Quercus: fiber tracheids of, 31 ; stem of, I79, 404; tracheids of, $3 \mathrm{I}$; vessels of, 99; wood of, 15; wood rays of, 76, I $78,18 \mathrm{r}$.

\section{$\mathrm{R}$}

Ranales, characteristics of, 386

Rays: aggregate, 78,82 ; compound, 78 , 82; fusiform, 69, 74; linear, 69; marginal cells of, 69; medullary, so called, 61 ; origin of, in Coniferales, 68 , 72 , in Lepidodendron, 62, 63, 64; relation of, to resin canals, 70 ; tracheids of, in Lepidodendron, 62, 64; types of, in Casuarina, 77; uniseriate, 67, 69; width of, in Cordaitales, 66, in Cycadales, 75 , in Cycadofilicales, 65

Recapitulation: in Coniferales, 235; in first annual ring, 237; in oaks, 235; in Phyllocladus, 235

Resin canals, relation of, to resin cells, 343
Reversion, 72, 73, 74, 75, 241

Rhizophore, 262

Rhodotypus, fibers and tracheids of, 33

Robinia Psendacacia, mucilaginous fibers of, 34

Root: absence of medulla in, 173; definition of, 137; development of, 145; exarch primary structure of, I45; exodermis of, 158 ; in Coniferales, 145 ; in dicotyledons, $15 \mathrm{I}$; in herbaceous dicotyledons, 156; in Lycopodium, I43; in monocotyledons, 159; in Osmunda, 150; piliferous layer of, ${ }^{58}$; primary and secondary structure of, 138 ; primary wood of, $145,150,151,157$, 159; radial organization of, I43; rays in, 147; spiral tracheids in, 161; velamen of, 159

Root cap or pileorhiza, 138

Root hairs, $146,150,158$

\section{$\mathrm{S}$}

Sachs, erroneous views of, as to origin of stem, I9I, 406

Safe lights, 466

Safranin, $45^{8}$

Salvia, origin of herbaceous type in, 189 Sapwood or alburnum, 55

Scalariform perforation of vessels, origin of, 97

Scolymus, oil canals in root of, $44 \mathrm{I}$

Scorzonera: laticiferous system of, 435; oil canals of, in root, $44 \mathrm{I}$

Secondary wood as indicator of climatic conditions, 418

Sectioning of material, 454

Seed: in angiosperms, chalazogamous, 230; in Archigymnospermae, 226; in Casuarina, 231; in dicotyledons, 232; in monocotyledons, 23I; in Pinus, 233; of Coniferales, 229; of Cycadales, 226; of Ginkgo, 228; of porogamous angiosperms, 230

Seedlike megasporangia, 223, 225

Selaginella, 216, 225, 254

Sequoia: anatomy of, 339; cone axis of, 340; marginal tracheids of, $73,34 \mathrm{I}$; parenchyma of, 339 ; tracheids of, 25 ; wound canals of, 34I

Sharpening of knives, 453

Shutter, photomicrographic, 465

Sieve plates: in the angiosperms, 120 , I23; in gymnosperms, I14; in herbaceous angiosperms, I 25 
Sieve tubes and vessels, comparison of, I 23

Sigillaria, primary wood of, 171, 259

Sigillarians, 259

Siphonostele, 163; collateral, 165; concentric, I63; in Filicales, 278; lacunae of, 164,278 ; reduction of, 165

Smilax, root of, I2, I38; stem of, 192

Solamum, origin of herbaceous type in, 190

Solidago, resemblance of stem of certain species to that of the oak, 405

Sphenophyllaceae, 25I, 264

Sphenophyllum insigne, 265

Sporangia, 142; in Abietineae, 219; in angiosperms, 220; in Coniferales, 219; in Cycadales, 216; in Ginkgo, 217; in Gnetales, 219; in Polypodium, 216; in Selaginella, .216

Sporangia and trichomes, 130

Staining methods, 457

Stangeria, reproductive axis of, 299

Starch: in phloem of Pinus, III; in phloem of Tilia, I1 8

Stem: definition of, I40; development of, in Pteris, 281; herbaceous, I86, 387 ; organization of, in Casuarina, 82, in Cupressus, 80, in dicotyledons, $8 \mathrm{I}$, in monocotyledons, I92, in Selaginella, 254; primary tissues in, 169; secondary tissues in, r69; structure of, in Cyathaceae, 282, in Marattiaceae, 282, in Pteris, 28r.

Stephanospermum, 3I 5

Stigmaria, 262

Stomata, I29

Strops, types of, 454

Substitute fibers, 35

$\mathrm{T}$

Tangential pitting in relation to climatic evolution, 424, 425

Taraxacum, laticiferous system of, 435

Taxineae, 344; parenchyma in, 346; reproductive structures of, 345 ; resin canals in, 346; tracheids of, 345

Taxodineae, 339, marginal tracheids of, 34I; wood parenchyma of, 342

Taxoxylon, structure of, 349

Teak, septate fibers of, 35

Terminal parenchyma, relation of, to climatic evolution, 426

Tissue systems, 8 , I0, I 2, I3
Tissues, 9; epidermal, Io; fibrovascular, Io; fundamental, ro

Torus, 6,68 ; position of, in heartwood, 56

Trabeculae, 68, 250

Tracheids, 25, 27, 29, 31; marginal, in Coniferales, $69,73,34 \mathrm{I}$; reticulated, I7; ringed, I7; scalariform, I7; spiral, 17; spring, 24, 26; summer, 24, 26; tylosis of, 106; types of, 25, 26, 31

Tragopogon, oil canals in root of, $44 \mathrm{I}$

Tree nails, 34

Trias, woods of, $42 \mathrm{I}$

Tsuga: parenchyma of, 46; resin canals in, 346

Tubiflorae, oil canals of, 434

Tyloses: in fibers of Liquidambar, 106; in resin canals of Coniferales, 56; in vessels of dicotyledons, 57

U

Urtica, organization of the stem in, 397

\section{V}

Vacuole, I

Vasicentric parenchyma, 5I

Velamen, $\mathbf{1} 60$

Vessels: absence of, in certain angiosperms, 373, and sieve tubes, I 23; in Ephedra, 94; in Gnetum, 95; in Pteris, 92; in Welwitschia, 95; lateral walls of, I03; scalariform, I03; terminal walls of, 93, 95; tylosis of, 104; types of, $94,95,100$, IOI; with pitted perforation, 94; with porous perforation, 96, 99; with scalariform perforation, 96

Vine type, origin of, $184,390,396,397$

Vitis, organization of stem in, r85, 391; thick and thin annual stem of, 397

Voltzia, 355

\section{W}

Welwitschia, 366; leaf of, 368; stem of, 366; vessels of, 366

Wood: cryptogamic, 222; holders for, 455; primary, I6, I8, 20, 22, 167; tangential pits of, 425

Wood parenchyma: origin of, in Abietineae, 4I, 43; in Araucariineae, 320,322 ; in Podocarpineae, 344 ; in Taxineae, 346; in Taxodineae, 339, 342 Woody dicotyledons, 379

Woody tissues, softening of, 447

\section{$\mathrm{X}$}

Xylem: endarch, 21, 167; exarch, I9, I67; mesarch, 20, I67 

This book is DUE on the last date stamped below
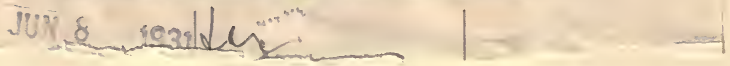
